\title{
Abstracts of the 65th Annual Conference of IACTS, February 2019
}

Impact of the intraoperative use of fibrinogen concentrate for hypofibrinogenemia during aortic surgery

Akihiko Usui, Toshihiko Nishi

\begin{abstract}
:
Hypofibrinogenemia is a major factor for coagulopathy in aortic surgery and prediction of hypofibrinogenemia at the termination of cardiopulmonary bypass (CPB) is essential for preparation of blood transfusion. The effectiveness of fibrinogen concentrate for hypofibrinogenemia also has to be clarified. We examined incidence and risk factor of hypofibrinogenemia and efficacy of fibrinogen concentrate in our experiences. Retrospective survey was performed on consecutive 268 patients undergoing aortic surgery at our institution from 2013 to 2016 . The mean age was $66.0 \pm 13.7$ years. The perioperative serum fibrinogen level (SFL) had routinely been measured. Fibrinogen concentrate was principally used for hypofibrinogenemia (\&lt;150 mg/dl of SFL) at CPB termination. Logistic regression analysis was performed to analyze the risk factor of hypofibrinogenemia. Fibrinogen concentrate were used in 184 patients $(69 \%)$ at doses of $3.2 \pm 3.3 \mathrm{~g}$ (FIB group). We compared the perioperative SFL and the amount of intraoperative blood transfusion between FIB group and non-FIB group $(n=84)$ to evaluate the efficacy of fibrinogen concentrate. The SFL were dramatically decreased with values of $157 \pm 64$ $\mathrm{mg} / \mathrm{dl}$ at CPB termination, compared to the preoperative SFL of 317 $\pm 111 \mathrm{mg} / \mathrm{dl}$. Hypofibrinogenemia was shown in 137 cases $(51 \%)$ at CPB termination. The predictors of hypofibrinogenemia were preoperative SFL (OR, 0.98; 95\% CI, 0.98-0.99), CPB time (OR, 1.005; 95\% CI, 1.002-1.01) and thoracoabdominal aortic surgery (OR, $0.016 ; 95 \% \mathrm{CI}, 1.3-10.9)$. In the FIB group, the intraoperative SFLs $(132 \pm 44 \mathrm{mg} / \mathrm{dl})$ was recovered quickly by the administration of fibrinogen concentrate to $254 \pm 50 \mathrm{mg} / \mathrm{dl}$. There were no significant differences in intraoperative blood transfusion volume between groups regardless of the SFL at the termination of CPB. There were neither significant differences between groups in re-exploration for bleeding, stroke and 30-day mortality. Hypofibrinogenemia frequently was observed at the termination of CPB during aortic surgery. Administering intraoperative fibrinogen concentrate appeared to be a useful option to treat coagulopathy.
\end{abstract}

Risk factors of Reoperative aortic root replacement after cardiac surgery.

Atsushi Shimizu

Daiki Saitoh

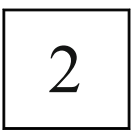

\author{
ABSTRACT: \\ OBJECTIVE: \\ Reoperative aortic root replacement (RARR) after cardiac surgery is high \\ mortality and challenging. Their risk factors are unclear. We evaluated \\ risk factors of RARR. \\ METHOD: \\ Between 2004 and 2017, we performed 109 RARR procedures.
}

The patients' mean age was 64 years (range 24-90 years), and 69\% were men. The main indication for operation was prosthetic valve endocarditis, root dissection and Annulo Aortic Ectasia. Cox proportional hazard regression modeling was performed to identify risk factors that adversely affected operative and overall mortality.

\section{RESULTS:}

The operative mortality was $12 \%$ (13 patients). Mean follow up period was $3.9 \pm 3.5$ years.

The risk factors of operative mortality were cardiogenic shock and Infective endocarditis. The risk factors of overall mortality were cardiogenic shock and 1 degree Ejection Fraction.

\section{CONCLUSION:}

Cardiogenic shock and Infective endocarditis were risk factors of operative mortality. On the other hand, cardiogenic shock and 1 degree Ejection Fraction were risk factors of overall mortality.

But previous root replacement and left heart surgery weren't risk factors both of overall and operative mortality.

Elephant trunk with "Eaves" technique versus Frozen elephant technique for acute type A aortic dissection Keijiro Katayama; Shinya Takahashi; Takanobu

Okazaki; Shohei Morita; Masazumi Watanabe;

Yoshitaka Yamane; Tatsuya Kurosaki; Taijiro Sueda

\section{ABSTRACT: \\ OBJECTIVE:}

This study aims to evaluate the outcome of elephant trunk (ET) technique using "Eaves" technique and frozen elephant trunk technique (FET) for acute type A aortic dissection (AAD).

\section{METHODS:}

Between April 2011 and July 2018, 146 consecutive patients underwent surgery for AAD. 54 patients who underwent total aortic arch repair with ET or FET technique enrolled this study (excluding hemiarch or aortic root repair and case with concomitant cardiac surgery). We compared early outcomes between the ET $(n=29)$ and FET $(n=25)$ groups.

\section{RESULTS:}

There were no significant differences in hospital deaths and stroke between the ET and FET groups (3.5\% [1/29] vs $12.0 \%$ [3/25]; $\mathrm{P}=0.23$ and $10.3 \%$ [3/29] vs $12.0 \%$ [3/25]; $\mathrm{P}=0.84)$. The postoperative spinal cord injury was occurred more frequency in the FET group $(0 \%$ [0/29] vs $16.0 \%[4 / 25] ; \mathrm{P}=0.02)$. The operation time, $\mathrm{CPB}$ time and cardiac arrest time (min) were significant shorter in the ET group compared with FET group (343.5 \pm 88.1 vs $408.8 \pm 84.0$ P\&lt; $0.01,183.6 \pm 41.7$ vs $241.0 \pm$ 84.9 P\&lt;0.01, $93.4 \pm 19.7$ vs $129.0 \pm 37.3$ P\&lt;0.01, respectively). There were no significant differences in additional TEVAR rate between the both groups $(17.2 \%$ [5/29] vs $36.0 \%$ [9/25]; $\mathrm{P}=0.11)$.

\section{CONCLUSIONS:}

The ET with "Eaves" technique for AAD had the potential to lower the morbidity and mortality rate. This technique could be performed single procedure including distal anastomosis, distal aortic stump and insertion of ET. 
Assessment of the arterial stiffness change after endovascular aortic repair

Katsukiyo Kitabayashi; Masayuki Sakaki; Shin Yajima; Akira Marumoto; Takashi Murakami

\section{4}

\section{ABSTRACT:}

BACKGROUND:

Endovascular aortic repair is widely accepted because of the minimal invasiveness compared to conservative open repair for the patients of AAA and TAA. Although the outcome of endovascular repair is excellent, some cases are reported to have the deterioration of exercise tolerance or rapid growth of aortic aneurysm in other part. Elevation of arterial stiffness is supposed to be a cause of such unfavorable outcomes after endovascular aortic repair.

Pulse wave velocity and cardio-ankle vascular index (CAVI) are recognized as parameters of the arterial stiffness. Compared to Pulse wave velocity, CAVI has an advantage, not depends on the systemic arterial pressure.

\section{METHOD:}

From 2015 to 2018, 29 patients undergone TEVAR for TAA and 41 EVAR for AAA. Among them, 12 TEVAR and 20 EVAR cases have the appropriate measurements of pre and post operative CAVI. We examined the change of CAVI after endovascular aortic repair.

\section{RESULT:}

Preoperative CAVI in TAA patients is $9.91 \pm 0.37$ and $10.93 \pm 0.68$ after TEVAR. Preoperative CAVI in AAA patients is $9.97 \pm 0.31$ and $9.60 \pm 0.30$ after EVAR. There is a significant difference between pre and post operative CAVI in TEVAR patients ( $\mathrm{p}=0.027$ ), while no significant difference was found between pre and post operative CAVI in EVAR patients.

\section{CONCLUSION:}

We examined the pre and post operative CAVI in the TEVAR and EVAR patients. CAVI is elevated after TEVAR but not after EVAR. Aortic stiffness is more severely affected by TEVAR, performed at more proximal part of aorta than EVAR.

\author{
Management of Large Fusiform Aneurysm of \\ Distal Aortic Arch with Contained Rupture \\ Using Midline Sternotomy: A Case Report. \\ Jignesh Kothari \\ Devvrat Desai
}

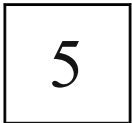

\section{ABSTRACT: \\ INTRODUCTION:}

Aneurysm of distal aortic arch is routinely repaired using left thoracotomy. This approach can be rather unsafe if presentation is with contained rupture in very large aneurysm. Here we are reporting a case of large fusiform aneurysm of distal aortic arch with contained rupture managed successfully using midline sternotomy.

\section{BACKGROUND:}

Fusiform aneurysms of distal arch are very uncommon entity compared to saccular aneurysms. In addition to the presentation with contained rupture is even more rare and lethal. It is very challenging to manage such cases with success.

\section{CASE:}

Our patient, a 54 year gentleman presented with dyspnoea and chest pain, which gradually progressed to NYHA class IV over a period of two to three years. CT Scan revealed $(11 \times 11.5 \times 12 \mathrm{~cm})$ fusiform aneurysm of distal aortic arch extending up to proximal descending thoracic aorta with contained rupture resulting in displacement of trachea towards right \& left main bronchus to inferior aspect with underlying collapsed lung. Elective total arch replacement was done via midline sternotomy under Deep Hypothermic Circulatory Arrest with continuous Selective Antegrade Cerebral Perfusion using right axillary artery and right femoral artery cannulation. The arch was replaced using $28 \mathrm{~mm}$ woven polyester graft impregnated with collagen. He remained stable in post-operative period $\&$ discharged on tenth post-operative day.

CONCLUSION:

Fusiform distal aortic arch aneurysm with contained rupture remains very lethal entity. Time of presentation \&amp; surgery remain the good prognostic factors. Peripheral cannulation \&amp; deep hypothermic circulatory arrest with selective antegrade cerebral perfusion provide good outcome without neurological complications.

A case of right sided aortic arch and mirror image branches with ductal aneurysm.

Aravind P. Raman; Vipin B. Nair

\section{ABSTRACT:}

an elderly female who presented with dysphagia. On evaluation with OGD scopy was found to have a extra luminal pulsatile lesion causing compression. She was evaluated with angiogram and found to have a right sided aortic arch with mirror image branches and an aneurysm distal to right subclavian artery ( kommerell diverticulam/ ductal aneurysm was suspected). She was operated upon using right thoracotomy approach. A dacron graft repair of the aneurysm was done after securing arterial and venous lines.

\section{PRESENTING AS AN ASCENDING AORTIC ANEURYSM}

K.N. Bhosle

Vignesh Ravikumar

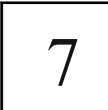

ABSTRACT:

\section{INTRODUCTION:}

Syphilitic aortic aneurysm is a rare occurrence in the antibiotic era, making the diagnose assumption even more infrequent. Nonetheless, this pathology can appear and should be suspected in patients with aortic aneurysm.

\section{CASE REPORT:}

We report a case of a 57-year old patient who presents with cardiovascular syphilis and, in the following study, a large ascending aorta aneurysm is identified. The authors discuss the diagnostic challenge, the epidemiologic concerns, surgical indication and treatment and subsequent follow-up.

\section{TREATMENT:}

The patient was evaluated for the aneurysm and found to be VDRL positive and subsequent confirmatory tests were done to confirm the same. The patient underwent replacement of the ascending aorta with $24 \mathrm{~mm}$ woven Dacron double velour graft without replacement of the aortic valve under cardiopulmonary bypass. Postoperative period was uneventful and patient was discharged on 10th post-operative day.

DISCUSSION:

These days, syphilitic aortic aneurysms are a rare entity, since effective treatments are available for this infectious disease. Only few case reports have been published on this disease in the literature over the last decade. In a study which was done on untreated Syphilis, $10 \%$ of the patients were found to develop cardiovascular Syphilis after an incubation period of 10-30 years [1,2,5], 16\% had gumma formation and $7 \%$ had neurosyphilis. 
Subclavian to carotid transposition for long segment subclavian artery stenosis: a case report

Neerav Bansal; Varun Bansal

\section{ABSTRACT: INTRODUCTION:}

Subclavian artery stenosis can remain asymptomatic or lead to vascular (upper limb claudication) or neurological disorders (vertebro-basilar insufficiency). Subclavian-carotid transposition for the treatment of subclavian artery occlusive disease was first described by Parrot in 1964 . Various other approaches for treatment of this disease have been tried since then, including carotid to subclavian bypass, subclavian-axillary bypass and endovascular stent placement.

\section{CASE REPORT:}

A 34 year old male presented with history of progressive pain in left upper limb and discoloration of distal digits for 2 weeks. Examination revealed mean arterial pressure difference of $20 \mathrm{mmHg}$ between the two upper limbs. CT angiogram revealed long segment critical stenosis of first and second part of left subclavian artery, with reformation in its third part and normal contrast opacification of subsequent limb arteries. He underwent surgical subclavian to carotid artery transposition through a single supraclavicular incision.

\section{CONCLUSION:}

Several studies have compared endovascular stenting versus open surgery for subclavian artery stenosis and have concluded that endoluminal therapy is effective, however open surgery carries better long term durability and should be preferred approach in low risk patients. Surgical repair also prevents late aneurysm formation at stent edges and eliminates the risk of stent thrombosis.

Case report- Rare case of Kommerrell's diverticulum with arch vessel anomaly underwent Hybrid procedure Ravi Shivdasani; Vijit K. Cherian

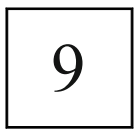

\section{ABSTRACT:}

This gentleman who is 48 years old, a farmer, presented with history of dysphagia and dyspnoea for 3 moths and on evaluation was diagnosed to have Kommerrell's diverticulum, with abscent left radial pulse and feeble right radial pulse. $\mathrm{CT}$ aortogtram with peripheral angiogram revealed first arch branch to be Right CCA, second branch as right SCA and kommerrell's diverticulam giving rise to left CCA and Left SCA.

The pateint was planned for staged Hybrid procedure:

Arch debranching

EVSR

He underwent arch debranching through sternotomy and bilateral neck incisions. Right CCA to Right SCA bypass was performed in neck using $6 \mathrm{~mm}$ interposition graft, a Y graft(12x6) was used to bypass ascending aortoa to right CCA and left CCA. Left SCA owing to its aberrant course was not reachable from either approaches. Post procedure the right upper limb BP was lower than left upper limb BP and hence another $6 \mathrm{~mm}$ interpostion graft was anastomesed between common limb of $\mathrm{Y}$ graft to right SCA after which the difference was eliminataed.

After confirming the neurological status, he was taken up for EVSR of Kommerrell's on next day. Diagnostic angiogram before EVSR showed good flow in Y graft to both carotids and right SCA. A 28x200 mm thoracic endograft was deployed through right femoral artery covering distal ascending aorta to mid DTA and a $12 \mathrm{~mm}$ plug was placed in left SCA origin. Check angiogram revealed total occlusion of kommerrell's with good flow in neck and upper limb vessels

All the peripheral pulses except Left radial were well palpable and patient had though prolonged but uneventful recovery in post operative period. We conclude that aneurysmal aortic diseases of congenital etiology are associated with multiple vascular anomalies and detailed evaluation with multidisciplinary approach is paramount and staged hybrid procedure is excellent option.
Interruption of Aorta with delayed presentation and its management without Cardiopulmonary

Bypass : A safe technique.

G.N. Lone; Farooq Ah Ganie

ABSTRACT:

BACKGROUND:

Delayed presentation of Interruption of Aorta in adult life is very rare ad its management is always a surgical challenge.

\section{CASE STUDY:}

A 28 years old man had uncontrolled hypertension with epistaxis. Echo revealed Bicuspid Aortic Valve, mild AR, and severe Coarctation. Aortogram revealed type A Interruption with multiple collaterals from posterior intercostal, internal mammary, inferior epigastric arteries. Patient was operated through left posterolateral thoracotomy with a standby cardiopulmonary bypass and deliberate hypotension. Complete interruption of aorta, $2.5 \mathrm{cms}$ like long fibrous cord was detected at the level of subclavian artery with hugely dilated arch and subclavian artery. A small patent ductus and major collaterals were identified. After heparinization, arch of aorta and thoracic aorta were dissected out, controlling the proximal arch, descending aorta and subclavian artery. Ductus was transected and pulmonary end was closed. Clamp was applied proximally at the level of left common carotid, distally, within $3 \mathrm{cms}$ of thoracic aorta and subclavian artery. Aorta was transected and fashioned at the level of subclavian-arch junction. Distally descending aorta was transected $3 \mathrm{cms}$ below the level of ligamentous arteriosus. The area of interruption was excised. Undersurface of arch was incised and upper end of a $16 \mathrm{~mm}$ Dacron graft was anastomosed to arch and contiguous portion subclavian artery. Distally, other end of Dacron tube graft was anastomosed to transected end of thoracic aorta with continuous 5-0 polypropylene. No extra-anatomic graft was required. Patient was discharged within 7 days from hospital.

\section{CONCLUSION:}

Resection and replacement is a viable and economical option to adult type interruption of aorta and can be performed without $\mathrm{CPB}$, with minimum morbidity, if attempted carefully.

Collateral Miracle - Reformation of Iliac and Femoral Arteries by Collaterals from Bilateral Subclavian Arteries following Contained Rupture of Abdominal Aortic Aneurysm in a Hypertensive Female

Suraj Nagre

Saptarshi Paul

\section{ABSTRACT:}

Ruptured abdominal aortic aneurysm is a surgical emergency with grave prognosis for the affected. However in a few cases, a chronic contained ruptured abdominal aortic aneurysm can present in a multitude of manners rather than as life threatening haemorrhage. In our case we are reporting a 41 year old hypertensive female who developed claudication pain in both her lower limbs. Imaging later revealed that she had a contained ruptured abdominal aortic aneurysm, a thing she was previously unaware of, with collaterals from the bilateral subclavian arteries supplying her femorals.

Single stage surgical approach to coarctation of aorta, bicuspid aortic valve and ascending aorta aneurysm. Rakesh Seetharaman; Giridhar Kamlapurkar Jeeva Vijayan

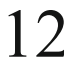

ABSTRACT:

Coarctation of aorta $(\mathrm{CoA})$ is a common congenital defect which may rarely go undiagnosed until adulthood. It can be associated with other cardiac 
defects which may require simultaneous or staged surgical correction. Surgery for such a complex pathology is technically challenging as there are no standard guidelines. Several staged or single step surgical approaches has been described but an extra anatomic ascending to descending aortic bypass grafting for $\mathrm{CoA}$ will allow simultaneous correction of other cardiac defects.

We report a case of 42-year-old man who presented with bicuspid aortic valve, severe aortic regurgitation, ascending aortic aneurysm, incidentally detected severe CoA distal to the origin of the left subclavian artery, who underwent single stage surgery consisting of Bentall's procedure and extra anatomic ascending to descending aortic bypass grafting using $16 \mathrm{~mm}$ collagen coated polyester tube graft, through median sternotomy. The immediate postoperative outcomes were favorable.

\section{GIANT PSEUDOANEURYSM OF ASCENDING AORTA RARE CLINICAL ENTITY \\ N.S. Hiregoudar \\ Sharan Hallad}

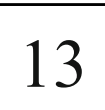

ABSTRACT:

\section{INTRODUCTION:}

Aortic pseudoaneurysm is rare life threatening complication after cardiac surgery or aortic root surgery. The incidence, risk factors, and natural history of aortic aneyrysm are unknown, because very few cases have been reported. BACKGROUND:

Ascending aortic pseudoaneurysm are associated with high morbidity and mortality rates. Progressive growth of aortic pseudoaneurysm can cripple cardiopulmonary system producing signs and symptoms of heart failures it exerts pressure on SVC, pulmonary artery, bronchus, RA. Rupture is imminent sequela of aortic pseudoaneurysm hence surgical correction was necessary.

CASE REPORT:

A 50 year man Hypertensive known case of CAD (stenting of RCA) presented with week's history of progressive dyspnea, cough with bout's of hemoptysis, puffiness of face. Chest X-Ray (Fig 1) showed large illdefined homogenous opacity in right paracardiac, hilar region. 2D Echo revealed large mass in the anterior mediastinum with to -and fro motion of blood inside -with good LVEF

CT-with Contrast agent showed giant pseudoaneurysm of ascending aorta arising from the the right lateral wall of proximal asending aorta rent measures $11-12 \mathrm{~mm}$ and seen $1 \mathrm{~cm}$ distel to sinotubular junction. Aneurysm measuring $14 \mathrm{~cm} / 11 \mathrm{~cm}$ in its greatest diameter -multilobulated with laminated thrombus, exerting mass effect on SVC right bronchus right pulmonary artery.(Fig. 2-5)

Before sternotomy CPB was established by femoral cannulation Hypothermic circulatory arrest was established. Targetted core temperature of 20 degree attained, sternum entered expedetiously. The organised clots evacuated-defect in the asending aorta repaired with dacron patch. To enable a better cerebral perfusion pressure flows were stoped for about 5 minutes. CPB was resumed patient rewarmed and weaned off CBP. The postoperatively patient had convulsions. Shortly before discharge CT showed no leakage but minimal residual hematoma. After 3 months patient recovered completely

\section{CONCLUSION:}

Safe entry into chest is challange. Methods for cerebral preservation of cerebral perfusion should be considered. The surgeons decision to institute axillary, femoral or carotid CPB should depend on the nature of each case. The appropriate use of HCA enables medistinal dissection and aortic control.

Our treatment strategy in a patient with Leriche's syndrome Ismail Yurekli; Levent Yilik; Yuksel Besir; Hüseyin Durmaz; Ali Gurbuz; Kazim Ergüneș
ABSTRACT:

BACGROUND:

Leriche syndrome is a rare event, which is potentially causing visseral and lower extremity ischemia. We presented a case with Leriche syndrome.

\section{METHODS:}

A 58-year old man was hospitalised in our clinic on September, 2018. He was a smoker. He had severe claudication in the both lower extremity. The digital substraction angiography showed atherosclerotic occlusion of distal abdominal aorta and iliac arteries and proximal occlusion of left profunda femoral artery.

\section{RESULTS:}

The patient was operated using the transperitoneal approach. Aortobifemoral bypass with $16 / 8 \mathrm{~mm}$ dacron graft. The reverse safenous vein was anastomosed end-to-end to the distal profunda femoral artery and end-to-side to graft anastomosing to left common femoral artery. The distal pulses of both lower extremity were palpabl, postoperatively. Pain in the both lower extremity diminished, postoperatively. The patient was discharged after six days with antiplatelet and antiagregant drugs.

\section{CONCLUSSION:}

Aorto-bifemoral bypass grafting is safe and effective treatment method in patient with Leriche' syndrome. Endovascular treatment is diffucult and has potentially high complication rate.

Incidental finding of penetrating atheromatous ulcer in the ascending aorta in a patient undergoing coronary artery bypass grafting and aortic valve replacement Sanjeev Malhotra

Himanshu Joshi

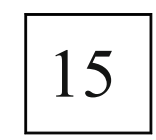

\section{ABSTRACT:}

A 70 year old male, hypertensive, with severe left ventricular dysfunction, atherosclerotic coronary artery disease and moderate aortic regurgitation was taken for elective CABG and aortic valve replacement. During surgery, on opening the aorta it was found that the ascending aorta was studded with multiple penetrating atheromatous ulcers. It was decided to replace the ascending aorta and it was replaced with $26 \mathrm{~mm}$ Dacron graft. AVR was done with $21 \mathrm{~mm}$ Hancock ultra porcine bioprosthesis. CABG was done with two reversed saphenous vein grafts to the LAD and distal right coronary artery. Patient took the procedure well and was shifted to CTVS ICU in a hemodynamically stable state.

Efficacy of ECG gated CT angiography to diagnosis Stanford type-A acute aortic dissection Susumu Manabe; Ryoji Kinosita; Daiki Hirayama Norihisa Yuge

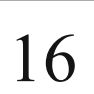

\section{ABSTRACT: \\ BACKGROUND:}

To diagnose Stanford type-A acute aortic dissection, entry-site imaging is important for determining the subsequent surgical procedure. However, accurate diagnosis is difficult due to heart-beat motion artifact. In electrocardiogram (ECG) gated computed tomography, dissected intimal flap can be evaluated in cardiac phases with less motion artifact (e.g., end diastole phase).

\section{METHODS:}

In 20 Stanford type-A acute aortic dissection cases between January 2017 to May 2018, pre-surgical CT was performed. Two cardiovascular surgeons, two radiologists and two medical interns examined anonymized CT scans $(n=20)$. Entry site was evaluated as either Valsalva sinus, proximal or distal ascending aorta, brachiocephalic bifurcation, aortic arch, or distal left subclavian artery bifurcation. 
The size of entry was based on distance (e.g., \&lt; $1 \mathrm{~cm}$, between $1-5 \mathrm{~cm}$, $\& \mathrm{gt} ; 5 \mathrm{~cm}$, unknown). All evaluators evaluated presence of dissection (e.g., three-point scale) and ease of interpretation based on the dissecting flap shape (e.g., five-point scale). Results were compared to intraoperative findings in those with asynchronous CT-ECGs $(n=11)$ or synchronous CT-ECGs $(n=9)$.

\section{RESULTS:}

Improved entry site localization $(0.82 \pm 0.43 ; 1.65 \pm 0.23)$, entry size ( 24 $\pm 15 \% ; 57 \pm 22 \%$ ), presence of dissection $(58 \pm 17 \% ; 80 \pm 15 \%)$ and CT readability $(2.8 \pm 0.6 ; 4.5 \pm 0.4)$ was seen in the synchronous group. Finding concordance was high, with easy interpretation.

\section{CONCLUSION:}

ECG gated CT angiography can accurately and easily diagnose an entry site by reducing the influence of motion artifact in type A dissection.

\section{Extra anatomical bypass for complex preductal coarctation of aorta in an adult-a case report Prashant Mishra \\ Harsh Seth}

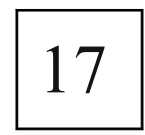

\section{ABSTRACT:}

Complex coarctation as well as coarctation proximal to the left subclavian was very rare and it accounts for less than $1 \%$. The surgical management of complex coarctation in an adult remains a challenge till date. Various surgical options are available in treating complex coarctation in adult including anatomical and extra anatomical corrections. Percutaneous intervention has very limited role, and an option is still uncertain. We are reporting a 20-year-old male with complex coarctation of aorta who underwent an Extra Anatomical Bypass using $16 \mathrm{~mm}$ Dacron graft from ascending aorta to descending aorta through midline sternotomy using posterior pericardial approach. He had uneventful postoperative period with acceptable gradients between both limbs. He remains asymptomatic on three months follow up with reduced need of antihypertensive medication.

\section{Suture Reduction Might be a Simple Alternative to Ascending Aorta Replacement for Borderline Ascending Aortic Aneurysm in Patients Undergoing Aortic Valve Replacement Hyuk Ahn \\ Yeiwon Lee}

\section{ABSTRACT: \\ BACKGROUND:}

The evidence supporting current guidelines recommending concomitant ascending aorta replacement during aortic valve replacement (AVR) is still weak. This study was conducted to evaluate the efficacy of simple suture reduction of the ascending aorta (SRA) performed with AVR in patients with borderline ascending aortic aneurysm.

\section{METHODS:}

170 patients (63 \pm 11 years) who underwent concomitant SRA with AVR were enrolled. Diameter of the ascending aorta was 45 $\pm 4.1 \mathrm{~mm}$. Ninety-one patients had bicuspid aortic valve (BAV). Median follow-up duration was 83 (interquartile $=31,146$ ) months. Last computed tomographic follow-up was performed at 71 $(26,127)$ months after surgery. Early and long-term outcomes were evaluated and growth rate of the repaired aorta was analyzed. Major aorta-related events (MARE) were defined as cardiac death including sudden death, aortic dissection or rupture, aortic reoperation and recurrent ascending aortic aneurysm \&gt;50mm.

\section{RESULTS:}

Mean ascending aortic diameter after surgery was $35.8 \pm 6.6 \mathrm{~mm}$. Early mortality rate was $2.4 \%$ (4 of 170 patients). No patients had early postoperative complications associated with SRA. The MAREs during the follow-up included only 2 cases of sudden death. No patients had aortic aneurysm greater than $50 \mathrm{~mm}$. However, 11 patients had recurrent aneurysm \&gt; $45 \mathrm{~mm}$. Median growth rate of the repaired aorta was $0.10 \mathrm{~mm} /$ year $(0-0.28)$. A multivariable analyses demonstrated that combined coronary artery disease was significantly associated with both the recurrence of aortic aneurysm ( $\& \mathrm{gt} ; 45 \mathrm{~mm}$; hazard ratio [95\% confidence interval] $=23.850$ [3.718-153.002]) and growth rate of the repaired aorta (beta, $95 \%$ confidence inter$\mathrm{val}=0.741,0.145-1.338)$. Neither preoperative diameter of the ascending aorta nor BAV was associated with recurrence of aortic aneurysm or growth rate of the aorta after SRA.

\section{CONCLUSIONS:}

Concomitant SRA might be a safe and effective surgical alternative to ascending aorta replacement for AVR patients with borderline ascending aortic aneurysm, regardless of aortic valve pathology.

Outcome of operative intervention in a case of chronic mesenteric ischaemia with peripheral arterial disease. S.S. Mahapatra; R. Rathi; S.B. Roy Soumya Darshan Sanyal

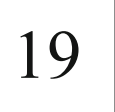

\section{ABSTRACT: \\ OBJECTIVES:}

Chronic mesenteric ischemia is a rare condition resulting from stenosis and occlusion of one or more visceral arteries. Its diagnosis requires a high degree of clinical suspicion and treatment presents a daunting therapeutic challenge. Medical management may serve to be palliative, but timely surgical intervention is not only curative, it also provides lasting symptomatic relief. Open surgical procedure to rescularise the gut and bilateral lower limbs, was carried out at our institution and has served to elicit the benefits of the same.

\section{METHODS:}

One patient with chronic mesenteric ischaemia and peripheral arterial disease underwent open surgical revascularisation at our centre. The period of recovery, duration of hospital stay, immediate post operative complications, return to normal activity, post operative dependence on medications and amelioration of symptoms were taken into consideration in order to gauge the benefits.

\section{RESULTS:}

The patient under consideration had an uneventful hospital stay followed by complete recovery. The subject had complete symptomatic relief and medicine dependence was significantly reduced.

CONCLUSION:

Timely surgical intervention provides excellent symptomatic relief. It also served to be a definite cure. The subject of this case had no complications in the post operative period.

\section{BENTALL OPERATION FOR MYXOMATOUS DEGENERATIVE AORTIC VALVE IN A CHILD OF 10 YEARS : RARE PRESENTATION Subodh Satyarthi \\ Ankit Jain}

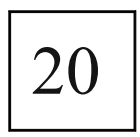

\section{ABSTRACT:}

Annuloaortic ectasia and aortic regurgitation in children have been reported but its management has a dilemma.

Hereby we report a case of 10 years old girl presented with dyspnea NYHA III was planned for REPAIR/BENTALL PROCEDURE. Valve showed myxomatous degeneration. Post operatively patient had satisfactory results. 
The role of epicardel fat in development atrial fibrillation and newel predictors LAA thrombosis or tromboembolic events in patient without structural heart diseases.

Elena Z. Golukhova

Mari Arakelyan

\section{ABSTRACT: \\ PURPOSE:}

To evaluate the AF mechanisms in patients without structural heart diseases and analyze clinical and instrumental parameters influencing left atrial appendage (LAA)/thromboembolic events.

\section{METHODS:}

We evaluated 151 patients with $\mathrm{AF}$ and without valvular and ischemic heart disease with normal or slightly enlarged LA. TDI and cardiac MRI with evaluating EF localization and thickness were performed in 39 cases. The myocardial fibrotic and proinflammatory biomarkers levels - matrix metalloproteinases (MMP-2, MMP-9), tissue inhibitor of matrix metalloproteinase-1 (TIMP-1) were also estimated. Contrast-enhanced computed tomography (CT) of LA was performed in 105 patients $(87 \%)$ to reveal of pulmonary veins abnormality and definition of morphological type of LAA. Transesophageal Echocardiography (TEE) was performed in 47 (39\%) patients with calculation of LAA blood velocity and identification of spontaneous ECHO contrasting.

\section{RESULTS:}

According to the MRI the majority of patients (86\%) had accumulation of epicardial fat along the anterior wall of LV, less frequently in the upper LV $(62 \%)$ and lateral wall $(22 \%)$. The EF thickness at anterior LV wall was significantly associated with TIMP- 1 level $(\tau=0,71 ; \mathrm{Tcr}=0,18)$. Correlation between EF thickness and MMP-9 $(\tau=0,65$; Tcr $=0,16)$. LAA thrombosis/ ischemic stroke in anamnesis were associated with:age older than 65 years $(\mathrm{p}=0.001)$, chronic AF ( $\mathrm{p}=0.003), 5$ and higher in CHA2DS2VASc score $(p=0,005)$, low LAA blood flow velocity $(p=0.001)$, the spontaneous ECHO contrasting $(\mathrm{p}=0.03)$ by TEE. According to $\mathrm{CT}$ the morphological LAA type III also had a predictive value $(\mathrm{p}=0.01)$.

\section{CONCLUSION:}

The increasing levels of MMP-9 and TIMP-1 are associated with EF thickness by MRI and LV local contractility disturbances by TDI, which could reflect initial manifestation presentation of atrial cardiomyopathy in patients with nonvalvular AF. In addition to CHA2DS2VASc score, we identified additional predictors of LA thrombosis/ TE events: chronic AF, low LAA blood flow velocity, the phenomenon of spontaneous ECHO contrasting in LA and the morphological LAA type III by CT.

\section{Surgery for adult patients with atrioventricular septal defects}

Masahide Chikada; Takeshi Miyairi; Hiroshi Nishimaki;

Makotao Ohno; Hirokuni Ono; Kiyoshi Chiba;

Tokuichirou Nagata; Misa Kougo; Hirotoshi Suzuki; Shota Kita

\section{ABSTRACT: \\ BACKGROUND:}

The number of adult patients with congenital heart defects in Japan is increasing. Surgery for adult patients with atrioventricular septal defects (AVSD) consists of initial repair for partial AVSD and reoperation for left AV valve regurgitation or infectious endocarditis. AVSD has morphologic characteristics such as common $\mathrm{AV}$ valve and inferiorly displaced $\mathrm{AV}$ node. In our institute, surgery for adult patients for AVSD was performed by cooperation between adult heart team and congenital heart team. In this study, we report our experience of surgery for adult patients with AVSD. METHODS:

Two cases were male and four cases were female. Ages ranged from 19 to 67 years old. Complete AVSD was one cases and others were partial
AVSD. Surgery for an adult patient with complete AVSD was reoperation of left $\mathrm{AV}$ valve replacement due to left $\mathrm{AV}$ valve regurgitation. Initial repair (patch closure of ostium primum defect and cleft closure of left AV valve) was performed in two cases. Two cases underwent reoperation of left AV valve repair. These repairs were performed by cleft closure, ring annuloplasty and placement of artificial chordae. One re-replacement of left AV valve was performed because of infectious endocarditis.

\section{RESULTS:}

There was no hospital death and no late death. No pacemaker implantation was performed after operation. Follow up periods raged 2 to 8 years. At last follow up all patients were in NYHA class 1. One patient after initial repair has mild left AV valve regurgitation. One patient after left AV valve repair has mild left $\mathrm{AV}$ valve stenosis.

\section{CONCLUSION:}

Outcomes of surgery for adult patients for AVSD are good. The cooperation between adult team and congenital team was considered to be important.

\author{
A unique case of Post ICR, Residual VSD with \\ severe RVOTO \\ Zubair Rashid
}

ABSTRACT:

A 14 years old girl post ICR(intracranial repair) for TOF( Tetrology of Falot), Infundibular muscle resection, Pulmonary valvotomy, Transannular patch with autologous pericardium developed Large residual VSD (Ventricular septal defect), Severe RVOTO(Right ventricular outlet obstruction) and mild AR(Aortic regurgitation. She presented with insidious onset of shortness of breath. Was managed conservatively for 6 months. Failing medical therapy, was referred for surgery.

Residual VSD closure with acellular bovine pericardium, Bileaflet pulmonary valve reconstruction, Transannular patch with bovine pericardium was done. Post operative course was uneventful with no RVOTO(Right ventricular outlet obstruction) no VSD (Ventricular septal defect) and discharged with normal heamodynamics.

Cardiovascular Surgery Training in Zambia:

A One Year Review

Edward Nyimbili

Emmanuel Liche

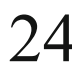

\section{ABSTRACT:}

\section{INTRODUCTION:}

In a low to middle income country like Zambia there are a constellation of factors against provision of consistent cardiac surgical services. These include lack of skilled labour, equipment and consumables and universal health coverage. Zambia's population consisting of children and adults in reproductive age has a burden in congenital heart diseases and rheumatic heart disease yet no locals to perform surgeries. Last year in October, Tokushima International Cooperation (TICO) introduced a 3-5 year training model of local personnel.

\section{MATERIALS AND METHODS:}

This is a one year retrospective study/audit to assess the impact of the training model introduced by TICO Japan. Three visits were made over one year by the Japanese Team.

\section{RESULTS:}

A total of 4 registrars in general surgery, one perfusionist, two theatre nurses and one anaesthesiology registrar were trained using wet laboratories and actual surgeries. A total of ten patients were operated on. Nine of them were children and 1 adult. Five open heart surgeries (ASD repair) and 4 PDA ligations were done with good outcomes. The adult patient was for pacemaker insertion. 


\section{CONCLUSION:}

The onsite training model by TICO has so far imparted skills to the local team who are the first Zambians to perform open heart surgery. The surgeons have taken a leading role right from the beginning and outcomes are comparable to other centres regionally and globally.

\section{Late Embolization of Atrial Septal Defect Occluder} Device, Presenting with Cyanosis. A Rare Case Report Chaitanya Raut

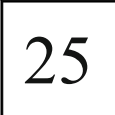

\section{ABSTRACT:}

A 29-year-old lady had undergone Atrial septal defect device closure 4 years ago and presented to us with central cyanosis and clubbing. She was investigated and found to have the device embolized to the septal leaflet of tricuspid valve. She underwent surgery and the device which was adherent to the septal leaflet was removed along with tricuspid valve replacement. Patient is symptom free at 1 year follow up.

\author{
A Rare Case Of Cyanotic ASD Due To \\ Prominent Eustachian valve \\ A. Rathinavel \\ K.J. Vignesvaran
}

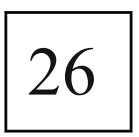

\section{ABSTRACT: \\ INTRODUCTION:}

Cyanosis in a case of ASD without pulmonary arterial hypertension is quite rare. A patient with ASD had a central cyanosis due to the presence of an overdeveloped Eustachian valve that directed IVC blood to the left atrium. In conclusion, ASD can present with cyanosis due to an over-developed Eustachian valve.

\section{CASE PRESENTATION:}

$17 y$ rs old female patient presents with complains of exertion dyspnoea for past 6 months. On examination patient had central cyanosis, grade I clubbing, with a wide and fixed split second heart sound. There was no murmur, SPO2 76\%. Chest $x$ ray, ECG \&amp; CT chest was normal. Trans thoracic Echo shows secundum ASD without any evidence of right to left shunt. TEE shows ASD with prominent Eustachian valve, sever TR with ATL prolapse, TRPG $25 \mathrm{mmHg}$. CATH study shows right to left streaming of IVC blood into LA by prominent Eustachian valve, PA pressure is normal. Patient was electively taken up for surgery. Intra operatively Eustachian valve was excised, DeVega procedure of tricuspid valve done, defect closed with gluteraldehyde treated pericardial patch. Post op period was uneventful, patient discharged on 5th POD. Saturation after correction was $99 \%$. CONCLUSION:

Central cyanosis can occur in ASD due to various causes. Most of the case it is due to right-to-left shunts resulting from pulmonary arterial hypertension. But in this case there was an abnormal over-developed Eustachian valve directed IVC blood to the left atrium is extremely rare and can result in significant confusion and misdiagnosis.

Risk factors for the development of stenosis of the right ventricular outflow tract and / or pulmonary artery and / or its branches in the remote period after arterial switch operation

Nefedova I.Y.

Lyazin Denis

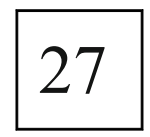

\section{ABSTRACT:}

Arterial switching operation is a standard of radical correction of simple transposition of the great arteries (TGA).

PURPOSE:

The aim of the study is to identify the most important risk factors for the development of right ventricular outflow obstruction, stenosis of the pulmonary artery and its branches in the remote period after the arterial switch operation.

\section{MATERIALS AND METHODS:}

The study included 248 patients with simple transposition of the great arteries who underwent arterial switch operation in the period from 2000 to 2015 in the neonatal emergency surgery department of A.N. Bakulev National Medical Research Center of Cardiovascular Surgery. Among patients who underwent surgery in stage 1 the average age was $13.4+/$ -2.4 days. In the group of children who underwent 2 -stage correction (with pulmonary artery banding), the average age at the time of radical correction was $6.9+/-1.47$ months. Reconstruction of the neo-pulmonary artery root during arterial switch in 187 cases was performed using autoperikard patch. In 61 patients xenopericardial patch was applied.

\section{RESULTS:}

To identify predictors that can cause the development of right ventricular outflow obstruction and pulmonary artery stenosis the method of multiple statistical analysis was used. In our study the frequency of right ventricular outflow obstruction, pulmonary artery stenosis and /or its branches was in 48 patients, which accounted for $19.4 \%$ of all operated children and $78.7 \%$ among patients in whom the reconstruction of the pulmonary artery trunk was performed with xenopericardial band.

\section{CONCLUSIONS:}

The most significant factors influencing the development of right ventricular outflow obstruction, stenosis of the pulmonary artery and its branches in the remote period after the arterial switch operation were reconstruction of the pulmonary artery trunk with xenopericardial band $(\mathrm{CS}=505,657$; $\mathrm{p}=0.001 ; 95 \% \mathrm{CI}, 62,644 ; 1284,508)$ and ascending aortic diameter (CS=1,636; p\&lt;0.001; 95\% CI 1,234 - 2,17).

Unusual Association of TOF with Pulmonary Atresia with Type 1 AP Window- Report of 2 cases and review of literature

Prateek Vaswani; Palleti Rajashekar; Devagourou Velayoudam

\section{ABSTRACT:}

Aortopulmonary(AP) window is a rare truncal anomaly with an incidence of $0.2 \%$ in patients with congenital cardiac disease. It is defined as a communication between the main pulmonary artery and the ascending aorta in presence of two separate semilunar valves. Its coexistence with tetralogy of Fallot(TOF) is rare. Even rarer, is its association with TOF with Pulmonary atresia(PA). So far only 7 such cases have been reported in literature. In the last few decades, the techniques for surgical repair of AP window and TOF have evolved. The simultaneous existence, however, is dealt with AP window repair followed by intracardiac repair for TOF. We report 2 such cases with Type $1 \mathrm{AP}$ window in association with TOF with PA. In the first case, AP window was opened and closed with anterior sandwich patch technique and pulmonary valve was reconstructed to avoid conduit. In the second case, the right coronary artery was high above the sinotubular junction in close proximity to the AP window, transpulmonary closure followed by intracardiac repair was performed. In both cases, pericardial patch was used for RVOT(right ventricular outflow tract) augmentation successfully avoiding conduit placement. In cases TOF with AP window, the branch pulmonary arteries are usually adequate sized being exposed to continuous systemic pressure. So, the AP window repair would be convenient via tranpulmonary approach which can be extended to reconstruct the RVOT. This approach significantly reduces the ischemic time as it avoids aortotomy incision. Although, the transpulmonary route of AP window repair has been found to result in late re-interventions of pulmonary arteries, it is preferable in cases with variant coronary anatomy and in cases of TOF variants like ours. The lack of evidence, however, fails to establish a standard surgical approach for this rare coexistence. 
Anomalous Coronary Artery Arising from the Wrong Sinus and Coursing Between the Great Arteries Tomoyuki Matsuba; Hideaki Kanda; Yuki Ogata; Shuji Nagatomi; Keisuke Kawaida; Kenji Toyokawa; Itsumi Imagama; Naoki Tateishi; Kazuhisa Matsumoto; Hiroyuki Yamamoto

\section{ABSTRACT:}

Anomalous coronary artery arising from the wrong sinus and coursing between the great arteries carries a risk of sudden death. However, operative indication and definite technique are still controversial.

CASES:

Our experience with 4 cases is reported. One patient (Case-1) with anomalous Lt. coronary artery (ALCA) from the Rt. sinus of Valsalva (SOV), and 3 patients (Case-2,3,4) with anomalous Rt. coronary artery (ARCA) arising from the Lt.SOV. The anomalous CA was coursing between the great arteries in all the cases. Case-1 is an asymptomatic 14-year-old boy. Coronary $\mathrm{CT}$ showed single coronary trunk originating from the Rt.SOV from which ALCA was branching. Although exercise-ECG and myocardial scintigraphy (MS) did not show any ischemic finding, surgery was indicated because of the high risk of sudden death. ALCA was transected and reimplanted to the Lt.SOV. Postoperative CT showed patent LCA without stenosis. Three years have passed without signs of myocardial ischemia. Case-2 is an 8-year-old boy with chest pain during swimming, but exercise-ECG and MS were negative. Surgery was not performed, and he is followed-up closely with exercise-restriction. Case-3 is a 11year-old boy with chest pain, but repeated exercise-ECG and MS were negative. He is followed-up closely with exercise-restriction and administration of beta-blocker without surgery. Case- 4 is a 46-yer-old male with effort angina. Severe arteriosclerosis in the LAD and CX along with ARCA from the Lt. SOV were detected by CT and CAG. Ischemia in the LAD and CX areas were detected but not in the RCA area. Only PCI for $\mathrm{LAD}$ and $\mathrm{CX}$ lesions were performed.

\section{CONCLUSIONS:}

Our strategies are, 1) Surgical treatment is indicated for ALCA coursing between the great arteries, 2) ARCA patients without definite ischemic sign in the exercise tests are followed-up without surgery. Surgery may be considered if the patients want active lifestyle.

\section{Optimal stage for intravenous administration of cardiac stem cells in univentricular heart disease; Experimental studies in novel piglet models of single ventricular circulation}

Daiki Ousaka

Takuya Goto

\section{ABSTRACT: \\ INTRODUCTION:}

We have recently reported that transcoronary infusion of cardiosphere-derived cells (CDCs) may improve cardiac function after staged palliation in patients with single ventricle physiology; however, the therapeutic efficacy of intravenous delivery of CDCs remains unanswered.

\section{HYPOTHESIS:}

We sought to determine whether intravenous injection of allogeneic CDCs in three novel models of single systemic circulation may result in sufficient CDC engraftment in pigs.

METHODS:

Surgical creation of univentricular circulation was attempted in 15 Yorkshire pigs $(20 \mathrm{~kg})$. Single ventricular model with modified Blalock-Taussig shunt $(\mathrm{BT})(\mathrm{n}=5)$, Bidirectional Glenn shunt (BDG) model $(\mathrm{n}=5)$, total cavo-pulmonary shunt (TCPC) model $(n=5)$ was created without mechanical support. Swine CDCs were labeled with superparamagnetic microspheres (SPMs) and directly injected into IVC. One hour after the administration of $1.0 \times 105 /$ $\mathrm{kg}$ CDCs, target heart was removed and T2* MRI was performed to detect CDC engraftment and distribution in host environment. RESULTS:

Models could survive for 4 to 6 hours after surgical construction. The SPM-labeled CDCs were readily detectable in the hearts underwent single systemic circulation or BDG procedure, while cell engraftment could be appreciated in pigs neither received TCPC nor with normal biventricular circulation $(n=5)$. Histological analysis revealed a concordant SPM-labeled CDC engraftment, manifested by iron-staining, within the targeted myocardium.

\section{CONCLUSIONS:}

Novel unassisted-models of single systemic circulation could be established in pigs with or without BDG or TCPC procedure. Our results suggest that intravenous injection of CDCs in patients with univentricular heart may be therapeutically applicable before the stage of TCPC.

Successful surgical management of a congenital giant right atrial appendage aneurysm: a case report on a rare cardiac anomaly

Anagha Tulsi T.

Utkarsh Sanjay Sanghavi

\section{ABSTRACT: \\ BACKGROUND:}

Aneurysms of the right atrial appendage usually are congenital in origin and only a handful have been reported. We report a case of giant right atrial appendage aneurysm in a 37 year old man with atrial flutter and right ventricular dysfunction. He underwent a successful aneurysmectomy and recovered in normal sinus rhythm.

\section{CASE REPORT:}

A 37-year-old man presented with complaints of frequent palpitations restricting his daily activities. On evaluation, he was found to have right atrial flutter with 2:1 conduction block. A transthoracic echo assessment revealed a large aneurysmal RAA extending upto RV apex and compressing it. Cardiac MRI showed a RAA aneurysm measuring $14.5 \times 6.8 \times 10 \mathrm{~cm}$ extending upto right ventricle, compressing the RA and RV and RVEF was $19 \%$.

\section{RESULTS:}

The postoperative period was uneventful and he was in normal sinus rhythm at discharge. On histopathological examination of the aneurysm sac wall-fibrosis and endocardial thickening were noted. At 3 months follow up patient is asymptomatic and transthoracic ECHO showed normal biventricular function and no residual RA aneurysm in normal sinus rhythm.

\section{CONCLUSION:}

Congenital right atrial aneurysm in asymptomatic patients should be considered for aneurysmectomy owing to the risk of arrhythmias and thromboembolism in these cases.

Surgical removal of a late embolized Patent ductus arteriosus occluder device causing occlusion of Left pulmonary artery and significant luminal compromise in Left main bronchus

Korah J. Kuruvilla

Deepak Narayanan A.

\section{ABSTRACT:}

\section{INTRODUCTION:}

The alternative strategies for non surgical closure of patent ductus arteriosus is well established. The disadvantages of this procedure include potential for misplacement and embolization of occlude device CASE REPORT:

We present a rare case of 3 year old child who underwent a patent ductus arteriosus device closure at the age of 8 months. Patient 
presented to cardiothoracic OPD with history of hemoptysis and recurrent respiratory tract infection. CT Thorax done revealed, PDA closure device in situ with surrounding soft tissue probably causing occlusion of left pulmonary artery and endobronchial soft tissue growth in left main bronchus possible arising from soft tissue adjacent to PDA closure device with significant luminal compromise. Surgical retrieval of PDA closure device was done under total circulatory arrest and PDA closure and pulmonary embolectomy was done. Post operative echocardiogram revealed normal PA flow and no flow across the ductus. The patients post operative course was uncomplicated.

\section{CONCLUSION:}

The technological advances in interventional cardiology have made non surgical closure of patent ductus arteriosus a routine procedure. Large PDA s are amenable to transcatheter closure with good results, but selection of appropriate device size and proper deployment are important for prevention of embolization. Although device embolization and surgical retrieval of an embolized device in acute stages have been reported frequently, the reported case is the only one in which an embolized device has caused occlusion of left pulmonary artery and also causing significant luminal compromise of left main bronchus.

\author{
Ruptured Sinus of Valsalva \\ S. Kumar \\ Vishak K.
}

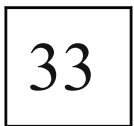

\section{ABSTRACT:}

\section{INTRODUCTION:}

A sinus of Valsalva aneurysms is a rare cardiac anomaly that may be congenital or acquired. If the aneurysm ruptures, it causes acute symptoms of dyspnoea, complete heart block and death. It occurs in between 0.09 and $0.15 \%$ of cases, and comprises up to $3.5 \%$ of all congenital cardiac anomalies. It may be associated with ventricular septal defect or aortic regurgitation. The treatment of choice is surgery

\section{CASE SERIES:}

\section{CASE 1:}

A 32 year old male, with history of breathlessness on exertion Class II was found to have a continuous murmur over left parasternal region. On Echocardiography, Ruptured Sinus of Valsalva (RSOV), RSOV rupture into RVOT, Small sub aortic VSD. Patient underwent Intracardiac repair with Goretex patch and Pericardial patch closure of VSD. Patient was doing well and was discharged.

CASE 2:

A 35 year old male, presented with breathlessness on exertion Class III. On examination, continuous murmur over left parasternal region. Echocardiography showed Sub aortic VSD, Restrictive left to right shunt, RSOV into RVOT, Mild AR, Moderate TR. He underwent Goretex patch closure of Ventricular Septal defect with Repair of RSOV with Goretex patch. Patient was doing well and was discharged.

\section{DISCUSSION:}

Sinus of Valsalva (SVA) is a rare cardiac anomaly, and arises mainly from a congenital defect of the aortic media or may follow bacterial endocarditis. Transoesophageal echocardiography plays a major role in identifying the shunt locations and other cardiac lesions.

\section{CONCLUSION:}

The optimal management for a RSOV is surgical repair. However, the best therapy for asymptomatic unruptured SVA is currently unknown. The mean survival in patients with an untreated ruptured SVA is about four years. Coexisting lesions should be repaired within the same surgical procedure. Today, the ten-year survival rate after surgical repair of a ruptured SVA is $90 \%$. However, aortic regurgitation might occur after surgical repair.
Conversion to Biventricular heart in Fontan failure. An alternative to Heart transplantation

K.R. Balakrishnan

Pradeep

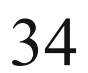

\section{ABSTRACT: \\ INTRODUCTION:}

In patients with Fontan failure, when anatomically feasible possibility of conversion into biventricular heart should be considered as an alternative to heart transplantation. we review our experience on 2 patients in last 6 months

\section{METHODS:}

In 2 patients 7 and 9 year old who presented with Fontan failure was referred for Heart transplantation have been thoroughly reviewed and possibility of Biventricular conversion was assessed. Their accesesibility to medical facilities were remote. Both patients had DORV. CT was done to assess the possibility of routability. Both Glenn and Fontan were taken down and converted to biventricular heart. Both patients were doing well in their 3 months follow up.

CONCLUSION:

Bi ventricular Conversion in feasible failed Fontan can be a better alternative to cardiac transplantation.

A rare case of Fungal Endocarditis in Pre-term neonate Jaikaran, Kathirvel

Rakesh Kumar

ABSTRACT:

\section{INTRODUCTION:}

The case has been presented for its rarity and only 5 per $1,00,000$ cases reported in one year. Fungal endocarditis is serious form of endocarditis, with mortality rate of about $50 \%$. It is mainly caused by candida and aspergillus species. In neonates, endocarditis commonly involves the right sided heart valves. Endocarditis occurs in neonates who are pre term, immune-compromised due to malignancy, HIV infection.

CASE STUDY:

A 90 days old male child was brought with complaints of respiratory distress, since birth and history of preterm birth. ECHO was done, which revealed the presence of hyper-echoic vegetation attached to tricuspid valve measuring about $1.4 \times 1.1 \mathrm{~cm}$, mild to moderate TR and PDA, Tricuspid inflow obstruction present with good ventricular function. Blood culture was done which revealed the presence of candida species. Patient was taken up for surgery, PDA was ligated, Right atrium was opened, which revealed the presence of vegetations measuring about $1.5 \times 1.2 \mathrm{~cm}$ in the anterior and septal leaflet of tricuspid valve. The vegetations are excised in toto and rent in the leaflet is repaired. Patient was extubated and post operative period is uneventful.

CONCLUSION:

Fungal endocarditis, though rare has high fatality rate. Early diagnosis and prompt surgical intervention are key for successful outcomes.

\section{1,1.5 or 2? : Contemplating biventricular repair in an adult DORV PS patient with severe \\ biventricular dysfunction. \\ Girish Warrier \\ Abid Iqbal}

\section{ABSTRACT:}

\section{INTRODUCTION:}

Biventricular repair is considered best treatment option for DORV patients. But late presentation and severe ventricular dysfunction often precludes a biventricular repair. 


\section{CASE REPORT:}

An 18 year old African national presented 2 years ago with NYHA class 4 dyspnoea. On evaluation, he was diagnosed as DORV VSD with severe PS and biventricular dysfunction. Since general condition was not favorable for any procedure on CPB, an Off pump BDG was performed. SPO2 improved to $80 \%$. Symptoms reduced to NYHA 2. His symptoms deteriorated over 2 years to Class 3. On evaluation, he was found to have $\mathrm{Hb}$ 24 and severe biventricular dysfunction. Serial phlebotomy was performed and $\mathrm{Hb}$ reduced to 18 after which ventricular function improved marginally. A VSD closure with Annulus preserving ICR was performed along with BDG take down. Patient had uneventful recovery. Ventricular function improved significantly after septation.

\section{CONCLUSION:}

A staged biventricular repair may be feasible in DORV PS with severe ventricular dysfunction. Hyper viscosity due to high hematocrit can be a contributing factor to ventricular dysfunction in such patients.

\section{The anomalous origin of the left pulmonary artery (LPA) in a case of TOF (Case report) Vikas Panwar}

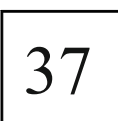

\section{ABSTRACT:}

Tetralogy of Fallot's (TOF) is a frequently encountered congenital heart defect, and the detailed diagnostic criteria are well established. There may be other anomalies associated with TOF, but the anomalous origin of the left pulmonary artery (LPA) from the ascending aorta is a rare one. The diagnosis of this anomaly, which may be mistaken for absence of the LPA, should be made in early life to avoid unilateral pulmonary hypertension and also for appropriate surgical planning. We are reporting a case of a 14yr- child, with the diagnosis of TOF associated with anomalous origin of the LPA from the ascending aorta who underwent a one stage surgical correction of this anomaly.

\section{A Novel Technique To Avoid Total circulatory Arrest} (TCA) in correction of Scimitar Syndromee in an adult. Saigopal Krishnan

Muthukumar Sundaram

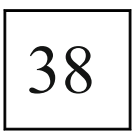

\section{ABSTRACT: \\ INTRODUCTION:}

Scimitar syndrome (SS) is a rare congenital malformation with an estimated incidence of approximately 2 in 100000 births.SS, first described in 1836 , consists of anomalous pulmonary venous drainage from the right lung to the inferior vena cava."https://www.ncbi.nlm.nih.gov/pmc/articles/PMC4342640/\#R2" There are often a constellation of findings associated with SS including hypoplasia of the right lung, pulmonary hypertension, dextroposition of the heart. We are reporting a case of 22 year old female was takenup for correction of scimitar syndrome in which a novel innovative technique is used to avoid TCA during the operation.

\section{CASE REPORT:}

This is a 22 year old female evaluated for dyspnoea and palpitation -NYHA -II. Her laboratory blood investigations are within normal limit. Chest $\mathrm{x}$ ray showed right hilar opacity. Echocardiogram revealed Atrial septal Defect with partial anomalous pulmonary venous drainage to the inferior vena cava. She was taken up for complete correction. Intracardiac Repair-Re -routing of scimitar vein to Left atrium and ASD closure was done Right atrium opened. IVC cannula taken out and replaced with a 6.5 flexo-metallic ET tube cuff inflated. IVC opening, scimitar vein opening with hepatic vein. Two additional pump suctions field was made clear and ASD closed. With rerouting of scimitar vein into LA. RA closure done and simultaneously rewarming started with 8-10 deg gradient. CPB
TIME 195 min, ACC 108 min. Post op course uneventful. Discharged on postoperative day 6.2 weeks Follow up. Doing well. $\mathrm{SpO} 2$ 99\% (Room air)

\section{CONCLUSIONS:}

TCA can be avoided if perfusion strategy is modified. Innovative idea of using Flexo metallic Endotracheal Tube is a novel technique and can be a substitute for IVC cannula in Scimitar Syndrome.

Usefulness of sternal closure with bioresorbable plate in respiratory function after coronary artery bypass grafting

Toshiyuki Maruyama; Syogo Sakurai

Kiyoshi Tamura

\section{ABSTRACT: \\ OBJECTIVE:}

The aim of our study is to investigate that sternal reconstruction using bioresorbable plate in median sternotomy may reduce postoperative respiratory dysfunction when compared with wire cerclage only.

\section{SUBJECTS AND METHODS}

We reviewed 100 patients (18 women, mean age 69.3 years) who were undergone coronary artery bypass grafting with median sternotomy. Patients were divided into two groups; patients underwent sternal reconstruction with bioresorbable plate and wire cerclage ( $\mathrm{S}$ group, $\mathrm{n}=50$ ), patients with wire cerclage only ( $\mathrm{N}$ group, $\mathrm{n}=50)$, and the following data were analyzed and compared between two groups.

\section{RESULTS:}

There was no significantly difference in perioperative respiratory function in both groups. However, in postoperative change rate of respiratory function, $\mathrm{N}$ group had significant decrease compared with $\mathrm{S}$ group in vital capacity (VC) $(\mathrm{N}: \mathrm{S}=74.9: 85.4 \%, \mathrm{p}=0.024), \mathrm{VC}$ as a percentage of predicated $\mathrm{VC}(\mathrm{N}: \mathrm{S}=75.0: 86.7 \%, \mathrm{p}=0.014)$, and forced expiratory volume in the first second ( $\mathrm{N}: \mathrm{S}=73.7: 84.6 \%, \mathrm{p}=0.021$ ). There were significantly more patients with score 4 of Prince Henry Pain Scale in $\mathrm{N}$ group compared with $\mathrm{S}$ group $(\mathrm{N}: \mathrm{S}=64.0: 38.0 \%, \mathrm{p}=0.002)$.

CONCLUSIONS:

Sternal fixation with bioresorbable plate could reduce impairment of postoperative respiratory function in comparison to wire cerclage only.

\section{Minimally Invasive Saphenous Vein Harvesting; Is This The Way Forward? Muhammad Ashraff Baruji; Taufiq Abdullah}

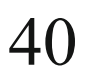

\section{ABSTRACT:}

\section{OBJECTIVE:}

The aim of this study is to compare the incidence of leg wound complication in patients who undergoing Coronary Artery Bypass (CABG) using traditional open method versus minimally invasive (bridging) method.

\section{INTRODUCTION:}

Traditionally, saphenous vein is harvested through long continuous skin incision. Moving forward, minimally invasive technique was introduced. It's many benefits include improved patient's quality of life in terms of shorter hospitalization duration, wound healing time and the superior cosmetic results and at the same time offering a conduit quality at par with the open technique.

\section{METHODS:}

A retrospective study from January to December 2017 conducted at Institut Jantung Negara. Patient demographic, risk factors, preoperative and intra-operative data were homogeneously grouped, and post-operative data was collected and analyzed. All patients underwent multivessel $\mathrm{CABG}$ were enrolled. Endoscopic vein 
harvesting, peripheral vascular disease, obese, uncontrolled diabetes and chronic kidney disease were excluded.

\section{RESULTS:}

Saphenous vein harvesting using open method (Group I) was performed in 471 patients $(78.2 \%)$, while bridging method (Group II) was used in 131 patients $(21.8 \%)$. There were no statistical differences in preoperative risk factors in both groups. The incidence of leg wound infection developed in Group 1 was $3.6 \%(n=17)$ versus $0 \%(n=0)$ in Group II, with the statistical difference $P$ value of 0.027 .

\section{CONCLUSION:}

The use of minimally invasive saphenous vein harvesting has been advocated in an effort to minimize leg wound morbidity. This study suggests that minimally invasive bridging technique reduces leg wound complications compared to the open technique and should be considered as method of choice for vein harvesting. However, follow-up study on long term survival or freedom from repeat revascularization will be needed to ascertain that the quality of conduits are not compromised with bridging technique.

\section{Congenital coronary abnormality found incidentally during work up for atherosclerotic coronary artery disease. \\ Willington Francis; Maryam Al Kuwar; C.S. Carr; Ihsan Rafie}

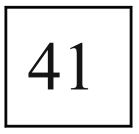

\begin{abstract}
:
OBJECTIVE:

Congenital coronary malformations can cause problems. We present a case where an unusual congenital anomaly was found in old age during work up for acquired coronary artery disease.

\section{METHODS:}

A 70 year old hypertensive, diabetic man with a positive family history for coronary artery disease presented with exertional dyspnoea. He had an ejection fraction of $43 \%$. Following a positive stress test he underwent coronary angiography.

\section{RESULTS:}

Coronary angiography showed all 3 coronary arteries emerging with separate origins from the right coronary sinus. The proximal right coronary artery had a tight stenosis which was stented. Computerised tomographic coronary angiogram showed a dominant right coronary artery with multiple lesions (stent clearly visible). The left circumflex was very large and tortuous with multiple large branches. The left anterior descending artery was very small with septal vessels visible, running a malignant course between the aorta and the pulmonary artery.

\section{CONCLUSIONS:}

Congenital coronary artery lesions can remain undiagnosed until old age when acquired coronary artery disease leads to investigation. Unusual anatomy can lead to challenges for the treatment of acquired disease.
\end{abstract}

\author{
Abnormal vascular malformation possibly preserving \\ myocardial function in the face of conventional acquired \\ coronary artery disease. \\ Maryam Al Kuwari; Willington Francis; \\ Cornelia Sonia Carr; Salem Abujalala
}

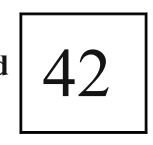

\section{ABSTRACT:}

\section{OBJECTIVES:}

Congenital coronary artery malformations can cause problems. We present a case where a vascular malformation may have preserved the left ventricular function in the face of conventional acquired coronary artery disease.

\section{METHODS:}

A 40 year old hypertensive man presented with an anterior myocardial infarction. Coronary angiography showed triple vessel disease with an occluded left anterior descending artery (LAD). He had an additional vessel emerging from the aorta above the left main stem, which entered a vascular malformation which drained into the LAD (distal vessel only visible on injection of this vessel) and the pulmonary artery (PA). He had an ejection fraction of $39 \%$. Computerised tomographic coronary angiogram (CTA) showed the malformation with feeding vessels from the ascending and descending aorta with a fistulous connection to the LAD and PA.

\section{RESULTS:}

At operation a vascular malformation was found on the surface of the main pulmonary artery with a large discrete vessel running into it; this was ligated in two places. Conventional bypass surgery (saphenous vein to ramus and right coronary arteries, and left internal mammary artery to LAD) was also performed. The patient made an uneventful recovery. 6 weeks post-operatively the patient underwent CTA where no vascular malformation or feeding vessels could be demonstrated.

\section{CONCLUSIONS:}

The fistulous connection to the LAD may have preserved the left ventricular function when the native proximal LAD occluded. At operation the large feeding branch was ligated, which appears to have led to the malformation regressing such that it was no longer visible on follow-up CTA. The patient remains well and asymptomatic.

\section{Management of suture piercel of intracoronary shunt at off pump coronary bypass surgery \\ Muntimadugu T. Babu \\ Sruthi Kurakula}

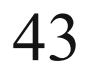

\section{ABSTRACT: INTRODUCTION:}

Insertion of intracoronary shunt (ICS) has been advocated by many during off pump coronary by-pass grafting $(\mathrm{OPCABG})$ that provides bloodless surgical field and of distal perfusion. time. Piercel of shunt is a known problem during OPCABG. Separation of shunt from suture carries risk of cutting or damaging the suture. We are describing our technique of managing the situation.

\section{METHODS:}

Full thickness piercel of shunt is dealt by stretching the shunt on both sides of suture entry site with two forceps. Partial thickness piercel is dealt by pulling the suture forcibly after stabilizing the shunt with forceps. When these measures fail 11 number blade is used to divide the shunt to release the suture from shunt to release the suture from shunt which carries risk of cutting the suture. This has been the practice at our institute for more than a decade.

\section{CONCLUSION:}

Shunt piercel by suture at off pump coronary bypass surgery happens occasionally and the techniques we have described could be made use of in managing removal of suture from the shunt.

Enter description here.

\author{
CORONARY CAMERAL FISTULA : \\ A RARE CASE REPORT \\ Jigar K. Shah \\ Divyesh Rathod
}

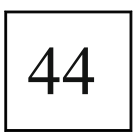

\section{ABSTRACT: INTRODUCTION:}

Coronary artery anomalies can be classified based on their origin, course or termination. Coronary artery fistulas $(\mathrm{CAF})$ are rare anomalies in which either a direct communication between the coronary 
artery and cardiac chamber (coronary-cameral fistula) or a segment of pulmonary and systemic circulation (coronary arteriovenous fistula). The huge right coronary artery aneurysm and posterior descending artery (PDA) draining into the LV is extremely rare.

\section{CASE DETAILS:}

A 52-year-old active, man presented with dyspnoea on exertion NYHA III-IV for 3 months in emergency unit. He was evaluated by trans-thoracic echocardiography and coronary angiography apart from routine biochemistry and haematological investigations. Investigations revealed single large coronary cameral fistula from posterior descending artery to left ventricle approximately $2.5 \mathrm{X} 2$ $\mathrm{cm} 2$. Rest of investigations were within normal limits. After preoperative routine investigations he was planned for surgical correction. Cardiopulmonary bypass was established with aorta and bicaval cannulation. After cardioplegic arrest, longitudinal arteriotomy was made on posterior descending artery, fistulous opening identified. Fistulous opening repaired with 6-0 polypropylene interrupted sutures. Arteriotomy repaired in two layers using 7-0 polypropylene sutures. Post-operative stay was uneventful. Patient was evaluated by coronary angiogram, which confirmed that there was no residual communication. He was discharged on 10th post-operative day in NYHA I. The Patient is in follow up in NYHA I.

\section{CONCLUSIONS:}

Coronary cameral fistula is an infrequently encountered entity, but with clinical suspicion, early diagnosis and timely intervention it can be successfully managed with excellent outcomes.

\section{Effort to the Standardization of Perioperative Nursing for Cardiovascular Surgery \\ Yu Shimizu; Azusa Sawa; Yukari Ishikawa; Fumiko \\ Aizawa; Kaori Tanaka; Hiroko Tanaka}

Can Off-pump coronary artery bypass grafting be a suitable option for patients with Low ejection fraction? Hiroyuki Nakajima

Yumeka Tamai

\section{ABSTRACT:}

\section{INTRODUCTION:}

Off-pump coronary artery bypass grafting (OPCABG) has improved its safety. However, outcomes in high-risk patients such as patients with low preoperative ejection fraction $(\mathrm{EF})$ remain unclear. We discussed the validity of OPCABG as the first choice in low EF patients.

\section{MATERIALS AND METHOD}

From June 2007 to June 2018, 147 patients with EF $\leq 30 \%$ underwent isolated CABG in our institution. Mean age was $66.9 \pm 8.9$ years, and 78.1 $\%$ were male. Mean preoperative EF was $23.8 \pm 4.5 \%$. Of these patients, 132 patients $(89.8 \%)$ underwent OPCABG, whereas 14 patients $(9.5 \%)$ underwent $\mathrm{CABG}$ with cardiopulmonary bypass (ONCABG).

\section{RESULTS:}

Emergency operations were $53.3 \%$ in OPCABG and $71.4 \%$ in ONCABG. $78.6 \%$ of ONCABG were cases converted from OPCABG during operation. 30-day mortality and cardiac mortalities were lower in OPCAB $(2.27 \%$ vs $21.43 \%, \mathrm{p}=0.006)$. OPCABG caused no delay of ICU stay ( 9.6 days vs 14.6 days, $\mathrm{p}=0.76$ ) or IABP removal ( 3.8 days vs 9.0 days, $\mathrm{p}=0.013$ ). Postoperative $\mathrm{EF}$ showed improvement in both groups. Unilateral internal thoracic artery (ITA) was used in all cases. There were no differences in the rates of bilateral ITAs use, complete revascularization and the numbers of anastomosis. Total arterial revascularization was achieved more in OPCABG (35.6\% vs $0 \%, \mathrm{p}=0.007)$.

\section{CONCLUSION:}

OPCABG showed better postoperative mid-term outcomes in patients with low EF than ONCABG. OPCABG with ITA grafts is considered to be first surgical option for patients with low EF. With an eye to longterm outcomes, operation strategies such as bilateral ITAs use and complete revascularization might be considered. 中高吉德栃泉金朝井

Postmyocardial Infarction Ventricular Rupture with Left Ventricular Aneurysm in a 70 years old woman : A surgical challenge.

G.N. Lone; Farooq Ah Ganie months ago and it became 43 cases in 15 months. Based on the acquirement of wide knowledge about cardiovascular surgery, I educated ICU nurses to develop practical thinking for nursing care depending on patient state. I report the result of the effort to the standardization of perioperative nursing for cardiovascular surgery in the ICU.

\section{METHOD:}

17 nurses in the ICU had targeted in this study from July 2017 to September 2018. I investigated the learning rate of the standard evaluation list for each operation stage, the rate of mastering a nursing skill checklist, and the number of incident reports in the cardiovascular surgery during one week after the operation.

RESULT:

The leaning rate of the standard evaluation list according to the stages in an ischemic heart disease, valvular disease, and aortic disease are $58 \%$. Especially in aortic disease is the highest among them with $61 \%$. The rate of a mastering nursing checklist is $56 \%$, and the highest rate of the general knowledge of after cardiovascular operation is $82 \%$. The number of incident reports in the cardiovascular surgery is one case.

CONCLUSION:

The ICU nurses learned the general knowledge after cardiovascular operations, however, it is indicated that they have experienced in difficulty to think practically depending on the situation. It can be inferred that the lecture of postoperative management in preoperative conference by doctor and case studies are efficient for ICU nurses to learn about the grounds of the judgment and an appropriate report as education through a specific scene.

\section{ABSTRACT: \\ BACKGROUND:}

Postinfarction Ventricular Rupture associated with LV Aneurysm is a lethal disease with high mortality rate. Such patients have an in-hospital mortality of more than $90 \%$ if treated without surgery. Even if surgically treated, their mortality rate is still $50 \%$ or more.

\section{CASE STUDY:}

A 70 year old woman, thrombolysed and treated in a Cardiac Care Unit of a Medical College hospital after sustaining myocardial infarction. She continued to be in cardiogenic shock and therefore, was referred to our hospital. She was re-evaluated and diagnosed to have "Coronary Artery disease with Ventricular Septal Rupture (VSR) with Pulmonary Edema, Severe LV dysfunction (15\%) and ventricular arrhythmias. In addition, she had multiple co-morbidities like uncontrolled Diabetes Mellitus, Acute Renal failure, Sepsis, Hypersplenism with Pancytopenia and Hypothyroidism. Coronary angiography showed proximal LAD and mid RCA blocks. She was aggressively managed, dialyzed and optimized under multidisciplinary approach. She was operated under IABP, and CABG, Ventricular Septal Reconstruction and Left Ventricular Aneuysectomy with LV reconstruction was done successfully. Cross clamp time was 90 minutes and total bypass time was 140 minutes. She was removed from ventilator after 24 hours and weaned off from IABP after 72 hours. She was shifted out of Cardiac ICCU after 7 days and discharged from hospital after 3 weeks. Renal failure disappeared 
completely. Latest ejection fraction is $45 \%$ and NYHA Class has improved to Class II.

\section{CONCLUSION:}

Postischemic left ventricular aneurysm with septal rupture continues to pose a surgical challenge with a high mortality. Prompt resuscitation, evaluation, and surgical correction under circulatory assistance is key to success.

\begin{tabular}{l|l|}
$\begin{array}{l}\text { Anomalous right coronary artery origin in a patient } \\
\text { with severe mitral stenosis } \\
\text { Rakesh Seetharaman }\end{array}$ & 48 \\
Vikram M.J. &
\end{tabular}

\section{ABSTRACT: \\ INTRODUCTION:}

Congenital coronary artery anomalies are rare and usually an incidental finding on cardiac imaging. Among coronary artery anomalies, anomalous coronary artery origin from opposite sinus poses a relatively higher risk of sudden cardiac death particularly in the young individuals. Recent angiographic studies have reported a relatively higher incidence of anomalous origin of right coronary artery $(0.17 \%)$ than left coronary artery(LCA) $(0.047 \%)$. Anomalous RCA origin from left sinus of Valsalva with subsequent coursing between aorta and pulmonary artery has been termed "malignant course". Although anomalous RCA origin from opposite sinus is prone to cause angina pectoris, myocardial infarction and sudden death, the risk is small when compared to anomalous LCA origin.

CASE:

We are reporting an unusual case of anomalous RCA origin from left sinus of valsalva with slit like orifice and malignant inter arterial course in a 47 year old gentleman with severe mitral stenosis of rheumatic etilology with severe PAH and systemic hypertension. MDCT showed significant compression of inter arterial course and was demonstrated intra-operatively. The patient underwent RCA translocation to anterior aspect of aorta along with mitral valve replacement and left atrial appendage ligation. Anomalous RCA was flush ligated to aorta and re-implanted to anterior part of aorta using great saphenous vein interposition graft. The patient had uneventful peri-operative and post-operative course and is on regular follow up.

\section{CONCLUSION:}

Anomalous origin of RCA from left sinus of Valsalva is often asymptomatic. Revascularization is recommended only if there is substantial atherosclerosis and documented ischemia. Our case had a unique presentation of anomalous RCA origin from left sinus with compression in the inter arterial course due to dilated great arteries secondary to severe mitral stenosis, PAH and hypertension. Hence the need to address this anomaly along with concomitant cardiac procedure.

\section{Minimally invasive Multivessel Total \\ Arterial Revascularisation (MVTAR) using BITAS in \\ diabetics after CARDia and FREEDOM trials- An observational study}

T.K. Lahiri

Sanjay Kumar

\section{ABSTRACT: \\ OBJECTIVE:}

Are reporting an unusual case of anomalous A minimally invasive Multivessel Total Arterial Revascularisation (MVTAR) technique uses BITAS through sternal sparing approach for diabetics.

\section{METHODS:}

From August 2011 to August 2016, 819 patients underwent off pump minimally invasive MVTAR using BITAS through left minithoracotomy. We analyzed our result of MVTAR with PCI cohort of the 2 prospective randomized tials - CARDia (Coronary Artery Revascularization in Diabetes) and FREEDOM (Future Revascularisation Evaluation in
Patients with Diabetes Mellitus: optimal Management of Multivessel Disease).

\section{RESULTS:}

819 patients comprising of $753(92 \%)$ diabetics had minimally invasive MVTAR using BITAS via a left minithoracotomy with 3.1 average grafts. There were $0.75 \%$ mortalities and $0.6 \%$ had re-exploration for bleeding. The average hospital stay was 3.1 days. Coronary angiograms in $44 \%$ at 12 months revealed patent grafts. $0.4 \%$ required reintervention angioplasty. CARDia trial showed that multivessel PCI is feasible in diabetics and was noninferior to CABG at 1 year. FREEDOM trial showed CABG was superior to $\mathrm{PCI}$ with reduced rates of death $(\mathrm{P}=0.049)$ and myocardial infarction(P\&lt;0.001).

\section{CONCLUSIONS:}

Our off-pump MVTAR using BITA offered excellent graft patency with reduced need of repeat revascularization in diabetics with sterum sparing approach.

Intra- and early postoperative glycemic control in patients with IHD and Diabetes Mellitus type 2 undergoing $\mathrm{CABG}$

Naida Bulaeva

Elena Golukhova

\section{ABSTRACT:}

OBJECTIVES:

The aim of our study was to evaluate glucose monitoring (CGMS) and insulin pump therapy efficacy in patients with IHD and diabetes mellitus type 2 (DM2) undergoing CABG in intra- and early postoperative periods and its impact on early and long-term results.

\section{METHODS:}

The study involved 97 patients (mean age $61+1$ years) with IHD after CABG They were divided into 2 groups: 48 patients with DM2 and 49 patients without DM2. We used CGMS during 72 hours in both groups. In 24 patients with DM2 CGMS was accomplished with insulin pump therapy (MiniMed Paradigm Veo 554/754) to successful glucose control. Also we took blood samples before and then one hour, 12 hours, 7 days after CABG to analyze high-sensitivity C-reactive protein (hs-CRP), leptin, adiponectin levels.

\section{RESULTS:}

During 48 hours after surgery we revealed a trend to high glucose levels according to CGMS in both groups of patients. In patients with DM2 the glucose level was significantly increased (p\&lt; 0,05 ). However, insulin pump therapy (Veo) resulted in glycemic control improvement in early follow-up (72 hours). Moreover, there were no hypoglycemic episodes in patients on insulin pump therapy. Hs-CRP level was significantly lower in patients with DM2 on insulin pump therapy compared to patients prescribed bolus insulin therapy in the early postoperative period (p\&lt; 0,05 ). It is most likely to confirm that insulin pump therapy decreases systemic inflammatory response syndrome (SIRS). We also revealed the trend to lower rate of postpericardiotomy syndrome in patients on insulin pump therapy compared to patients prescribed bolus insulin therapy in the early postoperative period $(\mathrm{p}=0,1)$.

\section{CONCLUSIONS:}

Thus, we demonstrated the CGMS and insulin pump therapy feasibility, safety, and efficacy in patients with DM2 undergoing CABG. We also suggested lower proinflammatory activity and as a result lower PCTS rate in patients on insulin pump therapy compared to patients prescribed bolus insulin therapy.

Flower technique for left ventricular free wall rupture after acute myocardial infarction

Muntimadugu T. Babu

Bavaji Konduri

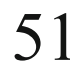

ABSTRACT:

INTRODUCTION:

LV free wall rupture is a dramatic complication of acute MI and presumably responsible for as much as 20 to $30 \%$ of infarct related deaths. Given 
the wide spread use of reperfusion therapy, the rate of occurrence of LV rupture and its clinical characteristics have changed in last one or two decades. Usually it carries high mortality and morbidity

\section{METHODS:}

We used an innovative surgical technique to repair blow out type of LV free wall rupture in these patients. it may be called a flower technique with dropped petals, as the sutures may cut through at the site of rupture after applying pledgetted 3-0 sutures at the site of rupture on either side, to prevent cutting through of these sutures, the area covered with a wide pericardial patch sutures to the heart surface with multiple pledgetted sutures to reduce tension on the site of rupture. The anchoring pledgetted sutures are avoided at site of coronary vessels. So that blood supply to the area covered by the pericardial patch is preserved.

\section{RESULTS:}

We operated three male patients for blow out type of left ventricular(LV) free wall rupture in last two years in our centre. Two of them survived, one patient succumbed to low cardiac output state due to massive infarct. CONCLUSION:

The LV free wall rupture, as a complication of acute MI. Rapid diagnosis and emergency surgery are crucial for successful treatment of patient with cardiac rupture. This technique is easily reproducible with acceptable mortality and morbidity. The most appropriate surgery remains controversial

Sternal wound infections after open cardiac surgery; Outcome and health care burden in a Sri Lankan tertiary care hospital.

Iresh Wijemanne

Dulan Irusha Samaraweera

\section{ABSTRACT: \\ INTRODUCTION:}

Sternal Wound Infection (SWI) after median sternotomy remains a dreaded complication of open cardiac surgery as it leads to patient morbidity, mortality and added burden to health care.

\section{OBJECTIVE:}

To determine the incidence and severity of SWI among patients undergoing open cardiac surgeries in Sri Lanka and to assess the outcomes.

\section{METHODS:}

Data of all open cardiac surgeries performed via median sternotomy by a single cardiothoracic unit at the National Hospital of Sri Lanka were collected prospectively for one year. Patient follow up completed for minimum of one month and SWI cases were defined according to CDC 2017 criteria. RESULTS:

Total of 190 patients underwent surgeries and 186(97.9\%) had their follow up completed. Patients median age was 53(16-74) years and $125(67.2 \%)$ were males. Co-morbidities included $\mathrm{DM}=45.7 \%$, BMI\&gt; $22.9 \mathrm{~kg} / \mathrm{m} 2=52.2 \%$, Smoking history $=30 \%$, Anaemia $=23.7 \%$ and LVEF\&1t;50\%=16.7\%. Coronary revascularisation was the commonest surgery performed $(\mathrm{n}=137,73.6 \%)$, followed by valvular surgeries $(n=27,14.5 \%)$ and congenital heart surgeries $(17,9.1 \%)$.

SWI was clinically diagnosed in $38(20.4 \%)$ patients (Superficial $=34$, Deep $=4$, Mediastinitis $=0)$. Only five $(2.6 \%)$ had infecting organism isolated. Median duration for SWI appearance was 6(2-15) days. Appearance of SWI resulted in use of antibiotics $(n=32$, Intravenous $=27$, oral $=5$ ), additional surgical interventions ( surgical wound debridement $=4$, secondary suturing $=4$ ) and prolonged hospital stay compared to the patients without SWI ( 25 vs 15 days, P\&lt;0.000). Patients with SWI also had higher WBC (17.28 vs $15.8 * 109 / \mathrm{L}, \mathrm{P}=0.08)$ and CRP levels ( 186.7 vs $160.1 \mathrm{mg} / \mathrm{L}, \mathrm{P}=0.017)$ during serial analysis. All achieved wound closure prior to discharge, except for one patient infected with MRSA. No one succumbed to SWI.

CONCLUSIONS:

Appearance of SWI results in additional antibiotic use, wound interventions and longer hospital stay for the patient with added financial cost to health sector. Utilisation of practices known to minimise the development of SWI, such as strict glycaemic control during perioperative period is important to lessen the impact.

Our strategy in a smoker patient with diabetes mellitus and hyperlipidemia having coronary artery disease and low ejection fraction

Levent Yilik; Yuksel Besir; Banu Lafci; Erturk

Karaagac; Gokkurt Yasar; Ali Gurbuz

Kazim Ergüneș

\section{ABSTRACT: \\ BACKGROUND:}

Coronary artery disease is an important factor affecting morbidity and mortality. We presented a smoker patient with diabetes mellitus and hyperlipidemia having coronary artery stenosis and low ejection fraction.

\section{CASE REPORT:}

A 40-years old man was hospitalised in our clinic in November,2017. The electrocardiography showed sinus rythm. In echocardiography, left ventricular end-diastolic diameter (LVEDD), LV end-systolic diameter were $55 \mathrm{~mm}$ and $45 \mathrm{~mm}$, respectively. The ejection fraction was $35 \%$. The angiography showed that left anterior descending and circumflex coronary artery had stenosis.

The patient was operated with general anesthesia. A median sternotomy was made. The pericardium was opened. The patient received a standard heparinization protocol of $300 \mathrm{IU} / \mathrm{kg}$ heparin bolus prior to cannulation, with an additional 10.000 I.U in the pump prime. Target activated clotting time greater than 400 was maintaned throughout bypass.

The aortic cannula was inserted. Cardiopulmonary bypass was established using a two-stage venous cannula inserted in the right atrial appendage, with a membrane oxygenator.

The systemic perfusion was performed using hypothermic bypass strategy of systemic cooling (280C). The systemic perfusion pressure was electively maintained at $55-65 \mathrm{mmHg}$. Cardiopulmonary bypass flow rates were maintained between 2.0 and 2.4 L.min -1 m-2.

Isothermic blood cardioplegia was initially administered antegradely and retrogradely, and thereafter continuosly retrogradely.

The saphenous veins grafts were anastomosed to left anterior descending and circumflex coronary artery.

The mean cross-clamp time and the mean CPB time were 40 min and $85 \mathrm{~min}$, respectively. The patient was discharged 7 days after operation with aspirin and clopidogrel treatment.

\section{CONCLUSION:}

On-pump coronary artery bypass grafting can be performed safely in the patients with diabetes mellitus and hyperlipidemia having coronary artery stenosis and low ejection fraction.

The image change of 28-year patency of saphenous vein graft bypassing to right posterior descending coronary artery (CABG)

Tsung-Po Tsai; Hao-Hung Tsai; Han-Chen Lee;

Chi-Chung Chen; Shou-Ho Tseng; Yi-Liang Wu; Chi Yu Lin;

Shao Lun Hung; Hsuan Chen Ho

Chien Tung lin

\section{ABSTRACT: PURPOSE:}

While a range of venous and arterial grafts are available for revascularization of the right posterior descending coronary artery (RPD), the optimum strategy remains uncertain. In this case, we demonstrated the extreme long-term patency of SVG bypassing to RPD. 


\section{CASE:}

A 76-year-old man with hyperlipidemia was diagnosed with severe triplevessel disease by cardiac catheterization on March 8, 1989. He had undergone CABG surgery with LIMA to LAD, RIMA to RCA, SVG(sequential) to diagonal (DG)/first circumflex (LCX)/distal circumflex (CXD) and SVG to RPD at age of 47 on June 1, 1989. In the following years, patency of all grafts was followed up by 64/640 Multislice Computed Tomography (MSCT) angiography between the years of 2005 and 2017. The patient had complained of shortness of breath when he played golf games since 2012. Repeated 640 MSCT angiography revealed SVG to DG/LCX/CXD occlusion on 2013/09/10. For some unknown reasons, the patient discontinued antiplatelet agents by himself between 2014 to 2015. Later, 640 MSCT angiography revealed the other SVG to RPD occlusion and BIMA graft remained patency on 2017/06/ 20.

\section{RESULTS:}

28-year patency of SVG to RPD and 24-year patency of SVG to DG/ LCX/CXD.

\section{CONCLUSION:}

SVG remains a safe conduit option for the distal RCA and careful evaluation of the SVG before surgery is also important. Besides, using SVG as a bypass conduit to RPD instead of arterial grafts is feasible especially in the elderly who can control his/her underlying diseases well with regular medication and exercise.

\section{Surgical and midterm results for post-infarction vetricular septal perforation repair using simple patch closure through infarcted $L V$ ventricle \\ Hironaga Ogawa; Ikuko Shibasaki; \\ Toshiyuki Kuwata; Yasuyuki Yamada \\ Hirotsugu Fukuda}

\section{ABSTRACT:}

We reviewed surgical and midterm results for VSP repair, mainly using the simple patch closure through infarcted left ventricle. We performed this procedure in 16 patients with VSP between 2009/7 and 2018/2. There were no 30 days mortality, but two hospital deaths because of intestinal bleeding and low output syndrome. Re-repair were performed in two pts for residual shunt. $14 \mathrm{pts}$ went home or transferred to nursing home. There were no late deaths and re-admissions for cardiac related events. Our surgical and midterm results were excellent.

\section{A comparative study on early clinical outcome of good, mild, moderate and severe EF after OРCAB Sudha \\ Ajay}

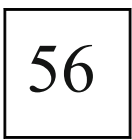

\section{ABSTRACT: \\ BACKGROUND:}

Preoperative left ventricular function is an important determining factor of post operative morbidity and mortality in patients undergoing coronary artery bypass procedure. The purpose of our study is to compare early clinical outcome in patients with good, mild, moderate and severe LV dysfunction after Off pump cardiac bypass surgery

\section{MATERIALS AND METHOD:}

We included 507 patients with $83 \%$ of male and $16 \%$ female, who underwent OPCAB during the period August 2014-2018. Patients were divided into four groups. Group I comprised 245 patients with EF \&gt;55\%, group II had 136 patients with EF55\%-45\%, group III had 94 patients with EF 45\%-35\%, group IV had 32patients with EF\&lt;35\%. All groups were compared on basis of pre-op variables like demographic feaures, intraoperative variables like number of grafts, Emergency conversion to on pump and ICU parameters like ventilation hours, inotropic supports, bleeding, Re-exploration, transfusion requirement, usage of IABP, hospital stay and mortality.

\section{RESULT:}

There were no demographic differences between groups. Intraoperatively, severe LV patients received greater number of grafts $(p=0.0001)$ and insertion of IABP $(p=0.006)$ than other groups. Post operatively, the hours of ventilatory support and post operative stay were longer in severe LV patients (mean 3.5days). There was no intergroup difference in other intra op and post op variables.

Impact of preop usage of Aspirin and clopidogril on clinical outcome of OPCAB patients Sudha, Deepa D, Theodore S, Siva Kumar R, Chokkalingam $\mathrm{M}$

\section{ABSTRACT: BACKGROUND:}

Antiplatelets are prescribed to CAD patients for reducing ischemic and thrombotic complication. The aim of this study is to assess the effect of aspirin and clopidogril on early outcomes in patients undergoing OPCAB. MATERIALS AND METHOD:

The study included 500 patients, who underwent OPCAB from august 2014 to august 2018. Group-A had 120 patients who stopped aspirin and clopidogril with in 3 days of surgery and Group-B had 380 patients who stopped aspirin and clopidogril more than 5 daysof surgery. Both the groups were compared for post operative bleeding, transfusion requirement, re-exploration and secondary parameters like pre-op and post-op $\mathrm{Hb}$, urea, creatinine and ventilator hours, ICU stay, hospital stay and mortality.

RESULT:

After analysis Group-A patients required higher transfusion and reexploration rate than that of Group-B but statistically it was not significant $\mathrm{p}-(0.92$ and 0.58 respectively). Pre-op and post-op Hb, BUN, creatinine and ventilator hours was higher in Group-A patients which was not statistically significant with $\mathrm{p}-(0.83,0.72,0.88$ and 0.92 respectively). The mean ICU stay and the mean hospital stay for Group-B was 1 or 2 days lesser than Group-A with no statistical significance $\mathrm{p}-(0.17$ and 0.10 respectively) with no significant mortality rate $(\mathrm{p}-0.72)$

\section{CONCLUSION:}

Pre op usage of antiplatelets does not have significant impact on early clinical outcome of OPCAB patients. However Group-A patients had higher transfusion rate, re-exploration and longer ICU and hospital staythat was statistically not significant.

Using multi-detector computed tomographic angiography to detect patency of right gastro-epiploic arterial graft utilized in minimally invasive direct coronary artery bypass surgery

Bo-Yang Sie; Tsung-Po Tsai; Yi-Liang Wu; Shou-Ho Tseng;

Chi-Chung Chen

Han-Chen Lee

\section{ABSTRACT: \\ PURPOSE:}

Coronary artery bypass grafting $(\mathrm{CABG})$ is a standard treatment for severe multi-vessel coronary atherosclerotic heart disease. Arterial grafts have been used to achieve a better long-term result. The right gastroepiploic artery (RGEA) will probably prove to be a valuable long-lasting conduit like internal thoracic artery (ITA) graft. We report a 15-year-old patent RGEA graft bypassing to right posterior descending 
coronary artery (RPD) assessed by using non-invasive multi-detector computed tomographic (MDCT) angiography.

\section{METHODS:}

An 84-year-old (1932-2016) female patient, with underlying diseases of hypertension and congestive heart failure (Fc:III), was diagnosed as triple-vessel coronary atherosclerotic heart disease in 1996. The patient had undergone minimally invasive direct coronary artery bypass (MIDCAB) with RGEA graft bypassing to RPD for the most stenotic culprit RCA lesion. At follow-up, non-invasive 64 slices MDCT angiography was performed to assess the graft patency on June 19, 2008. The image showed patent RGEA graft with anastomosis to RPD and mixed plaques at bifurcation of LM, LAD, and LCX. Doppler scan and colorcoded 2-D phonoangiography study were performed on the same day, revealing that the blood flow in RGEA graft was $20-30 \mathrm{~mL} / \mathrm{min}$. Another 64 slices MDCT angiography again demonstrated the patent RGEA graft on Oct. 24, 2011.

RESULTS:

A 15-year-old patent RGEA graft bypassing to RPD (MIDCAB)

CONCLUSION:

RGEA graft is an alternative valuable long-lasting conduit. Its graft patency can be assessed by 64 slices MDCT angiography.

\author{
Anomalous origin of Left Main Coronary Artery \\ from Right Sinus of Valsalva in young presenting \\ as STEMI : A diagnostic and therapeutic challenge \\ Rajeshwar Sharma \\ Abhishek Anand
}

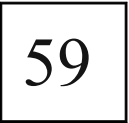

\section{ABSTRACT:}

A 18 year old defense recruit was brought to emergency and diagnosed to have STEMI with positive Trop -T. Upon further evaluation he was found to have anomalous origin of left main coronary artery from the right sinus of Valsalva. He was managed with deroofing of the intramural course of the left main coronary artery thus creating a neo ostia in the left sinus of Valsalva. The anomalous origin of left coronary artery from the right coronary sinus is a rare congenital anomaly which remains undiagnosed and is frequently implicated in cases of sudden cardiac death mainly in the young.

There is no consensus on management of these patients. The treatment offered ranges from purely medical including life style changes to surgery involving translocation of left main origin to coronary artery bypass grafting. We present this case to review this rare but eminently treatable congenital anomaly presenting in adulthood.

\section{Reversal of flow in vertebral artery npredicting post operative cerebrovascular accidents Koushik}

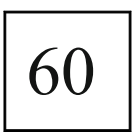

\section{ABSTRACT: \\ BACKGROUND:}

Cerebrovascular accident is one of the dreaded complications following Coronary Revascularisation. Various modalities like extracranial carotid doppler to CT Scan of brain has been tried to identify the at risk population.

\section{CASE REPORT:}

We report three patients who underwent coronary revascularisation. Their pre operative work up was normal. Extracranial doppler showed no disease of the carotid arteries but flow reversal in the left vertebral artery with no proximal subclavian stenosis. Operative and immediate post operative events were uneventful. 2 patients developed altered sensorium and 1 patient developed left upper hemiparesis on the 7 th post operative day. CT scan Brain showed Posterior circulation stroke.

CONCLUSIONS:

Reversal in vertebral artery might be a clue to predict post operative stroke. We recommend these set of patients to undergo pre operative evaluvation and be explained about a higher risk of Cerebrovascular accident post operatively.

Experimental of stent treatment of aortic dissection in a porcine ex-vivo model.

Kenji Aoki; Kazuhiko Hanzawa; Masanori Tsuchida

Yuka Okubo

\section{ABSTRACT:}

\section{OBJECTIVES:}

Stent-graft placement in the aorta has a risk of branch occlusion. Bare stent (BS) can expand the lumen like a stent-graft and keep blood flow in the branch. In some cases of aortic dissection, the false lumen (FL) spontaneously becomes thrombotic and gradually shrinks. The mechanism has not revealed yet. BS placement may create a situation similar to natural FL thrombosis without branch occlusions. We investigated morphological and hydrodynamic changes in the FL by BS placement in ex-vivo porcine models.

\section{METHODS:}

Twenty-six porcine aortic dissection models were used in this study. Under a pulsatile water circulation system, a homemade Nitinol BS was placed in the true lumen (TL) to cover the entry. Changes in morphology and flow velocity in both the lumens were evaluated by ultrasonography. Pressure waveforms in the two lumens were simultaneously monitored. RESULTS:

No significant changes were found in the pressure in both the lumens by the intervention. Mean diameter of the TL significantly increased after the intervention $(1.19 \pm 0.27$ vs. $1.67 \pm 0.20 \mathrm{~cm}, \mathrm{p} \& \mathrm{lt} ; 0.05)$. On the other hand, mean thickness of the FL significantly decreased $(0.83 \pm 0.26$ vs. $0.38 \pm 0.17 \mathrm{~cm}, \mathrm{p} \& 1 \mathrm{t} ; 0.05)$. Flow velocity in the FL significantly decreased $(183.8 \pm 55.5$ vs. $150.9 \pm 56.9 \mathrm{~cm} / \mathrm{s}, \mathrm{p} \& \mathrm{lt} ; 0.05)$.

\section{CONCLUSIONS:}

BS placement changed morphological and hydrodynamic balances in the two lumens. Narrowing with flow stasis may induce thrombosed formation in the FL. Further studies by in-vivo model are required.

Risk factors and outcome assessment of typeII endoleak after endovascular abdominal aortic aneurysm repair

Takeshi Okamoto; Kento Mishima; Ayako Nagasawa;

Yuka Okubo; Ryo Onishi; Takuma Muraoka; Shuichi

Shiraishi; Masanori Tsuchida

\section{ABSTRACT:}

PURPOSE:

Type II endoleaks is the risk factor of aneurysm enlargement in the long term. In this study, we evaluate risk factors of typeII endoleak after endovascular abdominal aortic aneurysm repair (EVAR) and performed coil embolization for the aortic side branches relate to early postoperative typeII endoleak.

\section{METHODS:}

Patency and diameter of inferior mesenteric artery (IMA) and lumbar artery (LA) were evaluated in 95 consecutive patients for three years with abdominal aortic aneurysm who were performed EVAR. Computed tomography was performed one week after EVAR for evaluation of endoleak.

\section{RESULTS:}

TypeII endoleak were noted at 1 week postoperatively in 14 patients (14.7\%). In all cases where type II endoleak was recognized, patency of the lumbar artery was the cause of endoleak. Patency and diameter of IMA was not associated with type II endoleak. The frequency of type II endoleak was significantly higher in patients with 4 or more patent LA $(24.4 \%$ vs $6.0 \%, \mathrm{P}=0.018)$. Diameter of LA was not associated with type II endoleak. Coil embolization of LA was performed for 3 out of 14 cases after EVAR, and these cases were successfully occluded. 


\section{CONCLUSION:}

The outcome of type II endoleak treatment at our hospital was good. 4 or more patent LA were associated with type II endoleak one week after EVAR. In these cases, it is considered that follow up is especially necessary.

\section{TEVAR IN THE TREATMENT OF AORTIC ANEURYSM WITH RIGHT HEMOTHORAX}

Amaresh Rao; Shrikant; P.S.S. Gopal; Nikhil Dixit

\title{
ABSTRACT:
}

A 40 year old male presented to with shortness of breath \&amp; chest pain. Sudden increase in shortness of breath. Vitals were stable. Auscultation revealed loss of breath sounds on the right side. CT scan of chest revealed a massive right pleural effusion and aneurysm of the descending aorta. TEVAR was done to the patient which resulted in complete resolution of the hemothorax. Patient settled well. The post TEVAR period was uneventful.

\author{
Non compliance with medication in cardiac transplant \\ recipient with suicidal intent \\ Bhagavathula K.S. Sastry \\ Sruthi Kurakula
}

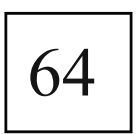

\section{ABSTRACT: \\ INTRODUCTION:}

Incidence of Non compliance with medications including immunosuppressants is a known to be around 20 percent among cardiac transplant recipients during first 12 months. We are reporting a case of non compliance with medications with possible suicidal intent.

\section{CAE REPORT:}

A 27 year old male software engineer who had end stage heart failure following massive anterior wall Myocardial infarction underwent cardiac transplant successfully at our centre. Pre transplant psychological assessment by psychiatrist did not reveal any psychological problems and had normal postoperative course

3 months following transplant he joined back his work and was able to function normally. He was on regular follow up. 11 months after transplant he was admitted to hospital with acute rejection and medical management as not successful and he died Death was secondary to consequences of acute rejection. Information from colleague at work place and family suggested that he had a failure of a relationship and possibly stopped medications with suicidal intent

DISCUSSION:

Non compliance with medications after transplant is a known and significant problem. It could be secondary to psychological issues, financial constraintas, alcoholism and inadequate family support. In our patient it appears to be related to be related to relationship issues and suicidal intent. Apart from pre operative psychological assessment post op on going psychological assessments and close relation with patient and family could help in recognizing issues which could have impact on post operative course and survival.

\section{Early Outcomes of Heart Transplantation:A Single Tertiary Center Experience in Less developed area in Western China \\ Liudaxing; Zhouhao; Liangguiyou; Dianke \\ Renyan}

\section{ABSTRACT: \\ OBJECTIVE:}

To summarize the experience of 14 cases of orthotopic heart transplantation for end-stage heart diseases in a single teriary center experience in less developed areas.

\section{METHODS:}

Retrospective analysis of 14 patients, include 12 males and 2 females who received orthotopic heart transplantation for end-stage heart disease in the first affiliated hospital of zunyi medical college from March 2017 to July 2018 and relevant data of pre-operative and post-operative monitoring were analyzed. The patients age from 12 to 57 years old, the weight from 40 to $80 \mathrm{Kg}$, there are 10 of dilated cardiomyopathy, 1 of hypertrophic obstructive cardiomyopathy, 1 of heart failure after valve replacement, 1 of right heart failure after Ebstein anomaly operation. The left ventricle ejection fraction are less than $25 \%$. The heart transplantation all were used double chambers transplantation. After transplantation, the anti-rejection drug use cyclosporin a/tacrolimus, MMF and dexamethasone.

\section{RESULT:}

13 cases include 1 paediatric patient were recovered and discharged. There are 1 patient had post-operation bleeding and undergone the second operation;1 patient had arrithmymia and acute renal failure, even cardiac arrest, but successfully rescued; 1 patient died due to acute rejection even with the ECMO assisted 2 weeks. At half a year of follow up after operation, the survival rate is $92 \%$, and the one year survival rate is $77 \%$. CONCLUSION:

Even in less developed areas to enhance the organ protection strict hemodynamic monitoring, rational use of immunosuppressive medicine aggressive treatment of the complications heart transplantation is an safely option to the end-stage heart disease patients.

Case report: hypothermia cause cardiac arrest after orthotopic heart transplant in child

Liudaxing; Zhouhao; Liangguiyou;

Dianke;

Renyan

\section{ABSTRACT:}

\section{RATIONALE:}

This case report describes an unusual case of hypothermia cause cardiac arrest after heart transplant in a child.

\section{PATIENT CONCERNS:}

A twelve years old boy complained about tired, shortness of breath, anhelation, edema of face and both lower limbs and recurrent vomiting for eighteen months.

\section{DIAGNOSES:}

The patient was diagnosed as dilated cardiomyopathy and whole heart failure. The whole heart enlargement, New York Heart Association function class IV and pulmonary arterial hypertension.

\section{INTERVENTIONS:}

The patient underwent heart transplant from a brain death donor. The operation went smoothly. On the second day after surgery, the patient gradually showed oliguria, increased CVP, Facial edama, secondary to acute renal insufficiency due to right cardiac insufficiency. we treated with continuous renal replacement therapy. After treatment, the CVP declined, and edama reduced. But after 14 hours, the patient suddenly appeared arrhymia, the heart rate slows, unstable blood circulation and eventually cardiac arrest happened. Immediately bedside chest open and heart pacemaker implantated, intrathoracic cardiac massage initiated, and intermittent epinephrine injected, but, the heart got no response, at the same time, the pupil dilated and light reflex disappeared, we fell the whole body very cold and the surface temperature of the heart is also low. The temperature coud not be measured. We assumed that the cardiac arrest was induced by the hypothermia, so immediately rewarmed the heart initiated by infusing warm water $\left(37^{\circ} \mathrm{C}\right)$ to the pericardial sac. After that, the patient was given intravenous epinephrine again and the cardiac autonomic heart rhythm recovered, the circulation gradually stabilized, the temperature was 35 degree centigrade. The cardiac pulmonary resuscitation was successful. After 12 hours, the consciousness of the patient recovered. After 1 week of strict hemodynamic monitoring, the patient successfully wean off the ventilator, finally wean off continuous renal replacement therapy. 


\section{OUTCOMES:}

After the rehabilitation, the patien recovered smoothely, and being discharged home uneventfull without cerebral complications.

Near-infrared spectroscopy (NIRS) monitoring for the early detection of limb ischemia in adult patients undergoing peripheral VA-ECMO

Sanghoon Jheon

Dong Jung Kim

\section{ABSTRACT: \\ OBJECTIVE:}

We previously reported our one-year experience of near-infrared spectroscopy (NIRS) monitoring. For the past five years, our institution has routinely used NIRS monitoring in VA-ECMO patients for the early detection of limb ischemia.

\section{METHODS:}

We retrospectively reviewed medical records of patients supported with VA-ECMO between September 2013 and August 2017. Distal perfusion catheterization was performed only in patients who exhibit a significant drop in the regional oxygen saturation $(\mathrm{rSO} 2)$ values provided by the NIRS system.

RESULTS:

120 adult patients were included and 36 patients underwent distal perfusion because their $\mathrm{rSO} 2$ values were significantly dropped. No patient underwent fasciotomy. There was no case requiring amputation.

\section{CONCLUSIONS:}

We think that NIRS monitoring is a useful and reliable method for the early detection of limb ischemia in adult patients undergoing peripheral VA-ECMO.

\author{
A rare case of fungal osteomyelitis of ribs and chest \\ wall abscess in a patient after orthotopic \\ heart transplantation \\ Alla Gopala Krishna Gokhale \\ Venkata Ramana Koduru
}

\section{ABSTRACT: \\ OBJECTIVE:}

To present a rare occurrence of fungal osteomyelitis of ribs and chest wall abscess in a heart transplant patient and it's management

CASE STUDY:

A 45 year old man underwent heart transplantation for dilated cardiomyoapathy with severe left ventricular dysfunction. He had a smooth post operative recovery. He came regularly for follow-up and tolerated medication well. 10 months after surgery he came with severe chest pain on right side of chest. Clinical examination was unremarkable and chest $\mathrm{x}$ ray and CT scan of the chest did not reveal any abnormality. He was treated conservatively. A month later he came to ER with history of seizures and severe right sided chest pain. A CT scan of the brain showed a chronic infarct in right MCA territory and acute bilateral cerebellar infarcts. Clinically, he had a large $10 \mathrm{cmx} 5 \mathrm{~cm}$ soft, fluctuating swelling over $5,6,7$ ribs in right mid axillary line. CT scan of the chest showed a large swelling in the right lateral chest wall and erosion of the right 6th rib. Needle aspirate of swelling showed fungal hyphae. At surgery, the chest wall muscles including healthy margins were removed, followed by partial excision of right 5,6,7 ribs. The infection did not invade the pleural cavity or the lung. The wounds healed well and patient was discharged with routine medication and anti fungal antibiotics. The pus in the abscess cavity, the necrosed muscle and the ribs showed fungal hypahae on microscopy. Histopathology of the ribs showed osteomyelitis.

\section{CONCLUSION:}

This is a rare presentation of fungal infection. Delayed infections in immunocompromised post heart transplant patients can occur and should be dealt with promptly and methodically.

Video Assisted Thoracoscopy- A viable approach to mediastinal ectopic Parathyroid Adenoma.

Shaji Palangadan

Vijay Cherian

ABSTRACT:

\section{INTRODUCTION:}

Primary hyperparathyroidism (PHPT) is the most common cause of chronic hypercalcemia. Patients present with varying symptoms ranging from kidney stones, peptic ulcer, decreased bone density to even anxiety disorders. Parathyroid adenomas cause $80 \%$ of PHPT. Among these, Mediastinal ectopic parathyroid adenoma(MEPA) are rare.

\section{CASES}

We present 2 cases of MEPAs. Both patients had elevated serum calcium levels and serum parathormone levels. The first case had history of kidney stones while the second case was asymptomatic. Diagnostic confirmation and localisation was done by MIBI scan. Ultrasound scan was done to rule out disease in the neck but the second case had features of MNG. We decided to proceed with VATS for resection and use IOPTH(INTRA OPERATIVE PARATHYROID HORMONE) assay to assess completion of surgery. The patients were successfully managed with VATS and IOPTH assay satisfying MIAMI criteria with follow up by calcium level monitoring. DISCUSSION:

High level of suspicion should be maintained while evaluating chronic hypercalcemia for the presence of parathyroid adenoma, especially at ectopic locations. MEPA is a rare diagnosis and requires thorough evaluation and proper localisation before planning surgery.

\section{CONCLUSION:}

A proper localisation of MEPA is of paramount importance for planning surgery. VATS is a safe, effective and feasible technique for minimally invasive resection of MEPA. IOPTH assay is a useful tool to confirm completion of resection. Armed with all these techniques VATS proves to be indispensable and should be offered as the first line treatment.

Combined Resection of Sub Aortic Membrane and modified Ravitch procedures with Minimally Invasive Technique in a patient with Pectus Carinatum Ratnamalika Kumar; Rajneesh Malhotra; Bhuvnesh Kansara; Anuj Sangal

\section{ABSTRACT:}

Pectus carinatum may exist as an isolated lesion or in association with genetic syndromes such as Marfan syndrome, Poland's syndrome, Di George's syndrome. Associated Subaortic membrane with Pectus carinatum deformity has not been documented in the literature.

Surgical correction of pectus carinatum is usually performed for cosmetic reasons and sometimes for physiological reasons. Cardiopulmonary complications resulting from mechanical compression by the deformed chest wall may be an indication for surgical correction, especially in patients with underlying cardiac disease. Cardiac compression can contribute to postoperative hemodynamic instability if the pectus deformity is left uncorrected.

We report the successful management of a simultaneous correction of Pectus carinatum Chondro-gladiolar symmetrial type, using Sternal AO plate at lower sternal osteotomy and KLS Martin plates at mid and upper sternal osteotomies and the resection of underlying Sub-aortic membrane performed through a minimally invasive approach (right anterior minithoracotomy and central cannulation). 
Comparative study of short-term cardiovascular autonomic control in cardiac surgery patients who underwent coronary artery bypass grafting or correction of valvular heart disease

Olga Bockeria; Anton Kiselev; Artak Ispiryan; Maria

Sokolskaya; Vladimir Shvartz

\section{ABSTRACT: \\ INTRODUCTION:}

Our aim was to perform a comparative study of short-term cardiovascular autonomic control in cardiac surgery patients who underwent coronary artery bypass grafting $(\mathrm{CABG})$ or surgical correction of valvular heart disease (SCVHD).

\section{METHODS:}

The synchronous 15 minutes records of heart rate variability (HRV) and finger's photoplethysmographic waveform variability (PPGV) were performed in 42 cardiac surgery patients ( 12 women) aged $61.8 \pm 8.6$ years (mean \pm standard deviation), who underwent $\mathrm{CABG}$, and 36 patients (16 women) aged $54.2 \pm 14.9$ years, who underwent SCVHD, before surgery and in 5-7 days after surgery. Conventional time and frequency domain measures of HRV and index S of synchronization between the slow oscillations in PPGV and HRV were analyzed. We also calculated personal dynamics of these indices after surgery.

\section{RESULTS:}

We found no differences $(\mathrm{P}>0.05)$ in all studied autonomic indices (preoperative and post-surgery) between studied patients' groups, except for the preoperative heart rate, which was higher in patients who underwent SCVHD $(\mathrm{P}=0.013)$. We have shown a pronounced preoperative and post-surgery variability (magnitude of inter-quartile ranges) of all autonomic indices in studied patients. In the cluster analysis based on cardiovascular autonomic indices (preoperative and post-surgery), we divided all patients into two clusters (38 and 40 subjects) which did not differ in all clinical characteristics (except for the preoperative hematocrit, $\mathrm{P}=0.038$ ), index $\mathrm{S}$, and all post-surgery HRV indices. First cluster (38 patients) had higher preoperative values of the $\mathrm{HR}, \mathrm{TP}, \mathrm{HF}$, and $\mathrm{HF} \%$, and lower preoperative values of the $\mathrm{LF} \%$ and $\mathrm{LF} / \mathrm{HF}$.

\section{CONCLUSION:}

The variability of cardiovascular autonomic indices in on-pump cardiac surgery patients (two characteristic clusters were identified based on preoperative indices) was not associated with their clinical characteristics and features of surgical procedure (including cardioplegia).

\section{Mutual Dynamics of Synchronization of Low-frequency Oscillations in Circulation Vegetative Regulation and Indicators of Variability of the Heart Rhythm in Patients after Operations with Artificial Circulation in the Early Postoperative Period Anton Kiselev; Artak Ispiryan; Maria Sokolskaya; Olga Bockeria Vladimir Shvartz}

\section{ABSTRACT: \\ OBJECTIVE:}

To investigate peculiarity of co-operative dynamics of autonomic circulatory control indices, including synchronization of low frequency oscillations in heart rate variability (HRV) and photoplethysmogram (PPG), in patients after surgery with cardiopulmonary bypass $(\mathrm{CPB})$ in the early postoperative period.

\section{METHODS:}

62 patients, who had survived surgery with CPB, were included in the study (40 men, 22 women, aged 57,9 7,6 years). The synchronous registration of cardiointervalogram and PPG during 15 minutes was performed in all patients before surgery and 5-7 days after surgery in the morning. We calculated the total percentage of phase synchronization of low frequency rhythms in HRV and PPG (index S), heart rate (HR) and some assessments of HRV (SDNN, TP, HF\% и LF\%). We calculated the dynamics of these indices after surgery: $\Delta \mathrm{S}, \Delta \mathrm{HR}, \Delta \mathrm{SDNN}, \Delta \mathrm{HF} \%, \Delta \mathrm{LF} \%$.

\section{RESULTS}

After surgery, index $\mathrm{S}$ decreased $(\Delta \mathrm{S} \& 1 \mathrm{t} ;-5 \%)$ in 26 patients, has not changed $(\Delta \mathrm{S}-5 \%$ to $+5 \%)$ in 18 patients and increased $(\Delta \mathrm{S} \& \mathrm{gt} ;+5 \%)$ in 18 patients. Patients with $\Delta \mathrm{S} \& 1 \mathrm{t} ;-5 \%$ had the highest value of the index $S$ and the lowest of TR, relatively to other patients (p\&lt;0.05). In all study phases, the strong correlation was shown for SDNN and TP ( $\mathrm{R}=0.86$ and 0.90 , p\&lt;0.05), and the medium correlation was shown for index $\mathrm{S}$ и $\mathrm{TP}(\mathrm{R}=-0.41$ and 0.53 , p\&lt;0.05). The multiple regression correlation was identified between $\Delta \mathrm{S}$ and following indices: $\mathrm{S}, \mathrm{HF} \%$, blood glucose, cardioplegia, $\triangle \mathrm{TP}, \triangle \mathrm{HF} \%$.

\section{CONCLUSIONS:}

We revealed the heterogeneity of patients with coronary artery disease, requiring surgery with $\mathrm{CPB}$, using source autonomic status and its dynamics in the early postoperative period. We showed multiple dependent postoperative dynamics of the index $\mathrm{S}$ with some indices, which characterize the initial status of the patient, peculiarities of the operation and dynamics of autonomic status in the early postoperative period.

Feasibility and Training of Cardiac Homograft Trimming by Surgical Assistant

Muhammad Ashraff Baruji; Sharifah Noraida Syed

Nong; Siti Laura Mazalan;

Mohd Nazeri Nordin

\section{ABSTRACT: \\ BACKGROUND:}

IJN cardiac homograft kick off its program in 1997 in respond to the need of a biological RV-PA conduit which size are small enough to suit the pediatric population. Institut Jantung Negara (IJN) is the only center that provide the cardiac homograft services in the country. Traditionally, cardiac homograft are trimmed into individual (aortic and pulmonary conduit) by the cardiac surgeon.

\section{OBJECTIVE:}

To highlight the training course and outcome of a surgical assistant in cardiac homograft training.

\section{METHODOLOGY:}

Steps taken to train a surgical assistant for cardiac homograft trimming are as follows:

Understanding of normal cardiac anatomy and morphology of different congenital abnormalities involving aortic or pulmonary artery.

Tagging along for harvesting of homograft at the donor hospital and assisting during the en bloc dissection, bagging the organ under aseptic procedure and transport of the organ to IJN.

Assisting and performing homograft trimming under supervision. Credentialing of the trained surgical assistant.

\section{RESULT:}

Single surgical assistant has been trained from 2015 to 2017 . Total number of homograft harvested is 8 (assisting $=5$, supervised $=3$ ), total number of homograft trimmed is 12 (assisting $=3$, supervised $=$ 4 , independent $=5$ ). Duration taken for harvesting under supervision is 60 minutes (mean) and duration taken for homograft trimming independently is 90 minutes (mean) with good conduit quality. Outcome of the training is feasible with good conduit quality and good valve quality.

\section{CONCLUSION:}

Cardiac homograft trimming requires a high anatomical knowledge combined with fine surgical skills due to its complexity and technical difficulties to shape the conduits into the good conduits. However, it can be safely and efficiently performed by surgical assistant after training and nurtured adequately. 
Primary intimal sarcoma of the left atrium

Yoshiei Shimamura; Kazuma Maisawa

\section{ABSTRACT: \\ INTRODUCTION:}

Intimal (spindle-cell) sarcomas are exceptionally rare and are highly aggressive cardiac tumors, which usually arise from the pulmonary artery or the thoracic aorta.

\section{BACKGROUND:}

We describe a case of a young woman which presented primary intimal sarcoma of the left atrium mimicking mitral stenosis.

CASE:

A 26-year-old woman with no previous medical history presented with exertional dyspnea, orthopnea, and fatigue. A systolic murmur was present at the apex, and the chest X-ray revealed pulmonary congestion and bilateral pleural effusion. Echocardiography revealed a large mass occupying the entire left atrial cavity, $60 \times 50 \mathrm{~mm}$ in size, prolapsing towards the left ventricle. The tumor caused mitral obstruction with preserved left ventricular ejection fraction. The patient underwent an urgent surgery under the diagnosis of decompensated heart failure. Gross appearance of the resected tumor was white, elastic, and egg-shaped with smooth surface. It was attached to the posterior wall of the left atrium just close to the orifices of right pulmonary veins. No stalk was found. Histologically, the specimen revealed a hypercellular malignant spindlecell neoplasm with fascicular growth pattern and areas of necrosis, with associated lymphocytic focal infiltrate. The immunostains were positive for Desmin, NSE, S100, CD56, CD34, CD117 and MDM2, and negative for Calretinin, Myoglobin, HMB45 and alpha SMA, compatible with a diagnosis of intimal sarcoma.

\section{CONCLUSION:}

Sarcomas should be considered in the differential diagnosis in patients with suspected mitral valve disease. Echocardiography should be performed as early as possible to establish a prompt diagnosis and management.

\section{RUPTURED FEMORAL}

\section{ARTERY PSEUDOANEURYSM - A}

RARE COMPLICATION POST

\section{FEMORAL CANNULATION IN OPEN HEART}

SURGERY: A CASE REPORT

Abu Yamin Khamis

Law Poh Suan

\section{ABSTRACT:}

Ruptured femoral artery pseudoaneurysm (FAP) is a rare yet lifethreatening complication after femoral cannulation. Here we present a case of ruptured FAP post femoral cannulation for redo coronary artery bypass grafting $(\mathrm{CABG})$.

\section{CASE REPORT:}

A 68 year old gentleman who had recent redo-CABG via femoral cannulation for cardiopulmonary bypass. He was presented with hypovolemic shock and bleeding from the groin wound 1 week after the surgery. On examination, the wound looked erythematous with profuse bloody discharge without pulsatile mass felt. He was brought to operation theatre immediately. Intraoperative finding was infected anterior wall of superficial femoral artery and ruptured of the aneurysmal wall. We performed embolectomy and external iliac artery to superficial femoral artery(EIA-SFA) bypass. The infected area was ligated proximally and distally. The infected wound was left open and healed by secondary closure.

\section{DISCUSSION:}

FAP is a devastating rare complication which may occurred after femoral catherization percutenously or via femoral cutdown. Ruptured FAP even cause higher mortality and morbidity. The incidence of ruptured FAP post femoral cannulation in open heart surgery is unknown. There was no similar case report found during literature search. The causes of FAP can be classified into 2 broad groups: patient or technical factors. The awareness of diagnosing FAP is crucial which can prevent ending in dreadful condition.

\section{CONCLUSION:}

High index suspicious of ruptured FAP is important while dealing with bleeding groin wound. Immediate surgical intervention is the cornerstone of treatment while managing ruptured FAP with hypovolemic shock.

A concomitant intramyocardial and pulmonary hydatid cyst: A rare case report.

Prashant Mishra; Kuntal Surana

\section{ABSTRACT:}

Cardiac hydatidosis has been reported infrequently even in countries where hydatid disease is endemic: $0.5-2 \%$ in comparison with liver $(65 \%)$ and lungs $(25 \%)$. The diagnosis is difficult because of long latency of disease. 1 However owing to increased travel all over the world, it can be found anywhere, including developed countries. Hence we report a case of a young lady with intramyocardial and pulmonary hydatid cyst, which was surgically removed.

Common tumor in uncommon location Vivek V. Pillai; Sriraman

\section{ABSTRACT:}

60 year old female patient presented to OPD with complaints of generalised weakness, facial puffiness, and palpitation since 2 months. On evaluation ECHO showed 70x48mm pedenculated RA mass, Obstructing tricuspid inflow, Mass moving in and out of tricuspid valve. Patient was taken up for emergency surgery, after excising the mass via RA approach tricuspid valve appeared to have dilated annulus with unhealthy annulus and leaflet tissue, severe TR was present, this brought in a suspicion of infective endocarditis. De Vegas annuloplasty was done for TR, Post operative TEE showed severe TR. Options of annuloplasty ring and valve replacement was ruled out in view of infective endocarditis features. Patient had an uneventful post operative course. Histopathology report turned out to be gram positive cocci in myxoma surface, infected cardiac myxoma. On first month follow up ECHO done showed decrease in TR and improvement in RV function hence patient is kept on routine follow up. Infected myxoma is in itself a rare condition only few cases reported in literature. Infected myxoma in RA is an extremely rare.

\section{MALIGNANT ROUND CELL TUMOR OF LEFT ATRIUM- CASE REPORT \\ Chaitanya Raut; Vijay Shewale}

\section{ABSTRACT:}

\section{INTRODUCTION:}

Primary tumor of heart is rare pathology with frequency on autopsy less than $0.02 \%$. Ninety percent are benign, out of which myxoma is commonest. Sarcoma is most common malignant tumor. Most malignant tumor are asymptomatic initially and becomes symptomatic when size is massive and causes heart failure and pulmonary edema. Primary line of management in such cases is surgical excision of tumor and adjuvant chemotherapy. We hereby presenting a case of malignant round cell tumor of left atrium.

\section{CASE REPORT:}

20 years old female presented with acute pulmonary edema in emergency room in april 2018, ECHO showed large mass occupying whole of left atrium and obstructing mitral valve with minimal blood flow across it, mitral valve normal, severe tricuspid regurgitation and severe pulmonary hypertension. Patient was immediately taken for left atrial mass excision. Intraoperative finding was large hard mass attached to left atrial surface densely, extending across mitral valve in left ventricle. Histopathology 
report showed malignant round cell tumor. IHC study done which showed CD 99 positive, vimentin positive, CD 45 - weak, desmin, myogenin and S 100 negative. Post operatively patient developed severe mitral regurgitation, underwent mitral valve replacement. Patient expired on day 3 because of persistent cardiogenic shock.

\section{CONCLUSION:}

Primary cardiac malignant tumor can be easily confused with a benign myxoma, therefore abnormal imaging features should raise suspicion for a cardiac malignancy. Due to the usually aggressive behavior of this tumor, early detection and aggressive surgical resection may result in a longer survival time.

\author{
Acute limb threatening ischemia following radial \\ coronary angiogram \\ Sujit Kumar Mohanti \\ Jaideep Trivedi
}

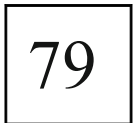

\section{ABSTRACT:}

Transradial access for cardiac catheterization is now widely accepted among the invasive cardiology community as a safe and viable approach with a markedly reduced incidence of major access related complications compared with the trans femoral approach. As this access technique is now being used more commonly for cardiac catheterization, it is of paramount importance to be aware of its complications and to trans radial access include asymptomatic radial artery occlusion, pseudo aneurysm and radial artery perforation are rarely reported complications of the trans radial approach.

We here report a rare complication in a patient who underwent routine coronary angiogram through radial route and develoed severe compartment syndrome and limb threatening ischemia following the procedure. After surgical consultation, immediate limb salvaging extensive fasciotomy was done followed by skin grafting two weeks later. Finally patient was discharged with viable functioning upper limb. Such limb threatening ischemia following radial angiogram is not reported till date.

\section{CONCLUSION:}

Access-site complications can be minimized by avoiding multiple punctures, selection of smaller sheaths, gentle catheter manipulation, adequate anticoagulation, use of appropriate compression devices and avoiding prolonged high pressure compression. In addition, careful observation for any ominous signs such as pain, numbness and hematoma formation during and in the immediate post procedure period is essential in the prevention of catastrophic hand ischemia. Alternate arterial access should be considered in patients with abnormal Allen test findings, poor hand perfusion, or physical examination results suggesting that the radial artery is too small for sheath insertion. Any symptoms or signs suggesting compartment compression should result in early surgical consultation for limb salvaging fasciotomy

\section{Del Nido Cardioplegia V/s St. Thomas Hospital Solution} II -Types of Cardioplegia in adult cardiac surgery Chaitanya Raut

Ritesh Mate

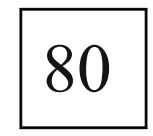

\section{ABSTRACT: \\ INTRODUCTION:}

St.Thomas' Cardioplegic solution No.2 (ST) has been a popular crystalloid cardioplegia among cardiac surgeons; however, it must be administered repeatedly at short intervals during the surgery.

Increases in myocardial acidosis between the doses have been noted, affecting the post operative outcome adversely.

In the early 1990s, Dr. Pedro del Nido and his team at the University of Pittsburgh developed a cardioplegic solution to address the specific needs of immature myocardium encountered in neonatal and pediatric cardiac surgery. DN's potential practical advantage is the fact that it provides along period of arrest before a subsequent dose is needed.
AIM:

The aim of this study is to evaluate the efficacy and safety of DN as compared to ST in adults undergoing elective coronary artery bypass grafting $(\mathrm{CABG})$ and double valve replacement (DVR) surgery.

\section{RESULTS:}

The aortic cross clamp $(110.15 \pm 36.84$ vs $133.56 \pm 35.66)$ and cardiopulmonary bypass $(158.60 \pm 39.92$ vs $179.81 \pm 42.36)$ times during both CABG and DVR surgery using delNido cardioplegia were significantly less as compared to the STgroup.

Administration of DN Cardioplegia resulted in a significantly lower number of required doses as compared to the ST group.

Postoperatively, mean LVEF showed a significant fall of $3.3 \%$ among the ST group and a non insignificant rise of $2.5 \%$ among the DN group as compared to preoperative values. This change in LVEF was mainly observed in the CABG group.

$52 \%$ patients in the ST group and $46 \%$ patients in the DN group needed Inotropic drug support but the difference between the two groups was not statistically significant.

\section{CONCLUSIONS:}

The use of DN Cardioplegia reduced the time of CC and CPB as well as the need for repeated doses of cardioplegia.

DN Cardioplegia provided potentially better myocardial protection in view of the preserved LVEF values, with a safety profile and mortality comparable to ST Cardioplegia.

\section{Re-expansion pulmonary edema after drainage} of less than 1 liter: A case report and literature review Clarence Pio Rey Yacapin

Alyza May T. Taguilaso

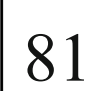

\section{ABSTRACT: \\ INTRODUCTION:}

Re-expansion pulmonary edema (RPE) is a rare complication that occurs after drainage of pleural effusion with an incidence rate of less than $1 \%$ but has a mortality rate of up to $20 \%$. Guidelines and expert opinion recommend limiting drainage of fluid to $1000-1500$ milliliters to prevent RPE. This case, however, presents a patient with symptomatic re-expansion pulmonary edema after drainage of less than 1000 milliliters of pleural fluid.

\section{CASE:}

A 47-year old female diagnosed with right breast cancer stage III who underwent modified radical mastectomy developed pleural effusion on the right hemithorax. Ultrasonography quantified the fluid amounting to 1200 milliliters. The patient underwent tube thoracostomy and up to 750 milliliters of serous fluid was immediately drained. An hour after the procedure, the patient developed dyspnea, tachypnea and oxygen desaturation of up to $88 \%$. Chest radiograph showed hazy ground-glass infiltrate on the right lung. Intubation and supportive management was initiated. After several days of supportive care, the edema resolved and the patient was taken off ventilator support.

\section{DISCUSSION:}

It is frequently stated in literature that large volume drainage may be a risk factor for RPE. However, several studies have reported the safety of large-volume thoracentesis. This case shows that even low volume drainage can still cause RPE. Other risk factors including chronicity of effusion, ischemia-reperfusion injury with subsequent cytokine release and increased capillary permeability should be further investigated.

\section{CONCLUSION:}

Identifying risk factors and early clinical signs even with lowvolume drainage is necessary in order to initiate immediate management. 
Left ventricular rhabdomyoma in adults - rare case report

Nishit Udapi

\section{ABSTRACT:}

\section{INTRODUCTION:}

Primary cardiac tumors are rare variety of cardiac lesions with reported incidence of $0.017 \%$ to $0.03 \%$ total number of autopsies studied; of which $50 \%$ are myxomas and $20 \%$ of rhabdomyomas.

\section{BACKGROUND:}

Rhabdomyoma most common cardiac tumors found in paediatric age group mainly during infancy. Literature reports rarely about the rhabdomyoma in adults. One case report of right ventricular rhabdomyoma is reported. In all these cases, presentation was either cardiac symptoms or incidental detection.

CASE:

We hereby report a rare case of rhabdomyoma of left ventricle in an adult presented as cerebro vascular accident. This presentation is not reported in the literature. We hereby discuss our approach and experience in treating the left ventricular rhabdomyoma.

\&nbsp;

\section{CONCLUSION/CLINICAL RELEVANCE:}

Non cardiac presentation especially stroke with left ventricular thrombus like lesion on echocardiography can be a rhabdomyoma. With aggressive surgical intervention, it is possible to achieve complete clearance of the tumor. It is necessary to keep this differential diagnosis in mind while dealing with suspected left ventricular thrombus. Hence after removing the lesion, a specimen should be sent for histopathological examination. \&nbsp;

Enter description here.

\section{COLD ALERT DURING}

\section{CARDIOPULMONARY BYPASS - CASE REPORT}

Vaibhav Shah

Vijay Shewale

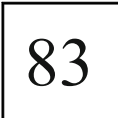

\section{ABSTRACT: \\ BACKGROUND:}

Cold agglutinins are immunoglobulin $\mathrm{M}$ antibodies that react with red cells aat low temperatures, resulting in cold hemagglutinin disease characterized by agglutination of red cells with resultant microvascular occlusion and hemolysis. This autoimmune response has significance in cardiac surgery where hypothermic cardiopulmonary bypass and cardioplegia is required. Activation of cold agglutinin antibody during cardiopulmonary bypass can lead to massive hemagglutination, hemolysis, and microvascular thrombosis. This can manifest intraoperatively as intracoronary thrombosis, incomplete cardioplegic delivery or high pressures in the circuit. Clinical manifestation can include cerebral or myocardial infarction, hepatic or renal failure, and hemolysis. We hereby presenting a case of mitral valve replacement in patient with cold agglutinin antibody positive.

\section{CASE REPORT:}

45 years old female known case of rheumatic heart disease severe mitral stenosis presented with acute pulmonary edema December 2017. She underwent emergency balloon mitral valvulotomy. Post procedure ECHO showed ruptured posterior mitral leaflet causing severe mitral regurgitation. Patient was taken for emergency mitral valve replacement. As it was emergency case blood cross match report was not ready when patient was taken in OT. Clumping was noticed in cardioplegia line which was suspicious of cold agglutinin antibody. It was immediately confirmed when $2 \mathrm{ml}$ of heparinised blood was kept in contact with ice cube. Patient was rewarmed to $34{ }^{\circ} \mathrm{C}$ on cardiopulmonary bypass. Use of warming blanket, hot air warmer, blood line warmer, $\mathrm{AC}$ was switched off to maintain normothermia. Clumping in cardioplegia line disappeared. Blood gas analysis done to rule out acidosis. Cerebral and renal oximetry readings taken to rule out ischemia. Patient was safely taken off cardiopulmonary bypass. Recovery was uneventful.

\section{CONCLUSION:}

Cold agglutinin antibody require individualized planning before cardiac surgery, including consultation with ahematologist. Careful temperature monitoring must be undertaken intraoperatively to avoid antibody activation causing catastrophic hemagglutination and hemolysis.

Multiple giant coronary artery aneurysms, an unusual presentation of a systemic disease. Willington Francis; Abdulwahid Al Mulla; Ehab Massad; Maryam AlKuwari; Karima Bacetti; CS Carr

\section{ABSTRACT: \\ OBJECTIVE:}

Giant coronary artery aneurysms are rare (incidence $0.02 \%$ angiographically) and are defined as having a diameter of over $2 \mathrm{~cm}$. The underlying aetiologies include genetic factors, Kawasaki disease, Takayasu's arteritis and other connective tissue disorders with vasculitis, and atherosclerosis. Complications of giant aneurysms include ischaemia (from thrombosis or embolism), infection, and rupture. Treatment can depend on the underlying cause.

\section{METHODS:}

A 23 year old man presented with fever and chest pain and was found to have a pericardial effusion. A serous effusion was drained percutaneously but reaccumulated. A CT scan suggested a mass on the right ventricle and so he underwent further imaging.

\section{RESULTS:}

He was found to have multiple giant coronary aneurysms in all three arteries with evidence of thrombosis in at least one. At this time he denied any other symptoms. Some days later he requested cream for a scrotal ulcer which had been apparent for 2 months but he had been too embarrassed to admit to. A diagnosis of Behcet's disease was made and appropriate immunosuppression was started.

\section{CONCLUSIONS:}

Giant coronary artery aneurysms are rare and usually present when a complication occurs. The underlying aetiology may be difficult to establish, especially when patients are reluctant to report unusual symptoms that they may perceive as carrying a social stigma (genital ulceration).

\section{RUPTURED SINUS OF VALSALVA ANEURYSM FROM LEFT CORONARY SINUS DISSECTING INTO LEFT VENTRICLE AND INTER-VENTRICULAR SEPTUM MIMICKING HYDATID CYST, A RARE AND ODD PRESENTATION \\ Abhilash J.; K.N. Bhosle}

\section{ABSTRACT:}

\section{INTRODUCTION:}

Sinus of valsalva aneurysms commonly involve the right or non coronary sinuses. Usually asymptomatic and rarely discovered before its rupture. We describe a patient with left sinus of valsalva aneurysm, ruptured into the left ventricle myocardium and inter-ventricular septum with no fistulous communication with the ventricle cavity and with severe aortic regurgitation.

\section{CASE REPORT:}

A 20 year old male patient with symptoms of chest pain, breathlessness and palpitations since 3 months. On examination patient had an early diastolic murmur in aortic area with peripheral signs of aortic regurgitation. Initial evaluation with 2 decho gave an appearance of multiple cysts in anterolateral and septal area prompting a differential diagnosis of ?hydatid cyst? unruptured sinus of valsalva aneurysm with severe aortic regurgitation. Cardiac CT was done for establishing the diagnosis. 


\section{TREATMENT:}

Patient underwent PTFE patch closure of the aneurysm neck with aortic valve replacement under cardiopulmonary bypass. Aortic valve was tricuspid with degenerated and prolapsing left coronary cusp, rest of the valve leaflets and commissures normal. Left coronary artery was seen arising separately and above the aneurysm. Postoperative period was uneventful and postoperative 2 decho showed prosthetic valve insitu with no para-valvular leak and regression in size of the aneurysm sac.

\section{DISCUSSION:}

Sinus of valsalva aneurysm is a very rare congenital cardiac anomaly ( $1 \%$ of all congenital cardiac anomalies) $70 \%$ of which affects the right coronary sinus, $20 \%$ non-coronary sinus and only $5 \%$ affects the left coronary sinus. Congenital deficiency of elastic and muscular tissue at the junction of aortic media and aortic annulus is the aetiology in most cases. Associated risks are rupture, compression of adjacent cardiac structures and aortic regurgitation. Contained rupture of this aneurysm arising from LCC into the left ventricle myocardium and inter-ventricular septum with no fistulous communication with ventricle cavity is an even more rarer and an odd presentation.

\section{Case report: a rare entity coronary cameral fistula left circumflex coronary artery to right ventricle Ashish M. Agrawal \\ Divya Arora}

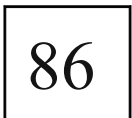

\section{ABSTRACT: INRODUCTION:}

Coronary cameral fistula (CCF) is rare, abnormal communication between coronary artery (CA) and cardiac chamber that usually results from aberrancy of normal embryological development. This case report signifies role of CT coronary angiography in making diagnosis and selection of treatment of choice.

\section{BACKGROUND:}

First successful surgical correction reported by Biorck and Crafoord in 1947. Sakakibara classified it in two types: Type A-proximal type, where the proximal coronary segment is dilated to the origin of the fistula and the distal end is normal; and Type B-distal type, where the coronary artery is dilated over its entire length terminating as a fistula mainly into the right side of the heart (end-artery type).

CASE:

A 14 years male child presented with dyspnoea NYHA class II, continuous murmur on auscultation, chest radiogram cardiomegaly present, echocardiogram CCF from left main coronary artery (LMCA) to right ventricle (RV), coronary angiogram revealed CCF from LMCA to coronary sinus, not correlated with echocardiogram. Cardiologist was not sure of fistula tract and diagnosis. Coronary $\mathrm{CT}$ angiogram showed $\mathrm{CCF}$ from left circumflex coronary artery (LCxA) to RV at crux of the heart with left dominant system, where sizes of LMCA $12 \mathrm{~mm}$, LCxA $11 \mathrm{~mm}$ and fistula $5 \mathrm{~mm}$. Surgical correction preferred over coil occlusion in our case due to distal type fistula opening at crux of the heart, was adjacent vessel at risk, large fistula, and young age. Intraoperative findings aneurysmaly dilated LCxA, thrill present. On cardioplegic arrest internal opening in RV was identified and closed with purse string suture with prolene 5-0. No thrill after procedure.

\section{CONCLUSION:}

CT coronary angiography is a gold standard procedure for defining $\mathrm{CCF}$ anatomy and aids in surgical correction.

\section{A Rare Case of Cardiac Paraganglioma with Mitral Valve Replacement \\ Parth Solanki}

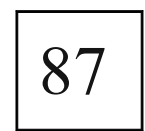

ABSTRACT:

One of the most unusual benign tumors affecting the heart is a cardiac paraganglioma is rarely observed in the mediastinum. Here with we are presenting a rare case of mitral valve disease along with mediastinal mass- paraganglioma.

\section{INTRODUCTION:}

Paraganglioma is a tumor arising from the extra-adrenal paraganglion system and is rarely observed in the mediastinum. It is a type of neurogenic tumour commonly occurring in posterior mediastinum.

Here with we are presenting a rare case of 50 year old male having mitral valve disease along with mediastinal mass-paraganglioma.

\section{CASE DETAILS:}

50 year old male presented with chief complain of dyspnea on exertion. A. Echocardiography was suggestive of moderate mitral stenosis with severe mitral regurgitation.ct thorax was suggestive of mediastinal tumour.. We planned for mitral valve replacement along with mediastinal mass excision.

\section{DISCUSSION:}

Total surgical resection of the mediastinal paraganglioma is the best treatment at present. Excision should always be planned on a cardiopulmonary bypass. However, the distinction between pheochromocytoma and paraganglioma is an important one because of implications for associated neoplasms, risk for malignancy, and genetic testing.

Concomitant Pulmonary And Cardiac Hydatid Disease: A Rare Case Report

Anil Jain

Deepti Kakkar

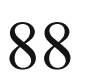

\section{ABSTRACT:}

\section{INTRODUCTION:}

The incidence of primary cardiac Echinococcosis is extremely rare. Only $0.5 \%-2 \%$ cases have been reported as compared to that of hepatic $(70 \%)$ or pulmonary $(20 \%)$. Here we report a case of concomitant intramyocardial and pulmonary hydatid cyst.

\section{BACKGROUND:}

Hydatidosis in man is caused by infection with the larval stage of the Echinococcus parasite, where man is an accidental host. The hexacanth embryo, passes through capillary networks and gets lodged in the myocardial tissues. CASE:

We describe a case of 20 year old lady, presenting with Dyspnoea and Angina on exertion NYHA II, of 3 month duration. X-Ray revealed a cystic lesions in cardiac silhouette and right lung. 2D Echo showed segmental pulmonary artery thromboembolism and hydatid cyst attached to interventricular septum of RV cavity. Concomitant intraparenchymal cyst was incidentally discovered in right lung. Patient was taken up for excision of the cardiac cyst. Cyst was excised from RV cavity. Hypertonic saline wash was given.

Communication of the cavity with RV was closed with purse string suture. Pulmonary hydatid was managed conservatively with albendazole for $2 \mathrm{cy}$ cles of 3 weeks duration each. Post operative period was uneventful, patient was discharged on day 6. On 6 month follow up, pulmonary cyst was noted to reduce in size with no evidence of recurrence of cardiac hydatid disease. CONCLUSION:

The diagnosis of cardiac hydatid disease must be made early to avoid complications and to achieve successful cure. Patients should be on regular follow up to look for recurrence of this endemic disease.

Successful management of thyroid tumor thrombus extending to right atrium through internal jugular vein and SVC

Nirmalkumar

P. Balasubramanian

\section{ABSTRACT:}

BACKGROUND:

Follicular carcinoma of thyroid has microscopic characteristic of angioinvasion but direct extension of tumour thrombus, i.e. collective 
form of intra-vascular metastases with thrombotic elements into caval veins and heart via cervical veins, is extremely uncommon. In the literature, only few cases have been reported where the patient underwent successful surgical resection of tumour thrombus

\section{CASE REPORT:}

We report a case where intra-cardiac extension of a follicular thyroid carcinoma

60 years old female came to us with neck swelling and class II dysponea. She had gross enlargement of both lobes of thyroid. Echo showed mass filling the whole right atrium. CT showed bilobular enlargement of thyroid with mass extending into the internal jugular vein, SVC and right atrium. Total thyroidectomy was done. ON CPB with bicaval and innominate vein cannulation, tumor thrombus was removed(750 grams) in totto from IJV to RA and sen for HPE which revealed follicular carcinoma. There was no invasion of tumour into the IJV wall or right atrial wall' Post operative radionuucleotide study was done. Radio ablation of residual and ectopic thyroid done with radio iodine and patient was put on thyroxin.

Two years post operatively, she is asymptomatic and no recurrence.

Post-traumatic Severe tricuspid regurgitation and rupture of interatrial septum : A Rare entity. Syed Wahid

G.N.Lone

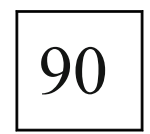

\section{ABSTRACT: \\ BACKGROUND:}

Post traumatic ASD with or without Tricuspid Regurgitation following intervention is not uncommon but rare following road traffic accidents or falls from trees or multistoried buildings with high mortality.

\section{CASE STUDY:}

We report a 45 years old young man after falling from a walnut tree, with massive chest trauma, lung contusion, multiple bilateral rib fractures, sternal fracture, bilateral hemothorax, supraventricular arrhythmias and shock. Patient was put on ventilator and optimized. He improved temporarily but developed progressive congestive cardiac failure. D-Dimer, CPK-MB, Troponin were high. Echocardiography revealed a large atrial septal defect with severe tricuspid regurgitation. Patient was operated through median sternotomy under moderate hypothermia by conventional CPB. Intraoperative TEE substantiated the provisional diagnosis. Pericardium was densely adherent to SVC, Aorta, right atrium and pulmonary artery. Right atrium was opened and whole of the interatrial septum was seen torn vertically from floor of SVC to the tricuspid valve extending towards floor of IVC. Anterior leaflet of tricuspid valve was torn vertically and septal leaflet was grossly regurgitant with 2 torn major chordae but intact annulus. Septum was reconstructed by autologous pericardium and tricuspid valve was repaired after refixation of major chordae to septal leaflet and primary repair of anterior leaflet. Rewarming was begun and cross clamp removed followed by deaeration and decannulation. Check TEE showed competent tricuspid valve and intact septum. Patient was discharged within 2 weeks after surgery.

\section{CONCLUSION:}

Cardiac injury should be suspected when a patient has high cardiac enzymes, arrhythmias or develops congestive cardiac failure after sustaining chest trauma, requiring prompt evaluation and intervention.

(Corresponding Author):

G.N.Lone, Professor and Head, CVTS, SKIMS, Srinagar

Email Id: gn_lone@yahoo.co.in

\author{
A Rare case of interventricular septal Hydatid cyst \\ causing right ventricular outflow tract obstruction \\ and complete heart block \\ Sai Chandran B.V. \\ Gautham A.
}

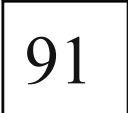

ABSTRACT:

\section{INTRODUCTION:}

Hydatid disease is a zoonotic disease, endemic in India and caused by ingestion of larval forms of Echinococcus granulosus tapeworm. Cardiac involvement is rarely seen constituting about 0.3 to $3 \%$ of cases in various series with interventricular septum involvement seen in $4 \%$ of cardiac cases. Clinical manifestations depend upon the site of involvement and include arrhythmias, heart block, ventricular septal rupture, outflow tract obstructions, various valve lesions, pericardial effusion etc. Cardiac involvement usually warrants surgical excision of the cysts followed by administration of larvicidal agents.

\section{BACKGROUND:}

A 29-year-old female presented with history of 1-month duration of shortness of breath with episodes of giddiness. On initial evaluation, patient was noted to have complete heart block with Echocardiography suggestive of Interventricular septal cystic lesion causing right ventricular outflow tract obstruction. Patient was referred to Department of CTVS, JIPMER for further evaluation.

CASE:

Patient was further evaluated with cardiac MRI and noted to have a cyst of size $4.5 \times 4.6 \mathrm{~cm}$ arising from the basal septum and protruding into Right Ventricle and left ventricle with internal septations suggestive of Hydatid cyst. Hydatid serology was inconclusive. CT Thorax and Abdomen was done and no cysts were noted elsewhere. Patient underwent Interventricular cyst aspiration with plication of cavity through median sternotomy under cardiopulmonary bypass. Post operatively patient continued to have complete heart block for which permanent pace maker implantation was done with pacing lead placed through left subclavian vein. Patient had further uneventful postoperative recovery and was discharged on POD 10 with oral Albendazole which was planned to be continued for 3 months.

\section{CONCLUSION/CLINICAL RELEVANCE:}

Hydatid disease of heart is a rare condition with multitude of manifestations and responds favourably to surgical management.

A case series (3) of Right Atrial Aneurysms repair. K.V. Krishna Kumar; Shalendra B.

Zubair Rashid

\section{ABSTRACT:}

A total of three cases of right atrial aneurysms in patients of multiple regions in the country including international patient.

42 years old lady from Tanzania asymptomatic, diagnosed on routine investigation having huge RA aneurysm.

56 years old gentleman from South India asymptomatic, diagnosed on routine investigation having RA aneurysm.

46 years old Lady from north India asymptomatic, diagnosed on routine investigation having RA aneurysm.

All three patients underwent repair of aneurysm with on pump and were discharged after post operative day 3 days.

\section{TUMOUR BLUSH -A RARE ANGIOGRAPHIC PICTURE IN ATRIAL MYXOMA Sreevathsa K.S.P.;

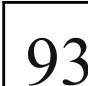
Jayakrishnan S.

\section{ABSTRACT: BACKGROUND:}

Atrial myxomas are common entities in cardiac surgery, demonstration of tumour blush is rare in Myxoma. 


\section{OBJECTIVE:}

This poster is for demonstrating the rare phenomenon of Tumour blush in Myxoma

\section{CASE:}

60 year old women presented with shortness of breath. Initial evaluation of patient with Echocardiography CT scan demonstrated a large left atrial mass, Coronary Angiogram showed no coronary artery disease and right coronary angiogram showed a branch supplying the tumour producing a "Tumour Blush" and also showed a large mobile filling defect of $5 \mathrm{~cm}$ in diameter in left atrium prolapsing into left ventricle in diastole. Patient underwent excision of tumour via biatrial approach. The raw area in septum where the stalk was attached was closed in two layers pledgeted prolene suture. IAS was repaired with pericardial patch,. Histopathological diagnosis being myxoma.

\section{CONCLUSION:}

Rare tumour blush in myxoma demonstrated.

\section{TUMOUR BLUSH -A RARE} ANGIOGRAPHIC PICTURE IN ATRIAL MYXOMA Sreevathsa KSP

Jayakrishnan S

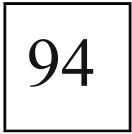

\section{ABSTRACT: \\ BACKGROUND:}

Atrial myxomas are common entities in cardiac surgery, demonstration of tumour blush is rare in Myxoma.

\section{OBJECTIVE:}

This poster is for demonstrating the rare phenomenon of Tumour blush in Myxoma

\section{CASE:}

60 year old women presented with shortness of breath. Initial evaluation of patient with Echocardiography CT scan demonstrated a large left atrial mass, Coronary Angiogram showed no coronary artery disease and right coronary angiogram showed a branch supplying the tumour producing a "Tumour Blush" and also showed a large mobile filling defect of $5 \mathrm{~cm}$ in diameter in left atrium prolapsing into left ventricle in diastole. Patient underwent excision of tumour via biatrial approach. The raw area in septum where the stalk was attached was closed in two layers pledgeted prolene suture. IAS was repaired with pericardial patch,. Histopathological diagnosis being myxoma.

\section{CONCLUSION:}

Rare tumour blush in myxoma demonstrated.

\author{
Mitral aortic intervalvular \\ fibrosa aneurysm rupture: rare but \\ life threatning condition \\ Subodh Satyarthi \\ Ankit Jain
}

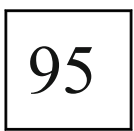

\section{ABSTARCT:}

MAIF aneurysm is a life threatning condition if not addressed early. We present a case of 24 year male presented with dyspnoea on exertion NYHA IV. Patient was diagnosed as a case of MAIF aneurysm rupturing into left atrium. Here, we present the case which was successfully managed with surgery.
Our surgical technique in a patients with non-infective sternal dehiscence

Levent Yilik; Yuksel Besir; Habib Cakir; Yasar Gokkurt; Utkan Tunca; Ali Gurbuz Kazim Ergüneș

\section{ABSTRACT:}

Enter description here.

\section{BACKGROUND:}

To overcome sternal dehiscence some surgical techniques and surgical equipment are designed. We presented a case with sternal dehiscence and multipl risk factors that underwent coronary bypass.

\section{METHODS:}

A 59-year old man was hospitalized in our clinic on November, 2018. He had coronary artery disease, hypertension, and chronic obstructive pulmonary disease. He underwent coronary artery bypass grafting. The sternal mobility was detected on sixth postoperatve day. Teleradiography showed displacement and rotation of sternal wires on the sternum. He was taken into operation room. The sternal dehiscence was repaired with steel wires and modified Robicsek technique. Vertical steel wires were placed to reinforce the lateral free margin of fracture line of the sternum. The figüre-of-eight steel wires were also used to provide rigid fixation in these regions of sternum due to strength of manibrium and above the level of the xiphoid process. Postoperatively, no problems occurred. The patient had an excellent sternal stability postoperatively. CONCLUSION:

The modified Robicsek technique is an important surgical method in the treatment of sternal dehiscence.

\section{Rare Case Of Pericardial Mesothelioma}

Gourav S. Shetty

Sambhram Shetty H.S.

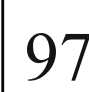

\section{ABSTRACT:}

Malignant pericardial mesothelioma is an extremely rare tumor with high lethality with a reported prevalence of $0.0022 \%$ at autopsy series. It is however, the most common primary malignancy of the pericardium. Malignant pericardial mesothelioma carries a poor prognosis with no successful treatment strategies and little benefit from radiation and chemotherapy. We report the case of a 38 year-old male without medical history, who was admitted in the department of cardiology for a dyspnea and chest pain due to a large pericardial effusion. A tamponade was confirmed by echocardiography and pericardial drainage was performed. Twelve months later patient developed a hemodynamically relevant pericardial constriction. Patient underwent a partial pericardectomy (Debulking) since pericardium was thick and adherent. The histological examination revealed a pericardial infiltration by malignant mesothelioma. After surgery five cycles of chemotherapy with carboplatine was given

The treatment methods of primary pericardial mesothelioma are limited. chemotherapy in addition to surgery seems to improve symptoms in case of constriction and non-response to pericardectomy

An Unusual Place To Be: Metastatic Pericardial Liposarcoma

Sivakumar Krishnasamy; Shahrul Amry Hashim;

Raja Amin Raja Mokhtar

Ashvin K. Nair 


\section{ABSTRACT: \\ BACKGROUND:}

Liposarcoma is the second most common type of soft tissue malignancy. Although this tumour usually metastasizes to various organs, cardiac metastasis especially to the pericardium is rare.

\section{CASE REPORT:}

A 55-year-old gentleman presented with increasing shortness of breath for 2 weeks. He has a history of left knee liposarcoma at the popliteal fossa which was surgically excised in 1990. An echocardiogram showed global pericardial effusion in tamponade. There was a mass noted lateral to the left ventricle. Urgent pericardiocentesis was done and 1.3litres of haemorrhagic fluid drained. CT thorax revealed left posterior pericardial mass measuring $4.6 \times 4.5 \times 5.0 \mathrm{~cm}$ initially thought to be a pericardial cyst. Surveillance CT thorax 6 months later showed the mass had enlarged to $6.9 \times 5.0 \times 5.7 \mathrm{~cm}$. He subsequently underwent a left VATS biopsy of the mass which turned out to be myxoid liposarcoma. Since the tumour was found densely adhered to the pericardium, cardiac MRI showed a soft tissue mass with involvement of the descending thoracic aorta, thoracic oesophagus and left hemidiaphragm. He then underwent 25 cycles of neoadjuvant radiotherapy and $\mathrm{CT}$ thorax a month later showed reduction in tumour size to $2.1 \times 5.6 \times 4.5 \mathrm{~cm}$ with no other distant mestastasis and a clear fat plane between the mass and adjacent structures. PET scan confirmed no FDG-avid lesions elsewhere. An excision of the left pericardial mass via thoracotomy with plication of left hemi-diaprhagm and implantation of prolene mesh over the pericardial defect was carried out. Histopathology confirmed a myxoid liposarcoma. The patient was discharged home well and CT thorax six months later showed no evidence of recurrence.

\section{CONCLUSION:}

Metastatic pericardial liposarcoma can be a diagnostic challenge and multimodal imaging is recommended to evaluate the differential diagnosis and deciding on appropriate treatment. Aggressive surgical resection combined with perioperative radiotherapy helps to prolong survival and avoid recurrence. However, continued surveillance with serial imaging is required for all cardiac liposarcomas.

\section{Left Atrial Myxoma-A Rare entity with a rarer presentation: A Case report \\ G.S. Nagi \\ Ravdeep Singh Sohal}

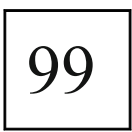

ABSTRACT:

Bilateral Lower limb compartment syndrome in Redo cyanotic congenital heart surgery done under peripheral cannulation.

Pradeep, Ganapathy subramaniam, V.K.swamy, Murali Krishna, Anitha,

Fortis malar Hospital, chennai

14 year old, case of pulmonary atresia (PA) with ventricular septal defect (VSD) who underwent classic BT shunt soon after birth and central shunt at later date, now underwent total correction in femoro femoral bypass. On POD 2 patient had compartment syndrome in Both legs and emergency fasciotomy was done. compartment syndrome in both legs following congenital cardiac repair is a rare phenomena and etiology in this case is still uncertain.

\section{CASE REPORT:}

14 year old female, with progressive deepening of cyanosis admitted for elective third cardiac surgery, for complete repair. Patient underwent surgery under peripheral cannulation (Right Femoral vessels ). Patient was on long bypass and was intermittently on low flow bypass to facilitate surgery. Shifted with chest open and closed the next day. Extubated on second day, Patient complained of pain in both legs. Both legs were edematous and localized there. Peripheral pulses were palpable. Doppler showed no thrombus in the deep veins and good bi phasic flow in the arteries. within few hours she was developing paraesthesia and foot drop bilaterally. diagnosed as compartment syndrome on clinical grounds underwent emergency fasciotomy in both legs. Patient had relieve of pain and better sensory perceptions post surgery.

\section{CONCLUSION:}

Post cardiac surgery compartment syndrome is an uncommon event. It can be a sequel of Long Cardio pulmonary bypass as few cases have been reported earlier, however there is no other definitive explanation in this case.

\section{A Rare Aneurysm}

Shubhendu Mahapatra; Ajil Joseph

\section{ABSTRACT:}

\section{INTRODUCTION:}

Renal artery aneurysms ( RAA) are rare in the general population. The widely accepted incidence of RAA is $0.1 \%$ in the general population. The natural history of RAAs is that of slow to null growth. Rupture rates is as high as $14 \%$ to $30 \%$ with associated mortality of $80 \%$.

\section{BACKGROUND:}

RAAs typically present in the sixth decade. Women are more commonly afflicted with RAA, likely due to the high incidence of associated fibromuscular dysplasia. Renal bruit or a palpable abdominal mass are inconsistent and unreliable physical findings. The majority of patients lack traditional cardiovascular risk factors other than HTN.

CASE:

A 55 Year old female patient complains of pain in abdomen since 2 months which is dull aching in the left lumbar region. It was non radiating, not associated with hematuria or fever or burning micturition. Patient developed pain after she underwent treatment for renal calculi 2 months back. Mild tenderness in right lumbar region on deep palpation and no palpable mass or bruit heard on auscultation. A large non obstructing calculus of size $19^{*} 14 \mathrm{~mm}$ is noted in lower calyx. A well defined, hyperdense, round SOL with rim calcification, measuring $18 * 16 \mathrm{~mm}$ noted at renal pelvis. CT scan was taken and showed wall calcification feature s/o renal artery aneurysm

\section{CONCLUSION:}

Currently, there is no consensus regarding the size at which an RAA should be repaired in an asymptomatic patient. In this case surgical treatment was opted as the patient was symptomatic. 
Repair of pericardio-cutaneous fistula from teflon felt- 10 years after $L V$ aneurysm repair.

Nicholas Kang; Jens Lund

Geevarghese Mathew

\section{ABSTRACT:}

58 year old man presented with a swelling in the epigastric region. He had a CABG with LV aneurysmectomy in 2007 for triple vessel coronary artery disease with severe LV dysfunction and apical LV aneurysm.

The swelling spontaneously ruptured and persisted as a chronically discharging, hyper granulating sinus after an initial attempt at wide excision. CT scan done revealed a track extending intrapericardially up to the LV apex in continuity with the Teflon felts used for the aneurysm repair. Coronary angiogram revealed patent LIMA and SVG grafts. In view of patent anterior grafts, an anterolateral thoracotomy was done and the site of LV aneurysm repair was exposed and the felt strips dissected. The sinus was dissected up to the felt strips and track laid open.

The Teflon felts were removed successfully without damage to the LIMA graft or LV and the thoracotomy closed over drains. The patient had an uneventful recovery and was treated with a prolonged course of antibiotics. Prosthetic material like Teflon felts are used commonly for reconstruction of cardiac chambers. Even though rare, they can be the source of delayed infection which may present as non healing sinuses. There are limited reports of similar cases in the literature.

The case highlights the need for a high index of suspicion and appropriate imaging to make the correct diagnosis. Complete removal of the foreign material is important for eradicating the infection. The presence of patent anterior grafts as in this case makes re-entry difficult and an anterolateral thoracotomy is a safe approach.

\section{Left ventricular cyst}

Narasimha Rao Ganta; Kiran Ganta

\section{ABSTRACT:}

A 50 years old male patient presented to hospital with shortness of breath NYHD grade 3 of 1 month duration, on evaluation the patient was found to have left ventricular cyst along with LAD ectasia. LV cyst was deroofed into LV via transaortic approach thus reliving patient from mechanical obstruction of LV. Patient LVEDV and ejection fraction improved significantly post surgery. Biopsy of the deroofed tissue was consistent with simple blood cyst.

\section{An unknown case of Hypertrophic Obstructive Cardiomyopathy having systolic anterior motion of posterior mitral leaflet and its surgical repair. Sujay Shad; Pankaj Kumar Gupta \\ Baryon Swain}

\footnotetext{
ABSTRACT:

Patients with hypertrophic obstructive cardiomyopathy demonstrate dynamic sub-aortic obstruction to left ventricular outflow. Various factors like redundant mitral leaflets, papillary muscle displacement, septal hypertrophy, small left ventricular cavity, reduced distance of coaptation between mitral leaflet and septum plays role in systolic anterior motion (SAM) causing left ventricular outflow tract obstruction. There is scarce literature about left ventricular tract obstruction due to SAM of posterior mitral leaflet.

In the present report, we describe a patient with hypertrophic obstructive cardiomyopathy in whom the abnormally elongated posterior mitral leaflet caused persistent dynamic Left ventricular outflow tract obstruction despite a satisfactory extended septal myectomy and underwent a posterior mitral leaflet shortening and mitral valve annuloplasty in the same operative setting to obtain a satisfactory correction.
}

A rare case of contained myocardial rupture

Suresh Babu Kale; Punithakumar Ramasamy

\section{ABSTRACT:}

Rupture of the free wall of the left ventricle occurs in approximately $4 \%$ of patients with infarcts and accounts for approximately $20 \%$ of the total mortality of patients with myocardial infractions. Rupture of the left ventricular free wall has been regarded as uniformly fatal unless emergency surgical repair is performed

Sixty five years old gentleman was diagnosed as lateral wall acute myocardial infarction and referred to our hospital with poor hemodynamics. Echocardiography showed large pericardial effusion with thrombus. In view of poor hemodynamics, patient was taken up for emergency exploration. Intraoperatively, there was a huge clot present in the pericardial cavity. After removal of the clot, there was active bleeding from lateral wall of left ventricle. Urgent cardiopulmonary bypass was established and in arrested heart, tear in left ventricle lateral wall was repaired with Goretex patch and hemostasis secured. Postoperatively patient was doing well and he was discharged in stable condition

Most ventricular ruptures occur within the first week after MI. In acute form, death ensues within minutes to hours. In subacute form, the presentation may evolve over hours, days, or even longer. In this case, the rupture is sealed by a hematoma in the epicardial surface of the heart, forming a contained myocardial rupture

Surgery is the cornerstone treatment of subacute left ventricular free wall rupture. The overall hospital mortality in patients with and without surgery was reported to be approximately $60 \%$. Surgery-related mortality is up to $33 \%$ but in those who survive the complicated operation, the longterm outcome is good.

Using ECMO (V-V Mode) to treat venous air embolism induced cardiogenic shock during pars plana vitrectomy for retinal retachment Han-Chen Lee; Chien-Tung Lin; Tsung-Po Tsai; Yi-Liang Wu; Nien Yi Wu; Chi-Chung Chen Shou-Ho Tseng

\section{ABSTRACT:}

A 59-year-old woman with hypertension had undergone 4 times of vitreoretinal surgeries for her right eye problem before. During her 5th pars plana vitrectomy and laser treatment for retinal detachment, she suddenly developed a sequence of ECG change (ST elevation, type II 2nd degree A-V block and then bradycardia), hypotension and acute oxygen desaturation 10 mintues after the air-fluid exchange procedure (with $23 \mathrm{G}$ trocar, air volume more than $30 \mathrm{ml}$ by $35 \mathrm{mmHg}$ of infusion pressure). Even under hand manipulated ambu bagging with 100\% O2 through endotracheal tube and TCP applied, the patient developed acute hypoxemic respiratory failure associated with cardiovascular collapse. TEE was done immediately, revealing lots of air bubbles accumulated and locked in the right atrium of heart. Therefore, ECMO (V-V Mode) was performed to treat the potentially fatal VAE. The patient woke up on the following morning and became hemodynamically stable with O2 Sat of $100 \%$ and ECMO was removed. Endotracheal ventilation was weaned on the 6th post-op day. She also had another cardiac catheterization and transthoracic echocardiography to ascertain good ventricular contractility, insignificant CAD lesion and no existence of patent foramen ovale. This patient survived to hospital discharge without any neurologic deficit at OPD follow-up.

\section{CONCLUSIONS:}

Rapid prompt diagnosis of VAE with acute hypoxemic respiratory failure after vitreoretinal surgery by real-time TEE and treated by ECMO are mandatory for this severe potentially fatal event. 
Hybrid closure of Post Infarct ventricular septal rupture with perventricular Amplatzer Septal occluder reinforced with a composite patch Ratnamalika Kumar; Rajneesh Malhotra Anuj Sangal

\section{ABSTRACT:}

We describe a rare interventional procedure in which an 77-year-old gentleman underwent a successful per-op hybrid closure of post myocardial infarction ventricular septal defect and coronary artery bypass grafting. Perventricular device closure of post-myocardial infarction VSDs (PIVSD) appears to be a safe and effective method to close PI-VSD. This approach has established itself for management of high risk posterobasal VSDs. It has advantages over both surgical and transcatheter techniques. With this approach, immediate complete closure of PI-VSD with complete coronary revascularization is feasible without the ill effects of cardiopulmonary bypass or challenges of arterio-venous looping. However, many more cases will be required before this hybrid procedure becomes as established a procedure as it has become for congenital muscular VSD closure. Post MI VSR carry high mortality rates and approach to posterobasal defects are very difficult to repair due to its close proximity to the mitral valve apparatus and location. Hybrid closure of the defect provided extra-strength and minimised chances of device embolisation or patch dehiscence in view of oedematous and infarcted myocardium along the defect.

\section{Primary Cardiac Osteosarcoma: A differential for calcified intra cardiac mass lesion. \\ Varghese T. Panikar; Kiran C. Kalappa}

\section{ABSTRACT:}

Primary cardiac tumors are uncommon, with incidence at autopsy of $0.02 \%$ to $0.05 \%$. Approximately $75 \%$ of primary cardiac tumors are benign, but the clinical presentation of benign cardiac neoplasms overlaps considerably with malignant cardiac masses. Imaging features of cardiac masses that suggest benignancy include a left-sided location; a uni focal intra cavitary or intramural mass with well-defined margins; and the absence of valvular, pericardial, or extra cardiac involvement.

Sarcomas are the most common primary cardiac malignancy. Cardiac osteosarcoma, a rare subtype of this tumor group has a poor prognosis. Osteosarcomas commonly involve the mitral valve. Reports of this rare lesion with detailed imaging features especially $\mathrm{CT}$ and MRI are less in available literatur. CASE REPORT:

A 52 year old gentleman came with complaints of dyspnoea on exertion with presyncopal episodes since 10 days. Patient visited a local hospital first where he was evaluated for presyncope and in echocardiography detected to have left atrial lesion which was initially thought to be LA myxoma. No history of chest pain or syncope.

Echocardiography showed LV ejection fraction of $58 \%$ with a $49 \mathrm{x}$ $28 \mathrm{~mm}$ heterogenous mass in left atrium occupying most of the LA. No RWMA. No pericardial effusion/clots/valvular vegetations noted.

\section{PATHOLOGIC EVALUATION:}

Gross specimen appeared grey white, firm to hard, calcified tissue measuring $6.5 \times 5 \times 0.5 \mathrm{~cm}$.

Microscopy showed plump spindle cells arranged in fascicles and storiform pattern interspersed by collagen bundles and slit like vascular channels. Immunohistochemistry revealed majority of the cells to be positive for vimentin and SMA.

\author{
Right Atrial Myxoma excision with Tricuspid \\ Valve Replacement \\ D. Janardhan Reddy; K.V. Muralidharan; P.S.N. Raju; \\ Masthanamma Chengali; Ramakrishnan; \\ C. Srinivasan; Srikanth Bhumana; Ashok Sampath \\ Thejaswi N Marla
}

ABSTRACT:

\section{INTRODUCTION:}

Cardiac tumors represent $0.2 \%$ of all tumors found in humans. These tumors are divided into primary and secondary or metastatic. Secondary or metastatic tumors are 20 to 40 times more frequent than primary tumors. Myxomas are located in the left atrium in $75 \%$ to $80 \%$ of cases and are almost always present with signs and symptoms of mitral valve disease or thromboembolic events.

\section{CASE REPORT:}

Myxoma arising from the heart valve is rare. We report here an unusual case of Large myxoma attached to the tricuspid leaflet, which was successfully treated by total excision of the tumor mass with tricuspid valve replacement. A 55-year-old man was admitted with a 1-year history of exertional dyspnea. Transthoracic and transesophageal echocardiograms showed a $5 \mathrm{X}$ $6 \mathrm{~cm}$ mass in the right atrium originating from the lower interatrial septum, and prolapsing into the right ventricle through the tricuspid orifice during diastole with no valvular incompetence. The patient underwent operation with the aid of cardiopulmonary bypass. The right atrium contained the giant tumor, which was no attached to the leaflet of the tricuspid valve. The mass was removed with excision of tricuspid valve leaflet at the base replaced with bioprosthesis.

\section{CONCLUSION:}

Primary cardiac valve tumors are uncommon, with an incidence of less than $10 \%$ of all cardiac tumors. In a recent review, 56 valve tumors occurring in 53 patients over a 58 -year period were documented. Fortyone of these tumors $(73 \%)$ were fibroelastomas, and five of them $(8.9 \%)$ were myxomas. Cole and associates summarized the 11 tricuspid valve myxomas. Five of them had tumor attachment on the atrial side of the valves like our case. Valve tumors should be managed surgically by complete excision to avoid recurrence of the tumor. In our patient, the location of the tumor on leaflet of the tricuspid valve and its tethering made conservation of the valve immpossible.

Open Pnumothorax a common presentation in Jallikattu Jason; J. Arul Vijaykumar

\section{ABSTRACT:}

Enter desIntroduction:

Open pneumothorax involves an unsealed opening of chest wall. It's rare in blunt injury chest, but can occur when there is significant loss of chest wall following trauma. In large defects, respiratory dynamics is altered and can eliminate ventilation on the affected side. Earlier and prompt surgical intervention can give good outcome to the patient. In this case study we present an adolescent male who sustained a blunt injury chest as a result of being run over by a tractor

\section{BACKGROUND:}

Open pneumothorax is rare in other parts of the world but is particularly common in south Tamil Nadu

CASE:

$>25$ years adolescent male was referred from a secondary care hospital as a case of open left side chest injury, An ICD was inserted through the wound in the outside hospital and was shifted here.. He had sustained injury after being run over by a tractor. On admission patient GCS was $15 / 15$ with stable vitals. Respiratory system examination showed no air entry in left lung. He had a defect of size $14 \times 10 \mathrm{cms}$ in left lateral chest wall through which heart was visible. After fluid resuscitation, Computed tomography of chest showed Left side collapsed lung with large defect in left lateral chest wall. Patient was immediately shifted to emergency operating room. Patient was intubated and connected to ventilator. Thorough wound wash was given. All devitalised tissues were removed. Sharp end of ribs were trimmed. Two chest tubes were placed, one in the base and one in the apex. Chest wall was closed in two layers with vicryl sutures. Muscles approximated with vicryl. Skin closed with proline sutures. Patient was shifted to post anesthesia care unit and was on elective ventilator for 10 hours. Post extubation patient was stable. Apical chest tube removed on 4th post op day. 
A novel technique in the use of endoscopically retrieved saphenous veins for femoropopliteal artery bypass. George Varghese Kurien; Manoj P. Nair

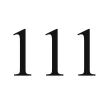

\section{ABSTRACT:}

\section{CASE REPORT:}

A 50 year old male smoker, labourer by occupation, was admitted with a non-healing ulcer of the anterior aspect of tibia with rest pain. Clinical evaluation suggested a Rutherford grade 2, category 4 lesion. 2 months later he sustained a left tibial fracture following a fall at his work place. Peripheral ct aortogram revealed a TASC II TYPE D lesion for which infra popliteal revascularisation was planned after discussion

\section{SURGICAL TECHNIQUE:}

After vein endoscopic harvesting the saphenous vein was tunneled through the submuscular plane. We introduced an autoclaved $5 \mathrm{fr}$ fogarty cover for threading across the tunnel. A standard atrium tunneler set was used in the sub sartorial muscular plane. The fogarty cover was threaded along the tunneler. An ethisteel n0 6 was used to thread along the fogarty cover. The end was made into a loop. The reversed end of the vein was attached to the loop end of the ethisteel and the vein was threaded along the whole length of the tunneler. Once the vein reached the desired length for the distal anastomosis the fogarty cover was removed.

\section{DISCUSSION:}

We at our centre routinely use the reverse saphenous vein grafting for the femoro popliteal bypass operations. We have quite commonly encountered the technical problem of vein avulsion and internal bleeding while tunnelling the reverse saphenous vein graft through the subsartorial tunnel. The use of a fogarty catheter cover as described can reduce the risk of intimal damage during traction while tunnelling, side branch avulsion and slipping of ligatures resulting in disastrous consequences of internal bleeding and conversion to an open wound exploration or resort to the use of prosthetic grafts which have reportedly lower graft primary, primary assisted and secondary patencies.

\section{Acute venous thrombosis of left upper extremity in a patient having port catheter and taking 5-fluorouracil due to breast carcinoma \\ Levent Yilik; Yuksel Besir; Habib Cakir; Erturk \\ Karaagac; Yasar Gokkurt; Ali Gurbuz \\ Kazim Ergüneș}

\section{ABSTRACT: \\ BACKGROUND:}

Venous thrombosis and carcinoma are important factor affecting morbidity and mortality.

We presented a patient having acute venous thrombosis of left upper extremity and implanted port catheter in the left upper extremity and receiving 5-fluorouracil due to breast carcinoma.

\section{METHOD:}

A 76-year-old woman admitted to the outpatient clinic of our hospital in November, 2018. She had pain and edema in the left upper extremity.

\section{RESULTS:}

Venous Doppler ultrasonography detected thrombosis in the deep venous system of the left upper extremity. The mamography and biopsy of breast showed breast carcinoma. Anticoagulant therapy with low-molecular weight heparin (LMWH) was immediately started. The patient's symptoms, particularly pain and edema continued to improve in the left upper extremity.

\section{CONCLUSSION:}

Low-molecular-weight heparin is important in treatment of acute deep venous thrombosis of the upper extremity in patients taking 5-fluorouracil due to breast carcinoma.
Management of a fractured peripheral intravenous cannula. Case report and review of literature

Babu T. Muntimadugu

S. Sabiha Nigar

\section{ABSTRACT:}

INTRODUCTION:

Fracture of peripheral venous cannula[PVC] is a rare event and can lead to complications like infection, migration and death secondary to embolisation.

\section{CASE REPORT:}

A 45yr old lady was admitted for the management of severe anemia with Haemoglobin 2.6gm. PVC was placed in the external jugular vein [Ejv[. She was diagnosed to have psychosis also. she pulled out the intravenous cannula forcibly and was noted that PVC was broken and partly retained in the neck. Ultrasound of neck was done which showed retained PVC in the vein. She was operated on urgent basis under local anesthesia. ultrasound was used to locate the lower end of retained cannula. PVC was removed with two transverse incisions in the neck over EJV. Lower incision just above the clavicle was used to control the lower end of EJV to prevent migration during manipulation of the EJV. She had uneventful recovery.

\section{DISCUSSION:}

Fractures of vein cannulae are known to be secondary to damage of the cannula with needle during insertion, poor quality and pronged kinking. Infection, embolisation to heart and pulmonary arteries is a known complication. Migration and embolisation could be prevented with early surgical intervention. Ultrasound is useful in planning the surgery.

Femoral pseudoaneurysm 30 years post gunshot: A rare clinical entity

Prateek Vaswani; Pradeep R. Reddy; Saurabh

Nanda; Milind P. Hote

\section{ABSTRACT:}

Pseudoaneurysms of the femoral artery are seen as saccular outpouching with a narrow neck resulting from a defect in the wall of vessel due to trauma or instrumentation with arterial flow seen in continuity surrounded by fibrous rim. There is presence of thrombus and calcification. This is a description of an uncommon delayed presentation of penetrating trauma of lower limb with history of inciting event (gunshot) being 30 years ago. There are only 4 such reported cases in published literature. Our case is a 55-year male with presenting complaints of progressively enlarging swelling over anterior aspect of right thigh with pain on walking and paraesthesias. Clinical signs consisted of a large non pulsatile lump in right anteromedial thigh. CT angiography revealed the presence of SFA (superficial femoral artery) pseudoaneurysm. The open surgical management consisted of excision and autologous vein patch angioplasty. The rarity of incidence and paucity of examination signs suggest that a high index of suspicion, careful clinical review and radiological investigations are indispensable in diagnosing and guiding route for intervention in order to optimise patient outcome.

Aorto Bifemoral Bypass Gastrointestinal Bleed

Murugan M.S.; Krishnanand Pai; Ganesan C.;

P.R. Murugesan

\section{ABSTRACT:}

\section{BACKGROUND:}

GI bleed secondary to aortoenteric fistula in post aortoiliac reconstruction for surgical abdominal aortic aneurysm repair/aortic iliac occlusive disease is a wellknown complication. The diagnosis \&amp; management is standardised. In this case we have difficulty diagnosing the source of bleed and on-table surprise finding. 


\section{CASE PROFILE:}

64 y/o M underwent Aortobiliac bypass for type I Aortoiliac disease with critical limb ischemia $8 y$ back, followup with oral anticoagulants for his thrombogenic status. Presented with persistent leukocytosis with generalized malaise. No abdominal symptoms. Bilateral foot pulses palpable. Routine workup including abdominal CT scan revealed perigraft collection with air pockets. Possibility of perigraft infection, treated with course of empirical antibiotics during followup over $3 \mathrm{M}$, Presented with persistent malena and significant anaemia $\mathrm{Hb} 6 \mathrm{gm}$. Workedup for GI bleed. Upper GIscopy revealed erosive gastritis, Contrast CT abdomen suggestive of perigraft collection, possible erosion of R-limb of aortoiliac bypass conduit to ileal loop for which exploratory laparotomy done, periappendiceal mass with distal ileal loop graft erosion managed with appendicectomy, graft limb excision, segmental resection of ileum, 3M followup satisfactory. Hospitalised with profound anaemia with new onset of malena. $24 \mathrm{hr}$ bleed scan revealed proximal jejunal bleed, option of endovascular stenting deferred, decided for reexploratory laparotomy and excision of entire thrombotic infected graft and bowel resection anastomosis. Limb perfusion-good, foot pulses-palpable, native arterial flow.

\section{CONCLUSION:}

Aorto biliac reconstruction synthetic conduit known for bowel erosion, even though aortoenteric fistula is common occurrence at anastomotic site. In rare situation graft erosion to bowel is to be considered and graft excision with bowel repair is appropriate if native vessel perfusion is acceptable. If limb perfusion is under jeopardy extraanatomical bypass need to be considered. Peritonealisation of anastomotic site and graft is mandatory to prevent bowel erosion and subsequent bleed. Perigraft collection is the lead for bowel erosion in late presentation.

\author{
A rare case of Hypothenar Hammer Syndrome \\ - case report \\ Arvind Kumar Bhisnoi; GuruPrasad Rai D; \\ Alankrit Ramesh Kashyap \\ Rajkamal Vishnu
}

\section{ABSTRACT: \\ INTRODUCTION:}

The hypothenar hammer syndrome (HHS) is a rare condition of vascular etiology, first described by Von Rosen (1934) and named by Conn (1970).

\section{BACKGROUND:}

The ulnar artery enters the palm at the wrist joint, below the palmar fascia and continuous through the guyons canal to from the palmar arches. In the guyons canal the ulnar artery is above the hook of the hamate bone and repetitive blunt trauma at this point, damages the ulnar artery, leading to thrombosis and ischemia of the digits. When aneurysm formation occurs it compresses the ulnar nerve. The symptoms are ischemia, paraesthesia to the 4th and 5th finger, lateral part of palm and painful hypothenar eminence. CASE:

43 year old male a bank employee who has to spend long hours on the computer, presented with painful swelling in the hypothenar eminence since two weeks and tingling and paraesthesia over the little and ring finger. He complained of milder symptoms since last 6 months. Clinically a pulsatile mass was felt in the line of ulnar artery just above the wrist and in the hypothenar eminence. Angiogram revealed a bilobed partially thrombosed aneurysm of the ulnar artery and compression of the ulnar nerve. The radial artery flow was normal. Patient was operated with excision of the aneurysm and ligation just proximal to the bifurcation to palmar arches. As collateral flow was adequate from radial artery ligation was sufficient.

Postoperatively his symptoms were relieved.

CONCLUSION:

HHS is a treatable cause of digital ischemia. Patient's with severe ischemic symptoms and entrapment of nerve, requires surgical excision. In the presence of doubtful collateral circulation revascularisation with a venous graft is essential. In our case the aetiology was presumed to be repetitive pressure on the hypothenar eminence due to continuous use of mouse for computer work.

Our surgical strategy in a case with hypertension having severe ulcerated right internal carotid artery stenosis

Orhan Goklap; Ismail Yurekli; Habib Cakir; Banu

Lafci; Hüseyin Durmaz; Utkan Tunca; Ali gurbuz

Kazim ergüneș

\section{ABSTRACT: \\ BACKGROUND:}

The severe ulcerated carotid artery stenosis is rare. We presented a case with hypertension having severe ulcerated right internal carotid artery stenosis.

\section{METHOD:}

A 55-year-old man was hospitalized in our clinic in August, 2017. He had hypertension and severe ulcerated right internal carotid artery stenosis. The electrocardiograph showed sinus rhythm. The angiography showed severe ulcerated right internal carotid artery stenosis.

\section{RESULTS:}

Carotid endarterectomy was performed with regional anesthesia. Five thousand units of heparin was administered by anesthesiologist intravenously. The right carotid artery was intensively fragile and ulcerated. Fagile and ulcerate segment of the carotid artery was dissected. A $5 \mathrm{~mm}$ PTFE tube graft was anastomosed between right common and internal carotid arteries by a continuous running stitch, using No.6 polypropylene suture material. The right external carotid artery was ligated since not suitable for anastomosis. The patient was discharged with clopidogrel and aspirin on 4th postoperative four day.

\section{CONCLUSSION:}

A tube graft between common and internal carotid arteries is effective and safe method in patients having severe ulcerated carotid artery stenosis. Careful and appropriate dissection of carotid artery is important to decrease morbidity.

Acute venous thrombosis of right lower extremity in a patient operating due to lombar hernia two months ago

Levent Yilik; Banu Lafci; Orhan Gokalp;

Koksal Donmez; Ali Gurbuz

Kazim Ergüneș

\section{ABSTRACT: \\ BACKGROUND:}

Venous thrombosis are important factor affecting morbidity and mortality. We presented a patient having acute venous thrombosis of right lower extremity that was operated due to lombar hernia two months ago.

\section{METHOD:}

A 65-year-old woman admitted to the outpatient clinic of our hospital in June, 2018. She had pain and edema in the right lower extremity.

\section{RESULTS:}

Venous Doppler ultrasonography detected thrombosis in the deep venous system of the right lower extremity. She was operated due to lombar hernia two months ago.

Anticoagulant therapy with low-molecular -weight heparin (LMWH) was immediately started. The patient's symptoms, particularly pain and edema continued to improve in the right lower extremity.

\section{CONCLUSION:}

Low-molecular -weight heparin is important in treatment of acute deep venous thrombosis of the lower extremity in patients undergoing surgery due to lombar hernia. 
Acute arterial thromboembolism of right lower extremity in a patient with atrial fibrillation in coexistence of diabetes, hypertension and cardiac failure Erturk Karaagac; Sahin Iscan; Ismail Yurekli; Habib Cakir; Bortecin Eygi; Ali Gurbuz

Kazim Ergüneş

\section{ABSTRACT:}

\section{BACKGROUND:}

Acute ischemia resulting embolic or thrombotic arterial occlusion is associated with morbidity and mortality. We presented a patient with atrial fibrillation in coexistence of diabetes, hypertension and cardiac failure.

\section{CASE REPORT:}

A 85-year old woman was referred to the emergency department of our hospital in October, 2016. She had pain and coldness in the right lower extremity. She had atrial fibrillation, diabetes, hypertension and cardiac failure. Pulses of the popliteal and trifurcation arteries were nonpalpable. The hand-held Doppler found no audible signals in the right popliteal and trifurcation arteries. The Doppler ultrasonography showed occlusion in the right superficial femoral, popliteal and trifurcation arteries.

He was emergently transferred to operation room. The patient was operated with general anesthesia.

The patient's electrocardiogram, and blood pressure was monitorized. 5000 units of heparin sodium was given intravenously before occlusion with nontraumatic vascular clamps of common, superficial, and deep femoral arteries. A Fogarty balloon catheter $(5 \mathrm{Fr})$ was then introduced into superficial femoral artery. A 4-Fr catheter was then introduced into the deep femoral artery. A 6-Fr catheter was then passed proximally toward the iliac artery for removal of a possible unsuspected thrombus. Abundant thrombus was thrown out from superficial femoral artery. Pulses of popliteal and trifurcation arteries were recovered postoperatively. Low-molecularweight heparin and warfarin was given postoperatively. The patient was discharged six days after operation with warfarin treatment.

\section{CONCLUSION:}

Acute arterial thromboembolism is important complication in patients having atrial fibrillation and co-morbitidies. The thromboembolectomy is effective and safe treatment method.

\author{
Interesting case of Chronic Thrombo Embolic \\ Pulmonary Hypertension (CTEPH) \\ G. Josephraj \\ B. Karthikeyan
}

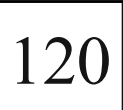

\section{ABSTRACT: \\ INTRODUCTION:}

Chronic Thrombo Embolic Pulmonary Hypertension (CTEPH) is one of the rare cases which needs surgical intervention in most of the cases without that survival rate is very low. But due to rarity of the disease and complex presentation of the disease surgical treatment should be correctly planned depends on the individual patient presentation.

\section{CASE REPORT:}

27 years old male patient developed dyspnoea and haemoptysis for one year duration. On evaluation he was found to have CTEPH and treated with medical management and IVC filter.

Patient started developing RV dysfunction and worsening of symptoms hence planned for Pulmonary Thrombo Endarterectomy(PTE). Under Cardio pulmonary bypass with Main Pulmonary Artery and Right Superior Pulmonary Vein venting PTE done under Total Circulatory Arrest (TCA). RPA Opened from the confluence upto the lobar divisions and endarterectomy done upto the segmental divisions after developing sub intimal plane. Patient cooled to 16 deg centigrade during TCA.

Immediate Post operatively patient developed severe pulmonary hypertension and treated with intravenous sildenafil and milrinone. Patient stabilized after 24 hours and extubated. Rest of the post operative period was uneventful. Post operative angiogram showed good pulmonary artery blood flow to the right lung.

\section{CONCLUSION:}

Although CTEPH is a rare disease, diagnosing and treating the disease early will improve the survival rate because this disease is having almost $90 \%$ mortality without surgical intervention.

Case report- Chronic pulmonary thromboembolism underwent pulmonary thromboendarterectomy

Vijit K. Cherian; Ravi Shivdasani

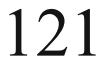

\section{ABSTRACT:}

This 57 years old, male patient, a farmer presented to us with progressive dyspnoea on exertion past six months. On evaluation 2D echo revealed moderate PAH with good biventricular function, normal valves and no RWMA. Chest X-ray showed bilateral pulmonary artery cut off and no changes on ECG. He had history of Spine surgery 3 years back and was bedridden for about 3 months after surgery. Based on history and other findings he was taken up for CT pulmonary angiogram which revealed chronic pulmonary embolism of LPA, lobar, segmental and subsegmental branches and RPA, RUL and RLL segmental branches.

He was taken up for Pulmonary Thrombo Endarterectomy through sternotomy. Heart was moderately enlarged, PA was tense. Standard CPB instituted by bicaval and aortic cannulation. Patient was cooled to 20 degree celcius and cardioplegic arrest achieved. After caval snaring TCA established and RPA opened and endarterectomy done with good back bleeding RPA closed using autologous vein patch. Circulation was resumed and than LPA was opened on low flows and endarterectomy was done. LPA also closed with autologous vein patch. He was fully rewarmed and and aorta declamped, weaned and decannulated. Their was no pulmonary hemorrhage or hypoxia. Patient had uneventful recovery postoperatively with no neurological or pulmonary complications and was discharged on 13th POD.

We conclude that early diagnosis, detailed evaluation, early intervention and excellent $\mathrm{CPB}$ management under deep hypothermia in patients with pulmonary thromboembolism is paramount. The strategy of performing endarterectomy over one side on TCA and than resuming circulation followed by endarterectomy over other side on low flows prevents neurological complications and adverse effects of TCA.

Surgery of acute pulmonary thromboembolism:

A single center experience

Won-Sang Chung; Hyuck Kim

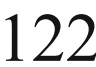

\section{ABSTRACT: \\ BACKGROUND:}

Pulmonary thromboembolism is potentially life threatening and rare in young and healthy adolescents. There are few cases reported in the literature with result of surgical treatment in patients with massive pulmonary thromboembolism.

\section{METHODS:}

From September 2006 to October 2018, 11 cases with acute pulmonary thromboembolism were retrospectively reviewed. All patients were in critical hemodynamic condition, with the patient deemed unlikely to survive.

\section{RESULTS:}

There was no early mortality.

\section{CONCLUSION:}

Although most of the treatment of acute pulmonary embolism is preceded by anticoagulant therapy, when the vital sign is unstable, considering surgical treatment is a viable option with safety. 
Surgical embolectomy for submassive saddle pulmonary embolism

Kristine Teoh; Leow Choong Kiat Lowell

\section{ABSTRACT:}

We describe a case of a 69 year old gentleman who presented with chest pain and shortness of breath on exertion secondary to a submassive saddle pulmonary embolism who successfully underwent surgical embolectomy with good outcomes.

A 69 year old gentleman was admitted to another hospital's cardiology unit for chest pain and dyspnoea of 2 days duration. He was treated as a non-ST elevation myocardial infarction (NSTEMI). A coronary angiogram was performed which showed triple vessel disease. However, on transthoracic echocardiogram, a long cylindrical mass measuring $1.4 \mathrm{~cm} \mathrm{x}$ $9.7 \mathrm{~cm}$ was seen in the right atrium prolapsing into the right ventricle causing right ventricular dysfunction. An urgent computed tomography pulmonary angiogram confirmed a saddle pulmonary thromboembolism with large filling defects extending from the right heart chambers into bilateral subsegmental pulmonary arteries. The patient was transferred to our cardiothoracic unit and underwent an emergent pulmonary embolectomy, right atrial embolectomy and coronary artery bypass graft (CABG) $x 3$. Bicaval cannulation was used with a left atrium vent. The main pulmonary artery (PA) was opened and saddle embolus extracted almost fully intact. Further fragments were flushed retrograde out through the right PA and left PA. Hypothermic circulatory arrest was used to extract the atrial component of the embolus and its superior venae cavae extension. Once all emboli were cleared, cardiopulmonary bypass was resumed and a routine triple bypass was performed.

The patient recovered smoothly post operatively with no complications. A deep vein thrombosis scan done showed only distal disease hence no inferior vena cava filter was placed. He was anticoagulated with warfarin on discharge.

Our case highlights the diagnostic challenges in detecting even massive pulmonary embolism, showcases interesting clinical images and demonstrates good outcomes associated with early surgical embolectomy for patients with saddle pulmonary embolism

\section{Bilateral Pulmonary Thromboendarterectomy for Chronic Pulmonary Thromboembolism developing Severe Pulmonary Hypertension - how do we do it. \\ Prasanta Kumar Chanda \\ Prodip Kumar Biswas}

\section{ABSTRACT: \\ INTRODUCTION:}

Chronic Pulmonary Thromboembolism (CPHE) occurs when emboli get trapped into pulmonary arteries either in single episode or subsequent episodes that get organized and become firm, fibrous tissue within the arteries resulting development of pulmonary hypertension.

\section{BACKGROUND:}

Approximately $1 \%$ to $5 \%$ of patient of acute pulmonary embolism results in CPHE. When Pulmonary Hypertensiondevelops mortality rate is high with fewer treatment option. Pulmonary thromboendarterectomy (PTE) is the treatment of choice. Here we have shown our operative approach for this condition.

\section{CASE DESCRIPTION:}

A 55year old gentleman came to our hospital with shortness of breath (NYHA III), occasional chest pain with a history of bone fracture 6 months back following a road traffic accident. Pulmonary computed tomography (CT) angiogram reveals both the branched pulmonary arteries completely occluded by thrombus with irregular outlines. Cardiac catheterization shows -severe pulmonary artery hypertension, non-critical coronary artery disease. We did bilateral pulmonary thromboendarterectomy with good outcome. After operation he was able to do his normal work comfortably, that was quit a night mare for him before operation.

\section{CONCLUSION:}

Pulmonary thromboendarterectomy offers a potential surgical cure for CTEPH and is the surgical procedure of choice for patients with this condition. Early recognition and referral to a centerspecializing in the management of pulmonary hypertension and with the necessary operative experience will allow timely intervention, resulting in improved operative mortality and long term outcome.

Keywords: Pulmonary thromboendarterectomy, pulmonary hypertension, pulmonary embolism.

${ }^{1}$ Registrar, Cardiac Surgery, National Heart Foundation Hospital and Research Institute; ${ }^{2}$ Associate professor, Cardiac Surgery NHFH and RI; ${ }^{3}$ Assistant professor Cardiac Surgery NHFH and RI; ${ }^{4}$ Assistant professor Cardiac Surgery NHFH and RI; ${ }^{5}$ Professor and Head of the department, Cardiac Anesthesia NHFH and RI.

Wedge resection as an alternative procedure for primary pulmonary carcinoma in poor-risk patients Hitoshi Igai; Mitsuhiro Kamiyoshihara

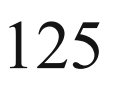

\section{ABSTRACT:}

\section{BACKGROUND:}

We present wedge resection as an alternative procedure for primary pulmonary carcinoma in poor-risk patients.

\section{PATIENTS AND METHODS:}

We examined the overall survival of 94 patients who underwent wedge resection for $\mathrm{pN} 0 \mathrm{M} 0$ primary pulmonary carcinoma over the last 20 years because of their intolerance of lobectomy.

\section{RESULTS:}

In the wedge resection group, the postoperative 5-year survival in all causes of death was $59.6 \%$, indicating significantly better prognoses in patients with adenocarcinoma aged less than 75 years old. The 5-year survival in the lobectomy group was $77.5 \%$, while the 5 -year survival in terms of primary causes of death in the wedge resection and lobectomy groups was $89.3 \%$ and $88.0 \%$, respectively. There was a significant difference between wedge resection and lobectomy in all causes of death, but not between groups in primary causes of death.

\section{CONCLUSION:}

Because there were many non-primary deaths in the wedge resection group, care should be taken to follow comorbidities that cause limited lung resection. Survival in the wedge resection group was not inferior to that in the lobectomy group in analyses of the primary causes of death. We suggest that wedge resection should be a favorable procedure for primary pulmonary carcinoma in poor-risk patients to obtain a large enough sample volume of tumor cells.

Is video-assisted thoracoscopic surgical decortication possible for stage III empyema?

Fauzi; Lok Yuh ing

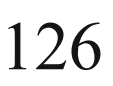

\section{OBJECTIVE:}

Video-assisted thoracoscopic surgical decortication (VATSD) is widely applicable in fibropurulent (Stage II) empyema, whereas organizing (Stage III) empyema requires conversion to open thoracotomy. However, existing reports reveal a lacuna in the management of Stage III empyema via VATSD. We evaluated the effectiveness of VATSD in both Stage II and Stage III pleural empyema and perioperative clinical outcomes.

\section{METHODS:}

A retrospective review of patients who diagnosed as empyema thoracis and treated with VATSD from March 2016 to July 2017 was performed. The perioperative outcomes including surgical effectiveness, duration of postoperative hospital stay, postoperative morbidity and mortality were analysed. 


\section{RESULTS:}

Total of 21 patients underwent VATSD in Penang General Hospital which mean age was 40.5 years and $16(76 \%)$ were male. Out of 21 patients, 10(48\%) had Stage II empyema and 11 (52\%) had Stage III empyema. The causes of empyema thoracis were pneumonia $(16,76 \%)$, infection secondary to prolonged tube thoracostomy $(2,10 \%)$ and undrained hemothorax $(2,10 \%)$. All Stage II empyema completed VATSD successfully. $9(43 \%)$ patients with Stage III empyema completed VATSD successfully whereas $2(10 \%)$ patients required conversion to open decortication. The length of postoperarative hospital stays for Stage II VATSD and Stage III VATSD were 7 days and 9 days respectively. Postoperative complications were significantly lower in patients treated with VATSD in terms of prolonged air leak, wound infection and postoperative pain.

CONCLUSION:

VATSD is an effective and safe treatment option for patient with Stage II empyema thoracis and feasible in most Stage III empyema thoracis with fewer postoperative complications.

\section{Deep cervical and paratracheal drainage is a good combination for treating descending necrotizing mediastinitis \\ Yoshikane Yamauchi; Takashi Sakai}

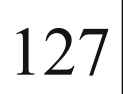

\section{PURPOSE:}

Descending necrotizing mediastinitis (DNM) is a life-threatening infection. DNM diffuses to the deep cervical fascia into the pretracheal, perivascular and retrovisceral, or prevertebral space. Deep cervical drainage and paratracheal drainage (DC-PTD) play important roles in DNM, and transthoracic mediastinal drainage is an essential treatment of DNM. METHODS:

Eleven patients who were diagnosed with DNM were treated with surgical treatment during April 2007 to December 2017 at Teikyo University Hospital, Tokyo Japan. Six patients who were diagnosed with extensive DNM had progressive extension of abscesses to the lower mediastinum below the level of the carina. The other five patients with localized DNM were infected in the upper mediastinum above the level of the carina. RESULTS:

DC-PTD was first performed in 10 patients who all recovered (5 extensive DNMs and 5 localized DNMs). One patient with extensive DNM had deep cervical drainage after transcervical drainage was performed, and showed no improvement of his clinical condition. He died because he developed severe sepsis.

\section{CONCLUSION:}

Fluid collection in the deep cervical fascia must be urgently performed. The transcervical approach for DC-PTD is less invasive treatment than thoracotomy/transthoracic approaches. However, we must always consider transthoracic mediastinal drainage when the transcervical approach cannot control infection. Thoracic surgeons and otolaryngologists must plan an effective treatment before approaching DNM.

\section{Primary Pulmonary Botryomycosis: A Rare Infection In The Antibiotic Era \\ Ganesh Sevagur Kamath; Guruprasad Rai D.}

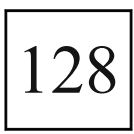

\section{ABSTRACT:}

Botryomycosis is a relatively rare pyogranulomatous disease and mostly observed cases have been of cutaneous or visceral type. We report a case of a $39 \mathrm{yr}$ female diagnosed with necrotising pneumonia. Microscopic examination of a transbronchial biopsy specimen revealed chronic suppurative inflammation and planned for lobectomy. Histological analysis of specimens taken after surgery revealed pulmonary botryomycosis. The diagnosis was made on the basis of characteristic eosinophilic granules in which the bacteria are surrounded by protein material (Splendore-Hoeppli phenomenon). Pulmonary botryomycosis may also be mistaken for pulmonary actinomycosis, tuberculosis or due to mass effect can resembles pulmonary carcinoma. Successful treatment often requires a combination of both surgery and long-term antimicrobial therapy. We report here a case of primary pulmonary botryomycosis occurring in a lung cavity.

Development of predictive nomogram for cause-specific mortality in surgically resected early-stage esophageal carcinoma:

a Surveillance, Epidemiology, and End Results analysis 129

Juwei $\mathrm{Mu}$

Xiangyang $\mathrm{Yu}$

\section{ABSTRACT:}

OBJECTIVE:

The objective of this study was to evaluate the probability of cancer-specific death and other causes of death for patients who underwent surgery with early-stage (T1N0) esophageal carcinoma (EC). We also built competing risk nomograms to predict prognosis for patients with EC.

\section{METHODS:}

Data were extracted from the Surveillance Epideiology, and End Results (SEER) database. We identified patients who underwent surgery with earlystage EC between 2010 and 2015. We estimated the cumulative incidence function (CIF) for cancer-specific death and other causes of death, and tested the differences by Gray's test. The Fine and Gray's proportional subdistribution hazard approach was applied to model CIF. We also built competing risk nomograms based on Fine and Gray's model.

\section{RESULTS:}

We identified 1144 stage T1N0 EC patients from 2010 to 2015 in the SEER database. The 5-year cumulative incidence of cancer-specific death for stage T1N0 EC was $15.7 \%$ and $11.2 \%$ for other causes of death. Variables associated with cancer-specific mortality included age, tumor length $(\mathrm{cm})$, T stage, grade, histology. The nomograms were well calibrated, and had good discriminative ability, with c-index of 0.663 for cancerspecific mortality model and 0.699 for competing mortality model.

\section{CONCLUSIONS:}

We evaluated the CIF of cause-specific death and competing risk death in patients with surgically resected early-stage (T1N0) EC using the SEER database. We also built proportional subdistribution models and the first competing risk nomogram to predicting prognosis. Our nomograms show a relative good performance and can be a convenient individualized predictive tool for prognosis.

Interesting case of Cervical Rib with Arterial

Thoracic outlet syndrome

A. Rathinavel

B. Karthikeyan

\section{ABSTRACT:}

\section{INTRODUCTION:}

Cervical rib is one of the most common causes of Thoracic outlet syndrome (TOS). $95 \%$ of the cases will present with Neurogenic TOS. Only $2 \%$ of cases will present with arterial TOS.

\section{CASE REPORT:}

55 years old female patient developed numbness and coldness of left upper limb for 6 months duration. On evaluation she found to have left side complete cervical rib with obstruction of subclavian artery with post stenotic aneurysm with thrombus upto proximal brachial artery and distal collateral flow. Right side incomplete cervical rib without any obstruction Through Left sided supra clavicular approach, cervical rib excised completely. Subclavian artery found to be completely thinned out hence planned for left side common carotid artery to left axillary artery bypass graft using $8 \mathrm{~mm}$ PTFE graft. Graft tunneled below the left clavicle. 
Fogarty's thrombectomy done through axillary artery and then anastomosis completed. Left brachial plexus neurolysis done and wounds closed with negative suction drain.

Post operatively patient adequate blood flow to left upper limb documented by Doppler monitoring. Post operative angiogram showed good flow to the upper limb

CONCLUSION:

Although cervical rib excision is enough in most of the cases to relieve obstruction to subclavian vessels, some chronic cases like this may need a bypass graft to maintain adequate blood flow to the limb.

\section{Giant esophageal GIST: Catch me if you can}

Santhosh Benjamin; Raj Kumar J.

\section{ABSTRACT: \\ INTRODUCTION:}

Gastrointestinal stromal tumours (GIST) are mesenchymal neoplasm commonly seen in stomach $(60 \%)$ and small intestine $(25 \%)$. It is rare in the esophagus $(2 \%)$. We intend to present a case of giant esophageal GIST and discuss the diagnostic dilemma and therapeutic challenges.

\section{CASE PRESENTATION:}

A 68-year-old male presented with chest pain and dyspnea for the last 18 months. Chest radiograph and CT revealed a large mass abutting the thoracic esophagus and causing mass effect. Upper gastrointestinal scopy did not show any mucosal lesion. CT guided biopsy was suggestive of a cellular neoplasm. Immunohistochemistry was inconclusive. In endoscopic ultrasound(EUS), involvement of the esophageal wall was not demonstrated. He underwent a thoracotomy where the tumour was noted to be involving the esophageal muscle layer. The mucosal layer was intact. The surgical specimen was reported was positive of CD117 and DOG with mitotic activity noted in 8 per 50 high power field. Adjuvant Imatinib therapy was initiated.

\section{DISCUSSION:}

Most common submucosal mass arising in esophagus is leiomyoma. Differentiating it from the GIST is difficult with CT and EUS as they have overlapping features. Obtaining a FNAC is controversial as there is a chance of tumor seeding, but in view of size and GIST being one of the differential diagnosis, it was attempted. Due to rarity of esophageal GIST there is a lack of clear recommendation for optimal surgical management. In our case, we resected the tumour with primary closure of the esophageal muscle layer. In view of size and location of the tumour, we had initiated Imatinib for this patient.

\section{CONCLUSION:}

Esophageal GIST may present with non-specific symptoms despite being large. Biopsy is not always definitive. Optimal surgical management is still debatable. Imantinib in the neo-adjuvant and adjuvant setting needs further evaluation.

\section{THORACIC WOMB-THE REAL CULPRIT: A CASE SERIES OF THORACIC ENDOMETRIOSIS SYNDROME}

Mohd Fauzi; N.H. Syed; Ahmadi Salleh; Abu Yamin; Basheer A. Kareem; M.K. Hamzah

Law Poh Suan

\section{ABSTRACT:}

Thoracic Endometriosis Syndrome (TES) is rare (3-20\%) which includes notably pneumothorax, haemothorax, hemoptysis and lung nodules independent of histological confirmation. It most probably has been underdiagnosed as the severity of symptoms can be variable. Here we describe 3 cases of TES have concomitant pelvic endometriosis.

CASE PRESENTATION:

All patients never had catamenial related cyclic symptoms. First case,31years old lady had right recurrent pneumothorax after laparoscopic bilateral salphingectomies. Second case,28years old lady had bilateral pneumothorax a month after open myomectomy. Third case,35years old lady had right recurrent pleural effusion. Diaphragmatic fenestrations were found in them during thoracoscopic surgeries. However, all histopathology reports were negative for endometrial stroma tissues. They have been asymptomatic with plans to complete 6 months of hormonal therapy.

\section{DISCUSSION:}

All 3 patients presented at age 28-35 which is similar to cases reported by various authors. Two of them had right hemithorax involvement which was reported as the most often affected site $(85-95 \%)$. Bilateral hemithoraces involvement are extremely rare. The incidence of having concomitant pelvic endometriosis was 50$85 \%$. In our cases, the patients were commenced on hormonal therapy which suppressed the menstrual cycles. Histological confirmation is ideal but elusive as the biopsy should be obtained just before or within 48 hours of the onset of menses as endometrial tissue may autolyze within this period. The characteristic lesions are diaphragm fenestrations, nodular and / or brownish endometrial deposits. Video-assisted thoracoscopic remains the gold standard tool for definitive diagnosis and surgical therapy. Patients with intraoperative findings suggest of TES should be referred to the gynaecologist for pelvic endometriosis assessment. The risk of recurrence reduced was reported as high as $50 \%$ in hormonal therapy and $30 \%$ in surgical therapy. Outcomes are however better in combined therapy $(8.3 \%)$.

\section{CONCLUSION:}

High clinical index of suspicion of TES in premenopausal women is important. Prompt diagnosis and commencement of combined therapy reduces the risk of recurrence.

Fibrous Dyaplasia and Anerysmal Bone Cyst of Rib. J.K. Das; Jayanta Kumar Das

\section{ABSTRACT:}

Enter description here - Fobrous Dysplasia of ribs is a benign cystic leson characterise by fibrous replacement. of the medulary cavity of the rib. It accounts for up to $30 \%$ of all the chest wall tumours and most commonly present as a solitary mass in lateral and posterior rib cage. Aneurysmal bone cyst accounts for less than $1 \%$ of chest wall tumours.

Reporting here a 28 years old male who presented with complaints of pain chest and on clinical examination found swelling of the isolated rib on the right side. Computed Tomography revealed a cystic lesion involving the 7 th rib right side suggestive of fibrous dysplasia. The tumour was succesfully removed en block through a right posterio-lateral thoracotomy. Histological examination confirmed fibrous dysplasia with secondary aneurysmal bone cyst.

Investigation of the factors prolonged length of stay after complete video-assisted thoracoscopic surgery

in clinical stage I lung cancer

Taijiro Mishina; Atsushi Watanabe; Masahiro

Miyajima; Yuki Takahashi; Ryunosuke Maki;

Makoto Tada; Miho Okawa; Wataru Arai

\section{ABSTRACT:}

OBJECTIVES:

We conducted a retrospective analysis of the complications of clinical stage I lung cancer after VATS procedure and investigated the factors of prolonged length of stay (PLOS).

\section{PATIENTS AND METHODS:}

813 patients with clinical stage I lung cancer who underwent VATS between January 2007 and July 2018 was retrospectively analyzed. In cases where more than the 3 rd quartile of the total was identified 
as PLOS, more than 13 days was the PLOS in our hospital. Postoperative complications were evaluated based on the ClavienDindo classification v2.0. The relation between PLOS and each factor was analyzed with t-test, Chi-square test, and added logistic regression model.

\section{RESULTS:}

$209(25.7 \%)$ cases of PLOS. The characteristics of the PLOS and non-PLOS groups were as follows: age 70.9 and 68.0 years (p\&lt;.001), duration of chest tube drainage 4.85 and 2.06 days (p\&lt;.001), blood loss 279 and $174 \mathrm{ml}(\mathrm{p}=.018)$, operative time 234 and $210 \mathrm{~min}$ (p\&lt;.001), and pathological maximum tumor diameter 24.2 and $22.2 \mathrm{~mm}(\mathrm{p}=.031)$, respectively. The preoperative factors were male ( $\mathrm{p} \& \mathrm{lt} ; .001$, odds ratio $(\mathrm{OR})=1.858)$, emphysema (p\&lt;.001, OR=1.940), and interstitial pneumonia $(\mathrm{p}=.006$, $\mathrm{OR}=2.037)$. The intraoperative factor was pleural adhesions (p\&lt;.001, OR=1.939). Postoperative factors, postoperative complications more than grade II(p\&lt;.001, OR=8.354), acute exacerbation of interstitial pneumonia $(\mathrm{p}=.005$, $\mathrm{OR}=5.921)$, bronchial fistula $(\mathrm{p}=.005, \mathrm{OR}=7.377)$, pneumonia ( $\& 1 \mathrm{lt} ; .001, \mathrm{OR}=17.747)$, pulmonary fistula (p\&lt;.001, OR=15.924), subcutaneous emphysema (p\&lt;.001, OR=20.078), and atelectasis (p\&lt;.001, OR=18.335), were associated with PLOS. In multivariate analysis, duration of chest tube drainage more than 5 days ( $\&$ \&lt;.001, OR=3.551), postoperative complications more than grade II (p\&lt;.001, OR=4.223), pulmonary fistula $(\mathrm{p}=.012, \mathrm{OR}=3.116)$, pneumonia ( $\& 1 \mathrm{t} ; .001$, $\mathrm{OR}=5.878$ )

\section{CONCLUSIONS:}

More than grade II postoperative complications, pulmonary fistula, and pneumonia extend the length of hospital stay. Be performed reliable closure of intraoperative air leakage, and control of postoperative complications, including pneumonia, will be prevent prolonged length of stay.

\section{Do blebs or bullae on high-resolution computed tomography predict ipsilateral recurrence in young patients with first episode primary spontaneous pneumothorax?}

Hyuck, Kim

Won Sang, Chung

\section{ABSTRACT: \\ PURPOSE:}

The relationship between bullae size and recurrence of pneumothorax is controversial. The aim of this study was to retrospectively evaluate the role of blebs or bullae in ipsilateral recurrence in young patients with first episode primary spontaneous pneumothorax (PSP) who underwent conservative treatment.

\section{METHODS:}

Two hundred ninety-nine cases with first episode PSP were enrolled. The status of blebs or bullae was reviewed from high-resolution computed tomography (HRCT). Dystrophic severity score (DSS) was calculated by HRCT and ranged from 0 to 6 points.

\section{RESULTS:}

The 5-year recurrence rate was $38.2 \%$. In univariate analysis, age (\&1t;20 years), BMI (\&1t;20 kg/m2), unilateral lesion, and intermediate risk (DSS 4 and 5) were associated with recurrence. Sex; smoking history; and presence, number, and maximal size of blebs or bullae were not related to recurrence. In Cox regression, age and intermediate risk were independent risk factors for recurrence. High risk (DDS 6) was not an independent risk factor.

\section{CONCLUSION:}

The presence, number, and size of blebs or bullae did not affect ipsilateral recurrence. DSS failed to show a positive correlation between severity and recurrence. The decision to perform surgery in the first episode of PSP might not be determined by severity of blebs and bullae.
Hydatid cyst of thorax extending to spine

Amaresh Rao M; Goutham Kollapalli

ABSTRACT:

Hydatid cyst of thorax extending to spine INTRODUCTION:

A case report of hydatid cyst of thorax extending to spine causing paraparesis and dyspnoea. Due to the rarity of presentation one must have hydatid cyst as a differential diagnosis in patients who are from endemic areas and present with focal neurological deficits and dyspnoea.

\section{CASE HISTORY:}

A 39 yrs old female presented to us with c/o of paraparesis since 5 months and dyspnoea since 3 weeks. O/E: a large swelling present on right para spinal region with decreased breath sounds on right side.

On HRCT- A large cystic mass of $16 \times 10 \times 10 \mathrm{~cm}$ in right upper part of lung extending into vertebral canal through neural foramina with destruction of right 5 th, 6 th ribs posteriorly

Surgery: posterior right sided laminectomy with the detachment of 5 th and 6th rib posteriorly and multiple hydatid cyst removed. A separate anterolateral thoracotomy incision was given for removal of the anterior intra thoracic extension of the cyst.

Post operative: the patient significantly improved with reduced dyspnoea on 2 nd day and was able to walk on 5th day. Patient was put on albendazole therapy and was followed up after 1 month with no symptoms.

\section{CONCLUSION:}

Any case of paraplegia and with severe dyspnoea with a cystic lesion suspect hydatid cyst more so in endemic area. Surgical removal with albendazole therapy has got excellent results but chances of recurrences are always present and to be watched for.

Primary Mediastinal Seminoma

Sreekar Balasundaram

Naveen George

\section{ABSTRACT:}

Primary pure Cell Seminoma of the Mediastinum is rare and potentially fatal lesion. Encroachment on or invasion of adjacent structures is common, as are distant metastasis. The differentiation between pure cell Seminoma and Mixed germ cell Tumour should be made since the extreme radio sensitivity of the seminoma improve the prognosis. A patient who was successfully treated for the mediastinal seminoma with excision and irradiation is presented here.

Giant Pulmonary Hamartoma- A case report

(Brig) Anurag Garg; Smruti Hindaria

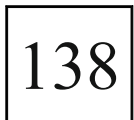

ABSTRACT:

We report a case of huge Pulmonary Hamartoma with collapsed lower lobe of left lung in a 32 years old woman. The woman presented with features of a space occupying lesion in left lung with deranged pulmonary functions. The Pulmonary Hamartoma was solid, $25 \mathrm{~cm} \times 15 \mathrm{~cm} \times 12.5 \mathrm{~cm}$ in size, with cross section revealing greyish-white glistening lobules of cartilage with firm to hard nodules. The tumour was compressing the left lung with no infiltration into the surrounding structures. The mass was successfully excised through left posterolateral thoracotomy, followed by complete expansion of left lower lobe and histopathology revealed lobules of mature cartilage separated by clefts lined with respiratory epithelium, with admixture of fibroconnective tissue and adipose tissue with placental villi differentiation. Patient did well post operatively.

This is one of the largest cases reported so far, successfully resected through left posterolateral thoracotomy. 
Total Sternectomy with chest wall reconstruction without using sternal implant for osteosarcoma sternum Neerav Bansal; Varun Bansal

\section{ABSTRACT: \\ INTRODUCTION:}

Less than $1 \%$ of bone tumors occur in sternum. Sternal osteosarcoma is a rare malignant mesenchymal tumor. It is resistant to radiation, so wide resection with neoadjuvant chemotherapy is the curative option.

\section{CASE REPORT:}

A 36 year lady presented with history of vague pain over anterior part of chest with gradually increasing swelling in middle of chest for one month. On evaluation it was found to be osteosacrcoma involving the body of sternum. Total sternectomy (body, manubrium and xiphoid) with removal of 3 rd to 6th costal cartilages was done. The sternal end of clavicles were fixed with a titanium plating and sternal gap was bridged by bringing the costal margins close with the help of intervening composite mesh of Dacron and polypropylene. Over the mesh double breasting of Pectoral major muscle was done. Specimen margins were clear of malignancy and patient did not have any recurrence at 18 month follow up.

\section{DISCUSSION:}

Chest wall osteosarcoma occurs in two age groups including young adults presenting with osseous type and patients over 50 years of age with extra osseous type. Frequently asymptomatic, patients sometime present with painful swelling in upper chest wall. Radiographic appearance is of irregular reactive bone and cortical destruction. MRI is helpful in surgical planning and to define extent of the lesion within the bone as well as extra osseous spread of the tumor and skip metastases. Surgical resection remains the cornerstone of treatment but poses challenges especially at places like sternum.

\section{Scimitar syndrome}

Goutham; Kalyanbabu

\section{ABSTRACT:}

Enter description here:scimitar syndrome is a rare anomaly of partial pulmonary venous drainage in to the inferior vena cava. Here we present a case of 22 years old female who present clinically with chest pain breathlessness and two episodes of haemoptysis in 3 months duration. The diagnosis obtain on $\mathrm{CXR} / \mathrm{CT}$ angiogram of chest and abdomen where we found partial anomalous pulmonary venous connection of Rt inferior pulmonary vein. A Rt antero lateral thorocotomy approach done the abnormal Rt inferior pulmonary vein ligated and devided. The post operative period was un eventful and patient was recovered well and discharged on 7 th post operative day.

\section{A case of tracheal diverticulam}

Ravikrishnan; Vipin B. Nair

\section{ABSTRACT:}

A $34 \mathrm{yr}$ female who presented with complains of dysphagia. On evaluation she was found to have atracheal diverticulam. she was operated upon and the specimen sent for biopsy whichconfirmed it to be a diverticulam. All over the world the reported cases are approx $2 \%$ which is a rare entity. Tracheal diverticulam are classified as congenital and acquired based upon the certain criterion.

Since this is a rare case, I would like to present this case.

\author{
Successful surgical management of atrioesophageal \\ fistula after radiofrequency catheter ablation : \\ a case report \\ HyoJun Jang \\ Won Sang Chung
}

\section{ABSTRACT:}

Atrioesophageal fistula (AEF) is a rare but lethal complication of radiofrequency catheter ablation of atrial fibrillation(AF). We report a case of successful surgical management of AEF.

A 60 year old male had catheter ablation for AF. He was admitted with high fever and altered mental status previously complaining of fatigu. Brain CT showed multifocal septic embolism and chest CT showed suspicious finding of AEF around junction between left atrium and left inferior pulmonary vein. He underwent emergency exploration. Intraoperatively, loculated pus was founded in posterior mediastinum. After dissecting of posterior mediastinum, fistula on the left atrium was founded, however fistula on the esophageal wall was not well observed because of severe infection. Normal side of esophageal wall was incised and fistula on the esophageal mucosa could be founded. Fistula on both side was repaired and intercostal muscle flap was interposited. After 4 weeks administration of antibiotics, He could be discharged with a little neurological deficit

\section{PRIMARY LUNG TERATOMA : CASE REPORT}

R.M. Krishnan

Pardhasarathi Reddy Allam

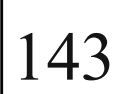

\section{ABSTRACT:}

\section{INTRODUCTION:}

Teratomas are tumors consisting of tissues derived from more than one germ cell line. Teratoma is a common tumor of mediastinum, but is very rarely found in the lung with less than 100 cases published to date. Criteria for pulmonary origin are exclusion of a gonadal or extra gonadal primary site and origin entirely within the lung.

\section{CASE REPORT:}

16 years old male presented with pain in right chest since 1 year, cough \&amp; breathlessness on lying left lateral position since 6 months. Chest radiograph showed complete opacity on right hemithorax. CT chest showed a huge well defined multi septate cystic lesion in right hemithorax with mediastinal shift. CT guided biopsy showed teratoma.

Postero lateral thoracotomy done through 5th ICS. Pleura opened \&amp; right thoracic cavity entered. Large mass adherent to the right lung \&amp; mediastinum, adhesions released, mass removed in total. Post excision of mass, right lung expansion was not satisfactory and hence proceeded with pneumonectomy. Post operative period was uneventful. Patient discharged on 9th POD. Patient on followup is doing well. Pathology of mass consistent with benign cystic teratoma

\section{CONCLUSION:}

Primary lung teratoma are exceptionally rare tumor. They originate from third pharyngeal pouch. Trichoptysis is most specific symptom but rare. $\mathrm{CT}$ chest is diagnostic. Complete resection is the treatment of choice with good long term prognosis

Sarcoidosis and Lung cancer, a coincidence? D.B. Aneez Ahmed; Aliakbar Fazuludeen

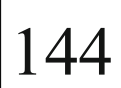

\section{ABSTRACT:}

A 53-year-old man presented with incidental findings of left lower lobe lung opacity on pre-employment chest radiograph.

A chest radiograph showed nodular shadowing in the left midzone. A computed tomography (CT) scan of the thorax illustrated A $1.6 \times 1.3 \mathrm{~cm}$ nodule with mild spiculated margin.

CT guided biopsy revealed that Nonsmall cell adenocarcinoma. He underwent Left thoracotomy and lower lobectomy. Intraoperative findings a mass in the left lower lobe. Mediastinal nodes were taken for sampling. Histology of the sample revealed double pathology. 1) moderately differentiated adenocarcinoma of the left lung. 2) non-necrotizing granulomatous inflammation - sarcoidosis. 


\section{DISCUSSION:}

There was no pre-existing lung condition to assess risk for sarcoidosis and the value of the CT guided biopsy can be questioned as it was unable to identify the presence of sarcoidosis preoperatively. This could also mean that the biopsy could have identified sarcoidosis instead and the absence of the identification of the lung cancer could mean delayed treatment of the lung cancer. It is essential to identify the key factors that link the pathogenesis of sarcoidosis and lung cancer. The association between sarcoidosis and lung cancer have long been debated. There are many mechanisms that have been described to understand the link.

1 It is a mere coincidence of such occurrences.

2 There is an association of T cell-mediated immune irregularities involved in the pathogenesis of sarcoidosis, with that of lung cancer.

3 Sarcoidosis results in fibrosis tissue, which is a precursor in the development of lung cancer.

4 Lung cancer causes an immunohistological reaction, resulting in sarcoidosis - sarcoid reactions.

Esophageal Tuberculosis presenting as Dysphagia G.N. Lone; Farooq Ahmad Ganie

\section{ABSTRACT:}

Esophageal tuberculosis is a rare in both immunocompromised and immunocompetent hosts with advanced pulmonary tuberculosis even in countries with high prevalence of tuberculosis like south East Asia. this study presents a case report of Esophageal tuberculosis presenting as Dysphagia.

\section{Ectopic Cushing's Syndrome in a hidden spot} Prem Prasath K.

\section{ABSTRACT: \\ Ectopic Cushing's Syndrome in a hidden spot INTRODUCTION:}

Ectopic Cushing's Syndrome is a form of Cushing's where a tumor outside the pituitary produces adrenocorticotropic hormone. We present here a case of ectopic ACTH secreting neuroendocrine tumor.

\section{CASE PRESENTATION:}

A 19yrs boy presented with complaints of cough and fever for past 1 month. HRCT was done to evaluate the same and was found to have left diaphragmatic mass. Serum ACTH levels were raised. Hence Dota PET CT was done, which revealed a Non dota avid lesion in the left supradiaphragmatic region. He underwent thoracotomy and excision of diaphragmatic mass and mediastinal lymphnodes. The surgical specimen showed findings of ACTH secreting high grade neuroendocrine carcinoma. Post operative ACTH levels declined.

\section{DISCUSSION:}

Ectopic ACTH secretion is a less common cause of Cushing's Syndrome, seen in 5 to $10 \%$ of cases with endogenous hypercortisolemia. It is associated with a high rate of infectious complications. Raised Serum ACTH levels and Ga68- DOTA PET CT were useful in the diagnosis of the ectopic ACTH secreting neuroendocrine tumor. Surgical resection of the ectopic tumor remains the mainstay of treatment.

\section{CONCLUSION:}

Ectopic Cushing's Syndrome in a hidden spot can be very hard to detect. We should first aim to lower blood cortisol medically or through surgery to avoid Cushing's complications such as severe infections, and then patients should be followed up through imaging studies to detect the tumor and resect it which Is the definitive treatment in such patients.

\section{Management of Rheumatoid Lung}

Alberto Oliaro; Pierluigi Filosso; Enrico Ruffini;

Paolo Olivo Lausi; Elisa Fontana

\section{ABSTRACT:}

Rheumatoid arthritis is one of the leading connective tissue diseases in Asia and Europe with an incidence rates of 54/100 000 for women and $25 / 100000$ for men.

Rheumatoid lung is a relative common form of involvement of pulmonary interstitium associated with rheumatoid arthritis.

The incidence of lung involvement in clinical evident rheumatoid arthritis varies from 10 to $70 \%$, and contributes up to $20 \%$ of the overall mortality.

Virtually any part of the respiratory tract can be affected.

The most common presentation consists of bronchiectasis and chronic bronchitis

The role of thoracic surgery in the management of this disease is limited to the treatment of complication as pneumotorax and, in some cases, to the performance of pulmonary biopsy, neccessary for diagnosis of uncertain lesions.

A 64-year-old man, non smoker, under treatment with Adalimumab for seropositive rheumatoid arthritis for the past 8 months was admitted at the emergency department of our institution for acute dyspnea and subcutaneus emphysema.

The patient had previously no signs or symptoms of pneumotorax, pneumomediastinum or spontaneus subcutaneus emphysema.

CT scan of the chest revealed pneumomediastinum, subcutaneus emphysema and diffuse honeycombing.

Broncoscopy and esophagoscopy were performed in order to exclude the presence of a laceration of the trachea, of the bronchus or of the esophagus.

The absence of a pneumotorax and the relative stable clinical conditions of this patient, lead us to the decision of a conservative management.

No chest tube was positioned and the patient was admitteed to a subintensive care unit in order to follow his clinical condition, and discharged at home after 4 days.

Clinical and radiological follow-up carried out 4 weeks later showed a full regression of subcutaneus emphysema and pneumomediastinum.

\section{Synchronous malignant pleural fibrous} tumor and NSCLC

Alberto Oliaro; Pierluigi Filosso; Enrico Ruffini; Paolo Olivo Lausi; Erika Passone

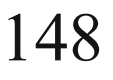

\section{ABSTRACT:}

The presence of synchronous neoplasm is a relative rare condition. The pleural fibrous tumor (PFT) are rare primary tumors whic originate from submesothelial mesenchymal tissue of the parietal, mediastinal, diaphragmatic and visceral pleura. Only $10 \%$ of the PFT are malignant. Both benign and malignat forms of PFT should be treated with radical surgicla resection, which may include wedge resection or lobectomy and chest wall resection. Surgery has been demonstrated as the unique effective therapy for PFT. We present the case of a 81 - year - old woman, non smoker, with synchronous malignant pleural fibrous tumor and lung adenocarcinoma. The patient came to our attention due to a persistent thoracic pain.

The chest radiograph demonstrated a paramediastinic epifrenic right mass. The enhanced CT scan confirm the presence of the lesion. The PET scan demonstrated a SUV value 6.9 on the main lesion in the right lower lobe, as well as another smaller nodule in the right upper lobe. A CT guided biopsy was performed; the cytological diagnosis was fibrous pleural tumor.

A right posterolateral thoractomy in VII intercostal space was performed. A $10 \mathrm{~cm}$ polilobulated lesion with strict adhesions with the right lower lobe was indentified. On the diaphragmatic pleura were identified some small nodules. A $2 \mathrm{~cm}$ lesion of the right upper lobe was identified. All the lesions were radical resected. The histopathological examination demonstrated that: 
The polilobulated RLL lesion was a MALIGNANT PLEURAL FIBROUS TUMOR. Immunohistochemical staining was positive for CD34 and CD 99, Ki67: 15\%

The lesione of right upper lobe was a well differentiated adenocarcinoma with an acinar pattern pT1a-N0

The diaphragmatic nodules were localizations of pleural fibrous tumor We have been monitoring our patient clinically and radiologically for 36 monts and have not recorded any recurrences or distant metastases.

\section{A case of Non Hodgkins T lymphoblastic lymphoma of thymus with secondaries in breast mimicking thyomoma}

Divakar Bhat; Bhagyashree Jagadeesh

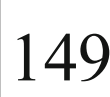

\section{ABSTRACT:}

Background In the anterior mediastinum, tumors originating from various tissue like thymic, epithelial, germinal or lymphoproliferative can occur. Sometimes it may be difficult to differentiate $\mathrm{T}$ lymphoblastic lymphoma(T-LBL) from a lymphocyte rich thymoma. T-LBL is uncommon tumor of anterior mediastinum, presenting in second to third decade, male predominance, in rapidly progressed stage. The 5 year survival is 45-55\%, prognosis worsens with age, high LDH level, advanced stage. Histologically, composed of cells with finely dispersed chromatin, scant cytoplasm, with round or highly convoluted nuclei and stain positive forCD3, CD99, TdT, neagtive for sudan black, Myeloperoxidase. Chemotherapy is the mainstay of treatment, surgical resection to be avoided. Case A 34 year old female, primarily evaluated for left breast lump, which was diagnosed benign fibrocystic disease, presented with recurrent breathlessness secondary to bilateral massive pleural effusion. $\mathrm{CT}$ thorax done revealed a large lobulated mass in anterior mediastinum /prevascular space with pericardial infiltration, medial quadrant lesion of breast. CT guided biopsy showed typeB1 thymoma, hence surgical resection planned. Intraoperatively, mass found adherent to under-surface of sternum, pericardium with lesions over diaphragm and lower lobes of lung. Debulking done along with left breast lump excision. HPE reported $\mathrm{T}$ lymphoblastic lymphoma of mediastinal mass and breast mass. Patient subjected to chemotherapy COP regime and endoxan with intercostal tube in-situ as she had persistant pleural effusion. She succumbed to rapidly spread disease after 1 1/2 month. Conclusion A differential diagnosis of T-LBL should be made when a rapidly growing anterior mediastinal mass with recurrent pleural effusion is encountered, as this needs Chemotherapy as against surgical excision for thymoma. This patient had unusual presentation of secondaries in the breast. Accurate histopathological diagnosis becomes important in determining the treatment and hence prognosis and remission.

[avatar user="bhagyashree01@gmail.com" size="original" align="center" link="file"]T lymphoblastic lymphoma of anterior mediastinum[/avatar]

\section{INTRATHORACIC PRESENTATION OF BRONCHOGENIC CYST WITH PARTIAL DEFECT OF PERICARDIUM} M. Vanithamani; V. Chitraleka

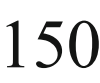

\section{ABSTRACT: \\ INTRODUCTION:}

Bronchogenic cyst a rare congenital malformation due to abnormal disturbance during development of primitive respiratory system during 4th week of gestation.

\section{CASE REPORT:}

66 years old gentleman admitted for the inguinal repair surgery in our hospital. Routine chest X ray showed large opacity in left lower zone causing mediastinal shift. CT Scan and MRI screening showed large cystic lesion of left lower zone with areas of calcification and mediastinal shift to right. Biochemical, haematological investigations normal. Hence referred to CTVS department. There was no h/o respiratory discomfort/chest pain / fever. Nondiabetic. Normotensive. Nonsmoker. Moderately built, Mildly decreased air entry on the left side of chest. Right side inguinal hernia present. PFT normal. No prostatomegaly. Fibrotic bronchoscopy normal. Thoracotomy planned. As thoracic cavity opened thick walled smooth cyst $-10 \mathrm{~cm} \times 10 \mathrm{~cm} \times 8 \mathrm{~cm}$ seen posteriorly adherent to hilar region. $1500 \mathrm{ml}$ of pus like thick fluid let out, sent for analysis and culure. Pericardium was absent over the pulmonary artery and left atrial appendage. Entire wall of the cyst excised except over adherent portion. Cut edges well cauterised. Routine closure. Post op period uneventful. Discharged on 10th post op day.

Histopathology report was suggestive of Bronchogenic cyst. Culture negative.

\section{DISCUSSION:}

Bronchogenic cyst though a rare congenital anomaly commonly located in lung parenchyma and mediastinum. Rarely localize outside. Extrathoracically in neck /carina /hilum. Usually asymptomatic. Complications include infection, obstruction /occlusion of SVC, pneumothorax, pleural effusion, arrhythmias and carcinomatous changes.

In cases with defective pericardium of herniation of cardiac contents, spread of infection, tamponade following rupture are possibilities.

Selective drainage, VATS surgery though very useful procedures Cyst resection via open thoracotomy is still a safe procedure.

\section{CONCLUSION:}

Though rare, asymptomatic cysts once diagnosed due to comparable malignant characteristics and possible complications excision is the best option. ption here.

Intrapulmonary teratomatous dermoid cyst - A Rare entity

Arun Beeman; Madhu Sankar; Jackin

Moses; Professor. Sarasa Bharathi; K.M. Cherian

Priyanka Jindal

\section{ABSTRACT:}

Teratoma of the lung is a rare tumor, and only few cases have been reported in literature since its first description by Mohr in 1839 . Benign cystic teratoma, if not excised may cause grave complications like life-threatening haemoptysis or malignant transformation with metastatic disease. 42 year old south Indian female, who was completely asymptomatic, was incidentally diagnosed to have right lower lobe collapse in her chest $\mathrm{x}$ ray, during her routine master health check up. Subsequent CT chest showed complete calcification of right lower lobe with mild fibrosis and minimal atelectatic changes. Further investigations to rule out any chronic infections or malignancies were unremarkable. Bronchoscopy revealed $75 \%$ narrowing of the right lower lobe bronchus and bronchial lavage was negative for malignancy or acid-fast bacilli. Hence we planned for right lower lobectomy through right posterolateral thoractomy. Dissection was complicated with dense pleural adhesions. The right lower lobe was firm, collapsed and stuck to the diaphragm. The right lower lobe was excised in toto. Patient had uneventful hospital stay. Histopathology reports showed a benign teratomatous dermoid cyst with no features of any malignant potential.

We write this case to increase awareness about this rare manifestation of teratoma, which often presents like commonly seen lung conditions, but needs accurate diagnosis and treatment by complete resection to avoid life-threatening complications.

Decortication of a prolong lung entrapment GS Nagi; Amit Pushkarna; Chuimei Keishing 


\section{ABSTRACT:}

Decortication is an invasive procedure of stripping of the constricting fibrous membrane off the pleural surface with an aim to reexpand the entrapped lung, hence, to relieve the symptoms of restrictive lung disease. However, prolong lung entrapment are difficult to manage and may not yield desirable result. We report a case of lung entrapment of seven years duration who achieved complete lung re-expansion after decortication.

\section{A RARE CASE OF RIGHT SIDED CHEST WALI AND LUNG AVM \\ Chuimei Keishing; Amit Pushkarna}

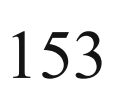

\section{ABSTRACT:}

A 43yrs old serving combatant reported for his annual medical board, when he was found to have a Right sided lower zone opacity on X RAY chest. The patient was asymptomatic except for occasional atypical chest pain on the right side. He was evaluated further by the pulmonologist and referred to CTVS for further management. CECT chest revealed AVM mass along the pleural surface adjoining the anterior aspect of infero lateral segment of middle and anterior basal segment of the right lung with arterial feeders from right SCA, IMA, Thoracodorsal, lateral thoracic and subscapular arteries and venous drainage into right sided pulmonary veins. He was deemed unfit for interventional procedure due to technical difficulties and drainage to the pulmonary venous system. He underwent "Non anatomical resection of Rt middle and Rt lower lobe with near total excision of AVM ( Venous end totally disconnected from AVM)". Post op recovery uneventful and imaging showed regression of the feeders with no connection left to the pulmonary venous circuit.

Cystic lung disease in tuberculosis with situs inversus totalis:unusual presentation

Shivakumar Swamy

Pramod H.J.

\section{ABSTRACT:}

Pulmonary tuberculosis may present as a cystic lung disease in rare instances and should be kept as a possible causes of acquired cystic lung diseases in appropriate settings. development of cysts is seen in tuberculosis often with varying severity, extent and unpredictable outcome

\section{Recurrent High Grade Chondrosarcoma Chest abutting Right Ventricular Outflow Tract Dhananjay Bansal, Anubhav Gupta, Sameek Bhattacharya, Sudesh Kumar, Gupta VK, Palash Aiyer}

\section{ABSTRACT:}

A 50-year-old man presented with recurrent anterior chest wall tumor with history of surgery 4 years ago for same. Now Radical en bloc resection of the tumour was performed including the sternal manubri, the first four costochondral joints bilaterally, underlying pericardium and the tumor mass. Chest wall reconstruction was done with polypropylene mesh and latissimus dorsi myocutaneous (LDMC) flap, with uneventful postop course. He is healthy 9 months postoperatively.
VATS: Current need in management of Thoracic Trauma

Sandeep Gautam; Dharmendra Kumar Srivastava;

Subash Singh Rajput; A.P. Jain

Anurag Rai

\section{ABSTRACT: \\ INTRODUCTION:}

Of all the causes of Traumatic injuries, thoracic trauma is considered to be an important reason of mortality and morbidity. The favoured management for thoracic trauma till date is intercostal drainage and to resuscitate the patient hemodynamically or to go for open thoracotomy in unstable patients as per indication. Video Assisted Thoracoscopic Surgery(VATS) is being increasingly utilized as diagnostic as well as therapeutic modality in management of stable patients of thoracic injury. This study was designed to evaluate the efficacy of Video Assisted Thoracoscopic Surgery (VATS) in the management of thoracic trauma in term of pain, dose of analgesic used, duration of hospital stay, restoration of pulmonary function and complication rate.

METHOD:

The study was conducted on 70 patients of thoracic injury (Blunt or Penetrating) who presented in Dr RML-IMS, Lcuknow. The patients were matched for age and sex and are randomly assigned in to two groups - Ist group treated by VATS, IInd group treated by prolonged ICD insertion only, and their outcome was analyzed on different aspects.

RESULTS:

Various procedures were done using Video Assisted Thoracoscopic Surgery(VATS) like Irrigation, Clot evacuation, electrocoagulation of bleeder, stapling of avulsed lung parenchyma \&amp; diaphragmatic repair). Pain improvement ( $\mathrm{p} \& 1 \mathrm{l} ; 0.001)$, early restoration of pulmonary function and radiological improvement were statistically significant in VATS treated group on 7th post procedure day with lesser duration of hospital stay (p\&lt;0.001).

CONCLUSIONS:

VATS (Video Assisted Thoracoscopic Surgery) is a better option than conventional ICD insertion only for management of thoracic injury, with better and early Clinico-radiological and functional improvement, and shorter duration of hospital stay.

\begin{tabular}{l|l|} 
GIANT SOLITARY FIBROUS TUMOUR OF PLEURA \\
$\begin{array}{l}\text { K.N. Bhosale } \\
\text { Vishal Sawkar }\end{array}$ & 157 \\
\hline
\end{tabular}

ABSTRACT:

Vishal Sawkar, Hrishikesh Parashi, K. N. Bhosale INTRODUCTION:

The solitary fibrous tumour of the pleura (SFTP) is a rare primary tumour arising from mesenchymal cells in the areolar tissue subjacent to the mesothelial-lined pleura. Giant solitary fibrous tumour of the pleura may cause serious and life-threatening conditions by causing compression of pleuropericardial and mediastinal sstructures with its expansive growth. The therapy of choice is radical surgical tumour removal.

\section{CASE REPORT:}

We report a case of giant fibrous solitary tumour of the pleura in 56 years old female admitted with complaints of progressively increasing breathlessness since 1 year and chest pain since 4 months. On examination air entry was absent on the left side of chest. On investigating her chest $\mathrm{X}$-ray show opaque left hemithorax with mediastinal shift to contralateral side, so CECT thorax was done which revealed $23 \times 17 \times 11 \mathrm{~cm}$ mass in the left hemithorax displacing mediastinum to right and causing complete collapse of left lung with right mediastinal displacement. It was completely encasing left common carotid and left subclavian arteries and partially encasing left inominate vein, arch of aorta and thoracic aorta. 


\section{TREATMENT:}

Patient underwent left posterolateral thoracotomy with excision of giant left intrathoracic tumour under general anaesthesia. Postoperative recovery was uneventful. Postoperative x-ray show complete expansion of the entrapped lung. Patient was discharged on POD-10.

\section{DISCUSSION:}

SFTP are a rare pathology of the pleural cavity, which most of the time develop from submesothelial fibroblasts of the visceral pleura. SFTP are usually diagnosed in the later stages of the development, when causing pressure on the surrounding structures on account of their size. Complete resection with negative margin is the treatment. Tumour may recur, which mainly depends on the histological characteristics of SFTP and radical nature of the surgical procedure.

\section{An unusual case of pleural hydatidosis M.U.N. Tejaswini; \\ K. Venkata Vijay}

\section{ABSTRACT:}

A 42 year old female came with the chief complaints of shortness of breath, left sided chest pain and fever since 2 months. She has been suffering from similar complaints since 4 years, aggravated since 2 months and was diagnosed in the past as pleural TB by doing pleural fluid aspiration cytology 2 years back and she had taken ATT for 9 months. Even with usage of ATT, her symptoms did not subside. She was investigated with chest $X$ ray PA view, showing - massive homogeneous opacification in left hemithorax compressing the left lung, trachea shifted to right with mediastinal shift to right. On CECT chest - non homogenous opacification of left hemithorax with multiple small circular shaped cysts with collapse of left lung and mediastinal shift to right side with probable diagnosis of hydatid cyst in left hemithorax. On ultrasound abdomen, no cysts were found in the liver, spleen. Routine blood investigations were normal except for leukocytosis. Surgical treatment was planned as excision of mass/cyst under general anaesthesia with double lumen endotracheal intubation with left lateral thoracotomy. Multiple daughter cysts were found and extracted. Thick pericyst was removed from the left lung, pleural cavity and diaphragm. Patient was kept on oral albendazole $400 \mathrm{mg}$ twice daily for 6 months and regularly followed up. The pericyst and daughter cysts were sent for histopathological examination and the diagnosis of Hydatid cyst was confirmed.

Echinococcosis can cause cyst formation anywhere in the body but primary pleural hydatid cyst is an uncommon mode of presentation of hydatid disease. It is important to keep hydatid disease in mind as one of the rare, but possible differential diagnosis in cystic lesions of the chest wall in the endemic zones and blind aspiration should be avoided.

\section{A rare case of primary Giant solitary benign schwannoma of the pleura \\ Sameer Kadam; Pavan Nagaraj Mulimani}

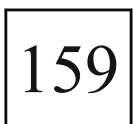

\section{ABSTRACT:}

Schwannoma, also called neurilemmoma, is a benign peripheral nerve sheath tumor. Most common locations are flexor surfaces of the extremities, neck, mediastinum, retro peritoneum, posterior spinal roots, and cerebello pontine angle. Pleural schwannomas are extremely rare neoplasms of the thoracic cavity. To the best of our knowledge, till date, \&lt;20 cases have been reported.

Herein, we report a case of primary benign pleura schwannoma in an elderly 50 -year-old female in whom the pleural mass was discovered incidentally on computed tomography of chest;. Highresolution computed tomography of the chest was done which revealed a large heterogeneously enhancing mass in the left thoracic cavity. She underwent left sided posteriolateral thoracotomy and was found to have a giant encapsulated and well-circumscribed mass arising from the pleura. pleural mass was resected which showed histopathological features of schwannoma.

\section{The Analysis of Prosthetic Valve in Patients with Dysfunction Valve Endocarditis \\ Hongwei Zhang; Jiayu Shen}

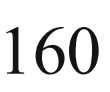

\section{ABSTRACT: \\ OBJECTIVE:}

To evaluate the outcomes of valve replacement of endocarditis using different valve types.

\section{METHODS:}

From 2005 to 2016, 10749 patients underwent valve replacement in west China hospital. Based on this database, 52 patients (mean age $48.3 \pm 6.8$ years, mean follow-up time $6.2 \pm 3.8$ years) with valve endocarditis receiving bioprostheses were comprised in this study. The control group were matched (3:1) with 156 patients with endocarditis receiving mechanical valves in the following variables: age $\pm 5, \mathrm{BMI} \pm 20 \%$, post-operation \pm 1 year, replacement position and sex ratio.

\section{RESULTS:}

The perioperative mortality of patients receiving bioprothetic and mechanical valves were $17.3 \% \pm 2.2 \%$ and $19.9 \% \pm 1.8 \%$, respectively, which was independent of valve type $(\mathrm{P}=0.27)$. Long-term survival were $56.1 \% \pm 5.2 \%$ and $61.2 \% \pm 8.1 \%(\mathrm{P}=0.58)$. Meanwhile, long-term complication-free survival were $75.0 \% \pm 3.2 \%$ and $82.3 \% \pm 4.4 \%(\mathrm{P}=0.29)$. For patients younger than or equal to 60 years of age, long-term reoperation for patients who receiving bioprothetic and mechanical valves were $41.4 \% \pm 7.2 \%$ and $30.5 \% \pm 5.4 \%(\mathrm{P}=0.22)$. For patients older than 60 years of age, however, reoperation rate were $24.1 \% \pm 8.5 \%$ and $14.7 \% \pm 5.7 \%$ $(\mathrm{P}=0.36)$.

\section{CONCLUSIONS:}

Perioperative mortality and long-term survival are independent of valve type in patients with endocarditis. Mechanical valve shows potential advantage for patients who are younger than 60 -year-old. The freedom from reoperation, however, is independent of valve type in patients older than 60 -year-old.

Clinical correlation research of Krebs von den Lungen-6 and perioperative pulmonary function in adults receiving continuing low-volume ventilation during valve surgery

Er-yong Zhang; Jia-yu Shen

\section{ABSTRACT: OBJECTIVE:}

This study was designed to investigate the influence of continuous low-volume ventilation used in cardiopulmonary bypass $(\mathrm{CPB})$ to the expression level of Krebs von den Lungen-6 (KL-6) and perioperative pulmonary function in adults undergoing valve surgery. And the relationship of KL-6 and perioperative pulmonary function was also identified.

\section{METHODS:}

From 2017 to 2018 , 60 patients who received valve replacement surgery in West China hospital were prospectively comprised in this study. We randomly allocated these patients into control group $(n=30$, non-ventilation) and experimental group $(n=30$, continuous low-volume ventilation during $\mathrm{CPB}$ ). At different perioperative time points, we identified the dynamic changes of pulmonary function and biomarkers which expressed in serum and bronchoalveolar lavage fluid (BALF). Meanwhile, we also compared perioperative clinical outcomes of the two groups.

\section{RESULTS:}

The expression of serum KL-6 and BALF KL- 6 in two groups were both statistically significant with Pvalue of 0.005 and 0.013 , respectively. The 
oxygenation indexes in both groups had a trend of descend with time $(\mathrm{P}=0.007)$, while $\mathrm{AaDO} 2$ in both groups were on the rise with time $(\mathrm{P}=0.377)$. According to linear correlation, the serum KL-6 level was negatively correlated with oxygenation indexes $(r=-0.525$, $\mathrm{P}=0.003)$, while it was positively correlated with $\mathrm{AaDO} 2(\mathrm{r}=0.489$, $\mathrm{P}=0.0061$ ). There were no significant differences between the two groups in the perioperative clinical outcomes, withP value of $0.237,0.301,0.693$ and 0.379 , respectively.

\section{CONCLUSION:}

Continuing low-volume ventilation during $\mathrm{CPB}$ could decrease the inflammation effectively, while it had no significant influence on perioperative outcomes. The expression level of KL-6 in patients who received $\mathrm{CPB}$ during the cardiac surgery was closely related to the damages of alveolar epithelial cells. Moreover, KL-6 could reflect the change of perioperative pulmonary function more effectively than other biomarkers do.

Our surgical strategy in a patient with severe mitral and tricuspit valve regurgitation and severe left ventricular dilatation

Banu Lafci; Yuksel Besir; Ismail Yurekli; Habib

Cakir; Erturk Karaagac; Huseyin Durmaz; Ali Gurbuz

Kazim Ergünes

\section{ABSTRACT: \\ BACKGROUND:}

Severe mitral and tricuspit valve regurgitation and severe left ventricular dilatation is an important factor affecting morbidity and mortality in patients undergoing cardiac valve surgery. We presented our case undergoing bipolar radiofrequency ablation with mitral valve replacement.

\section{CASE REPORT:}

A 54-year old woman has hospitalised in our clinic in November, 2018. He had severe mitral and tricuspit valve regurgitation and severe left ventricular dilatation.

Left ventricular end-diastolic and end-sistolic diameter, and left atrial diameter were $89 \mathrm{~mm}, 62 \mathrm{~mm}, 91 \mathrm{~mm}$, respectively. Pulmonary artery pressure was $56 \mathrm{mmHg}$. Ejection fraction was $60 \%$.

Cardiopulmonary bypass was established with bicaval canulation, with a membrane oxygenator. The systemic perfusion was performed using hypothermic bypass strategy of systemic cooling (280C). The systemic perfusion pressure was electively maintained at $55-65 \mathrm{mmHg}$. Cardiopulmonary bypass flow rates were maintained between 2.0 and 2.4 L.min $-1 \mathrm{~m}-2$. Plegisol cardioplegic solution was administered antegradely.

Mechanic mitral valve (33-Sorin) replacement and tricuspit De Vega annuloplasty were performed.. CPB time, and cross-clamping time were 100 minute and 45minutes retrospectively. Postoperatively, the patient was in sinus rhythm. The patient was discharged seven days after operation with wararin and salicylate.

\section{CONCLUSION:}

Mechanic mitral valve replacement and tricuspit De Vega annuloplasty was safe and effective method in patients having severe mitral and tricuspit valve regurgitation and severe left ventricular dilatation

\section{A unique case of Redo Ebsteins Anomaly of TV with severe low pressure TR \\ Sandeep A.J.; Zubair Rashid}

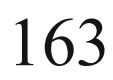

A 48 years old gentleman known case of Ebsteins anomaly, post VSD (Ventricular septal defect) and patent foramen ovale(PFO)repair years back. He presented shortness of breath and decreased exercise tolerance. Evaluated and found to have Mitral valve prolapse with mitral regurgitation with Ebsteins anomaly of Tricuspid valve with severe low pressure Tricuspid regurgitation, Atrial fibrillation with controlled ventricular rate. Redo Ebsteins anomaly repair was done.
Review Of Patients Who Underwent Pannus Removal Without Valve Replacement For Mechanical Prosthetic Valve Obstruction

L.F. Sridhar; Ashwene R.

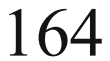

\section{ABSTRACT:}

The aim of our study is to review patient who underwent pannus removal without valve re-replacement. In these patients once the pannus was removed their prosthetic valve function remained good with no structural damage.

\section{METHODS AND METHODOLOGY:}

This is a retrospective observational study of 14 patients. They have undergone re-do surgery for pannus removal without valve re-replacement between 2014 and 2018 in our institute. These patients preoperative NYHA status and valve gradients were compared to postoperative values using Chi square and Student $t$ test. RESULTS:

All 14 patients underwent pannus excision without valve re-replacement. Average age of patients was 54.6 (42 to 73 years) and only five were males.

Patients presented in NYHA class II were 5 patients, NYHA III were 4 patients, and NYHA IV were 5 patients. The average duration from previous surgery was 12 years and 4 months.

Three patients had pannus in Mitral position and 11 patients in Aortic position. Medtronic hall valve was used in 6 patients and St Jude mechanical bi-leaflet valve in 8 patients. Postoperative mean gradient (MPG) in aortic valve was $21 \mathrm{~mm} \mathrm{Hg}$ and mitral MPG postoperatively was $5 \mathrm{mmHg}$. 7 patients in 3 months review were in NYHA I. 5 patients in for one-year review were in NYHA I. There was significant difference in MPG between the preoperative and one year follow up.

\section{CONCLUSION:}

Thus, pannus excision without valve re- replacement is a viable surgery for select few of the patients with prosthetic valve obstruction. It can be done in both aortic and mitral positions.

MASSIVE LA AND RA CLOT WITH NORMAL SINUS RHYTHM. A CASE REPORT

A. Farooqi; Sujit Kumar Mohanty; Afroz Farooqi

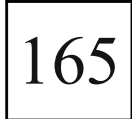

\section{ABSTRACT:}

\section{Key words: LA CLOT, RA CLOT, SINUS RHYTHM.}

LA clot usually associated with mitral valve disease in atrial fibrillation, very rarely it is seen in patient with normal sinus rhythm with mitral disease but it is very rare in a patient having sinus rhythm in a RA and LA clot. We also did phospholipid antibody test which was negative.

Early and Mid-term Hemodynamic Outcomes After Aortic Valve Replacement with the Magna Ease and the Trifecta

Ko Nakahara; Hideki NIshimura; Tatsuichiro Seto

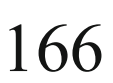

\section{ABSTRACT: \\ BACKGROUND:}

Pericardial tissue valves are widely employed for aortic valve replacement (AVR). The Perimount Magna Ease valve (Edwards LifeScience, Irvine, California) is the most frequently implanted bioprostheses worldwide, while the Trifecta valve (St Jude Medical, St Paul, Minnesota) is a new bioprostheses with an externally wrapped valve leaflet to maximize the valve opening. In this study, we compared the postoperative hemodynamics of the two bioprosthetic valves, the Magna and the Trifecta. 


\section{METHOD:}

A total of 124 consecutive patients with aortic stenosis (AS) undergoing AVR using either the Magna (group $M, n=83$ ) or the Trifecta (group T, n=41) between April 2009 to March 2018 were enrolled. Clinical and hemodynamic data were prospectively recorded and compared. Hemodynamic data was assessed using transthoracic echocardiography, including mean pressure gradient (MPG), effective orifice area index (EOAI), and left ventricular mass index (LVMI) at baseline, discharge, and late postoperative stages.

\section{RESULTS:}

The MPG (14.81 $\pm 5.08 \mathrm{mmHg}$ vs $10.94 \pm 6.25 \mathrm{mmHg}$; p\&lt;0.01) was significantly smaller in group $\mathrm{T}$ while the EOAI $(0.99$ $\pm 0.30 \mathrm{~cm} 2 / \mathrm{m} 2$ vs $1.11 \pm 0.31 \mathrm{~cm} 2 / \mathrm{m} 2 ; \mathrm{p}=0.06$ ) was not significantly different at discharge. In the mid-term outcomes (median follow up period; 38 months), the MPG $(13.1 \pm 3.50 \mathrm{mmHg}$ vs 16.0 $\pm 4.74 \mathrm{mmHg} ; \mathrm{p}=0.02)$, the EOAI $(0.95 \pm 0.19 \mathrm{~cm} 2 / \mathrm{m} 2$ vs 0.81 $\pm 0.21 \mathrm{~cm} 2 / \mathrm{m} 2 ; \mathrm{p}=0.02)$, and the LVMI $(128.5 \pm 38.2 \mathrm{~g} / \mathrm{m} 2$ vs $108.3 \pm 28.2 \mathrm{~g} / \mathrm{m} 2 ; \mathrm{p}=0.03)$ were significantly advantageous in group $\mathrm{M}$ in contrast to that in group $\mathrm{T}$.

\section{CONCLUSION:}

Altogether, the results demonstrated that the Magna ease valve is likely to be superior in comparison to the Trifecta valves in terms of late hemodynamic outcomes and preventing long-term cardiac failure and cardiac-related death.

\section{Management of severe valvular heart disease in third trimester pregnancy: Case series and algorithm for management \\ Vaibhav Shah; Chaitanya Raut}

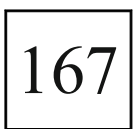

\section{BACKGROUND:}

Valvular heart disease is the most common heart disease complicating pregnancy. In a developing country, many patients are diagnosed with valvular heart disease, for the first time in the third trimester of pregnancy.

RESULTS:

We present a series of 8 patients presenting with severe valvular heart disease for the first time in third trimester of pregnancy over the last 5 years (Jan 2012 to Dec 2017). Six patients underwent combined Lower Segment Cesarean Section (LSCS) with valve replacement therapy successfully. The other 2 patients were managed medically with elective vaginal delivery after fetal maturity.

\section{CONCLUSION:}

As there are no specific guidelines to address severe valve during third trimester of pregnancy we present an algorithm for management of such patients. A combination of obstetrician, cardiologist, cardiac surgeon and anesthetist is required to manage such critical cases.

\section{Cor Triatriatum With Mitral Annular Calcification: A Rare Case. \\ Jignesh Kothari \\ Devvrat Desai}

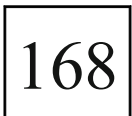

\section{ABSTRACT: INTRODUCTION:}

Cor-triatriatum sinister is an extremely rare congenital heart defect. It is even more uncommon in adults, and quite exceptional when associated with mitral valve disease. Very few cases have been reported for cor-triatriatum associated with mitral regurgitation. Here, we are reporting an exceptional case of cortriatriatum associated with calcified mitral valve resulting mitral stenosis.

\section{BACKGROUND:}

Cor-triatriatum is found in only $0.1 \%$ of cases of congenital heart anomaly. In classical form, there is a division of left atrium by a fibro-muscular membrane, band or tissue, resulting in development of two distinct chambers, a posterosuperior chamber \&amp; anteroinferior chamber. The posterosuperior chamber receives return of all pulmonary veins. The anteroinferior chamber is a true left atrium which communicates with mitral valve.

\section{CASE:}

A 65 year old lady presented with history of dyspnea \&amp; angina on exertion (NYHA class II) since past two years \&amp; progressed to NYHA class IV recently. Cardiac auscultation revealed mid diastolic murmur with pre-systolic accentuation. Trans-thoracic Echocardiography revealed cor-triatriatum, a membrane dividing Left atrium into two chambers. Colour \&amp; pulse wave doppler suggested moderate MS \&amp; mild MR with mild PAH with Ejection Fraction of 55\%. Multislice CT pulmonary angiography was suggestive of cor-triatriatum Loeffler's type 2 \&amp; small restrictive opening with calcification. All pulmonary veins were draining into posterior chamber. Mitral posterior annular calcification was present. The lesion was approached by midline sternotomy. After complete excision of membrane, mitral valve with severe stenosis \&amp; posterior annular calcification was identified, which was underestimated preoperatively. Mitral valve was excised and replaced with $25 \mathrm{~mm}$ St Jude mechanical prosthesis.

\section{CONCLUSION:}

Cor-triatriatum sinister remains a rare congenital heart disease despite of improvements in diagnostic imaging. Mitral valve pathology remains underestimated in presence of cor-triatriatum.

Suspected fat embolism referred for ECMO turned out to be LVF due to acute traumatic AR

Neerav Bansal; Varun Bansal

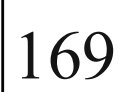

\section{ABSTRACT:}

\section{INTRODUCTION:}

Because of rarity, rupture of the normal aortic valve is not generally considered during primary assessment after polytrauma. Two of such cases were historically reported in 1830 by Penderleath and in 1841 by Bouillaud.

\section{CASE REPORT:}

We present a 21 year old male with history of polytrauma including fracture of left femur bone and blunt trauma head and chest. $\mathrm{He}$ presented with breathlessness within 12 hours of injury, initial diagnosis of suspected fat embolism was made at a peripheral center, and due to deteriorating condition was referred for ECMO salvage. However careful examination revealed a diastolic murmur. In absence of any previous history, echocardiography led to diagnosis of acute traumatic aortic valve rupture. Circumferential tear at non coronary cusp and one third of left coronary cusp including the commissure led to surgical decision of aortic valve replacement.

\section{DISCUSSION}

It is easy to miss cardiac injury due to lack of suspicion and other co-existent injuries. The most common injury after blunt trauma chest is myocardial contusion which itself is underdiagnosed. Aortic valve rupture is due to sudden increase in intrathoracic pressure during diastole or subadventitial rupture of ascending aorta causing prolapse of corresponding valve cusp. Any patient without history of heart disease presenting with a cardiac murmur should be evaluated with echocardiography. The timing of surgical intervention in the form of aortic valve repair or replacement remains an important issue of consideration. Deterioration of ventricular function, acute pulmonary edema and systemic diastolic blood pressure of less than $50 \mathrm{mmHg}$ are indications for immediate surgery. 
Case report- Stuck aortic valve with PPM as probable contributing factor

Ravi Shivdasani; Vijit K. Cherian

\section{ABSTRACT:}

We had a 56 years old, female patient, who is a housewife, resident of Sudan with weight $84 \mathrm{~kg}$, height $174 \mathrm{~cm}$ and BSA $2.02 \mathrm{~m} 2$ presenting to us with giddiness and syncopal attacks on minimal exertion past 6 months.

She underwent AVR in 2013 with 19mm mechanical bileaflet mechanical prosthesis elsewhere. She remained asymtomatic till mid 2014, later started developing symptoms on minimal exertion progressed in severity and frequency. She gave no history of irregularity in taking warfarin and maintained adequate INR with dose regulation. She was in sinus rhythm and no CAD on repeat angiogram.

2-D echo showed restricted movement of one leaflet with possible pannus at hinge of prosthesis, $\mathrm{PG}$ of $124 \mathrm{mmHg}$ and $\mathrm{MG}$ of $80 \mathrm{mmHg}$, severe concentric LVH and good biventricular function. Challenges here were Stuck valve, Large BSA, PPM, Small previous prosthesis, ? Pannus, Small aortic root and Redo.

We planned a Redo AVR with aortic root enlargement. We performed a Redo sternotomy, J shaped aortotomy and we observed stuck leaflet with pannus on ventricular side. We explanted previous prosthesis and debrided the annulus thoroughly. Only $19 \mathrm{~mm}$ valve sizers were passing freely and issue was root enlargement. However $21 \mathrm{~mm}$ TTK Chitra valve sizer was seating just well supraannularly. Since we were able to implant one size larger valve supraannularly, root enlargement was not performed. Techniques which helped were J aortotomy, thorough annular debridement, subannular pledgetted intermittent polyester sutures and aortotomy closure with PTFE felt reinforcement. Reimplanted prosthesis had no subannular extension.

We conclude that

Adequate sized aortic prosthesis is paramount to avoid PPM

Root enlargement in REDO is complex and risky

TTK chitra valve is an excellent choice in small aortic roots allowing one size larger prosthesis to be implanted in supra annular position specially in REDOs due to lowest profile.

\section{Doppler Echocardiographic assessment of TTK} Chitra Prosthetic Heart Valve in the Mitral Position Anurag Rai

Sandeep Gautam

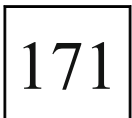

\section{ABSTRACT: \\ BACKGROUND AND AIM OF THE STUDY:}

TTK Chitra is a, made in India, Tilting disc cardiac valve prosthetic, which is low cost and proven efficacy. We present the echocardiography and Doppler evaluation of normally functioning valve in mitral position by using continuity equation (CE) and pressure half time (PHT).

METHODS:

We performed Doppler echocardiography on 61 patients with chitra heart valve in the mitral position. All patients were clinically stable without evidence of prosthetic valve dysfunction such as significant obstruction or regurgitation, endocarditis, left ventricular dysfunction or significant aortic regurgitation.

\section{RESULT:}

In the mitral position for valve size $23 \mathrm{~mm}, 25 \mathrm{~mm}, 27 \mathrm{~mm}, 29 \mathrm{~mm}$, the mean gradient (in $\mathrm{mmHg}$ ) were $6.00 \pm 00,3.91 \pm 1.31,3.55 \pm 1.34,3.45$ \pm 1.21 respectively and effective orifice area (in $\mathrm{cm} 2$ ) were $1.82,1.47$, $1.89,2.09$ respectively.

\section{CONCLUSION:}

TTK Chitra valve has comparable Doppler parameter in the mitral position with other prosthetic valves which are commonly in use. Areas by both methods were smaller then the EOA provided by the manufacturer as seen in other similar design valves.
Concomitant aortic regurgitation is a predictor

of outcome after surgical aortic valve replacement

in patients with severe aortic stenosis

Chaitanya Raut

Harsh Seth

\section{ABSTRACT:}

\section{BACKGROUND:}

Concomitant aortic regurgitation (AR) might worsen the prognosis of severe aortic stenosis (AS). But the prognostic value of concomitant AR in patients operated for severe AS is not addressed.

\section{OBJECTIVE:}

The aim of this study was to assess the impact and association of presence and severity of concomitant AR in patients operated for severe AS on long-term left ventricular (LV) function, functional capacity, and mortality.

\section{MATERIALS AND METHODS:}

Study group consisted of 200 patients operated due to severe AS retrospectively. The patients were divided into AS group (100 patients with AS without AR or with mild AR) and AS+AR group (100 patients with AS and moderate, severe or very severe AR). Follow-up included a clinical examination and echocardiography 5-30 months after AVR.

\section{RESULT:}

Patients in AS group had improved symptoms than AS+AR group. The patients with postoperative follow-up in AS group had lower LV volume indices than patients in AS+AR which was statistically significant (AS group; LVEDVi - 67 \pm 9.1 , LVESVi - 22 \pm 7.2 compare to AS+AR group; LVEDVi - 84 \pm 21.3 , LVESVi - 34 \pm 11 , p-value\&lt; 0.05). Postoperative mean LVEF was $61 \pm 11$ and $58 \pm 12$ in AS and AS+AR group, respectively. CONCLUSION:

Our study indicates that the preoperative presence and severity of concomitant AR has an impact on the outcome after aortic valve replacement for the patients having severe aortic stenosis symptoms on the basis of symptoms, LV remodeling, systolic and diastolic function.

Importance of bipolar radiofrequency ablation in patient with atrial fibrillation undergoing mitral valve replacement and tricuspit valve annuloplasty Levent Yilik; Orhan Gokalp; Yuksel Besir; Ismail Yurekli; Habib Cakir; Erturk Karaagac; Ali Gurbuz Kazim Ergüneș

\section{ABSTRACT: BACKGROUND:}

Atrial fibrillation is an important factor affecting morbidity and mortality in patients undergoing cardiac valve surgery. We presented our case undergoing bipolar radiofrequency ablation with mitral valve replacement. METHOD:

A 63-year old woman was hospitalised in our clinic in September, 2018. He had severe mitral stenosis, grade 3 tricuspit valve regurgitation and atrial fibrillation. Left ventricular end-diastolic and end-sistolic diameter, and left atrial diameter were $55 \mathrm{~mm}, 36 \mathrm{~mm}, 54 \mathrm{~mm}$, respectively. Ejection fraction was $60 \%$.

\section{RESULTS:}

The patient received a standard heparinization protocol of $300 \mathrm{IU} / \mathrm{kg}$ heparin bolus prior to cannulation, with an additional 10.000 I.U in the pump prime. Target activated clotting time greater than 400 was maintaned throughout bypass.

The systemic perfusion was performed using hypothermic bypass strategy of systemic cooling (280C). The systemic perfusion pressure was electively maintained at $55-65 \mathrm{mmHg}$. Cardiopulmonary bypass flow rates were maintained between 2.0 and 2.4 L.min $-1 \mathrm{~m}-2$. Isothermic blood cardioplegia was administered antegradely.

We conducted the ablation with bipolar clamp device (Cardioblate BP surgical ablation device; Medtronic Inc, Minneapolis, USA) at least twice 
for each line. Left atrial and pulmonary arteries ablation lesions were performed before mechanic mitral valve (29-St.Jude) replacement. The tricuspit Kay annuloplasty was performed. CPB time and cross-clamping time were 95 minute and 45 minutes retrospectively. Postoperatively, the patient was started on intravenous amiodarone $1.2 \mathrm{~g}$ for $24 \mathrm{~h}$, followed by oral amiodarone $200 \mathrm{mg}$ three times a day. Postoperatively, the patient was in sinus rhythm. The patient was discharged seven days after operation with wararin, salicylate and amiodarone treatment.

\section{CONCLUSSION:}

Intraoperative bipolar radiofrequency ablation was safe and effective method to cure atrial fibrillation in patients with atrial fibrillation undergoing mitral valve replacement and tricuspit valve annuloplasty.

\section{Our surgical approach in a patient having aortic valve stenosis and ascending aortic aneurysm Levent Yilik; Orhan Gokalp; Yuksel Besir; Erturk Karaagac; Banu lafci; Ali Gurbuz Kazim Ergüneș}

\section{ABSTRACT: \\ BACKGROUND:}

Aneurysm of the ascending aorta carry a high risk of aortic dissection or rupture in the absence of surgical treatment. We presented a patient having aortic valve stenosis and ascending aortic aneurysm.

\section{CASE REPORT:}

A 68-year-old man admitted to our clinic in September, 2018. He had hypertension, exertional dyspnea. The angiography and echocardiography showed ascending aortic aneurysm, severe aortic stenosis. Ejection fraction was $60 \%$. Left ventricle end diastolic diameter was $56 \mathrm{~mm}$. Aortic aneurysm was supravalvular. The proximal ascending aorta was $54 \mathrm{~mm}$. Cardiopulmonary bypass was instituted with one cannula for arterial return to the right remoral artery and a venous single 2 -stage cannula in the right atrium. The systemic perfusion was performed using hypothermic bypass strategy of systemic cooling down to $280 \mathrm{C}$. Isothermic blood cardioplegia was initially administered antegradely via coronary artery ostia and thereafter continuously retrogradely via coronary sinus ostium. Mechanic aortic valve (23-St-Jude) replacement was performed before supracoronary aortic tube graft conduit $(28 \mathrm{~mm})$ replacement. The mean cross-clamp time and the mean CPB time were $75 \mathrm{~min}$, and $140 \mathrm{~min}$, respectively. The patient was weaned from CPB with inotropic drug support.

Postoperative period was event-free. The patient was discharged on 7th postoperative day with warfarin treatment.

\section{CONCLUSION:}

Aortic valve stenosis associated with supracoroner ascending aorta dilatation can be treated by separete replacement of the aortic valve and supracoronary aortic tube graft conduit.

\section{The modified Ross procedure provides better} long-term outcome compared to mechanical valve surgery Siew-Pang Chan; Vitaly A. Sorokin; Smiris Konstantinos; Arash Mehdiani; Philip Minol; Artur Lichtenberg LI Yue

\section{ABSTRACT: \\ BACKGROUND:}

Mechanical aortic valve replacement (AVR) is a widely adopted solution for young patients however, Ross procedure provide autograft alternative for diseased aortic valve. The aim of this single-center study is to compare the early and late results of patients who underwent Ross procedure and mechanical AVR.

\section{METHODS:}

Between May 2010 and January 2014, 40 young adult patients underwent the Ross procedure in The Clinic for Cardiovascular Surgery, HeinrichHeine-University, Duesseldorf, Germany. Matched case control was used to compare Ross procedure patients with a mechanical AVR patients. The patients' data were collected and analyzed with generalized structural equation model.

\section{RESULTS:}

In the early post-operative period patients in the Ross procedure group had significantly reduced lengths of hospital stay $(p=0.002)$. There were significantly more para-valvular leaks in the mechanical AVR group $(p=0.027)$. There were no significant differences observed for non-valve-related complications such as reoperation for bleeding $(p=0.100)$. In the later period the risk of death was significantly higher in the mechanical AVR group $(\mathrm{p}=0.004)$. There were significantly fewer bleeding events $(\mathrm{p}=0.002)$ in the Ross procedure group. There were no difference for neither valverelated reoperations $(p=0.247)$ nor aortic position-related reoperations $(\mathrm{p}=0.500)$. Post-operative echocardiogram showed significantly lower trans-aortic valve velocity $(\mathrm{p}=0.001)$ and trans-valvular gradient ( $\mathrm{p}=0.013$ ) in the Ross procedure group. In quality of life assessment, the Ross procedure group outperformed the mechanical AVR group in terms of independent daily activities $(\mathrm{p}=0.028)$.

\section{CONCLUSIONS:}

The Ross procedure decreases risk of mortality after operation, lower bleeding events and improves quality of life related to independent daily activity. The valve in the aortic position after the Ross procedure offers lower trans-valvular gradient and blood velocity. Utilization of modified surgical technique with reinforcement of the original pulmonary valve on the annulus and sinotubular junction level could improve long-term result for this group of patients.

Recurrence Of Tricuspid Valve Regurgitation Following Various Tricuspid Valve Repair Techniques Sudha K; Theodore S; Siva Kumar R; Chokkalingam M

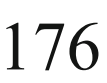

\section{ABSTRACT:}

\section{BACKGROUND:}

Tricuspid valve regurgitation secondary to left heart disease is the most common cause of TV inefficiency.TV repair is the primary treatment for Tricuspid regurgitation. We reviewed our experience with different surgical techniques, Devaga's, felt ring and commercial ring annuloplasty for the treatment of acquired TV disease.

\section{MATERIALS AND METHODS:}

80 patients who underwent TV Repair in Chettinad hospital during the period Aug2014-2018 were included in this study.36 patients had GradeIV TR,14 patients had GradeIII TR,29 patients had GradeII TR and 1 patient had GradeI TR. Results were evaluated by transthoracic echocardiography prior to discharge and at 6 month follow up. In the cohort we have followed up the degree of regurgitation, RV dysfunction and mortality to assess the efficacy of repair.

\section{RESULT:}

54 patients had Devega's (group1), 14 had commercial annuloplasty ring (group 2) and 12 patients had felt ring (group3). There was 1 mortality in group3. Out of 16 patients who had pre operative RV dysfunction, 3 continued to have RV dysfunction at discharge and at 6 month follow up and 1 patient developed RV dysfunction at 6 month review which was not there at discharge. On analysis there was no recurrence of TR, worsening of RV function and incidence of mortality in all three groups at early and midterm follow up $(\mathrm{p}=0.5)$

\section{CONCLUSION:}

All the three types of tricuspid repair technique seem to provide good results at early and mid term follow up. Further follow up and large sample is required to come to a definite conclusion as to the efficacy of the three techniques. 
Aquired gerbode defect with drop lesion on the tricuspid valve from aortic endocarditis Kim Chang

\section{ABSTRACT:}

47 year old man was investigated for fever. Echo showed aortic valve endocarditis with torrential AR and vegetations. He was taken up for urgent AVR.

Pre-op trans esophageal echo revealed a large vegetation on the tricuspid valve. Intra-operatively, the RA was opened and on removing the tricuspid vegetation, a pulsatile jet of blood was noted from the LVOT.

On further inspection of the aortic valve, there were large vegetations with a defect in the left ventricular outflow tract deep to the non-coronary cusp close to the membranous septum.

The aortic valve was excised and the annular abscess debrided. The LVOT defect and the annulus was repaired with a bovine pericardial patch and a mechanical valve implanted. The defect on the RA was closed with bovine pericardial pledgeted suture. the patient had a steady post op course and recovery. he did not develop any major conduction abnormalities.

The organism was identified as streptococcus pneumoniae and was treated with a prolonged course of antibiotics as per sensitivity. An acquired "Gerbode" defect (LV to RA shunt) with a "drop" vegetation on the tricuspid valve is a rare complication of aortic valve endocarditis. There are a few isolated case reports over the years. The role of transeosophageal echo in the proper diagnosis is to be stressed. Opening the RA prior to clamping the aorta facilitated the identification of the shunt.

Debridement of the infected annular and sub-annular tissue with patch repair is important for eradicating the infection. care should be taken to avoid the conduction system.

Total endovascular repair for double aneurysms (TAA \& AAA) with fenestrated and branching stentgraft Ryo Onishi; Takashi Enomoto; Yuka Okubo; Ayako Nagasawa; Takehito Mishima; Syuichi Shiraishi; Masanori Tsuchida Takeshi Okamoto

\footnotetext{
ABSTRACT:

Patient was 58 years old man. His past history were cerebral infarction, hyperlipidemia and angina pectoris. He had distal arch aneurysm of $55 \mathrm{~mm}$ (saccular type) and juxtarenal aneurysm of $65 \mathrm{~mm}$ (fusiform type). We determined the strategy of simultaneous endovavscular repair for double aneurysms considering the risk of aneurysm rupture. We chose an anatomically complete revascularization using fenestrated and branching stentgraft to avoid a paraplectic complication. TEVAR was performed Najuta (Kawasumi Laboratories, Tokyo, Japan) with fenestration for 3 cervical branches and branching stentgraft for LSA using VIABHAN (W.L.Gore and Associates, Flagstaff, AZ, USA) because of short distance between LSA and the aneurysm. At the same operation, EVAR was performed Zenith (Cook Medical, Bloomington, IN, USA) with fenestration and branching for bil. renal arteries using VIABHAN. Last DSA showed no endoleak. He was discharged 9 days after TEVAR. Endovascular repair is considered low invasive treatment. But, only just commercialbased devices (normal and no modification) have a limit in treatment for various aneurysms. Conventionally, some reconstructions for example an extra-anatomical bypass was required. The fenestrated stentgraft does not almost require reconstruction. Furtherore, the combination of fenestrated stentgraft and branching covered stent (VIABHAN) may make results high quality in treatment of complex aneurysms.
}

OUTCOME OF TRICUSPID VALVE REPLACEMENTA SINGLE CENTRE EXPERIENCE

Aayush Poddar;

Muralidharan Srinivasn;

Sundar Ramanathan; Chandrasekar Padmanaban

Muthukumar Sundaram

\section{ABSTRACT: \\ OBJECTIVE:}

In this study we reviewed our experience with TVR focusing on risk factors for operative mortality, long-term outcome and incidence of valve related complications and compared the results in tissue and mechanical valves.

\section{METHODS:}

A retrospective study of Tricuspid Valve Replacement (TVR) in 22 patients from 2002 to 2017. Compared mechanical valve versus tissue (GroupI/GroupII). The mean age was 41(15-64) years. Female:Male ratio was $14(63 \%): 8(36 \%)$. The underlying diagnosis were Congenital $8(36 \%)$, organic $5(22 \%)$, functional $5(22 \%)$, rheumatic $4(18 \%)$. Isolated TVR was performed in 7(31\%), 4 in GroupI/3 in GroupII and combine procedure was $15(68 \%), 7$ in GroupI/9 in GroupII. 7(31\%) had previous cardiac surgery.

\section{RESULTS:}

Overall mortality was $7(36 \%), 5(45 \%)$ in GroupI and $2(16 \%)$ in GroupII. $1(14 \%)$ mortality in isolated and $6(85 \%)$ in combine procedure. Inhospital mortality was 2(28\%) both in GroupII. Readmission was 3 in GroupI with elevated INR and 1 in GroupII with pulmonary oedema. $18(81 \%)$ were in New York heart Association class III or IV before surgery. 2(10\%) in GroupI had Major Adverse Cardiac and Cerebrovascular Event(MACCE) post surgery(Permanent Pace Maker Implantation and Subdural Haematoma). Mean follow up is 52 months(1-137). 3 lost follow up. Linearized survival rate at 3, 6 and 10 year is $68 \%, 58 \%$ and $42 \%$ respectively.

CONCLUSION:

Tricuspid Valve Replacement as assumed is a not so common surgery. We have performed 22 cases over the last 15 years with good results. Patients requiring TVR are usually in high-risk and also requiring concomitant cardiac procedures, and end-stage functional class. Operative and overall mortality remains high.

When valve replacement is necessary we recommend the use of biological prosthesis considering the higher incidence of readmission and valve related complications in mechanical valves.. Although there is no obvious guidelines which suggest advantage of bioprosthetic valve over mechanical valve in tricuspid position. We need to analyse more number of patients and follow-up them for logner period.

Study to assess the understanding of anticoagulation among patients after valvular surgery Rajshekhar P.; Agasti S.; Choudhary S.K. Parul

\section{ABSTRACT:}

\section{INTRODUCTION:}

There is an inverse relation between patient's understanding about anticoagulation and its accompanying complications. The present study was performed to assess the understanding of anticoagulation therapy in patients receiving oral anticoagulation for prosthetic heart valves.

\section{PATIENTS AND METHODS:}

A carefully drafted questionnaire was administered to consecutive 500 patients attending the outpatient department and receiving oral anticoagulants for valve replacement. Age ranged from 11 to 67 years and 183 were females. Duration of anticoagulation ranged from one month to 22 years.

\section{RESULTS:}

81 patients $(16.2 \%)$ didn't know that they were receiving anticoagulants. 301 patients $(60.2 \%)$ knew the name, and only $290(58 \%)$ knew the 
strength. 164 patients (32.8\%) knew that the drug can cause excessive bleeding after injury. 256 patients $(51.2 \%)$ patients had understanding of monitoring by PT/INR and 165 patients (33\%) knew the therapeutic INR. 170 patients (34\%) had, one or more time, missed the oral anticoagulant dose. Only 38 patients carried some sort of identification about receiving oral anticoagulant. Gender, education and place of residence (rural vs urban) were statistically significant determinants of anticoagulation knowledge in bi-variate analysis, whereas gender and education were significant determinants in multivariable analysis. Females were more likely to be ignorant about anticoagulation (OR: 3.1, (95\%CI: 1.8 5.4), $\mathrm{p}=0.001)$. The odds of not knowing anticoagulation decreased as level of education increased (OR: 0.14, (95\%CI: $0.06-0.36), \mathrm{p}=0.001)$. Besides this, female were more likely to miss the dose of the drug ( OR $1.67,95 \%$ CI 1.10-2.52, $\mathrm{p}=0.01$ ).

CONCLUSION:

Ignorance about anticoagulation is quite common in patients attending a public hospital in Northern India. There is a need to educate the patient and improve patients' understanding about oral anticoagulants.

\section{Our surgical strategy in a patient having} paravalvular leak of mechanical prosthetic aortic valve Levent Yilik; Ismail Yurekli; Yuksel Besir; Banu Lafci; Habib Cakir; Huseyin Durmaz; Ali Gurbuz Kazim Ergüneș

\section{ABSTRACT: \\ BACKGROUND:}

Paravalvular leak is a complication of aortic valve replacement and has a high morbidity and mortality. We present a patient having paravalvular leak and underwent mechanical prosthetic aortic valve replacement six years ago.

\section{CASE REPORT:}

A 65-year-old male was hospitalized in our clinic in November, 2018. He had undergone mechanical prosthetic aortic valve replacement six years ago. He had exertional dyspnea. Echocardiography showed dehiscence in mechanical prosthetic aortic valve annulus in region corresponding to right half of noncoronary and right coronary cusp. Left ventricular enddiastolic and end-sistolic diameter, and left atrial diameter were $71 \mathrm{~mm}$, $51 \mathrm{~mm}, 46 \mathrm{~mm}$, respectively. Pulmonary artery pressure was $50 \mathrm{~mm} \mathrm{Hg}$. Ejection fraction was $55 \%$.

Previous mechanical prosthetic aortic valve valves was removed. New mechanical prosthetic aortic valve was replaced. The mean cardiopulmonary bypass and aortic clamping times were 140 minutes and 100 minutes. CONCLUSION:

Surgical treatment of paravalvular leak of mechanical prosthetic aortic valve is an effective method.

Open conversion for type 2 endoleak after endovascular abdominal aneurysm repair Koichi Akutsu; Kensuke Ozaki

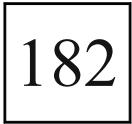

\section{ABSTRACT:}

Type 2 Endoleak(T2EL) is the most common complication of endovascular abdominal aortic aneurysm repair (EVAR). Open conversion for T2EL is one of the therapeutic options in addition to unsuccessful endovascular therapy. However, effect of open conversion for T2EL remains to be unclear because series of the patients with open conversion for T2EL could not be available. The purpose of this study is to examine the outcome of open conversion for T2EL.

\section{METHODS:}

Between 2016 April and 2018 July, 30 patients (79.1 \pm 6.1 years, male 20) were admitted for undergoing open conversion after EVAR with T2EL preoperative diagnosed. We approach abdominal aortic aneurysms by median laparotomy and make proximal clamp site against the possibility of type 1a EL. For T2EL, we ligate lumbar arteries to delete backflows. In the case we find concomitant type $3 \mathrm{~b}$ endoleak (T3bEL), we repair stent graft directly or replace with graft.

\section{RESULTS:}

Intraoperative findings were as follows; T2EL $(n=21,70 \%)$, T2EL+ T3bEL ( $\mathrm{n}=6,20 \%)$, and T3bEL $(\mathrm{n}=3,10 \%)$. Replacements of graft were done for 2 cases. There is no operative death. Operative time was 161.7 \pm 65.5 minutes. Hospital stay was $16.8 \pm 9.0$ days. On the 7 day after surgery, no residual endoleaks were found at $\mathrm{CT}$.

\section{CONCLUSION:}

Open conversion for T2EL with median laparotomy approach could be performed with acceptable early outcomes. In addition, open conversion is a reliable strategy to confirm and to repair T3bEL.

\section{MID-TERM RESULTS OF FROZEN ELEPHANT TRUNK TECHNIQUE FOR CHROIC AORTIC DISSECTION}

Takanobu Okazaki; Shohei Morita; Masazumi

Watanabe; Keijiro Katayama; Shinya Takahashi;

Tatsuya Kurosaki; Taijiro Sueda

Yoshitaka Yamane

\section{ABSTRACT:}

\section{OBJECTIVE:}

The usefulness of the Frozen elephant trunk(FET) technique for acute type A aortic dissection is widely reported. In regard to the chronic aortic dissection(CAD), the usefulness of the FET is unclear, however. In this study, we describe the mid-term results of the FET technique for the treatment of CAD.

\section{METODS:}

From January 2011, we performed total arch replacement(TAR) with FET technique for CAD with patent false lumen for 14 patients aged 43-80. 11 (78.6\%) patients were male. $10(71.4 \%)$ patients have had previous graft replacement, including ascending aortic replacement or aortic root replacement, for acute type A aortic dissection. The mean time between onset of the acute aortic dissection and treatment of TAR with FET was $62.2 \pm 43.8$ months and all cases were over 12 months from the onset of the aortic dissection. The mean greatest diameter of the aortic arch or the proximal descending aorta was $57 \pm 8.1 \mathrm{~mm}$.

\section{RESULTS:}

The average position of the distal edge of the FET was Th 6.3. One patient died within 30 days of the operation because of cerebrovascular event. One patient required additional thoracic endovascular aortic repair(TEVAR). Estimated overall survival at 1-, 3- and 5 years was $92.9 \%, 92.9 \%$ and $55.7 \%$ respectively. There were aortic reinterventions with Descending aortic replacement $(n=3)$, false lumen occlusion $(n=1)$ and TEVAR $(n=6) .6$ patients caused distal stent induced new entry(dSINE) and all 6 cases required unplanned additional TEVAR. 2 out of 6 dSINE cases occurred rupture of false lumen and 3 out of 6 dSINE cases occurred aorto-esophageal fistula. Estimated aortic-related event free rate at 1 and e year were $84.6 \%$ and $46.9 \%$.

\section{CONCLUSION:}

Mid-term results of the FET technique for CAD was not satisfied. we should be carefully to treat CAD using FET technique.

Early and Late Outcomes of Surgery for Acute type A aortic dissection with malperfusion

Tohru Ishimine; Akio Nakasu; Toshiho Tengan

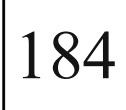

ABSTRACT:

OBJECTIVE:

The aim of this study was to evaluate early and late outcomes of surgery for acute type A aortic dissection with malperfusion. 


\section{METHODS:}

Between 1989 and 2017, 211 patients (mean age, $62.7 \pm 13.4$ years; 110 of the patients were men (52\%)) who underwent surgery for acute type A aortic dissection were reviewed. Perioperative and long-term outcomes were compared between patients with malperfusion (groupI, $n=38$ ) and those without it (group II, $n=173$ ). The mean follow-up period of the patients was $80.0 \pm 81.2$ months.

\section{RESULTS:}

Preoperative malperfusion were diagnosed in $38(18 \%)$ patients, $10(4.7 \%)$ patients had myocardial ischemia, $11(5.2 \%)$ had cerebral ischemia, $16(7.6 \%)$ had limb ischemia, $6(2.8 \%)$ had renal ischemia, and $2(0.9 \%)$ had mesenteric ischemia. In-hospital mortality was $31.6 \%$ in group I and $8.1 \%$ in group II (p \&lt; 0.001 ). Two patients (100\%) with mesenteric malperfusion and $5(50 \%)$ with coronary malperfusion died. Multivariate logistic analysis identified malperfusion as a significant risk factor for in-hospital mortality (Odds ratio $5.32,95 \%$ confidence interval1.97-14.40, p \& 1t; 0.001). Five year and 10 year survival rates of hospital survivors were $95.8 \%$ and $82.9 \%$ in group I, $93 \%$ and $80.1 \%$ in group $I I(p=0.579)$ respectively.

\section{CONCLUSIONS:}

Although malperfusion is a significant risk factor for in-hospital mortality, late outcomes of hospital survivors were acceptable. Treatment of malperfusion should be considered earlier than aortic repair especially in patients with mesenteric or coronary malperfusion to improve early survival after surgery.

Carotid Body tumors: clinical profile, surgical outcome and complications.

G.N. Lone; Syed Abdul Wahid

\section{ABSTRACT:}

\section{BACKGROUND:}

Carotid body tumors are rare and highly vascular neoplasms originating in the paraganglionic cells of the carotid bifurcation. The treatment is surgical excision. We studied the clinical profile, surgical outcome and complications of CBT patients.

\section{METHODS:}

A total of 72 patients were studied prospectively and retrospectively. Diagnostic tools were FNAC, CT/MR Angiography.

\section{RESULTS:}

Majority were females (48). Commonest age was 50 to 59 years. All had visible neck swelling of varying size. $91 \%$ patients had unilateral and remaining had bilateral lesions. Pain was present in 11, dysphagia in 6 patients. CT Angio was done in $70.83 \%$ cases, MRA in $19.44 \%$ and both CTA and MRA in remaining cases. All cases underwent complete excision. Mean operating time was 107 minutes. Of all, 17 (23.61\%) had Shamblin grade I, $46(63.88 \%)$ had grade II, and $9(12.5 \%)$ had Shamblin grade III. ECA repair was done in 5 cases $(6.94 \%)$, ICA repair with interposition graft in 2 patients $(2.7 \%$ ), and ECA ligation in 1 case. Postoperatively, 3 cases went to radiotherapy in view of being extensive or leaving residual tissue. No case had any long term neurological sequel postoperatively.

\section{CONCLUSION:}

Meticulous subadventitial dissection and excision is key to achieve complete cure with minimal morbidity. These tumors should be operated upon by experienced vascular surgeons to minimize complications.

\section{Aortic surgery in octogenarian population - a single} centre experience.

Syed Sadeque; Sajiram Sarvananthan

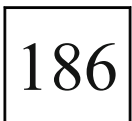

\section{ABSTRACT: \\ INTRODUCTION:}

Aortic surgery is challenging but this is even more of a challenge in Octogenarian patients. We carried out this study to review our experience in aortic surgery in octogenarian patients.

\section{METHODS:}

Retrospective data was reviewed to identify patients who underwent surgery on the aorta from 2000 to $2017(\mathrm{n}=135)$. The data was analyzed to find out the types of procedure, morbidity \&amp; mortality.

\section{RESULTS:}

We identified 3090 octogenarian patients, of which 135 had major aortic procedures which includes dissections. Most were male $(n=72,53.3 \%)$ with mean age of $82.3 \pm 2.23$ years. Their co-morbidities included hypertension ( $\mathrm{n}=91,67.4 \%), \mathrm{LV}$ impairment $(\mathrm{n}=40,29.7 \%)$, previous neurological events $(n=15,11.1 \%)$. The mean additive Euroscore was 12.5 \pm 2.3 . The procedures carried out were isolated ascending aorta replacement $(n=112,70.4 \%)$, aortic root replacement with or without ascending aorta $(n=34,21.3 \%)$ and arch replacement $(n=13,8.17 \%)$. Additional procedures were carried out in 88 patients (CABG 6, CABG+AVR 17, MVR 3, AVR 62). The priorities of surgery were elective 67 (49.6)\%, urgent 31 (23)\%, emergency $37(27.4) \%$.

The 30 day in hospital mortality in all cases were $11.8 \%(\mathrm{n}=16)$; in emergencies $27 \%(n=10)$ and elective/urgent cases were $6.1 \%(n=6)$. The mean length of stay was $11.8 \pm 7.9$ days. Postoperative complications were stroke $4(2.9 \%)$, renal failure $13(9.6 \%)$, deep sternal wound infection (0\%), PPM (3.7\%) and AF 23 (17\%).

\section{CONCLUSION:}

Although their mortality is marginally increased, the post-operative complications are comparable to overall treated group. The risk becomes higher in emergency and salvage procedures which is similar to any age group.

\section{Long-term outcome of open thoracoabdominal aortic aneurysm repair \\ Koki Yokawa}

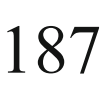

\section{ABSTRACT:}

OBJECTIVE:

Open repair of thoracoabdominal aortic aneurysm remains an important treatment option and continues to be challenging. The objective of this study was to investigate the results after open repair of thoracoabdominal aortic aneurysms by single surgeon.

\section{METHODS:}

A total of 236 patients underwent elective operation for thoracoabdominal aortic aneurysm by single surgeon from June 2000 and March 2018. The outcomes examined operative death, spinal cord injury and renal failure necessitating dialysis, as well as adverse event, a composite of these outcomes. The effects of preoperative, intraoperative, and postoperative risk factors were evaluated.

\section{RESULTS:}

The mean age was $62.6 \pm 13.5$ years old (range, 19-90), and 170 patients $(60.7 \%)$ were male. 130 patients $(55.0 \%)$ has dissective aortic aneurysm. 24 patients (57.9\%) presented with Crawford type I aneurysms, 92 patients with Crawford type II aneurysms, 91 patients with Crawford type III aneurysms, 29 patients with Crawford type IV aneurysms. 214 patients were used intraoperative motor evoked potential. The mean operative time and cardiopulmonary bypass time were $565 \pm 167 \mathrm{~min}, 185 \pm 77$ minutes. The operative death was $6.8 \%(\mathrm{n}=$ 16). The spinal cord injury rate the incidence of stroke were $10.6 \%$ (n $=25)$ and $3.8 \%(n=9)$. Postoperatively, 29 patients $(12.2 \%)$ required temporary hemodialysis. Incremental risk factor for operative death was degenerative aneurysm (Odds ratio: $3.62, p=0.03$ ), and for spinal cord injury were respiratory disorder (Odds ratio: $4.58, \mathrm{p}=0.003$ ) and coronary artery disease (Odds ratio: $4.40, \mathrm{p}=0.004$ ). Survival for 5 years, for 10 years and 15 years were $80.4 \pm 3.0 \%, 64.9 \pm 4.9 \%$ and $41.1 \pm 11.8 \%$. Freedom from aortic event for 5 years, 10 years and 15 years were $84.5 \pm 2.7 \%, 70.4 \pm 4.9 \%$ and $67.5 \pm 5.5 \%$.

\section{CONCLUSION:}

Survival remains acceptable, especially in the early years after thoracoabdominal aortic aneurysm repair. We need the strategy for thoracoabdominal aortic aneurysm with degenerative change. 
Blunt thoracic aortic injury : single-center comparison of open versus endovascular repair

Akio Nakasu; Hiroshi Horie

stroke $(11.4 \%$ vs $29.3 \%, p=0.001)$. Freedom from aortic event was no significant difference between two groups $(90.6 \%$ vs $88.2 \%$ in 5years, $74.6 \%$ vs $80.1 \%$ in 10 years, $p=0.987$ ), and survival rate was similar, too ( $78.2 \%$ vs $74.8 \%$ in 5 years, $59.9 \%$ vs $74.8 \%$ in 10 years, $p=0.683$ ).

CONCLUSIONS:

\section{ABSTRACT: \\ BACKGROUND:}

Blunt traumatic aortic injury (BTAI) is a rare but potentially lifethreatening entity. The mortality rate reaches $80 \%$ at the scene of the injury. The advances of the thoracic endovascular arterial repair (TEVAR) technique brought the paradigm shift in the treatment of BTAI. The aim of this study is to review our experience of two methods (open versus endovascular repair) for the treatment of BTAI.

PATIENTS AND METHODS:

Between March 1984 to March 2018, 55 BTAI cases were admitted to our hospital (mean age 41, range 19-57; male: female $=41$ : 14). The average Injury Severity Score (ISS) was 40.4 (range 35-47). 47 patients underwent open repair (OR) and 8 patients did TEVAR. We compared the baseline characteristics, the operative data and the outcome.

\section{RESULTS:}

The mean age in OR and TEVAR groups were $37.8 \pm 21.2$ and $59.6 \pm 12.3$ years $(\mathrm{P}=0.007)$. The mean blood loss during $\mathrm{OR}$ and TEVAR was $1709 \mathrm{ml}$ and $24.6 \mathrm{ml}$, respectively (P\&lt;0.001). The average time for OR and TEVAR was 305 minutes and 112 minutes, respectively (P\&lt;0.001). The mortality during admission in OR and TEVAR groups were $8 / 47(17 \%)$ and $0 / 8(0 \%)$, respectively $(\mathrm{P}=0.587)$. There was neither significant difference between each group about postoperative complication.

CONCLUSIONS:

Compared with OR, TEVAR can be performed with less blood loss and procedure time, which is favorable in cases of multi-organ injuries. However, OR also has an acceptable outcome and should be considered in younger patients who have risk of long-term complications of TEVAR because of the change of angulation and diameter of aortic arch.

\section{Optimal procedure for acute type A aortic dissection without intimal tear at aortic arch \\ Takashi Oshitomi; Ichiro Ideta; Kentaro Takaji; \\ Yukihiro Katayama; Toshiharu Sassa; \\ Hidetaka Murata; Shizuya Shintomi; Hideyuki Uesugi \\ Tomonori Koga}

\section{ABSTRACT: \\ BACKGROUND:}

Surgical strategy for acute type A aortic dissection (AAAD) is to resect the entry tear. When there is the entry tear at ascending aorta, ascending aorta replacement (AAR) is the minimum replacement and procedure of dissected aorta. But it is often performed total arch replacement (TAR) for the patients with the entry tear at ascending aorta or far descending aorta, because of worrying about long-term outcome. This study assessed whether limited or extensive aortic replacement make some difference in short-term and long-term outcomes.

\section{MATERIALS AND METHODS}

Between January 2001 and December 2017, 420 patients underwent surgical repair for AAAD at our hospital, and 285 patients of them could be followed-up. 54 patients of them who had the entry tear at the aortic arch or proximal portion of the descending aorta were excluded, because they had no choice but to undergo TAR or partial arch replacement (PAR) procedure for resecting the entry tear. After that, 231 patients were divided into two groups; group A: underwent AAR $(n=149)$ and group B: underwent TAR (include Open Stent added) or PAR $(n=82)$. We assessed complications such as stroke, survival rate, cause of death, aortic event (reoperation, recurrent dissection, and aortic rupture).

RESULTS:

30 days mortality was no significant difference between two groups (6.0\% vs $13.4 \%, \mathrm{p}=0.057)$. But group $\mathrm{B}$ had a higher rate of post-ope

Limited aortic replacement are optimal procedure for AAAD without intimal tear at aortic arch.

Total arch repair using frozen elephant trunk technique (TAR-FET) after thoracic endovascular aortic repair (TEVAR)

Hiroshi Yamamoto; Genbu Yamaura;

Fuminobu Tanaka; Daichi Takagi; Kentaro Kiryu;

Yoshinori Itagaki; Takeshi Arai; Kensuke Okuguchi

Takayuki Kadohama

\section{ABSTRACT: BACKGROUND:}

TEVAR has emerged as an effective therapy for a variety of aortic pathologies. However, secondary interventions are required as an open procedure in select circumstances such as retrograde type A dissection (RTAD) and endoleak. We present our technique of open proximal aortic repair using frozen elephant trunk technique (FET)

\section{METHODS:}

From 2015 to 2018, 161 patients underwent TEVAR and we performed TAR-FET in $4(2.5 \%)$ patients ( 2 males, mean age, 66 years, range, 54-71 years). The complications after TEVAR included RTAD $(n=3)$, Type Ia endoleak $(n=1)$. The median interval between TEVAR and repair was 216 days (range 18-681 days). All procedures were performed via a median sternotomy under hypothermic cardiopulmonary bypass (CPB) and selective cerebral perfusion. The left axillary artery was isolated and used for CPB routinely. After lower body circulatory arrest (rectal temp: $25^{\circ} \mathrm{C}$ ), the aorta was transected before the origin of the innominate artery (so called zone 0), proximal bare stents were removed (if necessary). A $10 \%$ over-sized $120 \mathrm{~mm}$ stent-graft system (J-graft Frozenix) was then implanted into the remaining stent-graft. Then proximal segment was anastomosed to distal aorta using sutures, incorporating the stented elephant trunk with full salvage of the stent-graft. After lower body re-perfusion, the proximal anastomosis was done and the aortic cross-clamp was released. Then the arch vessels were re-implanted with a separate branched graft in an end-to-end fashion.

RESULTS:

The mean operation time, aortic cross-clamp time and lower body circulatory arrest time were $402+/-50$ minutes, $103+/-32$ minutes and 45+/-9 minutes, respectively. There were no in-hospital deaths and major complications. Postoperative CT angiography demonstrated that all cases has been repaired successfully without endoleak.

\section{CONCLUSIONS:}

This technique is seemed to be useful and easy method for open proximal aorta repair leaving the previous stent-graft in place fully although further investigation is required.

Risk Prediction Score System for Late Aortic Events in Uncomplicated Stanford Type B Aortic Dissection Minoru Tabata; Wahei Mihara; Takeshi Shimamoto;

Tatsuhiko Komiya; Shuichiro Takanashi; Tetusya Tobaru; Tatsuya Nakao; Sunao Nakamura; Yasunori Sato; Akihito Matsushita

\section{ABSTRACT:}

OBJECTIVE:

Several risk factors for late aortic events in patients with uncomplicated type B aortic dissection (UTBAD) were reported. However, it remains 
controversial about definition of high risk UTBAD. We developed a risk prediction score system for late aortic events, and validated the model using multicenter data.

\section{METHODS:}

We reviewed 187 consecutive patients who were diagnosed with UTBAD from 2004 to 2017 at 2 centers (derivation cohort) and 219 consecutive patients who were diagnosed with UTBAD from 2012 to 2016 in 4 other centers (external validation cohort). We explored predictors of late aortic events using a Cox proportional hazards model, then developed a risk prediction score model for aortic events, and determined test reliability in the derivation cohort using receiver operating characteristic (ROC) analyses. Then, we validated the model using external multicenter clinical data with ROC analysis.

\section{RESULTS:}

The risk prediction score system was developed using the following independent predictors: initial aortic diameter $\geq 40 \mathrm{~mm}$ ( 2 points), false lumen diameter \&gt; true lumen diameter ( 2 points), ulcer like projection ( 1 point), and aged $\geq 70$ years old ( 1 point). ROC analysis showed that a cut-off value of total additive score was 2 points with sensitivity of $92.0 \%$ and specificity of $61.6 \%$ (AUC 0.83 ; $\mathrm{p} \& 1 \mathrm{t} ; 0.001$ ). In the validation cohort, the low risk group (score $\& 1 t ; 2$ ) demonstrated higher 1- and 3-year freedom rates from late aortic events than the high risk group (score $\geq 2$ ); $99.1 \%$ vs $67.5 \%$, $99.1 \%$ vs $52.6 \%$, respectively, p\&lt;0.0001). And, ROC analysis showed that a cut-off value of score was 2 points (AUC 0.85; p\&lt;0.001).

\section{CONCLUSIONS:}

We developed a simple risk prediction score system for late aortic events in patients with UTBAD and validated it. High risk patients can be identified using our model, and they should be closely monitored and considered for interventions at the appropriate timing.

\section{Analysis of the treatment for ruptured abdominal aortic aneurysm: open or endovascular repair Eitoshi Tsuboi; Yoshiki Endo; Akinobu Kitagawa; Yasuhisa Fukada; Yoshihito Irie, Hitoshi Nakanowatari}

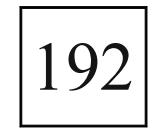

\section{ABSTRACT:}

\section{OBJECTIVE:}

Open repair have been the golden standard treatment for ruptured abdominal aortic aneurysm (rAAA), but recently many reports indicate endovascular aneurysm repair (EVAR) is effective for the rescue of the patients with rAAA. Our study aims to reveal the short and long term outcome of these two strategies for rAAA.

\section{METHODS:}

We retrospectively reviewed 47 consecutive patients who underwent surgical treatment for rAAA between January 2008 and Novenber 2018. Open repair was performed to 21 patients (O group), and EVAR was performed to 26 patients (E group). We analyzed 30-days mortality, rerupture and reintervention.

\section{RESULTS:}

Comparing two groups ( $\mathrm{O}$ and $\mathrm{E}$ groups), mean age ( $72 \pm 9$ vs $75 \pm 11$ years, $\mathrm{p}=0.35)$, pre-operative shock state ( 10 vs 7 cases, $\mathrm{p}=0.22)$ and mean diameter of aneurysm ( $71 \pm 12$ vs $72 \pm 12 \mathrm{~mm}, \mathrm{p}=0.75)$ wasn't different statistically. Reintervention ( 0 vs 6 cases, p\&lt; 0.05 ) was significantly different, but 30 -days mortality ( 19 vs $11.5 \%, \mathrm{p}=0.76$ ) and rerupture ( 0 vs 3 cases, $\mathrm{p}=0.31$ ) wasn't different. In E group, reintevention was performed for 2 stent-graft migration and 4 endoleak, and tre-rupture cases were 1 stent-graft migration and 2 endoleak.

\section{CONCLUSION:}

Both groups result in good short-term outcome. In the long term, E group tended to become rerupture and needed reintervention, but $\mathrm{O}$ group didn't at all. Even if we could rescue the patiens with rAAA by performing EVAR, we have to follow up strictly and perform adequate additional treatments if necessary.
Analysis of the Extent of Degenerative changes in Intra-pericardial Aorta of patients with Bicuspid Aortic Valve (BAV)

Reddy P; Arava S.K.; Ray Ruma; Talwar S.; Reddy P.

\section{ABSTRACT:}

OBJECTIVES:

To study the extent of degenerative process in intrapericardial aorta of patients with bicuspid aortic valve disease.

\section{METHODOLOGY:}

A prospective cohort of patients with bicuspid aortic valve, who were undergoing aortic valve intervention with or without ascending aortic replacement were included in this study. Biopsies taken from aortic sinus, mid ascending aorta and distal ascending aorta, were analyzed for degenerative changes using modified Schlatmann and Becker criteria and a composite histopathological examination (HPE) score was calculated. Preoperatively, all the patients were evaluated clinically, echocardiographically, and, also by computerized tomographic angiogram.

\section{RESULTS:}

Total 144 (94 male) patients were studied. The mean age was $39.3 \pm 15.6$ years (range $=11-78$ years). Aortic stenosis was the predominant lesion in $79(55 \%)$ patients, aortic regurgitation in $41(28 \%)$ patients, mixed lesion in $23(17 \%)$ patients. The mean diameter of the aorta was $29.59 \pm 6.43 \mathrm{~mm}$ (range $=17-55 \mathrm{~mm}$ ) at aortic annulus level, $39.48 \pm 7.83 \mathrm{~mm}$ (range $=23$ $66 \mathrm{~mm})$ at aortic sinus level, $45.53 \pm 11.93 \mathrm{~mm}($ range $=18-85 \mathrm{~mm})$ at the mid ascending aortic level and $36.26 \pm 7.05 \mathrm{~mm}($ range $=22-60 \mathrm{~mm})$ at the distal ascending aortic level. By linear regression analysis, there was statistically significant correlation between the aortic diameter and the degree of degenerative changes in the intrapericardial aorta at all three levels. At aortic sinus, ascending aortic and proximal arch level, for every one number increase in total histopathologic score, there is $0.9 \mathrm{~mm}$ increase in the aortic sinus diameter, $0.9 \mathrm{~mm}$ increase in the ascending aortic diameter and $0.6 \mathrm{~mm}$ increase in the proximal arch diameter. Individual components of the modified Schlatmann and Becker score showed statistically significant correlation with aortic sinus diameter and at variable levels at ascending aortic and proximal arch levels.

\section{CONCLUSION:}

There is significant correlation between the severity of degenerative histopathologic changes and the ascending aortic diameter at all levels with maximum correlation was found at the aortic sinus and ascending aortic level.

The early and midterm outcomes of one-staged or two-staged hybrid minimally invasive surgical and transcatheter ablation for long-standing persistent atrial fibrillation

Zhaolei Jiang; Ju Mei

\section{ABSTRACT:}

OBJECTIVE:

To explore the application and effectiveness of one-staged or two-staged hybrid minimally invasive surgical and transcatheter ablation for longstanding persistent atrial fibrillation (LSPAF). Methods: From Jun 2015 to Dec 2017, a cohort of 56 patients (18 female, mean age of $59.1 \pm 6.9$ years) with long-standing persistent atrial fibrillation underwent onestaged $(n=30)$ or two-staged $(n=26)$ hybrid minimally invasive surgical and transcatheter ablation. Mean AF duration was 5.9 \pm 3.0 years. Mean left atrial diameter was $45.4 \pm 4.2 \mathrm{~mm}$. Mean CHA2DS2-VASc score was $2.3 \pm 1.2$. Fourteen cases had a history of prior catheter ablation. All patients underwent continuous 24-hour or 48-hour holter monitoring at 3 months, 6 months, 1 year and yearly thereafter. Results: All patients successfully underwent one-staged or two-staged hybrid minimally invasive surgical and transcatheter ablation. During ablation, LSPAF was terminated in $80 \%(24 / 30)$ with one-staged hybrid ablation and $84.6 \%$ $(22 / 26)$ with two-staged hybrid ablation. At a mean follow-up of $20.3 \pm 8.2$ 
months, $89.3 \%(50 / 56)$ patients maintained sinus rhythm. Among them, $86.7 \%$ (26/30) patients with one-staged hybrid ablation maintained sinus rhythm, and $92.3 \%(50 / 56)$ patients with two-staged hybrid ablation maintained sinus rhythm. Six patients with recurrent AF continued to receive warfarin and amiodarone drug therapy. No death or cerebrovascular events occurred. No patient required permanent pacemaker implantation.

\section{CONCLUSION:}

One-staged or two-staged hybrid minimally invasive surgical and transcatheter ablation could be safely and effectively applied to the treatment of LSPAF. The early and midterm outcomes were satisfactory.

\section{Minimally invasive Cox Maze IV ablation procedure performed entirely by bipolar clamp concomitant to mitral valve surgery through right lateral minithoracotomy \\ Zhaolei Jiang; Ju Mei}

\section{ABSTRACT: \\ OBJECTIVES:}

To introduce the technique of performing minimally invasive concomitant Cox Maze IV ablation procedure entirely by bipolar clamp through right lateral minithoracotomy for patients with atrial fibrillation (AF) associated with mitral valve diseases. Methods: Sixty nine patients with mitral valve disease and long-standing persistent $\mathrm{AF}$ received minimally invasive Cox Maze IV ablation procedure combined with mitral valve surgery from June 2012 to January 2015. The etiology of mitral valve disease was rheumatic $(n=41)$ and degenerative $(n=28)$. Age at operation ranged from 52 to 71 years. There were 43 males and 26 females. AF duration ranged from 1.5 years to 13 years. Diameter of the left atrium ranged from 42 to $60 \mathrm{~mm}$. Diameter of the left ventricle ranged from 43 to $66 \mathrm{~mm}$. Left ventricle ejection fraction (LVEF) ranged from $45 \%$ to $67 \%$. Concomitant Maze IV ablation procedure was performed through right lateral minithoracotomy entirely by bipolar radiofrequency clamp. Results: All patients successfully underwent this minimally invasive concomitant Maze IV ablation procedure and mitral valve surgery. The mean cardiopulmonary bypass time was $130.3 \pm 17.7$ minutes. The mean aortic crossclamp time was $91.8 \pm 12.7$ minutes. No patient needed conversion to sternotomy during the surgery. There was no early death or pacemaker implantation in the perioperation. The average length of hospital stay was $9.8 \pm 3.3$ days. At discharge, 65 patients $(65 / 69,94.2 \%)$ maintained sinus rhythm. At a mean follow-up time of $21.0 \pm 8.6$ months, sinus rhythm was restored in 62 patients $(62 / 69,89.9 \%)$. Cumulative maintenance of normal sinus rhythm without AF recurrence at 2 years postoperatively was $85.1 \pm 5.8 \%$. Conclusions: The minimally invasive concomitant Maze IV ablation procedure performed entirely by bipolar clamp through right lateral minithoracotomy was a safe, feasible, and effective technique for patients with $\mathrm{AF}$ associated with mitral valve diseases.

\section{Effect of the size of the left atrium on sustained sinus rhythm in patients undergoing bipolar radiofrequent ablation and mitral valve surgery Miha Antonic; Harun Avdagic}

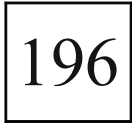

\section{ABSTRACT: INTRODUCTION:}

Over $58 \%$ of patients with mitral valvular disease have electrocardiographically documented atrial fibrillation with adverse hemodynamic effects before cardiac surgery. Radifrequent ablation has been established as an effective and safe method fort the treatment of atrial fibrillation. Many risk factors including the left atrial size have been correlated with a less favourable succes of ablation. The aim of this study was to evaluate the effect of the size of the left atrium on the outcome of surgical radiofrequency ablation.

\section{METHODS:}

The study was conducted in 40 patients scheduled for elective mitral valve surgery and radiofrequent ablation for permanent atrial fibrillation. The patients were divided in 2 groups regarding the size of the left atrium. Group 1 consisted of patients with a left atrium diameter $\leq 5 \mathrm{~cm}$ and group 2 of patient with left atrium diameter $\& \mathrm{gt} ; 5 \mathrm{~cm}$. The primary endpoint of the study was the fraction of patients in stable sinus rhythm 6 months after surgery.

\section{RESULTS:}

The groups were well matched for baseline demographics, preoperative medications, and comorbidities. The mean left atrium diameter was $45,5 \mathrm{~mm} \pm 4,6 \mathrm{~mm}$ in Group 1 and $62,4 \mathrm{~mm} \pm 6,8 \mathrm{~mm}$ in Group 2. No significant differencies between the study groups could be detected regarding the heart rhythm at the discharge from the hospital and at 3-month follow-up. However, after 6 months, significantly more patients were found to be in sinus rhythm in Group 1 compared to Group 2 (15 $(75 \%)$ vs $8(40 \%), p=0.025)$. Furthermore, the regression analysis proved the size of the left atrium as an independent predictor of the outcome of surgical radiofrequent ablation.

\section{CONCLUSIONS:}

The size of the left atrium is an important predictor of the outcome of radiofrequent ablation for atrial fibrillation.

The intra/extracardiac Fontan: preliminary results Shiv Kumar Choudhary; Sachin Talwar

\section{ABSTRACT:}

\section{OBJECTIVE:}

Since being popularized by Jonas in 2008, the intra/extracardiac Fontan (IECF), its claimed advantageous over the traditional extracardiac or lateral tunnel Fontan are simplicity, suitability for nearly all subsets, flow characteristics, low risk of sinus nodal artery injury and a possibly lower incidence of arrhythmias. In this paper, we present our early experience with this modification of the Fontan.

\section{METHODS:}

Between 2009-2018, 10 patients underwent IECF on CPB and cardioplegic arrest at our institute. Analysis of pre-operative, intraoperative with early follow-up results was performed. A polytetrafluoroethylene graft was sutured proximally to the orifice of the inferior vena cava and distally to the ipsilateral bidirectional superior cavopulmonary (BCPS) junction.

\section{RESULTS:}

Nine patients followed a previous BCPS and one patient had a primary IECF. Diagnoses were double outlet right ventricle $(n=2)$, unbalanced atrioventricular septal defect with associated atrioventricular valve regurgitation $(n=3)$, single ventricle with anomalies of cardiac situs $(n=2)$ and tricuspid atresia with borderline pulmonary arteries $(n=1)$ or borderline pulmonary artery pressures $(n=2)$. Median Clamp and CPB times were 42 minutes and 82 minutes respectively.

There were no early or late deaths. Median intensive care unit stay was 3 days (1 to 23 days). There were no arrhythmias. Mean duration of pleural effusions was 9.5 (median-5.5) days; There were no arrhythmias at a median follow-up of 5 years (range- 1 month to 9.3 years)

\section{CONCLUSION:}

The IECF is simple particularly at reoperations, in borderline patients and those needing concomitant intracardiac procedures. Early results are promising. These patients need constant surveillance.

Total Anomalous Pulmonary Venous Connection: improved early outcomes with an alternate management strategy

Nayem Raja; Tarun Raina Ramman; Sumir Girotra; Sushil Azad; Sitaraman Radhakrishnan; Parvathi U. Iyer; Krishna S. Iyer

Kuntal Roy Chowdhuri 


\section{ABSTRACT: \\ BACKGROUND:}

Surgical repair of TAPVC has been associated with significant mortality especially in developing countries, largely blamed on post-operative pulmonary hypertensive crisis. We believe that altered ventricular dynamics post-operatively are more responsible for mortality and have evolved a management strategy accordingly. The results of this strategy are presented.

\section{PATIENTS AND METHODS:}

308 patients (median age -93 days; median weight -4100 gms) with TAPVC underwent biventricular repair between Jan 2001 and Sept $2018.51 \%$ had supracardiac, $27 \%$ had cardiac, $10 \%$ had infracardiac and $12 \%$ had mixed connections. 155 ( 50.3\%) had pulmonary venous obstruction at some level. $17 \%$ required emergent surgery, $24 \%$ were semi-urgent and the rest were done electively on priority.

Operative strategies included 1) normothermic bypass with short crossclamp duration 2) posterior approach for supra and infra cardiac connections 3 ) routine ligation of the vertical vein.

Post-operative strategies included 1) routine left atrial pressure (LAP) monitoring instead of pulmonary artery pressure monitoring to track left ventricular function 2) aggressive afterload reduction 3) frequent echocardiographic assessment of bi-ventricular dynamics and 4) weaning from ventilator support only when LAP was consistently low.

\section{RESULTS:}

In-hospital mortality was $8 / 308(2.59 \%)$. Causes of death were severe drug resistant sepsis -5 , bronchomalacia-1, malignant arrhythmia- 1 , neurological complication-1. No patient required mechanical support or inhaled nitric oxide. Median ventilator duration and hospital stay was 52 hours and 11.2 days respectively. All survivors were discharged with documented unobstructed pulmonary venous drainage.

\section{CONCLUSION:}

Early outcomes following surgery for TAPVC can be improved by a strategy focused on tracking ventricular function rather than pulmonary artery pressure. Nitric oxide and mechanical support are easily avoidable.

Retrospective study of results of an alternate technique of completion of the total cavopulmonary connection Bharat Siddarth; P. Rajashekar; Srikant Sharma;

Rohan Magoon; Shiv Kumar Choudhary; Sachin Talwar

\section{ABSTRACT: \\ BACKGROUND:}

Total cavopulmonary connection (Fontan or TCPC) palliation is final stage of palliation for patients with a univentricular heart. Bidirectional cavopulmonary anastamosis (BDG) is used for staging in most of the cases in this pathway. There are different techniques for TCPC completion after a child undergoes BDG. It includes offpump as well as onpump palliation. We describe our technique of completing TCPC in an alternate way to reduce dissection and associated complications.

\section{METHODS:}

Between January 2018 and October 2018, 17 patients who underwent completion TCPC using the alternate surgical method at AIIMS.

\section{RESULTS:}

All patients had an uneventful intraoperative course. The mean cardiopulmonary bypass time was $60+/-16.8$ minutes (range, 39 to 102 minutes). The time of aortic clamping was not collected, because the cases of external conduit were performed without cardiac arrest. There were no deaths in the study. The mean postoperative fontan pressures were 15.6 $+/-1.2 \mathrm{mmHg}$ with no gradient between SVC and IVC pressures. Mean duration of hospital stay was $15.6+/-3.6$ days (Range from 10 to 22 days). There was no incidence of phrenic nerve paresis or palsy.

\section{CONCLUSIONS:}

Performing completion TCPC using our alternate technique is easy to perform as well as easy to teach. It avoids disadvantages associated with routine techniques.
Single coronary artery in transposition of the great arteries. Remote results.

Nefedova I.Y.; Lyazin Denis

\section{ABSTRACT:}

Arterial switch operation (AS) for transposition of the great arteries (TGA) has become the standard and optimal method of surgical correction of the simple TGA. Coronary reimplantation is the key to successful surgery. Coronary arteries are located "typically" in $67-72 \%$ of patients with TGA. PURPOSE:

The aim is to present an analysis of long-term results of TGA correction with a single coronary artery.

\section{MATERIALS AND METHODS:}

Between 1999 and 2016 AS operation was performed in 248 children in the neonatal emergency surgery department. The average age of patients at the time of AS operation was $9.9 \pm 3.5$ days (minimum 1 day, maximum 165 days). One-stage surgical treatment was performed in 235 (94.8\%) patients, two-stage correction of the defect - in $13(5.2 \%)$ patients. Of all patients who underwent AS operation $21(8.4 \%)$ had anatomy of a single coronary artery.

\section{RESULTS:}

In the long-term period 15 (79\%) patients out of 19 discharged from the neonatal emergency surgery department with a single coronary artery were observed. The median follow-up time was $2.9 \pm 1.9$ years (from 35 days to 12.5 years). Survival rate for all operated patients with a single coronary artery by 6 months was $95 \%$, by 10 year $84 \% .3$ (9\%) died children with a single coronary artery had the following anatomy: 2 the only right coronary artery, 1 - the left coronary artery. Only in 1 case out of 3 the child's death had coronary genesis.

\section{CONCLUSION:}

Anomalies of the coronary arteries could complicate the arterial switch operation. If during Echocardiography the absence of one of the coronary arteries is suspected, the examination of patients should be supplemented with an alternative research - an angiocardiography, either a computer tomography, or a magnetic resonance tomography.

\section{Biventricular repair in patients with hypoplastic left heart complex \\ Bockeria Leo; Kalashnikov Sergei}

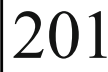

\section{ABSTRACT:}

\section{OBJECTIVES:}

To determine morphometric and hemodynamic criteria of successful biventricular repair in newborns and children under one year of age with hypoplastic left heart complex (HLHC). Methods: Retrospective analysis of 56 patients with HLHC treated at Bakoulev National Medical Research Center for Cardiovascular Surgery within period 2003 to 2015. Biventricular repair was performed in 56 patients divided into two groups. First group $(n=29)$ consisted of patients who underwent one-staged biventricular repair, second $\operatorname{group}(n=27)$ consisted of patients who underwent staged biventricular repair. Median age at hospitalization 35,9 $\pm 55,4$ days( 1 day to 7,5 months). Median weight $3,8 \pm 1,1 \mathrm{~kg}(2,17$ to 7,6$)$. BSA $0,24 \pm 0,047 \mathrm{~m} 2(0,16$ to $0,36)$. Mitral valve $z$-score in first group $-2.77 \pm 0.7(-0.84$ to -4.14$)$. Aortic valve $z$-score $-2.89 \pm 0.85(-0.58$ to -4.5$)$. Left ventricular outflow tract $\mathrm{z}$-score $-2.72 \pm 0.7(-1.15$ to -4.3$)$. End-diastolic left ventricular volume $21.3 \pm 3.99(16$ to 35$) \mathrm{ml} / \mathrm{m} 2$. Mitral valve $z$-score at hospitalization in second group $-3.54 \pm 0.74(-1.55$ to -4.7$)$. Aortic valve $z-$ score $-4.35 \pm 1.11(-2.63$ to -7.04$)$. Left ventricular outflow tract $z$ score $-3.77 \pm 1.06(-2.26$ to -6.45$)$. End-diastolic left ventricular volume $17.66 \pm 3.06(12$ to 22$) \mathrm{ml} / \mathrm{m} 2$. Patients who died after operation because of left heart failure $(n=8)$ became control group.

RESULTS:

In our investigation we revealed that if mitral valve had no morphological abnormalities and z-score at hospitalization \&gt;-3;aortic valve z-score 
and left ventricular outflow tract z-score \&gt;-3;left heart/heart axis ratio $\& \mathrm{gt} ; 0.8$;end-diastolic left ventricular volume $\& \mathrm{gt} ; 20 \mathrm{ml} / \mathrm{m} 2$ then all patients successfully underwent one-staged biventricular repair. If mitral valve had morphological abnormalities or z-score at hospitalization\&lt;4.5; aortic valve $\mathrm{z}$-score\&lt;-6;left ventricular outflow tract $\mathrm{z}$-score\&lt;$6 ;$ left heart/heart axis ratio\&lt;0.6;end-diastolic left ventricular volume \&lt $; 15 \mathrm{ml} / \mathrm{m} 2$ then biventricular repair failure risk was too high and univentricular repair was necessary. If morphological left heart parameters were less than described for one-staged biventricular repair and greater than described for univentricular repair then method of choice should be staged biventricular repair.

CONCLUSIONS:

In newborns and children under one year of age with HLHC, successful biventricular repair in case of correct assessment of morphometric left heart parameters is possible in at least $85 \%$ of cases.

Modified Double Decker Procedure: Loft Technique for Partial Anomalous Pulmonary Venous Connection Norihiko Oka; Takuma Fukunishi

\section{ABSTRACT: \\ BACKGROUND:}

The double decker technique is useful for PAPVC repair. In this study, we modified original double decker (loft technique) and report that using autologous tissue allowed for an anastomosis to be made over a greater distance than original procedure.

PATIENTS AND PROCEDURE:

We performed 4 cases (weight; $40.7 \pm 3 \mathrm{~kg}$, age; $11.7 \pm 4.8$ years) of PAPVC repair using the modified technique. In the Loft technique operation method: the Right atrium (RA) was incised from the RA appendage to before the SVC junction to incorporate the crista terminalis. Autologous pericardium treated with glutaraldehyde was used to baffle the intra-cardiac PV to connect the left atrium and close the ASD. One of the intra-cardiac PV return is completed to cover from ASD to SVC orifice using autologous pericardium treated with glutaraldehyde. A U-shaped incision was made in the superior wall of the SVC (as opposed to the original method), and the other intracardiac PV was baffled to cover the inferior wall of the SVC with autologous pericardium treated with glutaraldehyde. Finally, reconstruction of the systemic venous circulation was obtained by direct anastomosis of the RA and U-shaped incision of the SVC.

\section{RESULTS:}

With the modifed technique, operation, cardiopulmonary bypass (CPB), and aortic cross clamp (ACC) time was $420 \pm 104,200 \pm 71$, and $111 \pm 23$ mins respectively. The postoperative follow-up period was 111 \pm 23 months and revealed no evidence of mortality or complications arising from the procedure, such as the need for reoperation, SVC syndrome, or heart failure symptoms (NYHA I) in all patients. Post-operative electrocardiogram detected no evidence of SVC and PV stenosis, or incidences of supraventricular arrhythmia.

CONCLUSION:

This modified loft technique, which makes U-shaped incision for SVC that is opposite direction to the original double decker technique, enables the creation of systemic venous circulation by utilizing autologous tissue to create an anastomosis over a longer distance.

Mid-term results of pulmonary root translocation for transposition of the great arteries with ventricular septal defect and pulmonary stenosis Yasuhiro Kotani; Yasuyuki Kobayashi

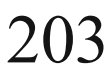

\section{ABSTRACT:}

OBJECTIVE:

Surgical treatment for transposition of the great arteries (TGA) with ventricular septal defect (VSD) and pulmonary stenosis (PS) remains still in debate. Pulmonary root translocation (PRT) is a less invasive procedure compared to Nikaidoh operation and may have a potential benefit on left ventricular outflow tract (LVOT) outcome. We sought to evaluate the mid-term results of this procedure.

\section{METHODS:}

A retrospective study was reviewed in 14 patients with TGA with VSD and PS performed Rastelli operation between 2005 and 2018. 6 patients underwent PRT. All had malposition of the great arteries as follows: TGA with VSD and PS ( $\mathrm{n}=4)$; congenitally corrected TGA with VSD and PS $(\mathrm{n}=2)$.

\section{RESULTS:}

Median age and body weight at PRT was 29 (23-70) months and 11.3 (10.2-15.3) kg, respectively. Median follow-up period was 69 (60-105) months. There were no early deaths and late deaths. After surgery, the distance between the top of the interventricular septum (IVS) and the aortic valve (AoV) by echocardiogram, where supposed to be LVOT, was decreased from $24.7(22.3-27.3) \mathrm{mm}$ to $19.5(18.2-20.9) \mathrm{mm}$. Reintervention was needed for only one patient who underwent Senning procedure to release obstruction of pulmonary vein. No patients had a significant LVOT obstruction.

\section{CONCLUSIONS:}

PRT provided very low operative risk with less required LVOT-related reintervention. This surgical option may be an excellent surgical alternative for TGA with VSD and PS.

Beginning of Pediatric Ventricular Assist Treatment in Korea

Yu Rim Shin; Han Ki Park 204

\section{ABSTRACT:} BACKGROUND:

In children with intractable heart failure, long-term mechanical circulatory support allows resuscitation of vital organs and provides bridge to transplantation for a long waiting period. This study was performed to review short-term outcomes of early experience of pediatric ventricular assist device (VAD) treatment in Korea.

METHODS:

Since November 2017, four pediatric patients received long-term VAD treatment. Three patients younger than 15 months had pulsatile paracorporeal device (Berlin Heart EXCOR $®$ ) and a 14 year old girl had implantable continuous flow device (HeartWare $\left.{ }^{\circledR}\right)$. Two patients were on pre-implant ECMO support. Biventricular assist was performed in a 15 month old patient with restrictive cardiomyopathy and the other 3 patient with dilated cardiomyopathy had left ventricular assist.

\section{RESULTS:}

All patients recovered from implantation without complication and the patient with HeartWare ${ }^{\circledR}$ could be discharged home and returned to school. Patient who was on biventricular assist had left side pump failure on post-implantation 2.5 month and died of rapidly progressed pulmonary edema and DIC. One patient could be weaned from VAD on postimplantation 7 months. Two patient post-implantation 3 month and 5 month are waiting for heart transplantation.

\section{CONCLUSION:}

In Korea, we are experiencing a beginning period of pediatric ventricular assist device treatment. Early experience with pediatric VAD demonstrated successful circulatory support to achieve stable hemodynamics and improvement in functional class. Watchful monitoring is required for pump related complications of VADs.

Outcomes of the Infants less than 2500g Performed cardiac Surgery under cardiopulmonary bypass in Eastern China: A 12-year Experience in a Tertiary Pediatric Medical center

Bing Jia; Yaping Mi 


\section{ABSTRACT: \\ OBJECTIVES:}

To summarize the outcome and the perioperative care experience of 134 cases with weight less than $2500 \mathrm{~g}$ performed open heart surgery under cardiopulmonary bypass in a single pediatric cardiac care unit (CICU) during the past twelve years.

\section{METHODS:}

We retrospectively reviewed the medical records of 134 consecutive small baby less than/ equal to 2500 grams who underwent open heart operation from January 2006 to January 2018.86 cases were male. $75.2 \%$ $(n=94)$ of the infants were premature. 5 cases were abandoned infants with unclear history. Diagnoses included ventricular septal defect (VSD) $(n=43)$ and complex defects $(n=91)$.

\section{RESULTS:}

All the low weight infants were directly sent into CICU or transferred from neonate unit. 22 infants $(16.42 \%)$ had been ventilated and 7 infants $(5.22 \%)$ had had digestive system surgery before the cardiac surgery. 2 cases $(1.49 \%)$ performed atrioseptoplasty. Early in-hospital infant surgical mortality ( $<30$ days) was $8.20 \%(n=11) .1$ late-death occurred 119 days postoperatively. 2 cases $(1.49 \%)$ added BT shunt. 57 (42.53\%) cases delayed closure of sternum. $2(1.49 \%)$ performed extracorporeal membrane oxygenation (ECMO) procedure for 7 to 11 days. $10(7.46 \%)$ needed nitric oxide inhalation. $9(6.72 \%)$ occurred capillary leak syndrome. $3(2.24 \%)$ patients required peritoneal dialysis. 2 (1.49\%) required diaphragmatic plication. $3(2.24 \%)$ patients developed postoperative bloodstream sepsis and $1(0.75 \%)$ occurred chylothorax. 9 infants $(6.72 \%)$ had reintubation. The median number of ventilation duration was $120 \mathrm{~h}$ and intensive care unit stay was $19 \mathrm{~d}$. There were no obvious neurological complications associated with cardiopulmonary bypass except a case underwent ECMO.

\section{CONCLUSION:}

The outcome of low weight infants underwent open heart operation is beneficial. The low weight might not be considered as high-risk variables for infant heart surgery in the experienced centers. Perioperative care should focus on the multiple organ support.

\section{Clinical outcome of adult patients with decreased ventricular function undergoing Fontan operation and TCPC conversion}

Yosuke Kuroko; Yasuhiro Kotani

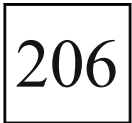

\section{ABSTRACT: \\ BACKGROUND:}

Ventricular dysfunction is known as a risk factor in Fontan circulation. We sought to see the clinical outcome of adult patients undergoing Fontan operation, especially in patients with decreased ventricular dysfunction.

\section{METHODS:}

Retrospective study was performed in 37 adult patients (primary Fontan operation in 18 patients and total cavopulmonary connection (TCPC) in 19 patients). Diagnosis included tricuspid atresia in 9 patients, single ventricle in 23 patients, pulmonary atresia in 2 patients, Ebstein disease in 2 patients, and arrhythmogenic right ventricular dysplasia in 1 patient. Median age at surgery was 27 years old. Decreased ventricular function was defined as ejection fraction (EF) of less than $60 \%$. Patients were divided into 2 groups depend on ventricular function (normal vs. decreased ventricular function).

\section{RESULTS:}

Seventeen out of 37 (46\%) had a decreased ventricular function, including 1 patient with EF of less than $40 \%$. PA index in patients with decreased ventricular function was significantly higher than that in patients with normal ventricular function ( 263 vs. $198 \mathrm{~mm} 2 / \mathrm{m} 2, \mathrm{p}=0039)$. There were no differences in mean pulmonary artery pressure $(7.0 \mathrm{vs}$. $10.5 \mathrm{mmHg}, \mathrm{p}=\mathrm{NS})$, pulmonary artery resistance $(2.25 \mathrm{vs} .1 .80 \mathrm{WU} / \mathrm{m} 2$, $\mathrm{p}=\mathrm{NS}$ ), and ventricular end-diastolic pressure ( 6 vs. $5 \mathrm{mmHg}, \mathrm{p}=\mathrm{NS}$ ) between the ones with decreased ventricular function and the ones without.
Early death was more often seen in patients with decreased ventricular function but Kaplan-Meier survival curve showed there was no difference in survival at 10 years after surgery between patients with decreased ventricular function and the ones without ( 82 vs. $83 \%$ at 10 year, $\mathrm{p}=0.76$ ).

\section{CONCLUSIONS:}

Approximately half of patients had decreased ventricular function at primary Fontan or TCPC conversion in adult population. Early death was observed in patients with decreased ventricular function but long-term survival was comparable with those with normal ventricular function.

A comparative study of Sung's technique of pulmonary valve augmentation and conventional transannular patch repair of Tetrology of Fallot.

Sanatanu Dutta; Asher George Joseph

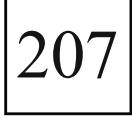

\section{ABSTRACT:}

Surgical repair of tetralogy of Fallot (TOF) with small pulmonic valve (PV) annulus requires the sacrifice of the PV and annulus resulting in a pulmonary regurgitation (PR) which may lead to acute and late functional deterioration of right ventricular performance. Conventionally, a monocuscp ventricular outflow patch has been used to reduce PR and the patient's own destroyed valve becomes nonfunctional or obstructive. Sung et al described a technique of repair by which, PR is reduced by augmentation of a PV cusp during transannular enlargement. We have operated on 12 patients using this technique. Patients with an existing BT shunt and pulmonary atresia were excluded from the study. The male to female ratio was 1.3:1. Post operatively, the number of hours on ventilatory support in this group was 10.5 hours, (it was 23.2 hours for the patients who underwent a conventional patch repair) with no significant post operative respiratory complications. The patients were discharged by 5.2 days (it was 7.6 days in the patients who underwent a conventional repair) and the follow up period of these patients were uneventful. Hence, the Sung's technique of repair provided better post operative results, however, a long term follow up is required to establish its advantage over the transannular patch repair.

Neurological Complications after cardiac Surgery in Children with Congenital Heart Disease- a single centre study.

Rahul Kumar Rai; Ravi Patel 208

\section{ABSTRACT: \\ INTRODUCTION:}

The incidance and pattern of neurological complication after pediatric cardiac surgery has been changing over the years due to improvement in surgical technique and peri operative management. Aim of this study was to evaluate incidanccec and pattern of neurological injury in our institute MATERIAL AND METHOD:

We retrospectively reviewed all pediatric patients who underwent non contrast tomography for suspected neurological injury occuring during first week after pediatric cardiac surgery between january 2015 to june 2018. we identified neurological injury as patients having ischemic infarct and intracranial hemorrhage

\section{RESUTS:}

Total 2971 pediatric cardiac surgeries performed at our institute. 67 patients $(2.25 \%)$ developed neurological injury.55 patients (82\%) developed ischemic infarct while 12 patients $(18 \%)$ had intracranial hemorrhage. pattern of ischemic infarct include global ischemic injury in 30 patients $(54.5 \%)$, posterior cerebral artery territory in 9 patients(16.3\%) middle cerebral artery terittory in 8 patients $(14.5 \%)$, multi territory involvement in 5 patients $(9 \%)$ anterior cerebral artery territory in 3 patients $(5.4 \%)$. in patient with intracranial hemorrhage, 5 patients $(7.4 \%)$ developed subarachnoid hemorrhage, 4 patients $(5.9 \%)$ developed subdural hemorrhage and 3 patients $(4.4 \%)$ developed intraparenchymal hemorrhage 


\section{CONCLUSION:}

Neurological complication account for significant morbidity and mortality after pediatric cardiac surgery. Ischemic infarct account for $82 \%$ cases. In ischemic infarct global ischemic injury is most common and carries high risk of mortality. In hemorrhage group subarachnoid hemorrhage is most common type

Autologous pulmonary artery tissue in Aortic reconstruction for coarctation repair- our experience K.R. Balakrishnan; Pradeep

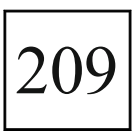

\section{ABSTRACT:}

Autologous pulmonary artery tissue in aortic reconstruction for c0arctation repair - our experience

Pradeep, Ganapathy Subramaniam, K.R.Balakrishnan, Suresh Rao

\section{INTRODUCTION:}

In Aortic reconstruction for coarctation or interrupted aortic arch repair, the choice of tissue we prefer is autologous pulmonary artery tissue to avoid shrinkage or aneurysm which is common in bovine or autologous pericardium. We present a series of 7 patients.

\section{MATERIALS AND METHODS:}

In a series of 7 patients including interrupted aortic arch, VSD with coarctation, we use part of anterior wall of the pulmonary artery as tissue of choice in re constructing the aorta. Usually the Pulmonary artery is thickened due to pulmonary hypertension and the wall thickness matches that of the aorta. Moreover the chance of shrinkage or aneurysm formation is less when compared to use of autologous pericardial patch or bovine pericardium.

\section{CONCLUSION:}

In 1 year follow up none of the patients developed re stenosis or aneurysm.

\section{An alternative technique for intracardiac exposure during transatrial repair of Tetralogy of Fallot \\ P. Rajashekar; Srikant Sharma; Shiv Kumar Choudary; Sachin Talwar}

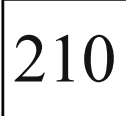

\section{ABSTRACT: \\ BACKGROUND:}

Tetralogy of Fallot (TOF) is a common cyanotic congenital heart disease for which intracardiac repair is now routinely performed. The commonly used technique to facilitate intracardiac exposure during its transatrial repair involves considerable retraction of the Tricuspid valve (TV) with the retractors. We describe an alternative surgical technique and advantages of such a technique are discussed.

\section{METHODS:}

Six patients who underwent VSD closure using the alternate surgical method at AIIMS.

RESULTS:

All patients had an uneventful intraoperative and post-operative course. Mean age of patients was 4.95 years ( range from 8 months to 9 years). The mean Aortic cross clamp time and cardiopulmonary bypass time was $61.2+/-40.5$ minutes (range, 21 to 139 minutes) and $94.5+/-44.3$ minutes ( range, 42 to 176 minutes) respectively. There were no deaths in the study. Post surgery $\mathrm{pRV} / \mathrm{LV}$ ( right ventricular/ left ventricular) was $0.5-0.6$ and there was no significant step up in oxygen saturation between right atrium and pulmonary artery (acceptable \&1t;5\%). Intraoperative transesopheal echocardiography and postoperative in the intensive care unit showed no residual VSD, no TV regurgitation, a wide open right ventricular outflow tract with gradient less than $30 \mathrm{mmHg}$.

Conclusions:

Theoretical advantages associated with retractor -free approach include no need for retractors, avoidance of excessive retraction of tricuspid valve, less chance of injury to ventricular myocardium and lesser incidence of junctional ectopic tachycadia. This technique does not prolong the aortic cross clamp or CPB time and is reproducible, safe and can be disseminated to junior colleagues and resident doctors.

Agreement of central venous saturation and mixed venous oxygen saturation in patients with pulmonary arterial hypertension after cardiac surgery Shuai Hu; Dianyuan Li

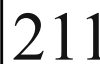

\section{ABSTRACT:}

\section{OBJECTIVE:}

To investigate the bias and the limits of agreement(LOA)of central venous oxygen saturation $(\mathrm{ScvO} 2)$ and mixed venous oxygen saturation $(\mathrm{SvO} 2)$ in patients with pulmonary arterial hypertension(PAH)after repair of congenital heart disease(CHD)

\section{METHODS:}

43 patients with CHD-PAH, 194 pairs of $\mathrm{ScvO} 2$ and $\mathrm{SvO} 2$, which were measured after the cardiopulmonary bypass(CPB)and 6, 12, 24, and 48 hours.

\section{RESULTS:}

Regression analysis of $\mathrm{ScvO} 2$ and $\mathrm{SvO} 2$ showed the correlation $\mathrm{R} 2$ $=0.369$, after CPB R2 $=0.515,6 \mathrm{~h}$ after admission to ICU R2 $=0.375$, at $12 \mathrm{~h} \mathrm{R} 2=0.605$, at $24 \mathrm{~h} \mathrm{R} 2=0.712$, at $48 \mathrm{~h} \mathrm{R} 2=0.252$. Bland-Altman analysis for the pooled measurements of $\mathrm{ScvO} 2$ and $\mathrm{SvO} 2$ showed that the mean bias is $-1.3 \%$, LOA is $-17.0 \%$ to $+14.4 \%$. Oxygen extraction rate $(\mathrm{O} 2 \mathrm{ER})(\mathrm{R} 2=0.03, \mathrm{P}=0.01)$, pulmonary artery systolic pressure $(\mathrm{SPAP})(\mathrm{R} 2=0.03, \mathrm{P}=0.03)$, heart rate $(\mathrm{HR})(\mathrm{R} 2=0.032, \mathrm{P}=0.01)$. But in patients with $\mathrm{ScvO} 2<70 \%$ the results are different: O2ER(R2=0.203, P\&lt;0.001), SPAP(R2 = 0.11, P = 0.005), HR (R2 = 0.15, $P \& 1 t ; 0.05)$, multiple linear regression showed that high correlations of O2ER (P\&lt;0.01), SPAP (P\&lt;0.05), HR (P\&lt;0.05) were observed with regard to $\triangle \mathrm{ScvO} 2-\mathrm{SvO} 2(\mathrm{R} 2=0.36, \mathrm{P} \& 1 \mathrm{lt} ; 0.01)$. In patients with a low $\mathrm{ScvO} 2$ there was a trend to underestimate $\mathrm{SvO} 2$ by using $\mathrm{ScvO} 2$, and $\mathrm{ScvO} 2$ will overestimate $\mathrm{SvO} 2$ with a high $\mathrm{ScvO} 2$. Bland-Altman analysis of $\mathrm{ScvO} 2$ and $\mathrm{SvO} 2$ with the mechanical ventilation: mean bias was $-1.3 \%$, LOA was $-16.1 \%$ to $+13.5 \%$. The only factor that influenced the $\triangle \mathrm{ScvO} 2$ $\mathrm{SvO} 2$ is O2ER(R2 $=0.122$, P \&lt;0.001). Bland-Altman analysis of ScvO2 and $\mathrm{SvO} 2$ without the mechanical ventilation: mean bias was $-1.3 \%$, LOA was $-19.5 \%$ to $+16.8 \%$, and the factors influencing $\triangle \mathrm{ScvO} 2-\mathrm{SvO} 2$ were as follows: O2ER(R2 = 0.32, P \&lt;0.001), arterial SO2(R2 = 0.32, P = 0.001). CONCLUSION:

In patients with $\mathrm{PAH}$ after repair of $\mathrm{CHD}, \mathrm{ScvO} 2$ and $\mathrm{SvO} 2$ should be monitored respectively in selective patients to exclude the hypoperfusion state.

Midterm performance of Contegra bovine jugular vein conduit as a right ventricle to pulmonary artery conduit Kuntal Roy Chowdhuri; Tarun Raina

Ramman; Sumir Girotra; Sushil Azad; Sitaraman Radhakrishnan;

Parvathy U. Iyer; Krishna S. Iyer, Nayem Raja

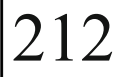

\section{ABSTRACT: INTRODUCTION:}

The bovine jugular vein conduit is an attractive option for reconstruction of right ventricular outflow tract. However, it has been associated with conduit stenosis and a significant incidence of infective endocarditis in western literature. This study aims to evaluate outcomes and performance of Contegra conduit at midterm follow up in an Indian institution.

\section{METHODS:}

A single centre retrospective review of 85 Contegra conduits implanted in 84 consecutive patients operated from 2007 to 2017 was completed. Underlying diagnosis were: VSD/PA (40\%); Truncus $(36.5 \%)$; DORV/VSD/PS (14.1\%), ccTGA/VSD/PS (5.9\%), TOF/PA (3.5\%). 
Median age and weight were 24 months and $10.7 \mathrm{Kg}$. Conduit diameter was $\leq 14 \mathrm{~mm}$ in 38 and $\geq 16 \mathrm{~mm}$ in 47 cases. Patients were followed up clinically and by echocardiography.

\section{RESULTS:}

Early mortality was $1 / 85$ due to severe acute pulmonary hypertensive crisis in a patient with Truncus. Median follow up duration was 59 months. Follow up was not available in 26 overseas patients and excluded from analysis. There were 5 late deaths. Conduit peak gradient at last follow up was $\leq 30 \mathrm{~mm} \mathrm{Hg}$ in 34, 31-50 $\mathrm{mm} \mathrm{Hg}$ in 6 and $\geq 51 \mathrm{~mm} \mathrm{Hg}$ in 4 patients. Moderate or severe Conduit insufficiency was seen in 10 patients. Catheter reintervention was required in 5 whereas redo surgery for conduit replacement was done in 3 cases while one is awaiting reoperation. LVOT reintervention was required in two cases. Overall survival and freedom from reintervention were $90 \%$ and $82 \%$ respectively.

\section{CONCLUSION:}

The Contegra conduit for RVOT reconstruction is an attractive option with acceptable early and mid-term outcomes. With better surgical techniques complications of conduit stenosis can be avoided resulting in longer periods of freedom from reintervention even in small sized conduits. In our experience incidence of conduit endocarditis is very low.

\section{Short and mid term outcomes of aortic arch repair : Single Surgeon Single Centre Experience Tapan Dash; Ashwin Uday Phadke; T. Vamshidhar; Prerna Singh}

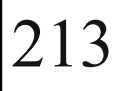

\section{ABSTRACT: \\ ABSTRACTINTRODUCTION:}

Aortic arch surgery has remained a technical challenge ever since its first description in 1956. Interrupted and hypoplastic aortic arch are rare congenital cardiac lesions which are uniformly fatal without surgical intervention. MATERIALS AND METHODS: Twenty patients (17 male, 3 female) mean age of 58.3 days, mean weight $3.8 \mathrm{kgs}$, underwent aortic arch repair from January 2013 to present. Twelve(60\%) patients had hypoplastic aortic arch while $8(40 \%)$ had interrupted aortic arch. Associated anomalies included coarctation of aorta, VSD, PDA, d-TGA and AP window. Hypoplastic left heart syndrome was excluded from the study. TECHNIQUE: After standard midline sternotomy and thymus excision, aorta and right atrium are cannulated. PDA is cannulated (in cases of IAA) through the main pulmonary artery (MPA) to perfuse descending aorta. CPB instituted and patient is cooled to $20^{\circ} \mathrm{C}$. Both carotids were clamped. PDA is ligated and divided from the pulmonary artery. Proximal cut end is anastamosed to ascending aorta, proximal to the origin of right brachiocephalic artery on the inner side.

\section{RESULTS:}

There were two early mortalities (PAH crisis and severe sepsis/ARDS). No late mortalities were reported. No early and late complications were seen. No patient required any reintervention. Sixteen patients $(88.9 \%)$ showed no flow gradients in follow up echocardiograms (6 months to 5 years). Mild turbulence (\&lt;10 $\mathrm{mm} \mathrm{Hg}$ ) was seen in two patients(11.1\%). All 18 patients are currently on regular follow up and are asymptomatic. CONCLUSION:

Aortic arch surgery still remains a challenge especially in multisegment pathologies. Apart from preoperative risk factors, intraoperative management and organ protection are the keys to success.

Early extubation after congenital heart surgery: A prospective observational study

Gaurav Patni, Debasis Das, Manish Kumar Sharma, Amitabha Chattopadhyay, Nilanjan Dutta, Rangan Koley

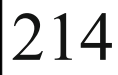

\section{ABSTRACT:}

Please correct as below Early extubation after congenital heart surgery: A prospective observational study Gaurav Patni,
Debasis Das, Manish Kumar Sharma, Amitabha Chattopadhyay, Nilanjan Dutta, Rangan Koley

\section{INTRODUCTION:}

Early extubation after congenital heart surgery has been described; The objective of this prospective observational study was to assess feasibility of early extubation after congenital heart surgery and identify factors which may hinder early extubation

\section{MATERIALS AND METHODS:}

This was a prospective observational study at a single institution between January 2016 and November 2018 which included 1728 consecutive patients undergoing congenital heart surgery. Early extubation was aimed for in all cases, except patients with body weight of \&lt; $5 \mathrm{kgs}$ and age \&lt; 6 months.

All patients were induced with midazolam $0.05 \mathrm{mcg} / \mathrm{kg}$, fentanyl $2.0 \mathrm{mcg} / \mathrm{kg}$, pancuronium $0.1 \mathrm{mg} / \mathrm{kg}$ and Sevoflurane $8 \%$. Anesthesia was maintained with Standard technique.

Data was collected for baseline characteristics and operative variables and was analyzed to assess outcome measures.

RESULTS:

1728 (936 males,792 females) patients underwent surgery for congenital heart disease. 920( $54 \%$ ) were extubated the same day with a mean ventilation time of $116 \min (\operatorname{Min} 10$, Max 365 $\mathrm{min}) .483(28 \%)$ were extubated in the operating room(OR). 315 (18\%) were ventilated overnight ( Mean 1234min, Max 5760, Min $540 \mathrm{~min}$ ).

There were 3 re-intubations due to respiratory causes in the early extubation/OR extubation group.

\section{CONCLUSION:}

Most children undergoing surgery for congenital heart disease can be extubated safely in the operating room. Early extubation facilitates good outcomes and early hospital discharge.

\section{TOTAL CORRECTION OF TETRALOGY OF FALLOT AT THE AGE OF 6 MONTH OR LESS: SURGICAL EXPERIENCE OF EIGHT YEARS}

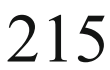

\section{AT SINGLE CENTER}

Sunil Kaushal; Yogendra Sharma; Abdul Razzak; Charu Dasratham; Sanjay Khatri; Rajeev Lochan Tiwari; Mohit Sharma

\section{ABSTRACT: \\ BACKGROUND:}

Age for total correction and surgical approach in tetralogy is still under debate. Purpose of this review was to evaluate functional status and outcomes of early repair of tetralogy (less than 6 month) by transatrial approach. Here we present a series of 33 patients of tetralogy correction at the age of 6 month or less.

\section{METHODS:}

This is a retrospective observational review of total correction of tetralogy of Fallot at single center from January 2011 to January 2018. Total 33 patients were operated by transatrial approach and patch plasty was considered for MPA, LPA or RPA enlargement if needed. All patients were age of 6 month or less. Total CPB (Cardio-Pulmonary By-pass) Time, Aortic cross clamp time, ICU stay, re-exploration, need of pericardial patch plasty of MPA, RPA and LPA were observed. Absent pulmonary valve patients were excluded. Functional status at follow-up was analyzed.

\section{RESULTS:}

Average age of patient was $5.01 \pm 1.246$ months, average height was $63.21 \pm 13.462 \mathrm{~cm}$, average body surface area was $0.32 \pm$ $0.093 \mathrm{~cm} 2$, average aortic cross-clamp time was $70.91 \pm 20.312$ minutes and average cardiopulmonary bypass time was 116.94 \pm 36.67 minutes and average ICU stay was $3.27 \pm 0.574$ days. There was no operative mortality, no re-exploration and good 
functional status of RV/LV (right ventricle and left ventricle) functions in follow-up echo-cardiography.

\section{CONCLUSION:}

Early correction (single stage repair) of tetralogy of Fallot (age of 6 month or less) offers the advantage of a more normal development and good functional status in follow

Comparison of early post op outcomes for arch repairs with or without vsd closure/aso/pa band/dks done on deep hypothermia on low flows versus additional 216 circulatory arrest

Hari Bipin Radhakrishnan Kattana; Smruti Ranjan Mohanty; Suresh G. Rao; Garima Bhag

\section{ABSTRACT: \\ En \\ BACKGROUND:}

Aortic arch anomalies are common conditions being treated in our unit. These include hypoplastic arches/ discrete coarcts with or without single/ multiple ventricular septal defects. Hypoplatic arches are also dealt with with transpositions and as a part of HLHS. While dealing with proximal arch or for vsd closure, and sometimes for coronary anastomosis in switches, to obtain a bloodless field, sometimes intermittent TCA is used when low flows with deep hypothermia doesn't suffice.

This is an attempt to compare the early post op outcomes between low flows and intermittent TCA at our institute.

\section{METHODS:}

Retrograde observational study of all the arch repairs done in 2017 was done. These cases were catagorised according to various criteria and analysed.

\section{RESULTS:}

14 cases were done on intermittent TCA in addition to low flows. Delayed chest closure was more common in TCA group which also were more complex neonatal cases. Median drain output in $24 \mathrm{hrs}$ was $90 \mathrm{ml}$ for the low flow group as compared to $60 \mathrm{ml}$ for TCA group. Packed cell requirement $440 \mathrm{ml}$ median in TCA and $420 \mathrm{ml}$ in low flows group. Cross clamp time 103 mins median in TCA group as compared to 88 minutes in low flows group. ICU stay was comparable 100 hours and 73 hours and ventilation time 43 hours and 29 hours for TCA group and low flows group respectively.

\section{CONCLUSION:}

Intermittent TCA is a safe technique, does not increase drain output or the quantum of blood transfusion, ventilation time and ICU stay is marginally more but that is insignificant when compared to the exposure and ease of surgery. er description here.

\section{Analysis of complications and evaluation of risk factors affecting the adverse outcomes due to IABP insertion in patients undergoing off-pump coronary artery bypass surgery \\ Vamshidhar Tirunagari}

\section{ABSTRACT: \\ INTRODUCTION:}

Intra-aortic balloon pump (IABP) is widely used mechanical assist device used for the treatment of low cardiac output in perioperative phase in patients undergoing coronary artery bypass surgery (CABG). Use of IABP is associated vascular, balloon related and other complications. There are many risk factors affecting adverse outcomes of IABP insertion. This study is intended to analyse the complications and evaluate the associated risk factors leading to complications in patients undergoing off-pump CABG with perioperative IABP.

\section{MATERIAL AND METHODS:}

This is a prospective observational study of 2years duration. 107 patients who underwent off-pump CABG with perioperative IABP support were included in the study. Demographic data, clinical features like diabetes mellitus, Hypertension, BMI, baseline creatinine and platelet count, peripheral vascular disease were analysed. Daily examination of IABP insertion site, peripheral pulses, serum creatinine, platelet count, duration of IABP and other complications are analysed.

\section{RESULTS:}

The mean age of all the patients was $59.7+$ SD 10.7, 85(79.4\%) patients were males, $22(20.6 \%)$ were females. Out of the 107 patient who had IABP insertion, $78(72.9 \%)$ patients had IABP inserted preoperatively, 21(19.6\%) intraoperatively and $8(7.5 \%)$ postoperatively. Average duration of IABP was 3+/- 1.5 days. Vascular complications including lower limb ischemia occurred in 4 patients(3.8\%). Local sepsis occurred in 2 patients(1.8\%). Post operative renal failure was seen in 12 patients(11.2\%).

CONCLUSION:

IABP is a valuable option in high risk patients undergoing off-pump CABG. Age, female gender, smoking, diabetes mellitus, severity of coronary artery disease were major risk factors affecting the adverse outcomes of perioperative IABP.

\section{EFFECT OF SHORT TERM USE OF METHYLPREDNISOLONE ON EARLY OUTCOME AFTER OFF-PUMP CORONARY ARTERY BYPASS GRAFTING \\ Muhammad Aslam Hossain; Mohammad Samir Azam Sunn

\section{ABSTRACT: \\ BACKGROUND:}

After commencement of Off-pump Coronary Artery Bypass Grafting (OPCAB) in mid 1990s, it has shown superiority to conventional Coronary Artery Bypass Grafting (CABG) with respect to better myocardial protection, less need of blood transfusion and shorter operative duration. However, duration of postoperative hospital-stay and frequency of postoperative paroxysmal atrial fibrillation (PAF) remain unchanged in both conventional and OPCAB. Many drugs and several measures have been tried to reduce the post-operative adverse outcomes after OPCAB. Among them, methylprednisolone has shown genuine promise to improve the outcome after OPCAB.

\section{METHODS:}

In this prospective study, population were randomly grouped into a control group (Group $\mathrm{C}: \mathrm{n}=25$ ) and a methylprednisolone group (Group M: $\mathrm{n}=25$ ). Group $M$ patients were administered intravenous $1000 \mathrm{mg}$ of methylprednisolone during anaesthesia induction, before incision of the skin and 500mg 12 hourly up to 48 hours postoperatively.

RESULTS:

Incidence of Post-operative Atrial fibrillation (POAF) was observed in 6 (24\%) in group $\mathrm{M}$ and $13(53 \%)$ in group $\mathrm{C}(\mathrm{P}-0.041)$. Post-operative blood sugar was higher in group M compared with group C (56\% vs $24 \%$, $\mathrm{P}-0.029 \%$ ). However, post-operative C-reactive Protein (CRP) levels were significantly lower in group $\mathrm{M}$ than in group $\mathrm{C}$. On postoperative day 3, mean CRP levels were in group M $5.62 \pm 1.68 \mathrm{mg} / \mathrm{L}$ vs. group C $8.58 \pm 2.05 \mathrm{mg} / \mathrm{L} ;$ P \&lt; 0.001).

\section{CONCLUSION:}

CRP level is significantly suppressed and POAF is prevented in postoperative period among $\mathrm{OPCAB}$ patients receiving preoperative methylprednisolone without increase in infectious complications.

Transit time flowmetry analysis for coronary endarterectomy for the diffusely diseased left anterior descending artery

Tomoya Uchimuro; Tomoya Uchimuro; Kohsaku Nishigawa 


\section{ABSTRACT: \\ BACKGROUNDS:}

Coronary endarterectomy (CE) is a surgical option for the diffusely diseased coronary arteries, while it carries potential risk of acute thrombosis. This study was aimed to evaluate whether the transit time flowmetry (TTF) values were predictive of acute thrombosis and clinical events after $\mathrm{CE}$ for the diffusely diseased left anterior descending artery (LAD).

\section{METHODS:}

Between July 2011 and July 2018, 108 patients underwent coronary artery bypass grafting with concomitant $\mathrm{CE}$ for the diffusely diseased LAD. All endarterectomized LADs were reconstructed using the internal thoracic artery. Graft flow was measured using TTF after the completion of anastomosis and 3 TTF values (mean graft flow [MGF], diastolic fraction $[\mathrm{DF}]$, and pulsatility index $[\mathrm{PI}]$ ) were obtained. Study endpoints were acute thrombosis of the endarterectomized LAD and LADassociated events, defined as graft occlusion including acute thrombosis of the LAD, perioperative myocardial infarction, and repeat revascularization.

\section{RESULTS:}

The median length of arteriotomy in the LAD was 6.5 (interquartile range [IQR], 5-7.9) $\mathrm{cm}$. The median value of MGF, DF, and PI was 38 (IQR, $25-58$ ) $\mathrm{ml} / \mathrm{min}, 72$ (IQR, 25-57) \%, and 2.2 (IQR, 1.8-2.9), respectively. Early postoperative angiography revealed acute thrombosis in 7 (6.5\%) endarterectomized LADs and these 7 patients underwent percutaneous coronary intervention. Apart from that, $7 \mathrm{LAD}$-associated events (graft occlusion in 1 , perioperative myocardial infarction in 3 , and repeat revascularization in 3) were occurred. Three TTF values were not associated with both acute thrombosis of the LAD and LAD-associated events. In the logistic regression analysis, longer arteriotomy in the LAD was an only predictor for LAD-associated events (odds ratio, 1.384; 95\% confidence interval, 1.072-1.808; $\mathrm{P}=0.013$ ).

\section{CONCLUSIONS:}

TTF values were not predictive of acute thrombosis of the LAD and LAD-associated events after CE for the diffusely diseased LAD. Longer arteriotomy in the LAD was a predictor for postoperative LADassociated events.

\section{ASSESSMENT OF FLUID OVERLOAD IN OFF PUMP CORONARY ARTERY BYPASS GRAFTING SURGERY PATIENTS BY BODY COMPOSITION MONITOR (BCM)}

Ashwene R.; Salgunan Nair; M.R. Girinath; Ashwene R.

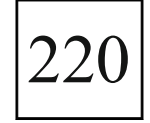

\section{ABSTRACT:}

Fluid accumulation occurs in most postoperative patients. There is no definitive clinical parameter to accurately quantify the fluid status of a patient in the perioperative period. This excessive fluid accumulation is associated with complications such as AKI, prolonged ventilatory support, prolonged intensive care, prolonged Inotropic requirement etc,. The postoperative OPCAB patients are observed to have fluid accumulation. Objective: To determine the incidence of fluid overload in the OPCAB patients and its influence on the postoperative complications. Material and methods: A total of 101 patients undergoing elective OPCAB in Apollo Main Hospital, Chennai during the period of October 2016 to April 2017 were studied prospectively. All the preoperative, intraoperative and postoperative details of the patient were recorded. The body composition monitor (BCM) was used to assess the fluid overload. The $\mathrm{BCM}$ measurement was done pre-operatively and again on 3rd and 6 post-operative day (POD). RESULTS: Sixty patients showed fluid accumulation of less than $5 \%$ of total body weight at 3rd POD. The presence of fluid overload, i.e fluid accumulation more than five $\%$ of body weight was found in 41 patients (40.6\%). 31 patients had fluid overload of $5-10 \%$ and 10 patients had fluid overload of more than $10 \%$ of body weight. The number of patients requiring inotropes and duration of requirement of inotrope in the post-operative period was significantly higher in patients with fluid overload than in patients without $(58.5 \%$ vs $30 \%, \mathrm{p}=0.004)$ (7 +/- 10.3 Vs $3+/-6.1$ hours, $p=0.015$ ) respectively. The occurrence of AKI was significantly more in patients with fluid overload (19.5\% Vs $6.7 \%$, $\mathrm{p}=0.05$ ). CONCLUSIONS: Significant fluid overload occurs in OPCAB patients. The presence of fluid overload is strongly associated with increased duration of requirement of vasoactive drugs. It is also significantly associated with the occurrence of postoperative AKI.

Evaluation of anxiety and depression in coronary surgery patients using the hospital anxiety and depression scale

Rubina Mariya Clara A; Devender Singh;

Shegu Gilbert

\section{ABSTRACT: \\ INTRODUCTION:}

Research indicates that about 30 to $40 \%$ patients undergoing cardiac surgery are affected by depression. Both depression and anxiety increase the risk of mortality and morbidity after CABG surgery.

AIM:

To analyse the incidence of anxiety and depression in patients undergoing CABG surgery both preoperative and postoperatively and its influences on patient outcomes.

MATERIAL AND METHODS:

We conducted a prospective observational study on 100 patients undergoing non emergent CABG surgery between the period of September 2017 to February 2018. These patients completed the hospital anxiety and depression scale (HADS) questionnaire preoperatively and postoperatively, one month after discharge. The patients characteristics and operative data, incidence of preoperative and post operative anxiety and depression were analyzed. Variables were expressed as mean +/- standard deviation and percentage.

\section{RESULTS:}

Preoperatively anxiety was observed in $18 \%$ of patients and depression was seen in $14 \%$ of patients. Postoperatively there was reduced incidence of anxiety and depression. Anxiety and depression had a negative correlation with the patient outcomes.

\section{CONCLUSION:}

Identifying anxiety and depression in patients groups undergoing $\mathrm{CABG}$ surgery helps early intervention in the form of a multidisciplinary approach that leads to improved patients outcomes including early discharge from hospital and quick return to work.

\section{CORONARY CAMERAL FISTULA : A RARE ENTITY}

Jignesh Kothari; Divyesh Rathod

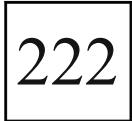

\section{ABSTRACT:}

OBJECTIVES:

The purpose of this study was to describe our experience with the presentation and management of surgical repair of coronary cameral fistula (CCF) and its outcome.

\section{PATIENTS AND METHODS:}

We performed a retrospective study from January 2015 to January 2018 in 8 patients operated for coronary cameral fistula. There were 5 men and 3 women; the age range was $18-55$ years (mean age, $36.2 \pm 11.7$ years). Among the 8 patients, 4 patient had CCF originating from Right coronary artery, 2 patients had from Left anterior descending artery and 2 patients had its origin from Left circumflex artery. All patients were evaluated by trans-thoracic echocardiography and conventional coronary angiography apart from routine biochemistry and hematological investigations.

RESULTS:

All operations were performed using cardiopulmonary bypass. Four patients with a CCF opening into RA and RV which was closed by right 
atriotomy, 2 patients had CCF at termination of coronary artery hence ligation done and 2 patients had CCF with dilated coronary artery amongst them dilated portion exposed, closed fistulous tract followed by arteriotomy closure. There were no postoperative complications. No recurrences have been observed so far in follow up visits.

\section{CONCLUSIONS:}

Coronary cameral fistulla is an infrequently encountered entity, but with clinical suspicion, early diagnosis and timely intervention it can be successfully managed with excellent outcomes.

\section{Minimal Invasive Direct Coronary Artery Bypass Grafting With Bilateral Internal Mammary Arteries. Our Experience. \\ Kaushal K. Tiwari; Parth Solanki; Parth Solanki}

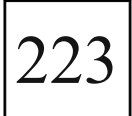

\section{ABSTRACT: \\ BACKGROUND AND AIM:}

Coronary artery bypass grafting $(\mathrm{CABG})$ is one of the commonly performed cardiac surgeries. CABG using bilateral internal thoracic arteries (BITA) through sternotomy is a well established procedure. However, minimal invasive CABG using BITA is not performed widely. We report our experience with this evolving method of CABG through left minithoracotomy.

\section{METHODS:}

We have enrolled 145 consecutive patients who underwent minimally invasive CABG using BITA at our institute between January 2014 and June 2018. In all patients, LITA-RITA-Y anastomosis with pedicled LITA inflow was made. A minimum of two distal anastomoses were done in every patient. The mean age was $59 \pm 7.8$ years. The mean number of distal anastamoses was $2.3 \pm 0.8$. LITA was used to supply LAD and diagonal vessels, whereas RITA was used for the remaining vessels. Retrospectively, we have analyzed Pre-operative, operative and three years post-operative outcomes. CT angiography at 1 week post surgery was performed for assessing the patency of grafts. Follow up of patients was done for a minimum period of one year and repeat CT coronary angiography was done to evaluate the patency of the grafts.

\section{RESULTS:}

All one hundred and forty five patients successfully underwent minimally invasive CABG using BITA. There was no need of conversion to sternotomy. There was no hospital mortality. Mean ICU and hospital stay were $1.45 \pm 0.89$ and $5.1 \pm 1.34$ days respectively. Requirement for iontropes and pain medicine were similar to the conventional surgery. Patients were mobilized early in the ICU. In post operative period, no new onset MI or arrhythmia detected. At the 1 week and one year follow up CT angiography all anastomoses were patent.

\section{CONCLUSION:}

Minimal invasive CABG using BITA is a safe, reproducible procedure with potential advantages of early return to work, better cosmesis, and elimination of sternal wound infection and other benefits of minimal invasive approach.

Role of the radial artery as second conduit for CABG

Takashi Kato; Masahiro Seki; Yuriko Kiriya;

Masahiro Tezuka; Yusuke Takei; Kouji Ogata;

Ikuko Shibasaki; Hirotsugu Fukuda; Hironaga Ogawa

\section{ABSTRACT: \\ BACKGROUND:}

It is still controversial to use radial artery(RA) as second conduit for coronary artery bypass grafting(CABG). The reasons are arterial spasm, cerebral infarction by aortic proximal anastomosis, et al. However, RA have many advantages, We use RA positively. We exam our experience with RA to CABG document the early results \&amp; grafts patency, 1 year \&amp; long term Major Adverse Cardiovascular Event (MACE) by retrospective study.

\section{METHODS:}

Harvest condition's: 1 Allen's test is not negative. 2 It is less than CKD stage2(GER $=60-89)$ in diabetics and is less than CKD stage $3 a(G F R=45$ 59) in non-diabetic patients. 3 Not a dialysis patients. Use condition: Don't perform Composite graft. Bloodstream was supplied from ascending aorta. Operation policy: on pump arrest CABG.Intra-luminal VG solution was used to prevent the spasm of RA grafts.

\section{RESULTS:}

RA grafts was used from June 2008 to March 2017 in 332 patients. Observation period was June 2008 to March 2018. Follow up $($ median $)=900$. age $=69$ years. Emergent Operation was performed in $29 \%$ patients. RA grafts was used sequentially in $9 \%$. Bilateral RA was used 43 patients. RA was most frequently placed to the circumflex marginal (218), Diagonal marginal (83), Posterior descending artery (109). Proximal RA anastomosis was directly to the aorta in all of patient's. Operative mortality was $0.9 \%$. Complications included stroke was $0.9 \%$. Re operation for graft failure in 1 patient, There was no patient that the new dialysis required. Angiography was done on 307 patients, before discharge of postoperatively. RA grafts patency was $97.7 \%$. MACE within one year after the CABG was $0.9 \%$. As for the MACE evasion rate until an observation period was $96 \%$, and the cardiac death evasion rate was $99.7 \%$.

\section{CONCLUSIONS:}

The early RA graft patency was relatively good. Use of RA to CABG extends the scope of arterial myocardial re-vascularization, and is safe.

Can Off-pump coronary artery bypass grafting be a suitable option for patients with Low ejection fraction? Hiroyuki Nakajima; Hiroyuki Nakajima; Yumeka Tamai

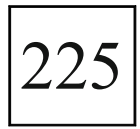

\section{ABSTRACT:}

\section{INTRODUCTION:}

Off-pump coronary artery bypass grafting (OPCABG) has improved its safety. However, outcomes in high-risk patients such as patients with low preoperative ejection fraction $(\mathrm{EF})$ remain unclear. We discussed the validity of OPCABG as the first choice in low EF patients.

\section{MATERIALS \&amp; METHODS:}

From June 2007 to June 2018, 147 patients with EF $\leq 30 \%$ underwent isolated CABG in our institution. Mean age was $66.9 \pm 8.9$ years, and 78.1 $\%$ were male. Mean preoperative EF was $23.8 \pm 4.5 \%$. Of these patients, 132 patients $(89.8 \%)$ underwent OPCABG, whereas 14 patients $(9.5 \%)$ underwent $\mathrm{CABG}$ with cardiopulmonary bypass(ONCABG).

\section{RESULTS:}

Emergency operations were $53.3 \%$ in OPCABG and $71.4 \%$ in ONCABG. $78.6 \%$ of ONCABG were cases converted from OPCABG during operation. 30-day mortality and cardiac mortalities were lower in OPCAB $(2.27 \%$ vs $21.43 \%, p=0.006)$. OPCABG caused no delay of ICU stay ( 9.6 days vs 14.6 days, $\mathrm{p}=0.76$ ) or IABP removal (3.8 days vs 9.0 days, $\mathrm{p}=0.013$ ). Postoperative EF showed improvement in both groups. Unilateral internal thoracic artery (ITA) was used in all cases. There were no differences in the rates of bilateral ITAs use, complete revascularization and the numbers of anastomosis. Total arterial revascularization was achieved more in OPCABG $(35.6 \%$ vs $0 \%, \mathrm{p}=0.007)$. CONCLUSION:

OPCABG showed better postoperative mid-term outcomes in patients with low EF than ONCABG. OPCABG with ITA grafts is considered to be first surgical option for patients with low EF. With an eye to longterm outcomes, operation strategies such as bilateral ITAs use and complete revascularization might be considered. 中高吉德栃泉金朝井

Comparative evaluatuion of BITA grafting in Acute MI vs Stable Angina patients - A single surgeon experience Rajiv Gupta; Sarju Ralhan 


\section{ABSTRACT: \\ OBJECTIVE:}

To compare the immediate and short-term outcome of BITA in patients presenting with acute myocardial infarction (AMI) or stable and unstable angina. METHOD: The study is based on primary data obtained from a single source and 1828 patients operated on by a single surgeon between 2014 and 2018. Outcomes of BITA CABG in Acute MI (STEMI \&amp; NSTEMI) and Stable Angina were studied. All statistical analyses were performed using SPSS 20. RESULTS: Out of the selected 1828 patients, 875 or 953 suffered from AMI or stable angina, respectively. The AMI patients had an average age of $60.30 \pm 8.89$ years and the stable angina patients had an average of $61.10 \pm 8.76$ years, with no statistically significant difference $(\mathrm{p}=0.055)$. There was a strong statistically significant difference in operation time or intraoperative blood loss $(618.92 \pm 299.5$ for AMI and $553.63 \pm 308.1 \mathrm{~mL}$ for NMI $)$ between the two groups $(\mathrm{p} \leq 0.00)$. The average length of hospital stay after surgery was 11.33 days for AMI and 9.28 days for stable angina patients, without any statistically significant difference $(p$ $\& g t ; 0.05)$. There was no statistically significant difference between the two groups (p \&gt; 0.05) in terms of mortality after surgery. Immediate improvement in ejection fraction was observed in AMI patients from an average of $43.04 \pm 11.47 \%$ to $48.75 \pm$ $9.97 \%$ and $47.51 \pm 11.18$ to $51.18 \pm 9.35 \%$ in stable angina patients. Comparison between AMI and NMI for improvement in ejection fraction did not reveal statistical significance ( $p$ \&lt; 0.05 ). CONCLUSION: With available data from a single center and operated by a single surgeon, a significant difference between the outcomes of the procedure could not be observed. The level of efficacy of BITA grafting in both AMI and stable angina patients remains similar.

Study on Perioperative Heparin resistance in CABG patients - Single center experience.

Ravi Krishnan J.; Manoop B.

\section{ABSTRACT:}

AIM:

To study perioperative heparin resistance and factors affecting the same in CABG patients, who were preoperatively anticoagulated with daily heparin intravenous injections.

\section{OBJECTIVES:}

To study perioperative heparin resistance in patients undergoing CABG, by calculating the amount of heparin required to achieve target Activated Clotting Time (ACT) of more than \&gt; 480s.

To study extra amount of heparin required to maintain target ACT \&gt;480s peroperatively.

To assess the excess protamine sulphate required to bring down target ACT levels to less than $10 \%$ baseline.

\section{METHODOLOGY:}

\section{Study design - Retrospective cohort.}

Study population - Patients with stable Coronary Artery Disease, admitted under Department Of Cardiovascular Thoracic Surgery, Government Medical College Thiruvanathapuram during 2017 - 2018.

Inclusion Criteria - All patients for elective $\mathrm{CABG}$, who were preoperatively anticoagulated with Heparin.

Exclusion Criteria - Patients who were not anticoagulated prior to CABG. LV dysfunction (\&lt;35\%) Moderate to Severe Mitral Regurgitation. Associated Liver Function Test derangement.

\section{RESULTS:}

136 patients were studied with 86 patients in heparin group and 50 patients in non heparin group. Patients in the heparin group had a mean ACT of $468 \mathrm{~s}$ after the first dose of heparin, and this value significantly $(\mathrm{P}=0.002)$ lower than mean ACT obtained in the control group of 554s. Also the total dose of protamine given to heparin group $($ mean $=407 \mathrm{mg}$ ) larger than non heparinised group (mean $=353 \mathrm{mg}),(\mathrm{P}=0.007)$. During $\mathrm{CPB}$ both groups had ACT values above target, though heparin group had mean higher total heparin (48100 IU) given to achieve and maintain target ACT compared to non heparinised group (42800IU), $(\mathrm{P}=0.007)$. Patients in heparin group had significantly $(\mathrm{p}=0.037)$ more packed red cell transfusions.

\section{CONCLUSION:}

Patients who where preoperatively anticoagulated showed significant perioperative heparin resistance and also intraoperative and postoperative transfusion requirement were higher.

\section{OUTCOMES AND SURVIVAL IMPACT OF INTRA- AORTIC BALLOON PUMP INSERTION BEFORE VERSUS AFTER CARDIAC SURGERY - SINGLE CENTER EXPERIENCE.}

Jacob Jamesraj; Jeswin Moses S.

\section{ABSTRACT: \\ BACKGROUND:}

The outcomes of intra-aortic balloon pump before versus after cardiac surgical intervention remains undefined.

\section{METHODS:}

We reviewed data of all patients undergoing cardiac surgical intervention from January 2016 to December 2017. Of all the 3965 patients who underwent cardiac surgical intervention, 93 patients required intra-aortic balloon pump insertion either pre-operatively or intra-operatively and in the immediate post-operative period. Of these 93 patients, intra-aortic balloon pump was inserted before cardiac surgical intervention and 62 patients required intra-aortic balloon pump after cardiac surgical intervention. We compared their outcomes and prognostic impact before versus after cardiac surgery.

\section{RESULTS:}

The after surgery intra-aortic balloon pump insertion group had higher mortality ( $43 \%$ versus $19.3 \%$ ) when compared with that of pre-operative insertion of intra-aortic balloon pump (P\&1t;0.05). Furthermore, the survival rates were lower in after surgery group when compared with the before surgery group.

\section{CONCLUSION:}

Patients who underwent intra-aortic balloon pump insertion prior to cardiac surgical intervention have better outcomes when compared with those who require during or after cardiac surgery. Moreover, patients had intra-aortic balloon pump insertion prior to cardiac surgery had better survival rates at the end of one year.

\section{Neurological Complications in the Immediate Postoperative Period After Cardiac Surgery- a single centre study}

Ravi Patel; Rahulkumar; Vishal Agrawal; Pankaj Garg

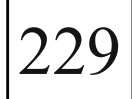

\section{ABSTRACT: \\ INTRODUCTION:}

Neurological complications related to cardiovascular surgeries are associated with significant morbidity and mortality. Here we retrospectively review our experience.

\section{MATERIAL AND METHOD:}

We retrospectively reviewed all computed tomography brain performed between January-2015 and June-2018 for patients with suspected neurological injury after cardiac surgery.

\section{RESULT:}

Total 12896 adult and pediatric cardiac surgeries were performed at our institute. Out of these, 1115 patients $(8.6 \%)$ underwent CT brain for suspected neurological injury. Total 215 patients $(1.6 \%)$ had positive 
finding on their NCCT head. Out of 215 patients, $148(68.9 \%)$ were adult patients while $67(31.1 \%)$ were pediatric patients. Total $156(72.5 \%)$ patients had ischemic infract (stroke) while hemorrhage present in 59 patients $(27.4 \%)$. In adult patients pattern of ischemic infarct comprised middle cerebral artery territory in 42 patients $(41.5 \%)$, posterior territory in 34 patients $(33.6 \%)$, anterior cerebral territory in 7 patients $(7.0 \%)$, multi-territory infract in 11 patients $(11.0 \%)$ and global ischemia in 6 patients $(6.0 \%)$. Intra parenchymal hemorrhage was present in 17 patients $(11.4 \%)$. In pediatric patients pattern of ischemic infarct included global hypoxic injury in 30 patients $(54.5 \%)$, posterior cerebral artery territory in 9 patients $(16.3 \%)$, middle cerebral artery territory in 8 patients $(14.5 \%)$, multi-territory involvement in 5 patients $(9.0 \%)$ and anterior cerebral artery territory in 3 patients $(5.4 \%)$. In haemorrhage group 5 patients $(7.4 \%)$ developed subarachnoid hemorrhage.

\section{CONCLUSION:}

In adult patients middle cerebral artery territory infracts was common in infract group while IPH in hemorrhage group. In pediatric patients Global hypoxic injury (GHI) was common in infract group and SAH in hemorrhage group.

Do blood transfusions affect the outcomes of patients who underwent coronary artery bypass grafting? Cheng K.M.; Lok Yuh Ing

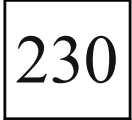

\section{ABSTRACT:}

\section{OBJECTIVE:}

Blood transfusion during hospitalisation for heart surgery has been shown to be associated with increased morbidity, mortality and healthcare costs. The objective of this study was to assess the outcomes perioperative blood transfusion who underwent coronary artery bypass graft surgery requiring cardiopulmonary bypass.

\section{METHODS:}

Data was collected from patients who underwent their first coronary artery bypass graft requiring cardiopulmonary bypass procedure in 2017, this study estimated associations between transfusion and complications after surgery, serious adverse events and death.

\section{RESULTS:}

There was statistically significant $(\mathrm{P} \leq 0.05)$ and strong relationship receiving transfused blood and serious adverse events, and death.

\section{CONCLUSION:}

Perioperative blood transfusion for coronary artery bypass graft requiring cardiopulmonary bypass was significantly associated with increased long-term post-operative morbidity, and mortality.

\section{Study to assess the impact of previous Percutaneous Transluminal Coronary Angioplasty (PTCA) on outcomes of after Coronary Artery Bypass Grafting (CABG) \\ Vamshidhar Tirunagari}

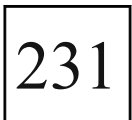

\section{ABSTRACT: \\ INTRODUCTION:}

The purpose of this study is to assess the impact of patient characteristics and procedural performance on peri-operative outcome of patients undergoing CABG after PTCA, and to analyze whether changes in these factors influence acute and long-term outcome.

\section{MATERIAL AND METHODS:}

Patients admitted to CARE Hospitals Banjara Hills for CABG with prior history of PTCA and matching the specific study criteria are potential subjects in this observational comparative study. Immediate postoperative period, data of duration of ICU stay and death and other primary outcomes was collected for morbidity and mortality. At one year, data about general condition, ECG for ischemic changes (new onset $\mathrm{Q}$ wave, LBBB), 2D Echo for RWMA, LV EF and mortality was collected.

\section{RESULTS:}

Out of 69 subjects enrolled for the study, 27 had prior PTCA done so they are included in study population and remaining 42 which are without any prior PTCA done are included in Control group. In Study Group, $88.9 \%$ of patients presents with In-stent restenosis or stent thrombosis. The percent incidence of Ischemic changes in study group is $18.5 \%$ and $2.4 \%$ in control group. In follow-up, three patients $(11.1 \%)$ in study group had new onset RWMA on 2D Echo in study group. In control group, one patient $(2.4 \%)$ had RWMA on follow-up.

\section{CONCLUSION:}

There is no statistical significant difference in post operative outcome between the two groups. But the patients with history of prior PTCA presents with more severe symptoms i.e.. $\geq$ grade III CCS angina, ischemic changes on ECG

Early and late mortality and morbidity after Post MI - ventricular septal rupture repair:

Predictors, Strategies and Results. Amber Malhotra; Kartik Patel

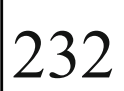

\section{ABSTRACT: \\ PURPOSE:}

There has been a shift in the paradigm of management of post-myocardial infraction ventricular septal rupture (MI VSR) with many authors reporting improved prognosis if the surgery can be 'optimally delayed'. Timing of the procedure is of critical importance and our management and prognosis scores have proven to be relevant. But long term outcomes and their correlation with our scores had not been analyzed. In this study we present our long-term results of post MI-VSR repair and their correlation with our prognostic score.

METHODS:

71 patients of post- MI VSR repair (2009-2017) were retrospectively studied. Patients were managed using according to standard institute protocols.

\section{RESULTS:}

The 30-day mortality was $56 \%(n=40)$. During a mean follow up of 4.91 \pm 2.43 years, there were 8 late deaths. Actuarial survival of 30-day survivors was $87 \%$ at 1 year, $74 \%$ at 5 years, and $69 \%$ at 10 years. Actuarial freedom from MACE was $82 \%$ at 1 year, $72 \%$ at 2 years and $72 \%$ at 8 years. Our prognostic score predicts late mortality with sensitivity of $75 \%$ and negative predictive value of $84 \%$.

CONCLUSION:

This prognostic score not only helps in predicting early mortality but also in identifying the patients who are likely to live longer. The management score also provides best timing for the procedure which is helpful in optimal utilization of resources in the developing world. The accuracy of these scores is reasonable and may be helpful in the decision making of low volume centers.

\section{COMPARISON OF DEL NIDO CARDIOPLEGIA AND ST. THOMAS SOLUTION- A RETROSPECTIVE STUDY \\ Abdul Rasheed; Krishna S.}

\section{ABSTRACT:}

Myocardial protection during open heart surgery is very challenging and the search for an ideal cardioplegic solution is still going on. This retrospective record based study done at Department of Cardiothoracic and Vascular Surgery, Government Medical College, Thiruvananthapuram analysed 200 patients who underwent CABG for triple vessel disease for the period January 1, 2012 to January 1, 2018 with an aim to 
determine whether del Nido (DN) cardioplegia, with longer periods of arrest, improves Post operative Ejection Fraction (as measured on day 5/ day 6) as compared to St Thomas Solution. Patients were divided into two groups based on the type of cardioplegia administered during surgery: 1) intermittent ST (ST, $\mathrm{n}=100)$ and 2) DN cardioplegia (DN, $\mathrm{n}=100)$, the aortic cross clamp time (CC) and cardiopulmonary bypass time $(\mathrm{CPB})$ times, and postoperative changes in left ventricular ejection fraction (LVEF) were compared and statistically analysed. On analysis, the average $\mathrm{CC}$ for $\mathrm{DN}$ group was 66.2 minutes while that of ST group was 79.4 minutes, CPB for DN group was 116 minutes while that of ST group was 133.4 minutes and post operative changes in LVEF for DN group was $7.4 \%$ while that of ST group was $5.4 \%$. The differences were analysed statistically and found to be significant. DN cardioplegeia when compared with ST solution was found to be better in reducing the $\mathrm{CC}$ and $\mathrm{CPB}$ times and showed greater improvement in LVEF. Based on the above findings DN cardioplegia offers better myocardial protection in patients undergoing $\mathrm{CABG}$.

\section{Moving towards delayed surgical correction using Right ventriculotomy in Post myocardial infarction(MI), Ventricular septal rupture(VSR) \\ Suraj Pai M.; Ramsankar Padmanabhan}

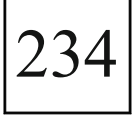

\section{ABSTRACT: \\ INTRODUCTION:}

Among mechanical complications of acute transmural MI, VSR results in catastrophic deterioration of a recovering patient needing patch closure of defect. Stability of patient at presentation depends on the quantity of left to right shunt. Myocardial dysfunction resulting from recent $\mathrm{MI}$ and pump run influences survival. We present our experience on VSR management in last 5 years as we have moved from emergency surgery to stabilisation and delayed surgery for a better scar which holds sutures and from Left ventriculotomy approach to right ventriculotomy approach.

Key words, Post myocardial infarction, Ventricular septal rupture, Right Ventriculotomy, Material and methods Between 2013 January and August 2018, among 23 patients referred with post MI VSR diagnosed on clinical grounds and transthoracic echo, 10 patients (5male,5 Female)underwent surgical repair. There were 6 deaths and 4 survivors. VSR was located at Apical septum in 6 patients(Simple type, AWMI, LAD involved), 4 had basal septal rupture(complex type VSR with multiple openings and serpiginous course $1.5 \mathrm{X} 2 \mathrm{~cm}$ largest size). The average mortality score predicted by EuroscoreII in 7 patients who were operated as emergency was 37.725 (Range 8.5 to 64) 3 had delayed repair(EuroII mortality risk 8.5 ). Left ventriculotomy, patch(Teflon Felt, Dacron) closure with exclusion of aneurysmal LV apex was done in 8. Right ventricular approach was used in 2 patients with basal septal defect with both patient surviving. Culprit vessel could not be grafted in 3, 30 day mortality was $60 \%$ in entire group with $100 \%$ survival in delayed repair patients. Delayed surgery was made feasible by appropriately optimising patient symptoms with diuretics, Levosimendan and IABP

\section{CONCLUSION:}

Post MI VSR has high mortality rate when operated in emergency and a patient in shock. Delayed surgery with optimisation and Right ventricular approach gives good results.

Off Pump Coronary Artery Bypass Grafting: where do we stand today? Harpreet Wasir; Imran Khan

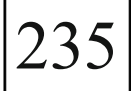

\section{ABSTRACT: \\ INTRODUCTION:}

Off pump coronary artery bypass grafting is usually criticised for incomplete revascularisation and fewer number of grafts compared to on-pump CABG. We tried to analyse our off pump CABG results prospectively with regards to completeness of revascularisation.

\section{MATERIAL AND METHODS:}

It is a prospective observational study.734 patients were studied from July 2017 to January 2018. Surgeons with more than 5 years experience with \&gt; 250 OPCABG cases experience were included. Inclusion criteria -all isolated OPCABG cases. Exclusion criteria-any concomitant procedure with OPCABG. Statistical analysis- paired ' $t$ ' test, 2 proportion $Z$ test, independent ' $t$ ' test. Aim-To analyse ICOR(index of completeness of revascularisation): Total grafts executed / total grafts planned.

\section{RESULTS:}

Age group: 46-55(20.4\%),56-65(43.9\%). Male-632(86.1\%), female-102(13.9\%). Ejection fraction:\&lt;35\%-16.9\%,\&gt;35\%$83.1 \%$ \&gt; . Diabetes mellitus-64.6\%, Hypertension-72.4\%. Coronary angiography: TVD-48.8\%,4 vessel disease-27.7\%. ICOR $=1$ in 709 patients $(96.6 \%)$, ICOR \&gt; 1 in 9 patients(1.2\%), ICOR \&lt; 1 in 16 patients(2.17\%). ICOR for lateral wall: $0.98+/ 0.06$. Average 3.2 grafts were done.

Intra-operative conversion to Cardiopulmonary bypass done in 14 patients(1.9\%)IABP used intra-operatively in 32 patients, pre-operatively in 9 patients and post-operatively in 11 patients. Post-operative stroke in 12 patients(1.63\%). Re-exploration done in 7 patients $(0.95 \%)$. Mean blood transfusion of 1.1 packed cells. 13 patients $(1.58 \%)$ were readmitted. Mortality of 14 patients(1.90\%) CONCLUSION:

Off pump coronary artery bypass grafting can achieve complete revascularision in experienced hands comparable to on-pump CABG. It also avoids aortic manipulation in total arterial revascularisation minimising the risk of stroke. By avoiding cardiopulmonary bypass circuit, post-operative systemic inflammatory response is absent.

Ten Year's Experience With Surgical Myocardial Revascularisation In Left Ventricular Dysfunction. Asit Baran Adhikary

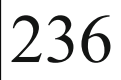

\section{ABSTRACT: \\ OBJECTIVE:}

Ischemic heart disease patients with left ventricular dysfunction, especially low ejection fraction is at higher risk for postoperative mortality and mortality. This study observed the clinical consequence of low ejection fraction following surgical myocardial revascularisation.

\section{METHODS:}

This retrospective cohort study involved 139 patients of severe left ventricular dysfunction patients ( $\mathrm{LVEF} \leq 30 \%)$ undergone coronary artery bypass graft surgery (CABG) in between 2008 and 2017 in single surgeons practice. All study population were evaluated preoperatively in respect to demographic characteristics, risk factors, and Echocardiography variables.

\section{RESULTS:}

The mean age of study population was $62.2 \pm 5.5$ years, and most common $(79.9 \%)$ were male. The mean pre-operative LVEF were $26.8 \pm 2.5 \%$. A total $97.1 \%$ of study patients underwent OPCABG surgery, and only $2.9 \%$ of patients required to conversion to on pump CABG surgery. Average number of grafts were 3.5 \pm 1.5 , and perioperative mortality rate was only $3.6 \%$. Post-operative mean extubation time was $12.5 \pm 2.5$ hours and mean ICU stay was $39.5 \pm 7.5$ hours. Follow up at 1 year, Echocardiography demonstrates left ventricular ejection fraction were improved in $92.1 \%$ of study patients. Moreover, freedom from all cause of death and angina was $94.8 \%$ and $90.5 \%$ at 1 year follow up respectively. 


\section{CONCLUSION:}

Surgical myocardial revascularisation in ischemic heart disease patients with severe LV dysfunction can be achieved safely, with significant improvement in LV function and the quality of life.

\section{Road to an unclamped all-arterial grafting strategy in off-pump coronary artery bypass grafting: \\ When and how to step forward \\ Jareon Cheewinmethasiri; Nuttapon Arayawudhikul}

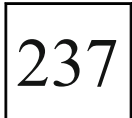

\section{ABSTRACT: \\ Enter description here \\ PURPOSE:}

Off-pump coronary artery bypass grafting(OPCAB) with total Arterial Revascularization (TAR) without aortic manipulation is thought to be better than other grafting procedure for high risk patients. However, this procedure is technically demanding, and takes a long time to learn. Here we evaluated the application of this strategy in our practice.

\section{METHOD:}

From April 2011 to December 2017, 777 patients underwent OPCAB. Of these procedures, 123(15.8\%) were performed with unclamped TAR. The patient demographics and surgical outcomes of the overall OPCAB and unclamped TAR groups were reviewed, and annual trends in these values were analyzed.

\section{RESULTS:}

In the unclamped TAR group, the mean patient age was $63+/-8.8$ years. The NYHA class was $2.7+/-0.7$ and ejection fraction (EF) was $50+/-15 \%$. The number of patients with low EF was 25(20.5\%). Composite Arterial grafts were used in $41.4 \%$ of the procedures. The rate of early mortality was $0.8 \%$. One patient $(0.8 \%)$ underwent on-pump conversion, and another patient $(0.8 \%)$ suffered from postoperative stroke 2 days after new on-set atrial fibrillation. There were no significant differences in the 30day mortality and secondary hard endpoints between the unclamped TAR and the overall groups.

\section{CONCLUSION:}

OPCAB using total Arterial grafts without aortic manipulation can provide favorable outcomes with a very low incidence of perioperative complications, and can become feasible by first incorporating OPCAB into the daily clinical practice.

Is use of anastomosis assist devices beneficial over side-clamps in preventing perioperative complications for those undergoing isolated CABG?

Hiraku Kumamaru; Hiroaki Miyata;

Noboru Motomura; Shinichi Takamoto; Aya Saito

\section{ABSTRACT: \\ OBJECTIVE:}

The use of an anastomosis assist device (Device) for proximal anastomosis in isolated $\mathrm{CABG}$ is considered less invasive for the ascending aorta than using side-clamp (Clamp), and is possibly associated with a lower incidence of postoperative stroke and other complications. However, this benefit has not yet been clearly demonstrated in with considerable population. This study was to evaluate whether the use of a Device will minimize postoperative stroke and other complications in patients undergoing off-pump CABG (OPCAB) in comparison to the use of a Clamp for proximal anastomosis of any type of free grafts.

\section{METHODS:}

The OPCAB cases registered to the Japan Adult Cardiovascular Surgery Database (2013-2016) were retrospectively reviewed. We performed a one-to-one matched analysis based on the estimated propensity scores of those who underwent OPCAB with a Device (HeartString, Enclose II) $(n=14213)$ or a Side-clamp $(n=7374)$, and obtained two cohorts $(n=7348$ each). The early outcomes were compared using Pearson's chi-squared test. P values of \&lt;0.05 were considered to indicate statistical significance.

\section{RESULTS:}

There were no significant differences in 30-day mortality (Side-clamp vs Device: $0.8 \%$ vs $0.8 \%, \mathrm{p}=0.93)$ or stroke $(1.4 \%$ vs $1.4 \%, \mathrm{p}=0.46)$. TIA RIND/delirium occurred more frequently in the Side-clamp group $(1.3 \%$ vs $0.9 \%, \mathrm{p} 0.002$ ), while new-onset atrial fibrillation (11.0\% vs $12.8 \%$, p\&lt;0.001) and prolonged ventilation $(2.0 \%$ vs $2.9 \%$, p\&lt;0.001) occurred more frequently in the Device group.

\section{CONCLUSION:}

Device use partially provided better results with regard to transient neurological complications; however, no overall benefit was observed in this study.

Trend of isolated CABG in Japan from 2013 through 2014: data analysis from the Japan Cardiovascular Surgery Database

Norimichi Hirahara; Noboru Motomura;

Hiroaki Miyata; Shinichi Takamoto; Aya Saito

\section{ABSTRACT: \\ OBJECTIVE:}

This study is to investigate the trends of isolated CABG of our whole country in the year of 2013/2014

\section{METHODS:}

The isolated CABG cases performed in 2013/2014 registered in the Japan Cardiovascular Surgery Database were retrospectively reviewed for preoperative characteristics, combination of grafts and graft choice for the LAD revascularization by age, and postoperative outcomes especially comparing the results of off-pump $\mathrm{CABG}(\mathrm{OPCAB})$ and on-pump $\mathrm{CABG}(\mathrm{ONCAB})$. RESULTS:

There were 31987 cases underwent isolated CABG during the 2 year period, and the OPCAB procedures were performed in $54.7 \%$ of cases. Those with chronic renal failure on hemodialysis $(10.7 \%)$ and previous PCI $(25.8 \%)$ were frequently observed. The single internal thoracic artery (ITA) were used in $55.3 \%$ and bilateral ITA in $40.7 \%$ for elective cases $(n=27629)$, whereas single ITA in $66.3 \%$ and bilateral ITA in $22.8 \%$ for emergency/urgency cases $(n=5429)$. The choice of graft for the LAD was dominantly left ITA $(74.3 \%)$ followed by right ITA $(15.6 \%)$ for overall cohort and similar distributions in different ages. Operative mortality was $2.0 \%$ in elective cases(ONCAB vs OPCAB: $3.0 \%$ vs $1.1 \%$ ), $8.2 \%$ in emergency cases $(12.0 \%$ vs $5.0 \%$ ), with $3.0 \%$ for overall cohort. Significant differences between elective and emergency/urgency cases were seen in incidence of renal failure $(2.9 \%$ vs $8.4 \%)$ and prolonged ventilation period $\& \mathrm{gt} ; 72$ hours $(4.3 \%$ vs $21.4 \%)$ with generally better results in OPCAB than in ONCAB.

\section{CONCLUSIONS:}

Nation-wide clinical results of isolated CABG were reviewed, and positive contribution of $\mathrm{OPCAB}$ in the short-term results were suggested.

Mid-term clinical outcomes of no-touch SVG

Chikara Ueki; Ken Yamanaka;

Masahiro Hirano; Yuta Kitagata,

Hiroshi Tsuneyoshi

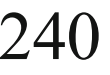

\section{ABSTRACT: \\ BACKGROUNDS:}

Saphenous vein is still an important graft in coronary bypass surgery. To improve the long-term patency of SVGs, no-touch harvesting techniques have been advocated. The present study showed the methods of graft harvesting and mid-term clinical outcomes of no-touch SVGs.

\section{METHODS:}

From August 2013 to October 2018, we have been using 83 no-touch SVGs in coronary artery bypass grafting. We retrospectively evaluated clinical outcomes of NT-SVG cases. 


\section{RESULTS:}

$82 \%$ of cases were performed by off-pump. Average age was $66 \pm 10$ years old. The short-term patency of NT-SVGs was $100 \%(83 / 83)$. The incidence of the wound problems in the SVG's harvesting site was $3.6 \%$ (3/83). 17 patients underwent mid-term graft assessments by coronary CT scan after surgery. The 3-year graft patency of NT-SVG was also $100 \%(17 / 17)$.

\section{CONCLUSIONS:}

Short and mid-term clinical outcomes of NT-SVGs were excellent. Future studies are needed to evaluate long-term superiority of NT-SVGs.

\section{Higher prevalence of common CYP2C9 and VKORC1 genotypes and its implications on warfarin (and acenocoumoral) therapy in Indian population. Manish Pungliya; Atul Mahadik; \\ Manoj Durairaj}

\section{ABSTRACT: \\ BACKGROUND:}

Warfarin/Acenocoumarol are widely prescribed anticoagulant drugs which are metabolized to its inactive form by CYP2C9. VKORC1 catalyzes the reduction of Vitamin $\mathrm{K}$, leading to the production of activated Vitamin K dependent clotting factors, Factors II, VII, IX, and X. Warfarin inhibits the VKORC1 subunit of epoxide reductase, thereby diminishing available vitamin $\mathrm{K}$ in the tissues.

Polymorphisms in the cytochrome P450 2C9 (CYP2C9) and vitamin K epoxide reductase complex subunit 1 (VKORC1) genes significantly alter the effective warfarin dose. The CYP2C $9 * 2$ (430C\&gt; T), CYP2C $9 * 3$ (1075A\&gt;C) and VKORC1 -1639 G\&gt;A polymorphisms affect warfarin dose through altered metabolism (CYP2C9) and sensitivity (VKORC1). PURPOSE:

We determined the frequencies of genetic variations in the CYP2C9 and VKORC1 genes in Indian patients undergoing mechanical valve replacement and its correlation with dose of warfarin/acitrom.

\section{MATERIALS AND METHODS:}

Genomic DNA was extracted from 35 individuals from peripheral blood or saliva using the QIAamp DNA Blood Mini Kit (Qiagen, Germany) according to the manufacturer's instructions. All subjects were genotyped for the CYP2C9*2 and *3 alleles and the VKORC1 -1639G\&gt;A polymorphism using real-time polymerase chain reaction (RT-PCR) taqman assay technology. To ensure the accuracy of the results, an internal positive control was utilized for each polymorphism (rare allele) in each real-time PCR run.

\section{RESULTS:}

Of the 35 patients, $4(11.4 \%)$ were heterozygous for CYP2C9*2, $2(5.7 \%)$ were heterozygous for CYP2C $9 * 3,9(25.7 \%)$ were heterozygous and 1 (2.9\%) was homozygous for VKORC1 $-1639 \mathrm{G} \& \mathrm{gt}$; A genetic variations. Minor allele frequency (MAF) were $5.7 \%, 2.8 \%$ and $15.7 \%$ for CYP2C9*2, CYP2C9*3 alleles and VKORC1 -1639G\&gt;A polymorphisms respectively.

\section{CONCLUSION:}

$46 \%$ of the patient population has at least one genetic variation making them sensitive to Warfarin/Acenocoumarol based therapy. This genetic information is imperative in dosing in order to a) potentially reduce the risk of bleeding complications and b) prevent higher INR at normal doses.

Validity of EuroSCORE II (European System for Cardiac Operative Risk Evaluation) \& STS Score (Society of thoracic Surgeons Score) to predict risk of mortality in patients undergoing coronary artery bypass grafting.

Sibashankar Kar; Sujay Shad; Ganesh Raj Shivnani; Sumir Dubey; Himanshu Goel
ABSTRACT:

\section{PURPOSE:}

Most of studies on EuroSCORE II and STS score were carried out in western population and showed good results and correlation with morbidity and mortality. There are however only a few studies which show efficiency of these scores in the Indian population. We aim to compare expected outcomes from these scores to the surgical results in the patient population being operated upon at Sir Ganga Ram Hospital.

\section{METHODS:}

Twelve hundred and fifty consecutive patients who underwent coronary artery bypass grafting (CABG) in our institution from March 2015 to April 2018 were included in this prospective study. The preoperative risk models, EuroSCORE II and STS scores were used to predict mortality. Patients were divided into three clinically relevant categories: low (0-3 $\%)$, moderate $(3-5 \%)$ and high (\&gt;5\%) risk groups. Observed and predicted mortalities were compared between groups, both for STS and EuroSCORE II models.

\section{RESULTS:}

Mean age was $61.23 \pm 8.99$ year with $81.2 \%$ male patients. For all 1250 patients undergoing coronary artery bypass grafting in our institution, the total mortality was 7 patients $(0.56 \%, 95 \%$ CI: $0.15-0.97)$. In the entire patient cohort the EuroSCORE II ranges from 0.5 to 31.5 (mean 2.1, 95\% CI: 1.96-2.23) having good discriminative capacity with AUC of 0.959. The STS score ranges from 0.18 to 24.01 (mean $1.29,95 \%$ CI: $1.19-1.38$ ) having good discriminative capacity with AUC of 0.973 .

\section{CONCLUSION:}

EuroSCORE II and STS score models, in their current forms, do not accurately predict outcomes in this group of Indian population for CABG patients. Both scores over-predict in low and moderate risk patients but EuroSCORE II over-predicts in high risk group while STS Score under-predicts in high risk group.

Early Reentry Closure Promotes Complete Remodeling of the Dissected Abdominal Aorta - Scheduled Stepwise Treatment for Type B Acute Aortic Dissection -

Kenji Toyokawa; Itsumi Imagama; Keisuke Kawaida;

Shuji Nagatomi; Yutaka Imoto; Hiroyuki Yamamoto

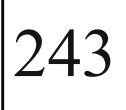

\section{ABSTRACT: \\ OBJECTIVE:}

Recently thoracic endovascular repair (TEVAR) has been widely performed for Type B acute aortic dissection (TBAD).TEVAR is highly effective in aortic remodeling with aneurysm sac stabilization or regression in dissections limited to the descending thoracic aorta. In more extensive dissections involving the entire distal aorta TEVAR is also effective in the thoracic aortic dimensions, but the abdominal aortic false lumen remains patent and is at high risk for expansion. We report a therapeutic strategy to stabilize dissection of the abdominal aorta and prevent aneurysmal change by stepwise treatment for remaining reentries. METHODS:

60 patients with TBAD were treated by TEVAR from 2014 to 2018 . We performed scheduled stepwise treatment in 6 cases by subacute phase. Their mean age was 57.1(range, 45-75) years, and 3 of them were women. All cases were uncomplicated TBAD. The treatment strategy is to perform TEVAR for entry closure early after onset of the aortic dissection and close the reentry of the abdominal aorta by EVAR several months later.

\section{RESULTS:}

Operative mortality was $0 \%$. Complete false lumen thrombosis and entire aortic remodeling were obtained immediately after the operation in 4 patients treated all reentry of the abdominal aorta. In 2 patients who were inadequate for reentry closure, thrombosis around the reentry was not obtained, but slow aortic remodeling was observed the site distant from reentry. CONCLUSIONS:

Entire aortic remodeling is induced early postoperatively by reentry closure following entry closure. Early disappearance of the false lumens has 
a potential to lead to the prevention of aneurysmal change of the thoracoabdominal aorta in the late phase and may improve long-term survival of patients with TBAD. In our series there are few cases and the observation period is short, further examination is necessary, but our success cases suggested the importance of closure of the reentry.

The false lumen occlusion concept after PETTICOAT technique for type B aortic dissection. Shinichi Higashiue; Masatoshi Komooka

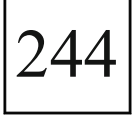

\section{ABSTRACT: \\ BACKGROUND:}

Recent studies have shown that the PETTICOAT (Provisional Extension To Induce Complete Attachment) technique alone for Stanford type B aortic dissection (TBAD) may fail to induce thrombosis of the thoraco-abdominal and the abdominal aortic false-lumen (FL) and late aortic positive remodeling. To accomplish false-lumen thrombosis (FLT), we developed 'the FL occlusion concept (FLOC)' which consists of selectively excluding FL blood flow with combination of stent-grafting and coil embolization.

\section{OBJECTIVE:}

This study objective was to evaluate the early clinical outcomes of FLOC. MATERIALS AND METHODS:

From October 2015 to September 2018, we applied the PETTICOAT technique in 18 nonconsecutive patients with TBAD, of which 5 patients' consent (all men, average age was 68 years) was obtained to perform FLOC procedures. All the ancillary procedures were done in a stepped approach to avoid spinal cord ischemia. The FLOC consists of procedures in the way hereinafter prescribed. When the branch artery is dissected with a distal re-entry or avulsed, a peripheral stent graft is deployed through the interstices of the aortic bare metal stent across the distal re-entry or the detached ostium of the branch. When there is no branch around the re-entry, the re-entry is simply occluded by stent grafts with or without FL coil emboli.

\section{RESULTS:}

Procedures were successful in all cases. The median interval between the PETTICOAT technique and the FLOC was 8.3 months (range 121). The median re-intervention was 1.6 times (range 1-3) and the median follow-up duration (1st re-intervention to last CT) was 9.8 months (range 2-16). No complications were found. Follow-up imaging showed that false lumen was totally thrombosed and consequent aortic positive remodeling in all cases.

\section{CONCLUSIONS:}

Although longer follow-up results are needed our FLOC may be the most simple procedure to induce FLT and consequent aortic remodeling after the PETTICOAT technique.

Retrograde popliteal approach for endovascular revascularization of flush SFA CTO

Vinay Krishna; Amit Singh

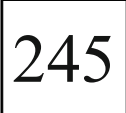

\section{ABSTRACT:}

\section{BACKGROUND:}

Superficial femoral artery (SFA) chronic total occlusions (CTO) are generally managed by a contralateral 'crossover' approach or an antegrade ipsilateral approach. In cases with a very short SFA stump or flush occlusion at common femoral artery bifurcation, antegrade recanalization from the contra lateral approach frequently fails due to an inability to engage the true ostium of the SFA. In such cases, retrograde revascularization can be performed via retrograde popliteal artery approach.

AIM AND OBJECTIVE:

To evaluate the efficacy and safety of retrograde popliteal approach for endovascular revascularization of flush SFA CTO.

\section{MATERIALS AND METHODS:}

Between January 2017 and January 2018, retrograde popliteal artery revascularisation was performed on 12 patients $(10$ males and 02 females; mean age $62+/-12$ years). All the patients had critical limb ischemia (Rutherford 4 to 6) with TASC A\&amp;C lesions. Indication for popliteal access was failure of antegrade recanalization.

Ipsilateral popliteal artery was punctured under usg guidance with patient in prone position with $5 \mathrm{~F}$ femoral sheath. CTO negotiation with guide wire and catheter was successful in all the patients either endoluminally or subintimally. Angioplasty and stenting were done via this sheath. Hemostasis after popliteal access was achieved by manual compression for 10 minutes. RESULTS:

Technical success rate was $100 \%$. Primary patency rates were $100 \%$ at 3 months and $83.33 \%$ at 1 year. Target lesion revascularization rate was $16.66 \%$ at $6 \mathrm{mths}(02$ patients with in-stent restenosis underwent angioplasty with drug coated balloon and both these cases were patent 6 months post procedure). There were no major access site complications. The symptoms improved post procedure (Rutherford 0 to 3), 04 patients underwent amputation of the gangrenous toes or forefoot with successful healing of the stump.

\section{CONCLUSION:}

Popliteal approach was effective in terms of technical success rate and primary patency rate. The approach was safe with no major acccess site complications.

Bi Ventricular mechanical circulatory support in Pediatric heart failure, a single centre experience. Pradeep; Ganapathy Subramaniam; K.R. Balakrishnan; Suresh Rao

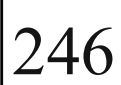

\section{ABSTRACT: \\ INTRODUCTION:}

Mechanical circulatory support ( MCS ) in pediatric heart failure patients is needed to stabilise them either from ciruculatory collapse or due to progressive end organ dysfunction. We review our experience in these subset of critically ill pediatric patients who required mechanical circulatory support as a bridge to transplant.

\section{MATERIAL AND METHODS:}

13 out of 70 pediatric heart transplantation were instituted on MCS pre operatively as an emergency or urgent procedure for imminent circulatory collapse. The youngest was 11 month old. Peripheral ECMO was instituted in 10 patients either during resuscitation or immediate post CPR, using graft on femoral vessels. Peripheral ECMO was changed to LVAD after one week using a Centrimag or central ECMO with atrial septostomy depending on the clinical condition.

RESULTS:

There were three deaths before they could get heart transplantation. Of the 10 patients who went on to have transplantation, there were three deaths in the postop period due to RV dysfunction and sepsis. The earliest transplantation was after 1 week and the longest period of preopsupport was for 6 weeks. The last 4 patients had central ECMO with atrial septostomy to reduce the PA pressure. There are 7 long term survivors(70\%) with good quality life

\section{CONCLUSION:}

Managing Pediatric patients on MCS raises complex clinical and ethical issues related to anticoagulation, neurological complications and accepting a donor organ. Central ECMO with atrial septostomy is currently our MCS of choice in patients with biventricular dysfunction which protects the lungs and increase the chances of succesful heart transplantation. 
Is Heart transplantation a solution for complex congenital cardiac disease ?! -Our Experience Pradeep; Ganapathy Subramaniam; K.R. Balakrishnan; Suresh Rao

\section{ABSTRACT:}

\section{INTRODUCTION:}

Heart Transplantation is sometimes the only solution possible for children with complex congenital heart disease with failed previous palliation or those who have severe ventricular dysfunction. We present our experience in 20 patients with complex congenital heart problems who underwent cardiac Transplantation..

\section{MATERIALS AND METHODS:}

From 2016 to 2018,20 patients in the age range 4 yrs to $40 \mathrm{yrs}$ who had complex congenital heart defects underwent cardiac transplantation at our unit. 5 had previous Glenn shunt, 2 had fontan, 4 had LSVC, 1 had left inferior venacava with separate hepatic venous opening. 2 had Total anomalous pulmonary venous connection with small left atrial chambers. 2 had LPA stent. One absent right pulmonary artery, 4 had malposed great artery.one had previous Ebsteins anomaly repair.one had ECMO for decompensated shock.

A variety of strategies were adopted, using graft from RSVC to donor atrium, augmenting the small LA chamber with recepient atrium, creating tunnel using autologous tissues, shifting the pulmonary anastomosis to the left, laeaving PFO open, using innominate vein to connect to LSVC. There were 3 early deaths and no late deaths. On multivariate analysis small recepient pericardial cavity and LPA stenting were risk factors for death. The number of previous surgeries, use of ECMO, age of the patient did not increase the risk of death.

\section{CONCLUSION:}

Transplantation can be offered to patients with complex congenital cardiac problems with acceptable early and good medium term outcomes. Previous pulmonary artery stent is a risk factor. Patients with protected pulmonary circulation and no previous palliation form the best subset of recipients.

\section{New therapy management of Severe Heart Failure on our Hospital \\ Takeki Ohashi; Norikazu Oshiro}

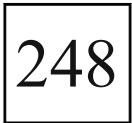

\section{ABSTRACT: \\ BACKGROUND:}

In Japan, Impella can be used for severe heart failure with cardiogenic shock since September 2017. We report 24 cases in which Impella was used in our hospital since December 2017.

\section{SUBJECTS AND METHODS:}

Subjects were 24 patients treated at our hospital between December 2017 and August 2018 were included. Their average age was $70.6 \pm 12.1$ years, 16 cases of males and 8 cases of females. The underlying diseases were 11 cases of AMI (including $\mathrm{AAD}+\mathrm{AAE}+\mathrm{AR}, \mathrm{VSP}$ ), 3 cases of OMI (including AS, left ventricular thrombus), 4 cases of UAP, and 4 case of Cardiomyopathy, and 1 case of Myocarditis, and 1 case of Prosthetic valve failure, all having severe heart failure.

\section{RESULTS:}

11 cases were Cardiopulmonary arrest. ECPELLA(VA-ECMO+ Impella) were 16 cases, isolated Impella were 8 cases. The average assistance period of isolated Impella was 5.26+3.78days, whitch of ECPELLA was 3.99 \pm 3.92 days. Within 24 hours after supported, Systolic blood pressure recovered to above $90 \mathrm{mmHg}$ in 18 cases $(75 \%)$, Urine recover ( \&gt; body weight $\times 0.5 \mathrm{ml} / \mathrm{kg}$ ) in 17 cases $(73.5 \%)$, Lactate recover in 16 cases $(66.7 \%)$, Pulmonary artery pressure down in 11 cases(45.8\%), Pulmonary congestion recover in 12 cases(50\%). It was possible to wean in 13 cases $(54.1 \%), 11$ cases demonstrated 30day mortality after weaning $(45.8 \%) .8$ cases underwent same-day PCI, 12 cases underwent open heart surgery. Complications were 9 cases of hemorrhages (gastrointestinal hemorrhage, rethoracotomy, subarachnoid hemorrhage, menorrhagia, nose bleeding), 13 cases of hemolysis DISCUSSION AND CONCLUSION:

Improvement in circulatory dynamics was observed in 16 cases within 24 hours after Impella supported, to wean in 13 cases. Impella is extremely effective in controlling severe heart failure in the acute phase.

\section{"Central ECMO with LV venting" circulatory support for acute critical heart failure including INTERMACS Profile 1}

Osamu Kinoshita; Kan Nawata

\section{ABSTRACT:}

\section{BACKGROUND:}

In Japan, mechanical circulatory support to treat refractory heart failure including implantable left ventricular assist device offers considerably acceptable results. However, the outcomes of treatment for acute critical heart failure categorized in INTERMACS Profile 1 are still dismal, especially when patients suffer from acute bi-ventricular failure with pulmonary edema. In our institute, central ECMO with LV venting via median sternotomy has been adopted for a treatment of sickest cases, avoiding biventricular assist device installation.

\section{PURPOSE:}

To evaluate the clinical results of central ECMO with LV venting strategy in our institute

\section{OBJECTIVES AND METHODS:}

Ten patients who underwent central ECMO with $\mathrm{LV}$ venting $(\mathrm{CE} / \mathrm{LV})$ in our or another institute between January 2016 and August 2018, and have been under our management, were retrospectively reviewed.

\section{RESULTS:}

The age at the CE/LV operation was $39+/-7$ years and 6 out of 10 were male. Etiologies of heart failure were fulminant myocarditis in 5 and acute deterioration of dilated cardiomyopathy in 5 . Preoperatively 5 patients were supported by IABP and peripheral ECMO, 4 were by IABP only and the other were just intubated and sedated. All had impaired LVEF less than $20 \%$ with significant lung edema. Support time of peripheral ECMO was $6.6+/-2.5$ (3-6) days. After installation of $\mathrm{CE} / \mathrm{LV}$, 4 patients weaned from the mechanical circulatory support, 5 had converted to durable ventricular assist device, and one was lost due to brain infarct two months later, after conversion to paracorporeal ventricular assist device. Thirtyday mortality was nil. Support duration by CE/LV was $38+/-34$ (7-108) days. Follow-up duration was $18+/-9$ months.

\section{CONCLUSION:}

Considering the sickest condition of acute critical heart failure, the results of CE/LV support strategy are good, and it is an acceptable intervention as a bridge to recovery or next durable circulatory support method.

Berlin Heart support during infancy:

The Melbourne experience.

Melissa G.Y. Lee; Bennett J. Sheridan; Yves d'Udekem;

Christian P. Brizard; Igor E. Konstantinov; Jacob Mathew; Johann Brink; Johann Brink

\section{ABSTRACT:}

\section{INTRODUCTION:}

Durable mechanical circulatory support as a bridge to transplantation or recovery for neonates and infants with end-stage heart failure remains a challenge. Our analysis aims to identify predictors for morbidity and mortality in this age group supported with Berlin Heart EXCOR (BHE) device. 


\section{METHODS:}

A retrospective analysis was performed on all neonates (\&lt;28 days) and infants (\&gt;28 days and \&1t;365 days) supported with BHE at the Royal Children's Hospital, Melbourne between 2013 and 2018.

\section{RESULTS:}

Eighteen infants (4 neonates and 14 older infants) under 1 year of age were implanted with BHE at a median age and weight of 0.4 years [range 0.040.85 ] and $5.65 \mathrm{~kg}$ [range 3.2-8.8] respectively. Diagnosis included dilated cardiomyopathy(13), myocarditis(2) and other(3). No patients had congenital heart disease. Seven patients required pre-implantation ECMO support for a median of 7 days [range 2-13]. Adverse events included sepsis in 7 patients (39\%) and stroke in 3 patients (17\%). Median duration of support was 72 days [range 14-252] with 3 patients still on BHE support at the time of the analysis. Successful bridge to transplantation or recovery occurred in $83 \%$. Three patients died with a mortality $17 \%$. Despite the statistical under powered nature of the analysis, similarities of fatal outcomes included neonates with myocarditis, sepsis and inadequate device function.

\section{CONCLUSION:}

More than $80 \%$ of infants supported with BHE will be successfully bridged to transplantation or recovery with low incidence of neurological events. Neonates with myocarditis and patients with severe sepsis may be at greater risk for mortality.

Utilisation of Extra-corporeal life support for refractory cardiogenic shock in awake patients

Anand Jothidasan; Prashant Mohite

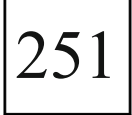

\section{ABSTRACT:}

\section{OBJECTIVE:}

Despite vast and continuous development in the field of Extracorporeal Life Support (ECLS), the outcomes after its utilisation in refractory cardiogenic shock (RCS) remains poor. Patients are usually are sedated and mechanically ventilated. In view of avoiding complications related to immobility and ventilation, we adopted 'Awake ECLS' strategy for the patients with RCS as a bridge to decision.

\section{METHODS:}

68 patients with RCS were supported by ECLS (All VA) in years 201014. Patients that could not survive 24 hours after ECLS implantation $(9$ patients) were excluded from the study. Study population constituted 59 patients- 'Awake' group ( $\mathrm{n}=18$; maintained awake without intubation) and 'Control' group $(\mathrm{n}=41$; intubated and required mechanical ventilation).

\section{RESULTS:}

Nine (50\%) patients were awake at implantation, with 5 of them remaining free of sedation and ventilator support through to explantation. Nine patients were ventilated at the time of implantation but subsequently extubated and remained non-intubated and ventilator free. Post-ECLS Survival at 1 month was $78 \%$ and $42 \%$ while the survival to discharge 78 and $37 \%$ in 'awake' and 'Control' group respectively.

CONCLUSIONS:

ECLS as a bridge to decision in RCS is effective in restoring adequate systemic perfusion and recovering end-organ function. ECLS can be initiated in awake patients with RCS and patients can be awakened on ECLS. The 'awake ECLS' strategy may avoid complications related to mechanical ventilation, sedation and immobilization. RCS patients supported on ECLS without severe metabolic acidosis, multi-organ failure, IABP or uncertain neurological status are more likely to be weaned from the ventilator. Patients that are awake at the time of ECLS implantation are more likely to remain awake during ECLS.

Outcome of rescue ECMO Support During Catheter Ablation of Ventricular Tachycardia

T.K. Lahiri; Sanjay Kumar
ABSTRACT:

OBJECTIVE:

In patients with ventricular tachycardia or ventricular fibrillation (VT/VF) electrical storm (ES) undergoing catheter ablation(CA), hypotension due to refractory VT/VF, use of anesthesia, and cardiac stunning due to repeated implantable cardioverter-defibrillator shocks might precipitate acute hemodynamic decompensation (AHD). Mechanical circulatory support (MCS) devices may facilitate haemodynamic stability and preserve end organ perfusion during sustained VT to permit mapping for long periods.

We evaluated the outcomes of emergent cardiopulmonary support with extracorporeal membrane oxygenation $(\mathrm{ECMO})$ to rescue AHD in patients undergoing CA of ES.

\section{METHODS:}

Between August 2010 and June 2013, 19 patients with ES (VT in 10 and premature ventricular complex-triggered VF in 9) had peri-procedural AHD during CA requiring emergent ECMO support.

\section{RESULTS:}

In 11 patients, AHD occurred a mean of $1.3 \pm 1.5$ days before the procedure. In the remaining 8 patients, AHD occurred during or shortly after the procedure. ECMO was started successfully in all patients. Ablation was performed in 18 patients ( 9 with VF and 9 with VT). In patients with VF, premature ventricular complex suppression was achieved in 8 of 9 $(89 \%)$. In those with VT, noninducibility was achieved in 7 of $9(78 \%)$. After a median follow-up of 10 days, 11 patients died ( 8 during the index admission). Death was due to refractory VT/VF in 2 patients, heart failure in 8 , and noncardiac cause in 1 patient. Seven patients survived beyond 6 months postablation; 5 remained free of VT/VF and 3 ultimately received a destination therapy (heart transplantation in 1 and left ventricular [LV] assist device in 2 .

\section{CONCLUSION:}

The outcomes of ECMO support as rescue intervention for AHD are poor In patients with ES undergoing CA,. Strategies to prevent AHD including preemptive use of hemodynamic support may improve survival.

VATS Left Ventricular Lead Insertion For Cardiac

Resynchronisation - Irelands Experience

James Curtin; Raluca Neagu; Vivek Sidiah;

Kishore Doddakula;

Joseph Mc Loughlin

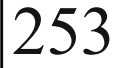

\section{ABSTRACT: \\ OBJECTIVES:}

Left ventricular (LV) lead placement for cardiac resynchronisation therapy is commonplace in the setting of severe cardiac dysfunction. It has been shown to reduce morbidity and mortality in heart failure patients with systolic dysfunction and ventricular electrical conduction delays. LV lead placement using a VATS (video assisted thoracic surgery) approach is a minimally invasive approach where endovascular placement has failed. We set out to analyse our initial outcomes.

\section{METHODS:}

This is a retrospective review of all patients that underwent VATS LV lead placement. The patient demographics, indications and outcomes were analysed. A left sided two port VATS approach was utilised with single lung ventilation. The pericardium was opened and the left ventricle exposed. Ventricular leads were implanted and the wires brought out to a pocketed device in a left subpectoral pocket. The device was tested and a single chest drain inserted.

\section{RESULTS:}

There were 6 cases performed in total. The group was comprised of 5 males and 1 female, the average age was 71. Average ejection fraction of $24 \%$ (15 - 35) Two patients required pre-operative dobutamine infusion. The average postoperative length of stay was 8 days $(3-14)$. Three patients were initially managed in cardiac ICU postoperatively. There was a $100 \%$ success rate in lead function. 
There was no surgically associated mortality. One patient required short duration continuous veno-venous hemofiltration.

\section{CONCLUSIONS:}

Our VATS approach provides an effective option to facilitate cardiac resynchronisation therapy in patients that have failed percutaneous placement. All our patients had severe cardiac dysfunction and were high risk surgical candidates. Without this approach, the alternative was via a thoracotomy, these patients were deemed medically unfit to undertake this. Our results demonstrate that this technique is safe, effective and has a low associated complication rate within this patient cohort.

\section{Results of orthotopic heart transplant at our center: 18 month follow up \\ Sarat Surapaneni; Sushant Panda; \\ Krishna Chaitanya; Prem; Ajeya Joshi}

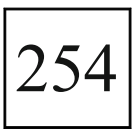

\begin{abstract}
:
Heart transplant is the established treatment for end stage heart failure not responding to maximal medical therapy. From May 2017 to November 2018, we have performed sixteen cases of orthotopic heart transplant. All patients are being followed up with an established protocol. Triple drug immunosuppression therapy was used in all patients. Endomyocardial biopsy is done at 1 month and repeated at one year after the surgery. Common complications observed in the postoperative period include tacrolimus toxicity (25\%), GI disturbances related to mycophenolate mofetil (35\%), steroid toxicity (7\%), pleural effusions (18\%). There were 4 deaths in the follow up, two cases due to pulmonary aspergillosis, one due to pneumonia and one due to rejection. Remaining patients are in NYHA class I functional status, responding well to the treatment. Frequent communication with the patient and regular follow up is essential to detect any complications and initiate early treatment, which will improve the long term survival of the heart transplant recipient.
\end{abstract}

\section{Minimally invasive surgery for hypertrophic obstructive cardiomyopathy with concomitantly significant mitral regurgitation through a single transaortic approach Min Tang; Ju Mei}

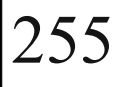

\footnotetext{
ABSTRACT:

Objective: To summarize the effect of minimally invasive surgery for hypertrophic obstructive cardiomyopathy (HOCM) with concomitantly significant mitral regurgitation (MR) through a single transaortic approach. Methods: From 2008 to 2017, 51 patients (female, n=22) with HOCM and significant MR underwent modified Morrow procedure and edge-to-edge mitral valvuloplasty through a single transaortic approach. Preoperative peak left ventricular outflow tract pressure (LVOTP) was $96.53 \pm 28.72 \mathrm{mmHg}$. Preoperative interventricular septum thickness (IVST) was $24.31 \pm 3.52 \mathrm{~mm}$. The left ventricular end-diastolic diameter (LVEDD) was $42.20 \pm 3.03 \mathrm{~mm}$. All patients had significant MR with SAM phenomenon. The modified Morrow procedure and edge-to-edge mitral valvuloplasty through a single transaortic approach was performed under $\mathrm{CPB}$ and ACC. An oblique incision was made on the anterior wall of the ascending aorta. Modified Morrow procedure was performed through the orifice of aortic valve. Then, mitral valve leaflet, annulus, chordae, and papillary muscles were also exposed through the orifice of aortic valve. Edge-to-edge mitral valvuloplasty was performed with suturing the prolapsed sites between the anterior and posterior leaflet. Results: All patients successfully underwent the minimally invasive surgery of modified Morrow procedure and edge-to-edge mitral valvuloplasty through a single transaortic approach. No early death and interventricular septal perforation were occurred. At discharge, postoperative peak LVOTP $(18.16 \pm 6.41 \mathrm{mmHg})$ and IVST $(14.33 \pm 1.99 \mathrm{~mm})$ were significantly decreased compared with the preoperative values (P\&lt;0.05), but LVEDD (44.27 $\pm 2.88 \mathrm{~mm})$ was significantly increased
}

(P\&lt;0.05). All patients had none or trivial MR. SAM phenomenon disappeared in all patients. During follow up, the peak LVOTP was $19.27 \pm 6.10 \mathrm{mmHg}$; the IVST was $14.67 \pm 1.87 \mathrm{~mm}$; the LVEDD was $43.98 \pm 2.30 \mathrm{~mm}$. All patients still had none or trivial MR. No SAM phenomenon occurred. Conlusion: Minimally invasive surgery of modified Morrow procedure and edge-to-edge mitral valvuloplasty through a single transaortic approach could be safely and effectively applied for patients with HOCM and concomitantly significant MR.

Total Arterial Multi vessels Minimal Invasive Direct CABG (MIDCABG) through Left

Minithoracotomy.

Vivek Wadhawa; Kaushal K. Tiwari

\section{ABSTRACT:}

BACKGROUND AND AIM:

MIDCABG is becoming popular and an important armamentarium for cardiac surgeons. MIDCABG with total arterial grafts is the best option for patients with coronary artery disease, who are at high risk for sternotomy. We aimed to evaluate the result of MIDCABG total arterial revascularization.

\section{METHODS:}

We have evaluated 221 patients who underwent MIDCABG multivessel total arterial revascularization through a left anterolateral mini thoracotomy.

LIMA harvested in every patient, RIMA or radial artery used as second conduit. LIMA-RIMA Y or LIMA-RADIAL Y was made to accomplish multivessel total arterial revascularization. Mean age of patients was $52.52 \pm 9.76$ yrs. Average number of anastomosis performed were $2.34 \pm 0.75$. All intraoperative and post-operative data were collected and evaluated. Post-operative graft patency was evaluated in all patients by CT coronary angiography at 6 months following discharge.

\section{RESULTS:}

Multivessel total arterial CABG was accomplished in all individuals. There was no mortality or wound infection. Re-exploration was done in one patient for bleeding. In one individual, conversion to sternotomy and $\mathrm{CPB}$ was required. Average ICU and hospital stay was $1.52 \pm 0.77$ and $4.92 \pm 1.46$ days respectively. Patients were mobilized earlier due to less pain. Wound healing of anterolateral thoracotomy was faster. In the follow up CT angiography all grafts were patent.

\section{CONCLUSIONS:}

Multivessels total arterial MIDCAB can be accomplished safely in selected individuals. RIMA can be harvested in long standing diabetic patients with no concern for sternal wound healing. MIDCABG patients experience less pain, better cosmetics and early return to daily activities.

Our experience of left atrial appendage occlusion for post-stroke patients with atrial fibrillation via a mini-thoracotomy

Daisuke Arima; Takaharu Shimizu

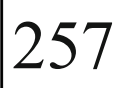

\section{ABSTRACT: \\ OBJECTIVE:}

Many cases have contraindications to anticoagulation, the gold standard for prophylaxis against cardiogenic stroke. Since 2018, we have performed left atrial appendage occlusion (LAAO) via a mini-thoracotomy (mini-thoracotomy LAAO) for post-stroke patients with atrial fibrillation (AF). In this study, we report on the evaluation of its short-term outcomes. METHODS:

We performed mini-thoracotomy LAAO using AtriClip ${ }^{\circledR}$ (AtriCure, Mason, Ohio, United States) for 13 cases (10 males) between March 
and July 2018, and conducted a retrospective analysis of the shortterm outcomes of this procedure. The average follow-up period was $103.4 \pm 41.9$ days.

\section{RESULTS:}

The mean age of the patients was $73 \pm 8$ years. The CHA2DS2-VASc score was $5.3 \pm 1.2$ points, and the HAS-BLED score, $3.4 \pm 1.1$ points. Hemorrhagic complications occurred in 4 cases $(30.7 \%)$, necessitating discontinuation of anticoagulation in 2 of these cases. Preoperative CT showed filling defects in 11 cases (84.6\%), indicating low LAA flow velocity.

The operation time and anesthesia time were $52.4 \pm 21.8$ and $128.1 \pm 29.9$ minutes, respectively. Femoral-femoral bypass was simultaneously performed in 1 case $(7.6 \%)$, due to arteriosclerosis obliterans. All cases were extubated in the operating room, before being sent back to the ICU. The chest drainage tube was removed on POD1 in 12 cases $(92.3 \%)$. The postoperative drainage volume was $50.7 \pm 39.4 \mathrm{~mL}$. Of the 13,11 cases $(84.6 \%)$ were discharged from the ICU on POD1.

In 4 cases (36.3\%), postoperative CT showed a thrombus in LAA, therefore treatment with a direct oral anticoagulants (DOAC) was initiated. We confirmed disappearance of the thrombus 3 months after, and discontinued the DOAC in all these cases. Three cases were transferred to the neurosurgery department for rehabilitation, and the postoperative hospital stay (days) of the others was $6.5 \pm 2.9$ days.

There were no postoperative complications or any cases of recurrent stroke.

\section{CONCLUSIONS:}

The short-term outcomes of mini-thoracotomy LAAO were satisfactory, without complications or recurrence of stroke.

\section{BILATERAL VIDEO ASSISTED THORACOSCOPIC SYMPATHECTOMY FOR MALIGNANT ARRYTHMIAS}

Preethi Kumar; Rakesh Naik L.

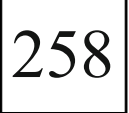

\section{ABSTRACT: \\ INTRODUCTION:}

High thoracic bilateral emergent VATS sympathectomy is a therapeutic option for patients with recalcitrant ventricular tachyarrhythmias and long QT syndrome not fully protected by antiarrhythmics. Here, we present our experience with VATS sympathectomy.

MATERIALS AND METHODS:

This study included 23 patients with symptomatic ventricular tachyarrhythmias refractory to antiarrhythmics, who underwent high thoracic sympathectomy in our institute from 2010 to 2018 . The mean age at HTLS was $31.25 \pm 3.12$ years (range 7-48 years). All underwent complete clinical evaluation. Out of 23 cases, eight were diagnosed clinically as long QT syndrome (LQTS), two were catecholaminergic polymorphic ventricular tachycardia (CPVT) and eleven were polymorphic ventricular tachycardia (PVT). One patient with long QT syndrome had an implantable cardioverter defibrillator (ICD) in situ, whose battery was discharging rapidly, and another patient with long QT syndrome had an associated ASD. The QT interval and the QTc were recorded in those patients with LQTS both preoperatively and postoperatively at 3 months and 12 months. Indications for surgery were recurrent episodes of syncope, symptomatic ventricular tachyarrhythmias unresponsive to antiarrhythmics, and excessive implantable cardioverter defibrillator discharges. All patients underwent VATS sympathectomy.

\section{RESULTS:}

There were no perioperative surgical complications. Two patients died due to recurrence of ventricular tachycardia storm refractory to all abortive measures. The mean follow-up period was $4.8 \pm 0.96$ years. All others returned to normal sinus rhythm and maintained so with additional $\beta$-blockers.

\section{CONCLUSION:}

VATS Sympathectomy is an effective therapeutic option and reduces arrhythmia burden in patients with recalcitrant ventricular tachyarrhythmias and long QT syndrome.
Minimally Invasive Aortic valve Replacement through Right Anterior Mini-thoracotomy approach: Is it a daunting operation? Gopichand Mannam; Venkata Satya Siva Sai Kiran Krishnamurthy

\section{ABSTRACT:}

\section{INTRODUCTION:}

Aortic valve replacement (AVR) is increasingly being done through non full sternotomy (FS) approaches. However, many surgeons have been hesitant to adopt a right anterior mini-thoracotomy (RAMT) for various reasons. We studied our experience of RAMT-AVR operated between January 2012 and October 2018 to audit the difficulties encountered with this operation.

METHODS:

All patients who underwent minimally invasive (MICS) AVR between January 2012 and October 2018 were eligible and their case records analysed retrospectively. Parameters which were retrieved and audited include:

Cardiopulmonary bypass (CPB) time and Aortic cross clamp (ACC) time

Re-exploration for any cause

Conversion to sternotomy

Mortality

Need for permanent pacemaker insertion (PPI)

Post operative cerebrovascular accident (CVA)

Wound complications

Post operative pain

\section{RESULTS:}

157 patients underwent RAMT AVR, including associated procedures like coronary artery bypass grafting $(\mathrm{CABG})$ in 6 and mitral valve replacement in 2. The mean CPB time $91.17 \mathrm{~min}$ and ACC time $68.17 \mathrm{~min}$ were slightly more than conventional surgery. Re-exploration for bleeding in 4 patients $(2.57 \%)$ due to minor chest wall or pacing wire site bleeding. None needed conversion to sternotomy. There was one late death $(0.6 \%)$ due to prosthetic valve endocarditis and $4(2.57 \%)$ had CVA post operatively. 11 patients needed permanent pacemaker $(7 \%)$. Superficial surgical site infection was seen in $5(3.18 \%)$ patients and 2 $(1.27 \%)$ had groin seromas of the vascular access site. Majority of the patients $(78 \%)$ complained of mild or no wound pain (Scored less than 6 on Wong Baker Scale) and were ambulant on day 2 after surgery.

\section{CONCLUSION:}

MICS AVR through RAMT approach is feasible with minimal complications, good outcomes and excellent patient satisfaction.

Minimally invasive right anterior mini-thoracotomy for repair of ventricular septal defects - A safe alternative Sunil Dixit; Jaikishan Suthar

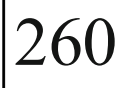

\section{ABSTRACT:}

Minimally invasive right anterior mini-thoracotomy for repair of ventricular septal defects - A safe alternative

Jaikishan Suthar, Anil Sharma, Sunil Dixit, Vikram Watti, Sourabh Mittal Department of Cardiac thoracic-vascular surgery Surgery, S.M.S. Medical college, Jaipur, Rajasthan

ABSTRACT:

\section{BACKGROUND:}

Minimally invasive cardiac surgical techniques are increasingly used in the treatment and management of a variety of adult cardiac surgery. However, repair of VSDs via right anterior mini-thoracotomy is rarely reported. The present study aimed to determine the feasibility and safety of the right anterior mini-thoracotomy for the repair of VSDs.

\section{METHODS:}

Twenty-three patients underwent repair of VSDs via right anterior minithoracotomy. Right sub mammary skin incisions given. The skin incision length was 5-8 cm (mean, $6 \pm 0.5 \mathrm{~cm}$ ). CPB was instituted from same 
thoracotomy incision. The closure of the VSDs was obtained through the right atrium under direct visualization.

\section{RESULTS:}

Successful repair of the defects was achieved in all the patients. No patients died or converted to median sternotomy. Average durations of cardiopulmonary bypass (CPB) and aortic cross-clamp were $96 \pm 12.5 \mathrm{~min}$ (range, 75-118 $\mathrm{min}$ ) and 55 $\pm 7.2 \mathrm{~min}$ (range, 45-68 $\mathrm{min}$ ), respectively. The average postoperative hospital stay was $5 \pm 0.8$ days (range, $4-7$ days). There were no postoperative complications related to the operative procedures. Postoperative pain and bleeding was significantly less. During the followup (3 months-2year), no patients were found to have residual VSD, wound infections, pericardial tamponade, neurologic complications.

\section{CONCLUSION:}

Our experiences demonstrate that minimally invasive cardiac surgical technique via right anterior mini-thoracotomy can be safe alternative for the repair of VSDs.

\section{TOTALLY ENDOSCOPIC NON ROBOTIC MINIMALLY INVASIVE MITRAL VALVE SURGERY- OUR EXPERIENCE \\ Prasanna Simha Mohan Rao; Rakesh Naik L.}

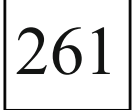

\section{ABSTRACT: \\ INTRODUCTION:}

Today, minimally invasive mitral valve surgery ranges from lower midline hemisternotomy, right thoracotomy, video assisted mini-thoracotomy, totally endoscopic non robotic surgery and robotic surgery. Very few centres in the world perform minimally invasive total endoscopic non robotic surgery. We hereby share our experience of the same.

\section{MATERIALS AND METHODS:}

We have performed minimally invasive non robotic mitral valve repair in 45 consecutive patients for mitral valve disease over a period of 2 years from 2016 to 2018. Peri-areolar incision in males and inframammary incision in females ranging from $2-3 \mathrm{~cm}$ were placed. Cardiopulmonary bypass was established with femoro-femoral and svc cannulation and a good myocardial perfusion was achieved.

\section{RESULTS:}

Of the 45 patients, average post operative stay was 3 days. Complications were seen in 3 patients primarily related to peripheral cannulation guide wire injury such as SVC injury and RV perforation which was managed by converting to lower midline hemisternotomy. In one patient, the periareolar incision was extended in view of lung hyperinflation for adequate exposure. One patient had severe MR on followup due to AML perforation which was reoperated.

\section{CONCLUSION:}

A totally endoscopic minimally invasive non robotic surgery is a state of art procedure which offers the patient a significant reduction of surgical trauma, is associated with limited post operative pain and accelerated recovery. However, it requires a steep learning curve and a prolonged operative and cardio-pulmonary bypass time. To conclude, it is painful for the surgeon but painless for the patient.

\section{Experience of first 126 cases with Minimally Invasive} Cardiac Surgery

S.S. Padhi; Arun Andappan; Alok Swain;

Nitin Kumar Rajput

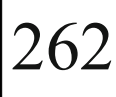

\section{ABSTRACT:}

\section{BACKGROUND:}

Minimally invasive cardiac surgery (MICS) has grown in popularity over the last decade. With the intentions to provide many benefits of minimal access surgery such as decreased pain, reduced surgical trauma and reduced fear of long sternotomy incision. Patience training and continuous learning ensures patients' safety and confidence with the minimally access surgery

\section{METHODS:}

One hundred twenty six (126) cases were operated between October 2016 to November 2018. All cases are included in this study. All patients were counselled about MICS, possibility of conversion and given a choice before admission between MICS \&amp; sternotomy.

\section{RESULTS:}

Out of 126 MICS cases 57 MICS Coronary Artery Bypass Graft (CABG )with mean age 59.6 years, 33 MICS Atrial Septal Defect (ASD) repairs, 24 MICS Mitral Valve Replacement (MVR), 12 MICS Aortic Valve Replacement (AVR), 1 MICS Subaortic Membrane Excision were done. All CABG done off pump on beating heart. Number of grafts done were one in 22 cases, two grafts in 23, three in 11 and four grafts in one patient. Cross clamp time for ASD closure, MVR and AVR were $30.5 \mathrm{~min}, 49.1 \mathrm{~min}$ and $46.8 \mathrm{~min}$ respectively. There is no in-hospital or early death. No conversion to sternotomy. Average blood transfusion 0.7 units per case. The average stay in ICU 2.3 days with average post op hospital stay of 5.7 days. One patient was re-explored to fix paravalvular leak of mitral prosthesis, one re-exploration for bleeding in CABG. One patient had surgical site wound infection after $\mathrm{CABG}$.

\section{CONCLUSION:}

Minimally invasive procedures can provide better outcome in terms of reduced bleeding, reduced pain, better cosmesis and reduced hospital stay. However, careful patient selection is important in the early phase to have successful outcomes.

\section{Sutureless Aortic Valve-A surgical Boon in challenging cases \\ Ratna Malika Kumar; \\ Rajneesh Malhotra; Anuj Sangal}

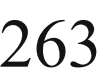

\section{ABSTRACT:}

In the last few years, surgical prostheses have been developed to incorporate sutureless fixation.

We demonstrate the efficacy and usefulness of these sutureless valves in challenging patients who are undergoing surgical AVR. We are presenting a case series of six patients who underwent successful sutureless aortic valve implantation. These were all high risk, multiple comorbidity surgical patients who benefitted with all the advantages of surgical AVR (vs TAVI), like complete excision of the diseased valve.

Sutureless aortic valves facilitate easier implantation by minimal invasive approach and also promise better clinical outcome in combined procedures with lesser CPB and aortic cross clamp timings. They have lesser gradients than standard tissue valves and benefit especially in patients with small and/or calcified roots.

All of the patients had an uncomplicated procedure and did well in intraoperative, peri-operative and post-operative phases and outcome was same as in conventional SAVR patients.

Cold-inducible RNA-binding protein as a novel target to alleviate blood-brain barrier damage induced by cardiopulmonary bypass

Bingyang Ji; Mingyue Liu

ABSTRACT:

\section{BACKGROUND:}

Hypothermia provides neuroprotection and alleviates cerebral injury after cardiopulmonary bypass (CPB). The mechanism of cold-inducible RNAbinding protein (CIRP), which has been reported to be facilitated by hypothermia and act as a critical regulatory protein in the brain, remains unclear in CPB. Hence, the role of CIRP on hypothermia CPB-induced brain injury was investigated in a rat model.

METHODS:

Cirp-/- rats were generated using the transcription activator-like effector nucleases (TALEN)-based genome editing technique. The animals were 
randomly allocated to three groups ( $\mathrm{n}=5$, each group): sham group, $\mathrm{CPB}$ group, and $\mathrm{CPB}+\mathrm{Cirp}-/-$ group (Cirp-/- group). Three biological replicates received RNA sequencing in the CPB and Cirp-/- groups. The relative protein expression of the hippocampus was detected. The integrity of the blood-brain barrier (BBB) was measured by transmission electron microscopy and IgG immunostaining. Glial fibrillary acidic protein (GFAP) in serum was detected. The brain was fixed for histopathological assessment.

\section{RESULTS:}

More differentially expressed genes (DEGs) of BBB leakage were clustered functionally by GO and KEGG pathway analyses. TGF- $\beta 1$, MMP-9, TNF- $\alpha$ and malondialdehyde in the hippocampus were higher in the Cirp $-/-$ group, while IL-4 level was opposite. Furthermore, more serious BBB disruption in the Cirp-/- group was demonstrated by transmission electron microscopy and IgG extravasation. Moreover, Cirp-/- enhanced the tight junction protein degradation and histopathologic injury in the hippocampus (pathological score, surviving hippocampal neurons, and TUNEL-positive neurons). Therefore, CIRP significantly alleviated neurologic injury.

\section{CONCLUSION:}

CIRP exerted important neuroprotective effect by alleviating BBB breakdown which might be associated with TGF- $31-M M P-9$ signals in hypothermia CPB.

\section{The factors influencing the satisfaction} with the treatment of cardiosurgical patients

Vladimir Shvartz; Olga Bockeria; Maria Sokolskaya

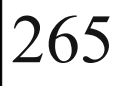

\section{ABSTRACT:}

To assess the quality of work of middle and junior medical staff to identify the key factors in their job that affect the level of patients' satisfaction inside the clinic.

\section{METHODS:}

All patients right before their discharge had been asked to complete a specially designed questionnaire, which was voluntary and anonymous. The questionnaire included the information relating to the quality of work of middle and junior medical staff. It also contained the general information about the patient such as sex, age, education, etc.

RESULTS:

There had been analyzed 1105 questionnaires. The frequency of refusal to complete the questionnaire was $4 \%$. The average age of the patients was $56(44 ; 64)$ years.

According to patients' opinion, the nursing staff treated them politely and respectfully in 59\% - "always", 28\% - "usually", 13\% - "sometimes".

Patients had worse evaluated the work of the junior medical staff. Only $76 \%$ of the patients gave a positive feedback about their work, such as "excellent" estimated $50 \%$ of patients and "good" only $26 \%$ of patients. A detailed analysis of the issues concerning the junior medical staff showed that the questions about the accompanying the patient to the toilet and using bedpans, had a direct correlation with the assessment of the work of junior medical staff $(r=0,77)$, nurses $(r=0,73)$. This issue also correlated with an evaluation of the work of the Department generally $(r=0.68)$, as well as with the question "Would you recommend this department to your friends?" $(\mathrm{r}=0.6)$.

\section{CONCLUSION:}

The most important issue in the quality of the care appeared to be the work of nurses and junior medical staff with patients, especially in the early postoperative period, when patients are in a limited mobility and social insecurity.

Trials, Toils and Triumphs - A Surgeon's Experience in Establishing a Cardiac Center in Semi-Rural India M. Vinod Kumar

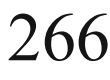

\section{ABSTRACT:}

\section{INTRODUCTION:}

The burden of cardiac disease in the India continues to rise at similar rates among urban and rural populations, but access to specialized health care remains inadequate in rural areas. Provision of comprehensive, costeffective and sustainable specialized cardiac services at a single center within the reach of the deprived is a challenging exercise. We present our experience in setting up a cardiothoracic surgery unit at a semi-rural location.

\section{AIMS AND OBJECTIVES:}

To present the challenges we faced, lessons we learnt in establishing a cardiothoracic surgery unit at Sri Narayani Hospital and Research Centre, Vellore, Tamil Nadu; and to share our vision for the future.

\section{MATERIALS AND METHODS:}

The basic structure, resources, processes and protocols required to set-up a state-of-the-art cardiothoracic unit were reviewed in detail. Patient data pertaining to the pre, intra and post-operative periods were also analyzed to understand the efficiency achieved and the limitations faced in providing appropriate care.

\section{RESULTS:}

About 150 cardiothoracic surgeries were performed in a year by a single surgeon, the details of which are presented. The spectrum included complex congenital, beating heart total arterial revascularization, minimally invasive valve replacement and lung surgeries. The most challenging aspects were encountered in training staff, establishing protocols, team building and creating patient awareness.

\section{DISCUSSION}

Cardiothoracic surgery is a labor-intensive but highly rewarding specialty. In order to gain proficiency to run an independent set-up, we must utilize our residency period to focus not only on developing surgical skills, but also on understanding the nuances of administration and equipment, strengthening interpersonal relationships and seeking the wisdom of colleagues and mentors.

\section{CONCLUSIONS:}

Establishment of a comprehensive cardiothoracic surgery unit needs extensive planning, rigorous quality-control measures and a dedicated team, while prioritizing patient safety above all else.

Correlation of intraoperative renal near infrared spectroscopy with the development of acute kidney injury in adult patients undergoing cardiac surgery with cardiopulmonary bypass Shakuntala Basantwani; Vijay Shewale

\section{ABSTRACT:}

\section{BACKGROUND:}

Cardiopulmonary bypass is known to cause renal damage; if total CPB time \&gt;140 min, and mean perfusion pressure is below $60 \mathrm{mmHg}$ and both increase the risk of developing Acute Kidney Injury(AKI). During $\mathrm{CPB}$, macro and micro embolic insults to the kidney, release of catecholamines and inflammatory mediators, increases in vascular resistance and decrease in glomerular filtration rates of $25-75 \%$, all lead to risk factors. Renal NIRS which non invasively monitors site specific adequacy of perfusion of kidney directly beneath its sensors can be employed for earlier detection of reduced renal perfusion leading to AKI.

\section{METHODS:}

Aim of this study was to correlate renal oximetry values with conventional biomarkers non-invasively for early detection of AKI in adult patients undergoing cardiac surgery with cardiopulmonary bypass. In this way renal NIRS can be used as real time device to prevent AKI.

INVOS (In-Vivo Optical Spectroscopy) oximeter system adult sensor will be applied on right side of spine at the lower border of L-1 (Transpyloric plane)(crossing of hilum of the kidney) before starting the induction.

We enrolled 100 adults patients undergoing cardiac surgery with cardiopulmonary bypass from april 2018 to july 2018. Renal NIRS was continuously measured intraoperative and was correlated with renal biomarkers and urine output post operatively.

RESULTS:

22 patients $(22 \%)$ developed AKI during this study. Out of these 21 (95\%) patients had fall in renal oximetry values compared to non AKI 
patients ( $\mathrm{p} \& \mathrm{lt} ; 0.05$ ). 3 patients needed dialysis in AKI group. 2 patients died in AKI group and 1 patient died in non AKI group.

\section{CONCLUSION:}

This prospective study has shown the renal NIRS to be valuable real time device for renal oximetry saturation values with perfusion parameters to correlate with $\mathrm{AKI}$ in post operative period and will help to prevent or reduce AKI.

Global cardiac surgery: access to cardiac surgical care around the world Bart Meuris; Dominique Vervoort

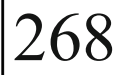

\section{ABSTRACT: \\ OBJECTIVE:}

Cardiovascular disease is the leading cause of death worldwide, responsible for 17.5 million deaths every year, of which $80 \%$ occur in low- and middle-income countries (LMICs). $75 \%$ of the world does not have access to cardiac surgery when needed, due to lack of infrastructure, human resources, and financial coverage. This study aims to map access to cardiac surgical care around the world.

\section{METHODS:}

A scoping review was done on access to cardiac surgery for an undifferentiated population. Workforce data was collected from the Cardiothoracic Surgery Network (CTSNet) database and used to calculate numbers and ratios of adult (ACS) and pediatric cardiac surgeons (PCS) to population.

\section{RESULTS:}

12,180 ACS and 3,858 PCS were listed in CTSNet in August 2017, equaling $1.64(0-181.82) \mathrm{ACS}$ and $0.52(0-25.97)$ PCS per million population globally. Large disparities existed between regions, ranging from 0.12 ACS and 0.08 PCS per million population (sub-Saharan Africa) to 11.12 ACS and 2.08 PCS (North America). Low-income countries possessed 0.04 ACS and 0.03 PCS per million population, compared to 7.15 ACS and 1.67 PCS in high-income countries (HICs).

\section{CONCLUSIONS:}

This study maps the current global state of access to cardiac surgery. Disparities exist between and within world regions, with a positive correlation between nations' economic status and access to cardiac surgery. Low early mortality rates in low-resource settings suggest the possibility of high-quality cardiac surgical care in LMICs. Policies should focus on increasing human and physical resources to improve access to cardiac surgery for the 4.5 billion people without.

The predictors of prognosis after pericardiectomy for chronic constrictive pericarditis; A single-center 38 patients experience.

Shuichiro Takanashi; Takahide Yoshio

\section{ABSTRACT:}

The purpose of this study was to evaluate the outcome of pericardiectomy for chronic constrictive pericarditis. The main objectives were long-term survival, recurrent of congestive heart failure, echocardiographic outcome, and identification of risk factors.

Previously, some literature reported the predictors of mortality and complication after pericardiectomy, which were small left ventricular dimension, high E velocity, use of cardiopulmonary bypass, and etiology of radiation or post-cardiac surgery.

We enrolled 38 consecutive patients who underwent pericardiectomy with or without concomitant other cardiac surgery at Sakakibara Heart Institute between 2006 and 2017.

Of these 38 patients, 11 (29.0\%) died during follow-up and 7 (18.4\%) were cardiac related death. The 11 deaths included 3 perioperative deaths and all of them were cardiac related death.

We generally try to achieve total pericardiectomy defined as radical phrenic to phrenic excision during pericardiectomy. Therefore, in the result, we could improve left ventricular end-diastolic volume (LVEDVI) at a year after operation than before operation (54.6 \pm 12.8 $\mathrm{ml} / \mathrm{m} 2,46.2 \pm 14.2 \mathrm{ml} / \mathrm{m} 2: \mathrm{p}=0.03$ ).

The 5-year overall survival was $65.4 \% \pm 9.6 \%$, and freedom from recurrent heart failure rate was $65.5 \% \pm 9.1 \%$. Univariate Cox regression analysis showed that small LVESVI, high E velocity, and high E/e' were significant predictors of mortality. Multivariate Cox proportional hazard analysis revealed that pre-operative E/e' was independent predictor of mortality after pericardiectomy (HR 1.137; 95\% CI, 1.035 to $1.249 ; \mathrm{p}$ \&lt; 0.01). Receiver operating characteristic curve analyses showed that the best cutoff value for pre-operative E/e' in predicting all-cause death after pericardiectomy was 7.1 (sensitivity of $81.8 \%$ and specificity of $59.3 \%$ ). There was a significant difference in survival between the two groups which was divided by the pre-operative E/e' 7.1 (p \&lt; 0.01). Pericardiectomy for chronic constrictive pericarditis presented high longterm mortality. Although this is the small number study, we could indicate a new predictor for mortality.

Study of effects of different weaning strategies for IABP Chaitanya Raut; Vaibhav Shah

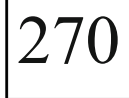

\section{ABSTRACT:}

IABP (Intra aortic counterpulsation Balloon Pump) currently represents the first-line temporary left ventricular assist device in patients with low cardiac output syndrome (LCOS), difficulties in weaning from cardiopulmonary bypass etc. Commonly used IABP weaning methods are reduction in rate ( most commonly used), volume and a combination of both. However, scanty data exist outlining optimal weaning protocols to optimize cardio circulatory functional restoration during IABP discontinuation. We aim to study the effects of weaning strategy of IABP on patient hemodynamics and determine an optimal strategy for weaning

\section{MATERIAL AND METHODS:}

The study group consisted of 124 patients who required an intra- or postoperative IABP because of LCOS refractory to inotropic or volume support at our institute from Jun 2015 to Oct 2018. The groups were divided according to the weaning strategy of IABP. 28 patients were weaned without any preset protocol, 58 by rate reduction (frequency), and 38 by a combination of volume (augmentation) followed by rate reduction. The data compared include the need for increment inotropic support, LCOS and the need for re-institution of IABP after withdrawal, acute myocardial infarction (AMI), interval from IABP withdrawal to ICU discharge and hospital mortality and were compared between the groups.

\section{RESULTS:}

LCOS after withdrawal, need for inotropic increment and IABP reinsertion was more common in those patients with no preset weaning policy. LCOS and inotrope increment was more in rate reduction alone as compared to volume-rate reduction group while IABP reinsertion was similar in rate and volume-rate reduction group. The vol-rate reduction group had lesser ICU stay after IABP withdrawal and quicker discharge from ICU than other groups. Mortality rates were comparable.

\section{CONCLUSION:}

The study shows that regardless of the weaning strategy, patient outcome following IABP removal with a documented policy had better outcomes. The volume followed by rate reduction had better outcome to rate reduction alone.

Challenges and solutions in establishing a new cardiac surgical unit. ( Brig) Anurag Garg; Ashish Dolas

ABSTRACT:

With the healthcare delivery system going through a major transition to ensure quality healthcare delivery at all levels a new paradigm appears to 
be emerging for establishment of tertiary care centers for the biggest killers of the future for heart diseases.

To start a new cardiac surgical unit with help and guidance of higher authorities and hospital management is definitely a new challenge.

Right from deciding an OPD chair to set up a Cardiac Recovery unit and ICU to recruitment of staff was a transforming and creating experience.

\section{Cardiac surgery in octogenarians and nonagenarian - single center experience Clifford Barlow; Sayed Sadeque}

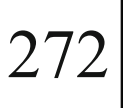

\section{ABSTRACT: \\ INTRODUCTION:}

The significant rise in life expectancy and advancement in medical therapy has contributed to more octogenarian and nonageneraian presenting with cardiac disease requiring complex cardiac surgery.

The aim of this study was to review our experience of patients above the age of 80 who underwent cardiac surgery; including major aortic surgery such as acute aortic dissection and compare our outcomes.

\section{METHODS:}

Our retrospective collected data was reviewed to identify patients who were above the age of 80 and underwent major cardiac surgery from 2000 to 2017 ( $n=3090)$. The data was analyzed to find out the morbidity and mortality.

\section{RESULTS:}

We analysed 3090 patients (octoagenarian $n=3039$ \&amp; nonagenarian $\mathrm{n}=51)$. Majority of patients were male $(58 \%)$ with median age of 83 years. Their co-morbidities included hypertension (64\%), LV impairment (Moderate-28\%; severely impaired-6\%), and previous neurological event $(10 \%)$, diabetes $(16 \%)$. The mean logistic Euroscore was $0.14 \pm 0.13$. The priority of surgery were elective $55 \%$, urgent $43 \%$ and emergency $2 \%$, The 30 day in-hospital mortality for the whole cohort was $5 \%(n=157)$. The mortality of elective patients was $3.5 \%$, urgent cases were $4.5 \%$ and emergency cases were $23 \%$. The mean ICU stay was $13 \pm 10$ days. Postoperative complications included stroke $2 \%$, renal failure $(10 \%)$, wound infection (1.1\%), neurological events (2\%), and PPM (4\%). CONCLUSION:

The octogenarians and nonagenarian should be considered for cardiac surgery. Despite a slight increase in in-hospital mortality compared to national average, the postoperative complications remain comparable. The use of frailty index in patient selection should improve outcome.

Cardiac Surgery in South Asia as of 2018

Dr.Farzana Amin, Dr.Nazmul Hosain

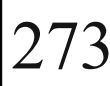

\section{ABSTRACT: \\ INTRODUCTION:}

The South Asian nations namely Afghanistan, Bangladesh, Bhutan, India, Nepal, Maldives, Pakistan and Sri Lanka collectively possess an area of 5.1 million square miles. This area is inhabited by a huge population of 1.8 billion. It is the most densely populated part of the World. South Asian countries have many historic, cultural, ethnic and genetic links. This brings the South Asian population together in war and peace, in disease and in treatment.

\section{METHODS:}

Record keeping is not well organized in this part of the World. It is often quite difficult to find authentic, genuine information. The best data often comes from an unusual source like Anesthetists' society or corporate houses. Thorough search of the printed and electronic materials has been made. The authors visited all these countries and contacted the eminent surgeons personally or through mails. All information is cross-checked and then compiled.

RESULTS:

Six of these South Asian countries namely Afghanistan, Bangladesh, Nepal, Pakistan and Sri Lanka have their cardiac surgical program.
Collectively they performed around 190000 cardiac operations in 2017, which is expected to cross two hundred thousand mark in 2018. India performs the highest number of cardiac operations in the region followed by Pakistan, Bangladesh, Sri Lanka, Nepal and Afghanistan in that order. CONCLUSION:

The number of cardiac surgical procedures in South Asia is increasing every year. Despite qualitative excellence South Asia is lagging behind the World standards in the numerical terms and has to make improvement to cater almost a quarter of the World population living here.

The Turbulent Journey from

Cardiac Surgery to Surgical Cardiology

Farzana Amin; Nazmul Hosain

\section{ABSTRACT: \\ INTRODUCTION:}

Cardiac surgery is one of the most difficult and sophisticated branches of medical science. Once thought an impossible task by many, cardiac surgery became a reality thanks to a series of inventions and innovations during the 1950s. This noble subject has probably reached its peak and now likely to take a downward numerical trend in future due to continued rapid expansion of medical and intervention cardiology. This might jeopardize the career of the cardiac surgeons in future. They might have to mold themselves to a different role.

\section{METHODS:}

The historic events of the development of Cardiac Surgery and Invasive Cardiology were explored. The trend of change in the number of cardiac surgery and Cardiac intervention was studied. Thorough search of the related printed and electronic materials has been made.

\section{RESULTS:}

The arena of Invasive Cardiology is rapidly expanding. Difficult newer coronary interventions are being practiced by the cardiologists. New trans-apical and trans-venous valvular devices are being introduced. The use of various percutaneous devices to close various congenital defects is increasing. The newer pharmacological tools are arriving to improve the scope of treatment. Al these are shrinking the area of Cardiac Surgery.

\section{CONCLUSION:}

The new technological developments in the area of pharmacological treatment and intervention cardiology are gradually reducing the area of Cardiac Surgery. The number of cardiac operations required is likely to be grossly reduced in next two decades. The cardiac surgical procedures would be much fewer in number but much difficult to perform. There would be requirement of a few surgeons with very high skill. The new generation of surgeons should acquire cathlab and wire skill as well as efficiency in minimal invasive surgery. They have to adopt themselves through a difficult journey from Cardiac Surgery to Surgical Cardiology.

The measurement of Ionized Magnesium for Personalized Care in Critically ill Cardiac Patients NA; Charbel Abou-Diwan

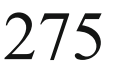

\section{ABSTRACT:}

Dysmagnesemia is common in critically ill patients and is associated with poor clinical outcomes. Hypomagnesemia is associated with several clinical conditions, ranging from cardiovascular disease to respiratory disorders and sepsis. Magnesium sulfate therapy is often used to correct hypomagnesemia but if administered without monitoring patient magnesium levels can lead to hypermagnesemia and poorer patient outcomes. Traditionally, total magnesium $(\mathrm{tMg})$ measured in the laboratory is used to identify patients with dysmagnesemia. However this delays the clinical management of critical care patients. Several studies have shown that iMg is more reliable than $\mathrm{tMg}$ for identifying and managing dysmagnesemia. As iMg is now available as a point of care test which can be used in conjunction with iCa this will benefit the management of these important 
cations in critically ill patients. Data will be presented on the clinical utility of the measurement of ionized magnesium, and the correction of ionized hypomagnesemia in cardiopulmonary bypass patients.

\section{Pulmonary thromboendarterectomy} experience across 15 years from a single centre Varun Shetty

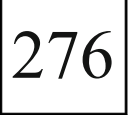

\section{ABSTRACT: \\ INTRODUCTION:}

Pulmonary thromboendarterectomy(PTE) is the potential and curative treatment option for pulmonary hypertension with chronic thromboembolic pulmonary hypertension(CTEPH). Patient selection and center experience are important for good outcomes. Here we present our PTE experience across 15 years from a single center.

\section{METHODS:}

We analyzed retrospective data of 493 patients who underwent PTE at our hospital between January-2004 and October-2018. We included patients' basic demographics, preoperative assessment, operative, and postoperative details and clinical outcomes. The statistical difference between preoperative and post-operative characteristics was compared by t-test.

\section{RESULTS:}

Out of 493 patients, 357 (72.4\%) were males. The mean age was $39.8 \pm 11.5$ years, $86 \%$ of patients survived to discharge after endarterectomy. The overall in-hospital mortality rate was $13.8 \%$. Mortality from 2004 to 2011 was $17.2 \%$ which has reduced to $11.4 \%$ in the later period (2012 to 2018). The reduction in mortality rate was $33.7 \%$ (RR 1.515 ; $95 \%$ CI 0.9754 to $2.3537, \mathrm{p}=0.06$ ). Since then our team has improved on the case selection, surgical technique, identification, and prevention of complications. After endarterectomy, there was a significant reduction in PASP $(85.91 \pm 24.2$ vs $39.27 \pm 12.7 \mathrm{mmHg}$; p\&lt;0.001) and significant improvement in 6 minutes' walk test $(259.6 \pm 141.1$ vs $449.0 \pm 201.4$ meters; p\&lt;0.001). Fifty-seven (13.6\%) patients required ECMO following PTE, the major indication for ECMO was reperfusion edema ( $44 \%$ of cases).

\section{CONCLUSIONS:}

PTE is a complex operation requiring expertise at every stage. A dedicated multidisciplinary team to CTEPH is crucial for good outcomes. Our experience is the largest in the country, currently performing an average of 40 cases per year. There is a statistically significant reduction in PASP and inhospital mortality, and a significant improvement in the six minutes' walk test, over the course of 15 years. This could be attributed to better case selection and improved handling of surgical complications.

\section{A Long-term Results of the Surgical \\ Treatements for Popliteal Venous Aneurysm \\ Makoto Mo; Naoki Hashiyama; Shinobu Matsubara; \\ Hiroko Nemoto; Kenichiro Aga; Yoshiyuki Kobayashi; \\ Yasuko Onakatomi}

\section{ABSTRACT: \\ BACKGROUND:}

Popliteal Venous Aneurysm (PVA) is localized dilation of venous without regurgitation. PVA occurs comparatively rare, however we recently encounter PVA more frequently, partly because of advancement of imaging technology. Because the prognosis of PVA after surgery is still unclear, we analyzed the cases of PVA with surgical treatment.

\section{METHODS:}

This is a retrospective analysis. We reviewed the case-records from 2004 to 2017 in our institution.

\section{RESULTS:}

We experienced 14 PVA cases which had been performed surgical treatments. Mean observation period is 72 months (10-167months). Mean age was 59 years old (18-77 years old). Eight cases were women. There was a case of bilateral. Tangential resection and aneurymorrhapy were underwent in 7 cases. Plication of PVA were performed in 3 cases. Resection and panel graft reconstruction with great saphenous vein (GSV) were done in 3 cases. A ligation for popliteal vein for long segment PVA was underwent in one case. In term of anticoagulants after operation, we used warfarin to 9 cases, edoxaban to 3 cases and rivaroxaban to 2 cases. Perioperative asymptomatic DVT was complicated in four cases. In remote period, we have 4 cases of PVA dilation. Two cases complicated with symptomatic PE. Re-operations was performed in three cases, one ligation and two panel graft reconstruction. DISCUSSION:

Our surgical results of PVA were satisfactory. However, carefully followup of PVA by duplex scan is required even after the surgery, because recurrences of PVA and PE may occur.

Juxta Renal Aortic Occlusion With Critical Limb Ischemia [JRAO] - Role of extra anatomical bypass Murugan. M.S; Krishnanand Pai; Ganesan. C; P.R. Murugesan

\section{ABSTRACT: BACKGROUND:}

Juxta renal aortic occlusion with critical limb ischemia demands supra renal aortic clamping and athero endarterectomy for aorto bi-iliac / femoral reconstruction. Heavily diseased infrarenal aorto requires an alternative surgical approach, we like to present a series of cases who had thoracic aorto bi femoral/ iliac bypass for JRAO with critical limb ischemia.

\section{MATERIALS AND METHODS:}

at PSGIMSR, Coimbatore from 2005 to 2018

Total number of 5 cases (Male: Female- 4:1 ), 4 chronic and 1 acute of JRAO. Analyzed retrospectively,

1 had septic abdomen secondary to Right Ilio psoas abscess and underwent Thoracic aortic Left femoral - cross over bypass, thoracic aorto bi-iliac bypass in 3, thoracic aorto bifemoral in 1 .

RESULT:

No operative mortality, No renal failure. All the 5 cases immediate recovery was uneventful. Graft patency over 6 months -Good. Follow up satisfactory on anti- platelet alone.

\section{CONCLUSION:}

Thoracic aorto bi-iliac/ femoral bypass on novel approach as on extra anatomical bypass. The long term patency was good because of the high pressure head and the proximal nature of the distal anastomosis in comparative axillo-femoral bypass.

Surgical managment of functional tricuspid insufficiency with leading aortic stenosis

Skopin I.I.; Latyshev M.S.

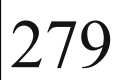

\section{ABSTRACT: \\ OBJECTIVE:}

Determining treatment methods of patients with functional tricuspid regurgitation (FTR) due to aortic stenosis (AS).

\section{MATERIAL AND METHODS:}

Based on the study "A.N. Bakulev National Medical Research Center of Cardiovascular Surgery", a multi-stage study of patients with FTR due to AS was developed. During the 1st stage, a retrospective analysis of the results of correction of FTR in patients with isolated AS performed in the last 11 years $(n=101)$ was analyzed. In The 2 nd stage, the prospective comparative analysis of preoperative parameters of $2 \mathrm{D}$ and $3 \mathrm{D}$ echocardiography and intraoperative measurements of the diameter of fibrous ring $(\mathrm{FR})(\mathrm{n}=35)$ were analyzed.

\section{RESULTS:}

Based on the results of the 1st study, the following predictors were identified: the diameter of the FR of the TV $\geq 35.25 \pm 2.37 \mathrm{~mm}$, the pressure in the PA $\geq 32.50 \pm 8.47 \mathrm{~mm} \mathrm{Hg}$, atrial fibrillation, mitral insufficiency $\geq$ 2nd degree. Prospective comparative analysis showed significant differences between TT 2D echocardiographic parameters of the FR of the TV 
and intraoperative measurements, as well as the differences in the diameter of FR of TV. When comparing the obtained data, a statistically insignificant difference between $3 \mathrm{D}$ echocardiography and intraoperative measurements was obtained $(\mathrm{p}-0.1)$.

\section{CONCLUSION:}

1. According to the results of the study, the main predictor of FTR was annuloectasia. 2. Patients with a diameter of the FR of the TV from 35 to $40 \mathrm{~mm}$ (2D echocardiography) should perfor 3D echocardiography of the $\mathrm{TV}$ to clarify the tactics of surgical treatment. If it is not possible to perform 3D echocardiography, taking into account the possible progression of TI and high lethality during reoperations, we recommend performing preventive correction of the TV. 3. We recommend performing TV annuloplasty in the presence of risk factors for the progression of TI.

The choice of rigid ring annuloplasty for functional tricuspid regurgitation: contour $3 \mathrm{~d}$ ring or mc3 ring? Abu Yamin; Lok Yuh Ing

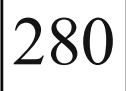

\section{ABSTRACT:}

\section{OBJECTIVE:}

We evaluated the outcomes of rigid ring tricuspid annuloplasty for functional tricuspid regurgitation by using Medtronic Contour 3D ring compared with Edwards MC3 ring in Penang General Hospital.

\section{METHODS:}

A retrospective review of patients with functional tricuspid regurgitation who underwent tricuspid annuloplasty by using Medtronic Contour 3D ring and Edwards MC3 ring from January 2014 to April 2018 was performed. RESULTS:

Medtronic Contour 3D rings were used in 43 patients whereas Edwards MC3 rings were used in 47 patients. Echocardiographic evaluation of tricuspid regurgitation was performed preoperatively and postoperatively in follow-up schedule. The degree of tricuspid regurgitation was reduced from $2.86 \pm 0.46$ to $0.02 \pm 0.15$ (regurgitation severity grade: 0 to 3 ) in the patients with Medtronic Contour 3D rings at discharge. It was from 2.68 \pm 0.70 to $0.06 \pm 0.24$ in the patients with Edwards MC3 rings. At thirty six months postoperative period, tricuspid regurgitation grades in patients with Medtronic Contour 3D rings and Edwards MC3 rings were 0.09 \pm 0.29 and $0.31 \pm 0.54$, respectively. Freedom from recurrent tricuspid regurgitation (grade 2 or 3 ) in patients with Medtronic Contour 3D ring and Edwards MC3 ring were $100 \%$ and $95.7 \%$, respectively.

\section{CONCLUSION:}

Both rigid rings provide low rate of recurrent tricuspid regurgitation. However, Medtronic Contour 3D ring might be more effective than compared with Edwards MC3 ring for decreasing functional tricuspid regurgitation in mid-term postoperative periods.

\section{Mid-term results of the Cingular Bovine Pericardium} heart valve and Carpentier-Edward Perimount valve in the aortic valve replacement

Yu Shi; Yao Wang

\section{ABSTRACT: \\ BACKGROUND:}

We compared valve replacement in aortic position using a trileaflet bioprosthetic heart valve manufactured by Shanghai Cingular Biotech Corporation, with a prosthesis manufactured by Edwards Lifesciences in patients with severe aortic stenosis and an increased risk of death during surgery.

\section{METHODS:}

From 2016 to 2017,425 patients underwent aortic valve replacement using bioprostheses artificial valves, such as the Cingular Bovine Pericardium heart valve, Carpentier-Edward Perimount, Hancock II and Epic were enrolled in the study. Among them, the outcomes of the Cingular Bovine Pericardium heart valve $(n=75)$ and the Carpentier-Edward Perimount $(\mathrm{n}=128)$ were reviewed retrospectively. Mean age at implant was $67.0 \pm 5.5$ and $65.8 \pm 4.2$ years in the male and female patient groups, ranging from 59 to 84 and 59 to 78 , respectively. The overall duration of follow up was $16.1+/-2.9$ months.

RESULTS:

The overall survival and re-operation free rate was $95.3 \%$ and $97.8 \%$ respectively. There was no re-operation required for the 75 Cingular Bovine Pericardium heart valve patients over 14.6+/-2.1 months. There was no statistical significance in the re-operation free rates between these 2 valves ( $p$-value $=0.51$ ). Preoperatively $89.9 \%$ of patients scored for New York Heart Association(NYHA) at class 3 or 4; at 30 days, post-surgery a decrease to $5.2 \%$ with an accompanying increase for class 2 from $10.1 \%$ to $91.2 \%$. These outcomes are consistent over time.

\section{CONCLUSIONS:}

The overall survival rate associated with two kinds of bioprosthetic heart valve was acceptable, and these preliminary clinical results indicate satisfactory clinical and hemodynamic outcome for the Cingular Bovine Pericardium heart valve.

The role of mitral valve plasty in Mitra-clip era - through our cases

Takashi Oshitomi; Shizuya Shintomi

\section{ABSTRACT: \\ BACKGROUND:}

Recently, endovascular treatment develops increasingly. The EVERESTIIrandomized trial evaluated the safety and efficacy of percutaneous mitral valve(MV) repair using the Mitra-Clip device relative to standard MV surgery, but it was less effective at reducing mitral regurgitation(MR). Our aim is to identify strategy of MR through our cases.

\section{PATIENTS:}

We enrolled 136 patients undergoing primary MV plasty from January 2012 to March 2018.

\section{METHODS:}

We analyzed our data according to the way described by the EVERESTII. Primary efficacy end points at 12 months were death, surgery for MV dysfunction, and higher than moderate MR. We categorized risk as low, mean, mean-high, high, and very high by estimating EuroSCOREII. We'll discuss which procedure is better to repair in each risk.

\section{RESULTS:}

In low risk, the result was perfect. Between mean and mean-high risk, 7\% were reoperated, but no patient died and surgery reduce MR below moderate to severe in $97 \%$. It was excellent result compared with Mitra-Clip. Between high and very high risk, $11 \%$ were died.

\section{CONCLUSION:}

We should choice surgical operation to repair MR except high or very high risk cases.

Strategy of aortic valve replacement in hemodialysis patients in valve-in-valve era

Hideyuki Uesugi; Shizuya Shintomi

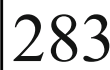

\section{ABSTRACT:}

\section{BACKGROUND:}

Hemodialysis patients(HD-P) are prone to aortic valve stenosis(AS). There is no obvious guideline about AS of HD-P. Our aim to identify strategy of AS in Valve-in-valve(VIV) era. PATIENTS:

We enrolled 36 HD-P undergoing aortic valve replacement from April 2009 to March 2018. 


\section{RESULTS:}

Between bioprosthetic valve and mechanical valve, univariate analysis(UA) showed that age, age of introduction of dialysis, hypertension were significant differences, but multivariate analysis(MA) showed that there were no significant differences. Between survival group and death group, both UA and MA showed that there were no significant differences. Prosthetic valve(PV) must be over $21 \mathrm{~mm}$ to use VIV in the future. Between over $21 \mathrm{~mm} \mathrm{PV}$ and under $21 \mathrm{~mm} \mathrm{PV}$, UA showed that gender, BSA, diameter of aortic valve, and diameter of STJ were significant differences, but MA showed that there were no significant differences. The prognosis of reoperation was poor. If possible, there's room for consideration to use over $21 \mathrm{~mm}$ PV to connect to the future VIV.

\section{CONCLUSION:}

We must choice PV depends on gender, BSA, and preoperative finding. But if possible, there's room for consideration to use over $21 \mathrm{~mm}$ PV to connect to the future VIV.

\section{Mitral valve repair for posterior leaflet prolapse: Fifteen-year experience}

Sachiko Yamazaki; Satoshi Numata; Tomoya Inoue; Satoshi Taniguchi; Tomohito Kanzaki; Kaichiro Manabe; Koki Ikemoto; Haruka Fu; Hitoshi Yaku

\section{ABSTRACT: \\ OBJECTIVE:}

The objective of this study was to clarify the long-term outcomes of mitral valve plasty (MVP) for posterior leaflet prolapse.

\section{METHODS:}

We retrospectively analyzed 158 patients (106 men; mean age $62 \pm 12$ years) with mitral regurgitation (MR) due to posterior leaflet prolapse who underwent MVP from 2003 to 2017. Functional mitral valve diseases (ischemic or atrial fibrillation) were excluded from our study. The long-term (median 79 months) results of reoperation for mitral valve was evaluated, and risk factors for recurrence of $\mathrm{MR}(\mathrm{MR} \geqq 2+)$ and mitral stenosis after MVP were analyzed.

\section{RESULTS:}

During the follow-up period, only one patient was performed reoperation for repaired mitral valve due to recurrence of MR. Kaplan-Meier analysis showed that 5- and 10-year freedom from reoperation were both $99 \%$. Follow-up echocardiography was performed for 120 patients, and 12 patients suffered from $\mathrm{MR} \geqq 2+$ at follow-up. According to univariable Cox-hazard model, use of artificial chorda $(\mathrm{p}=0.005$, Hazard ratio (HR) $5.50,95 \%$ confidence interval (CI) 1.67 - 18.1), concomitant tricuspid valve operation $(\mathrm{p}=0.004, \mathrm{HR} 5.95,95 \% \mathrm{CI} 1.76-20.2)$, preoperative left ventricular fractional shortening $(\mathrm{p}=0.031$, HR $0.92,95 \% \mathrm{CI} 0.85$ 0.99 ), and larger ring size devided by body surface area (ring size / BSA) $(\mathrm{p}=0.007$, HR $1.34,95 \%$ CI 1.08 - 1.66) were the risk factors for $\mathrm{MR} \geqq 2+$ at follow-up. Higher diastolic mean pressure gradient at follow-up was significantly correlated with smaller ring size $(\mathrm{p}=0.011, \mathrm{r}=-0.273)$.

\section{CONCLUSION:}

MVP for posterior leaflet prolapse provided excellent results. Larger ring size / BSA related to $\mathrm{MR} \geqq 2+$, and smaller ring predict higher mean gradient at follow-up.

\section{Original Technique of Artificial Chordae in Mitral Valve Repair \\ Alaaddine Yilmaz; Faouzi Safadi}

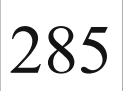

\section{ABSTRACT:}

OBJECTIVE:

The growing employment of different methods to create artificial chords has greatly contributed to make great number of Mitral Valve regurgitation responsive to repair rather than replacement. Despite this advancement, the common dilemma among these techniques remains how to determine the optimal length of neochordae. Our goal is to introduce new model of an adjustable neochordae, that we can modify the length by an unlimited, easy and smooth repositioning of the stitch handling the loop of the neochordae.

\section{METHODS:}

The use of neochordae reduces the need to large resections, therefore preserves more coaptation surface, hence a large effective orifice area. It is demonstrated that minimal variations in one chordae length can modify overall chordae tension. Chordae that are $10 \%$ longer than normal double the chordae tension, This means that even minor errors in length measurement can have dramatic consequences on the chordae tension. Our innovative neochordae is created in a simple loop model, having a single passage across the prolapsed leaflet and the papillary muscle, accordingly, the loop can rotate freely through the anchored tissues. The loop length modification is obtained by sliding down and up the stitch forming the loop "Dubai sliding-stitch". The decision of shortening or elongating the loop, to obtain optimal coaptation, is guided by the filling-water test. The mechanism of this artificial chord is inspired from the Neck-tie model.

\section{RESULTS:}

153 cases of Mitral repair using Dubai sliding-stitch, have been successfully performed, in six international centers, 145 cases were done with endoscopic and minimally invasive approach, 8 cases by sternotomy. The results are extraordinary satisfactory and promising, including Barlow's disease cases.

\section{CONCLUSION:}

This technique provides an easy, fast, accurate, and synchronized deployment of multiple independent simple loops, producing optimal coaptation with large effective orifice area.

Throughout this work, we encourage more surgeons to perform Mitral repair with more confidence.

\section{Early results of Rheumatic Mitral Valve Stenosis(MS)} repair in northern of Thailand

Jessada Methrujpanont; Sitichok Wachirasrisirikul

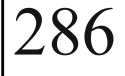

\section{ABSTRACT: \\ BACKGROUND:}

In patients with rheumatic mitral valve disease, the feasibility of repair or reconstruction of the deformed mitral valve and the progression of the underlying disease still challenging, and its quality, durability remain unknown. We evaluated the early outcomes of mitral repair focus on rheumatic mitral stenosis valve in etiology.

\section{METHOD:}

Retrospective data collection from January 2006 to August 2018, 141 patients underwent rheumatic mitral valve repair, 34 patients were caused by Mitral stenosis. Age ranged from 20 to 67 years, mean 50.70 years; 16 (47.58\%) patients were male. Mean preoperative NYHA was 2.61. mean preoperative right ventricular systolic pressure (RVSP) was 54.73 $\mathrm{mmHg}(10-141.07)$. 31(91.17\%) patients were in atrial fibrillation. Quality and durability of mitral valve configuration were assessed by echocardiography and clinically follow up.

\section{RESULT:}

The feasibility of repair rate was $32.69 \%$. Mean follow -up was 39.95 months $(0.75-137)$. The hospital mortality rate was 1 patient $(2.94 \%)$, there was 1 late deaths. 1(2.94\%) patient had redo mitral valve replacement due to technical failure. The initial postoperative echocardiography data result; MVA increase from 0.82 to $1.97 \mathrm{~cm} 2$, mean gradient decrease from 9.91 to $5.48 \mathrm{mmHg}$., RVSP decrease from 54.73 to $40.19 \mathrm{mmHg}$. The major adverse events; thromboembolic event, major bleeding event, rehospitalization were 0,2 patients $(6.45 \%), 1$ patients $(3.22 \%)$ respectively. CONCLUSION:

Rheumatic mitral stenosis valve repair was still challenging issue with lower feasibility rate than other etiology but the initial successfully 
operative repair has a satisfactory clinically follow up that might be the prime determinant of the expansion and reconsideration.

\section{FACTERS PREDICTING LEFT VENTRICULAR DYSFUNCTION AFTER MITRAL VALVE REPLACEMENT IN PATIENTS WITH RHEUMATIC MITRAL VALVE DISEASE. Vivek Wadhawa; Divyesh Rathod}

\section{ABSTRACT: INTRODUCTION:}

Rheumatic mitral valve disease remains one of the important heart diseases. There are many factors that influence the clinical outcomes, and little is known about how left ventricular (lv) dysfunction clinically affects the prognosis of the patient with rheumatic mitral valve disease after mitral valve replacement (MVR). We reviewed our clinical experiences of MVR in patients with rheumatic mitral valve

METHODS:

A single center retrospective observational study between January 2017 to July 2018, 458 patients with rheumatic mitral valve disease who underwent MVR were analysed and divided into three groups according to post operative ejection fraction (EF). 37 patients in Group $1(\mathrm{EF} \leq 30 \%)$ and 96 patients in group 2 (EF- $30-45 \%$ ) and 325 patients in group 3 (EF $\& \mathrm{gt} ; 45 \%$ ) were included.

\section{RESULTS:}

Operative mortality occurred in patients after MVR (group 1: $n=24,65 \%$; group $2: \mathrm{n}=3,3.1 \%$, group $3: \mathrm{n}=20.6 \%$ ) and its incidence was significantly higher in group 1 than in group 2 and 3 (p\&lt; 0.001). Preoperative LV dysfunction, atrial fibrillation, left atrial size, chordal preservation, severe $\mathrm{PAH}$, moderate to severe mitral regurgitation are main predisposing factors for it.

\section{CONCLUSION:}

This study identified predictable risk factors for post operative LV dysfunction post MVR in patients with rheumatic mitral valve disease after MVR, preventive care should be made in multiple ways, such as management of lv dysfunction, atrial fibrillation, and anticoagulation.

\section{Virtual Reality Image Analysis in Aortic Valve Leaflet Reconstruction \\ Kenichi Kamiya; Yuko Gatate; Tadamasa Miyauchi; Masaomi Fukuzumi; \\ Ryoi Okano}

\section{ABSTRACT: \\ PURPOSE:}

Aortic valve reconstruction with three same-sized autologous pericardial leaflets (AVLR) has been performed for patients with narrow aortic roots or contraindication for valve-prostheses. Since this procedure requires precise information of the aortic root including configuration of Valsalva sinus, we assessed physiologic-anatomical condition of the aortic root by Virtual Reality (VR) image in order to accomplish this technique with a reproducible fashion.

\section{METHODS:}

VR Imaging; Axial images using enhanced ECG-triggered cardiac CT with a slice thickness of $0.625 \mathrm{~mm}$ were obtained during mid-to-end diastole. Subtracted volume rendering data of the aortic root were converted to a $3 \mathrm{D}$ workstation. Basic surgical technique; 1) same-sized three leaflets from autologous pericardium were prepared by original templates referred by STJ diameter, 2) new commissures and nadirs were confirmed based on VR image in cases of unbalanced aortic root, like type 0 bicuspid valve, 3) three leaflets were sutured on cusp-suture-line, 4) commissure coaptation stitches were placed between each leaflet to prevent from minor leakage and coronary orifices obstruction.

\section{RESULTS:}

In 18 of 78 AVLR were evaluated by VR. In 11 cases, the Valsalva sinuses were unbalanced which required neo-commissure setting and adjustment of deviated nadir. Postoperative echocardiography revealed nicely opening of new-leaflet with no AR, although we had one case without 3D analysis who had taken down to AVR due to mild AR CONCLUSION:

VR image analysis had notably provides valuable information for understanding of precise anatomy of the aortic root for AVLR.

Results of reconstructive surgery of ischemic mitral regurgitation - immediate and long-term outcome

Alexander Vavilov; Alexander Vavilov

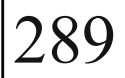

\section{ABSTRACT:}

\section{OBJECTIVE:}

The analysis of immediate and long-term results of surgical treatment in patients with $\mathrm{CAD}$ and concomitant mitral regurgitation of an ischemic genesis.

\section{METHODS:}

The research included 98 patients with CAD aged from 33 to 76 years operated from 2010 to 2018 . The majority of patients had III (79,5\%) and IV $(14,2 \%)$ functional class according to NYHA classification. Average logistic EuroScore was 6,43. The most part of patients had a dilatation of an LV, $50 \%$ of patients had symptoms of LV systolic dysfunction (EF\&lt;50\%) against a significant mitral regurgitation. All patients had severe MR. In all cases, CABG and MV repair were performed.

\section{RESULTS:}

The patients were divided into 3 groups according to Carpentier functional classification: 29 patients had isolated annulodilatation (type I), 26 patients - excessive mobility of the leaflets as a result of papillary muscle rupture or elongation (type II), 43 patients - limitation mobility of the leaflets (type IIIb). Hospital mortality rate was $6,2 \%(n=6)$. The main causes of deaths were a heart and multiple organ failure. Long-term mortality $-12,2 \%(\mathrm{n}=12)$.

\section{CONCLUSION:}

Mitral valve reconstruction should be aimed at restoring the normal anatomy of all components of the mitral valve apparatus and can achieve satisfactory immediate results. Risk factors of unsatisfactory results in the long-term postoperative period are LV EDD \&gt; 6,5 cm, EF \&lt; $40 \%$, atrial fibrillation. Long-term results of reconstructive surgery in type II demonstrate a tendency to more stable results than in type IIIb.A

The role of Modified Devega suture annuloplasty in secondary Tricuspid Regurgitation

Anjith Prakash R; Mithun Sundararaaja R

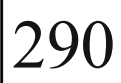

\section{ABSTRACT: \\ BACKGROUND:}

The aim of this study was to evaluate whether the patients with secondary tricuspid regurgitation benefit after modified Devega suture annuloplasty and to quantify any improvement in overall functional status.

\section{METHODS:}

From Jan 2013 to Dec 2017, 141 patients who underwent Mitral valve replacement and modified Devega suture annuloplasty were included and analyzed retrospectively. It was a single centre study. Mid-term survival and functional outcomes after revascularization were determined by follow-up studies obtained during the period of 5 years.

\section{RESULTS:}

Total of 141 patients who underwent mitral valve replacement with modified devega suture annuloplasty technique were included. No other concomitant procedure were included in this study. The mean follow up period was a minimum of 6 months to maximum of 60 months. Echocardiography obtained at regular follow up intervals with a 
minimum of 3 months to the maximum of 5 years showed significant improvement in reduction of TR.

\section{CONCLUSIONS:}

Modified Devega suture annuloplasty is the most commonly used technique nowadays amongst the suture annuloplasty techniques. It is an easy, fast and cost effective technique. Mitral valve replacement does decrease the severity of tricuspid regurgitation amongst those with secondary moderate tricuspid regurgitation by at least one grade, but Devega's annuloplasty confers a better repair result.

\section{Mitral valve replacement for chronic mitral regurgitation leads to left ventricular reverse remodelling postoperatively. \\ G.N. Lone; Mosin Mushtaq}

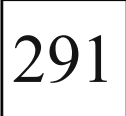

\section{ABSTRACT: \\ OBJECTIVE:}

Chronic mitral regurgitation (MR) results in volume overload followed by left ventricular (LV) remodeling. The aim of this study was to investigate the relation of clinical and echocardiographic findings to LV reverse remodeling early after valve replacement/repair for chronic MR.

\section{METHODS:}

We prospectively evaluated 50 patients who were surgically treated and mitral valve replacement done for severe chronic MR. Echocardiographic measurements were performed before surgery, 1 month after surgery followed by 3 and 6 months after surgery. Left ventricular reverse remodeling was assessed by looking at regression of LV diameters (systolic and diastolic) and LV mass index using echocardiography.

\section{RESULTS:}

Mean age of patients who underwent mitral valve replacement (MVR) was $42.42 \pm 13.63$ years and among them $36 \%$ were males and $64 \%$ were females. MVR surgery improved the functional class at 6 months of follow up $(p=0.008)$. LV end diastolic diameter decreased after MVR. LV mass index showed a statistically significant decrease in post operative period ( $\mathrm{p} \& \mathrm{lt} ; 0.005$ ). Preoperative high NYHA class, low LVEF and high LV end systolic diameter (LVESd) resulted with postoperative LV dysfunction ( $\mathrm{p}$ were \&lt; 0.001 , \&lt;0.001 and 0.006, respectively).

\section{CONCLUSION:}

In patients with severe chronic MR, MVR enhanced LV remodelling resulting in better NYHA function. Preoperative NYHA, LVEF and LVESd were significant predictors of postoperative LV function.

\section{Revisiting Aortic Valve Replacements with Cryopreserved Homograft - Long term follow-up study Priyanka Jindal; K.M. Cherian; Srinivas Aaluri}

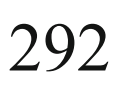

\section{ABSTRACT:}

Revisiting Aortic Valve Replacements with Cryopreserved Homograft Long term follow-up study

AIM:

While mechanical and bio-prosthetic heart valves are used routinely for aortic valve replacements (AVR), a subset of patients have had homografts AVR, especially in a setting of narrow aortic roots and infective endocarditis.

The objective of the study was to assess the longevity of cryopreserved homografts used for AVR and to define the role of homografts in our population.

\section{MATERIALS AND METHODS:}

The case records of patients who had undergone aortic valve replacement with cryopreserved aortic homograft at Frontier Lifeline Hospital, Chennai, between 2004 and 2013 were analysed retrospectively.

The demographic data, the indication for AVR, size of homograft used and follow-up echo data were collected.
A telephonic enquiry was made with the patients enquiring about survival and the current status of symptoms was made and the data was analysed.

A comparison was made with patients who had undergone AVR with mechanical valves (19 and $21 \mathrm{~mm}$ ).

\section{RESULTS:}

There were 23 patients who had survived to discharge after surgery, out of whom 18 were traceable on phone. The age at implantation ranged from 23 to 74 years. The common indications were narrow aortic root and infective endocarditis ( 5 patients).

There were 16 survivors of the remaining 18 (mean survival of 8 years and 6 months). The patients were in NYHA class 1 to 2. The peak gradients across the aortic homograft done during the last follow-up (3 months to 89 months) had a mean of $30 \mathrm{~mm} \mathrm{Hg}$ with 2 patients having a gradient\&gt; $50 \mathrm{mmHg}$.

\section{CONCLUSION:}

The durability of the cryopreserved homografts in patients who had been discharged after AVR is excellent, with good haemodynamics and allowing long term survival. Combined with the advantage of being anti-coagulant free, the homografts have been a better choice than mechanical valves for AVR for our patient population.

Assessment of a re-engineered 3-D printable poppet heart valve for the developing world C.J. Jordaan; Francis E. Smit

\section{ABSTRACT:}

\section{OBJECTIVE:}

After the development of the bi-leaflet carbon-pyrolite valve in the 1970's further development focused on the refinement of this design. The mismatch of a Cape Town poppet valve explanted in pristine condition more than 30 years after initial surgery on nonanticoagulation for patient prosthesis prompted a renewed interest in this design with the objective of refining hemodynamics and evaluating 3-D additive manufacturing processes.

\section{METHODS AND RESULTS:}

Phase 1: Using modern hydrodynamic principles and computational fluid dynamics (CFD), a new poppet valve was designed. A prototype frame design was machined from a single steel block and combined with the new poppet for pulse duplication and tested in a Vivitro pulse duplicator. Flow dynamics and shear stresses were evaluated by particle imaging velocimetry (PIV). Results were compared to those obtained in a similar sized bi-leaflet valve.

Phase 2: Six prototypes were implanted in the right ventricle outflow tract without any anti-coagulation in an ovine model for 180 days. None of the valves clotted and no thrombo-embolic event could be demonstrated.

Phase 3: The resulting CAD design was then 3D printed using Ti 6Al4V (ELI) heart valve frames via DMLS on an EOSINT M280 system. Six prototypes were tested in the as-built state. Failure was induced for quality and feasibility purposes. Mean failure load was $162 \mathrm{~N}$ (range 116-217N) before polishing and heat treatment.

\section{CONCLUSIONS:}

The re-engineered poppet valve has excellent hemodynamics and exceeds FDA requirements. Shear stress induced platelet activation remains below activation levels as demonstrated in CFD and PIV studies and confirmed in the animal study.

Manufacturing by 3-D printing is possible and strength requirements were satisfied although surface polishing remains a challenge. This methodology may enable mass production of a low-cost mechanical valve.

Predictive value of preoperative left ventricular ejection fraction on left atrial reverse remodeling after mitral valve replacement 
Prashant Mishra; Harsh Seth

\section{ABSTRACT: \\ BACKGROUND:}

Left atrium enlargement is commonly seen in patients with mitral regurgitation (MR). Left atrium reverse remodelling (LARR) occurs after surgical correction (repair or replacement of mitral valve) of MR. Preoperative left ventricle ejection fraction (LVEF), as a predictor of this phenomenon is not well known.

\section{METHODS:}

We retrospectively studied 200 consecutive patients who underwent mitral valve replacement (MVR) for MR in whom preoperative echocardiography report was available. The preoperative echocardiography parameters were compared. Follow up echocardiography was done during the study. LARR was defined as a reduction in left atrium volume index (LAVi) $\geq 15 \%$.

\section{RESULTS:}

LARR occurred in 163 patients (81.5\%), with the mean LAVi decreasing from $43.6 \mathrm{~mL} / \mathrm{m} 2$ to $29.7 \mathrm{~mL} / \mathrm{m} 2$ (p \&lt;0.001). These patients had a higher left ventricular ejection fraction $(\mathrm{LVEF})(\mathrm{p}=0.027)$. LVEF was identified as the preoperative predictor of LARR (odds ratio, 1.09; 95\% confidence interval, 1.008-1.12). A LVEF cutoff value of 53.6\% identified patients with LARR of $\geq 15 \%$ with a sensitivity of $86.8 \%$ and a specificity of $74.3 \%$.

\section{CONCLUSION:}

LARR occurs frequently after mitral valve surgery and is associated with preoperative LVEF higher than $53.6 \%$.

Clinical experience of aortic valve replacement using the SOLO SMART stentless bioprosthesis Mitsuaki Sadahiro; Tetsuro Uchida

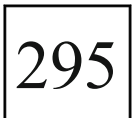

\section{ABSTRACT: \\ BACKGROUND:}

The SOLO SMART valve is a bovine pericardial stentless bioprosthesis which shows superior hemodynamic performance compared to the stented valves. It is designed for supraannular and subcoronary implantation. We report our surgical experience and results in patients undergoing aortic valve replacement (AVR) with this new bioprosthesis.

METHODS:

Since June 2016, 19 patients with various types of aortic valve disease (mean age: $72 \pm 7$ years, $58 \%$ males) have undergone AVR with a SOLO SMART stentless bioprosthesis. Fourteen patients (78\%) showed aortic stenosis (AS) and 9 patients $(47 \%)$ had concomitant procedures: coronary bypass grafting $(n=4)$, mitral valve surgery $(n=3)$, total arch replacement $(n=1)$, and pulmonary venous isolation $(\mathrm{n}=1)$. There was 1 patient with active infective valve endocarditis (IE) complicated with annular abscess and 1 patient was a redo AVR case. Surgical results of these patients were evaluated retrospectively. The hemodynamic performance was investigated with transthoracic echocardiography in pre- and postoperative period.

\section{RESULTS:}

There was no operative death and 1 hospital death (postoperative subdural hematoma; Heyde syndrome). One patient showed a mild trans-prosthetic valve leak. No patient required reoperation. Although postoperative transient thrombocytopenia was noted, it had cured at the time of discharge. At the last echocardiographic evaluation, the peak and mean gradients in the patients undergoing AVR for AS were $17 \pm 7$ and $9 \pm 5 \mathrm{mmHg}$, respectively. No prosthesis-patient mismatch (PPM) occurred in any patient.

\section{CONCLUSIONS:}

AVR using a SOLO SMART stentless bioprosthesiswas performed with acceptable morbidity and mortality. The SOLO SMART stentless bioprosthesis showed excellent hemodynamic performance and early results. Because of favorable effective orifice area, the valve is attractive for patients at risk for PPM. Furthermore, it is expected to contribute to more complex AVR with concomitant mitral valve surgery, active IE or redo situation.
To evaluate the clinical \& echocardiographic outcomes in patients with moderate to severe ischemic Mitral Regurgitation undergoing Coronary artery bypass graft surgery (CABG) with mitral valve repair with posterior annular band annuloplasty.

Sarju Ralhan; Rajiv Kumar; Gurmeet Singh; Sameer Kapur; Arun Garg; Bishav Mohan; Gurpreet Singh Wander; Vikram Pal Singh

\section{ABSTRACT:}

Ischemic mitral regurgitation due to unilateral PM displacement is the most diffuse cause of functional mitral regurgitation (FMR) of surgical interest. In ischemic mitral regurgitation there is a continuum between a normal mitral valve and a mitral valve with morphologic alterations. In general, FMR is related to displacement of one or both papillary muscles with increased tethering of the leaflets.

Surgery of IMR addresses the mitral annulus to reshape it in order to reduce the septolateral distance in such a way that the anterior leaflet could be able to cover the whole mitral orifice. Reshaping of mitral annulus is obtained by means of a support, that can be complete (ring, as it includes the whole length of the annulus) or incomplete (band, as it is applied only to the posterior annulus). A secondary purpose of annular reshaping is to maintain the systolic narrowing and to maintain the saddle shape to reduce the peak leaflet stress.

Our aim in the present study is to evaluate the clinical \&amp; echocardiographic outcomes in patients with moderate to severe ischemic Mitral Regurgitation undergoing Coronary artery bypass graft surgery (CABG) with mitral valve repair using $40 \mathrm{~mm}$ flexible posterior annular band. It was a Retrospective and Prospective study conducted in the Department of Cardiovascular and Thoracic Surgery, Hero DMC Heart Institute, Ludhiana from September 2015 to February 2017.

Retrospectively records of all patients $(n=80)$ who had undergone CABG with mitral valve repair from August 2010 till August 2015 were reviewed and patients diagnosed with coronary artery disease with ischemic mitral regurgitation planned to undergo $\mathrm{CABG}$ with mitral valve repair from September 2015 till 28th February 2017 were studied prospectively.

Rescue Balloon Aortic Valvuloplasty followed by Transcatheter Aortic Valve Implantation for low ejection fraction low-gradient severe aortic stenosis with preexisting mitral prosthesis

Hitoshi Nakanowatari; Yoshiki Endo; Eitoshi Tsuboi; Akinobu Kitagawa; Yasuhisa Fukada; Yoshihito Irie

\section{ABSTRACT:}

Transcatheter aortic valve implantation (TAVI) becomes a feasible method for patients with severe aortic stenosis at high surgical risks. However, those with low ejection fraction low-gradient (LEF-LG), preexisting mitral prosthesis, and severe congestive heart failure are considering high risks cases. We successfully performed a balloon aortic valvuloplasty (BAV) as a rescue procedure followed by TAVI for a LEF-LG case with preexisting mechanical mitral valve at congestive heart failure condition. CASE REPORTS:

An 81-year-old woman (weight $48.2 \mathrm{Kg}$, height $156 \mathrm{~cm}$ ) presented with progressive congestive heart failure symptom. Seventeen years ago, she underwent a mitral valve replacement with a mechanical prosthesis. In attempt to relief her symptoms, BAV was performed by local anesthesia. After transient improvement three months later, TAVI was scheduled. Echocardiography revealed severe aortic stenosis again with an AVA of $0.51 \mathrm{~cm} 2$, a mean gradient of $28 \mathrm{mmHg}$, and depressed left ventricular ejection fraction of $32 \%$ as a LEF-LG status. After evaluation a $23 \mathrm{~mm}$ Edwards Sapien 3 prosthesis was indicated. TAVI was successfully performed under general anesthesia with a very short time percutaneous cardiopulmonary support (PCPS). Post-operative course was uneventful. At follow-up of six months we observed an improvement of heart 
function with an AVA of $1.05 \mathrm{~cm} 2$, a mean gradient of $14 \mathrm{mmHg}$, and left ventricular ejection fraction of $61 \%$.

\section{CONCLUSIONS:}

We concluded that TAVI can be safely and successfully performed in patients with mechanical mitral prosthesis. We will show the procedure by video in this presentation.

Genetic polymorphisms and dosing of Vitamin-K antagonist in Indian patients after heart valve surgery Choudhary S.K.; Arun Mathew

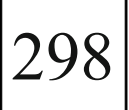

\section{ABSTRACT:}

\section{INTRODUCTION:}

Optimisation of Vitamin K Antagonist (VKA) dosage and attaining therapeutic INR is of primer importance in patients undergoing heart valve surgery. Besides clinical factors, polymorphisms of VKORC1 and CYP2C9 have been found to influence the VKA dosage.

\section{METHODS:}

In the current observational study, of 150 patients who underwent heart valve surgery and had stable INR were genotyped for VKORC1 (-1639 G\&gt;A), CYP2C9*2 and CYP2C9*3. The VKA dosage were estimated from published algorithms and compared to the clinically stabilised dosage.

\section{RESULTS:}

The results showed that around $44 \%$ of the population was intermediate metaboliser and $2 \%$ were poor metabolisers of VKAs. Individually, alleles of VKORC1 (-1639 G\&gt;A), CYP2C9*2 and CYP2C9*3 had mean dosage reduction effect on VKA dosage, which co-related to the clinically stabilised dosages (P\&lt;0.0001). Among VKORC1 (-1639 G\&gt;A) cohort the reduction in warfarin mean weekly dosage was $13.48 \mathrm{mg}$ as compared to wild type category ( $\mathrm{p} \& \mathrm{lt} ; 0.0001$ ) and similarly the reduction in mean weekly Acenocoumarol dose was $6.07 \mathrm{mg}$ (P\&lt; 0.0316 ) as compared to wild type after adjusting for age, gender and BMI.

\section{CONCLUSION:}

The findings suggest that VKORC1 (-1639 G\&gt;A), CYP2C9*2 and CYP2C9*3 genotypes have significant dosage lowering effects on Vitamin $\mathrm{K}$ antagonist based anti-coagulation therapy. The estimated dosages of VKAs significantly co-related to that of observed clinically stabilised dosage in the cohort. The calculators used to estimate the VKA dosages work well in Indian cohort and can be improved further.

Comparative evaluation of hemodynamic performance in early post-operative period of tilting disc vs. bileaflet mechanical valve at mitral position - A prospective study A.P. Jain; Dharmendra kumar Srivastava;

Sandeep Gautam; Subash Singh Rajput; Anurag Rai

\section{ABSTRACT: \\ OBJECTIVES}

In this prospective comparative study, we compared single tilting disc TTK - Chitra valve with Bi-leaflet valves St. Jude, ATS and ON-X valve for Hemodynamic Performance in the early post-operative period. METHODS:

Between January 2017 and October 2018, 142 patients were comparatively evaluated who had undergone Mitral Valve replacement. TTKChitra tilting disc valve or St. Jude, ATS and ON-X bi-leaflet valve. Echocardiography was used to compare the hemodynamic performance (transvalvular mean diastolic gradient and in-vivo mitral valve area) in the early post-operative period. The two groups were not significantly different with respect to age, sex, New York Heart Association (NYHA) Class, Left Ventricular ejection fraction, incidence and severity of mitral stenosis or insufficiency and preservation of subvalvular apparatus.

RESULTS:

Comparison of echocardiographic hemodynamic parameters obtained at the time of discharge from hospital demonstrated no significant differences between the prosthesis with valve size greater than or equal to $27 \mathrm{~mm}$. However, for small size mitral prosthesis $25 \mathrm{~mm}$, the mean diastolic pressure gradients were more in Tilting disc than bi-leaflet valves and effective orifice area was also smaller.

\section{CONCLUSIONS:}

Both tilting disc (TTK-Chitra) and bi-leaflet valves (St. Jude Medical, ATS and ON-X) give equallygood results for large size valves. But in case of small size valves $(25 \mathrm{~mm})$, Bi-leaflet St. Jude, ATS and ON-X valves at mitral position gave better results in terms of large effective orifice area and less gradient across the valve.

The three Deathly Hallows: Anaemia, Transfusions and Major Bleeding in Cardiac Surgery Sunil Dhar; Amit Agarwal; Anubhav Gupta; Jagdish

Prasad; Sunil Dhar

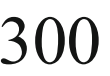

\section{ABSTRACT: INTRODUCTION:}

Postoperative bleeding is common after cardiac surgery. Major bleeding is a determinant of packed cell or whole blood transfusions, especially in patients with preoperative anemia. Preoperative anemia and excessive transfusions are recognized risk factors for operative mortality. The present study investigates the role of major bleeding as an independent determinant of operative mortality in cardiac surgery.

\section{METHODS:}

A single-centre retrospective study over the period of one year from July 2017 to July 2018 was conducted. Five hundred thirty-seven (537) consecutive adult patients undergoing cardiac surgery were analyzed. The impact of postoperative bleeding and major bleeding on operative (within 30 days) mortality was analyzed univariately and after correction for preoperative anemia, RBC transfusions, and confounders like history of stroke and uncontrolled diabetes.

\section{RESULTS:}

Postoperative bleeding was significantly (p \&lt; 0.001) associated with operative mortality. The main complications associated with major bleeding were surgical re-exploration and infections. Major bleeding remained an independent predictor of operative mortality. Preoperative anemia and $\mathrm{RBC}$ transfusions act with a multiplying effect when associated with major bleeding.

\section{CONCLUSIONS:}

Major bleeding in itself is a risk factor for operative mortality. However, its deleterious effects are strongly enhanced by blood transfusions and preoperative anemia. Major bleeding is a partially modifiable risk factor, and adequate preventive and treatment strategies should be applied to limit this event.

Early experience of $\mathbf{1 0 2}$ cases in Robotic Thoracic Surgery Praveen Varma; Balasubramoniam K.R.

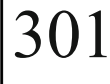

ABSTRACT:

BACKGROUND:

We report our initial experience of 102 Robotic Thoracic surgery cases METHODS:

Retrospective study of 102 consecutive patients who underwent Robotic Thoracic surgery at Amrita Institute of Medical Sciences, Kochi from March 2016 to Feb 2018

\section{RESULTS:}

All the procedures were performed by a single surgeon on the Da Vinci Xi surgical robot. 28 Lobectomies, 19 Thymectomies, 12 Lung wedge biopsies, 11 Lymphnode biopsies, 14 Bullectomies, 18 Mediastinal tumours and 2 diaphragmatic plications were performed. For Lobectomies median docking time, console time and operating time were $22 \mathrm{~min}, 185 \mathrm{~min}$ and 281 minutes respectively. There were 5 conversions (17\%), none for 
intraoperative bleeding. Median length of post operative stay was 6 days. For mediastinal tumours median docking time, console time and operating time were $20 \mathrm{~min}, 85 \mathrm{~min}$ and $155 \mathrm{~min}$ with one conversion. Median length of post operative stay was 4 days. For Bullectomies median docking time, console time and operating time were $19 \mathrm{~min}, 35 \mathrm{~min}$, and $85 \mathrm{~min}$ with no conversions. Median length of post operative stay was 5 days. For Thymectomies median docking time, console time and operating time were $20 \mathrm{~min}, 130 \mathrm{~min}$ and $199 \mathrm{~min}$ with no conversions. Length of stay was 4 days. Overall conversion rate 6/102 (5.8 \%), morbidity was $6 / 102(5.8 \%)$ with no mortality in our series.

\section{CONCLUSIONS:}

Robotics can be used in almost all thoracic surgical procedures with excellent results. There is a shorter learning curve for robotics in thoracic surgery, especially for a consistent team doing procedures at a regular frequency.

Two cases of extremely rare benign tracheal tumors. Rajiv Santosham; Ashwin Phadke

\section{ABSTRACT: \\ INTRODUCTION:}

Primary tracheal neoplasia is a rare phenomenon, accounting for less than $1 \%$ of all tumors. We present two cases of extremely rare benign tumors, a granular cell tumor and other probably the first reported case from India of Tracheal Rosai-Dorfman Disease

\section{MATERIALS AND METHODS:}

A 21 years old girl who presented with an endobronchial polypoidal mass at the lower end of trachea extending into carina and left main bronchus. She underwent tumor resection via a right thoracotomy and tracheotomy.

A 40 years old lady was diagnosed to have a large polypoidal mass $4 \mathrm{~cm}$ proximal to the carina. Initially, we attempted bronchoscopy guided diathermy-fulguration, but owing to development of severe stridor we had to do tracheal mass excision and anastomosis.

\section{RESULTS:}

Post-operatively, the first patient recovered uneventfully. Histopathology of the mass revealed it to be a Granular cell tumor. She is present asymptomatic.

The second patient required an emergency tracheostomy on 5th post-op day due to development of severe adductor spasm. She too is on regular follow-up and at last visit the mobility of vocal cords is restored fully and we plan to decannulate at the next visit. Histology revealed this mass to be sinus histiocytosis or Rosai-Dorfman disease which stained positive for S100 on immunohistochemistry.

\section{CONCLUSION:}

Benign tracheal tumors are rare and usually present as adult onset asthma like symptoms. They respond well to surgical management. But careful investigations, experience and teamwork are required for success.

\section{Using Extracorporeal Membrane Oxygenation as an} Adjunct in the Management of Iatrogenic Tracheal Injury Felice Granato; Muhammad Nawaz

\section{3}

\section{ABSTRACT:}

Iatrogenic injury to the tracheobronchial tree is a rare but life threatening event. The treatment options broadly include conservative or surgical options and ECMO can be a useful adjunct. Choosing a treatment route can be challenging.

We present first case series using ECMO as adjunct in tracheal injury management at tertiary unit. This shows diversity of its presentation, quick recognition and its management. This is followed by literature search.

Case 1: A 68 year old lady admitted electively for left upper lobectomy sustained an iatrogenic injury during intubation and underwent immediate primary repair with subsequent extracorporeal membrane oxygenation support.

Case 2: A 69 year old lady referred from local hospital after sustaining injury during an emergency intubation in the Accident and Emergency department. Delayed surgical repair undertaken with extracorporeal membrane oxygenation support.

Case 3: 69 year old lady diagnosed with iatrogenic injury during underwater seal check at the end of a right upper lobectomy. Underwent primary repair of defect and managed without extracorporeal membrane oxygenation.

ECMO was first introduced as adjunct in 1995, we found 94 relevant articles to our subject. After excluding articles not concerned with management of iatrogenic injuries we were left with 43 results. Recently, there been a shift toward conservative rather than operative management for iatrogenic tracheal injury. Tracheal stents are also a successful adjunct but role of antibiotics is controversial. The popularity of ECMO therapy in the management of iatrogenic tracheal injury is increasing but evidence is limited to isolated case reports. We demonstrate the first case series of ECMO in the management of iatrogenic tracheal injury.

Iatrogenic tracheal injury is an uncommon occurrence, however, carries high mortality and morbidity. The choice regarding surgical or conservative management is unclear. ECMO could be a viable rescue option for iatrogenic tracheal tear repair providing an excellent surgical field and safe anaesthetic management.

Sarcopenia is an independent unfavorable prognostic factor of non-small cell lung cancer after surgical resection: a comprehensive systematic review and meta-analysis

Han-Yu Deng

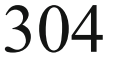

\section{ABSTRACT: \\ BACKGROUND:}

Whether sarcopenia has any impact on long-term survival of patients with surgically treated non-small cell lung cancer (NSCLC) remains unclear. We conducted a meta-analysis focusing on current topic comprehensively for the first time.

\section{METHODS:}

We systematically searched relevant studies investigating the impact of sarcopenia on patients with surgically treated NSCLC in PubMed, Embase, and Cochrane Library up to July 3, 2018. Data of 5-yeasr overall survival (OS) and disease-free survival (DFS) rates as well as hazard ratio (HR) of OS were collected for analysis by using the STATA 12.0 package.

\section{RESULTS:}

A total of 6 cohort studies consisting of 1213 patients (422 patients with sarcopenia and 791 without) were included for analysis. Meta-analysis showed that patients with sarcopenia had a significantly lower 5-yeasr OS rate $($ risk ratio $(\mathrm{RR})=1.63 ; 95 \%$ confidence interval $(\mathrm{CI})=[1.13,2.33]$; $\mathrm{P}=0.008$ ) than those without, which was more prominent in patients with early-stage NSCLC. Sarcopenia was found to be an independent predictor of poor OS in patients with surgically treated NSCLC (HR=2.85; $95 \% \mathrm{CI}=[1.67,4.86] ; \mathrm{P} \& 1 \mathrm{t} ; 0.001)$. With a limited sample size, there was no sufficient evidence of significantly different 5 -year DFS rate between the two groups $(\mathrm{RR}=1.14 ; 95 \% \mathrm{CI}=[0.59,2.17] ; \mathrm{P}=0.70)$. However, in the subgroup of patients with early-stage NSCLC, sarcopenia was associated with a significantly lower 5-year DFS rate $(\mathrm{RR}=1.59 ; 95 \% \mathrm{CI}=[1.01,2.52] ; \mathrm{P}=0.046)$.

\section{CONCLUSION:}

Patients with sarcopenia had a significantly worse prognosis than those without after surgical resection of NSCLC especially in those at early stage. Sarcopenia is an independent unfavorable prognostic factor for patients with surgically treated NSCLC. High-quality studies with appropriate adjustments for confounding factors are needed to confirm our conclusions. 
Diabetes mellitus and survival of non-small cell lung cancer patients after surgery: a comprehensive systematic review and meta-analysis Han-Yu Deng

\section{ABSTRACT: \\ BACKGROUND:}

Diabetes mellitus (DM) is one of the most common comorbidities in patients with non-small cell lung cancer (NSCLC) intended for surgery. It is well established that DM had a negative impact on short-term outcomes of patients with surgically treated NSCLC. However, the impact of DM on long-term survival of those patients remains controversial. Therefore, we conducted this updated meta-analysis focusing on current topic comprehensively.

\section{METHODS:}

We systematically searched relevant studies in PubMed and Embase up to September 6, 2018. Hazard ratios (HR) for the impact of DM on overall survival (OS) and recurrence-free survival (RFS) of patients with surgically treated NSCLC were extracted and analyzed by using the STATA 12.0 package.

\section{RESULTS:}

We included a total of 13 cohort studies consisting of 4343 patients (730 patients with DM and 3613 patients without) with surgically treated NSCLC for analysis. Meta-analysis showed that patients with DM had a significantly poorer OS (Random effects: $\mathrm{HR}=1.30 ; 95 \% \mathrm{CI}=[1.05$, 1.60]; $P=0.016$ ) than those without. However, with a limited sample size, there was no significant difference of RFS (Random effects: $\mathrm{HR}=1.06$; $95 \% \mathrm{CI}=[0.71,1.58] ; \mathrm{P}=0.786)$ between patients with $\mathrm{DM}$ and those without after surgical resection of NSCLC.

CONCLUSION:

$\mathrm{DM}$ is an independent unfavorable prognostic factor for patients with surgically treated NSCLC. High-quality studies with appropriate adjustments for confounding factors are needed to confirm our conclusions.

\section{Mediastinal Lymph Node Dissection for Early-stage (cT1) Non-small Cell Lung Cancers: Lobe-specific Lymph Node Dissection or Systematic Lymph Node Dissection? \\ Han-Yu Deng}

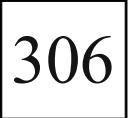

\section{ABSTRACT:}

\section{OBJECTIVE:}

In this study, we aimed to explore the lymph node ( $\mathrm{LN})$ metastasis pattern of early-stage (cT1) NSCLC.

\section{METHODS:}

We collected and analyzed pathologic data of patients with primary earlystage (cT1) NSCLC treated with anatomic lung resection with systematic LN dissection (SLND). We subgrouped the mediastinal LNs into three zones according to the IASLC recommendation: upper mediastinal zone group (2R, 3, and 4R for right lung; 4L, 5, and 6 for left lung), subcarinal zone group (7), and lower mediastinal zone group (8 and 9).

\section{RESULTS:}

A total of 611 patients with early-stage (cT1) NSCLC were finally included for analysis. The mean number of total dissected LNs was about $11.9 \pm 6.0$ and the rate of positive LN metastasis was $18.5 \%$ for all those early-stage NSCLC patients with a rate of mediastinal LN metastasis to $11.3 \%$. For NSCLCs in both upper lobes, about $8.2 \%$ and $10.2 \%$ of them respectively metastasized to the upper mediastinal LN group. However, NSCLCs in both upper lobes hardly metastasized to the subcarinal $(0.9 \%$ and $1.7 \%$ respectively) and lower mediastinal $(0.5 \%$ and $0.6 \%$ respectively) LN groups. For NSCLCs in right middle lobe, about $13.3 \%$ and $11.1 \%$ of them metastasized to the upper mediastinal and subcarinal LN groups respectively but none of them metastasized to the lower mediastibal LN group. For NSCLCs in both lower lobes, a relatively high rate of LN metastasis (around or more than 5\%) was observed in all those three mediastinal LN zones.

\section{CONCLUSIONS:}

Lobe-specific LN dissection (L-SND) could be applied in treating earlystage NSCLCs in the upper lobes, where only upper mediastinal LN zone should be dissected and for those in the right middle lobe, dissection of both upper mediastinal and subcarinal LN zones was required. However, for those in the lower lobes, SLND instead of L-SND should be performed.

Construction and validation of the mathematical models for predicting the pulmonary benign and malignant nodules

Yongbin Lin; Yingsheng Wen;

Xuewen Zhang; Lanjun Zhang; Xiangyang Yu

\section{ABSTRACT:}

\section{OBJECTIVE:}

The objective of this study is to construct the compound mathematical models for predicting pulmonary benign and malignant nodules, and validate their diagnostic efficiency.

\section{METHODS:}

From July 2016 to November 2017, a total of 100 patients with detected pulmonary nodules who received pathological diagnosis and treatment at department of thoracic surgery, Sun Yat-sen University Cancer Center, were prospectively enrolled in training group. Similarly, other 26 patients with detected pulmonary nodules were also prospectively enrolled in validation group from December 2017 to April 2018. RESULTS: First, based on convolution neural network, the diagnostic accuracy rate of Tencent AI is $79 \%$ in training cohort and $42.3 \%$ in validation cohort, respectively. And then, by using univariate analysis, these factors showed statistically significant differences: age, longest diameter, pleural retraction, lobulation, speculation, CEA, NSE, miRNA-17, miRNA-126, miRNA146a and miRNA-221 (P\&1t;0.10); In training cohort, the above mentioned statistically different factors were enrolled in multiple logistic regression analysis to construct $\mathrm{C}$ (clinical) model, B (biomarkers) model, $\mathrm{BC}$ (clinical + biomarkers) model, and $\mathrm{ABC}$ (AI + biomarkers + clinical) model step by step. The AUC of each model was $0.592,0.810,0.860$, and 0.955 , respectively. The predictive value of $\mathrm{ABC}$ model was significantly higher than other models in training cohort (all $\mathrm{P}$ less than 0.05 ). In validation cohort, the AUC of compound $\mathrm{ABC}$ model was still the largest one (0.621), but no significant differences between other models were observed (all $\mathrm{P}$ value greater than 0.05 ). In addition, although the accuracy $(69.23 \%)$ and sensitivity $(100 \%)$ of compound ABC model were also significantly higher than other models, but the specificity $(66.67 \%)$ and negative predictive value $(20.00 \%)$ were all the minimum values.

\section{CONCLUSIONS:}

Construction of multimodal mathematical models to make up the uncertainty of AI and improve diagnostic efficiency is effectual.

\section{Diaphragm surgery in chest wall neoplasms} Lanza Giovanni; Alberto Oliaro

ABSTRACT:

\section{AIM OF THE WORK:}

Chest wall tumors represent $5 \%$ of all thoracic tumors which may determine diaphragm infiltration. When in oncologic surgery it is necessary to proceed to a partial resection of the diaphragm along with thoracic wall traits, its reconstruction must be performed not only focusing the attention to the structional, functional and aesthetic aspect but it must be finalized to preserve this muscle's physiological function. There is no accordance on which technique represents the gold standard in diaphragm reconstruction. Direct suture must be done with detached stitches with non-absorbable material. 
Supporters of this technique focus on the infection risk related to the positioning of heterologous prosthesis and hence on the necessity of its removal. There are many alternative methods for diaphragm reconstruction: the most common are prolene, polypropylene, polyurethane, GoreTex prosthesis. This video shows the techniques for diaphragm reconstruction.

\section{MATERIALS AND METHODS}

Between 2014 and 2018, 15 diaphragm resections due to thoracic wall neoplasms were performed in the Department of Thoracic Surgery of the University of Torino. In 4 cases a direct suture of the diaphragm was performed. In the remaining cases, a prosthesis was placed. This video aims to point out the details of such surgical technique.

RESULTS:

Diaphragm direct suture and prosthesis use allowed an optimum anatomical and functional result. In one case of slight loss of substance and the underneath presence of the liver, Vycril prosthesis placement was not surgically successful.

\section{CONCLUSIONS:}

In diaphragm reconstruction a direct suture is to be preferred; otherwise the placement of a GoreTex and polypropylene-polyurethane prosthesis may be used.

Impact of epidural anesthesia on postoperative non-small cell lung cancer survival: analysis of a Japanese nationwide database

Kento James Fukumoto; Taisuke Jo;

\section{ABSTRACT: \\ OBJECTIVE:}

Recently, multiple retrospective studies have suggested that epidural anesthesia may increase postoperative survival by reducing the recurrence rate in colorectal, prostate, breast, and ovarian cancers. The biological rationale for the prolonged rate of survival is that epidural anesthesia decreases the surgical stress response, which induces transient immunosuppression in the perioperative period. Epidural anesthesia also reduces the need for perioperative immunosuppressive opioid analgesics.

The aim of this study was to reveal the probable impact of epidural anesthesia during radical lung cancer surgery on long-term postoperative survival or cancer recurrence.

\section{METHODS:}

Medical records of 34,694 patients from the Japanese Diagnosis Procedure Combination database, who received lobectomy or pneumonectomy for non-small cell lung cancer between July 2010 and March 2016, were retrospectively reviewed.

To adjust the differences in baseline characteristics, stabilized inverse probability of treatment weighting (IPTW) was performed based on the propensity score. The recurrence-free survival curves were estimated based on the Kaplan-Meier method.

\section{RESULTS:}

Among the patients, 27,810 received epidural anesthesia during lung cancer surgery and 6,884 underwent the surgery without epidural anesthesia. Adjusted Kaplan-Meier curve showed no difference in recurrencefree survival between the groups $(\mathrm{p}=0.89$ ). Stratification according to the cancer stage revealed no significant difference in recurrence-free survival depending on the type of anesthesia administered at each stage.

\section{CONCLUSION:}

Unlike cancer surgery in other organs, the use of epidural anesthesia during lung cancer surgery was not associated with better oncological outcomes.

$\begin{aligned} & \text { Sarcopenia is a predictor of poor prognosis of esophageal } \\ & \text { cancer after esophagectomy: a comprehensive systematic } \\ & \text { review and meta-analysis }\end{aligned}$
Han-Yu Deng

ABSTRACT:

\section{BACKGROUND:}

The impact of sarcopenia on long-term survival of esophageal cancer patients after esophagectomy remains unclear. We conducted an updated meta-analysis focusing on current topic comprehensively.

\section{METHODS:}

We systematically searched relevant studies investigating the impact of sarcopenia on survival of patients with surgically treated esophageal cancer in PubMed, Embase, and Web of Science up to July 20, 2018. Data of 3 -year and 5-year overall survival (OS) rates as well as hazard ratio (HR) of OS and disease-free survival (DFS) were collected for analysis using the STATA 12.0 package.

\section{RESULTS:}

A total of 11 cohort studies consisting of 1520 patients (795 sarcopenic patients and 725 non-sarcopenic patients) were included for analysis. Our meta-analysis showed that patients with sarcopenia had a significantly lower 3-year (51.6\% VS 65.4\%, P\&1t;0.001) and 5-year OS rates (41.2\% VS 52.2\%, P=0.018) than those without. Sarcopenia was found to be an independent predictor of poor $\mathrm{OS}(\mathrm{HR}=1.58 ; 95 \% \mathrm{CI}=[1.35$, 1.85]; $\mathrm{P} \& 1 \mathrm{lt} ; 0.001)$ and DFS $(\mathrm{HR}=1.46 ; 95 \% \mathrm{CI}=[1.12,1.90] ; \mathrm{P}=0.005)$ in esophageal cancer patients after esophagectomy. No obvious heterogeneities or publication bias were observed during analysis.

\section{CONCLUSION:}

Patients with sarcopenia had a significantly worse prognosis than those without after surgical resection of esophageal cancer. Sarcopenia is an independent unfavorable prognostic factor for esophageal cancer patients after esophagectomy. High-quality studies with appropriate adjustments for confounding factors are needed to confirm our conclusions.

The efficacy of the vessel sealing system for video-assisted lung cancer resection

Masahiro Miyajima;

Yuki Takahashi; Ryunosuke Maki; Makoto Tada;

Kodai Tsuruta; Taijirou Mishina; Atsushi Watanabe

ABSTRACT:

\section{INTRODUCTION:}

This study aimed to evaluate the efficacy of the LigaSureÔ vessel sealing system (VSS) when used for lung cancer resection.

\section{MATERIALS AND METHODS:}

The medical records of all patients who underwent an anatomical lung resection for primary lung cancer from 2004 to 2018 were reviewed. In this study, the operating time, amount of bleeding, and intraoperative complication of patients who had undergone lung resection in our hospital using the VSS for lung cancer resection and of the patients who had undergone lung resection by the conventional methods (CM) were compared.

\section{RESULTS:}

Of the 948 patients, 341 (36.0\%) patients who underwent anatomical lung resection for lung cancer by the conventional method and $607(64.0 \%)$ patients who underwent lung resection for lung cancer using the VSS were included. Eleven $(1.2 \%)$ patients developed postoperative chylothorax. The mean amount of intraoperative blood loss $(342.6 \pm 401.9$ vs. $194.9 \pm 375.2 \mathrm{ml}$, p\&lt;0.001) and the chest tube duration $(4.4 \pm 5.1$ vs. $2.6 \pm 3.4$ days, $p=0.003)$ were significantly lower in the VSS group than in the CM group. There were no differences in the mean operating time and the postoperative stay between the groups. The incidence of postoperative chylothorax in the CM group was $11(2.7 \%)$ patients, whereas it was $2(0.3 \%)$ patients in the VSS group $(\mathrm{p}=0.001)$. All patients who developed chylothorax in the VSS method group were cured with conservative therapy.

\section{CONCLUSION:}


We could reduce the chest tube duration, amount of intraoperative bleeding, and incidence of chylothorax in patients who had undergone lung resection using the VSS for lung cancer resection compared to patients who had undergone lung resection by the conventional methods.

Computed Tomography can predict the lymph node metastasis of recurrent laryngeal nerve but not survival time in esophageal squamous cell carcinoma Heng Huang; Jing-Ya Deng; Huan-Yu Tang; Dong Tian; Kai-Yuan Jiang

\section{ABSTRACT:}

Background and Objective: Accurate prediction with noninvasive methods of Recurrent laryngeal nerve lymph nodes metastasis (RLN LNM) may contribute to optimal treatment of ESCC. This study was to evaluate the predictive value of computed tomography (CT) in RLN LNM of esophageal squamous cell carcinoma (ESCC).

\section{METHODS:}

144 patients of ESCC with intro-operative RLN lymph nodes (LNs) dissection were collected between January 2015 and May 2018 retrospectively. The right long/short diameter of entire mass RLN LNs, right long/short diameter of maximum RLN LNs, left long/short diameter of entire mass RLN LNs and left long/short diameter of maximum RLN LNs were measured by CT preoperatively. The RLN LNM negative or positive were confirmed by histopathology. Correlation between CT and histopathology of RLN LNM was analyzed. Receiver operating characteristic (ROC) curve was drawn to find the different thresholds of the diameters of RLN LNMs. Cox proportional hazard regression analysis was used to compare survival differences with each threshold.

RESULTS:

Of these 144 patients, 38 (26.4\%) cases encountered RLN LNM. The diametral thresholds of right long/short diameter of entire mass RLN LNs, right long/short diameter of maximum RLN LNs, left long/short diameter of entire mass RLN LNs and left long/short diameter of maximum RLN LNs were $9.645 \mathrm{~mm} / 4.46 \mathrm{~mm}, 7.165$ $\mathrm{mm} / 4.46 \mathrm{~mm}, 8.14 \mathrm{~mm} / 4.205 \mathrm{~mm}, 6.355 \mathrm{~mm} / 4.205 \mathrm{~mm}$. With the eight different thresholds of RLN LNs diameters, the sensitivity, specificity, accuracy and area under the curve (AUC) reached $57.9 \% / 99.1 \% / 88.2 \% / 0.715,71.1 \% / 72.6 \% / 72.2 \% / 0.752,39.5 \% /$ $98.1 \% / 82.6 \% / 0.665,60.5 \% / 81.1 \% / 75.7 \% / 0.694,36.8 \% / 90.6 \% /$ $76.4 \% / 0.667,63.2 \% / 74.5 \% / 71.5 \% / 0.707,36.8 \% / 92.5 \% / 77.8 \% /$ $0.691,63.2 \% / 81.1 \% / 76.4 \% / 0.738$, respectively. The RLN LNM positive and negative of these eight thresholds were significantly different respectively (P\&lt;0.005). However, the results of multivariate Cox proportional hazard regression analysis showed that no diametral threshold was independent prognostic factors (P\&gt;0.05). CONCLUSION:

CT may predict the RLN LNM in ESCC while it cannot predict the survival time of ESCC patients.

\section{Dietary habits and medical knowledge of esophageal cancer in high-incidence area: 10-Year changes in Yanting county \\ Kai-Yuan Jiang; Heng Huang}

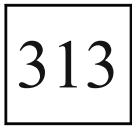

\section{ABSTRACT: \\ OBJECTIVE:}

To compare the differences in dietary habits, food group intake and medical knowledge of EC among residents of Yanting between 2007 and 2017. METHOD:

We investigated rural residents from Yanting county in the year 2007 and 2017. The 10-year changes of demographic features, personal dietary habits, food groups and medical knowledge of EC were compared. The results were then statistically analyzed.

\section{RESULTS:}

Participants in the year 2007 were more likely to be peasants, have a lower educational level, drink less tap water, and were less likely to use refrigerators and electric cookers than those in the year 2017 (P\&lt;0.05). Participants in the year 2007 were more likely to consume foods of lower toughness, have a slower speed of eating, have hotter temperature food, and have rice as a staple food less often (P\&lt;0.05). Residents in the year 2007 ate more preserved foods but fewer fresh foods than those in the year 2017 (P\&lt;0.05). Participants in the year 2017 improved their medical knowledge compared to those in the year 2007, which was conceptualized as a clear understanding of medical insurance for EC, common causes of EC, therapeutic measures for EC, preventive measures for EC, and government interventions (P\&lt;0.05).

\section{CONCLUSION:}

Residents in the year 2017 had healthier dietary habits and better medical knowledge of EC than those in the year 2007, while prevention and control measures in Yanting should be strengthened persistently.

Depth of Circular and Longitudinal Muscle Invasion in T2 Esophageal Squamous Cell Carcinoma Does Not Affect the Prognosis and Lymph Node Metastasis: A Multi-center Retrospective Study

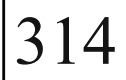

Kai-Yuan Jiang; Jing-Ya Deng; Huan-Yu Tang; Dong Tian; Heng Huang

\section{ABSTRACT: \\ OBJECTIVE:}

To investigate the relationship between depth of tumor invasion and the long-term outcomes, and identify the clinical significance of subclassifying T2 esophageal squamous cell carcinoma (ESCC).

\section{METHODS:}

Patients with T2 ESCC who underwent esophagectomy between January 2009 and December 2017 were reviewed retrospectively. According to depth of tumor invasion, the circular invasion was defined as T2a stage, and the longitudinal invasion was defined as T2b stage. Survival analysis was performed using the Kaplan-Meier method and the Cox proportional hazards model. Predict risk factors of lymph node metastasis (LNM) were evaluated by univariate and multivariate logistic regression analyses. Patients with $\mathrm{T} 1 \mathrm{~b}$ stage ESCC during the same period were selected to the cohort for comparisons to identify the prognostic significance of T2 sub-stage further.

\section{RESULTS:}

A total of 536 T2 ESCC patients were eligible for this study. The median follow-up period was $26.3 \pm 17.7$ months. $192(36 \%)$ patients encountered LNM. There was no significant difference in age, gender, $\mathrm{N}$ stage, length, differentiation, comorbidity, operation performed, postoperative hospital stay between groups of T2a $(n=219)$ and $T 2 b(n=317)(P \& g t ; 0.05)$, except tumor location $(\mathrm{P}=0.02)$. T2 sub-stage did not correlate with survival significantly on univariate and multivariate analysis $(\mathrm{P}=0.43, \mathrm{P}=0.79$, respectively). Kaplan-Meier analysis showed T2 sub-stage in T2 ESCC was not significantly different $(\mathrm{P}=0.42)$. Only $\mathrm{N}$ stage and comorbidity were independent prognostic factors (P\&lt;0.05). Logistic regression analyses also indicated $\mathrm{T} 2$ sub-stage did not affect LNM significantly on univariate and multivariate analysis $(\mathrm{P}=0.07, \mathrm{P}=0.13$, respectively). When T1b stage patients were considered, their survivals were significantly different from patients with T2a and T2b stage (P\&lt;0.001).

\section{CONCLUSION:}

Depth of circular and longitudinal muscle invasion in T2 esophageal squamous cell carcinoma did not affect the prognosis and LNM. It makes little sense to subclassify the circular and longitudinal muscle layers in T2 ESCC. 
Per-oral endoscopic myotomy (POEM) for treating \begin{tabular}{l|l} 
Jang Ming Lee; Chi Wei Lin; Pei Hsing Chen & 315
\end{tabular}

\section{ABSTRACT: \\ BACK GROUND:}

Per-oral endoscopic myotomy (POEM) is a novel technique to treat achalasia.

However, the experience is limited in Taiwan. In the current study, we evaluate the clinical outcome of POEM for treating the patients of achalasia in the NationalTaiwan University Hospital.

PATIENTS AND METHODS:

All of the patients of achalasia undergoing POEM in NTUH was evaluated. The Eckardt score for the degree of dysphagia before and after POEM was evaluated.

\section{RESULTS:}

From Nov 2011 to July 2016, there were 42patients of achalasia undergoing POEM in the National Taiwan University Hospital. 22 and 20 females and males enrolled in the current study. The mean age was 51 years-old and follow-up duration was 22.3 months respectiveely. Pneumothorax requiring transient chest tube drainage was encountered in two patients. There was no mortality in the series. The swallowing was improved for all of the patients with the preoperative and postoperative Eckardt score $7.6(+1.7)$ and $2.0(+1.9)$ respectively (p\&amp;t; 0.01$)$. The improvement of the patients was noted irrespective of the previous treatment or the subtype of achalasia of Chicago classification.

\section{CONCLUSION:}

POEM is a feasible and effective treatment option for patients of achalasia. The thoracic surgeons are therefore encouraged to engage this novel endoscopic minimally invasive procedure for treating achalasia in the future.

Intracavitary Photodynamic Therapy in Pleural Metastasis by Lung Cancer

Ke-Cheng Chen; Jang-Ming Lee

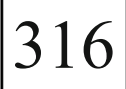

\section{ABSTRACT:}

\section{OBJECTIVES:}

Currently, the options of management of pleural metastasis in lung cancer include chemotherapy, target therapy, operation with pleurectomy, or/and photodynamic therapy. The aim of our study is to investigate the role of PDT in these patients

\section{METHODS:}

Between January 2005 and January 2017, we retrospectively reviewed the clinical characteristics, treatment course and outcome of 53 patients with pleural seeding by lung cancer. The goal was to have no visible or palpable tumor remaining in the affected hemithorax at the end of the surgical resection. PDT in seven regions of the pleural cavity: apex, anterior chest wall, posterior chest wall, posterior mediastinum, posterior costophrenic sulcus, anterior costophrenic sulcus, and pericardium. The chest cavity was filled with dilute intralipid solution $(0.01 \%)$ to act as a scattering agent to allow more homogeneous light delivery. The light was delivered with an optical fiber sheathed within an intravenous catheter. This delivery system was moved around the chest cavity until a measured dose of $40 \mathrm{~J} / \mathrm{cm} 2$ of $630 \mathrm{~nm}$ light was recorded at all seven sites.

\section{RESULTS:}

Thirty-nine patients enrolled in this study. There are 25 men and 28 women included in this study. The mean patient age was $54.8 \pm 12.8$ years. Using Kaplan-Meier survival analysis, the 3 -year survival rate and the 5-year survival rate were $70.8 \%$ and $63.7 \%$, respectively. There is one ARDS occurred immediately after the procedure. After medical treatment, the condition gradually improved. Other minor complications included prolonged air-leakage (eight patients) and skin redness (nine patients). The complications were successfully treated using medication. There was no procedure-related mortality.

\section{CONCLUSIONS:}

Photodynamic therapy and surgery for pleural dissemination in patients with non-small cell lung cancer is feasible and associated with a good outcome.

The Transsternal transpericardial approach for post pneumonectomy Broncho pleural fistulae

Kamran Ali; Sabyasachi Bal

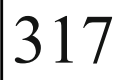

\section{ABSTRACT: \\ INTRODUCTION:}

Pneumonectomy is associated with considerable morbidity and definite mortality of $5-7 \%$. Bronchopleural fistula (BPF) is one of the most dreaded complications. Most large fistulae are large and need surgical treatment. This often needs a multi-pronged approach.

\section{METHODS:}

Between 2000 and 2017, 57 patients were seen with bronchopleural fistulae. All had inflammatory disease with at least one year follow up. The index operation had not always been done in our hospital. Of 397 pneumonectomies in our hospital, there were 34 BPFs. There were 3 patients with tube thoracostomy and 54 with Eloesser flaps (20 of these were referred from other institutions). Our BPF rate was $8.6 \%$. All patients had fistula closure attempted by the transsternal transpericardial approach (TP). This is the largest series in world literature.

\section{RESULTS:}

The interval between index surgery and development of fistula was 4 weeks to 15 patients. In all patients, the initial operation was the creation of an open window thoracostomy $(\mathrm{OWT})(\mathrm{n}=37)$ and packing. Once the cavity was ready, fistula closure was attempted. All fistulae were closed by the transsternal transpericardial route. One right sided fistula needed carinal resection and trachea-left bronchial anastomosis. All fistula closures were stapled. There were no deaths. One patient had a recurrence after 3 weeks and treated with muscle packing. In 16 patients, a simultaneous myoplasty and omentoplasty $(n=5)$ was done and the OWT was closed.

\section{CONCLUSIONS:}

We submit our experience with a relatively lesser known operation for bronchopleural fistula and underscore its value in difficult clinical situations. The transsternal transpericardial approach is a useful, safe and very effective procedure for the closure of post pneumonectomy bronchopleural fistula. It has a low recurrence rate and may be the best method for fistulae with very short bronchial stumps

Thoracotomies in children-An institutional experience Sivasankaran K.

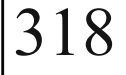

\section{ABSTRACT:}

Thoracotomies in children have been less extensively studied, as the incidence of diseases necessitating thoracotomies is low in the pediatric age group. This study reviews childhood thoracic diseases, thoracotomy approaches, indications, and complications. Surgical procedures and complications of a total of 482 children below 12 years of age who underwent thoracotomy for various reasons in the Department of Pediatric cardiothoracic Surgery, Institute of child health, between 2012 and 2017, were reviewed in this study. The most commonly encountered indications for surgery were ligation of patent ductus arteriosus $356(74 \%)$; pericardiectomy $52(10.8 \%)$; coarctation of aorta repair 18 (3.7\%).Other surgeries include those done for lung resections lobectomies \&amp; pneumonectomies; closed mitral commissurotomy for mitral stenosis; mediastinal mass excision; repair of vascular rings /slings; Impacted foreign body removal; thoracic empyemas. Out of the 481 patients, $423(87 \%)$ underwent posterolateral thoracotomy and $57(11.8 \%)$ patients underwent a Anterolateral thoracotomy incision and 
one child underwent a bilateral thoracotomy

The role of thoracoscopic decortication in chronic organised post pneumonic empyema thoracis Sabyasachi Bal; Kamran Ali

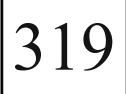

\section{ABSTRACT: \\ BACKGROUND:}

As per the American Thoracic Society (ATS), stage 3 pleural empyema is the phase of organisation which requires decortication of the thickened visceral pleural cortex to free the entrapped lung. In this study we evaluate the feasibility of a thoracoscopic decortication with open decortication

\section{METHODS:}

The outcomes of 216 consecutive patients undergoing decortication via thoracoscopy and thoracotomy for stage 3 ATS from 2015 to 2018 were reviewed and compared with respect to baseline characteristics, preoperative management, and operative and postoperative course

\section{RESULTS:}

The study group consisted of 177 Thoracoscopic and 39 open thoracotomy patients. The median operative time and median in-hospital length of stay were shorter for the Thoracoscopy group: 110 vs 165 minutes (p \&lt; 0.001), and 5 vs 8 days respectively. Thoracoscopy group had fewer post operative complications.

\section{CONCLUSIONS:}

Decortication for empyema, has promising results that are at par with conventional open decortication. They have fewer postoperative complications. It is a viable first option to put in a scope for most patients with complex chrnic pleural effusions and empyema.

\section{VATS Decortication for stage III empyema - our experience} Ajay Narasimhan; Arvind V.

\section{ABSTRACT: \\ BACKGROUND:}

Historically the definitive management for empyema has been surgical debridement. while this may be done via open procedures but in the early part of last decade, the minimally invasive approach of VATS with low operative morbidity has become the gold standard for fibropurulent pleural disease and thoracic trauma. We report our experience with VATS and review the role of VATS in the management of the same.

\section{MATERIALS AND METHODS:}

All the patients who underwent VATS from December 2016 to July 2018 at our institute were retrospectively studied.

\section{RESULTS:}

A total of 26 patients with mean age $45+/-5$ years received VATS decortication for their empyema. $21(81 \%)$ patients were male and $5(19 \%)$ were female. The major etiology of thoracic empyema was tubercular in origin. The main post operative complication was persistent air leak. 15 of 26 patients $(58 \%)$ had good reexpansion of affected lung after VATS decortication. 5(19\%) of them required conversion to open thorocotomy.

\section{CONCLUSION:}

Early referal for thorocoscopy has the advantage of the procedure being easy to perform, allowing better drainage, less perioperative bleeding, lower post operative narcotic requirement, shorter duration of chest drain in place and greater likelihood of obtaining full expansion of affected lung avoiding thorocotomy.

VATS decortication should be the gold standard for pleural empyema: higher magnification with precise debridement and breakage of fibrinous septa or band; identification of debris and lung parenchyma with a very meticulous decortication

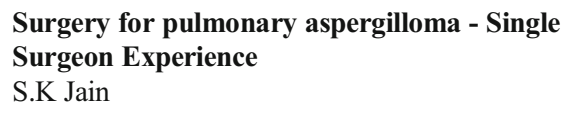

ABSTRACT:

\section{INTRODUCTION:}

With a heavy load of pulmonary tuberculosis in the country, surgical interventions are quite frequent for its complications such as destroyed lung, recurrent hemoptysis, chronic empyema, cavitary lesions with persistently positive sputum and aspergilloma. Generally, patients with aspergilloma present with recurrent hemoptysis of varying severity for many months or even years.

\section{METHODS:}

From Sept 2001 to Oct 2017, a total of 43 patients, 34 males and 9 females with age ranging from 19 years to 77 years formed the material of this study. Right upper lobe was involved in 23 and left upper lobe in 20 patients. Out of 28 patients operated, 7 had segmentectomy, lobectomy in 18 and pneumonectomy in 3 patients.

\section{RESULTS:}

No patient died in the series. Minor complications occurred in 7 patients, mainly in the form of persistent air leak requiring prolonged intercostal drainage. 4 patients had major complications, one requiring completion lobectomy after segmentectomy. One had a very poor expansion of residual lung and developed empyema with prolonged morbidity. Other two patients had significant air leak necessitating chest tube for many weeks to achieve lung expansion. None of the patients had major bleeding during or after surgery. Average blood loss was $180 \mathrm{ml}$. Only 2 patients required blood transfusion intraoperatively.

\section{CONCLUSION:}

Surgery for pulmonary aspergilloma, though technically very challenging, can be performed safely with acceptable immediate postoperative complications and good long-term results.

\section{Long-Term Outcome and 3D Reconstruction of Primary} Pulmonary Artery Sarcomas

Sertac Cicek; Christopher McGregor; Jennifer Boland;

Scott Okuno; Steven Robinson; Darin White;

Eunhee S. Yi; Shanda H. Blackmon; Nandita Nettu Mahajan

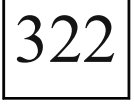

\section{ABSTRACT:}

\section{OBJECTIVES:}

To analyze long-term surgical management outcomes for primary pulmonary artery sarcomas (PPAS) and determine R0 resection strategy guide using 3D reconstructions.

\section{MATERIALS \&amp; METHODS:}

An institutional database search from January 1987 to August 2018 identified 14 patients with PPAS and 8 with surgical intervention were selected for retrospective medical record review along with $3 \mathrm{D}$ reconstructions. Surgical interventions were classified as: 1) Debulking/pulmonary endarterectomy, 2) Sleeve resection and reconstruction of main pulmonary artery (PA), 3) Sleeve resection and reconstruction of main PA and its branches, 4) PA angioplasty and 5) Pulmonary valve (PV) replacement.

\section{RESULTS:}

The median age was 65 years (range $=40-73$ years, $63 \%$ female) and the most common CT finding was a luminal filling defect distending the PA vasculature. Median follow up for all patients was 34 months ( $\mathrm{IQR}=15-85.3$ months). Eight patients underwent pulmonary endarterectomy. Four of these underwent more radical resection with PA reconstruction, PV replacement and/ unilateral pneumonectomy. Median postoperative survival for all patients was 47.5 months (range=13-156 months). Six patients received adjuvant therapy and 1 patient received neoadjuvant and adjuvant therapy (median survival=63 months, range=15-156 months). Three patients with radical resection and multimodality treatment are alive at 61 months, 66 months and 156 months. Six patients had tumor recurrence with a median relapse-free survival of 11.3 
months (range=3-30.5 months), of which 3 with tumor recurrence \&gt; 1 year after surgery had better outcome (median survival of 140 vs 17 months).

\section{CONCLUSION:}

In our small cohort of PPAS, radical surgical resection with multimodality therapy was associated with optimal survival.

\section{Robotics and VATS in Chest wall tumor resection Thombare B, Rane N, Khan AZ}

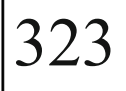

ABSTRACT:

\section{OBJECTIVE:}

To discuss the role of VATS and robotic surgery in resection of chest wall tumor

\section{METHOD:}

5 patients with chest wall tumors were diagnosed and presented for surgical resection. 2 had tumors in 2,3 rib and 3 had tumors in lower ribs straddling across two or more ribs.

\section{RESULTS:}

3 resections were carried out using VATS and 2 were done by robotics. The Rib was cut using a self designed locally made endoscopic rib cutter. Good clearance margin was achieved in all patients. 3 patients needed reconstruction using a synthetic mesh. The mesh was placed intrathoracic as an inlay technique. Fixation was achieved by using endoscopic tackers and sutures. One patient was operated using the video mediastinoscope as a tunneling visualizer.

Post op recovery was very good with no evidence of lung herniation. All patients were followed up with no evidence of recurrence.

\section{CONCLUSION:}

VATS and Robotics offers a minimally invasive technique for chest wall tumors. They cause reduced trauma and give good visualization for reconstruction

Robotics for Thoracic outlet tumors

Nikhil Rane; Bhushan Dinkar Thombare

\section{ABSTRACT:}

Robotics for Thoracic outlet tumors

Thombare B, Rane N, Khan AZ

Medanta, The Medicity

Gurgaon, India

\section{OBJECTIVE:}

To show the benefit of Robotics in resection of thoracic outlet tumor METHOD:

4 patients presented with thoracic outlet tumors, 2 on right side and two on left side. Diagnosis included

Schwannoma

Germ cell tumor

Ganglioneuroma

They presented with symptoms of pain, and weakness in arm and positive sign of thoracic outlet obstruction. MRI angiography of thoracic outlet did not show involvement of neurovascular tissue but evidence of pressure symptoms. All were resected by robotics.

\section{RESULTS:}

Drain was removed on first postoperative day. Patient was discharged on day 2. No neurovascular deficits were present postop. There was complete resolution of symptoms on follow up.

Robotic offered the following advantages over VATS

True 3D vision with good appreciation of depth of vision.

360 movements and 7 degrees freedom of Robotic endowrist. This allowed accurate dissection of the tumor off the subclavian and axillary vessels and off the brachial plexus with no injury to theses structures.

Ability to dissect the adhesions off the chest wall under magnified vision and with minimal blood loss.

Ergonomic superiority of surgery

Next day discharge with no morbidity leading to a saving in the overall cost of the surgery

Use of 3 arms instead of 4 arms gives extra cost saving of robotic disposables

High definition recording gives ability to make training video and ease of presentation in conferences.

CONCLUSION:

Robotics is a safe and effective technology that gives added advantage in resection of tumors off the thoracic outlet.

Eclipsing 3 decades of Lung Transplantation: Lessons learned from the event horizon Michael Wait; Matthias Peltz; Brian Kilmartin;

Lynn Huffman; Vaidehi Kaza; Fernando Torres; Steve Ring; John S. Murala

\section{ABSTRACT:}

\section{INTRODUCTION:}

Lung transplantation is considered as the gold standard for patients with chronic end stage pulmonary disease. Here we review survival up to 25 years at a university hospital.

\section{METHODS:}

We performed a retrospective review of prospectively collected transplant data from all lung transplants performed at our institution. We have follow up data for nearly all patients. Long term actuarial survival was determined using the Kaplan Meier method. RESULTS:

Between September 1990 and November 2018, we performed 761 lung transplants which include 561 bilateral lung, 106 right single and 94 left single transplants. Recipient ages ranged from 14 to 79 years (median 58). Etiology included Interstitial lung disease (349 patients), COPD (177 pts), Cystic Fibrosis (122 pts), Pulmonary Hypertension (46 pts) and others (67pts). Pre-operative ECMO was used in 28 patients. Ex Vivo lung perfusion was used in three patients. The overall median survival is 6.6 years. The actuarial survival rates at 30 days, 1 year, 5 years, 10 years, 15 years, and 20 years are $97.2 \%, 89.4 \%, 58.3 \%, 34.4 \%, 16.2 \%$ and $10.6 \%$ respectively. There are only two survivors beyond 20 years and one beyond 25 years. Cystic fibrosis patients, relatively younger, also have a median survival of 6.9 years. The cause of death in more than $50 \%$ of cases is chronic lung allograft dysfunction.

\section{CONCLUSION:}

The overall survival after lung transplantation is lower compared to other solid organ transplants. Improved long term outcomes depend on reducing chronic lung allograft dysfunction. It is to be seen if use of techniques such as EVLP and ECMO will have any bearing on long term survival.

Combined Repair of Total Sternal Cleft and Tetralogy of Fallot : A case study

Nilanjan Dutta; Gaurav Patni

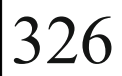

\section{ABSTRACT:}

Combined Repair of Total Sternal Cleft and Tetralogy of Fallot : A case study

Gaurav Patni, Debasis Das, Manish Kumar Sharma, Amitabha Chattopadhyay, Nilanjan dutta Rangan Koley.

Department of paediatric cardiac sciences, Narayana Superspeciality Hospital, Howrah 


\section{INTRODUCTION:}

Sternal cleft is an extremely rare congenital anomaly resulting from a failure of the fusion of sternal bars. The condition can be classified as complete or incomplete. It is $0.15 \%$ of all Congenital Chest wall deformities and usually associated with structurally normal heart. The repair in children beyond neonatal period is challenging as the hypoplastic nature of sternal edges may preclude primary approximation necessitating the use of prosthetic/autologous grafts or relaxing parasternal chondroplasties.

BACKGROUND:

A 2 years old child presented with severe cyanotic spell in Emergency Room of our hospital and got intubated. He was diagnosed at 3 months of age with Tertalogy of Fallot(TOF) with total Sternal Cleft but refused treatment at various hospitals. Echocardiography showed features of TOF, left pulmonary artery(LPA) origin stenosis. In addition there was complete absence of sternal cover with pulsating heart below the skin in mid-line. Computed-Tomography scan of chest confirmed Total sternal cleft. CASE:

Child was planned for Intra Cardiac Repair with LPA plasty and Chest wall reconstruction simultaneously. The patient underwent right ventricle outflow tract(RVOT) resection, Patch closure of ventricular septal defect and Transannular autologous pericardial patch augmentation of RVOT extending upto LPA origin. Mediastinum was covered with bovine pericardium. To overcome the gap between sternal plate, oblique bilateral chondrotomies of first four ribs after mobilisation of pectoralis was done. The two sternal plates were approximated with No.2 steel wires. Child was electively ventilated for 48 hours. He required intensive physiotherepy and discharged on 14 day.

\section{CONCLUSION:}

Combined surgical repair of both anomalies should be attempted soon after birth with optimal management. Direct approximation of sternal halves should be attempted whenever possible.

Transhiatal versus transthoracic esophagectomy for esophageal carcinoma: our experience SKIMS Srinagar. G.N. Lone; Mosin Mushtaq; Rouf Gul

\section{ABSTRACT: \\ BACKGROUD:}

There is a lot of controversy about the best surgical treatment for esophageal carcinoma.

\section{METHODS:}

In this retrospective study, 982 patients with carcinoma of the mid-todistal esophagus underwent transhiatal or transthoracic esophagectomy. Early morbidity and mortality were compared. Principal endpoints were disease-free survival (DFS) and overall survival (OS). A total of 560 patients underwent transhiatal esophagectomy, and 422 patients underwent transthoracic esophagectomy. Demographic characteristics and characteristics of the tumor were similar in the two groups.

\section{RESULTS:}

Perioperative and postoperative morbidity was higher after transthoracic esophagectomy. In-hospital mortality was also higher after transthoracic esophagectomy. The median follow-up was 4.5 years. Estimated 3 -year DFS rates were 47.36 and $34.74 \%$, whereas the 3 -year OS rates were 58.23 and $42.23 \%$ for the transhiatal and transthoracic groups, respectively (statistically significant). Also, the estimated 5-year DFS rates were 26.98 and $21.17 \%$, whereas the 5-year OS rates were 31.56 and $30.87 \%$ for the transhiatal and transthoracic groups, respectively (statistically not significant).

\section{CONCLUSION:}

Transthoracic esophagectomy was associated with higher perioperative and postoperative morbidity and in-hospital mortality than transhiatal esophagectomy. The DFS and OS were higher in the transhiatal group and were statistically significant at 3 years but insignificant at 5 years.
Mid-term results of TAVI with the use of a valve with PTFE leaflets.

Andrey Voevodin

\section{ABSTRACT:}

OBJECTIVE:

To evaluate the clinical and hemodynamic results of transcatheter replacement of the aortic valve with the "MedLab-KT" prosthesis.

\section{METHODS:}

MedLab-KT is the first model of a transcatheter prosthetic with PTFE leaflets. It is a balloon-expandable stent, the cusps of which are made of $0.1 \mathrm{~mm}$ thick polytetrafluoroethylene plates. The reason of choice of synthetic material was the hypothesis of the absence of biodegradation of polytetrafluoroethylene in the organism. The valve has passed the preclinical phases of the in vitro and in vivo tests.

The study included 69 patients who had undergone implantation of the MedLab-CT prosthesis. The survival rate and the frequency of a clinically significant stroke, as well as hemodynamic parameters according to echocardiography for up to 3 years, were evaluated. 39 patients were examined in person, the rest went through a telephone survey.

\section{RESULTS:}

The average follow-up was 9.6 months, the maximum - 3 years. The majority of patients belonged to the elderly group (mean age 73.3 years). For 51 patients (74\%) according to the EuroSCORE scale and for 37 (54\%), according to the STS scale, a high risk of surgical intervention was determined: $\geq 8 \%$.

Mortality was $13 \%, 6$ deaths were noted at the hospital stage, 3 patients died in the long-term period. Strokes not recorded. In the internal examination group, the average gradient on the aortic valve prosthesis is defined at $8.41 \pm 4.21 \mathrm{~mm} \mathrm{Hg}$; failure due to paraprosthetic fistulas not higher than I degree was noted in 7 patients (18\%), not higher than II degree in 1 case; transvalvular aortic insufficiency was not detected.

\section{CONCLUSION:}

The results of the studied parameters are comparable with the data provided by foreign randomized clinical studies of famous models of transcatheter aortic valve prostheses.

Total Arterial Multi vessels Minimal Invasive Direct CABG (MIDCABG) through Left Minithoracotomy. Vivek Wadhawa; Kaushal K Tiwari

\section{ABSTRACT:}

\section{BACKGROUND AND AIM:}

MIDCABG is becoming popular and an important armamentarium for cardiac surgeons. MIDCABG with total arterial grafts is the best option for patients with coronary artery disease, who are at high risk for sternotomy. We aimed to evaluate the result of MIDCABG total arterial revascularization.

\section{METHODS:}

We have evaluated 221 patients who underwent MIDCABG multivessel total arterial revascularization through a left anterolateral mini thoracotomy.

LIMA harvested in every patient, RIMA or radial artery used as second conduit. LIMA-RIMA Y or LIMA-RADIAL Y was made to accomplish multivessel total arterial revascularization. Mean age of patients was $52.52 \pm 9.76$ yrs. Average number of anastomosis performed were 2.34 \pm 0.75 . All intraoperative and post-operative data were collected and evaluated. Post-operative graft patency was evaluated in all patients by CT coronary angiography at 6 months following discharge.

\section{RESULTS:}

Multivessel total arterial CABG was accomplished in all individuals. There was no mortality or wound infection. Re-exploration was done in one patient for bleeding. In one individual, conversion to sternotomy and $\mathrm{CPB}$ was required. Average ICU and hospital 
stay was $1.52 \pm 0.77$ and $4.92 \pm 1.46$ days respectively. Patients were mobilized earlier due to less pain. Wound healing of anterolateral thoracotomy was faster. In the follow up CT angiography all grafts were patent.

\section{CONCLUSIONS:}

Multivessels total arterial MIDCAB can be accomplished safely in selected individuals. RIMA can be harvested in long standing diabetic patients with no concern for sternal wound healing. MIDCABG patients experience less pain, better cosmetics and early return to daily activities.

Less invasive bilateral anterolateral thoracotomy versus standard clam shell approach for bilateral sequential lung transplantation-an outcome analysis Manoras Chengalath Mathew; George Jose Valooran

\section{METHODS:}

Between January 2018 and October 2018, 17 patients who underwent completion TCPC using the alternate surgical method at AIIMS.

\section{RESULTS:}

All patients had an uneventful intraoperative course. The mean cardiopulmonary bypass time was $60+/-16.8$ minutes (range, 39 to 102 minutes). The time of aortic clamping was not collected, because the cases of external conduit were performed without cardiac arrest. There were no deaths in the study. The mean postoperative fontan pressures were 15.6 $+/-1.2 \mathrm{mmHg}$ with no gradient between SVC and IVC pressures. Mean duration of hospital stay was $15.6+/-3.6$ days (Range from 10 to 22 days). There was no incidence of phrenic nerve paresis or palsy.

\section{CONCLUSIONS:}

Performing completion TCPC using our alternate technique is easy to perform as well as easy to teach. It avoids disadvantages associated with routine techniques.

\section{ABSTRACT: \\ BACKGROUND:}

Bilateral anterolateral thoracotomy is a minimally invasive alternative to the standard clam shell incision in bilateral sequential lung transplantation. This approach has been cited to yield better post-operative lung function, pain scores and wound healing. The study aims to compare the outcomes between bilateral anterolateral thoracotomy and clam shell incision in bilateral sequential lung transplantation.

\section{PATIENTS AND METHODS:}

This was a single centre retrospective analysis of the perioperative variables and post-operative outcomes of patients undergoing bilateral sequential lung transplantation between January 2013 to June 2017 via bilateral anterolateral thoracotomies or clam shell incisions.

RESULTS:

The bilateral anterolateral thoracotomy arm had 26 patients while the clam shell arm had 52 patients. The mean follow up duration was 124.60 (SD 77.81) weeks. The pre-operative variables were comparable between the groups $(\mathrm{p}=\mathrm{NS})$. Mean donor cold ischemia time for the second lung was higher in thoracotomy group $(\mathrm{p}=0.007)$. The FVC at 1 month $(2.92 \mathrm{~m}$ vs $2.45 \mathrm{~m} \mathrm{p}=0.029)$, FVC $(3.41 \mathrm{~m}$ vs $2.79 \mathrm{~m} \mathrm{p}=0.007)$ and FEV1 at $3(2.82 \mathrm{~m}$ vs $2.36 \mathrm{~m} \mathrm{p}=0.035)$ and 6 months $(\mathrm{FVC}-3.68 \mathrm{~m}$ vs $3.02 \mathrm{~m} \mathrm{p}=0.002$, FEV1- $2.92 \mathrm{~m}$ vs $2.5 \mathrm{~m} \mathrm{p}=0.024$ ) were significantly higher in thoracotomy group. The operating times, re-explorations, ICU stay, ventilation hours, pain scores, wound infection rate, reinterventions and over-all survival were comparable between the groups.

\section{CONCLUSION:}

Bilateral anterolateral thoracotomy approach to bilateral sequential lung transplantation results in improved early post-operative lung function.

\section{Retrospective study of results of an alternate technique of completion of the total cavopulmonary connection Bharat Siddarth; P. Rajashekar; Srikant Sharma; Rohan Magoon; Shiv Kumar Choudhary; Sachin Talwar

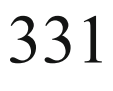

\section{ABSTRACT: \\ BACKGROUND:}

Total cavopulmonary connection (Fontan or TCPC) palliation is final stage of palliation for patients with a univentricular heart. Bidirectional cavopulmonary anastamosis (BDG) is used for staging in most of the cases in this pathway. There are different techniques for TCPC completion after a child undergoes BDG. It includes offpump as well as onpump palliation. We describe our technique of completing TCPC in an alternate way to reduce dissection and associated complications.
Early experience of 102 cases in Robotic Thoracic Surgery Praveen Varma; Balasubramonia K.R.

\section{ABSTRACT:}

BACKGROUND:

We report our initial experience of 102 Robotic Thoracic surgery cases METHODS:

Retrospective study of 102 consecutive patients who underwent Robotic Thoracic surgery at Amrita Institute of Medical Sciences, Kochi from March 2016 to Feb 2018

\section{RESULTS:}

All the procedures were performed by a single surgeon on the Da Vinci Xi surgical robot. 28 Lobectomies, 19 Thymectomies, 12 Lung wedge biopsies, 11 Lymphnode biopsies, 14 Bullectomies, 18 Mediastinal tumours and 2 diaphragmatic plications were performed. For Lobectomies median docking time, console time and operating time were 22min, $185 \mathrm{~min}$ and 281 minutes respectively. There were 5 conversions (17\%), none for intraoperative bleeding. Median length of post operative stay was 6 days. For mediastinal tumours median docking time, console time and operating time were $20 \mathrm{~min}, 85 \mathrm{~min}$ and $155 \mathrm{~min}$ with one conversion. Median length of post operative stay was 4 days. For Bullectomies median docking time, console time and operating time were $19 \mathrm{~min}, 35 \mathrm{~min}$, and $85 \mathrm{~min}$ with no conversions. Median length of post operative stay was 5 days. For Thymectomies median docking time, console time and operating time were $20 \mathrm{~min}, 130 \mathrm{~min}$ and $199 \mathrm{~min}$ with no conversions. Length of stay was 4 days. Overall conversion rate 6/102 (5.8\%), morbidity was $6 / 102(5.8 \%$ ) with no mortality in our series.

CONCLUSIONS:

Robotics can be used in almost all thoracic surgical procedures with excellent results. There is a shorter learning curve for robotics in thoracic surgery, especially for a consistent team doing procedures at a regular frequency.

Total Anomalous Pulmonary Venous Connection: improved early outcomes with an alternate management strategy

Nayem Raja; Tarun Raina Ramman;

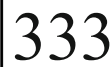

Sumir Girotra; Sushil Azad;

Sitaraman Radhakrishnan; Parvathi U. Iyer; Krishna S. Iyer; Kuntal Roy Chowdhuri

\section{ABSTRACT:}

\section{BACKGROUND:}

Surgical repair of TAPVC has been associated with significant mortality especially in developing countries, largely blamed on post-operative pulmonary hypertensive crisis. We believe that altered ventricular dynamics post-operatively are more responsible for mortality and have evolved a 
management strategy accordingly. The results of this strategy are presented.

\section{PATIENTS AND METHODS:}

308 patients (median age -93 days; median weight $-4100 \mathrm{gms}$ ) with TAPVC underwent biventricular repair between Jan 2001 and Sept $2018.51 \%$ had supracardiac, $27 \%$ had cardiac, $10 \%$ had infracardiac and $12 \%$ had mixed connections. 155 ( 50.3\%) had pulmonary venous obstruction at some level. $17 \%$ required emergent surgery, $24 \%$ were semi-urgent and the rest were done electively on priority.

Operative strategies included 1) normothermic bypass with short crossclamp duration 2) posterior approach for supra and infra cardiac connections 3) routine ligation of the vertical vein.

Post-operative strategies included 1) routine left atrial pressure (LAP) monitoring instead of pulmonary artery pressure monitoring to track left ventricular function 2) aggressive afterload reduction 3) frequent echocardiographic assessment of bi-ventricular dynamics and 4) weaning from ventilator support only when LAP was consistently low.

\section{RESULTS:}

In-hospital mortality was $8 / 308(2.59 \%)$. Causes of death were severe drug resistant sepsis -5 , bronchomalacia-1, malignant arrhythmia-1, neurological complication-1. No patient required mechanical support or inhaled nitric oxide. Median ventilator duration and hospital stay was 52 hours and 11.2 days respectively. All survivors were discharged with documented unobstructed pulmonary venous drainage.

CONCLUSION:

Early outcomes following surgery for TAPVC can be improved by a strategy focused on tracking ventricular function rather than pulmonary artery pressure. Nitric oxide and mechanical support are easily avoidable.

Uses of Bilateral internal mammary artery (BIMA)

in Coronary artery bypass grafting (CABG):

Comparison between Insitu vs Y-graft. A 500 cases study.

Sayedur Rahman Khan; Jahangir Kabir

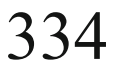

\section{ABSTRACT: \\ BACKGROUND:}

Coronary artery bypass grafting(CABG)using bilateral internal mammary artery(BIMA)is associated with improved long-term survival, event-free survival, and freedom from re-intervention. Despite their well-established advantages, bilateral internal mammary arteries(BIMA)are still largely underused because of time consumption, surgical skills and expertise, sternal wound complication, increased risk of early mortality and morbidity. We compared the early and midterm clinical outcomes after offpump coronary artery bypass grafting(OPCAB)using bilateral internal mammary arteries (BIMAs) in both configurations as in situ grafts with Y-composite graft.

\section{METHODS:}

A retrospective cross sectional study was conducted in which all patients underwent bilateral internal mammary arteries(BIMA)grafting for offpump coronary artery bypass grafting(OPCAB)at United hospital, Dhaka, Bangladesh from January 2014 to November 2018 were included. During this period total of 514 consecutive patients who underwent OPCAB using bilateral skeletonized IMAs as Y grafts(group I, $\mathrm{n}=$ 267) or in situ grafts(group II, $n=247$ )were studied.

\section{RESULTS:}

Patients had a wide range from 28 to 72 years with mean age $48.73 \pm 8.42$ years., Hypertension $(70.1 \%)$, diabetes mellitus $(41.8 \%)$, positive family history $(35.15 \%)$ and smoking $(56 \%)$ were the most common cardiac risk factors. Majority(64.3\%) of patients had triple vessel disease(TVD). Number of arterial graft per patient was $2.22 \pm .51$ and 2.33 \pm .61 in insitu and $\mathrm{Y}$ graft group respectively. There was no in hospital mortality in both groups. There were no statistically significant differences in operative time $(5.03 \pm 1.05$ vs $4.87 \pm 1.14$ Hours $)(\mathrm{p}=0.522)$. No statistically significant differences postoperative complications including re-opening for bleeding (3.64\% vs $3.05 \%)$, perioperative myocardial infarction $(3.8 \%$ vs $1.31 \%)$, sternal wound infection $(1.2 \%$ vs $0.8 \%)$, stroke $(0.0 \%$ vs $0.0 \%)$, renal failure $(0.0 \%$ vs $0.4 \%)$ and respiratory complication $(0.4 \%$ vs $0.8 \%)$ between groups I and II $(\mathrm{p}=\mathrm{NS})$. We found no significant difference in terms of mid-term survival or freedom from repeat revascularization between different configurations of BIMA use.

\section{CONCLUSIONS:}

Our results demonstrate that $\mathrm{OPCAB}$ using bilateral skeletonized IMAs is technically feasible with excellent graft patency.

Comparative study of diagnostic accuracy between CT-Angiography, Catheter-angiography and trans-thoracic ECHO in pre-operative evaluation of TOF Rana Sandip Singh; Manoj Kumar Rohit;

Harkant Singh; Anand Kumar Mishra; Madhusudan Katti

\section{ABSTRACT: \\ INTRODUCTION:}

Adequate preoperative assessment of TOF from both anatomical and functional points of view should be performed to plan the surgical approach and to avoid unpleasant surprises during the surgery. This study was aimed at analysing the accuracy of CTA, CATH and ECHO in preoperative assessment of TOF.

MATERIALS/METHODS:

Twenty patients undergoing intra-cardiac repair in Department of CTVS, PGIMER, Chandigarh

Exclusion: Renal dysfunction, Emergency surgery. All 20 patients underwent detailed ECHO, CTA and CATH to assess sizes of VSD, infundibulum, pulmonary valve annulus, MPA, RPA, LPA, number of MAPCAs and associated anomalies and were compared with intraoperative findings.

\section{RESULTS:}

VSD size: CTA showed 90\% accuracy. CATH and ECHO showed $80 \%$ and $70 \%$ accuracy, respectively. RV Infundibulum: CTA and ECHO were $90 \%$ accurate, CATH showed $85 \%$ accuracy. Pulmonary annulus: ECHO was more accurate (90\%) compared to CTA and CATH (both had $85 \%$ accuracy). CTA showed $95 \%$ accuracy for MPA, RPA, LPA size assessment. ECHO showed least accuracy in assessing branch pulmonary arteries. CTA showed $100 \%$ accuracy for assessing MAPCAs. CTA was most accurate in detecting associated extra-cardiac anomalies like Right Aortic arch, Coronary anomalies.

\section{CONCLUSION:}

Evaluation of RVOT and MPA, RPA and LPA was better with CTA, compared to ECHO and CATH. CTA was most reliable for evaluating MAPCAs and coronary anatomy.

\section{JUMP GRAFT, THE SAFE AND REPRODUCIBLE TECHNIQUE FOR COMPLEX LAD LESIONS Sayedur Rahman Khan; M. Zakir Khaled}

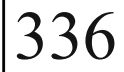

\section{ABSTRACT: INTRODUCTION:}

Special complementary techniques are required for complete revascularization of complex, left anterior descending artery (LAD) lesions like severely diffused, diffused calcified and multiple lesions. The maximum utilization of left internal thoracic artery (LITA) in coronary artery bypass procedure by jump fashion (sequential graft) is our method of choice to combat with such vessels. The purpose of this study is to follow-up the mid-term results of our procedure.

PATIENTS AND METHODS:

To observe the midterm outcome of our patient with jump graft in LAD, 23 out of 28 patients was operated from January 2014 to December 2015 
were selected for relook angiogram. The median period for relook CAG was 48 months.

\section{RESULTS:}

46 jump grafts by LITA in LAD are observed by relook angiogram. Among 23 LITA graft one LITA graft observed occluded both in proximal and distal anastomosis. One patient found $70 \%$ stenosis at the proximal graft and another patient found total occlusion of the distal anastomosis with LITA. Patients showed significant improvement in their NYHA and CCS classes. There is one mortality in follow up due to other than Cardiac Cause.

\section{CONCLUSION:}

Though we had a small series of population, still we can notice that it is an easy, safe and readily reproducible method to complete re-vascularize the lifeline LAD to perfuse the anterior myocardium as well as the septum.

\section{Ventral Aortic Repair for Coarctation of Aorta in Adults with associated Cardiac anamolies \\ Bhavin A. Brahmbhatt}

\section{ABSTRACT:}

\section{BACKGROUND:}

Coarctation of aorta and associated cardiac disease is complex medical situation. Both requires correction either simultaneous or in stages. A distinct approach is vital in order to bring down the perioperative mortality and morbidity.

\section{OBJECTIVE:}

To study the different strategies for simultaneous repair of aortic coarctation and associated cardiac diseases in adult patients.

\section{MATERIALS AND METHODS:}

We have operated seven patients with coarctation associated with other cardiac diseases, who were treated by various surgical approaches. Developments in endovascular technology over the past decade may potentially reduce the morbidity from open surgical repair. However, some cases are unsuitable for endovascular repair, and open surgical techniques continue to play a part in the management of these patients. Coarctation and associated diseases were treated in a single stage by an ascending-to-descending bypass ( $\mathrm{n}$ =6). One patient underwent a two stage operation for his treatment. RESULT:

Postoperative recovery of all the nine patients were uneventful. Their ICU parameters were within acceptable limits. All the three patients showed equalization of blood pressure between the upper and lower extremities and discharged within 8 to 9 days. They showed postoperative CT scan suggestive of normal functioning graft without any leak or aneurysm.

\section{CONCLUSION:}

In spite of the progress in the development and popularization of endovascular techniques, surgical intervention is becoming a more preferable option for the treatment of co-arctation accompanied by cardiac diseases. Two-stage and single-stage (extra-anatomical bypass) procedures are the alternative techniques that are performed depending upon the experience of the surgeons in the center; however, we prefer the single-stage (simultaneous) technique.

\section{Dysphagia Lusoria- Ambit of cardiovascular and Thoracic surgeon}

Durga Prasad Rath; Gautham A

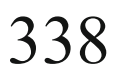

\section{ABSTRACT: \\ INTRODUCTION:}

Dysphagia Lusoria is an uncommon entity which occurs due to coursing of aberrant subclavian artery posterior to the oesophagus. The anomaly most commonly involves the right subclavian artery $(0.5-2.0 \%$ of the population), or rarely the left subclavian artery when there is a right-sided aortic $\operatorname{arch}(0.05-0.1 \%)$. The majority of patients with ARSA remain asymptomatic. Therefore, dysphagia lusoria is exceedingly rare, and a clinician may not encounter even one case during his or her entire career.

\section{BACKGROUND:}

Here we report 2 cases presented with dysphagia of recent onset with no other complaints. Patients were evaluated elsewhere and referred to CTVS Dept, JIPMER. Patients were further evaluated and diagnosed to have ARSA.

CASE1:

CT Neck and Thorax showed ARSA arising from DTA. Patient was operated in 2 stages, via supraclavicular approach in the first stage, dividing the aberrant artery with carotid subclavian transposition, later left thoracotomy was done and Aneurysmal stump was divided at the origin as it was compressing the esophagus. Patient had complete resolution of symptoms post procedure.

\section{CASE 2:}

Patient underwent single stage procedure. Carotid subclavian transposition via Right supraclavicular approach after dividing the aberrant artery was done. Post procedure patient had complete resolution of symptoms. CONCLUSION/CLINICAL RELEVANCE:

Open ligation and transposition to the right carotid artery via a right supraclavicular approach has been described as a minimally invasive method. However, approaching the origin of the aberrant right subclavian artery through this incision can be extremely challenging.

Axillary Versus Femoral Cannulation for Acute Type A Aortic Dissection: Single Center 32 years experience Toru Ishimine; Akio Nakasu

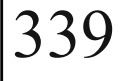

\section{ABSTRACT:}

The answer to the question about the better cannulation site for acute Type A aortic dissection is still controversial. Some studies showed a significantly lower complication rate with axillary artery cannulation than femoral artery cannulation. We usually did femoral artery cannulation for all aortic dissection repair before 1997. After 1997, cannulation site was dependent on the patient's condition, but the first choice was axillary artery for antegrade cerebral perfusion. The goal of this study was to compare early postoperative outcomes including stroke and hospital death between patients who underwent repair of acute Type A aortic dissection with axillary or femoral artery cannulation. From January 1985 to December 2017, a total of 213 patients underwent surgery for acute Type A aortic dissection in our institution. We excluded the patient who was treated with both axillary and femoral cannulation at the same time and centrally cannulated patients. The patients of 84 and 114 patients were cannulations via femoral (FG) and axillary artery (AG), respectively. We did not have any patient who troubled with malperfusion by pseudolumen cannulation. There was no statically significance on the patient's baseline characteristics between the two groups. Only renal failure was higher in FG $(p=0.040)$. There was no significance in the type of surgery. Hemodynamically unstable patients before surgery were higher in FG (p \&lt; 0.001). Stroke occurred in $14.2 \%$ of patients in FG and $14.0 \%$ of patients in $\mathrm{AG}(\mathrm{p}=1.000)$. Hospital death rate was higher in $\mathrm{FG}(19.0 \%)$ than AG $(9.6 \%)$, but it was not statically significant $(p=0.063)$. The selection of the cannulation site is dependent on the patient's condition. Both sites are favorable, but it should be individualized based on different patient risk profiles.

Timely surgical management of abdominal aortic aneurysm Dasari Kalyani Rama

\section{ABSTRACT:}

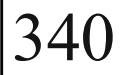

\section{INTRODUCTION:}

Abdominal aortic aneurysm (AAA) is aortic diameter at less than one and one-half times the normal diameter at the level of the renal arteries, which is approximately $2.0 \mathrm{~cm}$. 


\section{MATERIAL AND METHODS:}

Patients 5 of which 2 female, 2 male were referred to our hospital (Nov 2017 to oct 2018.)

With dyspnoea(NYHAIII), palpitations, loud continuous murmur, pulsatile abdominal mass.

Diagnosed with ultrasound abdomen(costeffective).

Underwent open abdominal aortic aneurysm resection and grafting.

\section{RESULTS:}

Male 3, Female 2 patients with average of age of 55 to 75 years, with Aneurysm size of $\& \mathrm{gt} ; 5.5 \mathrm{~cm}$ with variable pathology (Fusiform, Saccular, Pseudo aneurysm) with comorbid factors Underwent open abdominal aortic aneurysm resection and grafting. Graft flow achieved intra operatively and patent post operatively and also follow up with screening by ultrasound abdomen. No intra or postoperative mortality with average hospital stay of 10 to 12 days.

CONCLUSION:

Risk of death from an AAA rupture be reduced through early diagnosis(USG), monitoring, and surgical repair of the aneurysm if it is $\& \mathrm{gt} ; 5.5 \mathrm{~cm}$ in diameter.

Supravalvular and valvular Aortic stenosis with coronary artery disease in hyperlipedemia and it's surgical management

Dwarkanath Kulkarni; Amrin Sheikh

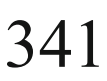

\section{ABSTRACT:}

Familial hyperlipidemia is associated with valvular and supravalvular aortic stenosis and premature coronary artery disease. Surgery in such patient is challenging due calcification of aorta and narrow aortic root. Surgery in such patient require aortic root enlargement with AVR with bypass graft. A 25 year female known case of familial hyperlipidemia on lipid lowering medication, presented with syncope and dysnea. ECHO showed severe aortic stenosis and norrow aortic root. On computed tomography finding were same and additionally she had $100 \%$ RCA occlusion. Patient underwent Aortic root enlargement ( Nick's technique) using bovine pericardial patch and AVR done with Medtronic aortic valve no. 16 with coronary artery bypass grafting. Patient recovered completely with no cardiovascular or cerebrovascular complication. Surgical management of these cases is challenging, we should be well versed with Aortic root enlargement procedures, gentle handling of aorta and myocardial protection to prevent complication.

Key words: Supravalvular Aortic stenosis, Hyperlipidemia, Coronary artery disease.

\section{Management of ascending aortic aneurysm: an observational study \\ Prashant Patil}

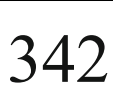

\section{ABSTRACT:}

\section{BACKGROUND:}

Ascending aortic aneurysm is common incidental finding on transthoracic echocardiography performed for unrelated indications. Usually seen in patients with underlying connective tissue disorders.

\section{METHODS:}

This was retrospective observational study involving ascending aortic aneurysm patients requiring surgical intervention during the period of Jan 2015 to Jan 2018

\section{RESULTS:}

Total 30 patients of ascending aortic aneurysm requiring surgical intervention were studied. Males outnumbered females with ratio of $5: 1$. Their age ranges from 14-72 years (mean=43.6years). Most of the patients of ascending aortic aneurysm also present with aortic regurgitation, $43 \%$. Hypertension being the most commonly associated co-morbidity.
Bentall's procedure, $67 \%$, is most commonly needed surgical intervention. Bleeding, $16.67 \%$, is the most common and independent risk factor for mortality with percentage of $26.67 \%$

CONCLUSION:

Bleeding is independent risk factor in ascending aortic surgery and careful application of hemostasis skills can bring down mortality associated with ascending aortic surgery.

Successful aortic arch replacement using intermittent pressure-augmented retrograde cerebral perfusion (IPA-RCP) for acute aortic dissection type A coexistent dissected aortic arch vessels and aortic arch 343 saccular aneurysm

Tomohiro Murata; Akiko Mano; Takashi Nishimura; Shunei Kyo Mitsuhiro Kawata

\section{ABSTRACT:}

\section{OBJECTIVE:}

Surgical treatment of acute aortic dissection type A (AAD-A) coexistent dissected aortic arch vessels is associated with higher peri- and postoperative mortality and morbidity. Clinical effectiveness of intermittent pressure-augmented retrograde cerebral perfusion (IPA-RCP) for brain protection has been reported in previous studies. We report a woman with AAD-A coexistent dissected aortic arch vessels and aortic arch saccular aneurysm, treated successfully with surgical treatment using IPA-RCP.

\section{METHODS (IPA-RCP protocol):}

Body temperature was cooled down to 18 degree centigrade. Open distal anastomosis using open stentgraft was performed with IPA-RCP. The oxygenated blood was perfused through SVC cannula at base line pressure of $15 \mathrm{mmHg}$. The pressure augmented at $45 \mathrm{mmHg}$ was attempted and was then lowered. The duration of the augmentation was 30 seconds. CASE PRESENTATION:

A 72-year-old woman was referred to our department for surgical treatment of AAD-A coexistent aortic arch saccular aneurysm, $52 \mathrm{~mm}$ in diameter. CT scan revealed the brachiocephalic trunk and left common carotid artery were also dissected. Therefore, we decided to perform aortic arch replacement under IPA-RCP in order to avoid aortic arch vessels' cannulations.

\section{RESULTS:}

Circulatory arrest time was $74 \mathrm{~min}$. IPA-RCP time was $74 \mathrm{~min}$. The clinical data were shown as time to regain consciousness, 3 hours; mechanical ventilation time, 30 hours; and no neurological complications. She discharged on foot 24 th post- operative day.

CONCLUSIONS:

IPA-RCP was effective and safe for brain protection of AAD-A surgical treatment in a patient who even if had dissected aortic arch vessels and needed aortic arch replacement.

Ventral Aortic Repair in Co-arctation of Aorta in adult patients with different comorbidities Bhavin A. Brahmbhatt

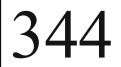

\section{ABSTRACT:}

\section{BACKGROUND:}

Coarctation of aorta and associated cardiac disease is complex medical situation. Both requires correction either simultaneous or in stages. A distinct approach is vital in order to bring down the perioperative mortality and morbidity.

\section{OBJECTIVE:}

To study the different strategies for simultaneous repair of aortic co-arctation and associated cardiac diseases in adult patients. MATERIALS AND METHODS:

We have operated seven patients with coarctation associated with other cardiac diseases, who were treated by various surgical approaches. 
Developments in endovascular technology over the past decade may potentially reduce the morbidity from open surgical repair. However, some cases are unsuitable for endovascular repair, and open surgical techniques continue to play a part in the management of these patients. Coarctation and associated diseases were treated in a single stage by an ascending-to-descending bypass $(n=6)$. One patient underwent a two stage operation for his treatment.

RESULT:

Postoperative recovery of all the nine patients were uneventful. Their ICU parameters were within acceptable limits. All the three patients showed equalization of blood pressure between the upper and lower extremities and discharged within 8 to 9 days. They showed postoperative CT scan suggestive of normal functioning graft without any leak or aneurysm.

\section{CONCLUSION:}

In spite of the progress in the development and popularization of endovascular techniques, surgical intervention is becoming a more preferable option for the treatment of co-arctation accompanied by cardiac diseases. Two-stageand single-stage (extra-anatomical bypass) procedures are the alternative techniques that are performed depending upon the experience of the surgeons in the center; however, we prefer the single-stage (simultaneous) technique.

Open technique of Aneurysectomy for Infrarenal Abdominal Aortic Aneurysms : Does it have a scope in the current scenario ?

Haroon Naqshi; G.N.Lone

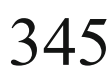

\section{ABSTRACT: \\ BACKGROUND:}

Abdominal Aortic aneurysm is most frequent during 5th and 6th decade with Atherosclerosis as the commonest cause. We have conducted Aneurysectomy by open technique which still is believed to have better long-term benefits and lesser chances of re-interventions in contrast with Endovascular technique, given the current scenario.

\section{METHODS:}

20 patients were operated for Inrarenal abdominal aortic aneurysms. Investigative tools included USG, CT Angiography, MRA and Echo. Carotid Doppler, Coronary Angiography and Renal Doppler were selectively done as per situation. Abdomen was opened through midline or paramedian incision. Proximally, Inrarenal and exceptionally suprarenal aorta, and distally the common iliac arteries were controlled. Aneurysm was opened after fine dissection and isolation of branch vessels and systemic heparinization. Distal anastomosis with common iliac was done first followed by proximal anastomosis. IMA was reimplanted in 3 patients due to poor return. For ruptured aneurysms patients were well optimised before exploration. Aorta was controlled proximally and distally. Aneurysmal sac was opened and aorta reconstructed by a Dacron tube graft of appropriate size. Retroperitoneal hematoma was always evacuated after completing the primary procedure.

\section{RESULTS:}

5 of 20 operated patients had ruptured aneurysm and rest were operated electively with intact aneurysms. One patient developed torsion of small gut while another had duodenal perforation, upper GI bleed and peritonitis and died after 4 months of surgery. Third patient developed distal thromboembolism of left lower limb. 1 of 5 patients with ruptured aneurysms died of exsanguination during exploration. 17 patients went home with satisfactory results.

\section{CONCLUSION:}

Management of Abdominal aortic aneurysm continues to be a surgical challenge. Open surgery continues to be a gold standard, especially in centers where endovascular procedures are not done.

Corresponding Author:

Dr. G.N.lone, CVTS, SKIMS, Srinagar(Kashmir) Email ID: gn_lone@yahoo.co.in
Dysphagia Lusoria- Ambit of cardiovascular and Thoracic surgeon

Durga Prasad Rath, Gautham A

\section{ABSTRACT:}

\section{INTRODUCTION:}

Dysphagia Lusoria is an uncommon entity which occurs due to coursing of aberrant subclavian artery posterior to the oesophagus. The anomaly most commonly involves the right subclavian artery $(0.5-2.0 \%$ of the population), or rarely the left subclavian artery when there is a rightsided aortic arch $(0.05-0.1 \%)$. The majority of patients with ARSA remain asymptomatic. Therefore, dysphagia lusoria is exceedingly rare, and a clinician may not encounter even one case during his or her entire career.

\section{BACKGROUND:}

Here we report 2 cases presented with dysphagia of recent onset with no other complaints. Patients were evaluated elsewhere and referred to CTVS Dept, JIPMER. Patients were further evaluated and diagnosed to have ARSA.

\section{CASE1:}

CT Neck and Thorax showed ARSA arising from DTA. Patient was operated in 2 stages, via supraclavicular approach in the first stage, dividing the aberrant artery with carotid subclavian transposition, later left thoracotomy was done and Aneurysmal stump was divided at the origin as it was compressing the esophagus. Patient had complete resolution of symptoms post procedure.

\section{CASE 2:}

Patient underwent single stage procedure. Carotid subclavian transposition via Right supraclavicular approach after dividing the aberrant artery was done. Post procedure patient had complete resolution of symptoms.

\section{CONCLUSION/CLINICAL RELEVANCE:}

Open ligation and transposition to the right carotid artery via a right supraclavicular approach has been described as a minimally invasive method. However, approaching the origin of the aberrant right subclavian artery through this incision can be extremely challenging.

Extra-anatomical Ascending-to-Descending Bypass for Simultaneous Surgery of Aortic Coarctation with Other Cardiac Pathologies

Saravan Krushna Raja; Aravind M.

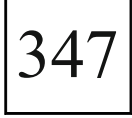

\section{ABSTRACT:}

The combination of cardiac diseases with aortic coarctation in adults is very rare and is usually combined with an aortic valve defect, frequently caused by a bicuspid anatomy.

A $40 \mathrm{yr}$ old female presented with complaints of left sided weakness, slurring of speech. She is known hypertensive on treatment with history of CVA 6yrs back. Clinical examination showed features of left CVA, UMN Facial nerve palsy and differential blood pressure, higher in upper limb than lower limb Echocardiography showed bicuspid aortic valve and left ventricular hypertrophy. MRI Brain confirmed acute infarct. CT Angiogram showed Tight stenosis for a length of $1.1 \mathrm{~cm}$ noted in descending thoracic aorta distal to left subclavian artery with fusiform dilatation of ascending aorta

Through median sternotomy and after instituting cardiopulmonary bypass, hypothermia, aortic cross clamp, cardioplegic arrest procedure was performed. An After opening posterior pericardium, Dacron graft was anastomosed to descending thoracic aorta, end to side. The graft was then tunneled was tunneled through the oblique sinus between IVC \&amp; right inferior pulmonary vein and anastamosed to Ascending aorta end to side. Simultaneous repair of bicuspid aortic valve was also done. Postoperatively patient had significant decrease of differential blood pressure. CT Angiogram showed good contrast opacification in graft. 
Anatomic repair often requires extensive mobilization of the aorta and control of collateral blood vessels; potential complications include bleeding from adhesions, parenchymal lung injury, damage to the recurrent laryngeal or phrenic nerves, chylothorax, and spinal cord ischemia. Paraplegia is dreaded complication.

Thus, single stage extra-anatomic bypass from ascending to desceding aorta with repair of other cardiac lesions seems a reasonable strategy as it avoids complications of anatomic repair, additional and interventional procedures

Repair of saccular aneurysm of descending thoracic aorta using homograftC S VARMA PONNAMANDA, MADHU SANKAR N, AJITH, SRINIVAS AALURI, ANTO SAHAYRAJ, KM CHERIAN

Srinivas Aaluri; Chandrasekhar Varma E.

\begin{abstract}
:
Saccular aneurysm of descending thoracic aorta is rare and early repair is indicated in symptomatic patients. Most of them can be repaired with a patch graft, sparing intercostals.

A 63 Yrs old male, diabetic, hypertensive and known case of CKD presented with complaints of severe left sided upper backache, fever. He had complaints of par aesthesia of both lower limbs, intermittent fever with chills and rigors. blackish discoloration left little toe for 1 week. Earlier he was admitted elsewhere and CT angio done there showed saccular aneurysm of descending thoracic aorta. His blood culture showed growth of gram positive Cocci. ECG was normal. He underwent repair of saccular aneurysm of descending thoracic aorta through left posterolateral thoracotomy. On opening the chest, lung was densely adherent which was released. Femiro-Femoral bypass was established. Descending thoracic aorta was looped proximal and distal to the aneurysm. Aneurysmal sac was opened, contents were evacuated and the defect was closed with cut open cryo preserved aortic homograft. Post operative recovery was smooth and uneventfull. Patient was discharged on 7 th post operative day. Cryopreserved homograft for patch repair is a viable alternative to traditional repair particularly in patients with mycotic aneurysm.
\end{abstract}

Total Arch Replacement with Concomitant TEVAR via the Ascending Aorta: Report of two Cases Hitoshi Nakanowatari; Eitoshi Tsuboi; Akinobu Kitagawa; Yasuhisa Fukada; Yoshihito Irie; Yoshiki Endo

\section{ABSTRACT:}

We're going to report two cases of total arch replacement with concomitant TEVAR via the ascending aorta. Case1: The patient was a 66 year-old male. CT angiography showed a huge aneurysm in the aortic arch and chronic type B aortic dissection. Echocardiography showed patent ductus arteriosus (PDA). Because of pulmonary hypertension due to PDA, it was considered unacceptable to put him under general anesthesia twice. After establishment of cardiopulmonary bypass, a stentgraft was inserted via the ascending aorta to cover the dissection site of descending aorta. The aorta was opened under circulatory arrest and PDA was suture closed. The proximal end of the stentgraft was anastomosed to the distal end of the prosthetic arch graft and arch branches were reconstructed as usual. Case2: The patient was a 49 year-old male. CT showed type B acute aortic dissection and the descending aorta ruptured at Th8 level. The diameter of aortic arch was $50 \mathrm{~mm}$ that cannot be used for stentgraft landing. We performed the same operation as Case1. Postoperative courses of two cases were acceptable. Before and after deploying the stent, we tried to lower the risk of paraplegia by keeping blood pressure under heartbeat and under the same situation as TEVAR.
Complete Vascular rings presenting in infancy with compressive symptoms: our surgical experience in 3 cases Mohd Javed Banday; Dhananjay Bansal

\section{ABSTRACT:}

A vascular ring is a rare congenital aortic arch anomaly that may cause trachea-esophageal compressive symptoms. Vascular rings may be double aortic arch, right aortic arch with left ligamentum or rarely LPA sling. Herein we share our experience with three such cases of vascular rings.

We present our series of three patients done over 2 years. Two patients presented in pediatric emergency for trachea-esophageal compressive symptoms and third presented in cardiology for cyanosis. Detailed investigations revealed complete vascular rings in all 3 cases.

One had LPA sling with left ligamentum, when LPA was translocated to MPA with left ligamentum division.

Second had, Right Aortic Arch with left ligamentum. Simple division of ligamentum with pexing of kommerell diverticulum to rib resolved the symptoms.

Third had Double Aortic Arch, left arch was divided along with ligamentum. CT imaging best delineates the anatomy of the vascular ring and associated trachea-esophageal compression. Echocardiogram and bronchoscopy are adjunctive investigations to find associated cardiac anomalies or tracheomalacia and bronchomalacia respectively.

Here in our series, we recommend that early diagnosis and intervention provides the best long term outcomes.

Valve-sparing Aortic Root Replacement Surgery: A single centre experience over five years

T. Periyasamy; Vivek Shrihari

\section{ABSTRACT:}

\section{INTRODUCTION:}

Patients who presented to our hospital and were subjected to replacement of the ascending aorta using a prosthetic graft for the management of ascending aortic aneurysms and aortic dissections using valve-sparing technique (David Procedure) between January, 2014 and November, 2018 were included in the study.

\section{MATERIALS AND METHODS:}

25 patients (17 males and 8 females) who presented to our hospital and were managed by valve-sparing Aortic Root Replacement Surgery (David Procedure) for ascending aortic aneurysms and aortic dissections for a study period of 5 years were included.

RESULTS:

The patients' particulars were recorded. All surgeries with their indications, procedure details, post-operative I.C.U. care, post-operative progress in the ward were documented in detail. Post-operative complications were all noted. Follow-up assessment was done for all patients. Time to get discharged, post-operative period required to start ambulating, period required to return to activities of daily living, period required to return to work and post-operative morbidity and mortality were all recorded.

\section{CONCLUSION:}

We found that valve-sparing Aortic Root Replacement Surgery (David Procedure) for the management of ascending aortic aneurysms and aortic dissections is a safe and effective procedure with an acceptable morbidity and mortality.

Cannulation strategy In Aortic Surgery-our experience at NHFH\&RI.

Prasanta Kumar Chanda; M.A. Gafur 
Proper cannulation is essential first step in establishing CPB, in Aortic Surgery. The choice of the optimal arterial cannulation strategy for surgery on proximal aorta and arch remains a controversial area and a subject of intense debate. Several cannulation strategies have been proposed to establish cardiopulmonary bypass (CPB) for aortic surgery and each one of these has its pros and cons.

We have done 100 cases of aortic surgery (aortic root, ascending aorta, arch \&amp; descending aortic pathology) from March 2012 to November 28, 2018. There age ranges from 7 to 67 years and male were 106 and female were 14 . We have used various cannulation sites, as deemed appropriate by the surgeon, for each patients. Femoral artery caulation-10, Right axillary artery -8 , Right common carotid artery -18 , Arch cannulation 28 , Left common carotid artery -6 , Right brachiocephalic artery 30 .

Initially we preferred femoral artery cannulation, aortic dissection forced us to change to axillary artery cannulation. Axillary artery proved time consuming and less satisfactory, so we switched to right brachiocephalic artery cannulation. Of Late we are also using right common carotid for this purpose.

Most centers, worldwide, axillary cannulation is rapidly emerging as the preferred strategy for both acute as well as chronic cases. Our experience with this was less favorable, so our choice is right common carotid or brachiocephalic artery

\section{Congenital Heart Block: A Rare Emergency ( Case Report)}

Rajeev Vashisth

\section{ABSTRACT: \\ INTRODUCTION:}

Isolated Congenital Heart Block (CHB) affects 1:15000 - 20000 live births. It can occur in the fetal life due to maternal disease \{maternal antibodies to SSA (Ro) and SSB (La) $\}$ or due to a congenital heart defect in the fetus.

\section{DISCUSSION:}

Various therapeutic regimes have been used for intrauterine treatment of CHB including; sympathomimetics, plasmapheresis, steroids, intravenous immunoglobulin, digoxin, diuretic and in-utero pacing. The usefulness of these treatments still remain controversial. The use of temporary epicardial ventricular pacing wires implantation at times can be used successfully as a bridge to a permanent pacemaker.

\section{CASE REPORT:}

Our team at Medanta Hospital Sriganganagar, had the opportunity to come across such a case recently.

A near term pregnancy, mother diagnosed as anti-Ro/SSA and anti-La/ SSB antibody positive, IUGR and CHB presented to us in labour on 24/ $08 / 18$ evening. The delivery was via emergency C-section ( weight $2.1 \mathrm{kgs})$, the newborn was shifted to Neonatal ICU. The child had Complete Heart Block with an Escape Rate of $40 \mathrm{bpm}$.

Rate enhancing drugs were started but desired response was not achieved. It was then decided to do Temporary Epicardial Pacing(TPI) as arrangements for Permanent Pacing(PPI) were being made.

TPI was done as an emergent procedure and after 2 days PPI was done electively. The baby was discharged after 5 days in satisfactory condition.

\section{Comparative study of cryomaze and cauterymaze for the treatment of chronic atrial fibrillation associated with mitral valve disease - freezing or burning? Vamshidhar Tirunagari}

\section{ABSTRACT:}

\section{INTRODUCTION:}

Atrial fibrillation is present in approximately $50 \%$ of all patients undergoing mitral valve surgery. Many simplified procedure using the basic "Maze" principle are in use like cryo-ablation, cauteryablation and radiofrequency ablation. This study compares CRYOMAZE AND CAUTERYMAZE for atrial fibrillation associated with mitral valve disease.

\section{MATERIAL AND METHODS:}

This is a prospective randomized comparative study. Patients undergoing mitral valve replacement with Maze procedure with or without associated procedures, at CARE hospitals, for 3yrs, shall be the potential subjects. preoperative clinical characteristics, procedural characteristics, major and minor complications after surgery, preoperative and postoperative left ventricular ejection fraction (LVEF) at discharge and 1 year follow up, rhythm by electrocardiogram at the time of discharge and at 6 weeks, 12 weeks and 1 year follow up will be studied

\section{RESULTS:}

In immediate post operative period $19(76 \%)$ in cryomaze and $16(64 \%)$ in electrocauterymaze group were reverted to sinus rhythm. In cryomaze group, out of converted patients, 3(12\%) of them reverted back to AF that required medical cardioversion. All of them converted back to sinus rhythm after cardioversion and correction of underlying cause. Cryomaze and electrocauterymaze are similar in terms of morbidity and mortality, effect on cardiac function, reduction in LA mean and quality of life.

\section{CONCLUSION:}

Cryomaze group has superiority over electrocautery maze in terms of providing freedom from atrial tachyarrythmias. Cryomaze and electrocauterymaze are similar in terms of morbidity and mortality, effect on cardiac function, reduction in LA mean and quality of life

Single center experience of permanent pacemaker insertions in pediatric patients: A 6-year retrospective study K.V.S.S. Sai Kiran; P. Sairam; Gopichand Mannam

\section{ABSTRACT:}

\section{INTRODUCTION:}

Complete heart block (CHB) is a rare condition which can occur either congenitally or post cardiac surgery and requires permanent pacemaker implantation (PPI). Pediatric PPIs constitute less than $1 \%$ of all PP implantations.

\section{METHODS:}

A total of 40 patients who underwent permanent pacemaker insertion from March 2012 to October 2018 were retrospectively analyzed with regard to cause of pacemaker insertion, device failures and replacements, prosthetic site infection and mortality. The mean age of the patients was $25.7 \pm 45.03$ months and the mean follow-up duration was $50.3 \pm 24.5$ months with 5 patients were lost to follow up. All patients had 6 monthly or yearly pacemaker evaluation.

\section{RESULTS:}

Out of 40 patients, $47.5 \%$ of patients (19) had Congenital CHB with PPI insertion being the primary procedure. $52.5 \%$ (21) had PPI insertion for post-surgical CHB with an incidence of $0.024 \%$ of all cases operated during the study period. Of these, $25 \%$ had VSD closure, $12.5 \%$ had TOF repair, $7.5 \%$ had ASO + VSD closure, $5 \%$ had AV canal repair and $2.5 \%$ underwent DORVrepair. Among the patients with congenital $\mathrm{CHB}, 84.2 \%$ had structurally normal hearts with the rest having one each case of PDA, severe LV dysfunction and CCTGA. A total of five mortality were noted among 35 patients followed with one mortality in the congenital CHB group occurring in a case of CCTGA and 4 in the post-surgical CHB group. 4 deaths were not related to pacemaker malfunction and one sudden death of uncertain etiology occurred in a case of VSD closure. There were no prosthetic site infections. One patient underwent pacemaker exchange for malfunction and 4 patients are awaiting battery replacement. 


\section{CONCLUSION:}

Incidence of post surgical PPI is very low. Usage of dual leads is associated with lower thresholds and longer PPI life. Techniques of implantation and management protocol will be further discussed.

\section{Effect on natural course of pulmonary vascular diseases in isolated Atrial septal defect at high altitude \\ Nabi Lone}

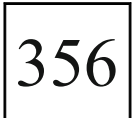

\section{ABSTRACT:}

BACKGROUND:

Development of pulmonary vascular disease has been considered a risk factor in long term course of ASD. lung biopsy remains the gold standard to confirm the degree of severity.

\section{OBJECTIVE:}

To determine the effect on natural course of pulmonary vascular disease in isolated ASD at high altitude. and to assess the histopathological degree of pulmonary vascular disease.

MATERIAL AND MANAGEMENT:

This study was carried in department of CVTS, Sheri-i- kashmir institute of Medical sciences. patients residing at more than $3,500 \mathrm{ft}$ above sea level. all patients were subjected to cardiac Cath prior to surgery. pulmonary vascular resistance was calculated in all patients. lung biopsy was taken before patient was put on CPB and send for histopathology. histopathology was done by single histopathologist. severity of pulmonary vascular disease was assigned a score between 1-4.

\section{RESULTS:}

Total of 18 patients histopathological score of one was seen in none wherease two patients had score of 2

8 patients had score of 3 .

8 patients had score of 4 .

$44 \%$ of patients had score of 3 .

\section{CONCLUSION:}

No formal study has been conducted so far to analyse the effect of high altitude on pulmonary vascular disease in atrial septal defect. Pulmonary disease as proved by histopathological scoring and Cath data was quite high. this is in contrast with those residing at low altitude as reported in literature. therefore it seemes seems that high altitude has a strong bearing on the natural course of disease.

\section{Surgical ligation on hemodynamically significant} patent ductus arteriosus in very low birth weight infants Jun Ho Lee; Hyo Jun Jang; Won-Sang Chung

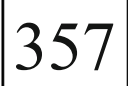

\section{ABSTRACT: \\ BACKGROUND:}

We aimed to evaluate the feasibility of early surgical ligation (before 2 weeks of life) in very low birth weight infants (VLBWIs) with hemodynamically significant (hs) patent ductus arteriosus (PDA) and to investigate parameters helpful for decision of early surgery

\section{METHODS:}

From January 2010 to December 2017, 231 VLBWIs with hs PDA were retrospectively reviewed. Of them, $125(54 \%)$ infants underwent surgical ligation and were subdivided into early ligation group (61 (48\%); EL; before 2 weeks of life) and late ligation group (64 (52\%); LL; 2 weeks or later).

\section{RESULTS:}

The mean gestational age $(26.2 \pm 2.2$ vs $27.2 \pm 1.7$ weeks, $\mathrm{p}=0.001)$ and birth weight $(881.4 \pm 228.1$ vs $1039.3 \pm 251.2$, p \&lt; 0.001) were significantly lower in the PDA-ligated group compared with the non-ligated group. The morbidities (BPD; $\mathrm{p}=0.858$, IVH; $\mathrm{p}$ \&gt; 0.999, sepsis; $\mathrm{p}=$ 0.716, NEC; $p=0.563$, ROP; $p=0.203$ ) and mortality ( $p$ \&gt; 0.999 ) did not differ significantly between the EL and LL groups.
After adjustment for confounders, echocardiography classification of hs PDA $(\mathrm{p}=0.013)$ and histologic chorioamnionitis $(\mathrm{p}=0.044)$ were significantly associated with EL group, which were consistently related to early ligation of hs PDA in our multivariable logistic regression analysis. CONCLUSION:

EL was not significantly associated with increased morbidities and mortality in VLBWs with hs PDA. The echocardiography classification of hs PDA and histologic chorioamnionitis can be related to the need for early surgical ligation of hs PDA.

Arterial switch operation - Modified trapdoor technique for coronary artery transfer

Prashanth Shah; Harun

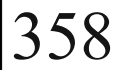

\section{ABSTRACT:}

\section{INTRODUCTION:}

The arterial switch operation has been the most accepted procedure for the repair of transposition of great arteries. Many techniques and its modification have been developed over the decades. The transfer of coronary arteries is the challenging and crucial part in the procedure.

\section{AIM AND OBJECTIVE:}

In our early experience the classical trapdoor technique which is performed widely for coronary artery transfer was associated with little tension on the anastomosis. In recent years we noticed that slight modification in the trapdoor technique gave better results without tension on the anastomosis

\section{METHODS:}

In arterial switch operation the coronary buttons harvested from the native aorta and transfer to the neoaorta. The corresponding anastomotic site is marked with stay sutures above the level of neoaortic valve commissures. Instead of using aortic punch in classical trapdoor technique, we incised the aortic wall without excising the aortic wall for the coronary anastomosis. The advantage of this technique is that we can extend the incision length in any direction depending upon the orientation of the coronary buttons, so that we can avoid even little kinking and stretching of the coronary arteries.

\section{RESULTS AND CONCLUSION:}

This approach for coronary artery transfer facilitates the precise transfer of the coronary buttons into the neoaortic root. It minimizes the risk of tension on the anastomosis and facilitates proper alignment. After weaning of cardiopulmonary bypass it reduces the incidence of ischemia induced ST elevation, improves contractility and faster recovery of the patient.

An interesting case of Bland White Garland syndrome Sujit Kumar Mohanty

\section{ABSTRACT:}

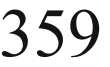

Anomalous origin of the left coronary artery from the pulmonary artery (ALCAPA) syndrome, also known as Bland-White-Garland syndrome, is a rare congenital abnormality that affects 1 of every 300,000 live births and accounts for $0.25 \%-0.5 \%$ of all congenital heart defect. $90 \%$ of patients with ALCAPA syndrome die within the 1st year of life. In patients who live to adulthood, ALCAPA syndrome may cause myocardial infarction, left ventricular dysfunction and mitral regurgitation, or silent myocardial ischemia, which can lead to sudden cardiac death. Early diagnosis and prompt surgical intervention is the key to success.

\section{CASE:}

35years male previously operated(MVR) for rheumatic mitral regurgitation presented with chest pain and breathlessness. His ECG showed frequent ventricular ectopics with LBBB pattern. Echocardiogram showed well functioning mitral valve with normal gradients and severe LV dysfunction. His coronary angiogram was done which revealed dilated 
tortuous dominant right coronary artery and anomalous origin of left coronary artery from pulmonary artery. Findings were reconfirmed by CT angiogram. A redo surgery was planned. PTFE patch closure of ALCAPA done along with CABG (LIMA to LAD \&amp; SVG to Ramus). Patient recovered well his LV function improved and is asymptomatic and following with us since last 6 months.

CONCLUSION:

Bland White Garland syndrome(ALCAPA syndrome) is very rare entity. Redo surgery in this case makes it more complex and rare. All patients presenting with severe LV dysfunction and mitral regurgitation should be properly evaluated before surgery. Coronary angiogram is must even in young rheumatic mitral valve cases.

\section{Low Requirement of Surgical Intervention in Postoperative Chylothorax after Pediatric Cardiac Surgery}

Yu Rim Shin

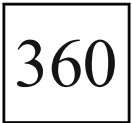

\section{ABSTRACT: \\ BACKGROUND:}

Chylothorax after congenital heart surgery is a common complication with associated morbidities, but consensus treatment guidelines are lacking. We have implemented management protocol for postoperative chylothorax to reduce its incidence and to improve treatment outcomes.

\section{METHODS:}

All corrective and palliative operations for congenital heart disease performed in our institute between January 2008 and April 2018 were retrospectively reviewed.

\section{RESULTS:}

Incidence of chylothorax was $1.9 \%$. Sixty percent of the patients could be managed with low-fat diet and $27 \%$ of the patients required NPO. Thoracic duct embolization was performed in 2 patients and chest tube drainage decreased immediate after procedure. No patient required thoracic duct ligation or pleurodesis. After implementation of institutional management protocol, chests tube drainage date and hospital stay decreased from ( 24 vs 17 days and 37 vs 21 days), but it did not reach statistical significance.

\section{CONCLUSION:}

Utilization of strategy to reduce postoperative chylothorax resulted in acceptable incidence of postoperative chylothorax. Institution of a clinical practice guideline might help to reduce the treatment duration and to decrease the requirement of surgical treatment. Image guided embolization of thoracic duct is effective treatment for postoperative chylothorax.

Outcomes in tetralogy of fallot - our experience from a pediatric tertiary care center - chennai. G.K. Jaikaran

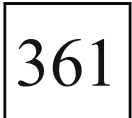

\section{ABSTRACT: \\ INTRODUCTION:}

Tetralogy of Fallot (TOF) is one of the conotruncal family of heart lesions in which the primary defect is an anterior deviation of the infundibular septum (the muscular septum that separates the aortic and pulmonary outflows. The degree of pulmonary outflow obstruction varies, with the severity of the obstruction determining the degree of the patient's cyanosis. Corrective surgical therapy consists of relief of the right ventricular outflow tract obstruction by removing obstructive muscle bundles and by patch closure of the VSD. The surgical risk of total correction in major centers is \& $1 \mathrm{t} ; 5 \%$.

\section{MATERIALS AND METHODS:}

We conducted a retrospective study in our institute of paediatric CVTS, Egmore, regarding the outcomes and the predictive factors of mortality following TOF repair between 2014 -2018. study population 50 cases.
RESULT:

Our overall mortality during the study period is $10 \%$, with improving expertise and perioperative management, our mortality over the past two years has reduced to zero and is comparable to international standards.

Surgical outcome of repair for Tetralogy of Fallot with absent pulmonary valve

Yasuhiro Kotani

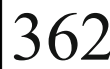

\section{ABSTRACT:}

\section{OBJECTIVES:}

Tetralogy of Fallot with absent pulmonary valve (TOF/APV) is a rare congenital anomaly and little is known about surgical outcome. The purpose of this study is to review the surgical outcome in patients with TOF/APV.

\section{METHODS:}

11 patients underwent surgical repair of TOF/APV at our institution from 1991 to 2012. Median age and body weight at surgery was 9 months and $5.5 \mathrm{~kg}$, respectively. 4 patients had a respiratory symptom preoperatively with 1 patient required a mechanical ventilation. Our surgical techniques included 1) creation of monocuspid pulmonary valve using autologous pulmonary arterial wall and 2) Aggressive plication of the bilateral pulmonary arteries.

\section{RESULTS:}

There was no early and late death. 1 patient required surgical reintervention due to pulmonary stenosis/regurgitation 21 years after initial surgery. At median follow-up of 12 years, median pressure gradient across the right ventricular outflow tract was $34 \mathrm{mmHg}$ and pulmonary regurgitation was mild in 3 patients, moderate in 6 patients, and severe in 2 patients. All 11 patients improved their respiratory syndrome after surgery and no one required a prolonged mechanical ventilation.

\section{CONCLUSIONS:}

Surgical outcome after repair of TOF/APV was excellent. Our surgical approach has a benefit not only to address respiratory symptom but also preventing RVOT-related reintervention.

\section{Use of virtual reality in congenital} heart diseases-a Primer

John S.K. Murala; Animesh Tandon; Paige Mckenzie;

Vasu Gooty; Gerald Greil; Robert Jaquiss; Tarique Hussain

\section{3}

\section{ABSTRACT:}

\section{INTRODUCTION:}

In the fast pace of technological advances in the management of congenital heart disease (CHD) we describe the role of Virtual reality (VR) technology in diagnosis, planning and teaching. We present our early experience and the possible spectrum of this technology.

\section{METHODS:}

3D CT or MRI datasets from patients with CHD were segmented and 3D masks generated. A shell was calculated around the blood pool mask, and the shell was converted to a 3D file. These shells were then imported into a custom, patient-specific, virtual reality (VR) environment designed (Varyfii Imaging, UT Southwestern) to allow interaction with 3D datasets, including distance measurements, scaling and sectioning. The VR space, enables the operator to view the internal anatomy and plan surgical or catheter interventions and develop teaching modules.

\section{RESULTS:}

This methodology was used for: (1) the planning of complex double outlet right ventricle cases, and was found useful in surgical planning, (2) planning Fontan conduit placement in the setting of hetrotaxy, (3) virtual fitting of left ventricular assist device in pediatric recipients to check device fit, and (4) virtual assessment of device parameters in device closure of sinus venosus defect. We are currently using this technology to create $3 \mathrm{D}$ virtual models of preserved cardiac specimens for teaching purposes. 


\section{CONCLUSIONS:}

This study describes the early experience in the medical use of VR technology. We believe that this technology greatly enhances further treatment of patients with congenital heart disease and replaces the need for $3 \mathrm{D}$ printing in the majority of cases. We feel that this is a valuable tool for teaching cardiac morphology. This technology also helps in digitization of cardiac specimens and making it freely available for training purposes.

\section{Effect of Monocusp Valve Construction on Tetralogy} of Fallot repair - Early and long term outcomes. Manoj Durairaj

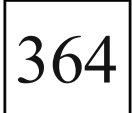

\section{AIM:}

ABSTRACT:

The aim of this study are a) to compare the early outcomes of pulmonary valve sparing vs Transannular patch(TAP) with monocusp valve reconstruction using PTFE membrane or TAP alone and b) evaluate the long term results after monocusp valve reconstruction.

\section{METHODS:}

Between 2007-2013, 112 patients underwent Total Correction. 44 patients (39.2\%) underwent pulmonary valve sparing procedure (Group 1), 55 patients (49\%) underwent PTFE monocusp valve reconstruction (Group 2) and 13 patients $(11.6 \%)$ underwent TAP only. The early outcomes were measured by RV/LV ratio, RV outflow tract gradient, tricuspid and PV function and ICU parameters. Group 1 patients were also evaluated at the mid term for clinical/ Echo parameters with specific focus on monocusp function.

\section{RESULTS:}

The mean age, weight and PV Z-value of Group 2 were lower ( 38 months, $12.5 \mathrm{kgs}$ and -4 respectively). Post operative $\mathrm{RV} / \mathrm{LV}$ ratio was \&lt; 0.5 in all groups. In Group 3, the mechanical ventilation time and total ICU stay was significantly longer. The in hospital mortality was $2.6 \%$ (3 pts). The pulmonary incompetence was moderate or severe in $2 \%$ of Group 1 pts, $2.5 \%$ of Group 2 pts and $55 \%$ of Group 3 pts. The long term follow up of Group 2 pts revealed $85 \%$ had normal function or mild monocusp incompetence. There were overall 3 mid term deaths.

\section{CONCLUSIONS:}

A competent pulmonary valve ( either valve sparing/ monocusp ) improves early and mid term results after TOF repair. The RV function is preserved in the long term with this strategy.

\section{Closure of atrial septal defect through back Mahendra Bafana}

\section{ABSTRACT:}

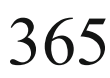

Atrial septal defect can be closed surgically by multiple approaches. We closed the ASDs in pediatrics age groups through the back. The anterior part of incision does not cross the right posterior axillary line. We report 75 cases done successfully. The weight of patient s range from $8 \mathrm{~kg}$ to 21 $\mathrm{kg}$. Advantage_scar is never visible, in females does not hamper breath development. Summary Surgeons armamentarium to compete the device, easily reproducible, cost effective, no extra costly instruments required.

\section{Biventricular conversion after single ventricular palliation in a patient with protein losing enteropathy: An alternative to transplantation Ganapathy Subramaniam; \\ K.G. Suresh Rao; K.R. Balakrishnan}

\section{ABSTRACT:}

\section{INTRODUCTION:}

Fontan circulation is associated with a variety of long-term sequelae like thromboembolic events, hepatic dysfunction, protein-losing enteropathy, etc which are not seen in a biventricular circulation. Transplantation is an option in such a subset but may not be feasible due to various reasons. The indications, timing and outcomes after transplantation remain undefined.

\section{BACKGROUND:}

Data on Biventricular repair after Single ventricle palliation are limited. Patients who initially undergo single ventricle palliation due to complex anatomy not amenable to biventricular repair may eventually be bailed out from late complications with a biventricular repair. We describe our experience in one such case.

\section{CASE:}

14 years old boy with previous Glenn and Fontan procedure for DORV, Non-routable VSD, PS presented with anasarca and ascites from proteinlosing enteropathy. Echocardiography and CT scan revealed that biventricular repair was possible.

A $22 \mathrm{~mm}$ PTFE valved conduit was prepared. The groin vessels were exposed. Due to an aortic injury during dissection, emergency CPB was established. The thickened Dacron conduit was dissected out completely from IVC and PA and whole of it was excised. Intra-cardiac repair was done by closing the VSD with a large patch routing the VSD to the aorta. Systemic venous blood was diverted to RA using $18 \mathrm{~mm}$ PTFE Interposition graft. RV-PA continuity was established with the $22 \mathrm{~mm}$ valved conduit.

Post-operative TEE showed satisfactory repair with unobstructed systemic venous return, good LVOT/ RVOT pathways, no residual VSD. He is asymptomatic with good biventricular function on follow-up.

\section{CONCLUSION:}

Biventricular conversion should be considered whenever feasible, to alleviate the long-term complications of Fontan circulation. It is a worthy alternative to transplantation.

Outcome of cardiac surgery in low birth weight neonates - a single centre experience Charumathi Dasarathan

ABSTRACT:

OBJECTIVE:

Irrespective of weight or prematurity, neonates with congenital heart disease (CHD), require surgery, in view of their deteriorating clinical condition. This study reviews a single centre experience with cardiac surgeries in low birth weight neonates.

\section{METHODS:}

From August 2011 to august 2018, 178 cardiac surgeries were performed in infants, out of which 65 infants (45 males and 25 females) weighing less than $2500 \mathrm{~g}$, who underwent palliative or corrective surgeries with or without cardiopulmonary bypass (CPB) were analyzed.

\section{RESULTS:}

Peri-operative morbidity was $8 \%$ in LBW patients in CPB group and $1 \%$ in non CPB group. There were five early deaths. Mean survival at one year was $90 \%$ and at five years was $88 \%$. Peri-operative morbidity and mortality were significantly high in patients with non-cardiac abnormalities and premature babies.

\section{CONCLUSION:}

Cardiac surgery in LBW infants can be performed safely and effectively. However post operative stay in premature and low birth weight babies are comparatively high.

Grown-up Congenital Heart Disease - our Institutional Experience

Sarava Krushna Raja; Manimaran

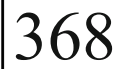

ABSTRACT:

\section{BACKGROUND:}

Congenital heart disease patients have cardiac and thoracic great vessel structural malformations at birth. The incidence of CHD is about 8-10/ 
1000 live births. Small ASD and VSD spontaneously resolve. Grown-up Congenital Heart (GUCH) disease comprises of patients who are diagnosed to have CHD in their adulthood or those who have been followed up or being treated conservatively and present with new onset symptoms. MATERIALS AND METHODS:

All patients above the age of 14 yrs, diagnosed with congenital heart disease and admitted in our tertiary care centre from january 2017 were studied. They undergo investigations like 2-D echo, cardiac cathstudy, CT thorax.

\section{RESULTS:}

A total of 77 patients were studied of which 6 were in-operable. In those who underwent surgery, 53 had ASD, 3 had Tetrology of Fallot, 8 had VSD, 2 had Coarctation of aorta, 3 had Double chambered right ventricle, 2 had Patent ductus Arteriosus.

Mean age at surgery were 25.1 yrs for ASD, 22 yrs for TOF, 23.1yrs for VSD, $38 \mathrm{yrs}$ for DCRV, 43 yrs for COA.of all patients who underwent surgery, 1 of TOF, 1 of COA, 2 of DCRV expired within few days of surgery.

\section{CONCLUSION:}

Identification of $\mathrm{CHD}$ at early age and regular follow up can decrease the age at diagnosis and initiate prompt intervention. Patient education at the time of diagnosis about natural history of disease and identification of symptoms and advice to seek early intervention at the onset of symptoms can reduce post op complications.

Registry of Congenital Heart disease patients to be maintained and shared treatement groups.

Hand made conduit for right ventricle to pulmonary artery connection Benefits and outcome in our experience Prashant Shah; Harun

\section{ABSTRACT:}

\section{INTRODUCTION:}

A significant proportion of congenital heart defects have RVOT abnormality. Depending upon the type of the RVOT abnormality the surgical option differs. It is indicated for more complicated form of RVOT abnormality such as $\mathrm{RV}$ - PA discontinuity. There are various reconstructive options are available for RV - PA conduits.

\section{AIM AND OBJECTIVE:}

Majority of congenital heart disease patients in Indian population are from lower social economic group. The affordability for the commercial valved and valveless conduits are low. Our aim is to reduce the cost of the conduit as well as not to compromise the efficacy of the available ready made conduits. MATERIALS AND METHODS:

Patients who have absent RVOT like pulmonary atresia, unsuitable RVOT like D-TGA, VSD, PS. LTGA, PS. complicated DORVand ross procedure are the candidates for RV - PA conduit. We used autologous pericardium for first time replacement and bovine pericardium, PTFE graft for redo cases. According to BSA the size of the graft was calculated. The calculated size of the graft prepared by using Dr. Shah's template.

\section{RESULTS AND OUTCOME:}

5 patients were operated. 3 patients were first replacement and 2 patients were redo replacement. Post operative echocardiography showed well functioning valved conduit. Mild to moderate pulmonary regurgitation was accepted. CONCLUSION:

Hand made valved conduit is a good alternative to readily available commercial conduits and it is very cost effective and well functioning conduit as same as the manufactured valve conduits.

A Comparison of Short Term Results of Bidirectional Glenn Procedure with or without Cardiopulmonary Bypass support

Kuntal Surana; Chaitanya Raut;

Vaibhav Shah; Harsh Seth
BACKGROUND:

Bidirectional Glenn Shunt i.e. superior cavopulmonary anastomosis (BDG) is a palliative surgical procedure for anatomical or physiological univentricular hearts, in which the superior vena cava is anastomosed to the ipsilateral pulmonary artery in an end-to-side manner with or without cardiopulmonary bypass (CPB). The objective of this study was to compare the short term results of the BDG procedure performed on $\mathrm{CPB}$ or without $\mathrm{CPB}$

\section{METHODS:}

From September 2016 to September 2018, 40 patients with anatomical or physiological univentricular hearts (mean age $36 \pm$ 12 months) underwent BDG procedure and were randomly assigned to either group I: using CPB ( $n=22$ patients; mean age $36 \pm 14$ months) or group II: without using CPB ( $n=18$ patients; mean age $38 \pm 10$ months). In group II two techniques were used (A) With using a temporary veno-atrial shunt $(\mathrm{n}=9$ cases mean age $38 \pm 10$ months), (B) Without using a venoatrial shunt $(\mathrm{n}=9$ cases mean age $38 \pm 10$ months $)$. We monitored the superior vena caval (SVC) pressure, O2 saturation, Operative time, mechanical ventilation period, intensive care unit (ICU) stay, hospital stay, operative mortality and postoperative complications for comparison between the two major groups.

\section{RESULTS:}

There was significant decrease in the operative time in group II $(100 \pm 20$ $\mathrm{min})$ in comparison to group I $(140 \pm 15 \mathrm{~min})$. The differences in the period of mechanical ventilation, ICU stay, hospital stay, postoperative complications and mortality were insignificant between both the groups. CONCLUSIONS:

The BDG procedure can be performed with no significant differences in operative mortality, morbidity, or use of resources, with or without $\mathrm{CPB}$ support and with considerably less operative time without CPB support.

Intraoperative thoracotomy is a risk factor for prolonged pleural drainage after the Fontan operation Satoshi Matsuo

Naoki Masaki

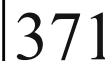

\section{ABSTRACT: \\ BACKGROUNDS AND OBJECTIVES:}

The postoperative prolonged pleural effusion (PPE) has remained as one of the challenging problems after Fontan procedure. The aims of this study were to describe the risk factors for PPE after Fontan operation, and to clarify the impact of the intraoperative thoracotomy for the drainage tube insertion on PPE.

\section{METHODS:}

We retrospectively reviewed 43 consecutive patients who underwent Fontan operation from 2008 to 2018. Drainage tubes were usually placed in the thoracic space or pericardial space by surgeon's preference. In the present study, we divided the patients into the following 3 groups: group A ( $n=7)$ : no thoracotomy; group B $(n=15)$ : unilateral thoracotomy; and group $\mathrm{C}(\mathrm{n}=21)$ : bilateral thoracotomy, and data was analyzed.

\section{RESULTS:}

Median age was 26 months old, and median body weight was 10.5 $\mathrm{kg}$. Total amount of the pleural effusion at day 1, 2, and 3 were significantly lower in group A than those in other groups. Median duration of drainage was significantly shorter in group A than that in other groups $(3,4$, and 5.5 days, respectively, $\mathrm{p}=0.03)$. There were 12 patients $(28 \%)$ who need the chest tube drainage for 7 days or more. Multivariate analysis revealed that intraoperative thoracotomy $(\mathrm{p}=0.048)$ and postoperative water balance $(\mathrm{p}=0.04)$ were independent predictors for PPE.

CONCLUSIONS:

Intraoperative thoracotomy and post-operative water balance were the risk factors for the prolonged pleural effusion after Fontan operation. To 
avoid intraoperative thoracotomy for the drainage tube insertion may contribute to the reduction of postoperative pleural effusion and morbidities after Fontan operation.

\section{Beating Heart Surgery in Atrial Septal Defect with Pulmonary Arterial Hypertension \\ Uday Jadhav, Dr. Kalpesh Agrawal}

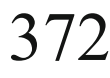

We present our experience at our institute of repairing all types of atrial septal defect(ASD) with moderate pulmonary arterial hypertension (PAH), on pump beating heart with normothermic blood perfusate.

PAH is a major risk factor for morbidity and mortality in patients undergoing cardiac surgery. Myocardial dysfunction and PAH after cardiopulmonary bypass (CPB) are usually mild and do not lead to postoperative circulatory failure. However, in vulnerable patients, patients with abnormal pre-operative RV myocardial relaxation, $\mathrm{CPB}$ may contribute to postoperative right ventricular failure (RVF).

From 2016 to 2018, 13 patients (Male - 6 and Female - 7) age more than 40 years with moderate PAH underwent ASD repair with on pump beating heart technique. Among these 3 patients underwent minimal invasive ASD repair. We had 8 patients with ostium secondum, 3 with sinus venosus and 2 with ostium primum defects. Pre operative diagnosis was established by 2D ECHO and TEE. ASD was closed using autologous pericardial patch. Heart was kept beating throughout the procedure with normothermic blood perfusion at 4 to $5 \mathrm{~L} / \mathrm{min}$ flow. There was no complication.

Aim of this technique is to avoid ischemic - reperfusion injury and RVF, with additional benefit of monitoring and avoiding any cardiac conduction injury and assessing mitral and tricuspid insufficiency during the procedure. Post-operatively patients required less inotropic support and ventilatory support with early discharge.

Beating heart surgery provides a good method for preventing RV failure in patients of ASD with pre-operative PAH.

\author{
Surgical outcome and treatment of \\ TOF with absent pulmoary valve \\ Balaji Aironi \\ Harendra Ojha
}

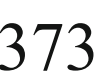

\section{ABSTRACT: \\ OBJECTIVE:}

Tetralogy of fallot and absent pulmonary valve(TOF/APV) is associated with significant pulmonary artery dilatation and airway compression. Treatment of children of \&lt; $3 y$ rs of age presenting with respiratory symptoms is associated with high mortality (10 to $20 \%$ ). We aim to report our results and identify factors associated with survival and prolonged ventilation. METHODS: We performed a retrospective review of 38 consecutive patients following repair of TOF/APV(2008 to 2018). Median age at repair was $3 \mathrm{yrs}(1.5 \mathrm{yr}$ to $35 \mathrm{yrs})$.

\section{RESULTS:}

38 patients underwent complete repair. The right ventricular outflow tract (RVOT) was reconstructed with valved conduit $(\mathrm{n}=25)$, and mechanical prosthetic valve $(\mathrm{n}=8)$, transanullar patch $(\mathrm{n}=5) .5$ And 10 yrs survival was $9 \& \mathrm{gt} ; 1+/-4 \%$ and $85+/-5 \%$. Mean ventilatory requirements for children more than $1 \mathrm{yr}$ of age were $2.2+/-1.1$ days(p\&lt;\&lt;0.0001). Freedom from RVOT re operation was $95+/-3 \%$ and at $5 y$ rs there were no significant risk factors for time related survival or RVOT reoperation on multi variable analysis.

\section{CONCLUSION:}

In contrast to adults with TOF/APV, children of age less than 3 yrs presenting with respiratory symptoms require prolonged ventilation. Our current surgical approach which includes reconstruction of a competent RVOT, and aggressive postoperative ventilator management to relieve airway obstruction offers satisfactory outcomes.
Our experience in oriental type of tetralogy of fallot Prashanth Shah; Harun

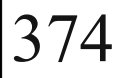

\section{ABSTRACT:}

\section{INTRODUCTION:}

Tetralogy of fallot is a congenital cardiac malformation that consists of four components ventricular septal defect, overriding of aorta, right ventricular outflow tract obstruction and right ventricular hypertrophy. Each component can vary in its severity, with the variation directly affecting the manifestation and management of the disease.

\section{AIM AND OBJECTIVE:}

Our aim is to outline the features of a variant of tetralogy of fallot so called oriental type. This variant requires different approach in closing the VSD. MATERIALS AND METHODS:

Total of 288 patients were operated between 2009 to 2018.38 patients were associated with classical features of oriental type which are slightly different from regular tetralogy. The features of oriental type of tetralogy includes large conoventricular VSD, absent infundibulum, aorta and pulmonary artery at the same level that is aorta bulging into RVOT, good sized MPA and MPA continues as RPA. Majority cases of oriental type confirmed on table.

\section{RESULTS AND OUTCOME:}

The closure of the VSD differs from other regular cases. In regular cases most of the VSD closed through transatrial approach. But in oriental type the VSD closed through transpulmonary or transinfundibular incision. The anterosuperior portion of the defect closed by passing the sutures through the pulmonary valve leaflets to annulus. Because the anteroseptal margin of the VSD is not formed by the muscular outlet septum and it is formed by the fibrous continuity between the leaflets of aortic and pulmonary valves.

\section{CONCLUSION:}

Understanding the features of oriental type of TOF helps in identify the correct anatomy and changing the approach for surgery. This helps in proper intra cardiac repair and better outcome of the patients.

Ebstein's anomaly presenting with acute coronary syndrome-a rare combination R.P. Mahapatra

Jaideep Kumar Trivedi

\section{ABSTRACT:}

Ebstein's anomaly is a relatively rare congenital heart disorder characterized by downward displacement of septal and posterior leaflets of the tricuspid valve into right ventricle cavity. The usual presenting symptoms are cyanosis, right sided heart failure and arrhythmia. Progressive heart failure or tachyarrhythmia may worsen cyanosis. Acute coronary syndrome is rarely reported in Ebstein anomaly. We report here a patient of Ebstein anomaly who was apparently asymptomatic and undiagnosed and presented with acute coronary syndrome.

\section{CASE REPORT:}

44years male previously asymptomatic presented with breathlessness and chest pain of sudden onset. His ECG showed $T$ wave inversion and LBBB pattern. His coronary angiography showed severe triple vessel disease. Echocardiogram confirmed ebstein anomaly. Patient was operated, CABG was done on beating heart then taken on $\mathrm{CPB}$ and Cone repair of tricuspid valve was done successfully. Patient recovered well and was discharged on 9th day. Post surgery echocardiogram showed improved LV function. Patient is in regular follow up since last 6 months and completely asymptomatic

\section{CONCLUSION:}

The present case describes an atherosclerotic acute coronary syndrome in a relatively young active adult presenting Ebstein's Anomaly, not diagnosed during the early ages of life, without any complications 
during childhood and any related symptoms in the adult age. This is an unusual evolution of that rare disease and, as far as we are concerned, not previously described association with coronary atherosclerosis in the scientific literature.

Ebstein's anomaly correction and CABG combined procedures and its risks were not described previously in the literature

\section{Intermediate outcomes of direct aortic implantation during repair of Anomalous left coronary artery from pulmonary artery \\ Goutami Vejendla; Anil Kumar Dharmapuram}

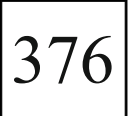

\section{ABSTRACT: \\ OBJECTIVE:}

During repair of Anomalous left coronary artery from pulmonary artery (ALCAPA), direct aortic implantation is the preferable method and can be achieved in all cases.

\section{METHODS:}

Between September 2012 to April 2018, 16 patients underwent repair of ALACPA. The age ranged from 1 month to 120 months (median 5 months) and the weight ranged from $3 \mathrm{~kg}$ to $21 \mathrm{~kg}$ (median $4.5 \mathrm{~kg}$ ). In all patients, the ALCAPA button was harvested after transecting the pulmonary artery. It was carefully dissected and aggressively mobilized to gain adequate length to reach the aorta. It was implanted directly to the aorta on the left lateral aspect by creating an anterior flap to avoid kinking. One patient aged 5 years had severe mitral regurgitation due to a bulky and prolapsing anterior leaflet; a quadrangular resection of the bulky leaflet was done and an artificial goretex neo-chorda was inserted. Perioperative management included using of milrinone as a inodilator. In all patients less than $10 \mathrm{~kg}$, the chest was kept electively open in the ICU and closed only after hemodynamics and lactates stabilised.

RESULTS:

There was one hospital mortality in a 4 month old infant due to renal failure secondary to systemic infection. The median follow up of the survivors was 36 months. All the survivors were doing well with normal ventricular function and no mitral regurgitation. The patient in whom the mitral valve was concomitantly repaired had good leaflet co-aptation without prolapse and mild mitral regurgitation.

\section{CONCLUSIONS:}

During repair of ALCAPA, it is always possible to implant the coronary button directly to the aorta. This technique is easily reproducible and does not cause kinking or distortion of the coronary button. The intermediate outcomes with this experience are encouraging.

\section{Intermediate outcomes of complete routing of the anomalous pulmonary veins in mixed type of connection \\ Sandeep Janardhan Alamandha \\ Anil Kumar Dharmapuram}

\section{ABSTRACT: \\ OBJECTIVE:}

In mixed type of TAPVC, complete re-routing is always preferable for better long-term outcomes.

\section{METHODS:}

Between May 2012 to January 2018, 16 patients underwent complete rerouting of the mixed type of anomalous pulmonary venous connection. The age ranged from 1 month to 36 months, (median 3 months) and the weight ranged from $2.5 \mathrm{~kg}$ to $9.5 \mathrm{~kg}$ (median $3.5 \mathrm{~kg}$ ). In all patients, the entire right pulmonary veins and the left inferior pulmonary vein drained to the coronary sinus; the left upper lobe and the middle lobe veins drained separately to the left vertical vein. Intraoperatively, the intra cardiac variety was routed to the left side after unroofing the coronary sinus and using a baffle of pericardial patch to route them to the left atrium. The left vertical vein was carefully dissected and anastomosed widely in an end to side fashion to the left atrial appendage.

\section{RESULTS:}

Two babies expired after discharge due to complications related to lung infection. The median follow up of the survivors was 33 months. At follow up, all the patients were doing well without pulmonary hypertension and are free from any cardiac medication. Four patients had mild gradient at the anastomosis of the left vertical vein to the left atrial appendage, but there was no evidence of any significant narrowing by $2 \mathrm{D}$ echocardiography evaluation.

\section{CONCLUSIONS:}

Complete re-routing in mixed type of TAPVC is always preferable. Intermediate follow up showed good outcomes without pulmonary hypertension and with acceptable growth of the anastomosis to the left atrial appendage without obstruction. This can be achieved even in small weight babies by careful and wide anastomosis using fine and delicate suturing of the anomalous veins to the left atrial appendage.

Ventricular Septal Defects with near systemic pulmonary pressures - Institutional Experience Kathirvel; Sivasankaran K.

ABSTRACT:

Ventricular Septal Defects (VSD) with severe pulmonary hypertension (PHT) patients are the difficult ones to intervene as they carry increased risk of postoperative morbidity and mortality especially when pulmonary pressures are near to systemic pressures. We share our experience of 31 such cases managed by double flap technique and use of pulmonary vasodilators.

Warm blood perfusion as a novel way of myocardial preservation with biochemical correlation in beating heart with cross clamp viz a viz arrested heart in adults. G.N. Lone; Syed Abdul Wahid

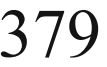

\section{ABSTRACT: \\ OBJECTIVE:}

To study warm blood perfusion as a novel way of myocardial preservation in cardiac surgery with biochemical correlation in beating heart with cross clamp on viz a viz arrested heart in adults.

\section{PATIENTS AND METHODS:}

From September 2011 to November 2016, 25 patients underwent ASD repair on beating heart with continuous antegrade normothermic blood perfusion with aortic cross clamp on. An equal number of patients were subjected to ASD repair on arrested heart. Patients' age and size of defect were similar in both the groups. Preoperative diagnosis was established by 2D echo and color Doppler study. The normothermic perfusion was kept at 4-5 $\mathrm{ml} / \mathrm{kg}-\mathrm{BW} / \mathrm{min}$ in the beating heart group.

\section{RESULTS:}

The mean aortic cross clamp time in the beating heart and arrested heart group were $20.72 \pm 7.08$ and $30.56 \pm 5.83$ minutes respectively. The mean bypass time in the beating heart and arrested heart group were $32.80 \pm 7.48$ and 47.12 \pm 6.28 minutes respectively. There were no differences in blood products transfused. ICU and hospital stay were significantly less in beating heart group. There was no hospital mortality in either group, and no statistically significant difference was detected in CPK-MB and Troponin-I levels. No major complications were observed during hospital stay. All patients were successfully discharged. Post operative echocardiography showed normal LV function and no residual shunt in either group.

\section{CONCLUSION:}

Beating heart surgery using normothermic blood is a safe and effective technique for the closure of ostium secundum ASD. The heart is operating under more physiological conditions. One can evaluate the severity of any associated 
mitral or tricuspid valvular insufficiency and identify iatrogenic conduction injuries.

Redo surgery for Ebstein's anomaly- A Case report Sunil Dixit; Jaikishan Suthar

\section{ABSTRACT:}

380

Redo surgery for Ebstein's anomaly- A Case report

Jaikishan suthar, Vikram Watti, Anil Sharma, Sunil Dixit, Sourabh Mittal Department of Cardiac thoracic-vascular surgery Surgery, S.M.S. Medical college, jaipur, Rajasthan

Ebstein's anomaly is a rare cause of congenital heart disease. Multiple reparative and replacement techniques of surgical correction have been presented. We report a case of reoperation for severe tricuspid valve regurgitation with biventricle dysfunction in a patient who was previously underwent modified Danielson repair for Ebstein's anomaly at the age of 14 years with anuloplasty ring. A 25 years-old female was presented to us with dyspnea (NYHA class 4), congestive hepatomegaly, gross ascites, pedal edema. Patient remained asymptomatic for 5 year after than she develops symptoms that was gradually increased. Serial echocardiography shows gradually increasing severity of tricuspid regurgitation for last 5 year and ATL become tetherd and immobile. Chest xray shows $\mathrm{CT}$ ratio was 0.80 . CT chest suggest right atrium and pericardium was adhere to posterior table of sternum. Patient kept on medical management for last 5 year than planned for Redo surgery. We went for surgery after institution of peripheral femoro-femoral CPBP, valve inspection revealed anuloplasty ring at True Tricuspid annulus with severely tethered immobile ATL. Prosthetic mechanical bileaflet Valve $31 \mathrm{~mm}$ was replaced with anuloplasty ring remain insitu. RA reduction done from $10 \mathrm{~cm}$ to $7 \mathrm{~cm}$. Patient rapidly improved symptomatically and discharged on 7 th postoperative day. Post operative serial echocardiography revealed a perfectly functioning valve with improved biventricular function. Our experience in such case is to reoperate patient as early as possible instead keep on medical management and preferably from peripheral CPBP to avoid catastrophe and valve replacement is better choice in such case.

\section{ECMO Experience in Pediatric Cardiac Unit in a Tertiary Care Institute \\ Hari Bipin Radhakrishnan; Simran Kundan}

\section{1}

\begin{abstract}
:
AIM:

ECMO is a support therapy for patients with cardio-respiratory failure who are failing maximal conventional therapy with inotropes and ventilatory support. In children ECMO is used as long term cardiorespiratory support for those who fail separation from cardiopulmonary bypass and low cardiac output or as respiratory support in ARDS not amenable to ventilatory therapy. METHODS:

From 2011, we retrospectively analysed our data for outcomes.

RESULTS:

Fourty two ECMO runs were carried out. Twenty two were for children below 1 year of age, 7 were 1-5 years, 9 were 5-10 years and 3 were older than 10 years. Average weight was $13 \mathrm{~kg}$. Out of the children who required it for cardiorepiratory support 18 were having LV dysfunction and 17 had $\mathrm{RV}$ or biventricular failure. Seven children required it for respiratory support. Average duration of support was 134.7 hour (SD $179.5 \mathrm{hr}$ ). 30 (71.4\%)successfully decannulat. $12(28.5 \%)$ children developed end organ damage which led to withdrawal of ECMO support and none of these children survived. Out of the children decannulated 18 (42.8\%) were successfully discharged home. Out of the 7 children who required ECMO for respiratory support only one child was successfully decannulated and discharged home.
\end{abstract}

RESULTS:

In our experience of ECMO, end organ damage whistle on ECMO, portented a worse outcome. And in patients who required it for respiratory support, the outcomes have been poor, a major reason being the delay in referral for ECMO support, rather than early in the disease process.

Mid-Term Outcomes of the Surgical Management of Absent Pulmonary Valve Syndrome Ashvin Krishna Nair; Sivakumar Sivalingam; Ibrahim Mukhtar

\section{ABSTRACT:}

\section{BACKGROUND:}

Absent pulmonary valve syndrome (APVS) is a variant of Tetralogy of Fallot characterised by aneurysmal pulmonary arteries which causes compression of the tracheobronchial tree, leading to respiratory symptoms. We report the mid-term outcomes of surgical correction of patients with APVS.

\section{METHODS:}

A review of 27 patients who underwent surgery between 2001 and 2015 was conducted and they were followed up for up to 14 years (mean 5.87 years). Out of the 27,14 patients $(51.9 \%)$ were infants. The median age at repair was 0.82 years (range, $0.4-4.6$ years). Pre-operative intubation was required in 6 patients $(22.2 \%)$ and 11 patients $(40.7 \%)$ had symptoms of respiratory distress. The pulmonary valve was replaced with a valved conduit in 15 patients $(55.6 \%)$, monocusp valve in 6 patients $(22.2 \%)$ and valveless repair with a transannular patch in 6 patients $(22.2 \%)$. Reduction pulmonary arterioplasty was done in 26 patients (96.3\%).

\section{RESULTS:}

The overall 10 -year survival was $82.1 \%$, where there was 1 early and 2 late mortalities. There was also $81.1 \%$ overall freedom from reintervention at 10 years. No significant difference was found in long term survival $(p=0.464)$ and re-operation rates $(p=0.129)$ between valved conduit, monocusp, or transannular patch techniques. Older children had a significantly longer survival $(\mathrm{p}=0.039)$ and freedom from reintervention $(p=0.016)$ compared to infants. Patients without respiratory complications had $100 \%$ 10-year survival and $93.3 \%$ freedom from re-operation at 10 years compared to $55.6 \%$ and $60.1 \%$ respectively for patients with respiratory complications.

\section{CONCLUSION:}

There has been improvement in surgical results for APVS over the years. However it still remains a challenge to manage infants and also patients with persistent respiratory problems. Patient selection is crucial in a resource-limited setting.

End to side anastomosis for infants with coarctation of aorta- a single center study

Tapan K. Dash; Sailaja Vasireddy

\section{ABSTRACT:}

\section{INTRODUCTION:}

To study the outcomes, incidence of recoarctation in infants who underwent End to Side anastomosis for coarctation of aorta

\section{MATERIALS AND METHODS:}

From 2015 to 2018, 62 patients underwent end to side anastomosis for coarctation of aorta. We did a prospective study on these infants by analysing early and mid term complications. 32 cases were male. Coarctation with PDA and bicuspid aortic valve were included. Pre operative analysis of the patient was done by assesing the clinical condition, need for inotropic and ventilatory suppot, two dimensional Echocardiography to assess left ventricular function and size of PDA.

\section{RESULTS:}

Mean age and weight of patients was 45 days and $3.3 \mathrm{~kg}$ respectively. Minimum and maximum follow up period was 2 months and 3 years. 
All the repairs were approached through left posterolateral thoracotomy from the third intercostal space by the same surgeon. Mean cross clamp time was 23.16 minutes. All the patients had a gradient less than $12 \mathrm{~mm}$ $\mathrm{Hg}$ at the end of surgery. There were two in hospital deaths(sepsis). No complications like recurrent laryngeal nerve palsy, bronchial compression and chylothorax were observed.

Follow up data was available for 57 patients. One patient died 3 months later due to unknown cause. 2 patients were lost to follow up.

During follow up two dimensional Echocardiographic gradient of $20 \mathrm{~mm}$ $\mathrm{Hg}$ was considered as recoarctation. All the survivors and the followed up cases were free of recurrence.

\section{CONCLUSON:}

Repair of coarctation with end to side anastomosis has a low early and late mortality, freedom from reintervention, excellent mid term outcomes. However long term follow up is required to assess the rates of reintervention and late outcomes.

\section{Surgical closure of VSD in infants: Results in our centre over 4 years}

Dhananjay Bansal; Narender Singh Jhajhria;

Mohd Javed Banday

Ventricular septal defect (VSD) is the most commonly recognised congenital heart defect presenting with failure to thrive in neonatal period and infancy. Surgical closure of VSD before first year of life is the treatment of choice for these patients. We present our experience of 84 patients treated in our hospital over period of four years.

Between January 1, 2014 and Dec 31, 2017, 84 patients underwent isolated VSD repairs at a median age of 10 months ( range of 14 days to 11.5 months) and median weight of $5 \mathrm{~kg}$ ( range $2.5 \mathrm{~kg}$ to $8 \mathrm{~kg}$ ). The following VSD types were found: 71 perimembranous $(71 \%), 8$ supracristal $(9.5 \%)$, 2 inlet $(2.3 \%)$ and 3 muscular (3.5\%). Most common indication was recurrent pneumonias and failure to thrive. Severe pulmonary artery hypertension was present in 55 patients $(65 \%)$.

No patient underwent reoperation for residual VSD. Median extubation time was 12 hours (range 6 hours to 30 hours), Median ICU stay was 3 days ( range 2 days to 15 days) and median hospital length of stay was 5 days ( range 4 to 15 days). There were 2 early mortalities(2.3\%) and 2 late deaths $(2.3 \%)$ occurred in follow up. One patient developed complete heart block requiring permanent pacemaker implant. No patient was discharged on antiarrhythmic medications. 50 patients were discharged on medication for pulmonary hypertension. On median follow up of 2 years 80 patients (95\%), were asymptomatic. Pulmonary hypertension regressed over median time of 2 months (range 2 days to 3 months). None exhibited greater than mild new onset tricuspid valve regurgitation. No aortic valve injury noted.

Early surgical closure of VSD in infancy is treatment of choice for patients with VSD with minimal risk of death, complete heart block and reoperation.

\section{Ascending aortic and arch replacement in a child with Bicuspid aortopathy \\ Arum Bheeman; K.M. Cherian; Srinivas Aaluri}

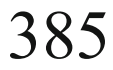

\section{ABSTRACT: \\ INTRODUCTION}

Bicuspid aortopathy can afflict the aorta of patients with bicuspid aortic vale disease. We report a 10 year-old child who had ascending aortic with Type A dissection along with aneurysm of the arch of aorta who underwent ascending aortic and aortic arch replacement.

\section{CASE REPORT:}

Following balloon aortic valvotomy for severe stenosis of bicuspid aortic valve and subclavian coarctoplasty 2 years back, a 10 year-old boy presented with type A dissection with aneurysm of ascending aorta and aortic arch up to the previously repaired coarct segment.

In view of small aortic annulus (14mm), competent aortic valve with less than $20 \mathrm{~mm}$ gradient and lack of involvement of the aortic root, it was decided to spare the aortic valve. CPB was established through grafts sutured to the innominate artery and the right femoral artery. $\mathrm{He}$ underwent ascending aortic and aortic arch replacement with a gelatin impregnated dacron graft with an island patch of the arch vessels implanted to the graft.

He had an uneventful recovery and was discharged on post-operative day 7 . CONCLUSIONS:

Bicuspid aortic valve disease with aortic involvement is a well known pathology with well established guideline for follow-up and intervention for aortic involvement and to prevent aortic dissection. Serial follow-up and appropriate surgical intervention can lead to adequate management of this disease.

Direct Closure of ASD without patch

Abhay Jain; James Thomas

\section{6}

ABSTRACT:

Traditionally Atrial Septal Defects are closed with Dacron or pericardial patches. In some cases pericardial patch had lysed producing minor leak. Last ten years we have closed ASD s secundum type of all sizes $80 \%$ directly using 40 sutres and others with dcaron patch. Sinus venosus defects were closed with patch so was primum defects. Follow up showed no leak on either of the techniques. With large RA and LA even ASD with 2.5 to $3 \mathrm{~cm}$ large defects can be directly closed without compromising the both chambers. Most of the septal defects we see are around $2 \mathrm{~cm}$ sixze only. Reuslts presented.

\section{Pulmonary valve sparing predicts better Right ventricular function after Tetralogy of Fallot Repair in early follow up. \\ Anuj Mehta}

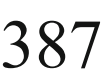

\section{ABSTRACT: \\ INTRODUCTION:}

The right ventricular(RV) dysfunction after Tetralogy of Fallot repair (TOF) is usually due to the long standing effect of pulmonary regurgitation. We assessed RV function after TOF repair and the impact of pulmonary valve preservation on the early outcome.

\section{METHODS:}

Forty six consecutive patients, undergoing intracardiac repair of TOF between January 2015 to December 2016, were divided into Group 1: Valve sparing TOF repair $(n=31)$, Group 2: Non valve sparing $(n=15)$. 2D Echo with Tissue Doppler(TDI) was used for assessment of post operative RV function.

\section{RESULTS:}

The mean age was $48.94 \pm 78.82 \mathrm{Vs} 23.93 \pm 35.32$ (months). The mean body weight was $12.5 \pm 9.4 \mathrm{Vs} 9.6 \pm 4.32(\mathrm{~kg})$. The mean Pulmonary valve annulus $\mathrm{Z}$ score $-0.6+1.3 \mathrm{Vs}-4.0+1.7$. The Mean post repair $\mathrm{RV} / \mathrm{LV}$ pressure ratio was $0.47+0.12$ Vs $0.50+0.14$. The Mean ICU stay was $3.23+6.1$ Vs 7.7 +6.4 days $(\mathrm{p}=0.025)$. There was only one $(2.1 \%)$ mortality. Tricuspid annular plane systolic excursion at one year follow up was $(2.08+2.4 \mathrm{Vs} 1.56+0.26$, $\mathrm{p}=0.387$ ). Fractional area change was found to be lower in group 2 but, the difference was not significant $(\mathrm{p}=0.232)$. Pulmonary valve gradients were higher in group $1(\mathrm{p}=0.800)$. TDI at one year follow up showed Late diastolic tricuspid annular velocity $(8.81 \pm 1.37 \mathrm{vs} 7.37 \pm .79$, p\&lt;0.05); Systolic tricuspid annular velocity(10.32 $\pm .90 \mathrm{vs} 9.43 \pm .35$, p\&lt;0.05); Isovolumetric relaxation time $(59.58 \pm 4.65$ vs $75.60 \pm 7.15$, p\&lt;0.001) Isovolumetric contraction time(52.29 $\pm 4.25 \mathrm{vs} 69.33 \pm 9.46)$ and significantly higher Myocardial Performance Index in valve sparing group $(\mathrm{p}=0.008)$ suggestive of better RV function in group1. Early diastolic tricuspid annular velocity and ejection time did not showed significant difference. In both groups, $86.7 \%$ are in NYHA class1. Re intervention occurred in 2 patients $(6.4 \%)$ in group 1. CONCLUSIONS: 
Pulmonary valve sparing approach has better early outcomes as compared to transannular patch and provides better RV function. Subclinical RV dysfunction can be assessed by $2 \mathrm{D}$ echo with TDI.

\section{Atrial Septal Defect Causing Angina}

Javed Banday; Dhananjay Bansal; Anubhav Gupta

\section{ABSTRACT: \\ INTRODUCTION:}

Prolonged left-to-right shunt in patients with ASD promotes right heart and PA dilatation causing extrinsic compression of the LMCA (left main coronary artery). In 1957, Corday et al, first described proximal LMCA compression by enlarged PA in patients with severe pulmonary hypertension.6 The optimal management of LMCA compression caused by a dilated pulmonary artery secondary to ASD is yet to be clarified. This formed the premise of this case report.

\section{METHOD:}

A 39 yr old woman presented with complaint of chest pain with dyspnea on exertion since 18 months. AOE II. NYHA III.

Detailed investigations revealed Sev MR, Sev TR, Large Os ASD with sev PAH, dialated MPA causing critical LMCA osteal stenosis. Dacron patch closure of ASD with surgical revascularization of coronary was done.

\section{RESULT:}

Post-op course was uneventful. Patient recovered well and is asymptomatic in followup.

\section{DISCUSSION:}

Various Risk Factors for dialated PA causing LMCA compression have been found like, origin of the LMCA from the right sinus of valsalva, young women of short stature, large PA (\&gt;40 mm; normal: 25-30 mm), PA trunk/aorta ratio \&gt;1.2 (normal: 1.0), LMCA angle of takeoff from the left sinus of Valsalva of \&1t;30 $30^{\circ}$ Both intravascular ultrasound and fractional flow reserve have been validated to guide the treatment of LMCA disease by Pina et al, and recommended that revascularization should be performed if the intravascular ultrasound minimum lumen area is \& 1 t; $5.9 \mathrm{~mm}$ and/or abnormal fractional flow reserve is \&lt; 0.80 .

\section{CONCLUSION:}

ASD closure with $\mathrm{CABG}$ should be performed in large ASD with reversible PAH with LMCA compression, vis a vis Percutaneous revascularisation in Large ASD with irreversible PAH with high operative risk.

\section{ALCAPA with lobar emphysema \\ Periyasamy; Ashwanth}

\section{ABSTRACT: \\ INTRODUCTION: \\ ALCAPA patient presented with lobar emphysema} MATERIAL AND METHODS:

Series of 3 casess age of around 7 to 11 months presented with lobar emphysema 2 casess with bilateral lobar emphysema and one with unilateral lobar emphysema

\section{RESULTS:}

All three patients were operated and discharged.

\section{Papillary muscle approximation suture as an adjunct} to $\mathrm{AV}$ valve closure in single ventricles K.M. Cherian; Anto S.R.

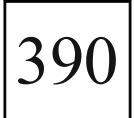

\section{ABSTRACT:}

\section{INTRODUCTION:}

Closure of a dysfunctional (regurgitant) $\mathrm{AV}$ valve in single ventricle hearts is an established technique. However, closure of the AV valves with patch material is prone to complete heart block and patch dehiscence. Further if the ventricle below the dysfunctional valve is dilated and compressing the sub-systemic ventricle (as in severe forms of
Ebstein's) the dilated ventricle is not addressed completely by closure of the AV valve. We report a novel technique to aid in the closure of AV valve and to reduce the size of the dilated ventricle.

\section{METHOD:}

Following a failed repair of Ebstein's anomaly of the tricuspid valve, a 14 year-old boy presented with right heart failure and severely dilated dysfunctional right ventricle compressing the left ventricle. He had a right BDG as part of his prior repair of Ebstein's anomaly.

Intra-operatively the right ventricle was thinned out and significantly dilated. A ligature was passed around the chordal elements of the dysplastic tricuspid valve abutting the papillary muscle heads and tied. This led to the obliteration of most of the dilated right ventricle. The AV valve cuspal tissue was approximated with sutures and a patch pf decellularised bovine pericardium was sutured over the leaflets in the region of the triangle of Koch and along the rest of the previously reconstructed annulus. Extracardiac Fontan surgery was sompleted with a $16 \mathrm{~mm}$ PTFE tube graft.

Post-operative echo showed a small right ventricle with no compression effect on the left ventricle.

\section{CONCLUSION:}

Suture ligature of the chordal apparatus of the AV valve of a dysfunctional ventricle during Fontan surgery effectively reduces the deleterious effect of the dilated ventricle on the sub-systemic ventricle and is an adjunct to more easier and effective AV valve closure in single ventricles when deemed necessary during repair.

Isolated double orifice mitral valve without supporting chordae in a young girl Ganapathy Subramaniam; K.G. Suresh Rao; K.R. Balakrishnan; Anitha Chandrasekhar

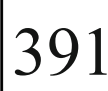

\section{ABSTRACT: \\ INTRODUCTION:}

Double-orifice mitral valve (DOMV) is a rare cardiac malformation. It can either be an isolated malformation or concomitant with other congenital anomalies. It consists of two anatomically distinct mitral orifices that are divided by an accessory bridge of fibrous connective tissue.

BACKGROUND:

DOMV is classified into 3 types- Complete bridge, Incomplete bridge and hole type. Our present case was an incomplete bridge type in which the connection was seen only at the leaflet edge

The hemodynamic effects of DOMV vary depending on whether the leaflets are normal or regurgitant or stenotic. We report a case of a 13 years old girl with congenital DOMV associated with mitral regurgitation. CASE:

Patient presented with breathlessness. Echocardiography reveled Severe MR and TR. She was taken up for valve repair. Superior septal approach was used to access the mitral valve. There was a smaller competent anterolateral orifice and a larger regurgitant posteromedial orifice separated by a central bridge of leaflet tissue connecting the middle of AML to PML, not supported by any chordae

The valve was repaired by plication of the prolapsed A3 segment, closure of medial commissure, Alfieri repair of A2-P2 segment at the site of bridge between $\mathrm{A} 2$ and $\mathrm{P} 2$ area of the leaflets. The tricuspid valve was repaired by sectoral annuloplasty and looked competent on saline testing. TEE showed no MR, no MS, No TR, Mild LV dysfunction.

\section{CONCLUSION:}

Successful repair of DOMV requires tailoring of surgical approach to individual anatomy of the tensor apparatus.

Endarterectomy in a case of Fontan Associated Liver Disease due to IVC \& hepatic vein thrombosis Ganapathy Subramaniam; K.G. Suresh Rao;

K.R. Balakrishnan

Anitha Chandrasekhar 


\section{ABSTRACT: \\ INTRODUCTION:}

Fontan-associated liver disease (FALD) comprises a wide spectrum of structural and functional alterations of the liver caused by hemodynamic disturbances following Fontan surgery. It can be due to multiple reasons like high venous pressure, thrombosis, ventricular dysfunction etc.

BACKGROUND:

We report a case of a 10-year-old girl who presented with thrombosed extracardiac Fontan conduit, thrombosed hepatic veins and hepatic nodules.

CASE:

A 10-year-old girl with tricuspid and pulmonary atresia, S/P Fontan procedure 3 years back, presented with abdominal distension, pedal edema and abdominal pain. Imaging revealed thrombosis of extracardiac Fontan conduit extending into the inferior vena cava (IVC) and hepatic veins akin to a Budd-Chiari equivalent picture. The suprarenal IVC was thrombosed while the infrarenal IVC drained into the azygos and hemiazygos systems through multiple collaterals. She had multiple veno-venous hepatic collaterals and multiple hepatic nodules.

Thrombosed conduit was excised and endarterectomy of the IVC \&amp; Hepatic veins was done. This required a brief period of total circulatory arrest. An $18 \mathrm{~mm}$ conduit was anastomosed from IVC to RPA. A fenestration was created between the conduit and the right atrium.

She developed disorientation and obtundation, typical of hepatic encephalopathy in the post-operative period. She had postoperative neurological issues and renal shutdown, both of which gradually recovered. A CT scan taken 6 months after surgery showed complete resolution of the hepatic nodules with patent suprarenal IVC, hepatic veins and Fontan conduit.

\section{CONCLUSION:}

The systemic complications of Fontan are many. Endarterectomy is a useful option for thrombosed veins. Fontan patients need to be on close follow up for early recognition and treatment of complications.

\section{Youngest neonate(16hours) undergoing} successful biventricular repair for pulmonary atresia with intact ventricular septum

Charumathi Dasarathan; Charumathi Dasarathan

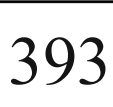

\section{ABSTRACT:}

We present a case of 16 hour old male baby born at 35 weeks of gestation, weighing 2200 grams, with imperforate pulmonary atresia with intact ventricular septum, large secundum atrial septal defect (ASD), moderate infundibular stenosis. Pulmonary circulation was dependent on tiny PDA. Ongoing prostaglandin infusion, baby was taken up for surgery. Biventricular repair was performed consisting of pulmonary valvotomy, infundibular muscle resection, closure of ASD and ligation of a patent ductus arteriosus (PDA). At 36 hours, the adjustable left open patent foramen ovale (PFO), for decompression of right ventricle (RV), was partially closed, saturating $88 \%$ at $55 \%$ fio 2 . At one year follow up echocardiogram revealed $1.5 \mathrm{~mm}$ PFO and baby is saturating $95 \%$ on room air.

\section{Vascular ring due to double aortic arch -a rare cause of dysphagia : A case report \\ Charumathi Dasarathan}

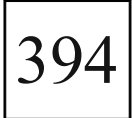

\section{ABSTRACT:}

A 5-year-old male child, known case of congenital heart disease (CHD) ventricular septal defect (VSD) since birth, was presented to our institution, with a history of difficulty in swallowing, failure to thrive and recurrent respiratory tract infection. On investigations, cardiac computed tomography revealed double aortic arch (DAA) with rudimentary right aortic arch, presenting as vascular ring producing trachea-esophageal compression. After division of the non-dominant part of the DAA, the child was relieved of symptoms.

\section{Management of scmitar syndrome and mesocardia mimciking cardiac herniation postoperative period}

Sreevathsa; Jayakrishnan S.

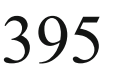

\section{ABSTRACT: BACKGROUND:}

Scmitar syndrome is in itself a rare congenital cardiac anomaly The scimitar syndrome consists of several changes. An anomalous vein drains thelower lobe or all of the right lung into the inferior vena cava at or below the diaphragm.

\section{CASE REPORT:}

We report a 16year old female, presented with progressive dyspnea on exertion. The pulmonary symptoms began in 2014 with chronic cough, and right chest pain, occasional hemoptysis. 2D ECHO -OSASD L-R shunt, Right pulmonary vein not seen. CT Angiogram Right pulmonary vein draining into IVC, right pulmonary lower lobe hypoplasia. Diagnosis of Scmitar syndrome was made Rerouting of SCMITAR VEIN to Left Atrium via Right Atrium was done. Scmitar vein $(7 \mathrm{~cm})$ was identified to go through diaphragm to join IVC. RA was opened. ASD measuring $2.5 \times 2 \mathrm{~cm}$ was created. 3 PVs draining into LA observed. Scmitar vein was divided and distal end closed, proximal end anastomosed to RA, patch closure of created ASD was done with rerouiting of scimitar vein to LA. On POD 1 patient developed dyspnoea and hypotension, since pericardium was harvested for t6he patch closure and rerouting, Chest X Ray showed mediastinal shift to the right suspecting cardiac herniation. Reexploration done with hitching of pericardium to parietal wall, followed by bronchoscopic assessment of bronchi and retrival of clots. There was no cardiac herniation on reopening. Repeat HRCT and CT-Pulmonary Venogram done and was found to be satisfactory.

\section{CONCLUSION:}

Preoperative X-ray and post op X-rays compared, only mild increase in shift of heart to right, It was mesocardia, absent pericardial support on right side and hypoplasia of right lower lobe, sudden hypotension with dyspnoea made us suspect cardiac herniation. Reopening is justified in view of poor hemodynamics and symptoms.

\section{Hypertrophic obstructive Cardiomyopathy (HOCM)}

M.Q.I. Talukder; Saikat Dasgupta;

Prodip Kumar Biswas

D.M.A. Kabir

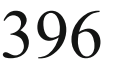

\section{ABSTRACT:}

\section{INTRODUCTION:}

Hypertrophic obstructive Cardiomyopathy HOCM is mostly autosomal diseases representing about 1 in every 500 population. About $70 \%$ of all the cases involve a subaortic area of interventricular septum (IVS) and rests are diseases of the mid and apical part of IVS. The aim of this paper is to project our efforts to manage this sort of pathology in our setup as it is still less commonly performed surgery in our country.

\section{Materials And Methods:}

During the period from July 2014 to November 2018, 15 patients underwent septal myectomy for HOCM. The male and the Female ratio was 9:6 which is an incidental finding. Our patient's age range was 19 years to 60 years. Our 2 patients had septal myectomy along with Mitral Valve Replacement and Coronary Artery Bypass Graft (CABG). One patient had septal myectomy along with the release of LAD due to the myocardial bridge. One patient had septal myectomy along with plication of LA appendage due to Atrial fibrillation. 


\section{RESULTS:}

Our longest cross-clamp time was 123 min and lowest cross-clamp time was $40 \mathrm{~min}$. Postoperatively all the patients were reviewed with color Doppler which revealed an acceptable gradient at LVOT. One patient underwent Permanent Pacemaker (PPM) implantation due to complete Heart block (CHB). However, still we are in the initial phase of our service in this field and our patients are yet to be followed up properly.

\section{CONCLUSION:}

Septal myectomy for HOCM is very rewarding surgery as a consequence of this disease is very detrimental including sudden death also. So once the diagnosis is established surgery should be performed whenever possible.

Risk factors for bleeding requiring re-exploration after Coronary artery bypass grafting Subrata Dey; Subhajit Sharma

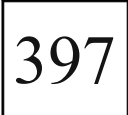

\section{ABSTRACT: \\ INTRODUCTION:}

The proportion of patients undergoing coronary artery bypass grafting that require re-exploration for bleeding reported 2-6\% worldwide. We aimed to identify the risk factors re-exploration for bleeding after surgical revascularisation.

\section{METHOD:}

Between April 2015 - January 2019, 718 patient undergoing CABG in our institution, the pre-operative data, intra-operative finding, the timing, estimated blood loss for 19 patients undergoing re-exploration were analysed.

\section{RESULTS:}

Out of 719 patients undergoing CABG 21 patient had re-exploration shortly after operation. males are more $16(76.1 \%)$ as compared to female $5(23.9 \%)$. Mean age was $59.4+/-14.6$ years. The mean time interval between the primary surgery and re-exploration is $3.2+/-1.5$ hours. Mean chest tube drainage just before re-exploration was $796+/-25.6$ $\mathrm{ml}$. On pump surgery was $11(52.4 \%)$, off-pump surgery was $10(47.6 \%)$. On exploration in $18(85.7 \%)$ patient definitive bleeding points identified, no active bleeding identified in 3(14.3\%)cases. Indication for re-exploration were as follows -Reactionary hemorrhage $16(75.9 \%)$, cardiac tamponade $4(19.1 \%)$, both reactionary hemorrhage and tamponade 1 (4\%)cases. Associated emergency surgery, more distal anastomosis, increased age and associated diabetes increased risk and statistically significant.

\section{CONCLUSIONS}

There is strong evidence supporting early re-exploration in patients after coronary artery bypass grafting, complicated by reactionary hemorrhage, cardiac tamponade, early re-exploration when indicated may help reduce the mortality and morbidity.

\author{
"An unusual \& interesting case of coronary \\ cameral fistula". \\ Raman Kumar; Jaideep Trivedi
}

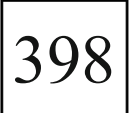

\section{ABSTRACT:}

Coronary cameral fistula is a sizeable communication between a coronary artery, bypassing the myocardial capillary bed and entering a chamber of heart. It constitutes $0.2 \%-0.4 \%$ of congenital cardiac anomaly. We present a case of a young female who was Coronary symptomatic and was evaluated for the same. The fact that she had a coronary cameral fistula from the left main coronary artery(LMCA) terminating into the SVC right atrium (RA) junction made the case an unusual one.

28 yrs Female who was asymptomatic previously presented to us with atypical chest pain, palpitation and breathlessness on exertion since 6 months. On CVS examination-loud second heart sound and continuous murmur in the precordium. X-ray suggestives of cardiomegaly. In echocardiogram good LV function, LMCA was dilated and a fistulous tract could be traced from LMCA to right atrium. Coronary angiogram showed dilated LMCA with a fistulous tract terminating into right atrium. Sternotomy was done, pt. was taken on $\mathrm{CPB}$, fistulous tract was identified dissected and doubly ligated on pump beating heart. Pt. was extubated within few hours of surgery. Precordial continuous murmur completely disappeared. Pt.was discharged on 5th day. After 22 months of surgery she is completely asymptomatic and following up with us.

\section{CONCLUSION:}

Coronary Cameral Fistula from LMCA to SVC-RA junction is very rare and unusual.

Surgery was successfully done on pump beating heart without arresting heart, avoiding cardioplegia and cardiotomy.

\section{A New Practical Classification for Sternal \\ Wound Infection Management: Medanta Classification \\ Rakesh Khazanchi; Hardeep Sing}

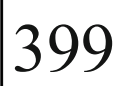

\section{ABSTRACT: \\ INTRODUCTION:}

Sternal wound infection is an important cause of morbidity in patients undergoing $\mathrm{CABG}$. There are numerous classifications based on duration and depth of wound. These classifications doesn't help in management of the wound. We propose a practical classification for management of sternal wound infections.

According to our experience, the most important factor for patients having sternal wound infection is the stability of sternum. A stable sternum on one side limits the spread of infection to mediastinum and on the other hand is important for normal ventilation of patient hence limiting atelectasis and pneumonia formation.

All 24 patients in last one year presenting with sternal wound infection of various duration underwent CECT to know the extent of the infection. All the patients underwent radical surgical debridment and were followed with VAC dressing till the wound is nicely granulating and the TLC, CRP had come normal.

\section{RESULTS:}

10 patients who has stable sternum underwent secondary suturing and 12 of the 14 patients with unstable sternum underwent flap cover from omentum and pectoralis major muscle. 2 patients were managed with VAC closure as they refused any further surgery. One patient in deep infection group expired while all other recovered completely.

\section{CONCLUSION:}

We propose a classification and algorithm based on stability of sternum. The infections not involving the sternum are termed as superficial and those involving are deep. The deeper infections form dead space on debridement and hence require a flap to fill the defect.

On pump iabp verses off pump iabp in cabg a rare study early outcome

G.V. Rama Krishna; Sujit Kumar Mohanty

\section{ABSTRACT:}

Abstract Title: ON PUMP IABP VERSES OFF PUMP IABP IN CABG A RARE STUDY WITH EARLY OUTCOME

Main Presenter: Dr.Sujit Kumar Mohanty Sub Author: Dr.Afroz Farooqi, Jaydeep Trivedi Institute: Apollo Hospitals, Visakhapatnam Intraaortic balloon pump(IABP) is now lifesaving for many patients in operating theatre, ICU and Cath lab. The IABP will help to support the heart \&amp; recovery if it will be place in proper time followed by timely further management. It may be elective or emergency. It is for coronary bypass surgery or other heart diseases like acute MR, bridge to transplant etc. 
Here we present a small study between Jan 2016 To Nov 2017 for coronary artery bypass surgery. Total 43 patients studied retrospectively.

All patients inserted with IABP out of 43 , off pump CABG done is 23 patients \&amp; On pump CABG done is 20 patients.

The patient operated by off pump CABG all survived.

But on pump with IABP 8 patients died. The mortality and morbidity on pump CABG with IABP may be due tomore sick patient, post post operative drain little more, nephrology, neurological compilation and more no. of blood transfusion.

\section{CONCLUSION:}

If we are planning the patient for $\mathrm{CABG}$ on pump then we should avoid IABP and if it is require we can put later on or if we inserted IABP before procedure we should avoid on pump surgery, better go for off pump coronary artery bypass surgery.

\section{Lateral left ventricular aneurysm: Incidental \\ finding during off pump \\ Coronary artery bypass grafting surgery \\ A. Farooqi; Sujit Kumar Mohanty}

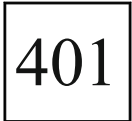

\section{ABSTRACT:}

It is common left ventricular aneurysm in coronary artery disease patients with low ventricular ejection fraction. Most commonly it is located left ventricular apex area and some cases it is found in posteriorly. Majority of these patients present with congestive heart failure, systemic embolism and mitral insufficiency. Left lateral aneurysm particularly in between ramus and obtuse marginal coronary vessels are extremely rare.

Here in we report a case of 62 year male patient with medical history of coronary artery disease and myocardial infarction with a lateral left ventricular aneurysm measuring approximately $7 \times 5 \times 5 \mathrm{~cm}$ in diameter. He underwent successful surgical ventricular restoration (Dor procedure) and coronary artery bypass grafting to left anterior descending artery (LAD)and right posterior descending artery.

After surgery patient developed motor aphasia and with right sensorimotor deficit but improves with physiotherapy and conservative management and discharged home after 15 days.

$\mathrm{He}$ is doing well after 1 month of follow up.

Risk stratification by EuroSCORE II in Indian patients Arokiya Priya. A.; Devender Singh; Shegu Gilbert

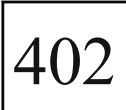

\section{ABSTRACT: \\ INTRODUCTION:}

A mutiple of risk stratification scores for cardiac surgery are available. The European system for cardiac operative risk evaluation II (EuroSCORE II) is used world wide as a model for prediction of operative mortality in cardiac surgical patients.

AIM:

To evaluate the operative risk by calculating EuroSCORE II for 100 patients undergoing cardiac surgery. To compare the predictive mortality and the observed mortality in our group of patients.

\section{MATERIALS AND METHODS:}

Prospective data analysis of 100 patients in our cardio thoracic unit from December 2017 to July 2018. All the patients, above 18 years of age who underwent $\mathrm{CABG}$, valve replacement procedure \&amp; CABG with valve procedures were included. Statistical analysis was done. Variables were expressed as mean $+/$ - standard deviation and percentage. The patients characteristics \&amp; operative data were analyzed. We calculated the EuroSCORE II using the Euroscore II calculator which is available on the website. The primary end point was In-hospital mortality.

\section{RESULTS:}

The overall mortality was $6.3 \%$. Mortality for CABG, valve and combined procedures were $6.3 \%, 5.4 \%, 9.3 \%$ respectively. The observed mortality and the predictive mortality in the various risk groups were analyzed.

\section{CONCLUSION:}

Risk stratification in cardiac surgical patients for quality monitoring is useful tool for assessing the outcomes, counselling the patient $\&$ relatives about the mortality risks \& preparing the surgical team for the procedure.

Off pump coronary artery bypass grafting in patients with significant left ventricular dysfunction:

Experience from Tier III city.

Nitin P. Kochar; Prashant Kothari

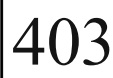

\section{ABSTRACT: \\ OBJECTIVE:}

Off-pump coronary artery bypass grafting $(\mathrm{OPCAB})$ is known to preserve left ventricular function better than conventional coronary artery bypass grafting (CCAB). This study was carried out to investigate the safety, feasibility and efficacy of off-pump coronary artery bypass grafting in patients with significant left ventricular dysfunction.

METHODS:

One thousand two hundred and thirty three patients who underwent CABG procedure consecutively between March 2014 till June 2018 with preoperative EF \&lt;30\% were included in this retrospective study. We analysed their post-operative outcomes, of these 70\% (863) were males, 85\% (1048) had type II diabetes, and 87\% (1073) were having NYHA class III symptoms. Anaesthetic techniques were similar for all patients, with fentanyl, propofol and midazolam as induction agent

RESULTS:

Number of grafts per patient was $3( \pm 1)$, out of these patients $70 \%$ (863) were total arterial (LIMA-Radial-Y). Left Internal Thoracic Artery (LITA) was anastomosed to LAD in $99 \%$ (1220) of these patients. 5 (61) patients had EF less than $10 \%$. Operative mortality was $1 \%$ (13) which was early in ICU. $2 \%$ (24) patients were lost during follow up. $25 \%$ (61) patients underwent endarterectomy of one or more coronary vessels

CONCLUSION:

Off-pump coronary artery bypass grafting is feasible and safe in patients with depressed left ventricular function.

Gender Differences in Arterial conduits for CABG in an Urban Indian Population

Biju Sivam Pillai

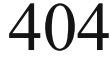

\section{ABSTRACT:}

\section{BACKGROUND:}

There are significant differences in arterial revascularization strategies in coronary surgery. This study addressed these differences to determine whether discrepancies are related to underlying risk profiles of the patients or systematic gender bias.

\section{METHODS:}

Changes in gender-specific rates of revascularization approaches were determined in 700 patients undergoing isolated coronary artery bypass grafting by a single team.

\section{RESULTS:}

In the entire study population, male gender was associated with increased bilateral internal thoracic artery use (BITA) (odds ratio, 1.467 ; 95\% confidence interval [CI], 1.154 to $2.243 ; \mathrm{p}$ [0.005). Multiple-arterial grafting increased yearly in both genders, but the rate of increase in BITA use was greater in men (3.59\%/year [95\% CI, 1.506 to 1.985 ] vs $1.57 \% /$ year [ $95 \%$ CI, 1.30 to 1.45$]$, p \& It; 0.001 ). The use of radial artery was independently assessed as a subset designated as the use of 3 arteries (2.14\%/year [95\% CI, 
1.23 to 1.40 ] vs $0.98 \% /$ year [ $95 \% \mathrm{CI}, 0.78$ to 1.26$), \mathrm{p} \& \mathrm{lt} ; 0.001$ ) and the use of any 2 arteries (2.34\%/year (95\% CI, 2.32 to 2.35 ) vs $1.92 \% /$ year [ $95 \%$ CI, 1.90 to 1.95], p \&lt; 0.001). BITA use in low-risk patients (male: $53.4 \%$, female: $31.0 \%$; $\mathrm{p}$ [0.135), or radial artery use (male: $46.5 \%$, female: $36.1 \%$; [0.886), but use of 3 arteries was greater in men (14.5\% vs 5.3\%, p [0.028). CONCLUSIONS:

In our clinical practice we have noticed that women receive less complete multiple arterial coronary revascularization than men. This difference is likely related to later presentation of coronary artery disease in women with higher comorbidity profiles.

\section{Analysis of our experience and outcomes of patients with renal dysfunction undergoing CABG \\ Vijit K. Cherian; Ravi Shivdasani}

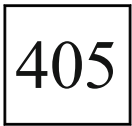

\section{ABSTRACT:}

Renal dysfunction is independent predictor of morbidity and mortality in patients requiring $\mathrm{CABG}$. Despite increased early mortality for $\mathrm{CABG}$, it appears to have improved long term survival and need for lesser repeat procedures. There is significant room for decreasing early mortality and morbidity in patients with $\mathrm{CKD}$ requiring $\mathrm{CABG}$. As a large population is diagnosed with $\mathrm{CKD}$ we sought to study the preoperative variables and perioperative outcomes of patients with renal insufficiency undergoing CABG.

AIMS AND OBJECTIVES:

Evaluate the high risk population with CKD and assess pre and perioperative factors that can be modified to achieve better outcome

Left atrial appendage ligation in coronary artery bypass grafting : a prospective randomized study Akshya Kumar Bisoi; Pratik Manek

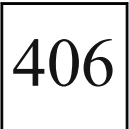

\section{ABSTRACT:}

\section{OBJECTIVES}

Incidence of Post Op Atrial Fibrillation (POAF) following CABG is high particularly within first 7 days. The left atrial appendage (LAA) is the site of more than $90 \%$ of detected thrombi in patients with AF. Primary outcome is to assess the incidence of Stroke/Transient Ischemic Attacks post on-pump coronary artery bypass grafting with or without LAA ligation.

\section{METHODS:}

All the consecutive patients who underwent On-Pump Coronary Artery Bypass Grafting (CABG) from September 2016 to June 2017 who are at high risk of developing POAF based on the CHA2DS2-VASC score (\&gt;=2) were randomized to CABG either with or without Left Atrial Appendage (LAA) ligation. Patients were followed up post operatively to rule out presence of AF or TIA or Stroke at 1 week, 1 month, 3 months, 6 months and 12 months.

\section{RESULTS:}

A total of 140 patients were randomized into two groups of which 8 patients in Group I and 15 patients in group II developed AF ( $p$ value 0.1 ). The Risk Difference in the incidence was 0.1 between the two groups. Thus the Number Needed to Treat (NTT) so as to prevent the risk of $\mathrm{AF}$ and subsequent stroke in patients post $\mathrm{CABG}$ by LAA ligation is 10 . None of the patients had clinically evident Stroke/ Transient Ischemic Attack during the follow up.

\section{CONCLUSION:}

The technique of surgical epicardial suture LAA ligation during CABG is a safe and effective technique of LAA occlusion for reducing the incidence of POAF related Stroke/TIA.

LAD endarterectomy on beating heart : Our experience Sanjeev Khulbey; Raviraju Chigullapally

\section{ABSTRACT:}

\section{INTRODUCTION:}

Many patients referred for CABG today have diffusely diseased coronary vessels, and most of them may require coronary revascularization using endarterectomy. Endarterectomy of LAD especially is difficult because of important septal and diagonal branches. Most reports of coronary endarterectomy describe an on-pump procedure. As off-pump coronary artery bypass graft has become safer and more routine, there is renewed interest in off-pump coronary endarterectomy. We report on our series of patients who underwent off-pump coronary endarterectomy of the left anterior descending (LAD) artery.

\section{METHODS:}

All patients undergoing open heart surgery at The Apollo hospitals Hyderabad were included in the study. Retrospective review of our database revealed total number of CABGs performed 13985 and among those $1119(8.1 \%)$ patients required endarterectomy of any vessel for revascularization including limited endarterectomy. 46 Patients required LAD alone or combined with other vessel endarterectomy on beating heart.

\section{RESULTS:}

There were a total of 46 patients, with a mean age of $58 \pm 4$ years. 31 $(67.39 \%)$ were male and $15(32.60 \%)$ were females. Comorbidities included hypertension in $22(47.82 \%)$ patients, dyslipidaemia in $29(63.04 \%)$, diabetes in 40 (86.9\%), chronic kidney disease in $14(30.43 \%)$. The mean number of diseased coronary territories was $3 \pm 0.4$ (range $2-3$ ), and the mean number of coronary bypass grafts performed was $4 \pm 0.8$ (range 2-5). IABP support was required in 7(15.21\%) patients.3(6.52\%) patients had perioperative $\mathrm{MI}$ and $2(4.34 \%)$ patients had postoperative MI. The mean intensive care unit stay was $3 \pm 2.8$ days, and the mean hospital length of stay was $7 \pm 4.3$ days. Postoperative follow-up early at $7 \pm 1.2$ days and mid term and late follow up at 3 months, 6 months and 1 year is complete. Postoperative CAG was done in selective patients.

\section{CONCLUSIONS:}

We conclude that off-pump endarterectomy of the LAD is a viable option for patients with diffuse $\mathrm{LAD}$ disease.

Analysis of our experience and post operative outcomes of DM patients undergoing CABG

Vijit K. Cherian; Ravi Shivdasani

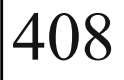

\section{ABSTRACT:}

Diabetic patients have higher morbidity and mortality and higher incidence of renal failure, stroke, infectious complications and post operative low cardiac output. They have prolonged ICU and in hospital stay which adds to the cost burden. DM patients with CABG are more likely to require repeat revascularisation procedures and have higher incidence of readmissions for cardiac and non cardiac related issues. These outcomes were thought to be irrversible since $\mathrm{DM}$ patients have more diffuse $\mathrm{CAD}$, LV dysfunction, and have impaired endothelial function leading to reduced graft patency ultimately perioperative mortality, reduced long term survival and less freedom from recuurent ischemic events. Since a large population with $\mathrm{CAD}$ requiring $\mathrm{CABG}$ are $\mathrm{DM}$ we sought to evaluate the preoperative parameters and perioperative variables which can be modified to achieve better post operative outcomes.

\section{AIMS AND OBJECTIVES:}

Preoperative variables and perioperative factors are compared in context of operative morbidity and in hospital mortality

To assess the impact of pre operative, intra operative and post operative glycemic control to achieve post operative course.

The dynamic expression profiles of long noncoding RNA in a rat vein graft model

Chen Lu; Jiayu Shen; Jia Hu; Hongwei Zhang;

Peng Yang; Yao Huang

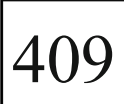

\section{ABSTRACT:}




\section{INTRODUCTION:}

Improvements in molecular mechanism of restenosis after coronary artery bypass grafting $(\mathrm{CABG})$ are urgently needed to explore novel biomarkers involve in the progression. Long non-coding RNAs(lncRNAs)have been proved to be key regulatory molecules involved in numerous biological processes and human diseases, yet the functional effects of these lncRNAs on vein graft failure remain unclear.

\section{MATERIALS AND METHODS:}

Twelve rats were randomly assigned to four groups while three of them accepted external jugular vein-carotid artery bypass graft surgery serving as comparison groups. Microarray analysis was performed to measure RNA expression, including lncRNAs and mRNAs, in the rat autologous vein grafts at $0,1,2,4$ weeks post-surgery.

\section{RESULTS:}

A total of 2,199 lncRNAs and 4,229 mRNAs have significantly different expression $(p<0.05$, fold change $\geq 2$ ). The RT-qPCR results were in consistent with the microarray data. We utilized bioinformatics approaches, including lncRNA classification, gene ontology $(\mathrm{GO})$ analysis and target prediction to investigate the functional effects of these dysregulated lncRNAs and mRNAs on vein graft failure. We predicted several lncRNA-mRNA pairs may be involved in biological processes related to vein graft failure, containing MRAK083052-Nrpland MRAK029384-Tpm1.

\section{CONCLUSION:}

This is the first systemic screening and analysis of the rat autogenous vein grafts related lncRNAs expression profile which significantly indicated that lncRNAs may play a potential role in the pathogenesis of vein graft failure. Future studies are demanded to determine whether these lncRNAs could serve as a diagnostic biomarkers or therapeutic targets in vein graft failure.

$100 \%$ successes of one thousand plus consecutive OPCAB surgeries- our experience

Shankar M. Gieengia; P. Balasubramanian

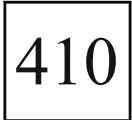

\section{ABSTRACT:}

BACKGROUND:

CABG surgery is one of the commonest life saving procedures done throughout the world. OPCAB surgery is practiced widely in India. With increasing experience, almost all CABG patients can be done as $\mathrm{OPCAB}$ with complete revascularization and excellent results.

\section{PATIENTS AND METHODS:}

From January 2017 to September 2018, 1093 CABG surgeries performed in our unit out of which 1092 where OPCAB. Age ranged from 30 to 92 years. $78 \%$ male patients. Ejection fraction ranged from 25 to $60 \% .14 \%$ where LMCA, 5\% emergency surgeries,. At least one mammary artery used in $92 \%$ of patients. Bilateral mammary arteries used in $63 \%$ of patients. Doppler probe was used to check the patency of grafts and to locate buried coronaries. No one pump conversion. One patient wit critical left main, small vessels and hemodynamic instability was put on CPB electively.

\section{RESULTS:}

We achieved hundred percent successes rate in one thousand plus consecutive $\mathrm{OPCAB}$ surgeries. Complete revascularization was our aim. Average number of grafts 3.2 . Vessels bigger than $1.25 \mathrm{~mm}$ was grafted in any territory. Average ICU stay was two days and hospital stay was 8 days. One patient needed IABP support following venous graft thrombosis due to hyperhomocystinemia, for whom venous grafts where redone on OPCAB. One patient had reversible ischemic stroke on 4th POD. None developed renal impairment requiring dialysis. None of the patient had sterna dehiscence.

\section{CONCLUSION:}

With experienced surgical team and good anesthetic support, OPCAB gives excellent results, even in patients with small vessels, diffuse disease, severe LV dysfunction, impaired renal, pulmonary function, octo and septo genarians
Coronary artery size and patient outcomes in off pump CABG

Devender Singh; Shegu Gilbert

\section{ABSTRACT:}

Small coronary artery size increases the risk for CABG. We evaluated the outcomes of Off pump CABG for different coronary artery sizes In our retrospective study of 1000 patients who underwent $\mathrm{CABG}$ we calculated the average coronary artery size and its relation to the patient outcomes like hospital duration of stay, low cardiac output syndrome, mortality and morbidity. Statistical analysis was done. Patients were divided into groups according to coronary size and patient outcomes were evaluated for each group and comparison was done.

One Stage CABG plus Aorto-Bifemoral Bypass a Study of 13 patients with 10 year follow up Purushottam Deshpande; Swapnil Deshpande

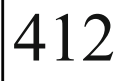

ABSTRACT:

Atherosclerosis is a systemic disease. The area which is maximally affected, the patient goes to that specialist. It is very common that the patient may have more than one system significantly affected and presents as difficult subset to treat. We are prsenting our unique study of 13 patients who had Significant Coronary Artery Disease and Aorto-Iliac disease requiring treatment for both to be done. We performed simultaneous Off Pump CABG and Extra Anatomic Ascending Aorta to Bifemoral Bypass. Total of 13 Patients have been treated with the first being operated in 2005 and last in 2017.

All the patients had Coronary artery disease and Bilateral AortoIlliac disease except on who had unilateral Illiac artery occlusion. On patient also had Bilateral Renal Artery Stenosis. The conventional treatment is to perform a Axillo-Bifemoral Bypass or Abdominal Aorta Bifemoral Bypass but to do them along with CABG need additional Incisions and also the long term results are poor. Ascending aorta is used as the site of proximal grafting area available is big. $8 \mathrm{~mm} \times 8 \mathrm{~mm} \times 8 \mathrm{~mm}$ T shaped ringed graft is used to do the anastamosis. Simultaneous CABG and Aorto Bifemoral Grafting is performed which does two things. 1. relieves the Debilitating Claudications and 2. Relieves the Coronary Artery Symptoms. The patient who had Bilateral Renal Artery Stenosis underwent Trans Brachial Bilateral Renal Artery Stenting along with the Procedure. All the patients are given Oral Vit K Antagonists for 1 year keeping INR between 2-3. A one year CT Angiogram performed to check for the patency and if found patent continued on Dual Antiplatelet therapy.

All the patients have been followed up for last 10 years and till now no graft occlusion is observed. This type of complete therapy definitely helps but judicious and meticulous planning is definitely required to avoid any complications.

A study of octogenarian patients who underwent CABG; Risk factors, course in the hospital and early outcomes.

Deepak Arkalgud

\section{ABSTRACT:} OBJECTIVE:

To study octogenarian patients who underwent CABG; Risk factors, course in the hospital and early outcomes.

\section{BACKGROUND:}

With improved medical management of coronary artery disease and demographic changes leading to easier access to medical care and availability of tertiary cardiac treatment, more older patients are undergoing $\mathrm{CABG}$ for 
coronary artery disease. Elderly population contrary to belief successfully undergo CABG and achieve satisfactory recovery at hospital discharge.

\section{METHODS:}

From 2015 Jan to 2018 Nov, 22 patients aged 80 years or more underwent isolated $\mathrm{CABG}$ for coronary artery disease. Retrospective review of the patients' medical records were performed, tabulated and analysed.

\section{RESULTS:}

The mean age of the patients was $80.5+-3.5$ years. Eighteen $(81.8 \%)$ were males and 4 were females $(18.2 \%)$. Each received a minimum of 3 grafts and a maximum of 5 grafts. (Mean 3.95). Nine patients had history of recent MI (40.9\%). Nine (40.9\%) patients had AF during their hospital stay. Average ICU stay was 80.5 hours and ward stay was 6.15 days. Four patients $(18 \%)$ needed IABP support and 3 deaths $(13 \%)$ occurred in the immediate postoperative period.

\section{CONCLUSIONS:}

Patients at the age of 80 years can be considered as suitable candidates for CABG procedure, but noting that they have longer period of Intensive Care Unit and/or ward stay. They have more co-morbidities and risk factors for surgery. Morbidity and mortality seem to be in higher range. Hence, CABG may be considered in these patients with slightly higher risk than in younger patients with lesser co-morbidities.

\section{Effects of low renal dose dopamine on patients with deranged renal function undergoing cabg. Anurag Garg; Ashish Dolas}

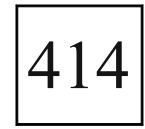

\section{ABSTRACT:}

\section{OBJECTIVE:}

A prospective randomized study undertaken to assess the effect of postoperative "renal-dose" dopamine on patients with deranged renal functions post-cabg clinical outcome.

\section{METHODS:}

Fifty consecutive patients undergoing cabg were randomized to receive either 3-5 microg $/ \mathrm{kg} / \mathrm{min}$ dopamine $(\mathrm{n}=25)$ or not given dopamine $(\mathrm{n}=25)$ for 48 postoperative hours. Clinical outcome parameters were collected for four postoperative days. Post operative parameters for assessment were post operative: Blood urea and serum creatinine, urine output, recovery of patient post-operatively.

\section{CONCLUSIONS:}

Prophylactic dopamine administration after open heart surgery improves patient hemodynamic and renal status, improves urine output, reduces the need for additional medical support Inotropes and Furosemide and thus, provides stable postoperative course and recovery.

\section{Use of Retrograde Cardioplegia in On Pump CABGs}

Abhay Jain; James Thomas

\section{ABSTRACT:}

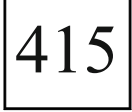

Last thirty years in four institutions we have used Retrograde Cardioplegia in all patients undergoing Coronary Artery Bypass Surgery with On Pump technique. $20 \%$ of patients in between patients were done Off Pump. Patients with Left Main, and near total occlusions of the coronary arteies would be most benefitted by Retrograde Cardioplegia. We have used St Thomas Hospital Cardiolegia solutions ans wella s DelNido. The technique of inserting Retrogarde Cardioplegia is important best done after insertion of Single venous drain and before going on bypass. Heart comes back with hardly any arrhythmias with the use of Retogarde. Our Technique and experience described.

A rare delayed pituitary apoplexy post coronary artery bypass surgery - a review and management Kofidis Theodoros; Hari Kumar Sampath
ABSTRACT:

Pituitary apoplexy is a rare entity, commmonly seen in postpartum as Sheehans's syndrome. Even rarer in patients going for surgery, but can be fatal if not intervened early.

Any patient with pituitary adenoma functional or non functional must be screened for the size and function before cardiac surgery in view of complications secondary to long surgery, especially on cardiopulmonary bypass, expected hypotension or haemorrhage during bypass and anticoagulation post surgery.

Here we present an interesting case of delayed pituitary apoplexy in a patient with pituitary adenoma who underwent coronary artery bypass surgery, with coronary endarterectomy.

Patient is a 60 years old male with known history of pituitary adenoma, with low testosterone levels otherwise normal functioning pituitary on conservative management. He underwent Coronary artery bypass surgery. Preoperatively he was investigated and had prophylaxis to prevent an anticipated introperative or immediate perioperative complication of pituitary apoplexy.

Endocrinologist opinion sought and he was given steroid prophylaxis preoperatively and post operatively to prevent life threatening adrenal crisis and hypopituitarism post operatively.

Immediate post operative period was uneventful, patient recovered well but had pituitary apoplexy with neurological and ophthalmological symptoms secondary to haemorrhage after one week post surgery on initiation of anticoagulation for coronary endarterectomy. Eventually diagnosed early, in view of anticipation pre operatively and timely excision of pituitary adenoma saved the patient with further permanent debilitating complications.

We will discuss about the strategies of investigation, prediction of pituitary apoplexy, appropriate consenting pre-operatively and medicolegal issues and involvement of multidisciplinary team approach in patients with pituitary adenoma in view of potential pituitary apoplexy post cardiopulmonary bypass surgery, to achieve a favourable results.

\section{Post Infarction VSD Repair}

Abhay Jain; James Thomas

\section{ABSTRACT:}

Ventricular Septal Rupture is a rare complication of Myocardial Infarction but lethal. Usuallly rupture happens 2-3 days after rupture often precipitated by cardiogenic shock. Free wall ruptur and papillary rupture is not included in presentation. Delay in surgery Our experience with trree patients presemnted. Delay in surgery in stable patients gives better esults. surgery done three weeks after IABP was not required. Approach on CP Bypass, left ventricular aneurysma area incised. VSD edges identified. Dacros velour patch used wit interrupted Ethibond 20 sutures from R V Side and securely tied on Lv Side. In one patient one side of patch secured on to LV side outside with pldgetted suture, LV aneurysmal area was sutured with felt and 20 ethibond.

Patients made good recovery Delayed surgery and technique emphasised.

Outcome of early CABG in acute myocardial infarction Ganesh Kamath; Sakthivel Kumaresan

\section{ABSTRACT:}

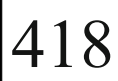

\section{AIMS AND OBJECTIVES:}

The aim of this study was to examine outcome in early coronary artery bypass graft surgery (CABG) for non-ST-segment elevation myocardial infarction (NSTEMI) patients, stable ST segment elevation myocardial infarction (STEMI) and chronic stable angina (CSA). Although practice guidelines recommend delaying $\mathrm{CABG}$ after presentation for ST-segment elevation myocardial infarction patients, current guidelines for NSTEMI patients do not address optimal CABG timing. 


\section{MATERIALS AND METHODS:}

We evaluated outcome in early CABG among NSTEMI, stable STEMI and CSA patients treated at our hospital from January 2016 to September 2018. The clinical characteristics and outcomes of CABG were studied. Patients requiring additional valve repair or replacement were excluded from the study.

RESULTS:

The composite outcome of death, arrhythmias, need for prolonged ventilation, transfusion requirement, ionotropic requirement and duration of hospital stay, requirement for IABP and CPB were similar between patients undergoing early $\mathrm{CABG}$ in acute myocardial infarction (MI) in comparison with CSA.

\section{CONCLUSIONS:}

Most NSTEMI and stable STEMI patients undergo late CABG after hospital arrival. They have the same risk of adverse clinical outcomes compared with patients who undergo early CABG. Thus, delaying CABG routinely after NSTEMI and stable STEMI may not improve outcomes.

\section{Comparison of short term outcome between total arterial CABG and conventional CABG \\ Mayank Acharya; Dharmendra Joshi; \\ Niraj Bhattarai; Samir Azam Sunny \\ Mohammed Aslam Hossain}

\section{ABSTRACT: \\ BACKGROUND:}

Coronary artery bypass grafting $(\mathrm{CABG})$ is an established treatment for coronary artery disease. There have always been debate on the superiority of total arterial $\mathrm{CABG}$ versus conventional CABG (arterial and venous graft). METHODS:

This studywas conducted between April 2017 to September 2018 (1.5 years). 3 months short term outcome of total arterial CABG (LIMA, RIMA/Radial) and conventional CABG (venous and arterial graft) has been observed, and compared. A total of 130 patients were studied among them 62 patients were studied in the total arterial group and 68 patients in conventional group.

RESULTS:

The mean age of our patient was $58.9 \pm 7.5$ years of which $105(80.7 \%)$ were males and $25(19.2 \%)$ were females. 111 (85.4\%) cases were TVD, 17(13.1\%) were DVD and 2(1.5\%) were SVD. 7 cases $(5.4 \%)$ required cardiopulmonary bypass. There were similar incidences of most observed postoperative complications in both groups as well as similar length of ICU and hospital stay, however some findings favored arterial than conventional CABG.

\section{CONCLUSION:}

Total arterial graft has a few advantages to conventional CABG. However since most postoperative complications are similar in both groups the choice of conduit can be chosen based on surgeons choice.

\section{A rare case of Myocardial Abscess Post Chronic Coronary stent infection \\ Hiren Dholakiya; Chintan Seth; Niren Bhavsar Dhiren S. Shah}

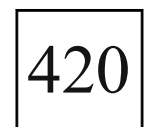

\section{ABSTRACT: \\ INTRODUCTION:}

Percutaneous transluminal coronary angioplasty (PTCA) with metal stent placement has become a well-developed treatment modality for coronary stenotic lesions. Although infection involving implanted stent is rare, it can, however, occur with high morbidity and mortality.

\section{BACK GROUND:}

We describe an inserted coronary stent that was infected and complicated with recurrent stent thrombosis, sepsis and myocardial abscess formation. In an effort to raise awareness of the possible fatal outcome of this situation, we herein report our experience.
CASE:

We describe a rare case of infected coronary stents complicated with recurrent stent thrombosis, sepsis and myocardial abscess formation after 2 years of PCI. Using multi-modality imaging like 2D Echo, Cardiac MRI, PET Scan, final diagnoses to evaluate the precise location, extent and morphology of myocardial abscess (MA) was done. "On pump" coronary artery bypass graft (CABG) was performed, left anterior descending (LAD) artery intramyocardially was opened up, about $7-10 \mathrm{ml}$ of pus was evacuated and 2 drug eluting stents (DES) were removed. The isolated organism was Pseudomonas Aeruginosa which had remained dormant and restricted to the stent area for almost 2 years thinning the myocardium.

CONCLUSION:

Myocardial abscess (MA) is a spurious infection of the myocardium, endocardium, native or prosthetic valves, perivalvular structures, the cardiac conduction system and very rarely stents. The present case exemplifies the high index of clinical sensitivity with early multi-modality diagnosis, aggressive medical therapy, multidisciplinary care and timely surgical intervention saving the patient's life in otherwise fatal condition.

Routine use of Harmonic Scalpel for skeletonized Internal mammary and Radial artery harvesting.

Devapriya Marik

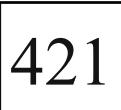

\section{ABSTRACT: \\ INTRODUCTION:}

Use of skeletonized arterial grafts has lot of advantages. Harmonic scalpel can be used to skeletonize various arterial grafts. Use of this technology has lots of advantages over the conventional technique of electrocautery and clips.

\section{Material and Methods:}

Starting from February 2012, the Harmonic scalpel ( Ethicon) along with the Synergy hook was used to harvest all arterial grafts used during CABG. A total of 1158 LIMA, 281 Radial arteries and 149 RIMA were harvested. The time taken to harvest, the number of injuries to the graft during harvesting, the total length of the grafts harvested, the diameter of the conduits after harvest, flow in the grafts after harvest were measured. Clinical results including incidence of perioperative MI, total blood loss, total blood products used, incidence of sternal and forearm infections were recorded.

\section{RESULTS:}

Results were compared to historical controls in whom the conduits were harvested using conventional electrocautery and clips.

\section{CONCLUSIONS:}

Harmonic scalpel has several advantages when used to harvest arterial conduits and should be used more frequently.

Early Outcomes of OPCAB for LMCA

Sudha; Ajay

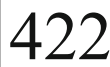

ABSTRACT:

BACKGROUND:

Left main coronary artery (LMCA) stenosis is a risk factor for morbidity and mortality in patients undergoing coronary artery bypass grafting (CABG). Although improved outcomes of off-pump CABG have been well documented, LMCA stenosis is often perceived as a contraindication for off-pump CABG. In this study, we compared off-pump techniques in high-risk patients with LMCA disease with patients without LMCA disease.

\section{METHODS AND MATERIALS:}

Study included 819 patients who underwent off-pump CABG at Chettinad hospital from august 2014 to 2018. Group A included 100 with LMCA disease and Group B included 719 with non LMCACAD. Variables like percentage of male vs female, age $>60$ and $<$ 
60 years, good LV versus Poor LV, emergency Vs elective, use of IABP peri op and post op period, number of days in ICU and hospital, inotropic support and transfusion rate, total blood loss and mortality rate were studied.

\section{RESULTS:}

On analysis, both the Groups were similar in terms of preoperative and intraoperative variables such as age, sex, nature of disease, conversion to on pump and usage of IABP, except for LMCA patients who received significantly more grafts than non LMCA patients. Despite a higher risk profile of LMCA patients there was no significance mortality. Both groups had a lower requirement for postoperative inotropes, ventilation hours, blood product transfusion, and reduced postoperative stay. Except for patients severe LV dysfunction and LMCA group, ICU stay was high. Mean ICU stay for both group was 2.5 days.

\section{CONCLUSIONS:}

Offpump coronary artery bypass grafting is safe and effective in patients with critical LMCA stenosis.

\section{Evaluation of genetic variation in SCLO1B1 gene $(521 \mathrm{~T}>\mathrm{C})$ in patients receiving statin therapy: An observational study. Manish Pungliya; Atul Mahadik; Manoj Durairaj}

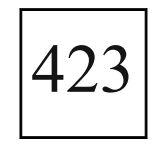

\section{ABSTRACT: \\ BACKGROUND:}

Statins are commonly prescribed drugs used for cholesterol reduction and maintainence. Approximately 1-5 percent of exposed individuals may experience a dose-dependent myopathy (skeletal muscle toxicity).

SLCO1B1 is an influx transporter that moves active statin acid from the blood stream into the liver. Statins are one of the most common classes of medications affected by this transporter.

Variations in SLCO1B1 result in decreased transporter activity that may result in increased statin blood levels and increase the risk of adverse effects, such as myopathy, myalgia and rhabdomyolysis.

Statin-induced myopathy is reported to be significantly associated with the SCLO1B1 (521T\&gt;C) genetic polymorphism.

\section{PURPOSE:}

To date, SCLO1B1 (521T\&gt;C) genetic epidemiologic data for the Indian population is still lacking. This study was aimed at assessing the genotype and allele frequencies of the SCLO1B1 (521T\&gt;C) variant in Maharashtra and correlate the same findings with clinical implications on patients who are on statin therapy.

\section{MATERIALS AND METHODS:}

Genomic DNA was extracted from peripheral blood from 69 individuals who were receiving statins and was analyzed for the SCLO1B1 (521T\&gt;C) polymorphism by using the real-time polymerase chain reaction (RT-PCR) taqman assay method.

\section{RESULTS:}

Of the 69 total patients, $10(14.5 \%)$ were heterozygous $(\mathrm{C} / \mathrm{T})$ and 1 $(1.5 \%)$ was homozygous $(\mathrm{C} / \mathrm{C})$ for the SCLO1B1 (521T\&gt;C) polymorphism. The SCLO1B1 (521T\&gt;C) minor allele frequency was $8.7 \%$ in our population $(\mathrm{HWE}=0.46)$.

\section{CONCLUSION:}

This is the first report exploring the frequency of SCLO1B1 (521T\&gt;C) genotype in Maharashtrian population. Compared to other populations like Japanese, Chinese \&amp; Europeans the presence of this polymorphism is lower in our population. From our study it may be presumed that about $8 \%$ of the population potentially could be at risk of statin induced myopathy.

\section{Y grafts in cabg \\ Kushant Gupta; Milind Anand Umre}

\section{ABSTRACT:}

\section{INTRO:}

Cabg as an operation for revascularization has been there for nearly $60 \mathrm{yrs}$ ( since 1 successful Cabg was done on humans in 1964 by Kolesov), every now and then we get know some development in this operation. Y grafts both venous and arterial have been used for Cabg $(1,2)$

Like wise we have started using Y grafts in Cabg since past few years from 2014 onwards

Results have been excellent.

We intend to present here the same.

\section{METHOD:}

100 Patient were chosen at random. No of grafts usually varied from 4-5 per patient. Total no of grafts used were 65 LIMA-Y and 35 SVG -Y. 35 simple LIMA, 160 simple RSVG, Total no of anastomosis used were 405. Grafts used were LIMA -Y, RSVG -Y. They were used to Vascularize Lt side arteries, that is LAD, Diagonals, RI, OMs. LIMA was used in $100 \%$ patient RSVG -Y was used to mostly revascularise OM, diagonal. Only in1 case RSVG was used to graft PD and distal LAD. LIMA-Y was used to graft LAD, Diagonal, RI occasionally to OM1.

\section{RESULTS:}

Of 100patients that underwent Cabg using Y grafts, nearly 60 had only LIMA -Y, 30 had RSVG -Y and only 10 patient had both LIMA-Y, and RSVG -Y.

All the above patient are under follow up for 4 years, with no adverse complaint.

In $25 \%$ of the patients grafts were evaluated with CTAngio.

\section{CONCLUSION:}

Use of Y grafts in CABG has been used for long time nearly 10 years, It is safe to use. Although long term patency needs to be evaluated, short term follow-up in our cases do not show any adverse results. LIMA-Y, RSVG$\mathrm{Y}$ grafts are safe and can be used without any adverse results.

Coronary Bypass surgery in young adults : - analysis of their risk factors and midterm follow-up.

Hiren Dholakiya; Niren Bhavsar; Cintan Seth;

Amit Chandan

Dhiren Shah

\section{ABSTRACT:}

\section{OBJECTIVES:}

Coronary artery disease is considered to exhibit very malignant course and is presumed to affect young adults in India. Our Aim was to analyse the 16 variables about their preoperative factors and their perioperative course in last 8 years. We have also analysed their short term and midterm outcomes post $\mathrm{CABG}$.

\section{METHODS:}

We included in this retrospective analysis, all patients aged 40 years or younger undergoing CABG at our centre from September 2010 till 2018 October.

\section{RESULTS:}

There were 84 patients operated for CABG in last 8 years who were 40 years and less. Average age was 33.3 years with $91.67 \%$ ( 77/7) male patients. Average cases were only $1.6 \%$ patients of the total CABG cases annually. $91.67 \%$ patients were in the age group of 30-40. Risk factors analysis for CAD showed, $22.62 \%$ patient had diabetes, $27.38 \%$ patients had Hypertension, 32.14 patients had history of smoking and only $3.57 \%$ had obesity, $65 \%$ were from rural area against $28.57 \%$ from urban area. $41.67 \%$ patients presented with Acute MI or ACS( Acute coronary syndrome). $19.03 \%$ patients were having EF less than $30 \%$ and $61.90 \%$ were more than $40 \%$. $14.29 \%$ patient had single vessel disease, $15.48 \%$ had DVD and majority (70.24\%) patient had TVD. On average followup of 5 years there was 1 in hospital mortality and 2 mortality were cardiac related and one died in followup due to non-cardiac reasons. Overall actuarial freedom from any cardiovascular lethal or morbid event was estimated at $86 \%$

CONCLUSION: 
Traditional risk factors doesn't seem to be the etiology of CAD in young adults. Peri-operative mortality and morbidity is low in young patients undergoing coronary bypass surgery, with good freedom from MACE in midterm followup.

\author{
Miraculous survival from all three concurrent \\ mechanical complications of acute MI: a case report \\ Neerav Bansal; Biju Sivam Pillai; Neerav Bansal
}

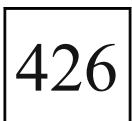

\title{
ABSTRACT:
}

\section{INTRODUCTION:}

Acute myocardial infarction (AMI) is responsible for a significant proportion of morbidity and mortality in patients with ischemic heart disease. With incidence of less than $1 \%$, mechanical complications like ischemic mitral regurgitation (IMR), infarct ventricular septal rupture (PMI-VSD), left ventricular free wall rupture (LVR) or left ventricular aneurysm (LVA) further worsen survival of such patients.

\section{CASE REPORT:}

61 year old male presented with history of sudden onset rapidly progressive breathlessness for 3 days with history and ECG suggestive of inferior wall AMI 2 weeks ago. He was being managed medically at a peripheral health center. Clinical examination and two-dimensional echocardiography (2D Echo) revealed posterior VSD, adjacent inferior wall LV aneurysm, severe IMR and LVEF $25 \%$. Promptly supported by inotropes, vasodilators and intra-aortic balloon pump (IABP), he underwent early corrective surgery in the form of CABG, MV repair, VSD closure and LV aneurysm repair with a prolonged post-operative course. His 2D echo after 3 months shows LVEF $40 \%$, no left to right shunt, trivial MR, no LVA and he is in NYHA-I.

\section{CONCLUSION:}

For the diagnosis of mechanical complications, high degree of clinical suspicion is necessary. Besides the physical examination, 2D Echo is useful with high specificity and it can be performed bedside. Preoperative optimization, with an IABP and vasodilators, may help to reduce the afterload on the compromised ventricle and may improve cardiac output in the short term, but should not delay surgical intervention. Surgery remains the mainstay of treatment in such patients, with poor outcomes for patients treated medically.

How to deal with late brain infarction after off pump coronary artery bypass grafting. "The efficacy and safety of anti-coagulant therapy."

Kohei Abe; Hiromi Yanagisawa; Manabu Yamsaki; Hiroyasu Misumi; Kunihiko Yoshino

\section{ABSTRACT: \\ BACK GROUND:}

A brain infarction is a devastating complication after coronary artery bypass grafting. Although the intraoperative managements to prevent neurological complication have been improved, some patients are complicated with brain infarction which is associated with postoperative atrial fibrillation (POAF). The reported incidence of perioperative brain infarction after off pump coronary artery bypass grafting (OPCABG) were 1.6 to $3 \%$. At our institution, we use anticoagulant drugs after OPCABG. In current study, the efficacy and safety about using of anticoagulant drugs after OPCABG were examined.

\section{MATERIALS AND METHODS:}

313 patients underwent isolated primary OPCABG from January 2011 to April 2018 at our institution. Anticoagulant strategy after OPCABG had consisted of continuous heparin infusion and subsequent warfarin administration postoperatively. We assessed the occurrence rate of brain infarction after OPCABG, which was was classified to early (the onset was less than 24 hours after surgery) or delayed (the onset was 24 hours to 30 days after surgery).

\section{RESULTS:}

269 patients $(86 \%)$ were male. 33 patients $(11 \%)$ had previous brain infarction. The average CHADS2 score was $2.0( \pm 1.2)$ and average HASBLED score was $2.9( \pm 1.0)$ points. POAF was occurred in 87 cases (28\%). Warfarin was administered to 236 patients $(75 \%)$ at the time of discharge. After OPCAB, we had just two new onset brain infarction $(0.6 \%)$, one in early period and another one in delayed period respectively. Bleeding complications occurred in 15 patients $(4.8 \%)$ although those were not fatal.

CONCLUSION:

The use of prophylactic anticoagulant drugs after OPCABG is effective toprevent perioperative brain infarction.

Does addition of mitral valve intervention to coronary artery bypass surgery adversely affect the post operative outcomes

Manish Banshal; Nita Bharti

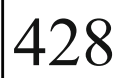

\section{ABSTRACT:}

Approximately $30-50 \%$ of patient will develop ischemic mitral regurgitation (IMR) after myocardial infarction, which is a result of progressive left ventricular remodeling and dysfunction of the subvalvular apparatus. Surgical treatment is centered on mitral valve repair utilizing a restrictive annuloplasty or valve replacement with preservation of the subvalvular apparatus. The optimal surgical strategy for the management of significant IMR $(\geq 2+$ grade) remains controversial and mitral valve surgery (MVS) at the time of coronary artery bypass grafting (CABG) is still debated. The primary objective of the present study is to clarify the effects of additional MVS on the post operative morbidity and prognosis in the patients with significant IMR, compared with isolated CABG.

In the present study, we included patients admitted with the diagnosis of coronary artery disease (CAD) with or without Mitral Regurgitation and underwent Coronary Artery Bypass Grafting with or without mitral valve intervention. Data was collected and analyzed. Both the groups (first group was $\mathrm{CAD}$ patient who underwent $\mathrm{CABG}$, second group was $\mathrm{CAD}$ plus IMR who underwent $\mathrm{CABG}+$ Mitral valve intervention) were compared in terms of intraoperative and postoperative morbidity and mortality.

\section{Anomalous origin of Left Main Coronary Artery from Right Sinus of Valsalva in young presenting as STEMI : A diagnostic and therapeutic challenge Rajeshwar Sharma; Abhishek Anand}

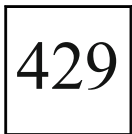

\section{ABSTRACT:}

A 18 year old defense recruit was brought to emergency and diagnosed to have STEMI with positive Trop -T. Upon further evaluation he was found to have anomalous origin of left main coronary artery from the right sinus of Valsalva. He was managed with deroofing of the intramural course of the left main coronary artery thus creating a neo ostia in the left sinus of Valsalva. The anomalous origin of left coronary artery from the right coronary sinus is a rare congenital anomaly which remains undiagnosed and is frequently implicated in cases of sudden cardiac death mainly in the young.

There is no consensus on management of these patients. The treatment offered ranges from purely medical including life style changes to surgery involving translocation of left main origin to coronary artery bypass grafting. We present this case to review this rare but eminently treatable congenital anomaly presenting in adulthood.

Coronary Endarterectomy - Early Outcome Richard Saldanha; Mohan D. Gan; Nischit Udapi Ravi Ghatanatti 


\section{ABSTRACT: \\ BACKGROUND:}

Coronary endartectomy $(\mathrm{CE})$ is performed as an adjuvant to coronary artery bypass surgery(CABG). However, the efficacy of this technique is still debated. We aim to evaluate the effect of $\mathrm{CE}$ and its early outcome. METHOD:

Patients who underwent CABG between January 2016 and November 2018 were included. 147 of 1209 patients $(12.15 \%)$ underwent CE in addition to CABG. Early results were compared with isolated $\mathrm{CABG}$ and predictors of adverse outcome were measured in step wise multivariate logistic regression analysis.

\section{RESULT:}

The incidence of co-morbities including prior myocardial infarction, diabetes mellitus and triple vessel disease in CE patients was higher however, mortality (3.4\% Vs control 3\%; $\mathrm{p}=0.762)$ and post-operative complications were not significantly increased in this group of patients. Although female gender, impaired ejection fraction, intra-operative intra-aortic balloon pump and prolonged cardiopulmonary bypass time were important predictors of adverse outcomes, CE was not associated with increased mortality or post-operative morbidity. On the contrary, there was lesser incidence of arrhythmia and ST segment changes.

\section{CONCLUSION:}

CE can be performed safely in high risk patients without any adverse post-operative outcome.

\section{Comparitive analysis of EuroSCORE II and STS score in patients undergoing isolated $\mathrm{CABG}$ Koushik}

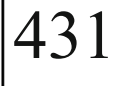

\section{ABSTRACT:}

\section{BACKGROUND:}

Risk stratification in cardiac surgery has become important for both surgeon and the patient for predicting perioperative mortality and morbidity AIM:

To analyze the predictive values of these scoring systems and their applicability in indian population

\section{MATERIALS AND METHODS:}

Prospective non randomnized study of 390 consecutive patients undergoing isolated CABG ( both off and on pump ). Patients were followed up for duration of ventilation, length of hospital stay, renal failure, deep sternal wound infection, stroke, MACCE and mortality

RESULTS:

EuroSCORE II and STS score have good calibration indicating satisfactory model fit in the study population

Both the scores in this study showed poor discriminatory power indicating overestimation of mortality in high risk groups

STS morbidity accurately predicted post operative stroke and deep sternal wound infection.

Early outcomes of CABG for left main coronary artery disease: Single center analysis of factors influencing outcome

Neethu Krishna; Rajesh Jose; Murukan P.;

Praveen Varma; Kirun Gopal

\section{ABSTRACT: \\ INTRODUCTION:}

Left main Coronary disease is among the few subsets of patients, which currently remain within the treatment domain of the cardiac surgeon. However with the recent advances in stent technology more and more left main patients are being enrolled in trials to establish percutaneous coronary interventions as a viable alternative to surgery. In such a setting, continuous evaluation and refinements of surgical procedure and post operative management and analysis of surgical outcomes is needed to ensure our outcomes remain the gold standard in care for left main disease.

\section{METHODS:}

288 consecutive patients with left main disease who came for coronary artery bypass surgery to our department from 2014 to 2017 were retrospectively analyzed for short term and early midterm outcomes to 1 year. Statistical analysis was done to compare factors contributing to mortality using multivariate analysis.

\section{RESULTS:}

9 out of the 288 patients died during this study period. Analysis of the data comparing patients who survived and those who died by univariate analysis showed emergency surgery, presence of pre or postoperative renal dysfunction and recent MI as being predictors of mortality. On multivariate analysis, postoperative renal dysfunction, use of therapeutic antibiotics, IABP use and presence of moderate LV dysfunction were predictors of mortality. Early Mid term multivariate analysis at one year showed the same 3 variables were predictive of mid term mortality.

\section{CONCLUSIONS:}

Surgical outcomes for left main coronary artery disease are very good. Risk factors for immediate and early mid term mortality are postoperative renal dysfunction, need for therapeutic antibiotics and IABP use. CABG surgery should remain the gold standard in care for these patients and with further refinements to decrease the incidence of post operative renal dysfunction, infections and myocardial protection may even set the standards higher.

\section{Left ventricular free wall rupture following acute} myocardial infarction

Ganesh M.; Prasad Surendran

Rupture of the left ventricular free wall following acute myocardial infarction is a rare complication which carries a very high mortality rate. Timely surgical repair is essential for a successful outcome. We describe here our experience with successful repair of 4 cases of post-MI left ventricular free wall rupture over a two and half year period.

Bilateral internal mammary artery grafting and sternal wound infection- a myth on decline Sadiq Sheriff; Vikram M.J.

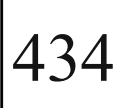

\section{ABSTRACT:}

Coronary artery bypass grafting (CABG) is one of the most common cardiac surgical procedures over half a century. Landmark paper from Cleveland clinic group reported left internal mammary artery (LIMA) as a conduit to left anterior descending artery (LAD) with good surgical outcomes at 10 years. LIMA graft is the gold standard of CABG. Suzuki et al published the first report about bilateral internal mammary artery (IMA) for revascularization in CABG in 1973. Several observational studies have reported the use of bilateral IMA is associated with significant long term survival benefit over single IMA graft irrespective of age, LV function and diabetes. Meta-analysis of studies by Taggart and colleagues revealed the benefits of bilateral IMA grafts increase with duration of follow up with reference to need for redo-CABG ( $8 \%$ bilateral IMA vs $40 \%$ single IMA) in matched patients at 12 years. In spite of substantial evidence of long term survival benefit, this technique remains underutilized, especially in view of the risk of deep and superficial sternal wound infections. Meticulous surgical techniques have been adopted for bilateral IMA harvesting. These strategies are associated with lesser devascularization of sternum and good sternal stability, thus lowering the incidence of sternal wound infections. Our study aims to prove safe use of bilateral IMA even in high risk population (DM, COPD, Obesity, renal dysfunction) who have undergone CABG from 2009 onwards, with negligible incidence of deep sternal wound infection. 
Endovascular management of critical hand ischemia by 'palmar arch loop' technique

A.P. Jain

\section{ABSTRACT: \\ BACKGROUND:}

Critical hand ischemia with advancing gangrene of digits requires urgent intervention to salvage as much tissue as possible. Endovascular intervention strategies aim at establishing inline flow to the palmar arch via both radial and ulnar arteries to safeguard digital perfusion. Frequently, antegrade recanalization of the radial artery or the ulnar artery fails due to flush occlusion at brachial artery bifurcation or dense fibrotic occlusions. AIM AND OBJECTIVE:

To evaluate the efficacy of 'palmar arch loop' technique for endovascular management of critical hand ischemia by establishing inline flow to the palmar arch via both radial and ulnar arteries.

\section{MATERIALS AND METHODS:}

Between April 2017 and May 2018, PTA for critical hand ischemia was performed in 06 patients (04 females and 02males; Mean age $39+$ +12 years). Causes for critical hand injury were: Iatrogenic injury due to intraarterial injection in 03 patients $(50 \%)$ and thromboembolic event in 03 patients (50\%). Mean time of presentation post event was $24+/-12$ days. All the patients had some degree of tissue loss or gangrene of fingers and all of them had severe rest pain.

All the patients were approached via right $\mathrm{CFA} / 5 \mathrm{~F}$ femoral sheath. After unsuccessful attempt at antegrade revascularization of either radial artery or ulnar artery, the patent artery was negotiated antegradely, the guidewire with a pta balloon or support catheter was negotiated through the palmar arch and then through the distal part of occluded vessel and sequential angioplasty carried out successfully.

\section{RESULTS:}

Retrograde PTA of radial artery in 03 patients and ulnar artery in 03 patients was done successfully. $100 \%$ technical success achieved in all patients, ensuring inflow to palmar arch via both the arteries with improved vascularity distally. Primary patency rate was $83.33 \%$ at 6 months. All patients showed subjective improvement with healing of lesions and/ or reversal of pregangrenous changes.

\section{Angiosome-targeted BTK angioplasty without stenting for CLI: A single centre experience \\ Amit Chaudhary}

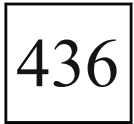

\section{ABSTRACT: \\ BACKGROUND:}

Recent development in endovascular intervention hardwares has made it the first choice strategy for the management of complex below-the-knee (BTK) and below-the-ankle (BTA) disease in critical limb ischemia (CLI).

\section{AIM AND OBJECTIVE:}

To evaluate the immediate and long term clinical results of angiosometargeted BTK percutaneous transluminal angioplasty (PTA) without stenting in patients with CLI at a single centre.

\section{MATERIALS AND METHODS:}

Between January 2017 and May 2018, BTK PTA was performed on 36patients presenting with CLI (Rutherford 4 to 6): Ischemic rest pain (08 patients), non healing ulcer (12 patients) and gangrene toes (16 patients). The etiology was buerger's disease (24 patients) and atherosclerosis (12 patients). The mean lesion length was $280 \mathrm{~mm}+/$ $90 \mathrm{~mm}$ (CTO and stenosis\&gt;70\%). 14 patients had significant femoropopliteal disease which was dealt by PTA. Comorbidity factors were: diabetes (06patients), hypertension (04 patients) and renal impairment (04 patients).

Approach was antegrade CFA (24 patients) and contralateral crossover (12 patients) using 5F femoral sheath. Angiosome targeted PTA with semicompliant balloon catheters (4F/0.014" guidewire compatible, 80 to $200 \mathrm{~mm}$ length, 2.0 to $3.0 \mathrm{~mm}$ diameter) was done successfully. Pedal to plantar loop technique was used in 04 patients for retrograde revascularization of occluded vessel when antegrade revascularization failed.

\section{RESULTS:}

A total of 48 procedures was performed in 36patients. Technical success rate was $87.5 \%$. Primary patency rate at 6 months was $85.7 \%$. Complete wound healing was $89 \%$ at 6 months. Subjective improvement in pain was observed in 31 patients. Angiosome targeted BTK PTA has good limb salvage potential in patients with CLI. Presence of diabetes, hypertension and renal impairment were negative predictors for long term patency.

An End stage Renal disease patient on hemodialysis with severe Left ventricular dysfunction coming for CABG

Praveen Kumar

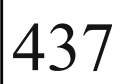

\section{ABSTRACT:}

51 years old Diabetes Mellitus and Hypertensive patient with known history of Chronic Kidney Disease with raised creatinine on medical management had an episode of acute dyspnoea and was diagnosed to be having Acute Left ventricular failure due to acute MI. Since he had Acute Renal Failure ( S. Creatinine $8.6 \mathrm{mg} / \mathrm{dl}$, ESRD) he was initially resuscitated with hemodialysis. On cardiac evaluation 2D Echocardiography revealed severe Left Ventricle dysfunction (LV EF $26 \%$ ) and severe Mitral regurgitation and severe PAH. Coronary Angiogram revealed critical Left Main disease and DVD. He was stabilized with medication and was given hemodialysis at regular intervals. Patient has undergone conventional Coronary Artery Bypass Grafting with 3 grafts ( LIMA to LAD, RSVG to OM and RSVG to Diagonal ) and intraoperative conventional ultra filtration and Modified Ultrafiltration. He was extubated on first Post operative day( $23 \mathrm{hrs}$ ventillatory support). Post operatively hemodialysis was resumed on second day and managed as per protocols. Peritoneal Dialysis was started from fourth day and was sent home in stable condition with Peritoneal Dialysis support.

\section{Long Term Mechanical Circulatory}

Supprot - Case presentation

Vivek Rao

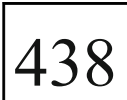

Long term Mechanical circulatory support - case presentation

Presenting two cases of long term mechanical circulatory support. Case 1: Percutaneous Trans Catheter Management of Aortic Insufficiency in Continuous flow left Ventricular assist device

60year old gentleman with Ischemic cardiomyopathy and severe left ventricular dysfunction (LVEF 20\%). Refractory to medical therapy with NYHA class IV symptoms. PMHx - hypertensive, chronic kidney disease, insulin DM and in in atrial fibrillation.

PET scan showed minimal viability. ICD was inserted in January 2016. 2D ECHO - severe LV dysfunction, LVEF 20\%, RV function mildly reduced, mild MR \&amp; AR, moderate TR, RVSP 61mmhg.

PET scan showed minimal viable myocardium, surgical revascularization was deferred.

Plan: Destination Therapy left Ventricular assist device (DT-VAD)

Post Op: dyspnea and fever. Infective endocarditis was rule out with TEE and blood cultures.

Trans esophageal ECHO - severe aortic insufficiency, functioning VAD. Plan:

Percutaneous TAVI with 34mm Medtronic Evolut R.

BACKGROUND:

Increased use of LVAD as destination therapy, rate of de-novo progressive aortic valve insufficiency have increased. Mechanism of aortic insufficiency is due alteration in blood flow kinetics, sustained high pressure 
results in aortic root dilatation, commissural fusion and myxoid degeneration of aortic valve.

Percutaneous TAVI or occluder devices is an option as the mortality for open surgical procedure is very high.

Case 2: Right Ventricular dysfunction in continuous flow LVAD CARDIAC STAND STILL

58 year W, with refractory heart failure secondary to dilated cardiomyopathy with sever LV dysfunction (LVEF 16\%)

She is a known hypertension, chronic kidney disease, hypothyroid and also had AICD inserted.

Trans thoracic ECHO - severe LV dysfunction with LVEF 16\%, moderate RV dysfunction, moderate TR with RVSP 50mmhg

She was planned for LVAD as a bridge to transplant

Surgery: LVAD - HeartWare and Tricuspid ring annuloplasty as a BTT. Had post-operative RV dysfunction and was on Inotropes.

Trans thoracic ECHO - Ventricular stand still

Plan: Heart Transplant

\section{In vitro prediction of the lower-critical hemorrhage} index

R.S. Bharath; Nichith Chandrasekaran;

Charlie Oommen; B.N. Raghunandan;

Shiv Kumar Choudhary; Pradeep Kumar Radhakrishnan

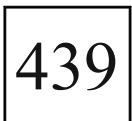

\section{ABSTRACT:}

Of late we have reported that the biofluid (blood) heat capacity ratio and the critical blood pressure (BP) ratio are the key deciding factors for the myocardial infarction, with and without symptom of plaque, due to the formation of shock waves leading to abnormally high transient pressure overshoot (AIAA 2018-3962). Note that the boundary layer blockage, Venturi effect and cavitation will invite internal flow choking causing shock waves in the arteries with divergent/bifurcation regions and/or plaque leading to aneurysam and/or hemorrhage, and myocardialinfarction. In this paper in vitro studies have been carried out, the first in the world, for predicting the lower critical hemorrhage index (LCHI) of human being from the estimated heat capacity ratio of the blood collected from the healthy subjects. The in vitro studies are carried out, using a well calibrated Differential Scanning Calorimeter - Perkin Elmer DSC 8000 at the Indian Institute of Science. We inferred that for a healthy-life the subjects with high $\mathrm{BP}$ ratio (SBP/DBP) necessarily have blood with high-heat capacity ratio. Therefore, increasing the heat capacity ratio of blood can reduce the risk on myocardial infarction for the cases with the extreme swings in BP, which is corroborating the closedform analytical findings of V.R. Sanal Kumar (2018). Note that the choked flow condition will occur in the transition / blockage region of an artery when the axial Mach number of the biofluid reaches unity. At the choked flow condition, the SBP/DBP ratio is a unique function of the heat capacity ratio of the blood, which dictates the LCHI of all subjects. We conjectured that the gene editing and the transfusion of blood, having high heat capacity ratio, in high-risk heart-patients for maintaining the unchoked biofluid flow condition in the artery can also negate the risk factor of hemorrhage and massive heart-attack.

Immediate graft dysfunction in marginal Donor Heart - A case report

Dhaval Naik; Niren Bhavsar; Hiren Dholakiya

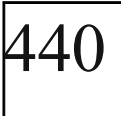

\section{ABSTRACT: INTRODUCTION:}

Heart Transplant program in India is on the rise. For successful Heart Transplant surgery and better long term survival multiple factors are important of which optimal Donor heart condition is utmost important. Because of lack of awareness of donor optimization in the peripheral centers and limited donor heart availability, sometimes marginal donor heart have to be used for transplantation

\section{BACKGROUND:}

Brain Dead Donor has to optimized haemodynamically and metabolically and with Humoral supplementation. We describe a case where we transplanted a heart which was marginal donor and had immediate graft dysfunction which was managed with ECMO support and medically.

\section{CASE:}

A male 21 years old case of Dilated cardiomyopathy on Inj. Milirinone therapy was listed for Heart Transplant on urgent basis due to his clinical condition. A donor was in Surat Civil Hospital who was brain dead due to severe brain injury. Donor was Haemodynamically very unstable and donor optimization was not done because of lack of knowledge about it. Heart was transplanted and and cold ischemia time was 4 hrs and 30 minutes. Immediate post unclamping Heart did not beat and was standstill. So central ECMO was initiated and along with that Various other Pharmacological treatment to replenish the myocardium started. Patients heart recovered within 36 hours and patient was discharged on 21 day. In one year followup patient is doing fine with normal LV function.

\section{CONCLUSION:}

Inspite of Marginal donor heart, with proper management primary graft dysfunction can be treated with good short term outcome. Long term outcomes are awaited.

Ventricular septal rupture repair with Endocardial patch by Infarct exclusion technique

D. Janardhan Reddy; K.V. Muralidharan; P.S.N. Raju;

Masthanamma Chengali; Srinivasan C.;

Srikanth Bhumana; Ashok Sampath

\section{ABSTRACT: \\ INTRODUCTION:}

A novel operative technique for postinfarction ventricular septal defect has been used in 64 consecutive patients.

\section{AIM:}

The operation consists of excluding rather than excising the infarcted septum and ventricular walls. To present the technique of VSR repair.

\section{MATERIALS \&amp; METHODS:}

This is accomplished by performance of a left ventriculotomy through the infarcted muscle and securing a polytetra fluoro ethylene patch to the endocardium of the left ventricle all around the infarcted myocardium. The ventriculotomy is closed over the pericardial patch. There were 20 men and 44 women whose mean age was $64 \pm 5$ years. Sixty-two patients were in cardiogenic shock at the time of operation. All patients had Doppler echocardiography and coronary angiography before operation. All but two patients were operated on during the acute phase of the myocardial infarction. There were no operative deaths. Postoperative complications included renal failure in 20 patients and respiratory failure in 28 .

\section{RESULTS:}

Renal and liver function were found to be independent predictor of operative mortality. The actuarial survival at admission was $75 \% \pm$ $7 \%$. Only one patient had a small residual ventricular septal defect. Late postoperative assessment of ventricular function by echocardiography revealed that most patients had normal or mild impairment of right ventricular function and mild or moderate impairment of left ventricular function.

\section{CONCLUSION:}

Repair of acute postinfarction ventricular septal defect by endocardial patch with infarct exclusion of the left ventricle probably 
avoids additional damage to the right ventricle and the organ systems, remodels the acutely infarcted left ventricle, and enhances survival.

Pulmonary tuberculosis in lung transplant recipients: a report of 2 cases

Anil kumar, Madhu Sankar,

Jakin Moses, Ananthraman, C S Varma,

K M Cherian

\begin{abstract}
:
Post transplant Lung infection is one of the leading causes of mortality and morbidity. Tuberculosis is not rare in lung transplant recipients and can be managed with anti tubercular therapy.

CASE: 1

19 years old male, a known patient of complex congenital heart disease, diagnosed as tricuspid atresia, hypoplastic RV, large VSD with bidirectional shunt and severe PAH, Class IV NYHA symptoms. He underwent heart and lung transplant in March 2018. Four months later he presented with right pleural effusion, $\mathrm{CT}$ chest showed right lower lobe consolidation. Sputum AFB studies revealed tuberculosis, Bronchoscopic alveolar lavage was done and real time PCR confirmed the same. The patient was treated with ATT. Currently he is doing well with resolution of consolidation.

CASE: 2

A 58 years old female Ms.S, from Kuwait was suffering from idiopathic pulmonary fibrosis with end stage Respiratory failure. She was on home oxygen therapy. She underwent right lung transplant in April 2016 and was on regular follow up. She presented with cough and fever in august 2017, sputum showed negative for AFB but positive with gene expert for MTB. Patient was initiated on ATT drugs and she is doing well.

The diagnosis of tuberculosis is more complex in transplant recipients. With use of immunosuppressive drugs, they are not only predisposed to primary tuberculosis infection but they are also at risk for reactivation of latent infection acquired prior to transplant or transmitted via the donor organ.
\end{abstract}

\section{Heart transplantation- Long term survival at a} single center

Matthias Peltz; Frans Van Wagenberg; Michael Wait; Lynn Huffman; Pietro Bajona; Mark Drazner; Michael

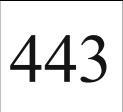

Jessen; Steve Ring

\section{ABSTRACT: \\ INTRODUCTION:}

Heart transplantation is still considered the gold standard for patients with end stage heart failure. Few centers have reported long term survival after cardiac transplantation. Here we review survival up to 30 years at a university hospital.

\section{METHODS:}

We performed a retrospective review of prospectively collected transplant data from all heart transplants performed at our institution. We have follow up data for nearly all patients. Long term actuarial survival was determined using the Kaplan Meier method.

\section{RESULTS:}

Between June 1988 and November of 2018 we performed 663 heart transplantations. Recipient ages ranged from 15 to 77 years (median 56). The etiology of heart failure included ischemic (364 patients), idiopathic (222 pts), familial (21 pts), valvar (20 pts), congenital (19 pts), and other cardiomyopathies (17 pts). Pre transplant circulatory support consisted of inotropes (294 pts), LVAD (127 pts.), IABP (49 pts), ECMO (7 pts.) and Bi-VAD (5 pts). The 30 day mortality is $2.4 \%$. Median survival for the whole cohort is 13.6 years. The actuarial survival rates at 1 year, 5 years, 10 years, 15 years, 20 years, and 25 years are $91.8 \%, 81.4 \%$, $66.6 \%, 42.6 \%, 27.6 \%$ and $16.4 \%$, respectively. There are 15 patients who are still alive more than 25 years after cardiac transplantation.

\section{CONCLUSION:}

Heart transplantation for end stage heart disease has excellent long term survival. It is to be seen if the use of interventions such as ECMO and VAD have any impact on the long term survival.

Early experience with direct vision MICS - Is there a learning curve ?

Sudha

Sanjay Theodore

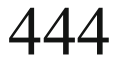

\section{ABSTRACT: \\ INTRODUCTION:}

MICS has gradually become the technique of choice for the vast majority of patient with valve disease and selected patient with coronary artery disease. with the development of standardized techniques and better equipment it has emerged as a safe and possibly better alternative to conventional sternal splitting surgery.

\section{MATERIAL AND METHODS:}

This is a retrospective study evolving 80 consecutive patients operated by the MICS technique over a two year period from January 2016 to November 2018. Patient demography, clinical parameters, surgical and post surgical parameters were compared with a historical cohort of patient undergoing similar surgical procedures. Surgical and post surgical parameters who compared with results of other surgical series.

\section{RESULTS:}

Patient in the MICS group tended to be younger (36.2 \pm 15.69 vs $35.87 \pm 20.8)$, ASD patients were older $(26 \pm 12$ vs $11 \pm 9)$. There was a statistically significant difference in CPB and cross clamp time. However, when compared to times of other series, this group had a significant shorter $\mathrm{CPB}$ and cross clamp time ( $\mathrm{p} \& 1 \mathrm{lt} ; 0.05)$. There was significant reduction in ICU, transfusion and hospital stay. Pain score was less in MICS patients. Major morbidity included stroke and femoral artery injury in one patient each with no mortality.

\section{CONCLUSION:}

In centers with significant experience with conventional surgery, MICS an be performed safely with good results without any apparent 'learning curve'. MICS can be applied in majority of valve cases and in selective coronary patients with reduced ICU and hospital stay, less pain and with no mortality.

Feasibility of Minimally Invasive Cardiac Surgery for Atrial Septal Defect Closure in a Government Set up.

Chaitanya Raut;

Ritesh Mate

\section{ABSTRACT: \\ INTRODUCTION:}

The technical ease of ASD closure has triggered interest in minimally invasive closure (MIC) in many centers. We retrospectively reviewed the results of right anterolateral thoracotomy compared with conventional median sternotomy (CMS) for ASD closure at our institute. In addition, we compared the cost of surgery, postoperative cost, and overall resource utilization and cost effectiveness in both the groups.

\section{METHODS:}


We retrospectively analyzed 49 adult patients who underwent isolated ASD closure from January 2011 to October 2018. The primary surgical out-comes analyzed aortic cross clamp time, CPB time, length of ICU and hospital stay, and post-operative complications (including pain). The operative cost was primarily determined by the consumables used during surgery. The postoperative cost was calculated by considering the number of consumables used, number of blood transfusions and cost of per day ICU and hospital stay. RESULTS:

The MICS group was younger ( 18 years vs. 14 years, $\mathrm{p}=0.002)$ and included more females $(66.7 \%$ vs. $94.4 \%, \mathrm{p}=0.025)$ than the $\mathrm{CMS}$ group. Operation time (188.4 minutes vs.286.7 minutes, $\mathrm{p}<0.001)$, cardiopulmonary bypass time (72.7 minutes vs.125.8 minutes, $\mathrm{p}<0.001$ ), and aortic cross-clamp time ( 25.5 minutes vs. 45.6 minutes, $\mathrm{p}<0.001$ ) were significantly longer in the MICS group. However, there were no significant differences in the postoperative complications and pain relief. The cost of surgery was significantly higher in the MIC group. However the MIC group had comparable postoperative cost and better resource utilization. The overall cost in the MIC group was higher.

\section{CONCLUSION:}

MICS via right anterolateral thoracotomy is excellent alternative choice to conventional approach to ASD closure. It demonstrated excellent results with a favorable resource utilization profile. Greater surgical cost and overall cost were the main limiting factors for use of MIC in a government hospital. Efforts should be taken to make MICS ASD closure available even to the underprivileged patients of the society.

\section{Complications, procedural difficulties and limitation observed during minimal invasive cardiac surgery Uday E. Jadhav; Yashasvi Sharma}

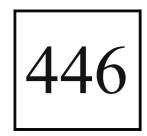

\section{ABSTRACT:}

This study was undertaken to assess the complications and procedural difficulties observed during MICS(minimally invasive cardiac surgery), to delineate the mechanisms of the complications and devise strategies for avoidance of the same.

A retrospective study of 75 patients undergoing MICS for various indications was undertaken. Data analysed included demographic variables, associated comorbidities, ventilation techniques, cannulation techniques, operative time and cardiopulmonary bypass time, rates of intraoperative and postoperative complications and mortality.

Of the 75 MICS procedures, 14 were MVR, 8 MVR with TVA, 11 ASD closure, $22 \mathrm{CABG}$ and $10 \mathrm{AVR}$. The mean age was $48+/-4.5 \mathrm{yrs}$. 48 patients were ventilated using double lumen endotracheal tube. 43 patients underwent peripheral cannulation and full extrathoracic $\mathrm{CPB}, 22$ patients were operated off-CPB. Mean operative time was $323+/-68$ minutes and mean CPB duration was $136+/-42$ minutes. One patient had conversion to full sternotomy, 2 patients had retrograde aortic dissection, 1 patient underwent re-exploration. Post operative complications were right ventricular failure, groin seroma, femoral artery thrombosis, unilateral chylothorax. 30 day moratlity was $3.9 \%$. MICS is gaining popularity among both doctors and patients with a number of studies showing it produces equivalent results to conventional open heart surgery with faster postoperative recovery, less pain and better cosmesis. However the minimally invasive approach does pose its own unique set of challenges and and further studies into its complications will be helpful in improving outcome.

\section{A case series of mics mitral valve replacement}

Thiyagarajamurthy; Sujith

\section{ABSTRACT:}

\section{BAKGROUND:}

Since we started mics mitral valve replacement in our institute, it has become routine surgical option for mitral valve disease. This prospective study is on perioperative clinical outcome of our patients.

\section{METHODS:}

Total of 24 patients who underwent mics MVR for mitral valve disease by anterior mini thoracotomy approach from 2017 january to 2018 july were included in this study The preoperative demographics, intraoperative findings, postoperative outcome and 3 months followup results were analysed.

RESULTS:

Among all patients $\mathrm{n}=35$, Mean CPB time 105 min, Mean clamp time 69 min, no immediate residual MR, $3(8.8 \%)$ patients underwent reexploration for bleeding, 3 patients had long ICU stay, no mortality, no wound infection, satisfactory echo finding on discharge and 3 month followup. One patient had residual MR on discharge as this patient had cardiac arrest postoperatively revived by internal CPR. At 3 months echo one patient had residual MR with history of fever and diagnosed as IE, later patient underwent coiling of residual MR.

\section{CONCLUSION:}

MICS MVR is a safe surgery with acceptable clinical outcome with low early postoperative mortality and morbidity.

Minimally invasive hybrid coronary revascularization in high risk patients with multi vessel disease Dhamodaran K.; Sengottuvelu G.; Refai Showkath Ali; Dheeraj Reddy; KCS Lakshmana Kumar

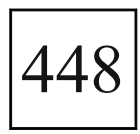

\section{ABSTRACT:}

Minimally invasive hybrid coronary revascularization in high risk patients with multi vessel disease.

\section{BACK GROUND:}

With the increasing number of high risk cases, minimally invasive cardiac surgery is gaining importance because of its low peri operative complications, morbidity and early recovery. Literature evidence shows IMA has patency around $90 \%$ at 20 years and $56 \%$ of stents and $53 \%$ of SVG grafts have re stenosis by $8-10$ years. Hence Minimally Invasive Hybrid Coronary Revascularistion (MIHCR) is increasingly performed as safe approach to treat Triple vessel coronary artery disease.( TVCAD)

\section{METHOD:}

From Nov 2017-Nov 2018, more than 25 patients underwent MIHCR. Of these 8 patients with euro score II \&gt; $4 \%$, with multi vessel coronary artery disease (MVCAD) and multiple co morbidities underwent MIHCR by MIDCAB with LIMA anastomosed to LAD, PCI to RCA/PDA/LCx. Patients with CKD underwent PCI by contrast free, Intra vascular Ultra sound (IVUS), Fractional Flow Reserve (FFR) guidance.

\section{RESULTS:}

8 Patients with MVCAD, euroII score more than 4, requiring total of $1 / 2$ stents for LCx / RCA /PDA lesions were selected. Of these 5 patients had CKD, 3 patients had COPD, 2 patients had CKD and COPD, 4 patients aged \&gt; 70, 2 were \&gt; 75 and 1 patient was obese. MIDCAB done with LIMA - LAD anastomosis. PCI done on post-operative day $4+/-2$ days. 4 patients required 2 stents and 4 patients required 1 stent.

CONCLUSION:

MICHR can be better option than conventional CABG for high risk cases.

Endoscopic radial artery harvesting with a watch band incision

Shivalli Rai; Manoj J. Pradhan

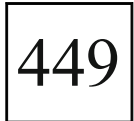

\section{ABSTRACT:}

The Radial artery has been used as a second arterial conduit for composite arterial grafting since the last 20 years. However, the deterring factors were the long and totally visible forearm incision and the propensity of spasm of the conduit using conventional clip and cautery method. To avoid these, we have been using a single $2 \mathrm{~cm}$ incision at the wrist (watchband) and with the use of endoscopic vessel harvesting instruments (Storz) and a harmonic scalpel, harvested the radial artery in the last 63 patients with this technique. 
The detailed technique with a video and tips and tricks for a successful harvest will be discussed in this presentation. We have encountered one instance requiring conversion to a two incision technique due to bleeding from the proximal radial after division, and one patient having a hematoma that was evacuated on day 2 through the wrist incision. There were no complaints of thumb paraesthesias or wound infections and the pain score for the incision and the forearm was between 0-3 (none to mild). We believe, this is an extremely desirable, cosmetically appealing and easy technique for harvesting specially as it does not need any insufflation of gas or application of a tourniquet and the conduit is delivered spasm free and in a ready to use state.

Continuous retrograde cardioplegia ensure safe minimally invasive aortic valve replacement through thoracotomy

Takashi Azami; Shunsuke Sato

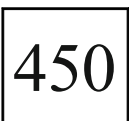

\section{ABSTRACT:}

OBJECTIVE:

Minimally invasive aortic valve replacement through thoracotomy (MICS AVR) has difficulties due to complication of selective cardioplegic perfusion and difficulty of tie-down of biological valve to aortic annulus. We present a safe approach of MICS AVR using continuous retrograde cardioplegia through anterior thoracotomy. Continuous retrograde cardioplegia allows excellent continuous homogenous cooling of the heart during the ischemic period. Anterior thoracotomy facilitates cannulation of ascending aorta, and allows our all manipulations using the fingertips without the aid of a knot pusher or long-shafted surgical instruments.

\section{METHOD:}

We entered thoracic space through anterior 3rd intercostal space with resection of 4th costal cartilage, and resection of 3rd costal cartilage if aortic cannulation was required.

Cardiopulmonary bypass was established with the femoral artery /or the ascending aorta, and the right femoral vein and inferior vena-cava. After clamping of ascending aorta, right atrium was opened and continuous retrograde cardioplegia was administered through a coronary sinus cannula.

We performed MICS AVR using this method in 5 patients between June 2018 and November 2018. There were 3 women. Median age of patients was 71 years (range: 43-77 years). Femoral artery cannulation was performed in 2 cases, and ascending aorta in 3 cases. Two patients required ascending aortic cannulation had shaggy descending aorta, and one patient had severe atherosclerosis obliterans. One patient required patch plasty of Valsalva sinus because of severe calcification on ascending aorta.

RESULTS:

There was no patient requiring defibrillation or extra weaning time after declamping of aorta. Knot-pusher was not required.

Extubation in operative theater was in 2 patients. Hospital stay was 8-14 (median 12) days after operation. There was no hospital death.

CONCLUSION:

MICS AVR using continuous retrograde caridoplegia is safe technique.

\author{
Minimally Invasive Mitral Valve Replacement in a \\ case of Marfan's Syndrome-Post Bentall's procedure \\ and Pectus Excavatum Reconstruction \\ Ratnamalika Kumar; Rajneesh Malhotra \\ Anuj Sangal
}

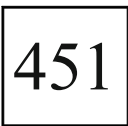

\section{ABSTRACT:}

We describe a rare interventional procedure in which a 30 -years gentleman, diagnosed case of Marfan's syndrome (post Bentall's procedure for dilated aortic root and surgically corrected Pectus excavatum 15 years back) who presented with mitral valve prolapse causing severe mitral regurgitation; underwent a Minimally invasive Mitral valve Replacement.
Minimally Invasive technique was opted in view of Redo procedure and right anterior thoracotomy incision was done through fourth intercostal space to avoid previous plates of pectus excavatum reconstruction.

In view of previous cardiac surgery and dense pericardial adhesions, the native mitral valve was distant and difficult to approach, hence "Cor-knots" were used to implant a 31/33 On-X metallic mitral valve prosthesis.

\section{Minimally Invasive Coronary Artery Bypass Grafting for Multi-vessel Coronary Artery Disease}

T. Periyasamy; Vivek Shrihari

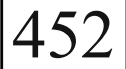

\section{ABSTRACT:}

INTRODUCTION:

Patients who presented to our hospital and underwent Minimally Invasive Coronary Artery Bypass Grafting for Multi-vessel Coronary Artery Disease (2 or 3 grafts) were included in this study.

\section{MATERIALS AND METHODS:}

The duration of this study was from November,2016 to October, 2018. It was a prospective randomized study.

All patients included in this study required 2 or 3 grafts for adequate revascularization.

The approach in all the surgeries was via a left anterior thoracotomy incision (left sub-mammary incision). Minimally invasive Cardiac Surgical instruments were used to perform the surgeries.

The data were analyzed using SPSS software.

RESULTS:

25 patients underwent Minimally Invasive Coronary Artery Bypass Grafting for Multi-vessel Coronary Artery Disease in which 2 or 3 vessels were grafted.

Follow-up assessment was done in the post-operative period in the Intensive Care Unit and later in the ward. Patients were also contacted later by telephone to assess the period required for return to activities of daily living and also the time period required for return to work.

\section{CONCLUSION:}

It was found that patients who underwent Minimally Invasive Coronary Artery Bypass Grafting for Multi-vessel Coronary Artery Disease had a shorter time to get discharged, had less blood loss, had less post-operative pain, required less time to return to their normal physical activities and had less incidence of wound infection as compared to conventional Coronary Artery Bypass Grafting Surgery.

Closure of atrial septal defect through back

Mahendra Bafana

ABSTRACT:

Atrial septal defect can be closed surgically by multiple approaches. We closed the ASDs in pediatrics age groups through the back. The anterior part of incision does not cross the right posterior axillary line. We report 75 cases done successfully. The weight of patient $\mathrm{s}$ range from $8 \mathrm{~kg}$ to $21 \mathrm{~kg}$. Advantage scar is never visible, in females does not hamper breath development. Summary Surgeons armamentarium to compete the device, easily reproducible, cost effective, no extra costly instruments required.

Local epicardial application of amiodarone-releasing hydrogel: experimental and clinical study results Vladimir Shvartz; Teymuraz Kanametov; Leo Bockeria

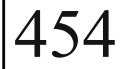

\section{OBJECTIVE:}

To evaluate the safety and efficacy of the local epicardial application of the hydrogel with amiodarone in the preclinical setting; to determine the optimal dose of amiodarone; to determine preliminary results of usage in clinical practice. 


\section{METHODS:}

Epicardial application of amiodarone-releasing hydrogel was performed on 46 rabbits (3-5 kg). There were 5 groups: $\mathrm{G} 1$ - dose of amiodarone in hydrogel $1 \mathrm{mg}$; G 2 - 3 mg; G 3 - 6 mg; G 4 - hydrogel without amiodarone; G 5 - amiodarone intravenously $60 \mathrm{mg}$. Pathomorphological study of the heart was performed on the 3-rd, 7-th and 14-th day.

\section{RESULTS:}

No systemic inflammatory response following the application of the amiodarone-releasing hydrogel was shown, as well as any significant change in the WBC and neutrophils level before and after the application $(\mathrm{p}=0.735)$. G 2 marked a significant decrease in the heart rate (before operation: $158 \pm$ 16 , after $130 \pm 11$, (p \&1t;0.001), without any conduction disorders. Significant conduction disorders (70\%) were in G 3 with an increased amiodarone dose and no effect on heart rate was observed in $\mathrm{G} 1$ with a decreased amiodarone dose. Hydrogel without amiodarone does not have any effect on the conduction system $(\mathrm{G} 4)$. The native medication nebulizer was tested in the trial as well.

\section{CONCLUSIONS:}

The experimental study has shown the overall safety and efficacy of amiodarone-releasing hydrogel. The optimal dose of amiodarone in the hydrogel was defined as $1 \mathrm{mg} / \mathrm{kg}$. The design of clinical trial has been created; the local ethical committee approval has been obtained as well. First 3 patients have shown no complications. The dose of $1 \mathrm{mg} / \mathrm{kg}$ was defined as an optimal for clinical study. In series of 3 patients no complications were detected. Clinical study is now carrying out.

Right atrial mass: Tumour or Thrombus?

Sunil Dhar; Siddharth Kunwar; Jagdish Prasad

\section{ABSTRACT:}

The prevalence of primary cardiac tumours is approximately $0.02 \%$. About $75 \%$ are benign, and $50 \%$ are myxomas, commonly occurring on the left side in particularly the left atrium. Although myomas are the most frequent primary tumours, right atrial is an atypical location leading to a diagnostic dilemma. A far more common cause of right atrial tumours are metastatic tumours that arise in the abdomen and pelvis, especially hepatoma and renal cell carcinoma. Secondary infiltrative tumours of the heart usually extend into the right atrium from the IVC and from the right atrial free wall.

We present a case of 42-year old woman, who presented to us with gradually worsening dyspnea, paroxysmal palpitation and several syncopal episodes. Echocardiography revealed a large, soft, hyperechoic mass present in an enlarged right atrium, collapsing in the right ventricle through the tricuspid valve. The patient underwent surgical excision of the mass on $\mathrm{CPB}$ via median sternotomy. The excised mass was pedunculated, mobile, lobulated, and necrotic-appearing. attached to the right atrial roof near the commissure of the anterior and septal leaflet of the tricuspid valve. There was no invasion of nearby structures.

Histopathological report of the mass showed hypocellular myxoid polypoid mass with multinucleated polygonal shaped cells in perivascular arrangement with finely vacuolated eosinophilic cytoplasm suggestive of the diagnosis of myxoma. A majority of myxomas are found to be attached to the interatrial septum, usually at the border of the fossa ovalis but in this case the myxoma was found to be attached by a thin stalk to the annulus of the tricuspid valve; an unusual site.

Though a rare location, a high index of suspicion of a myxoma is needed in a right atrial mass presenting with a variety of unexplained symptoms. The patient should be counselled regarding possible recurrence once diagnosis is confirmed.

\section{A study on the incidence and management of Heparin Induced Thrombocytopenia (HIT) post-cardiac surgery in a tertiary care hospital Sanjay O.P.}

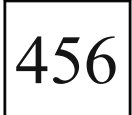

\section{INTRODUCTION:}

Heparin and cardiac surgery are inseparable. Heparin Induced Thrombocytopenia (HIT), though is a well-recognised complication of wide-spread heparin use post-cardiac surgery, has a global incidence of $0.5-5 \%$. There is paucity of domestic data on the incidence of HIT despite more than 60,000 cardiac surgeries being performed annually in India. Our study aims to bridge this lacuna. Apart from identifying patientrelated and procedure-related risks of developing HIT, we endeavour to establish management guidelines for HIT in South-East Asian population. MATERIALS AND METHODS:

Our study includes all patients who underwent cardiac surgery between January 2017 and December 2018 at our hospital. HIT was diagnosed in patients with a clinical suspicion of HIT and immunoassay (PaGIA method) positivity. Among these HIT-positive patients, we intend to prospectively study the impact of factors such as age, sex, race, usage of CPB, total dose of heparin, baseline/nadir platelet counts, pre-operative antiplatelet agent usage, transfusion requirements, HIT-related complications including HIT-T (HIT-Thrombosis) on morbidity indicators.

\section{RESULTS:}

Our study includes 13,685 patients who underwent cardiac surgery at our hospital between January 2017 and October 2018. Among 202 clinically suspected cases of HIT, we had 42 cases of immunologically-proven HIT. The overall incidence of HIT was $0.31 \%$. There were no clinically proven cases of HIT-T in our series. The data is being analysed statistically. We would like to discuss our institute's HIT management policy including suspicion index, multidisciplinary involvement, use of heparin alternatives, long term anticoagulation policies and novel strategies.

\section{CONCLUSION:}

Prompt recognition and effective multi-pronged management of HIT is key for positive patient outcomes despite major life/limb-threatening thrombotic complications associated with HIT-T. The relatively lower incidence and higher sero-positivity rates of clinically suspected patients with HIT should prompt any physician dealing with the cardiac surgical patient to consider HIT on top of the differentials' list in the diagnosis of thrombocytopenia in the post-operative period.

\section{"Atypical right atrial myxoma from atypical site with atypical presentation" \\ D.K. Barua}

\section{ABSTRACT:}

Primary cardiac tumours are rare with estimated incidence of $0.0017 \%$ $0.19 \% .75 \%$ of them are myxomas. $80 \%$ of myxomas arise in left atrium and only $15 \%-20 \%$ in right atrium. Myxomas usually arise from intra atrial septum. We present a rare case of right atrial myxoma from lateral wall of right atrium which presented to us with symptoms of superoir vena cava (SVC) syndrome and syncope.

50 years female presented to us with syncope and breathlessness. Patient was apparently alright one month back when she developed progressively increasing swelling over face, abdomen and lower limb. Her ECG was normal, x-ray showed features of pulmonary oedema. Echocardiogram showed large right atrial mass with LV dysfunction. Mass was occupying whole of right atrium. We decided to go for early surgery. Large right atrial mass of $7 \mathrm{cmx} 5 \mathrm{~cm} \times 3 \mathrm{~cm}$ was excised under cardiopulmonary bypass. Intra atrial septum was intact and mass was arising from lateral wall of right atrium from pectinate muscles. Patient was extubated next day, post op recovery was uneventful. Patient was discharged on 7th day. His histopathology confirmed myxoma.

\section{CONCLUSION:}

This case is a rare case as right atrial myxoma of such a big size arising from lateral wall of right atrium from pectinate muscles. Patient presenting with SVC syndrome, IVC obstruction, syncope and CCF right atrial mass should be ruled out. 
Surgical Treatment Of Cardiac Tumors : Our Experience

Kamlesh Jain

\section{ABSTRACT:}

Cardiac tumors are uncommon, mostly intracavitary includes both benign and malignant tumours. Here we summarize a retrospective study of intracardiac tumours at our centre in past 3 years from August 2015 to August 2018. Clinical histories, perioperative data, surgical findings, efficacy and follow-up data were reviewed in our study.

19 patients (Female - 13 and Male - 6) age between 1 year to 64 years were diagnosed with cardiac tumors in this period and all underwent surgical excision. Of the 19 patients 1 patient were diagnosed as having rhabdomyoma, 1 patient had metastatic secondaries and rest 17 were diagnosed as having myxoma on histopathology examination.

1 patient was known case of Tuberous sclerosis with rhabdomyoma, another presented with stroke and was diagnosed as having left ventricular mass. 1 patient with secondary metastasis presented with dyspnoea and were admitted with pulmonary embolism, underwent pulmonary thrombectomy. Pre operative diagnosis was done using $2 \mathrm{D}$ Echocardiography, MRI and CT pulmonary angiography in patient with pulmonary embolism.

All patient underwent surgical excision with stable post-operative hemodynamics. The most common postoperative complication was bleeding and infection. The early diagnosis and appropriate treatment are often curative, with very low risk of recurrence. Postoperative survival is high.

\section{A Retrospective analysis of Planned Versus Unplanned Mediastinal Reexplorations for Bleeding. Chaitanya Raut}

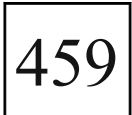

\section{ABSTRACT: \\ BACKGROUND:}

Mediastinal reexplorations are associated with significant morbidity and mortality.

\section{METHODS:}

100 patients were retrospectively analysed who underwent cardiac surgery via midline sternotomy and who required mediastinal reexploration for bleeding. No redo sternotomy cases were included in this study who had reexploration for bleeing. Reexplorations were classified as planned (temporary chest closure for a planned "second look") or unplanned (initial sternal closure and subsequent reexploration). The primary outcome was 30-day mortality. Secondary outcomes were hospital length of stay, duration of mechanical ventilation, and incidence of postoperative pneumonia and cardiac arrest.

\section{RESULTS:}

Among 100 patients who underwent reexplorations, 70 patients had planned $(70 \%)$ and 30 patients had unplanned $(30 \%)$ mediastinal reexplorations. The operative mortality rates were ( $37 \%$ vs $37 \% ; p=1.00$ ) between unplanned and planned reexplorations. There were no differences in rates of deep sternal wound infection, renal failure, postoperative hospital length of stay, pneumonia, or cardiac arrest, with the exception of a higher rate of prolonged intubation (93\% vs 53\%; p \& $1 \mathrm{t} ; 0.01$ ) in the planned reexploration group.

CONCLUSIONS:

Delayed sternal closure is a safe alternative to initial definitive chest closure when chances of postoperative bleeding is expected.

ECMO as salvage therapy in Aluminium phosphide poisoning: Our experience and review of literature Neerav Bansal

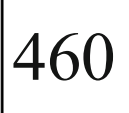

\section{ABSTRACT:}

INTRODUCTION:

Aluminium phosphide (Alp) is an effective and cheap pesticide commonly used worldwide. The active ingredient - phosphine gas is highly lethal.
It carries high rate of mortality due to refractory myocardial depression, severe metabolic acidosis and acute respiratory distress syndrome. Extracorporeal membrane oxygenation (ECMO) is an effective method of providing temporary cardio-respiratory support and thus we evaluated its use in subset of patients presenting with such history.

\section{CASE REPORTS}

Between March 2017 and September 2018, four patients (three males and one female) with acute Alp poisoning presented to our hospital emergency. Patients aged 21, 34, 56 and 63 years, with history of Alp intake between 30 minutes to 6 hours. All patients had severe metabolic and lactate acidosis and refractory cardiogenic shock with severe left ventricular dysfunction (\&lt;25\%). All received veno-arterial ECMO support within 30 minutes of admission; however one of them could not survive despite the treatment. The mortality observed was in patient who had all the adverse denominators: he was most elderly, had presented very late, and had worst lactic acidosis

\section{CONCLUSION:}

Veno-arterial ECMO has shown to improve survival in patients with acute Alp poisoning by providing adequate hemodynamic support in absence of any proven antidote or treatment modality. Delay in presentation was found to be the single most important factor influencing survival in patients with no previous history of any cardio-respiratory illness.

\section{Prophylactic Topical Mupirocin in prevention of SSI} after sternotomy

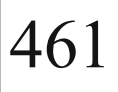

Biju Sivam Pillai

\section{ABSTRACT: \\ BACKGROUND:}

Sternal wound infection is one of the dreaded complications after cardiac surgery. Various topical agents have been assessed from time to time for peri-operative skin preparation. Our team evaluated the efficacy of topical mupirocin versus standard betadine ointment in 1000 consecutive patients undergoing open-sternal procedures.

\section{METHODS:}

A retrospective review of all coronary artery bypass graft and valve surgery cases performed at a single institution between 2016 and 2018 was performed (n [1,012). Appropriate preoperative intravenous antibiotics were administered for all patients. Patients were randomized into two groups based on a random number generator into the betadine and Mupirocin group. Mupirocin topical antibiotic ointment was routinely applied to the sternal surgical incision after skin closure for all patients during this period. The incidence of sternal wound infection was assessed.

\section{RESULTS:}

During this 3-year experience, no episodes of deep sternal wound infections were observed in mupirocin group, compared with a rate of $0.33 \%$ in the Betadine subgroup. The world-wide average risk based on The Society of Thoracic Surgeons National Database risk calculator was $0.28 \% .2$ episodes of superficial sternal wound infections were noted in the betadine sub-group; gram-positive organisms were cultured in these cases. Mupirocin ointment was well tolerated by patients, with no serious adverse effects reported.

\section{CONCLUSIONS:}

Mupirocin is relatively low cost adjunct that can be added to standard surgical care of surgical skin incision after cardiac surgery.

Our experience in patients requiring cardiac surgery with collagen vascular diseases

Vijit K. Cherian

ABSTRACT:

Collagen vascular diseases have extensive cardiovascular menifestations because of ubiquitous and crucial function they serve in body. Connective 
tissue supports of the heart and vascular structures play an integral role in normal cardiovascular performance and connective tissue disorders produce important physiological processes that affect cardiac and vascular elements. With increasing incidence of cardiovascular menifestations of Collagen vascular diseases we sought to study the extent of disease, imaging modalities, investigational modifications, perioperative morbidity and mortality and post operative complications in such patients to achieve better understanding of disease process and improve post operative outcomes.

AIMS:

The primary aim is to understand the extent of cardiovascular involvement in collagen vascular diseases, optimise the medical management, diagnostic modatilies, prognostic implications, to reduce perioperative morbidity and mortality and prevent post operative complications.

Scimitar syndrome variant (meandering pulmonary vein) Amaresh Rao M.

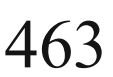

\section{ABSTRACT: \\ INTRODUCTION:}

Scimitar syndrome is a rare congenital anomaly with wide clinical spectrum governed by age of presentation. In infants it presents with cyanosis, poor growth, PAH and with other cardiac defects. In adults it generally presents with recurrent chest infection and hemoptysis. Here we present adult women with hemoptysis whose symptoms relieved from surgical correction of the defect.

\section{CASE HISTORY:}

A 22 year female c/o of hemoptysis, shortness of breath, shivering and cold extremities, pain in right hypochondrium. O/E pulse rate- 108/min, blood pressure $-100 / 70 \mathrm{~mm}$ of $\mathrm{Hg}$, respiratory rate- $26 / \mathrm{min}$ with decreased sounds on right side.

CT angiogram: Found to have partial anomalous pulmonary venous connection of right inferior pulmonary vein with dual drainage into left atrium and infra diaphragmatic inferior venacava scimitar variant meandering pulmonary vein with no other features of scimitar syndrome.

\section{SURGERY:}

Right anterolateral thoracotomy done. right inferior pulmonary vein identified which is arising from the lung parenchyma and piercing diaphragm. 2-3 collaterals branches present along with right inferior pulmonary vein. All branches draining into inferior venacava are ligated inferiorly and superiorly and divided. Hemostasis secured anterior and posterior drains kept. wound closed in layers.

\section{POSTOPERATIVE PERIOD:}

Patient recovered well. Discharged on 6th postoperative day in stable condition.

\section{CONCLUSION:}

Surgical correction is to be considered in the presence of significant right to left shunt and pulmonary hypertension. The anomalous vein can be directly reimplanted into the left atrium. Alternatively in meandering pulmonary vein the vein draining into inferior venacava is ligated to divert the flow into the left atrium.

One year experience of a new cardiac surgery unit - An untold story

Shahriar Moinuddin

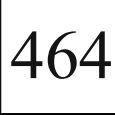

\section{ABSTRACT:}

In a developing country like Bangladesh, we have to face many challenges to start a new cardiac surgery unit as a young surgeon. To achieve patients trust for a junior most surgeon to perform as a chief surgeon in a new unit is the most vital factor, others are a shortage of an adequate number of trained manpower, lack of adequate infrastructure (hospitals), lack of adequate training facilities, lack of modern surgical instruments etc. After starting our journey on 9th August of 2017, we have done 110 cases successfully with minimal complications. We believe we can do much better in future with the available resources, within an affordable cost to serve the people of our country.

Coronary cameral fistula: a rare entity

Jignesh Kothari

ABSTRACT:

OBJECTIVES:

The purpose of this study was to describe our experience with the presentation and management of surgical repair of coronary cameral fistula (CCF) and the outcomes.

\section{PATIENTS AND METHODS:}

We performed a retrospective study from January 2015 to January 2018 in 8 patients operated for coronary cameral fistula. There were 5 men and 3 women; the age range was $18-55$ years (mean age, 36.2 \pm 11.7 years). Among the 8 patients, 4 patient had CCF originating from Right coronary artery, 2 patients had from Left anterior descending artery and 2 patients had its origin from Left circumflex artery. All patients were evaluated by trans-thoracic echocardiography and conventional coronary angiography apart from routine biochemistry and hematological investigations.

\section{RESULTS:}

All operations were performed using cardiopulmonary bypass. Four patients with a CCF opening into RA and RV which was closed by right atriotomy, 2 patients had CCF at termination of coronary artery hence ligation done and 2 patients had CCF with dilated coronary artery amogst them dilated portion exposed, closed fistulous tract followed by arteriotomy closure. There were no postoperative complications. No recurrences have been observed so far in follow up visits.

\section{CONCLUSIONS:}

Coronary cameral fistula is an infrequently encountered entity, but with clinical suspicion, early diagnosis and timely intervention it can be successfully managed with excellent outcomes.

Relationship between High Red Cell Distribution Width and Systemic Inflammatory Response Syndrome after Extracorporeal Circulation Chaitanya Raut

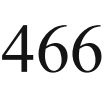

\section{ABSTRACT: \\ OBJECTIVE:}

Cardiac surgical operations involving extracorporeal circulation may develop severe inflammatory response. This severe inflammatory response syndrome (SIRS) is usually associated with poor outcome with no predictive marker. Red cell distribution width (RDW) is a routine hematological marker with a role in inflammation. We aim to determine the relationship between RDW and SIRS through our study.

\section{METHODS:}

A total of 1250 patients who underwent cardiac surgery with extracorporeal circulation were retrospectively analyzed out of which 26 fell into the SIRS criteria and 26 consecutive control patients were taken. RDW, preoperative clinical data, operative time and postoperative data were compared between SIRS and control groups.

\section{RESULTS:}

The demographic profile of the patients was similar. RDW was significantly higher in the SIRS versus control group ( $15.5 \pm 2.0$ vs. $13.03 \pm 1.90)$, respectively with $\mathrm{P}$ value \&lt; 0.0001 . There was significant mortality in the SIRS group, $20(76.92 \%)$ as compared to 2 (7.6\%) in control group with a $P$ value of \&lt;0.005.

Multiple logistic regression analysis revealed that there was significant association with high RDW and development of SIRS after 
extracorporeal circulation (OR for RDW levels exceeding $13.5 \%$; $95 \% \mathrm{CI}$ 1.0-1.2; P\&lt;0.05).

\section{CONCLUSION:}

Increased RDW was significantly associated with increased risk of SIRS after extracorporeal circulation. Thus, RDW can act as a useful tool to predict SIRS in patients undergoing cardiac surgery with extracorporeal circulation. Hence, more aggressive measures can be taken in patients with high RDW to prevent postoperative morbidity and mortality.

\section{Advantages of early re-exploration for post operative hemorrhage following cardiac surgery Subhankar Bhattacharya}

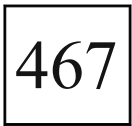

\begin{abstract}
:
Surgical re-exploration due to postoperative bleeding still has a frequent incidence, and is accompanied by various subsequent complications leading to an impairment of the postoperative course and increased morality. Various studies have included early re-exploration for bleeding as a risk factor in analyzing adverse outcomes after cardiac operations. A prospective study was done for a period of 22 months, which included all post operative cardiac patients who underwent re-exploration for mediastinal hemorrhage. A total of 74 patients were part of the study population, with a mean age of 40.9 years and male to female ratio of $2.5: 1$. The study showed that those patients who underwent re-exploration within 12 hours of surgery required statistically significant less transfusion. Among those who were re-explored after 12 hours, half of them required massive transfusions. Similarly, statistically significant difference was shown in inotrope requirement based on the timing of re-exploration, wherein, $91.6 \%$ of patients with delayed re-exploration required prolonged inotropic support. It was also shown that patients undergoing early re-exploration required less blood products viz. FFP\&amp;PLC (20\% v/s 41.7\%). Finally, mortality in patients undergoing early re-exploration was $12 \%$ as opposed to $41.7 \%$ in those re-explored late. Hence, to conclude, we can say that timing of reexploration is an important, but often neglected parameter affecting the patient's outcome. We should have a low threshold for re-exploration for better post-operative outcomes.
\end{abstract}

\author{
A Decade's Analysis on Primary Cardiac Tumours from \\ a Tier II City \\ A.R. Raghuram
}

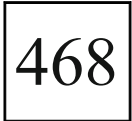

\section{ABSTRACT: INTRODUCTION:}

Tumours of the heart are extremely rare entities with a prevelance of about $0.001-0.03 \%$. They are usually metastatic in origin. Primary cardiac tumours are mostly benign in nature, with malignant tumours accounting for $25 \%$ of cases. They have varied presentations and are usually present during the 4th to 6th decade of life. Echocardiogram is the primary modality of diagnosis. Early surgical intervention has proven to have favourable outcome. We describe our experience with managing cardiac tumours.

\section{PATIENTS AND METHODS:}

A Retrospective analysis of cases presented to us between September 2008 and September 2018. We came across 21 patients. All patients were managed with the same protocol. They underwent surgical excision of the mass under cardiopulmonary bypass. Mean follow up period range from 5 months to 10 years. Case records were analysed and data collected for results given below.

\section{RESULTS:}

Atrial Myxoma was the most common primary neoplasm of the heart (90.5\%). Left atrium was most common site of attachement (85.7\%). The mean age at the time of presentation was 50.9 years. Males and Females were more or less equally affected in our study. Even though patients presented with a wide spectrum of symptoms, Dyspnoea (57.2\%) was overall the most common mode of presentation. A good number of patients (33.3\%) developed complications due to mass before undergoing surgery. Complications were seen more in patients who delayed surgery due to various reasons. Another interesting observation was that most patients were from Tirunelveli district (57.1\%). However more research is needed to substantiate this observation. Out of the 21 patients, 2 patients were diagnosed to have extensive and metastatic disease. They succumb to their primary illness a year after surgical resection. All other patients are asymptomatic and are on follow up. No recurrence has been reported in our study so far.

\section{Primary Chest Packing and Delayed Sternal Closure: Our Experiences \\ Allen James; Ross Mejia; Taranpreet Singh; Peng Seah}

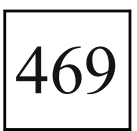

\section{ABSTRACT:}

Intractable bleeding after cardiac surgery remains a cause of high mortality and morbidity globally. Primary chest packing and delayed sternal closure, although examined positively in a dozen or so observational studies, is approached with caution by the vast majority of cardiac surgeons. We present our experiences with primary chest packing and delayed sternal closure, looking at end points of superficial/deep sternal wound infections, 're-openings' in ICU and overall mortality; along with comparisons to global literature.

Various uses of Harmonic Scalpel in cardiac surgery. Devapriya Marik

\section{INTRODUCTION:}

Harmonic scalpel provides simultaneous incision and coagulation by transfer of mechanical and thermal energy to tissues. It has been used to harvest coronary bypass grafts, in skeletonized and non skeletonized fashion. It can be used to locate and dig out intramyocardial coronary arteries. It has also been used to release adhesions during redo cardiac surgery and doing pericardiectomy.

\section{MATERIAL AND METHODS:}

Short edited video presentation of various uses of Harmonic scalpel in cardiac surgery will be presented.

Harvesting of skeletonized internal mammary arteries.

Harvesting of skeletonized and non skeletonized Radial arteries.

Locating intramyocardial coronary arteries.

Entire pericardiectomy.

\section{RESULTS:}

To show the technical feasibility of using this very useful tool in the various situations in cardiac surgery.

Clinical outcomes of redo-cardiovascular surgery Hiroshi Tsuneyoshi; Chikara Ueki; Ken Yamanaka; Yuta Kitagata

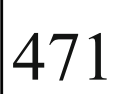

\section{ABSTRACT:}

\section{BACKGROUND:}

As surgical techniques advance, cases of redo-cardiovascular surgery have been increasing. We reviewed cases of redo-surgery in our hospital. METHOD:

From April 2013 to August 2018, 68 patients underwent redo-surgery after past median sternotomy.

We assessed re-entry risk by CT scan. We exposed access artery before resternotomy in case of middle risk. In high risk case, cardiopulmonary bypass (CPB) was established before resternotomy. As one of reference, we measured RV branch movement distance using 4D-CT. It was measured horizontal distance on a plane perpendicular to RV branch. 
Patients were $65.3 \pm 16.3$ years old. There were 40 cases of valvular disease (60.3\%), 4 CABGs (5.9\%), 17 aortic surgeries (25.0\%), and 6 congenital cases $(8.8 \%)$. In the preoperative 4D-CT, RV branch movement tended to large in case pericardium was closed at previous surgery. Before resternotomy, we exposed femoral artery in 15 patients $(25.9 \%)$, and established CPB in 2 cases (2.9\%). At the time of resternotomy, RCA was injured in one case, and pseudoaneurysm was ruptured in another case. But there was no other problem in both case because CPB was already established.

In the postoperative course, hospital mortality was $2.9 \%$ (2case). There were 3 cases of cerebral infarction $(4.4 \%), 3$ cases of reoperation for bleeding (4.4\%), and 4 cases of tracheotomy $(5.8 \%)$. Long-term mortality (mean follow-up 18.9 months) was $13.2 \%$ (9 cases).

CONCLUSION:

Although redo-cardiovascular surgery is always challenging, meticulous planning and managements could reduce the risk of redo-surgery.

Re Exploration in the Intensive Care Unit After Cardiac Surgery, PSGIMSR Experience

Murugan M.S.; Krishnanand Pai; Ganesan C.

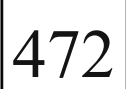

\section{ABSTRACT: \\ BACKGROUND:}

Cardiac surgical re-exploration is necessary in approximally $5 \%$ of all cardiac surgical procedures. The impact of unplanned re-exploration at cardiac surgical intensive care unit remains ill defined. This study evaluate the outcome of such procedures.

MATERIALS AND METHODS:

At PSGIMSR, Coimbatore from 2000 to 2018

All patient who underwent unplanned re-exploration at Cardiac surgical ICU were included in this study. Out of 210 re-exploration of 5000 Open Heart surgeries, 7 ( Male : Female - 5:2 ) were re-explored in the cardiac surgical ICU as an unplanned extreme emergency. Indication: Sudden crash massive bleeding : 2, tamponade : 3, Lethal ventricular arrhythmia : 1 , and impending cardiac arrest $: 1$. All required internal cardiac massage.

RESULTS:

Out of 7, 2 could not be salvaged because of Atrio ventricular disruption ans exanguination of Post MVR status.

Remaining 5, 1 required IABP support.

The post operative recovery of these 5 was uneventful.

No significant infection or sternal wound complication.

Series of un noticed hepatic vein draining into left atrium in post operative atrial septal defect patients Periyasamy

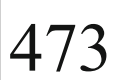

\section{ABSTRACT:}

\section{INTRODUCTION:}

Un noticed hepatic vein draining into left atrium

\section{METERIALS AND METHOD:}

Case series of 6 patients presented with cyanosis after 4 to 6 years post atrial septal defect closure, on investigation it was diagnosed as un noticed hepatic vein was draining into left atrium

RESULTS:

All six patients were re operated improved and discharged

CONCLUSION:

patient presenting with cyanosis post atrial septal defect un noticed hepatic vein draining into left atrium should be ruled out.

\section{INTRAVENOUS LEIOMYOMATOSIS WITH IVC AND INTRA CARDIC EXTENSION - A RARE PHENOMENON}

Dinesh Chandra

\section{Abstract:}

Enter description he

Uterine leiomyoma is a common benign disease in women, however intravenous leiomyomatosis of uterine origin extending into IVC, right atrium (RA), right ventricle (RV) and main pulmonary artery is a rare clinical entity. Such unusual presentation of a common disease poses a diagnostic challenge in the field of cardiac surgery.

A 33 year old lady with uterine fibroid, presented with complaints of breathlessness and palpitation on exertion. Computed tomography pulmonary angiography (CTPA) showed thrombus in main pulmonary artery, RA and RV. Patient was managed with thrombolysis without any resolution of symptoms, so open resection was planned. The resected mass was sent for histopathology that showed leiomyoma with hyaline degeneration. A two stage surgery was performed for complete resection of tumor involving multidisciplinary team approach. The patient was discharged from hospital in improved health state.

Intravenous leiomyoma is rare entity as such the diagnosis seeks a watchful mind, multimodality team and multistage resection.

Atrial myxomas with varied manifestations- a single Institute experience

Pares Bandyopadhyay

\section{ABSTRACT:}

\section{INTRODUCTION:}

Atrial myxomas are the most common benign primary tumor of the heart and account for at least $30 \%$ to $50 \%$ of benign tumors. Despite significant advances in cardiac diagnostics leading to early recognition of myxomas, the potential for deleterious effects secondary to embolic complications remains high. They may present with a wide variety of symptoms, majority present with embolic and neurological symptoms. Hematological manifestations and thrombocytopenia are also described. These tumors can result in high patient morbidity and mortality. They mainly seen in the left arrium often attached to the atrial septum but can occur in the right atrium and may be multiple as described in familial myxoma syndromes. We have retrospectively studied these cases operated in our institute.

\section{METHODS:}

A total of 10 consecutive patients atrial myxomas were operated in our Institute of N R S Medical College and Hospital in the Dept of Cardiothoracic and Vascular surgery from 2010 to 2018. These cases were retrospectively studied.

\section{RESULTS:}

We had a total of 10 cases of which 7 were male and 3 were female patients. Right atrial myxomas were 3 cases and all cases had Ideopathic Thrombocytopenic Purpura. Seven cases had Left atrial myxomas of which five cases had neurogenic manifestations apart from dyspnoea, All cases showed relief of symptomps after surgery and there were no mortality in our series.

\section{CONCLUSIONS:}

Atrial myxomas are rare forms of Cardiac disease. The presentations are also diverse. More importantly early diagnosis and management can cure these cases with minimal morbidity. Right atrial myxomas appear to be commonly associated with hematologic manifestations like thrombocytopenia.

Successful excision of recurrent plunging mediastinal liposarcoma Sreevathsa
ABSTRACT: BACKGROUND: 
Primary mediastinal liposarcomas are a rarity on their own (less than $1 \%$ of mediastinal tumors) but plunging reccurent liposarcomas of mediastinum are still rarer. We report a case of plunging mediastinal liposarcoma successfully managed by complete surgical excision.

\section{CASE REPORT:}

Recurrent neck swelling since last 22 yrs, operated suspecting thyroid swelling 20 yrs back, reoperated for same swelling in 2007. Now recurrence of swelling in the neck. No pain or dysphagia, dysphonia. On examination $10 \times 5 \mathrm{~cm}$ bilobulated swelling in neck with smooth surface. CT: Large lipoma arising in the left side of the neck as high submandibular region and extending into the mediastinum behind the innominate vessels, aorta reaching till carina. APPROACH:

Cervical +sternotomy

FINDINGS:

Lipoma arising in the left side of the neck from submandibular region to the mediastinum behind the innominate vessels, aorta reaching till carina. PULMONARY ARTERY, AORTA displaced by the tumour. Right pleural adhesions.

\section{PROCEDURE}

Horizontal neck incision was given. Subplatysmal dissection was done and strap muscles were divided. Lipoma was identified. Tissue was sent for frozen section, reported as benign tumour, Lipoma. Cervical part of the lipoma was freed from surrounding structures except for the part plunging into the mediastinum. Median sternotomy was done for access to the mediastinal part of the lipoma. Mediastinal part was dissected off from surrounding structures and was pulled into the neck. Tumour was excised. he was found to have left vocal cord palsy and adviced speech thearapy.

Biopsy - well differentiated liposarcoma, margins are free of tumor

Discussion:

Liposarcomas are tumors of adipose tissue from mesenchymal origin with malignant potential. Well differentiated liposarcomas (ATYPICAL LIPOMATOUS TUMORS) are not associated with metastasis. Complete surgical excision is the mainstay of treatment. In our case previous two excisions done were possibly incomplete and tumor reccured. Post operative CT SCAN showed no evidence of reccurence after 6 months.

\section{HYDATID CYST OF TRICUSPID VALVE, A RARE PRESENTATION}

Dinesh Chandra

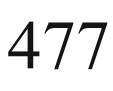

\section{ABSTRACT:}

Hydatid cyst is a common disease in human being, the organ affected in order of frequency, as follow, liver, lung, central nervous system and heart. Cardiac involement is . 5 to $2 \%$. In heart most common location is left ventricle $(46 \%)$, followed by rt ventricle(21\%), intraventricular septum(19\%), rt atrium $(9.7 \%)$, left atrium and sinus of valsalva( $1.6 \%)$.

This is a case presentation of 60 years male diagnosed case of hydatid cyst of lung and aortic regurgitation. In CT chest incidental finding was non enhancing filling defect in RV. Intraoperative RA was open and cystic lesion was found in tricuspid valve which was resected, histopathology proven the diagnosis of hydatid cyst.

Primary calcified myxoma tumor of the Superior Vena Cava extending into the Right atrium masquerading as RA myxoma - a case report C.S. Hiremath

\section{ABSTRACT:}

\section{INTRODUCTION:}

Primary tumor arising from the SVC is very rare. Here, we present a case of SVC tumor extending into RA treated surgically successfully. CASE:
A 49 years old female patient presented with dyspnoea and atypical chest pain since 2 years. Her vital parameters were within normal limits. ECG showed heart rate of $80 / \mathrm{min}$ in sinus rhythm with RV strain pattern. CXR showed mild cardiomegaly. ECHO showed RA myxoma of size $4 \times 3 \mathrm{~cm}$. Contrast CT pulmonary angiogram showed RA tumor with chronic calcified thrombus in bilateral lower lobar arteries with features of early pulmonary arterial hypertension. Coronary angiogram revealed normal coronaries.

Intraoperatively, there was a tumor stalk arising from the posteromedial aspect of upper third of SVC which is extending into the RA and of size $9 \times 4 \times 3 \mathrm{~cm}$ and was fully calcified. The tumor was excised under CPB with deep hypothermia of 18 degrees with aortic and right IJV, innominate vein and IVC cannulae. After tumor excision, SVC was reconstructed and proximal RA augmentation with untreated pericardial patch. Postoperatively, patient had features of right lower lobe collapse with features of PAH which was treated conservatively and settled over a period of two weeks.

Histopathology report showed features of degenerated myxoma with dystrophic calcification with bone formation.

CONCLUSION:

Secondary SVC obstruction causing SVC syndrome is common. Primary calcified myxoma SVC tumor extending into RA with features of PAH is rarely reported and was treated surgically successfully.

\section{An Adult Patient with Simple Aortic} Coarctation Associated with a Collateral Aneurysm who Underwent Staged Endovascular Therapy Shunichirou Fujioka; Toshiaki Mishima; Yuta Murai; Hirotaka Ishido; Haruna Araki; Takuma Fukunishi; Nobuyuki Inoue; Tadashi Kitamura; Takashi Miyamoto; Shinzou Trii; Kagami Miyaji Hirotoki Ohkubo

\section{ABSTRACT: BACKGROUND:}

Aortic coarctation is a congenital disease. Most adults with this disease have already been treated, but previously untreated patients who have simple aortic coarctation are rarely encountered. In high-risk patients, endovascular therapy with no influence on curative surgery can be selected as initial treatment.

CASE:

The patient was a 42-year-old woman with meningitis and mental retardation. The patient had right chest discomfort and intermittent claudication. CT angiography revealed that the distal portion of the aorta was focally narrowed to $5 \mathrm{~mm}$. In addition, an aneurysm, $(20 \mathrm{~mm} \times 15 \mathrm{~mm})$ was found in the prevertebral anastomosis between a branch of the right vertebral artery and the right sixth intercostal artery. Aneurysmal embolization was initially performed because the patient had right chest discomfort, and second-stage surgery was scheduled for to dilate the coarctation site. Endovascular therapy was performed to treat the aortic coarctation. The pressure gradient was measured and found to be $52 \mathrm{mmHg}$. An Omnilink Elite ${ }^{\circledR}$ Vascular Balloon-Expandable Stent $(8 \mathrm{~mm} \times 19 \mathrm{~mm})$ was placed in the site to dilate the artery. The pressure gradient was measured again and was found to be $36 \mathrm{mmHg}$. The risks and benefits were taken into account, and treatment was terminated without performing additional dilation.

\section{DISCUSSION:}

Endovascular therapy is less invasive than surgery and is considered useful. Excessive dilation carries the risk of vascular injury and postoperative aneurysm formation. Therefore, to safely dilate the coarctation site, factors such as the pressure gradient should be considered when performing the procedure.

\section{CONCLUSION:}

We described our experience with an adult patient with simple aortic coarctation who underwent endovascular therapy. 
Role of liberal primary Fasciotomy in traumatic vascular injury.

G.H. Nabi Lone

\section{ABSTRACT: \\ BACKGROUND:}

Vascular injuries represents less than $1 \%$ of all injuries. but deserves special attention because of its severe complications like amputation or retention of painful function less limb. thus vascular injury needs a judicious and multidimesional approach.

\section{OBJECTIVE:}

The study was done to asses the outcome of minor modifications of methodology of extremity Fasciotomy by making it liberal with respect to time and length of incision

MATERIAL AND METHODS:

We divided patients in two group.

Group A. ( Had no Fasciotomy or limited)

Group B( Had primary liberal Fasciotomy).

Total of 55patients out of which 45 patients had either no Fasciotomy or limited 45 patients had ( group B) had primary liberal Fasciotomy.

Results: in group A we had 5amputations and one death. in group B there were no amputation or death.

\section{CONCLUSION:}

Blunt and distal traumatic vascular injury of extremities and its repair should always combined with primary liberal Fasciotomy which although increases manageable morbidity however avoids disability functional as well as anatomical.

Clinical study of deep venous thrombosis

Dasari Kalyani Rama

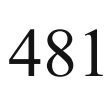

\section{ABSTRACT:}

Title: Clinical study of deep venous thrombosis

Author:DR.D.Kalyani Rama. MS Mch (CVTS), Department of CT Surgery, GGH-Guntur

\section{INTRODUCTION:}

Deep vein thrombosis (DVT) is the formation of thrombi in the deep veins such as the calf veins, femoral vein, or popliteal vein) or the deep veins of pelvis.

MATERIAL \&amp; METHODS:

50 patients with DVT were admitted to our hospital between nov 2017 and oct 2018. The mainstay of treatment is anticoagulant therapy. Lowmolecular-weight heparin, unfractionated heparin, and vitamin $\mathrm{K}$ antagonists, compression stockings.

\section{RESULTS:}

All were treated with therapeutic dose of UFH or LMWH, or with fondaparinux to achieve a critical therapeutic ratio within the first 24 hours of treatment achieving an activated partial thromboplastin time (aPTT) that is 1.5 times the mean of control value or the upper limit of normal aPTT range (aPTT ratio) of 1.5 to 2.5 .

No hospital mortality. Average stay is 10 days.

\section{CONCLUSION:}

The goal of therapy for DVT is to prevent the extension of thrombus, acute PE, recurrence of thrombosis, and the development of late complication such as pulmonary hypertension and post-thrombotic syndrome.

Comparison of surgical versus medical management of acute and chronic limb ischemia: a prospective study Sachin Talwar

\section{ABSTRACT:}

\section{BACKGROUND:}

During the course of management of limb ischemia, there is need of embolectomy some times. The patient responded well to medical management too. This study is intended to study the timing of surgical intervention, complications related to it as well as advantage and disadvantage of both.

\section{PATIENTS AND METHODS:}

Bewteen July 2017 to September 2018, 200 consecutive patients undergoing treatment for limb ischemia were included in this prospective cohort study. All the preoperative and postoperative parameters were noted in the described format including age of patient, duration of disease, NYHA status, rhythm, cardiac illness etc. Simultaneously a note was made of all the patients pre-operative characteristics, aetiology, associated cardiac anomalies, echocardiogram findings, cardiac catheterization and angiography data (if performed), intra operative details, Comparison of medical versus surgical intervention and its outcome, post operative course, follow up and follow-up echocardiographic data.

\section{RESULTS:}

Two hundred patients of limb ischemia \{acute/chronic\} were included in this study. They reported to our hospital between July 2017- September 2018. We selectively divided the patient into two group, suitable for each category. It was found that the patient of both the category responded well.

\section{CONCLUSIONS:}

Timely intervention is the key factor for success, both the group responded well to therapy. No gross differences between outcomes following medical or surgical intervention in the respective category patient. Though surgical intervention has risk of reperfusion injury.

Surgery for pseudoaneurysm in intravenous drug abusers:our experience I. Mir

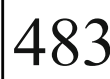

\section{ABSTRACT: BACKGROUND:}

Pseudoaneurysm formation is a serious life threatening complication commonly seen in intravenous drug abusers. Surgical options are often limited by severe local sepsis, abscess formation and/or gangrenous changes in the distal limb.

\section{MATERIAL AND METHODS:}

Since October 2014, out of 18 patients presenting with peripheral artery pseudoanerysms secondary to i.v drug abuse, 14 patients had local abscess formation and 2 patients had gangrene of the distal limb on presentation. Two patients presented with ruptured pseudoaneuryms with hemorrhagic shock.

\section{RESULTS:}

All the patients underwent excision of the pseudoaneurysm and ligation of femoral artery. Revascularization was done in 16 patients in the same sitting. Amputation was done in 2 patients who had gangrenous changes on presentation. There was no in-hospital mortality.

\section{CONCLUSION:}

Excision along with revascularization is safe and effective approach in the management of femoral artery pseudoaneurysms.

Single stage thoracotomy approach : simultaneous coronary artery bypass grafting and the descending aorta to bifemoral bypass

Sudhir Adalti

\section{ABSTRACT:}

\section{INTRODUCTION:}

Coronary artery disease and peripheral arterial occlussive disease frequently coexist. Concomitant revascularization procedures may be required because harvest of the internal thoracic artery (ITA) in patients with PAOD carries a risk of leg ischemia. For single stage approach, median sternotomy incision is most commonly used. This study reports our experience with combined coronary and femoral revascularization using the descending aorta to bifemoral bypass through Left posterolateral thoracotomy. 


\section{METHODOLOGY:}

A single centre prospective study from July 2016 to January 2018, 23 patients who have underwent single stage thoracotomy revasculaisation of peripheral (lower extremity)and coronary vessels were investigated and analysed. In operative techniques Left Postero-lateral Thoracotomy incision, Saphenous vein graft used for both single and double vessel coronary artery disease patients. Proximal source of vein graft anastomosed to descending thoracic aorta. Peripheral Revascularisation achieved by using Ringed PTFE grafts.

RESULT:

21 patients were recorded to be asymtomatic postoperatively with biphasic flow on colour arterial doppler and no fresh changes on ECG and 2D Echo. The number of bypass grafts were $1.4+/-0.6$. Average duration of surgery was 2.o-2.5 hours and blood loss was around $100-400 \mathrm{~mL}$. Operative mortality of 2 patients due to non surgical causes were recorded.

\section{CONCLUSION:}

The descending aorta is a good source of inflow to femoral arteries and the descending aorta to bifemoral bypass did not require an intraperitoneal and sternotomy procedure. Therefore the simultaneous operation can be performed in shorter time, and it is an interesting alternative in cases with ischemic heart disease and leg ischemia.

\section{Aorto popliteal bypass: A limb salvage technique for critical ischaemia. \\ Meghna Kinjalk}

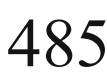

\section{ABSTRACT: \\ INTRODUCTION:}

Concurrent iliac and femoral arterial occlusive disease disease with critical ischaemia has traditionally been treated with an aortobifemoral bypass.

\section{BACKGROUND:}

With unilateral iliac artery occlusive disease a majority of patients undergo endovascular stenting with good outcomes. If stenting is not feasible, the surgical treatment options are ipailateral or contralateral iliofemoral bypass, femorofemoral bypass or axillofemoral bypass.

If the distal revascularisation by the profunda femoris arterial collaterals are inadequate, a further outflow procedure is required. In such situations with extensive iliac, common femoral and superficial femoral arterial occlusive disease with inadequate distal outflow, an aorto popliteal bypass can be an alternative to amputation and can provide a solution and relief to these aggrieved patients.

\section{CASE REPORT:}

A 42 year male, smoker, presented with acute on chronic left lower limb ischaemia with rest pain and non healing ulcers. Patient had no other comorbidities. CT angiography showed thrombus from the origin of the common iliac to the popliteal artery. Below knee popliteal artery was reconstituted by collaterals with 3 vessel run off to the ankle. Doppler ultrasound showed trickle flow in the crural vessels and complete occlusion from iliac to popliteal artery. CT angiogram confirmed the doppler findings and showed collaterals arising from the contralateral internal iliac artery filling the profunda femoral artery which in turn provided collaterals to the below knee popliteal artery. Interventional radiologists advised surgery for such a long TASC D lesion. The patient underwent an aorto-popliteal (below knee) bypass with PTFE graft and venous cuff. Post operatively, the patients symptoms were relieved and Doppler showed biphasic flow in the popliteal and all three crural vessels. Gradually the ulcers healed over a few weeks and the patient could walk pain free at 8 weeks follow up.

\section{CONCLUSION:}

Aortopopliteal bypass is a suitable intervention to relieve patients of ischaemic rest pain and non healing ulcers.

Intraoperative distal end arterial and venous thromboembolectomy during an above knee amputation.
Pallavi Kadam

\section{ABSTRACT: \\ INTRODUCTION:}

Above knee amputations (AKA) are commonly performed for critical irreversibly ischaemic limbs.

\section{BACKGROUND:}

Occasionally the arterial embolus can extend upto the iliac arteries compromising the viability of the thigh musculature and healing of the stump suture line as there is no flow through the profunda femoris artery (PFA). An embolectomy of the distal cut end of the superficial femoral artery(SFA) with a Fogarty catheter during amputation may restore flow in the PFA which may prevent further ischaemic breakdown and revision of stump length.

Acute irreversibly ischaemic lower limbs with arterial emboli rarely present with concurrent extensive deep venous thrombosis extending upto the iliac veins. Operating on such a limb runs the risk of a fatal massive pulmonary embolism. Ideally, an IVC filter should be placed before operating. This depends on the logistics of finances and the availability of trained personnel in a timely fashion. However, in an emergency with concurrent irreversible acute limb ischaemia, the operation has to be undertaken without the IVC filter with high risk due to time constraints. In such situations a careful and gentle venous thromboembolectomy can free the remaining deep venous system of extensive thrombus burden and can reduce the chance of a post operative massive pulmonary embolism.

\section{CASE REPORT:}

A 70 yr lady presented with acute lower limb ischaemia with gangrene. A doppler examination revealed extensive arterial embolus extending to the iliac arteries as well as extensive DVT extending to the iliac veins. The limb was unviable and she underwent a primary above knee amputation. Intraoperatively, venous and arterial thromboembolectomy was performed with Fogarty catheter removing $30 \mathrm{cms}$ of clot. She made an uneventful recovery and her stump healed completely.

CONCLUSION:

Concurrent arterial and venous thromboembolectomy can be performed during AKA to prevent stump ischaemia and reduce the chance of pulmonary embolism.

Management of Brachial artery occlusions (traumatic
and non traumatic)-report of 3 year experience
in JIPMER
Sreevathsa

\section{ABSTRACT:}

BACKGROUND:

Although the management of vascular occlusion in tertiary care centers have been reported many times in both civilian and warfront population, the experience of managing Brachial artyery occlusions ( traumatic and non traumatic )were not a frequent topic of discussion.

\section{METHODS:}

From July 1, 2015, to June 30, 2018, all BRACHIAL ARTERY CUCCLUSIONS treated in Emergency Operation theatre in JIPMER, Puducherry, were registered and retrospectively reviewed with doppler study was done immediate post operatively and after 3 months.

\section{RESULTS:}

34 BRACHIAL ARTERY OCCLUSION were treated in emergency operation theatre. Most of which were traumatic thrombus occluding the vessel and some were non traumatic emboli or thrombus. Embolectomy was done in most cases, some cases had traumatic transection with occlusion, they were repaired. Autologous vein was the conduit of choice for these vascular reconstructions. We studied the post operative out come of these cases, 16 Cases were traumatic injuries, all of which had good Doppler signals and palpable pulses immediate post operatively. 18 cases of non traumatic vascular occlusions include 10 embolisms and 8 thrombotic occlusions. Post operatively 4 of them had reccurent thrombosis of vessels, requiring redo embolectomy. 2 cases had gangrene and went in for amputation. 


\section{CONCLUSIONS:}

This study is the first large upper extremity vascular occlusion management report in JIPMER.

\section{DISCUSSION:}

Early diagnosis and immediate intervention are mandatory to save the extremities and lives of the patients. Factors, such as extent of soft tissue damage, the capacity of collaterals and pre-existing arterial disease may affect the clinical outcomes, while the ischaemia time is more critical, and more than six hours of ischaemia time are associated with increased rates of limb loss.

Factors associated with Arteriovenous Fistula Functional Maturation in Patients with Chronic kidney disease

Solomon

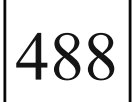

\section{ABSTRACT: \\ INTRODUCTION:}

With increasing numbers of patients diagnosed with $\mathrm{CKD}$, arteriovenous fistula (AVF) maturation has become a major factor in improving both dialysis related outcomes and quality of life of those patients. Compared to other types of access it has been established that a functional AVF access is the least likely to be associated with thrombosis, infection, secondary interventions to maintain patency, hospital admissions and death.

AIM:

Study of demographic factors implicated in the functional maturation of arteriovenous fistulas. Also, to explore any possible association between preoperative haematological investigations and functional maturation.

\section{METHODS:}

We performed a retrospective chart review of 115 patients with $\mathrm{CKD}$, of which male were $95(82.6 \%)$ and female were $20(17.4 \%)$ who were referred to the Cardiothoracic department in Tirunelveli Medical College Hospital for creation of vascular access for HD. We included patients with primary AVFs; and excluded those who underwent secondary procedures. RESULTS:

Overall AVF functional maturation rate in our study was $53.91 \%(62 / 115)$ and the failure was $46.09 \%$ (53/115). Female gender showed significant association with non-maturation $(\mathrm{P}=0.004)$ and lower the systolic and diastolic blood pressure, more the failure. Patients who had relatively lower haemoglobin levels showed better functional maturation rates. Blood vessel diameters were also the important factors of arteriovenous fistula failure. The greater the diameters, the lesser the risk of failure.

\section{CONCLUSION:}

Female gender and lower systolic and diastolic BP was found to be associated with functional non-maturation, and low haemoglobin levels and large diameter vessels were all associated with successful functional maturation. In view of the conflicting evidence in the literature, large prospective multi-centre registry-based studies with well-defined outcomes are needed.

\section{Pulmonary Artery Thrombectomy and IVC} Filter Placement - A New Approach

Purushottam Deshpande

Swapnil Deshpande

\footnotetext{
ABSTRACT:

Pulmonary Artery Thromboendarterectomy is a difficult and dreaded surgery world over. early diagnosis and complete treatment is the key to proper patient outcome. We present our series of 4 patients operated at our Centre between October 2013 to July 2018.

Average age of Patients was 64.5 years ( 33 years to 76 years). 3 of the 4 patients had acute on chronic pulmonary thromboembolism with associated DVT of bilateral thigh veins. one patient had Chronic Pulmonary thromboembolism with no DVT.
}

All the patients underwent CT pulmonary angiogram and Coronary angiogram if required. All the patients were admitted in emergency Worked up and operated in emergency.

In view of DVT the thigh veins which can cause high chances of ReEmbolism, the patient was also planned for Placement of IVC filter.

All the patients were operated in Hybrid Operation theatre where they were intubated. underwent Coronary angiography and then underwent Pulmonary Artery Thrombectomy and then underwent Trans Right Atrial IVC filter placment in the same sitting. The idea was to avoid re-Admission and avoid the femoral vein or the Internal Jugular vein access as additional puncture site. The filters used were placed retrogradely and retrievable. All the patients are doing well with no mortality for average follow up of 3 year (3 years to 4 years). All the patients were given Oral Vitamin K Antagonists and Aspirin for 1 year and $2 \mathrm{D}$ Echo, Bilateral Lower limb Venous Doppler and IVC doppler done at 6 months, 12 months, 18 months and 24 months and then on yearly basis. All the patients had resolved DVT and hence IVC fliter was removed via trans Jugular route at 1 year.

Performing Pulmonary artery Thrombectomy in hybrid lab definitely helps in adequate diagnosis, proper surgical cure and easy placement of IVC filter in high risk cases. Our Institutional experience on managing
Chronic Thrombo Embolic Pulmonary

Hypertension (CTEPH)

A. Rathinavel; B. Karthikeyan

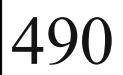

\section{ABSTRACT: \\ INTRODUCTION:}

Chronic ThromboEmbolic PulmonaryHypertension (CTEPH) is one of the rare cases which need surgical intervention in most cases.

\section{CASE SERIES:}

We have evaluated 10 patients of CTEPH in last one year. Three patients were treated surgically by PulmonaryThromboendarterectomy(PTE). One PTE was done under Total circulatory arrest(TCA). Other 7 patients are under medical management.

Eight patients are males. Four among 10 patients gave clear history of DVT. Five had only RPA involvement. Two had involvement upto the confluence. Two patients had extensive bilateral disease, one patient had right side distal segmental disease alone. One patient was already on IVC filter.

First 2 patients were operated under CPB with deep hypothermia. Both the patients had thrombus from the level of the confluence of the MPA upto distal segments. One among them had severe bleeding from the endotracheal tube and succumbed post operatively.

Third patient, 27 years old male found to have CTEPH and treated with medical management and IVC filter. PTE done under Total Circulatory Arrest (TCA). RPA Opened from the confluence upto the lobar divisions and endarterectomy done upto the segmental divisions after developing sub intimal plane. Patient cooled to $16 \mathrm{deg}$ centigrade during TCA.

Immediate Post operatively patient developed severe pulmonary hypertension and treated with intravenous sildenafil and milrinone. Post operative angiogram showed good pulmonary artery blood flow to the right lung.

\section{CONCLUSION:}

Although CTEPH is a rare disease treating the disease early will improve the survival rate because this disease is having almost $90 \%$ mortality without surgical intervention.

Pulmonary endarterectomy for chronic thromboembolic pulmonary hypertension - early post operative experience in a tertiary cardiothoracic care centre - a series of ten cases 
Manoj Joshi; Saptarshi Paul

\section{ABSTRACT: \\ OBJECTIVES:}

Pulmonary endarterectomy is a documented surgical treatment available worldwide for chronic thromboembolic pulmonary hypertension (CTEPH). CTEPH usually develops in patients who have a prior history of acute pulmonary embolism (PE) or deep venous thrombosis. The objective of our study is to document our initial experience regarding the effectivity of pulmonary thromboendarterectomy as a definite curative surgical procedure for CTEPH.

\section{METHODS:}

Ten patients with CTEPH underwent pulmonary thromboendarterectomy between March 2015 and August 2017 in our institution, Grant Medical College, Mumbai. The patients were evaluated on the basis of their pre operative symptoms and the alleviation of the same symptoms one year post operatively; and also quantitatively on the basis of comparison between three parameters viz. 6 minute walk test (6 MWT), brain natriuretic peptide (BNP) and PASP (pulmonary artery systolic pressure) pre and post operatively.

\section{RESULTS:}

All 10 patients had breathlessness, with 4 having pedal oedema and 2 having jaundice. All underwent pulmonary thromboendarterectomy under cardiopulmonary bypass. One year into the post operative period, there was a general improvement in the overall quality of life. The 6MWT increased post operatively with a simultaneous decrease in the values of BNP and PASP and all these changes were statistically significant.

CONCLUSION:

Pulmonary endarterectomy is a potentially curative surgical treatment for CTEPH and 6MWT, BNP and PASP are definitive prognostic predictors of the same.

\section{Inflammatory Myofibroblastic Tumor of the Lung}

Alberto Oliaro; Pierluigi Filosso; Enrico Ruffini; Paolo Olivo Lausi; Elisa Fontana

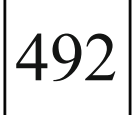

\section{ABSTRACT:}

Inflammatory myofibroblastic tumor (IMT), also known as inflammatory pseudotumor, is the most common pulmonary neoplasm in children, although rather uncommon in adults. It represents less than $1 \%$ of all lung neoplasms. We present a case of 66-year-old female, past smoker, without any previous oncological disease, and without signs or symptoms for respiratory disease. CT scan showed a $1.5 \mathrm{~cm}$ lesion, with heterogeneous contrast enhancement, suggestive of neoplasm.

PET scan demonstrated an increased FDG uptake with SUV value of 3.8. A VATS wedge resection was performed, after an intraoperative exclusion of malignancy.

Histologically, the tumor consisted of bundles of spindled cells and inflammatory cells in a myxoid stroma.

Immunohistochemically observed, spindled cells were the following: actin, desmin, bcl2, ALK positive, and P53, CD 34, TTF1, S100, CD117, HMB45, HHV8, ER negative.

A final diagnosis of IMT was therefore made based on the morphological and immunohistochemical findings.

The usual nature of this disease is a slow-growing tumor without local invasion or distant metastases. Occasionally, it can show a locally invasive or distant metastatic spread.

The histopathological diagnosis of an IMT is a challenge. Often CT guided biopsy and frozen section provide inconclusive results.

Approximately half of all inflammatory myofibroblastic tumors show a rearrangement of the anaplastic lymphoma kinase (ALK) gene locus secondary to ALK-gene (2p23).

Surgery is the treatment of choice for IMT. Usually wedge resection is the gold standard treatment for non - invasive forms, on the contrary an anatomical resection is required and recommended for the invasive forms.
In some cases is necessary to perform an extensive resection including diaphragm, chest wall or pericardium.

Some studies report the use of Crizotinib, an ALK inhibitor, as an effective therapy in ALK positive pulmonary IMT.

Other treatment modalities such as radiotherapy, chemotherapy, or NSAID as Colecoxib have to be further evaluated.

Does sarcopenia have any impact on survival of patients with surgically treated non-small cell lung cancer?

Han-Yu Deng

ABSTRACT:

A best evidence topic in thoracic surgery was written according to a structured protocol. The question addressed was 'Does sarcopenia have any impact on survival of patients with surgically treated nonsmall cell lung cancer (NSCLC)?'. Altogether, 342 papers were found using the reported search, of which 9 cohort studies represented the best evidence to answer the clinical question. The authors, journal, date and country of publication, patient group studied, study type, relevant outcomes and results of these papers are tabulated. Eight of those cohort studies measured skeletal muscle or total psoas cross sectional area at the level of the first or third lumbar vertebra using computed tomography images normalized for the square of height for defining sarcopenia while only one study used absolute measurement of total psoas cross sectional area at the level of the third lumbar vertebra. Seven cohort studies with adjustment for confounding factors found that patients with sarcopenia had a significantly worse overall survival than those without after surgical resection of NSCLC while only one study without adjustment found that there was no difference of overall survival between sarcopenic patients and non-sarcopenic patients. Two studies found that patients with sarcopenia had a significantly worse disease-free survival than those without while other three studies found that there was no significant difference of disease-free survival between sarcopenic patients and non-sarcopenic patients. Another one study found that sarcopenia was an independent predictor of early recurrence of NSCLC patient after surgical resection. Therefore, we conclude that sarcopenia is a predictor of poor prognosis of patients with surgically treated NSCLC.

\section{Does liver cirrhosis have any impact on esophageal cancer patients treated with esophagectomy? Han-Yu Deng}

\section{ABSTRACT:}

A best evidence topic in thoracic surgery was written according to a structured protocol. The question addressed was 'Does liver cirrhosis have any impact on esophageal cancer patients treated with esophagectomy?'. Altogether, 293 papers were found using the reported search, of which 9 cohort studies represented the best evidence to answer the clinical question. The authors, journal, date and country of publication, patient group studied, study type, relevant outcomes and results of these papers were tabulated. Six cohort studies found that liver cirrhosis was correlated to increased risk of postoperative complications especially for the risk of pulmonary complications and pleural effusion. Five cohort studies found that cirrhotic patients had a higher risk of postoperative mortality than non-cirrhotic patients after surgical resection of esophageal cancer. Moreover, for cirrhotic patients with child grade B or high model for end-stage liver disease (MELD) score $(\geq 10)$ the risk of postoperative complications and mortality was extremely high. However, there seemed to be no significant difference of overall survival between cirrhotic patients and non-cirrhotic patients, but patients with high-MELD score yielded a significantly poor overall survival than those with low-MELD score. Therefore, we conclude that for cirrhotic patients with Child grade A or low-MELD score surgical resection of esophageal cancer can be the preferred option while for those with Child grade $\mathrm{B} / \mathrm{C}$ or high-MELD 
score surgery may not be suggested.

Mediastinal tumour-clinical features, diagnosis, treatement, and complications Subrata Dey; Subhajit Sharma

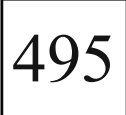

\section{ABSTRACT:}

\section{INTRODUCTION:}

Mediastinal tumor, heterogenous entity encompasses variety of benign, malignant, inflammatory disease. Treatment of such tumor could be medical, surgical or multi-modal. Definitive diagnosis is essential leading to adequate prompt therapeutic strategy.

\section{AIMS AND OBJECTIVES}

a) To know the incidence and etiology in different compartments.

b) To know the modes of presentations, and clinical spectrum in different ages.

c) To know the mode of treatment and complications.

\section{RESULTS:}

Most common compartment involved is anterior(57.1\%),

Most common anterior tumor is thymoma(25\%) and lymphoma(25\%)

Most common middle mediastinal tumor is malignant mediastinal lymphadenopathy $(40 \%)$

Most common posterior mediastinal tumor is nurogenic tumor $(75 \%)$

Overall most common tumor is lymphoma(23.8\%)

Most common in pediatric age group are lymphoma( $(40 \%)$ and nurogenic tumor $(40 \%)$

Slightly male predominance( $1: 1.1)$

Most common modes of presentations are fever(23.8\%) and chest $\operatorname{pain}(23.8 \%)$

Mediastinal widening is seen in CXR(PA) in 80.9 cases

Associated plural effusion seen in $38 \%$ cases.

They are mostly approached via thoracotomy $(71.4 \%)$

Tumor completely resected in $61 \%$ cases.

Most common complications are arrythmias(14.2\%) and wound infections $(14.2 \%)$

Recurrence after 1 year of follow up is $4 \%$ cases

\section{CONCLUSIONS:}

Mediastinal tumor are mostly malignant, most common mode of presentations are fever and chest pain. Mostly approached via thoracotomy, some needs multimodal treatment, prevalent in all age and sex.

\section{BRONCHIAL FISTULA AND MYOPLASTY}

Lanza Giovanni; Oliaro Alberto

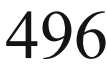

\section{ABSTRACT: \\ OBJECTIVE:}

The myoplasty is not a frequent procedure in thoracic surgery. The indications for the use of myoplasty for trachea and main bronchi are the closure of bronchial fistulas and the treatment of oesophago-tracheal fistulas. The direct closure of broncho-pleural fistulas after pneumonectomy is often impossible and has a high risk of failure due to the presence of fibrotic tissue. The muscle flap is used to achieve an indirect suture of the bronchial fistula. We present a video in which in the presence of a large and complete fistula of left main bronchus after pneumonectomy we achieved a complete closure of the bronchial fistula utilizing the serratus anterior and the latissimus dorsi muscles.

\section{METHODS:}

In the first time we used the serratus anterior muscle flap in considering that the patient have undergone a posterior-lateral thoracotomy with the division of the latissimus dorsi muscle. Moreover, we performed a multiple costal resections to permit the use of the muscle flap in the residual pleural space. Unfortunately, at the CT-scans control, due to a persistent air-leak, the muscle flap not covered efficiently the fistula. Thus, we performed a further surgical revision of the pleural space and we insert a latissimus dorsi muscle flap to complete the coverage of the fistula.
RESULTS:

In the post-operative days, we observed a complete resolution of air leak and the CT-scans control demonstrated a complete closure of the bronchial fistula. At the 1 year follow-up the patient is alive and in optimal clinical conditions. CONCLUSIONS:

The use of a combination of the serratus anterior and the latissimus dorsi muscle flaps could contribute to an optimal closure of the broncho-pleural fistulas after pneumonectomy.

An unusual cause of cerebrovascular accident after lung resection

Mundayat Gopalakrishnan, Amit

Kiran, Manjunath Kamath, Rishab KS

\section{ABSTRACT: \\ INTRODUCTION:}

Cerebrovascular accident (CVA) following lung resection is very rare. We have encountered such an event after lung resection in a female following left upperlobectomy.

\section{MATERIALS AND METHODS:}

A 51 year old female patient was admitted with complaints of recurrent respiratory infection. Her CT thorax revealed features of intralobar sequestration of left lowerlobe. She underwent uneventful left lowerlobectomy and was discharged on postoperative day 8 . Her Histopathology report was congenital cystic adematoid malformation ofmultiple segments of leftlower lobe She was readmitted after 10 days with rightsided hemiplegia. There were no changes in her higher mental functions or speech. Her CT and MRI brain revealed acute cerebral infarct in the left internal capsule. Her transthoracic Echo revealed evidence of clot near the left pulmonary veins and spontaneous echo contrast in the leftatrium.

RESULT:

She was started on anticoagulants and physiotherapy. Her left lung was fully expanded with no residual deadspace or effusion. Her neurological status slowly improved and was able to walk with support by the end of 2 weeks.

CONCLUSION:

CVA after lung resection is very rare. Possibility of thrombus formation in the left atrium should be considered in these patients and a routine echo evaluation before discharge will help to identify this rare pathology.

Pleurodesis: a comparision of two sclerosing agents for pleural effusion

A. Rathinavel; S. Naveen

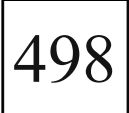

\section{ABSTRACT: \\ INTRODUCTION:}

Pleural effusion constitute one of the most frequent pathologies encountered. Malignant pleural effusions will recur after simple thoracocentesis within 5-10days. Repeated thoracocentesis in these cases are not recommended as this process can increase the risk of metastatic spread at the site of the puncture, empyema, loss of protein. placement of pleural drainage tube and instillation of sclerosing agents in the pleural space is the preferred approach for these recurrent effusion.

AIM:

Comparision of effectiveness of sodium tetradecyl sulphate and talc as the sclerosing agent for recurrent pleural effusion.

\section{METHODS:}

Recurrent pleural effusion (malignant and non malignant) cases were randomly selected for pleurodesis using talc and sodium tetradecyl sulphate $(10 \mathrm{ml})$. clinical data regarding general complications, rate of success and lung expansion were noted for every patient and compared.

\section{RESULTS:}


Total of 40 patients were included in the study, of which 20 cases were used talc and 20 cases were used sodium tetra decyl sulphate as the sclerosing agent. patient characteristics were balanced between the two groups with majority of pleural effusions malignant in etiology. No statistical significant difference between the both in general, local complications and clinical response. Lung expansion rate was better with sodium tetra decyl sulphate ( 0.0024 significant)

\section{CONCLUSION:}

Sodium tetra decyl suphate was better than talc in the lung re expantion in pleural effusion cases.

\section{Large Hamartoma of Left Lung}

A .Rathinavel

K.J. Vignesvaran

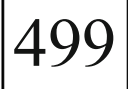

\section{ABSTRACT: \\ INTRODUCTION:}

Pulmonary hamartomas are benign mesenchymal tumors and represent $6 \%$ of lung lesions treated by thoracotomy, usually coin sized lesion or nodules, large lesions are rare.

\section{CASE PRESENTATION:}

78 yrs old male with complaints dyspnea for past 4 months. On evaluation patient found to have hypo dense $16 * 10 * 10$ well defined mass lesion in left lower lobe with chunk of calcification and loss of left lung parenchyma. Intra operatively mass was densely adhering to the lower lobe with thick capsule and cartilaginous matrix. Debulking of tumor done due to dense adhesions. Post operative periods uneventful with no complaints of dyspnea patient discharged on 5th POD. Biopsy shows fibrocollagenous tissue with cartilage and bone formation.

\section{CONCLUSION:}

Pulmonary hamartoma usually small size and usually asymptomatic but requiring differentiation from lung cancer due to relatively large size and respiratory symptoms in the patient.

Efficacy of tranexamic acid in reducing blood loss during and after Transhiatal Oesophagectomy.

G.N. Lone

Nadeem U1 Nazeer

\section{ABSTRACT:}

\section{BACKGROUND:}

Transhiatal oesophagectomy is associated with peri and postoperative blood loss. This study was aimed to evaluate the clinical efficacy and safety of tranexamic acid in reducing peri and postoperative blood loss in patients undergoing transhiatal oesophagectomy.

\section{MATERIALS AND METHODS:}

A total of 74 patients, who underwent elective transhiatal oesophagectomy were studied. They were divided into two groups. In the study group of 40, tranexamic acid 1 gram intravenous was given before starting dissection and the control group did not receive tranexamic acid. Intra and post-operative blood loss, operation time, transfusion of blood products, pre- and post-operative haemoglobin, number of days of hospitalisation and in hospital mortality was studied for both groups.

\section{RESULTS:}

Tranexamic acid significantly reduced the volume of blood loss during the surgery when compared with the control group. Considering the duration of operation and the treatment groups only, the mean total blood loss in the control group was more than that in the tranexamic acid group. Eight patients in the control group required blood transfusion post-surgery due to significant drop in haemoglobin. There was no difference in the length of hospital stay between the two groups.

\section{CONCLUSION:}

The prophylactic administration of tranexamic acid has effectively reduced the blood loss and transfusion needs during transhiatal oesophagectomy without any adverse effects or complication of thrombosis.

Effect of normal saline flush injection into bronchus on lung decellularization

Kentaro Kitano

Masahiro Yanagiya

\section{ABSTRACT:}

OBJECTIVE:

Lung decellularization is necessary for bioartificial lung regeneration. The aim of this study was to evaluate effect of normal saline flush injection into bronchus on creation of decellularized lung scaffolds utilized for lung transplantation.

\section{MATERIALS AND METHODS:}

Native lung grafts $(\mathrm{N}=9)$ were harvested from 15 to $25 \mathrm{~kg}$ pigs. The lung decellularization was conducted by sodium dodecyl sulfate (SDS) and Triton X-100 method: the main pulmonary artery was perfused with $0.5 \%$ SDS in 30 hours, deionized water in 12 hours and $1 \%$ Triton X-100 in 12 hours at $2 \mathrm{ml} / \mathrm{min}$. Three grafts were pre-treated with $2 \mathrm{~L}$ of normal saline flush injection into bronchus before SDS and Triton X-100 method (PreTx+; N=3). Other three grafts were conducted with SDS and Triton $\mathrm{X}-100$ method without pre-treatment (PreTx-; N=3). The others were treated as normal grafts (Normal; $N=3$ ). Staining and quantitative analyses were performed.

\section{RESULTS:}

Hematoxylin eosin staining apparently revealed scarce cells in PreTx+ and PreTx- groups, compared with abundant cells in Normal group. Quantitative DNA analysis demonstrated a significant reduction of DNA content in PreTx+ and PreTx- groups compared with Normal group $(35.1 \pm 17.1 \mu \mathrm{g} / \mathrm{mg}$ wet tissue vs. $53.0 \times 102 \pm 25.3 \times 102 \mu \mathrm{g} / \mathrm{mg}$ wet tissue, P \&lt; 0.001). DNA content was significantly lower in PreTx+ group than in PreTx- group $(21.6 \pm 5.46 \mu \mathrm{g} / \mathrm{mg}$ wet tissue vs. $48.7 \pm 12.3 \mu \mathrm{g} / \mathrm{mg}$ wet tissue, P \& 1 t; 0.05).

\section{CONCLUSION:}

The normal saline flush injection combined with SDS and Triton X-100 method of decellularization could be a promising procedure in creating decellularized scaffolds effectively. The scaffolds would be utilized for lung transplantation.

Prognosis of preoperative high Carcinoembryonic antigen level in node-negative lung adenocarcinoma Khashchuluun

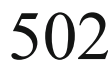

\section{ABSTRACT:}

\section{PURPOSE:}

Carcinoembryonic antigen (CEA) is a well-known tumor marker of lung adenocarcinoma. Although high CEA level is correlated with advanced stage, it has been found in node-negative lung adenocarcinoma. This study evaluates the clinicopathological characteristics and prognosis of patients with preoperative high CEA in node-negative lung adenocarcinoma.

\section{METHODS:}

Data of 1,269 patients with node-negative lung adenocarcinoma were prospectively collected between 2004 and 2016. The clinicopathological variables were compared between high CEA and normal CEA groups. Kaplan-Meier survival analysis with Log-rank test and Cox proportional hazards regression analysis were performed for recurrence-free survival (RFS) and overall survival (OS).

RESULTS:

There were 1,151 (91\%) patients in the normal CEA group and $118(9 \%)$ patients in the high CEA group. Old age, low DLCO, high SUVmax, large tumor, presence of visceral pleural invasion, lymphovascular 
invasion, aerogenous spread, and high histologic grade were more common in the high CEA group. The median follow-up duration was 49.2 (range, 6-163) months. Recurrence and cancer-related death were observed in $130(11 \%)$ and $97(8 \%)$ patients in the normal CEA group, and $37(31 \%)$ and $27(23 \%)$ in the high CEA group. The 5-year RFS and OS were statistically different between the two groups. Multivariable analysis showed high CEA, high SUVmax, presence of VPI, and aerogenous spread were independent prognostic factors for RFS. In 118 of high CEA patients, CEA level was normalized in 92 patients, reelevated in 14 patients, and still high in 12 patients after complete resection. Patients whose CEA level was re-elevated showed worst RFS among them.

\section{CONCLUSIONS:}

In node-negative lung adenocarcinoma, patients with preoperative high CEA level had well-known poor prognostic factors and significantly worse RFS and OS. Adjuvant chemotherapy should be considered for this group.

Vasculogenic Thoracic Outlet Syndrome

Murugan M.S.; Krishnanand Pai;

Ganesan C.

P.R. Murugesan

\section{ABSTRACT: \\ BACKGROUND:}

Post stenotic subclavian aneurismal dilatation and embolic distal limb ischemia is the common presentation of cervical rib to vascular surgeon, the procedure of choice cervical rib excision, subclavian artery repair by interposition graft and distal embolectomy. We like to present the role of subclavian reduction angioplasty and its followup results.

\section{CLINICAL PROFILE:}

Symptomatic cervical rib with limb ischemia. 6 cases all females 18 to 40 years of age. Preop $\mathrm{Ct}$ angiogram revealed Post stenotic aneurismal dilatation of sub clavian artery with distal embolisation. Supraclavicular approach cervical rib excision with scalenotomy and distal vessel embolectomy done with exclusion of redundant aneurysmal sac and repair of subclavian artery.

\section{RESULTS:}

Post operative recovery satisfactory, no residual brachial neuropraxia. Limb perfusion good with palpable brachial pulsation.

\section{CONCLUSION:}

In selected cases of subclavian aneurysm of poststenotic dilatation can be managed with exclusion (reduction) angioplasty with good long term outcome by which one can minimize the need for synthetic graft interposition and its related complication.

\section{Intercostal Muscle Flap in Pneumonectomy for Inflammatory Lung Disease Ramaswamy Rajendran; R.K. Dewan; Klein Dantis; Sanam Jindal}

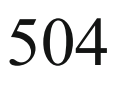

\section{ABSTRACT: \\ OBJECTIVE:}

The development of a postoperative Bronchopleural fistula (BPF) is a serious complication with significant morbidity and mortality. There are various risk factors for post operative BPF including diabetes, perioperative steroid therapy, preoperative chemotherapy and radiotherapy. The risk of BPF increases with resections for inflammatory lung diseases especially in cases of tuberculosis. The study evaluated the use of an intercostal muscle flap to reinforce the bronchial stump after Pneumonectomy as a prophylactic measure from post Pneumonectomy BPF.

\section{METHODS:}

Between May 2017 to May 2018, 33 patients were taken up for Pneumonectomy for Inflammatory Lung diseases in the Department of Thoracic Surgery, National Institute of Tuberculosis and Respiratory Diseases. Patients were divided into two Groups. Group A: 18 patients who underwent Pneumonectomy without bronchial stump coverage. Group B: 15 Patients in whom an intercostal muscle flap was used to buttress the bronchial stump. The Mean age of patients in group A was 36.6 years (range $21-60$ years) and in group B, it was 29.8 years (Range 19 to 65$)$.

\section{RESULTS:}

Bronchopleural Fistula developed in three patients in Group A (16.6\%) and in one patient in Group B (6.6\%); Empyema in one patient in Group $\mathrm{A}(5.5 \%)$ and in one patient in Group $\mathrm{B}(6.6 \%)$; Wound Infection in one patient in Group A (5.5\%) and in one patient in Group B(6.6\%). Patients were followed up for a period of six months. An elective bronchoscopy was performed three months after surgery to look for BPF, if any.

\section{CONCLUSION:}

The addition of an intercostal muscle flap to buttress the bronchial stump possibly reduces the occurrence of Bronchopleural fistula following Pneumonectomy for inflammatory lung diseases.

Massive haemorrhage due to erosion of the internal mammary artery by chest wall tubercular abscess R.K. Dewan; Ramaswamy Rajendran; Pallavi Purwar; Saraansh Bansal; Utsav Gupta

\section{ABSTRACT:}

Chest wall involvement in Tuberculosis is an uncommon phenomenon. Very rarely, this involvement can cause erosion of the internal mammary artery and massive hemorrhage as a consequence there of. We encountered two such cases in the last 2 years. The first case presented in emergency with moderate to severe bleeding taking place from the site of chest wall sinus on left hemithorax anteriorly. The second case presented with a chest wall abscess in left parasternal region which got ruptured and this was followed by massive hemorrhage from the site. Both the cases were taken up for emergency thoracotomy and left internal mammary artery was found to be eroded and bleeding. Control of the artery was taken in both cases. Patients did well in the post operative period. Clinical details of the cases will be presented.

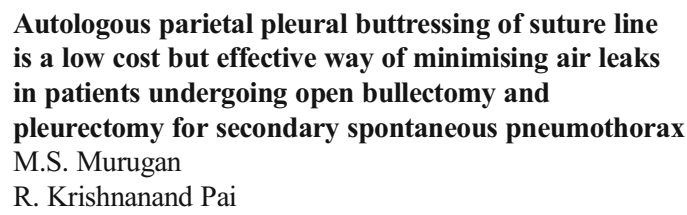

ABSTRACT:

\section{INTRODUCTION:}

Patients with Secondary Spontaneous Pneumothorax [SSP] and persistent air leak in spite of tube thoracostomy are referred for surgical Bullectomy and Pleurectomy. Economic circumstances dictate that many patients need open bullectomy. The underlying diseased lungs are difficult to suture and the persisting air leak albeit minimal takes many days of hospitalisation to improve. We describe the use of harvested autologous parietal pleura to buttress the lung suture line resulting in minimal air leak, faster recovery and discharge.

\section{METHODS:}

Between 2016 to 2018 we used a technique of harvesting the parietal pleura in 12 patients undergoing surgery for SSP. After open bullectomy the lung was sutured with 4-0 poly propelene, The suture line was reinforced with another set of sutures buttressed with the autologous parietal 
pleura harvested initially as a part of radical pleurectomy during the procedure. Clinical outcomes were compared with historic controls.

\section{FINDINGS:}

There was less that $25 \mathrm{ml}$ per breath air leak in 4 patients and no air leak in remaining 8 patients on completion of the procedure. Clinical and radiological recovery was quick, efficient and event free in all patients with tube thoracostomy drains removed by 72 hours in all patients with discharge effected in 5 days after surgery. Over a follow up from 24 days to 18 months there as no recurrence of SSP on either side and all patients have returned to gainful employment. While the technique of using heterologous pericardium reinforced staplers is well described in thoracoscopic LVRS surgery, we report our initial experiences with open surgical procedure.

\section{CONCLUSIONS:}

Use of the patients own parietal pleura obviates need of using expensive products like bovine pericardium or sealants to buttress lung suture lines and at the same time is effective in preventing leaks thus speedening recovery and minimising hospital stay. It is cost effective and can be harvested initially during surgery.

\author{
Risk factors of lymph node metastasis in $\mathrm{T} 1$ \\ Esophageal squamous cell carcinoma \\ Kai-Yuan Jiang \\ Dong Tian
}

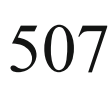

\section{ABSTRACT: \\ OBJECTIVE:}

Lymph node metastasis (LNM) is a poor prognostic factor for esophageal squamous cell carcinoma (ESCC). LNM in T1 ESCC is not rare, but the predictive factors of LNM for T1 ESCC are unclear. This study aimed to evaluate clinicopathologic and hematological predictive factors for LNM in T1 ESCC.

\section{METHODS:}

We retrospectively analyzed 81 patients of T1 ESCC who underwent esophagectomy and lymph node dissection between January 2015 and December 2018. The clinicopathologic and hematological characteristics were evaluated by univariate and multivariate logistic regression analyses. Odds ratios (OR) with $95 \%$ confidence interval (95\% CI) were calculated. We further examined the relationship between the positive number of significant predictive factors and LNM rate.

\section{RESULTS:}

Of the 81 patients with T1 ESCC, $23(28.4 \%)$ cases encountered LNM. LNM rate of stage T1a was $7.1 \%(2 / 28)$, T1b was $39.6 \%(21 / 53)$. The different thresholds of alanine aminotransferase/aspartate aminotransferase ratio (LSR) and high-density lipoprotein cholesterol (HDL-C) were 0.5656 and $1.105 \mathrm{mmol} / \mathrm{L}$ from the receiver operating characteristic (ROC) curve. The $\mathrm{T} 1$ subclassification $(\mathrm{OR}=6.772,95 \% \mathrm{CI}: 1.222-$ 37.526, $\mathrm{P}=0.029)$, tumor size $(\mathrm{OR}=5.773$, 95\% CI: $1.239-26.906$, $\mathrm{P}=0.026)$, preoperative LSR $(\mathrm{OR}=6.109,95 \% \mathrm{CI}: 1.032-36.154$, $\mathrm{P}=0.046)$, and preoperative HDL-C $(\mathrm{OR}=7.668,95 \% \mathrm{CI}: 2.466-$ 195.305, $\mathrm{P}=0.006$ ) were found to be independent risk factors for LNM on multivariate analysis.

\section{CONCLUSIONS:}

Clinicopathologic and hematological parameters of T1 sub-stage (T1b), tumor size $(\geq 2 \mathrm{~cm})$, LSR $(\geq 0.5656)$ and HDL-C $(\geq 1.105 \mathrm{mmol} / \mathrm{L})$ may predict the risk of LNM in T1 ESCC.

Emergency thoracotomy, our experience over 4 years. Subrata Dey

Subhajit Sharma

ABSTRACT:

INTRODUCTION:
Trauma to the thorax represents significant portion of injuries. Although most of people may be successfully managed without thoracotomy, a certain proportion require operative intervention immediately.

\section{METHOD:}

Out of 541 patients of significant chest injury,,48 patients underwent emergency thoracotomy following preliminary resuscitative treatment. AIMS:

a) To know the indications of emergency thoracotomy.

b) To know the timing of emergency thoracotomy.

c) To know mortality.

\section{RESULTS:}

493 patients $(91 \%)$ required only good supportive measures such as tube thora-costomy, temporary ventilatory support, and careful observation. 48 patients (9\%) required immediate operation following initial resuscitative effort. Penetrating injury was 17 cases $(35.4 \%)$ blunt trauma in 31 cases (64.4\%)cases. Indications was hemmorrhage(21) cardiac tamponade(13), injury to great vessels(7) major lung injury(3) diaphragmatic injury(1), oesophagal injury,(1) no definitive bleeding identified(2). Overall mortality was $14.5 \%(7)$ cases.

CONCLUSIONS:

Thus, although it may be clear which patients require immediate operation, only careful and continuous monitoring can identify who initially appear to be in stable condition but eventually will require exploration.

Thoracoplasty for Postpneumonectomy Empyema: An uncommon case report

Ravindra Dewan

Sanam Jindal

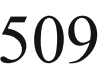

\section{ABSTRACT:}

Thoracoplasty is a historical procedure, initially devised for the treatment of refractory tuberculous empyema. Advances in medical treatments have nearly eliminated the need for this surgical procedure in pulmonary tuberculosis and it is rarely performed or taught in modern day surgical practice. However, a few indications still exist, like postpneumonectomy empyema with or without bronchopleural fistula. We present a case of a 53 years old male patient who underwent multiple surgeries - right upper lobectomy to begin with followed by completion pneumonectomy. Then he developed postpneumonectomy empyema which was treated by a limited thoracoplasty. However, he had to undergo two more completion procedures for the same. Management of postpneumonectomy empyema and need for thoracoplasty will be discussed thorough this case report.

Interesting cases of mediastinal masses.

Sreekar Balasundaram;

Sathish Kumar

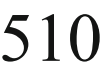

\section{ABSTRACT: \\ INRODUCTION AND BACKGROUND:}

Mediastinal tumours are uncommon lesions encountered in clinical practice. There has been a significant increase in the incidence of malignant mediastinal tumours over the past four decades. The presentation varies from asymptomatic lesions detected incidentally on imaging to severe life threatening presentations. This study is a description of a single center experience, outlining the clinical characteristics, types, treatment modalities and outcome of mediastinal masses.

\section{METHODS:}

Retrospective study of 9 cases of mediastinal masses of varying pathology who underwent surgery between December 2017 and September 2018 in St.John's Medical College Hospital, Bangalore.

\section{CASES}

We describe nine patients who presented with mediastinal mass. We wish to showcase the range of diagnosis possible in these situations. The pathological diagnosis include Thymoma, Thymic cyst, Seminoma, Synovial sarcoma, 
Hodgkins lymphoma, Schwannoma and Ganglioneuroma arising from various divisions of the mediastinum.

\section{CONCLUSIONS:}

A high index of suspicion and good clinical acumen is necessary for early diagnosis and management of these conditions along with judicious use of imaging and investigations. Histopathology and IHC play an important and crucial role.

Diagnosis and treatment of pulmonary hydatid cysts: Our experience.

G.N. Lone

Nadeem U1 Nazeer

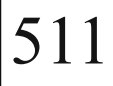

\section{ABSTRACT:}

\section{OBJECTIVE:}

To report our experience of surgical procedures in the management of hydatid cysts of the lung and to assess the effect of postoperative chemotherapy.

\section{MATERIALS AND METHODS:}

In our 154 consecutive patients who presented with hydatid cysts of the lung, the main parameters studied were: characteristics on presentation, operative techniques, postoperative morbidity, and the outcome of treatment.

\section{RESULTS:}

The mean age was 21 years (range $4-65$ years). The most common symptoms were cough, chest pain, fever, and hemoptysis. Chest radiographs and computed tomograms (CT) were the main tools of diagnosis. Chest radiographs and CT scans showed a smoothly outlined spherical opacity in $89 \%$ of patients. Other radiographic findings included ill-defined shadow (3\% patients), pleural effusion (3\% patients), air fluid level (2\% patients), and hydropneumothorax in $3 \%$ patients. High vacuum suction $(-30 \mathrm{~cm} \mathrm{H} 2 \mathrm{O})$ was employed in every case. Pulmonary cystotomy and capitonnage were performed in $94 \%$ of patients. Some cysts were removed by means of different surgical techniques such as segmentectomy, lobectomy and atypical pulmonary resection. Simultaneous combined resection of hydatid cysts through thoracotomy with transdiaphragmatic removal of liver cysts was performed in one stage in $7 \%$ of patients. There were $13 \%$ cases of immediate postoperative complications. These occurred mostly in patients who had cysts larger than $10 \mathrm{~cm}$. All patients were treated by a 3-month course of albendazole chemotherapy. Three patients had recurrence of the disease during the follow-up period

\section{CONCLUSION:}

Conservative surgical methods are the preferred surgical techniques. Postoperative chemotherapy with albendazole for 3 months is recommended.

A novel indigenous technique of chest wall
reconstruction for Pectus Excavatum - 2 years follow up 512
A. Rathinavel
B. Karthikeyan

\section{ABSTRACT:}

\section{INTRODUCTION:}

Pectus Excavatum is the most common chest deformity presenting in 1 in 1000 live births. It usually presents with asymmetric depression of lower chest. Right side depressed more than left. Symptoms of compression will present in few cases. Null Bar and Ravitch procedure are the available surgical options for deformity correction.

\section{CASE REPORT:}

Our patient is a 11 years old female child presented with pectus excavatum deformity, NYHA class II dyspnoea, and intermittent palpitation. Imaging studies showed severe mediastinal shift to right side with compensatory emphysematous changes on the left lung. Echo showed compression of both ventricles.

Chest deformity correction was done using lower middle hemi sternotomy and osteotomy of bilateral 4th to 7th ribs. Sternum everted anteriorly and stabilized with Dynamic compression plates and fixed with stainless steel wires. Two plates fixed at 5th and 6th rib level and midline sternal defect covered with prolene mesh.

Post operative period uneventful. Haller's index at D8 level reduced from 4.23 to 3.6 and D10 level 4.25 to 3.16. Post operative follow up showed good sternal stability with relief of compression of cardiac chambers and patients symptoms improved.

Two years after the procedure, the plates are removed and patient is symptom free now.

\section{CONCLUSION}

The case is presented here because of the good post operative outcome with this new novel approach of chest deformity correction (similar to modified Ravitch procedure) using locally available cost effective plates and wires used in orthopaedics department with limited resources in government setup.

Slipping Rib Syndrome: A rare case report

Ravindra Dewan

Sanam Jindal

ABSTRACT:

Slipping rib syndrome is a rare and usually underdiagnosed cause of lower chest and upper abdominal pain. Pain can be quite debilitating and impairs the quality of daily life. Patients can go undiagnosed for months to years and undergo several investigations. We present a case of a 20 year old man, having upper abdominal pain for more than 2 years. He underwent a battery of investigations several times without any benefit. Finally, he was diagnosed with slipping rib syndrome. He underwent surgical treatment at our center following which his symptoms completely resolved. The diagnostic workup and treatment options for the condition will be discussed.

Hydatid disease of the Lung: single centre experience Sreekar B.

Sheeja Varghese

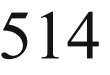

\section{ABSTRACT:}

BACKGROUND:

Hydatid disease is a parasitic infestation which is endemic in many sheep and cattle raising areas and still an important health hazard in the world. This study is a description of a single center experience, outlining the efficacy and complications of surgical interventions in the management of pulmonary hydatidosis.

\section{METHODS:}

Retrospective study of 5 cases of pulmonary hydatid disease who underwent surgery between January 2018 to September 2018 in St.John's Medical College Hospital, Bangalore.

\section{RESULTS:}

All the cases considered in this study were young adults (ranging from 16-35 years) with two of them having a past history of pulmonary tuberculosis. All the patients presented with cough $(n=5,100 \%)$ followed by chest pain $(n=4,80 \%)$. those with past history of TB presented with hemoptysis. Two patients had hydatidosis of both lung and liver and in them upper abdominal pain was a significant presenting complaint. Most commonly performed surgical intervention was pericystectomy $(n=3,60 \%)$ followed by enucleation $(n=1,20 \%)$ and lobectomy $(n=1,20 \%)$. In both the patients with Liver hydatid disease, Laparotomy and evacuation of the cyst was done. There was no significant Post operative complications. CONCLUSIONS: 
Surgical management in patients with Pulmonary hydatid disease provided good outcomes, with no recurrence noted in our study.

\author{
A Rare Case of Sequestration Lower Lobe of Right \\ Lung with an Abnormal Arterial Supply from the \\ Celiac Trunk \\ Abdul Rasheed \\ Rajendran M.C.
}

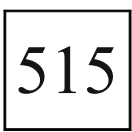

\section{ABSTRACT:}

Bronchopulmonary sequestration is an important differential diagnosis of Lower lobe broncheictasis. It is more common on the left side. Another salient feature of lower lobe sequestration is that the arterial supply can be from infra diaphragmatic aorta. This awareness is absolutely necessary for a surgeon who is planning lower lobectomy for symptomatic bronchiectasis. Missing this crucial fact may lead to concealed haemorrhage into the abdominal cavity which may in turn endanger the life of the patient. Our case report is unique because of its right sided nature and its abnormal arterial feeding vessel from the abdominal aorta.

Tracheal reconstruction versus rigid bronchoscopy in benign tracheal stenosis - our institutional experience N. Ajay; Rakesh Kumar

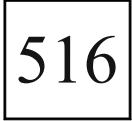

\section{ABSTRACT: \\ INTRODUCTION:}

Tracheal stenosis may be congenital or acquired. The most common cause is due to iatrogenic injury following intubation. It is mandatory to evaluate and precisely analyze the length and grade of tracheal stenosis. Flexible bronchoscopy and Computed Tomography were reliable diagnostic tools for evaluating the site and length of the stricture, helps in proper planning for surgery and good outcome.

\section{AIMS AND METHODS:}

To study about the outcome of tracheal resection and anastomosis \&amp; tracheal dilatation with rigid bronchoscopy based upon the grade of stenosis MATERIALS AND METHODS:

21 cases of benign tracheal stenosis treated at Rajiv Gandhi Govt General Hospital, Chennai from August 2016 to August 2018 were analyzed retrospectively based upon the type of management which includes resection of stenosed trachea and anastomosis \&amp; dilatation of trachea with rigid bronchoscopy in our institute.

\section{RESULTS:}

From our institutional experience we conclude that tracheal reconstruction is far superior when compared to rigid bronchoscopy and dilatation of trachea in terms of improvement in orifice diameter and symptom free interval. But In cases of emergency, rigid bronchoscopy still plays a vital role.

Pneumonectomy for an unusual case of Cyanosis: Multiple diffuse pulmonary arteriovenous malformations

Sabyasachi Bal

Kamran Ali

\section{ABSTRACT: \\ INTRODUCTION:}

Diffuse pulmonary arterio-venous malformations (PAVM) is a very rare vascular anomaly of the lung characterized by abnormal direct communication between the pulmonary arterial and venous systems. We present a case of multiple diffuse PAVM managed by a pneumonectomy not reported before in literature.

MATERIALS \&amp; METHODS:
A 7- year girl was brought with a history of cyanosis and failure to thrive for last 2.5 years and a short history of hortness of breath. On examination she was plethoric, had digital clubbing and room air oxygen saturation $(\mathrm{SpO} 2)$ was 70 $75 \%$. On Xray Chest she had multiple nodules in the right lung field. Cardiac catheterization and angiography revealed diffuse PAVM of the right lung

\section{RESULTS:}

She underwent a right pneumonectomy via a posterolateral thoracotomy. On table she documented an improvement in $\mathrm{SpO} 2$ from $75 \%$ to $95 \%$ after ligation of all pulmonary veins. She was discharged on day 4 after surgery. At one year follow up she has gained weight, her clubbing has reduced and maintains normal ambient air oxygen saturations.

DISCUSSION:

Surgery for a diffuse PAVM restricted to a lobe or segment is usually parenchyma preserving. But in this case as the AVM were present in all lobes of the right lung in a diffuse fashion, we had to perform a pneumonectomy. To the best of our knowledge this is the first case of pneumonectomy for diffuse multiple PAVM.

Pulmonary sclerosing emangioma

Alberto Oliaro; Pierluigi Filosso; Enrico Ruffini; Paolo Olivo Lausi; Elisa Fontana

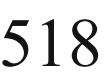

\section{ABSTRACT:}

Pulmonary sclerosing emangioma, is an unfrequent benign pulmonary disease, of wich the pathogenesis and the clinical behavior is still not fully understood.

The most frequent presentation of the disease is a solitary pulmonary nodule, often asymptomatic.

The vaste majority of the PSE are smaller than $3 \mathrm{~cm}$, although it can grow up to $7 \mathrm{~cm}$ in diameter.

Enhanced CT of the chest demonstrates a round to oval lesion with smooth margins, without any sign of calcification or cavitation that can easely be interpretated as a carcinaoid or an amartocondroma.

In the overwhelming majority of the cases, PSE show increased FDG uptake, exceedeling more than 2.5 SUV max value, which reflect a malignant metabolic fingerprint.

We present the case of a 62 years old woman, HCV positive, never smoker, without any respiratoy symptoms or history of smoking, which visited our hospital for evaluation of a solitary pulmonary nodule in her righ upper pulmonary lobe.

The CT scan confirm the presence of a 2,8 cm lesion in the right upper lobe, suggesting the neoplastic nature. There was no sign of enlarged lymph node or pleural involvement.

A PET - CT scan was performed and demonstrated uptake of FDG at the nodule SUV max 2.9.

The patient undergone a wedge resection of anterior segment of the right upper lobe through a uniportal VATS acces.

No malignant cells were evident in the frozen sections of tissue retrieved during the operation, but at the same time was not clear the nature of the lesion. It was performed a nodal sampling as precautionatory mesasure. Immunohistochemical stains were positive for: thyroid transcription factor 1 (TTF-1), estrogen receptor (ER), CD15, Epithelial Membrane Antigen (EMA), Ep-CAM/Epithelial Specific Antigen (Ber-EP4), Vimentin.

High-resolution CT scans were peformed as follow-up for 5 years and have not detected any recurrence or distant metastasis.

A rare case of a pleuro-pulmonary neoplasia Alberto Oliaro; Pierluigi Filosso; Enrico Ruffini; Paolo Olivo Lausi; Erika Passone
ABSTRACT: 
We present the case of a 35 - year - old man, non-smoker without any previous oncological disease, admitted to our department for chest discomfort, dyspnea and temperature.

The CT scan, performed in an other institution, demonstrated the presence of vast pleural effusion occupying the entire right pleural cavity.

The laboratory findings on admission showed thrombocitosis, and leukocytosis.

In accordance with the hypothesis of an empyema in a young non-smoker patient, we have performed a thoracoscopic uniportal procedure.

During the VATS procedure we have identified a large solid mass invading the visceral pleura, the pulmonary parenchima, the mediastinum and, probably the large vessels.

The frozen sections demonstrated an highly anaplastic mesenchymal lesion, reported as pleuro-pulmonary blastoma.

The cardiovascular-surgical team was involved for the possibility of an hemodynamic instability during the procedure.

The unanymous opinion was the impossibility of gain a controll of the disease with a surgical resection.

On this basis we have decided to perform a sort of pleural toilet, through a limited thoracotomy, with the aim of obtaining the best lung expansion possible.

The patient stayed in intensive care unit for eleven days.

The chest tube was removed in IV postoperative date.

The final histopathological diagnosis was: biphasic malignant neoplasia, related with malignant immature teratoma with foci of yolk sac tumor and fetal adenocarcinoma.

A multidisciplinary thoracic tumor board decided to treat this extremely rare neoplasia as a sarcoma.

Ifosfamide and Gemcitabine were used as first-line chemotherapy.

Nine months after six cicles of chemotherapy the patient was alive with stable disease.

In the common practice of Thoracic Surgery it is necessary to consider the possibility of treating an extremely rare pathology.

In particular it is necessary to underline the possibility of missunderstading the neoplastic nature of a lesion due to radiological characteristics of the pathology and the clinical presentation of the patient.

\section{Two cases of aortoesophageal fistula associated with thoracic aortic aneurysm \\ Hiroyuki \\ Tomoyuki}

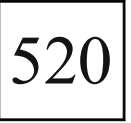

\section{ABSTRACT: \\ INTRODUCTION:}

Aortoesophageal fistula (AEF) is categorized as either primary or secondary. In addition to primary AEF associated with a thoracic aortic aneurysm, due to the increase in the frequency of stent-graft operations, more cases of secondary AEF have been reported. The incidence of secondary AEF was reported to be $1 \%$ to $5 \%$, and the prognosis is extremely poor. In this meeting, we report 2 cases of AEF along with a review of relevant literature.

In our cases, prognosis after AEF was extremely poor, and the possibility of saving life with temporary general treatment was low. Therefore, it is difficult to save life without complex invasive surgery.

Many reports have confirmed that deconcentrating invasion by gradual operations provides good results.

However, deciding which operation to perform at which phase, especially the timing of an infected graft removal operation after the removal of the esophagus, is difficult.

This is because the infection in the aorta remains after esophageal removal, which is the most invasive surgery among gradual surgeries.

In our experimental cases, normalization of C-reactive protein level and white blood cell count on blood collection and normalization of albumin level were used as indicators. However, we consider that the surgical timing should be judged comprehensively on the basis of infection control and nutritional status improvement.

In addition, some reports indicated that esophageal removal and infectedgraft replacement should be performed simultaneously. However, gradual operations seem to be more advantageous in terms of size of invasion and prevention of infection through graft replacement.

As the frequency of TEVAR increases, an increase in the incidence of secondary AEF is expected.

In case of AEF, prompt treatment by understanding early symptoms and executing gradual operations might increase the chance of survival.

Case of Empyema mimicking CA Lung

Gowri Shankar

S. Dinesh

ABSTRACT:

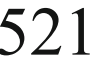

50 year old male came with complaints of cough with expectoration and chest pain for 3 months.

CT Chest done somewhere revealed a mass of about $10^{*} 8 \mathrm{cms}$ in $\mathrm{Rt}$ lower lobe with 2 to 3 mediastinal lymph nodes

CT guided biopsy done 2times by a reputed Hospital revealed large cell carcinoma Lung one time and the biopsy was negative for malignancy in second time. but showed only inflammatory and necrotic tissue in that site. so they did a PET CT which confirmed the malignancy in Rt lower lobe and positive nodes inmediastinum.

Pt came to me and I planned for Rt lobectomy.

on opening through Rt posterolateral thoracotomy, there was only multiloculated empyema with franck pus with few nodes.

there was no mass or suspicious tissue but only some necrotic tissues with nodes.

did only Rt decortication with pus culture with biopsy of necrotic tissue and nodes. we deferred Lovectomy or pneumonectomy.

post operative CXR revealed full expansion of Rt Lung with and biopsy negative for malignancy with only inflammatory tissue.

pus culture no growth

pt discharged and doing well.

the purpose of presenting this case is to demonstrate that even both tissue diagnoses and PET CT can misguide us and a clear decision with intuition prevents unnecessary lung resection

Empyema as a surrogate presentation of pleural malignancy

Bhushan Thombare; A.Z. Khan

Nikhil Rane

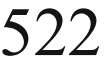

\section{ABSTRACT:}

OBJECTIVE:

To report histopathological confirmation of malignancy in patient operated for drainage of empyema and VATS decortication

\section{METHODS:}

842 patients underwent drainage of empyema and VATS decortication at a single centre. Histopathological reports were analysed to identify the incidence of malignancy.

\section{RESULT:}

8 patients were found to have pleural malignancy. These included mesothelioma, metastatic adenocarcinoma, metastatic hepatocellular carcinoma, metastatic colorectal carcinoma, metastatic ovarian cancer. Most patient were confirmed to be tuberculosis or pyogenic empyema needing antibiotic or anti tuberculosis therapy. The malignancy patients were send for palliative chemotherapy. Prognosis was severely impacted due to histopathology.

\section{CONCLUSION:}

All empyema is not infective. A high index of suspicion of metastatic cancer has to be kept in cases with previous malignancies. 
Role of PET scan for staging mediastinum in lung cancer Bhushan Thombare;

A.Z. Khan

Nikhil Rane

\section{ABSTRACT:}

AIM:

Retrospective analysis of impact of PET scanning in management of lung cancer in Indian patients.

METHODS:

355 patient underwent surgery for lung cancer. PET scan was done preoperatively for assessment of primary tumour and metastatic staging as per British thoracic surgery guidelines. Histology of mediastinal lymph nodes was performed to understand the impact on staging.

\section{RESULTS:}

$12 \%$ patients underwent down-staging in a PET positive mediastinum. Essentially these patients were found to have concurrent tuberculosis or sarcoidosis in addition to lung cancer. They were thereby down-staged and had improved prognosis and did not need adjuvant therapy. $10 \%$ of patients had a positive histology for lung cancer in a PET negative mediastinum. They were upstaged and needed further adjuvant therapy.

\section{CONCLUSION:}

PET positivity and negativity of mediastinum should be interpreted with caution in Indian scenario. There may be associated concurrent tuberculosis or sarcoidosis.

\section{Does Radiotherapy make VATS impossible}

Bhushan Thombare;

A.Z. Khan

Nikhil Rane

\section{ABSTRACT:}

OBJECTIVE:

To present data and video evidence that Video Assisted Thoracic Surgery and Robotic Thoracic surgery is possible in the presence of previous chemotherapy and radio therapy in the management of lung cancers

\section{METHODS:}

10 patients underwent surgery for lung cancer with previous history of stage IIIA cancers. They were primarily treated with chemoradiotherapy. A restaging PET scan confirmed reduction in size and activity of the tumour thus making them amenable for surgery. Access was gained by Video assisted Thoracoscopic surgery and also by Robotic Thoracic surgery.

\section{RESULTS:}

The entire resection was done by minimally invasive thoracic surgery and tumour was delivered using endoscopic bag. Surgery included left upper lobectomy, Right upper lobectomy, segmentectomy, Middle lobectomy, Resection of mediastinal mass (thymomas, Germ cell tumour) and systematic mediastinal nodal dissection.

The advantages of these techniques include

1. Smaller incisions

2. Less pain

3. Early discharge

4. Less morbidity

5. Less immunological / inflammatory response

6. Better visualisation and better ergonomics due to robotics

7. Less bleeding due to better adhesiolysis as compared to open thoracotomy

\section{CONCLUSION:}

Our video evidence abolishes the myth that previous radiotherapy and Chemotherapy are a contraindication to minimal access surgery. Prior to doing a thoracotomy, we strongly believe that the thoracic surgeon must at least put in a camera into the chest to assess the situation.

Use of VATS placement of Intra thoracic Inlay Mesh

for Lung herniation

Bhushan Thombare; A.Z. Khan

Nikhil Rane

\section{ABSTRACT:}

OBJECTIVE:

To demonstrate a new technique of intrapleural placement of Inlay mesh by video assisted thoracoscopic surgery (VATS) for treatment of Lung herniation.

\section{METHOD:}

65year old patient presented with history of fall and injury to right chest wall. This was followed by chest infection and bouts of severe cough. That led to displacement of fractured $6,7,8,9 \mathrm{rib}$. Subsequently he developed a cough on impulse and painful herniation of right lung.

Instead of a thoracotomy and fixation of ribs, a decision was taken to perform the procedure by VATS. An rolled up Intrapleural mesh was delivered via a port into the thoracic cavity. The mesh was unfolded and fixed to anterior and lateral chest wall using tackers. transdermal stitches were also applied at 4 corners to ensure fixation of the mesh. The knot was pushed subdermally by a small nick on the skin.

\section{RESULTS:}

Herniation stopped immediately and patient was able to be discharged on day 2 post surgery. The rib fractures healed on subsequent X-rays. Follow up at 1 year showed an intact repair. Pain was well controlled and patient was satisfied by repair.

\section{CONCLUSION:}

Intra thoracic placement of inlay mesh allows control of herniation. The mesh stays anchored to the chest wall due to tackers and outward movement of the lung. Hence there is no risk of mesh migration.

\section{Surgical fixation of flail chest using sternal wires reduces duration of ventilation and hospital stay to patients and cost effective to government hospitals Gautam Sengupta; S. Bhattacharya \\ Rohit Kumar Rathi}

\section{ABSTRACT:}

\section{OBJECTIVES:}

The standard treatment of flail chest includes pain control with analgesics, intercostal drains, epidural analgesia and mechanical ventilation when needed. Mechanical ventilation, while potentially lifesaving, carries risks of pneumonia, septicaemia, lung injury, lung collapse and tracheostomy. Surgical stabilization of flail chest has been found to reduce length of stay and complications and to improve long term quality of life when compared with standard methods of care. Sternal wires are easily available in cardiothoracic departments and are being used in our institution for rib fixation with good outcomes and has effectively reduced the cost of surgery.

\section{METHODS}

Between September 2016 and September 2018, 16 patients with flail chest undergoing rib fixation were matched with 14 patients with conservative management. Surgery was done in first 48 hours.

\section{RESULTS:}

There was no significant differences between groups for age, gender and trauma prognostic score. However the mean ventilator time (18 vs 74 hours, P\&lt; 0.01 ) and ICU stay (4 vs 8 days, P\&lt;0.01) was significantly lower for operative patients. Mortality and overall hospital stay were also decreased in patients with surgical stabilization.

\section{CONCLUSION:}

Rib fixation with sternal wires is feasible and decreases the ventilator timing, length of ICU and hospital stay and improves the pulmonary function test of patients with flail chest. However, sternal wires being 
easily available in Cardiothoracic department and much cheaper in comparison to titanium plates makes it more feasible and also cost effective to government hospitals.

\author{
Intrathoracic Giant Thymolipoma - A Series of \\ case reports \\ Murugan M.S.; Krishnanand Pai; Ganesan C. \\ P.R. Murugesan
}

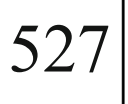

\section{ABSTRACT: \\ BACKGROUND:}

Intrathoracic lipoma is a rare condition. Giant intrathoracic lipoma presents with breathlessness mimicking like pleural effusion is the usualo presentation. Although intrathoracic lipoma is a benign tumour, it should be completely resected in favour of diagnosis \&amp; therapeutic perspectives. We would like to present a series of 3 cases.

\section{MATERIALS AND METHODS:}

All 3 cases presented with R sided chest discomfort, effusion, breathlessness for few months duration. Male : 1 Female : 2 Avg age : 40y. Workup revealed - Lung function test : Restrictive lung function, CT Chest : Large fatty density ( - 105 Hounsfield units ) with underlying lung compressive atelectiasis. Right thorocotomy \&amp; complete excision done.

\section{RESULTS:}

Post-op uneventful. Followup after 6 months satisfactory. Lung expansion good. No residual pleural disease.

\section{CONCLUSION:}

Intrathoracic lipoma never evolves towards liposarcoma. Surgical resection is still necessary to exclude malignancy and other mechanical complications during surgery. One should remove the lipoma in toto \&amp; take care of the vital structures.

\section{Surgical management of benign tracheal stenosis-our institutional review \\ G. Sengupta; Rohit Rathi \\ Chaina Paul}

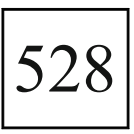

\section{ABSTRACT: \\ OBJECTIVES:}

Post-intubation injuries and tracheostomy are the most frequent conditions leading to tracheal stenosis. Tracheal surgeries are very challenging and requires meticulous surgical techniques. Single-stage resection and reconstruction by sound surgical techniques results in good or satisfactory results in $92.54 \%$ of patients, with a failure rate of $5.97 \%$ and a mortality rate of $1.49 \%$. We review our experience with surgical management of tracheal stenosis.

\section{METHODOLOGY:}

Over a 5-year period from august 2013 to august 2018, 67 patients aged $14-64$ years with moderate (50\%-70\% of the lumen) to severe ( $\& \mathrm{gt} ; 70 \%$ ) tracheal stenosis underwent resection and primary anastomosis. Patients were followed up to assess the surgical outcomes.

\section{RESULTS:}

Resection and primary anastomosis was performed via either cervical incision (65 patients) or median sternotomy(2 patients). In 34 patients with subglottic stenosis, complete resection of the tracheal lesion and anterior portion of cricoid cartilage was performed, in 5 patients hyoid drop performed and in 2 patients hilar mobilization performed and the remaining trachea was anastomosed to the thyroid cartilage. 2 patients had restenosis, of which 1 required repeated dilation and other required repeat surgery. 2 patient had wound infection., There was only one perioperative death in a patient with a tracheo-innominate fistula.

\section{CONCLUSION:}

The result of resection of stenotic segment and primary anastomosis is highly successful in the treatment benign tracheal stenosis Fewer complications like-restenosis, trachea-innomonate fistula, anastomotic dehiscence etc may occurs in the post operative period depending on various factors.

VATS or RATS, is the jury out?

Bhushan Dinkar Thombare

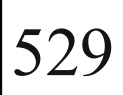

\section{ABSTRACT:}

AIM:

To identify the benefits of VATS and to define the additional benefits of RATS in the modern era

\section{METHODS:}

Personal practice and published literature was reviewed to quantify the benefits of VATS versus RATS

\section{RESULTS:}

VATS offered the following benefits

1 Smaller incisions

2 Less pain

3 Early discharge

4 More turnover of patients

5 Early improvement in lung function

6 Less inflammatory response, less immune response and less infection Robotics added the following features

1 True HD 3D vision

2 High dexterity of surgical instruments with 7 degrees of freedom of endowrist

3 Better ergonomics

4 Less assistance

5 Dual console for teaching

6 Diathermy in both hands hence ambi dexterous

7 Tremor filtering

8 No mirror image, intuitive, shorter learning curve

9 Increased ability for suturing- hence good for sleeve resection, plication of diaphragm, ligation of thoracic duct

10 Better and safer movement s at the thoracic outlet

The disadvantages and complications will be discussed.

CONCLUSION:

VATS has become the gold standard in treatment of thoracic diseases. Robotics adds significant advantage in diseases of mediastinum, thoracic outlet, esophagus and complex lung resections.

The learning curve for robotics is shorter.

\section{Designing New instruments to facilitate}

Minimally Invasive Techniques in third world countries: Strategies for moving room table to market Thombare B.; Rane N.; Khan A.Z.

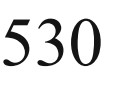

\section{ABSTRACT:}

AIM:

The ability to perform VATS is limited by availability and cost of expensive laparoscopic instruments. Endemic diseases like tuberculosis necessitate a need for redesigning instrument to suit specific operations.

We describe a method of creating ones own indigenous instruments to facilitate video assisted thoracoscopic surgery in any setting.

\section{METHODS:}

Need for a specific instrument is identified. A rough drawing is made on a plain paper. 3D designs are created using AutoCad software. Files are transferred to online $3 \mathrm{D}$ printing services. A prototype is prepared and send to international and local companies. Our designs have been accepted for production by local companies at substantially reduced cost for local market. International companies have also accepted the designs for international market. 


\section{RESULTS:}

Our designs have been accepted for production by local companies at substantially reduced cost for local market. International companies have also accepted the designs for international market. This gives exposure to Indian doctors to international fame and income potential.

\section{Role of Multidisciplinary tumor board in management} of thoracic malignancies

Bhushan Dinkar Thombare

\section{ABSTRACT:}

AIM:

To analyse the impact of multidisciplinary tumor board on management of patients with thoracic malignancies

METHODS:

All patients undergoing treatment for thoracic malignancies were discussed in a multispeciality tumor board. Analysis of decision was made from the medical records and minutes of the meeting. The impact was to define a change in treatment for the patient. Many decisions were changed due to the input of the respective specialities and review of current literature.

\section{RESULTS:}

These changes happened as a direct impact of multidisciplinary team opinion:

needed metastatectomy for Sarcoma preferentially over radiotherapy preoperative chemotherapy for down-staging thymoma and then rediscussing for surgery

Multispeciality input to management of germ cell tumour.

Need for preoperative mediastinoscopy prior to chemotherapy

cancellation of liver transplant due to preoperative VATS diagnosis of metastasis

Need for neoadjuvant radiotherapy for tracheal tumours

Decision to give SBRT for unfit surgical patients.

CONCLUSION:

Discussion in a multidisciplinary tumour board impact patient care in thoracic malignancies. The treatment is now evidence based.

\section{Unusual endobronchial foreign bodies}

Nikhil Rane

Bhushan Dinkar Thombare

\section{ABSTRACT:}

\section{OBJECTIVE:}

We set out to present our experience of extracting unusual endobronchial foreign bodies

METHODS:

A total of 11 cases were diagnosed roentogenographically and bronchoscopically at our hospital. These patients consisted of 10 men and 1 woman with a mean age of 48.5 years (range 10 to 77 years). Symptoms on presenting were usually cough, sputum, or chest pain. Three patients were not aware that they had aspirated a foreign body. Rigid bronchoscopy and retrieval of foreign body was performed in all the cases.

RESULTS:

The articles removed were betelnut, peanuts, denture, antihypertensive tablet, plastic button, coin. There were no severe complications during or after the endoscopic removal of the foreign bodies.

\section{CONCLUSION:}

In conclusion, rigid bronchoscopy is useful for the diagnosis and treatment of endobronchial foreign bodies.

Plastic reconstruction of chest wall defects after tumour excision - how we do it.
Smruti Prakash Sahu;

Mohanraj R.

\section{ABSTRACT:}

Defects in the chest wall following oncological surgery need reconstructive procedures, to avoid paradoxical chest wall motion. We present our experience in chest wall reconstruction in 10 such patients over the last 9 years. Eight were females. Age ranged from 20 years to 58 years. The histopathology of resected tumours included malignant sarcoma (1), benign chondroma (1), malignant chondrosarcoma (1), malignant phyllodes tumour (2), malignant invasive ductal carcinoma (1), benign fibromatosis (1), malignant Ewing's sarcoma (2) and fibrous dysplasia of rib (1). Chest wall defects after oncological resection were reconstructed using a contoured double layer Polypropylene mesh (Bard Mesh subsidiary of C.R.Bard,Inc) with bone cement ( SmartSet HV by DePuy International Ltd.) sandwiched between the two layers of the mesh. Chest wall reconstruction was successful in all the ten patients and all survived the operation. One patient died 2 year after surgery due to chemotherapeutic complication. Duration of follow up in remaining patients ranged from 2 months to 9 years. Three patients lost to follow up since 2014, 2015 and 2016. Two patients developed infection of the composite prolene mesh needing removal.

Chest wall reconstruction with bone cement sandwiched between prolene mesh is a robust and safe method for reconstruction of chest wall defects with low complication rate and satisfying short and medium term result.

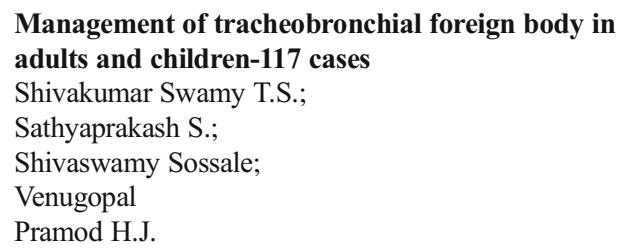

\section{ABSTRACT:}

Accidental aspiration is the commonest cause of tracheobronchial foreign body in children as well as adults, high index of suspicion is required in all cases and subjected to diagnostic bronchoscopy even though radiological signs are negative for foreign bodies. Early diagnosis and intervention are at most priority to avoid further complications. In paeditric patients with recurrent, non resolving lung infections should be evaluated for tracheobronchial foreign body. Rigid bronchoscopy is an essential prerequiste in majority cases of foreign body removal, complimented by flexible bronchoscopy. Only a few patients required surgery, who presented with complications who were miss diagnosed with fewer symptoms during inital period due to small size of trachebronchial foreign body.

Penetrating knife Injury to the heart: Does cardiopulmonary bypass help?

Amit Aggarwal; Anubhav Gupta; Jagdish Prasad

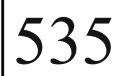

\section{ABSTRACT:}

We report a case of penetrating cardiac injury seen in our hospital. The patient sustained penetrating chest trauma due to a homicidal attempt, and had borderline haemodynamics on arrival at the emergency department. We suspected cardiovascular injuries from the clinical picture and imaging and echocardiography findings. The case was operated six hours after injury. Approach was via median sternotomy. During the operation, we found that 
the patient had pericardial injury with a rent in the right ventricle extending to atrioventricular groove. The tears were repaired using pledgeted sutures on cardiopulmonary bypass. Patient was discharged after 5 days.

We believe that patients with cardiac rupture who are alive when they reach hospital can often be saved by prompt diagnosis and immediate, adequate surgical repairs. \&nbsp;

Giant anterior mediastinal thymic cyst - a case report C.S. Hiremath

Naveen Pandarinathan

\section{ABSTRACT:}

\section{INTRODUCTION:}

Thymic cysts are uncommon lesions. They may be located in the cervical region or mediastinum. It may be of common congenital unilocular type or rare acquired multilocular type. Rarely develops symptoms when large in size and due to pressure effect. The characteristic CT findings include a homogeneous mass of low attenuation value and an indistinct capsule.

\section{BACKGROUND:}

The presence of thymic tissue within the wall is essential for diagnosis. Treatment options for unilocular cyst include observation, aspiration under CT guidance or surgical excision via open method or VATS. There is a possibility of development of squamous cell carcinoma in thymic cyst. Hence, excision is preferred for both diagnosis and cure.

\section{CASE:}

A 35 years old female patient presented with symptoms of dyspnoea, atypical chest pain, odynophagia since 3 months. Chest $\mathrm{x}$ ray showed a massive radioopaque lesion in the left hemithorax. CT thorax revelaed a $11 \times 8 \mathrm{x}$ $15 \mathrm{~cm}$ well defined hypodense lesion in the left anterior mediastinum extending from 1st to 6th intercostal space cloacking along the left cardiac order and causing mass effect on the left upper lobe and lingular segments with a differential diagnosis of pericardial or thymic cyst. Intraoperatively, the cyst was arising from the left thymic lobe. Through midline sternotomy, excision of the cyst with intact capsule arising from the left lobe of the thymus along with right lobe was done. Histopathology revealed squamous to cuboidal epithelium in the cyst wall with thymic tissue.

\section{CONCLUSION:}

Thymic cysts are uncommon congenital unilocular mediastinal lesions. Rarely symptomatic due to pressure effect. Surgical excision is useful for diagnosis, prevent rare development of carcinoma and for cure.

\section{Combined management of coronary artery disease and lung cancer - a challenge foreseen and reviewing the literature \\ Arvind Kumar \\ Navdeep Singh Nanda}

\begin{abstract}
: to manage these difficult situations. year disease free survival at follow up. combined cardiac and lung resection scenario and safe outcomes.

\author{
A two year Experience in Management of posterior \\ mediastinal Schwannoma. \\ VijayKumar
}

Combined management of coronary artery disease and lung cancer is a challenge foreseen. Various surgical strategies have been described in the literature from 1 stage to 2 stage surgical procedure or opting for a nonsurgical management for either. Still no consensus guideline is there to direct the pathway

We present the case of a patient operated for left pneumonectomy and off pump CABG through mid-sternotomy incision in 1 stage procedure with 1.5

A critical look on the literature to pave pathway for similar situations of

\section{Samuel John Nehemiah}

\section{ABSTRACT:}

Posterior mediastinal schwannoma is the most common posterior mediastinal tumour and has a variety of clinical presentations which could be symptomatic or asymptomatic regardless of the size of the tumour. We present an experience of 8 cases of schwannoma over 2 years, with size ranging from $3 \mathrm{~cm}$ to $15 \mathrm{~cm}$. The various locations of the tumours found being in the thoracic inlet to tumour extending across the diaphram in to the abdominal cavity. Clinical diagnosis was made by radiology, either CT or MRI or both and rarely required FNAC.

These required various approaches like posterolateral thoracotomy, Sternotomy and Thoracoabdominal approach. This often required multidisciplinary approach including Neurosurgery for the safe removal of the tumours.

Early diagnosis, Prompt treatment and Successful outcome of Iatrogenic tracheal injury: a case report Vinay Krishna

Anumeet Bagga

\section{ABSTRACT:}

\section{INTRODUCTION:}

Iatrogenic tracheal injuries are rare complications. The literature reports only five cases of tracheal injury which were caused by median sternotomy during cardiac surgeries. Among these, one case was caused by the electro- cautery prior to sternotomy, other causes being orotracheal intubation, tracheal dilation, tracheostomy, internal tracheal stenting or esophagectomy.

\section{BACKGROUND:}

Laryngotracheal injuries are uncommon and can be very deceptive. Serious injury to laryngotracheal anatomy could exist even in the absence of any visible external injury.

\section{CASE:}

18 year female was operated for ASD (atrial septal defect) under cardiopulmonary bypass and was extubated after 24 hours post surgery. Her vitals and arterial blood gas were normal though there was air leak in her right pleural drain so the drain wasnot removed. On 8th postoperative day, she spontaneously developed subcutaneous emphysema on her neck and upper chest with right drain still in situ. The main wound showed movements coinciding with respiration. Chest $\mathrm{X}$ ray showed bilaterl pneumothorax and pneumomediastinum. Alarm was raised regarding upper airway tract injury. The patient was immediately shifted to operation theatre. Sounds indicative of air leak were heard from the operating field, following which the surgical team identified an approximately $2 \mathrm{~mm}$ area of tracheal injury. The tracheal tube was advanced and placed just above the carina, so that the cuff was located below the injury site. The site of injury was directly repaired using absorbable sutures (PDS 3-0; Johnson \&amp; Johnson) over teflon pledgets using interrupted horizontal mattress and covered with mediastinal fat tissue. Following repair of the injury, no air leaks were observed. The patient recovered fully and was later on discharged.

\section{CLINICAL RELEVANCE:}

One needs to be very cautious in recognising tracheal injury keeping high degree of suspicion and immediate repair should be cosidered.

VATS in large recurrent pneumatocele with onlay mesh Dasari Kalyani Rama

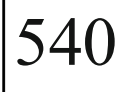

ABSTRACT:

\section{INTRODUCTION:}

Pneumatoceles are air-filled lung cysts as a result of bronchial injury or inflammation most commonly in children as sequelae of severe pneumonia

Material and Methods: 
A 2-year-old girl presented with past $\mathrm{H} / \mathrm{O}$ severe pneumonia with pneumatocele managed by VATS outside and with recurrence with cough and dyspnoea admitted in our hospital in Nov 2018 and underwent VATS with closure of pneumatocele $(7.8 \mathrm{~cm} \times 2.6 \mathrm{~cm})$ multiloculated with onlay mesh.

\section{RESULTS:}

Pneumatocele is diagnosed with ultrasound, chestxray. On CT scan with large pneumatocele comprising lung parenchyma. underwent VATS, marsupialization followed by onlay mesh. Lung expansion achieved with $100 \%$ saturation. Child extubated on table and discharged on 8thday. postop CXR, U/Scan normal.1st follow up CXR, U/scan normal and recovered well.

\section{CONCLUSION:}

VATS with onlay mesh seems to be effective and safe for large pneumatocele.

\section{Hydro-dissection aided uniportal thoracoscopic pleurectomy for spontaneous pneumothorax Sabyasachi Bal Kamran Ali}

\section{ABSTRACT: pleura. sequent stripping of the pleura easy and fast. \\ Thoracoscopic ultrasound: a good supplement in video-assisted GGO surgery \\ Zhang Hongbin}

Pleurectomy forms an important component of surgical management of recurrent primary spontaneous pneumothorax for inducing pleural symphysis, following a bullectomy. Uniportal video assisted thoracoscopic surgery (VATS) is frequently being employed for performing a pleurectomy but may pose certain anatomic challenges, owing to various angulations of the chest wall and thinness of parietal

We describe our subtle modification of uniportal pleurectomy using hydro-dissection, for the inexperienced surgeon. This is a simple and easy technique utilizing hydrodissection which helps in creation of an avascular plane between the pleura and endothoracic fascia which makes sub-

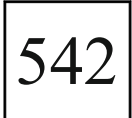

Localization of GGOs with more than $1 \mathrm{~cm}$ distance to the pleura are still an challenge to thoracic surgeon during VATS surgery. The efficiency, non-damage, safety are uppermost factors to be considered when choosing the location technique.

Our center tried the thoracoscopic ultrasound as a complementary means during VATS GGO surgery. We evaluated 40 GGO patients divided with group A (VATS-US) and group B (VATS).

We found that the use of ultrasound during VATS surgery could Improve the success rate of localization(p\&lt;0.05), especially in the patients hard to conduct the Preoperative CT guided localization(p\&lt;0.01). The operation time, bleeding in operation, postoperative complications between the two groups showed no statistical difference $(\mathrm{p}>0.05)$.

We concluded that the thoracoscopic ultrasound could be an excellent supplement to the Preoperative CT localizing technique if not replacement at present.

Chondroblastoma of the sternum - report of a rare case Rejith Mathews Philip

\section{INTRODUCTION:}

Benign tumors of the sternum are exceedingly rare, as is chondroblastoma among bone tumors, and sternum as the location of a primary bone tumor. A combination of all three was found in a 22 year old male. The details of clinical, radiological and surgical findings, and the reconstructive technique used are reported here.

AIMS AND OBJECTIVES:

To report a case of chondroblastoma of the sternum and describe the treatment performed.

\section{MATERIALS AND METHODS:}

A 22 year old male presented with a rapidly growing, painful swelling over the body of the sternum about $9 \times 5 \mathrm{~cm}$ in size. An expansile osteolytic lesion occupying the body of the sternum, extending to the adjacent costal cartilages was found on imaging. Pre-operative core needle biopsy was suggestive of a giant cell tumor, a differential diagnosis of chondroblastoma.

\section{RESULTS:}

Tumor resection was performed along with the involved portion of the lower half of the manubrium, body of the sternum, xiphisternum and involved portions of the costal cartilages. The defect was reconstructed in layers with a titanium mesh over a prolene mesh, and covered with bilateral pectoralis major muscle advancement flaps. The tumor was diagnosed to be a chondroblastoma on final histopathology. Adequate resection margins were achieved. The patient recovered well with no paradoxical chest wall movements.

\section{DISCUSSION \&amp; CONCLUSION:}

Tumors of the sternum are usually considered malignant unless proven otherwise. The surgeon should be aware of the spectrum of benign tumors, though uncommon, as tumors like chondroblastoma might be locally aggressive and mimic malignancy. An excellent functional outcome and recurrence free survival can be provided with good surgical clearance and efficient reconstructive techniques.

Tricuspid regurgitation after mitral valve replacement surgery with st.jude mechanical valve at 108 military central hospital

Do Xuan Hai; Hoa Nguyen Thi; Nam Nguyen Van; Mai Van Vien

\section{ABSTRACT: \\ INTRODUCTION:}

We all agreed that systemic mitral valve repair should be done in tricuspid valve (TR) surgery (Rheumatic fever). We corrected the TR according to the guidelines of AHA and ESC with TR measured in echocardiography and measured in surgery from $35 \mathrm{~mm}$ and using PTFE strips. The rate of tricuspid regurgitation after mitral valve replacement with SJM mechanical valve and the effectiveness of PTF stripping technique at 108 military center hospital

\section{SUBJECTS AND METHOD:}

Including 122 patients received surgical mitral valve replacement with SJM mechanical with or without tricuspid valve repair in $05 / 2010$ to $12 / 2014$ Technique for using PTFE strips:

(1) Build the pillar at the posteroseptal like Kay method using PTFE.

(2) Build the pillar at the anteroseptal commissure by ethibond 2.0 stitches into anterior cusp of TR.

(3) Bypass after pillar and before pillar by a PTFE strip (the length even tricuspid annulus valve of septal leaflet

\section{RESULT:}

Mean age was $48.1 \pm 9.2$, no difference in mean age of male and female. Rate of tricuspid regurgitation valve medium - heavy is $51.6 \%$ and $46.7 \%$ were repair by PTFE strips.

Mainly in the group with lesions coordination mitral regurgitation and stenosis valve $(80,3 \%)$ 
Early results after surgery in the group with repair TR: tricuspid regurgitation valve medium - heavy is $12.2 \%$ and in the group with not repaired TR: tricuspid regurgitation valve medium - heavy is $12.3 \%$ (before the surgery are not tricuspid regurgitation)

Followed at 1 month, 6 months, 12 months, 24 months, 36 months, 48 months: In the group with not repaired tricuspid is increased tricuspid regurgitation and heart failure compare to group with repair tricuspid valve. CONCLUSION:

It is important to fix the TR systematically in mitral valve replacement surgery to reduce postoperative heart failure.

Using PTFE strips in the repair tricuspid valve is good after surgery.

Enter description here.

\begin{abstract}
Should we re-think about minimal age criteria for coronary angiography in patients with rheumatic heart disease undergoing valvular intervention? Jigar Shah; Vivek Wadhawa
\end{abstract}

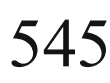

\section{ABSTRACT: \\ BACKGROUND:}

Our aim of this study was to find the prevalence of $C A D$ and various CAD risk factors in RHD patients below 50 years.

\section{MATERIALS AND METHOD:}

It was a single center observational retrospective study of 6324 rheumatic heart disease patients, who underwent coronary angiography (CAG) prior to surgical valvular replacement from January 2011 to December 2017, a period of 7 years. Among them 3920 patients were less than 50 years of age, they were included in this study. Patients with age more than 50 years (2404) were excluded. Among them significant CAD group patients (stenosis $\geq 50 \%$ Group A), were compared with similar age group matched patients of RHD without CAD (Group B).

\section{RESULTS:}

The overall prevalence of $\mathrm{CAD}$ in the patients undergoing valvular intervention was $5.68 \%$ (61.88\% males, $38.12 \%$ female). The CAD incidence in patients with mitral, aortic and both valve replacement were $4.20 \%$, $6.5 \%$ and $5.3 \%$ respectively. The prevalence of smoking habit $(41.7 \%$ vs. $27.48 \%)$, diabetes $(18.83 \%$ vs. $3.62 \%)$, hypertension $(69.05 \%$ vs $13.52 \%)$ and family history of CAD $(78.02 \%$ vs. $3.8 \%)$ was significantly higher in group A as compared to group B.

\section{CONCLUSION:}

Prevalence of CAD in patients with Rheumatic valvular heart disease in western population of Asian Indians with age less than 50 years is $5.68 \%$. Thus Coronary angiography should be performed in such patients only when there are associated CAD risk factors. No need of doing prophylactic angiography for those rheumatic heart disease patients who do not having any CAD risk factors in young patients age less than 50 years. Keywords: Rheumatic heart disease, Coranary Angiography, Coronary artery disease, Incidence, Risk factors

\section{Mitral Valve Replacement-Comparative study with or without posterior mitral leaflet preservation technique \\ Subrata Dey \\ Subhajit Sharma}

\section{ABSTRACT: \\ OBJECTIVE:}

The papillary muscle and chordae tendinae of both mitral leaflets contribute to the preservation of left ventricular function. In this study we compared long term results with or without posterior mitral leaflets preservation in patient of mitral valve replacement in terms of left ventricular ejection fraction and left ventricular dimensions.

\section{METHODS:}

In a group of 160 patients, 80 of them underwent mitral valve replacement with leaflet preservation, 80 underwent mitral valve replacement without leaflet preservation. All of these patient had mechanical valve with St jude bileaflet mechanical valve.

\section{RESULTS:}

Ejection fraction and left ventricular dimetions for group 1 shows significant improvement (p\&lt;.05) as compared to group 2

Post operative complications (bleeding, arrythmia, Congestive cardiac failure, ventilator stay) also less in group 1 as compared to group 2 .

Our experience in mitral valve repair for mitral valve disease in children and adolescents

Prashant Shah; Harun

\section{ABSTRACT:}

AIM:

Mitral valve repair is the preferred technique of choice in recent years for mitral valve disease. We aim to outline our experience in mitral valve repairs performed by a single surgeon in various centers.

\section{MATERIALS AND METHODS:}

All patients underwent mitral valve repair for isolated mitral valve disease from 2009 to September 2018. All patients were subjected to preoperative trans thoracic echocardiography, intraoperative saline test, post CPB and post operative echocardiography. Various operative techniques have been performed depending upon the type of the lesion.

\section{RESULTS AND OUTCOME:}

Total of 91 patients were operated between age 2 to 18 years with mean age of 9.7 years. Underlying pathology were rheumatic $(n=76)$, myxomatous $(n=12)$, degenerative $(n=2)$ and traumatic valve disease $(n=1)$. The techniques chordal shortening $(n=17)$, neochordal formation( $(n=17)$, thinning of the leaflets $(n=24)$, triangular plication( $(n=14)$, triangular resection $(n=7)$, scallop repair $(n=8)$, suture annuloplasty $(n=53)$ and ring annuloplasty $(n=34)$ were performed. No patient underwent mitral valve replacement in the same sitting. Post repair mild to moderate regurgitation was accepted. Mean CPB time was 123 minutes and mean ACC time was 67 minutes. Mean hospital stay was 7.4 days. There were no intraoperative and perioperative mortality. 3 patients required mitral valve replacement after 2 years. 5 patients developed grade II MR from grade I.

\section{CONCLUSION:}

Mitral valve repair is the most preferred technique for mitral valve disease. Thorough on table examination of the native valve and modification of the techniques is required to achieve better results.

Lactate level during cardiopulmonary bypass and immediate post operative period as a predictor of postoperative outcomes in patients undergoing open heart surgery

A. Rathinavel

B. Karthikeyan

\section{ABSTRACT:}

\section{INTRODUCTION:}

Hyperlactemia in the immediate post cardiac surgery patient will adversely affect the early and late outcomes in terms of morbidity and mortality. CASE SERIES:

We have prospectively evaluated 50 cases who were undergone open heart surgeries in the past 6 months. First 3 months of the study period Average serum lactate level is $7.2 \mathrm{mmol} /$ litre on the start of CPB, 9.5 $\mathrm{mmol} / \mathrm{litre}$ at the end of CPB and $7.5 \mathrm{mmol} /$ litre on ICU arrival.

The mortality rate in 4 among the 25 patients (first 3 months)studied. The mortality was due to unexplained severe acidosis with low cardiac output state. Then we have started using the different formula for calculating blood volume and we have started using oxygen blender. 25 cases studied 
in the Last 3 months of the study period Average serum lactate level is 4.1 $\mathrm{mmol} /$ litre on the start of CPB, $6.2 \mathrm{mmol} /$ litre at the end of CPB and 4.2 $\mathrm{mmol} /$ litre on ICU arrival.

2 immediate post operative mortality among the 25 patients studied. One mortality is due to post operative Cerebro vascular accident and one other is due to bleeding from the thinned out diseased aorta following aortic valve replacement with prolonged pump time.

\section{CONCLUSION:}

Reduction of lactate levels by avoiding haemodilution (maintaining $25 \%$ haematocrit for adults and 30\% for paediatric cases) and using oxygen blender reduces mortality to $50 \%$ in the last 3 months which directly correlates with the lactate levels on $\mathrm{CPB}$ onset, $\mathrm{CPB}$ end and at the arrival on ICU.

\section{Outcomes of surgery for Infective endocarditis:}

Our experience

R.V. Kumar

Chaitra K. Bhat

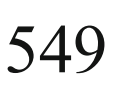

\section{ABSTRACT:}

\section{INTRODUCTION:}

Infective endocarditis (IE) is being increasingly diagnosed and referred for surgery. The presence of unusual organisms is also on the rise. In this background, we share our experience with surgery for IE.

\section{MATERIALS AND METHODS:}

Patients operated for IE between 2011 through 2017 were selected from database. Infecting organism, risk factors, perioperative subsystem status were analysed. Morbidity and mortality were studied and compared with Indian and international studies. EuroSCORE II was used to calculate predicted mortality.

\section{RESULTS:}

30 patients operated for IE were included. Mean age was 36.7+/-11.7 years. $73 \%$ patients were males. Staphylococcus isolated in $26.6 \%$ patients, streptococcus in $16.6 \%$, Klebsiella and enterococcus spp found in $10 \%$. One case each of M. tuberculosis, Brucella and B. pseudomallei were found. $96 \%$ had native valve IE. $56 \%$ patients had previous rheumatic heart disease. Mitral valve was involved in majority of cases (40\%). The mean hospital stay was $9+/-3.7$ days. The in-hospital mortality rate was $30 \% .50 \%$ patients had pre-operative renal insufficiency. $40 \%$ and $43.3 \%$ had higher bilirubin levels and baseline liver enzymes. Complications of heart failure $(55 \%)$ and sepsis $(44 \%)$ were associated with poor outcome of in terms of survival. Predicted mortality by EuroSCORE II was $26.6 \%$, $23.3 \%$ and $50 \%$ as against no deaths in the low and medium risk category and $30 \%$ in the high risk category respectively.

\section{CONCLUSION:}

Population at risk for IE in our country remains younger. Perioperative multiorgan dysfunction is predictor of poor surgical outcome. Early surgery with appropriate antibiotics and duration may reduce mortality.

\section{"Successful management of a rare case of Aspergillus Prosthetic Valvular Endocarditis" Raman Kumar Jaideep Trivedi}

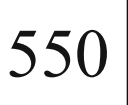

\footnotetext{
ABSTRACT:

Cardiac Aspergillosis is a severe and extremely life threatening entity with a very few reported cases. We present a case of an atypical early presentation of Aspergillus Endocarditis(AE) with an echogenic valvular mass which got embolized and detected after femoral embolectomy in a previously operated male for mitral valve replacement. He was treated successfully with complete prosthetic valve explantation, re valve replacement and anti fungal therapy.
}

52years male presented to us with fever, chills and rigor since 1 day. $\mathrm{He}$ had undergone mitral valve replacement 3 months back. His echo was done which showed a vegetation of 1.5 to $2 \mathrm{~cm}$ in prosthetic valve. His blood culture was negative and was started on Vancomycin, Gentamicin and Rifampicin. In hospital patient developed severe pain in buttocks and lower limbs. Colour doppler of lower limbs suggested pulses tardus with proximal obstruction. CT angio suggested multiple embolus in iliac, femoral and renal vessels. Embolectomy was done and embolus was sent for histopathological examination, which suggested Aspergillus colonies, meanwhile patient deteriorated in ward and developed sudden breathlessness and hypotension. Echocardiogram showed severe MR with destruction of prosthetic valve, we decided to do redo mitral valve replacement. The prosthetic valve was completely destroyed and had black coloured vegetation over it. Complete emplantation of valve along with vegetation was done and was replaced with 27 NO. Perimount valve. In post op. patient was treated with intra venous lypophilic AmphotericinB and Voricanazole for 2 weeks then on oral voriconazole. Histopathology confirmed Aspergillus niger. Pt. recovered very well and was discharged on 15 th post OP day. He is on tab. Voriconazole since 2 years completely asymptomatic and following up with us.

\section{CONCLUSION:}

Aspergillus Endocarditis is a rare and dreadful clinical entity. Echocardiographic assessment of suspected AE is critical. Early administration of appropriate anti fungal treatment and prompt surgical intervention is the key to success. How long to continue azoles in such cases is still a matter of debate.

Internal vs external ligation of left atrial appendage during Mitral valve repair/replacement

Sachin Talwar

Rakesh Kumar

\section{ABSTRACT:}

\section{BACKGROUND:}

During the mitral valve repair/replacement operation, there is need of ligation of left atrial appendage. The LAA may be ligated internally or externally. This study is intended to study complications related to both as well as advantage and disadvantage of both procedure.

\section{PATIENTS AND METHODS:}

Between July 2017 to September 2018, 50 consecutive patients undergoing the mitral valve operation were included in this prospective cohort study. All the preoperative and postoperative parameters were noted in the described format including age of patient, duration of disease, NYHA status, rhythm, mitral valve morphology, LAA clot, cardio-thoracic ratio etc. Simultaneously a note was made of all the patients pre-operative characteristics, aetiology, associated cardiac anomalies, echocardiogram findings, cardiac catheterization data (if performed), intra operative details, Histo-pathological examination of native mitral valve, comparison with internal ligation versus external ligation of LAA, post operative course, follow up and follow-up echocardiographic data.

\section{RESULTS:}

Fifty patients had mitral valve repair/ replacement between July 2017 September 2018. We did internal versus external ligation of LAA, compared it with each other in the finding described in the literature. It was found that internal ligation is easier to perform, less time consuming as well as having low risk, though it requires good exposure as well as it is technically demanding and has a learning curve. Hence it is better to do internal LAA ligation during mitral valve surgeries.

\section{CONCLUSIONS:}

There were no gross differences between outcomes following surgery but internal ligation of LAA requires less time and there is complete obliteration of LAA. Entire procedure is under vision; therefore it is wiser to go for internal LAA obliteration. 
Pseudoaneurysm of Aortomitral contuinity- study of 2 rare case

Balaji Aironi

Kapil Prafull Patel

\section{ABSTRACT:}

Aortomitral contuinity is an avascular fibrous structure between the non coronary and left coronary cusp and anterior mitral leaflet. Pseudoaneurysm of the aortomitral continuity is a rare clinical entity. Its common causes are infective endocarditis and valve surgery. Its progression and enlargement is inevitable and may rupture leading to temponade. Hence it has to be managed surgically.

We present a clinicopathological study of two different cases of pseudoaneurysm of aortomitral contuinity at our institute. This article shows the highly variable clinical features of the same disease entity and its different complications. The pathoanatomy of both the lesions is also different. It also shows how routine echocardiography is at times unable to detect this rare but fatal condition. It also shows different management strategies that can be used for this completely cureable disease.

\section{MITRAL VALVE THROMBECTOMY : A NOVEL APPROACH FOR PROSTHETIC MITRAL VALVE THROMBOSIS}

Anil Jain

Jigar Shah

\section{ABSTRACT: \\ OBJECTIVE:}

To evaluate and compare the outcome of Mitral valve thrombectomy versus Redo Mitral Valve replacement for prosthetic mitral valve thrombosis in the early postoperative period.

\section{PATIENTS AND METHODS:}

It is a Retrospective observational study of 104 patients admitted for prosthetic mitral valve thrombosis in emergency department from January 2016 to January 2018. Group A ( N-26) patients underwent thrombectomy and Group B ( N-78) patients were treated by Redo mitral valve replacement. They were diagnosed with transthoracic echocardiography and fluoroscopy. All patients were operated under general anesthesia through midline redo sternotomy and CPB. Preoperative demographics, size of thrombus, mean gradient, Intraoperative CPB and total cross clamp time were recorded. Postoperative hemodynamic status, ionotropic support, ventilation time, ICU and Hospital stay were assessed in both groups. 2D echocardiography done in all cases post operatively.

\section{RESULTS:}

No statistical difference was found on the basis of gender, age, interval between initial mitral valve replacement (MVR) and redo operation or thrombectomy, anticoagulation status, NYHA functional class, INR level, trans thoracic echocardiography and fluoroscopy. The mean CBP time ( $76.38 \pm 24.42$ minutes) \&amp; cross clamp time ( $40.29 \pm 16.48$ minutes) was significantly less in group A than group B. Similarly ionotropic support, mean ventilation time and mean ICU stay in group A was significantly less than in group B. Morbidity and mortality was not statistically significant in both groups.

\section{CONCLUSION:}

Though prosthetic valve thrombosis after mechanical mitral valve replacement is dreaded complication and night mare for surgeons, if its managed and operated timely with proper surgical planning it will result in wonderful outcome. Thrombectomy is better cost effective option in emergency and it is recommended because of better outcomes in early and late post operative period.

The efficacy and safety of del nido cardioplegia in valvular heart surgery : an institutional report Ashish M. Agrawal
Divya Arora

ABSTRACT:

\section{INTRODUCTION:}

The del Nido cardioplegia provides 60 - 90 minutes of cardiac arrest in single dose with adequate efficiency is gaining popularity among cardiac surgeons. Its role has been well established in paediatric cardiac surgeries yet to be proven in adult cardiac surgeries. We are reporting our observation in support of its efficacy and safety for use in valvular cardiac surgery.

\section{MATERIALS AND METHODS:}

A total 50 consecutive patients those underwent surgery for the correction of mitral valve pathology with or without concomitant Tricuspid or AF ablation surgery from January 2017 to June 2018 were evaluated. Perioperative data patient NYHA class, comorbidities, diagnosis, ejection fraction, aortic cross clamp and total cardiopulmonary bypass time, cardioplegia volume, pre bypass TROP I, return to spontaneous rhythm, defibrillation requirement, haemodynamic instability, change in blood troponin levels (at 2, 12, 24 hours post-CPB), mechanical ventilation, inotropic requirement, postoperative clinical outcomes, ejection fraction at termination of $\mathrm{CPB}$ and hospital stay were collected.

\section{RESULTS:}

The included patient had, age $33.7 \pm 15.6$ years, female $66 \%$, moderate to severe MR $56 \%$ with associated functional tricuspid regurgitation, severe MS 70\% with or without LA clot, moderate to severe PAH 54\%, preoperative ejection fraction $56.18 \pm 5.39 \%$. Cardiac Trop I post bypass at 2 hrs, $12 \mathrm{hrs}$ and $24 \mathrm{hrs}$ intervals, mean values were $7.23 \pm 2.11,5.25 \pm 2.56$ and $3.58 \pm 2.82$ respectively. Post operative ejection fraction was $47.7 \pm$ $7.63 \%$. Clinical outcomes were satisfactory with mortality rate of $6 \%$, prolonged ventilation $10 \%$, re-exploration rate of $2 \%$, short hospital stay $34 \%$.

\section{CONCLUSION:}

The del Nido cardioplegia solution is safe to use in isolated or complex mitral valve surgery. It has the advantage of less interruption for surgical procedure with spontaneous recovery of rhythm. Caution is still warranted for adopting its optimal use.

\section{ROLE OF INTRA- AORTIC BALLOON PUMP IN INTRACTABLE CARDIOGENIC SHOCK FOLLOWING VALVULAR SURGERY \\ Vivek Wadhawa \\ Jigar Shah}

\section{ABSTRACT: BACKGROUND:}

Mechanical circulatory support may be necessary to prevent irreversible end-organ damage in cardiogenic shock. Post valvular surgery incidence of severe LV dysfunction is around $8.6-11 \%$. Our aim is to evaluate the role of intra-aortic balloon pump (IABP) in intractable cardiogenic shock following valvular surgery, assess prognostic factors and patient survival. PATIENT AND METHODS:

A single center retrospective study was performed in 19 (3.37\%) patients with cardiogenic shock following valvular surgery supported firstly by IABP in the year 2017.

\section{RESULTS:}

Out of all valvular surgery patients (N-567), 64 patients developed severe LV dysfunction. Amongst them 19 patients underwent IABP insertion. In all patients after putting IABP at 24 hour, lactate decreased from 3.7 (2.87.4) to $1.6(1.4-2.5) \mathrm{mmol} / \mathrm{L}$ ( p \&lt; 0.001). Twelve patients $(63.15 \%)$ defined as IABP responders were successfully weaned from IABP in 2-3 days with ionotropic support and discharged to home. IABP failed in seven patients (non-responders, $36.85 \%)$ who either died $(n=4)$ or needed support by extracorporeal membrane oxygenation $(n=3)$. At 24 hour of IABP support, urinary output was higher $(2,890[1,730$ $4,625]$ vs. $1,150[680-2,125] \mathrm{mL} ; \mathrm{p}=\& 1 \mathrm{lt} ; 0.001)$ and fluid balance more 
negative $(-1,450[-2,572$ to $-1,134]$ vs. $-500[-965$ to +255$] \mathrm{mL}$; $\mathrm{p} \& \mathrm{lt}$; $0.001)$ in responders than non-responders.

\section{CONCLUSION:}

In most patients, first-line support by IABP is safe and cost effective in intractable cardiogenic shock following valvular surgery is associated with improvement in organ perfusion and clinical stabilisation for at least 24-48 hours allowing time for decision making on next therapies.

Single Suture Continous Technique in Mitral

Valve Repalcement

Abhay Jain

James Thomas

\section{ABSTRACT:}

Continous Suture with one 20 Prolene for Mitral Valve Insertion has been our preferred technique for the last 15 years. Everting Sutures wit atrial side pledgets gave way to LV side non pledgeted sutures. Then we now prefer debriding the annulus wella nd one single suture continous tachnique for mItral Valve insertion. Starting at 9 o Clock coming down to 60 clock position and pushing the valve to $\mathrm{LV}$ and taking rest of the sutures in a continous manner. Over 600 mitral valve followed up with ECHO studies showed less than 5\% patients with minimal or trivial Leak. Details presented

\author{
Mitral Valve replacement in a case of Sickle cell \\ anemia \\ Sarava Krushna Raja \\ Ezhilan K.S.
}

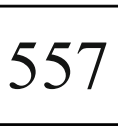

\section{ABSTRACT:}

Sickle cell anemia is a genetic hemoglobinopathy where abnormal hemoglobin chains are produced due to missence mutation. The mutation occurs in $\beta$ - globin chain producing Hemoglobin-S which has a tendency to sickle in conditions like acidosis, hypoxia, hypothermia, dehydration, physical exertion, leading to vasoocclusive episodes, hemolysis and anemia.

Sickle cell trait is a condition where patients have a heterozygous beta gene and is less severe than sickle cell disease.

Patients with sickle cell anemia present unique challanges while undergoing valve replacement surgery using Cardiopulmonary Bypass (CPB). We present a case of Rheumatic heart disease with sickle cell trait with anemia who underwent mitral valve replacement. We used a combination of techniques including pre op blood transfusion and interop exchange transfusions. Adequate hydration with CVP monitoring, moderate hypothermia during Cardiopulmonary bypass.

We present this case for its rare clinical presentation and challenges in management.

\section{GDMT versus Early SAVR for asymptomatic severe aortic incompetence with dilated left ventricle with normal ejection fraction \\ T.K. Lahiri \\ Sanjay Kumar}

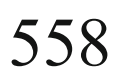

\section{ABSTRACT: \\ OBJECTIVES:}

The management of patients with asymptomatic severe aortic incompetence $(\mathrm{AI})$ remains unclear. The aim of the present is to compare the clinical outcome between early surgery and guidelines directed medical therapy (GDMT) for asymptomatic severe AI in patients with normal left ventricular (LV) systolic function and LV dilatation.

METHODS:

This retrospective cohort study included 39 consecutive patients with severe AI with left ventricular ejection function (LVEF $\geq 50 \%)$ and left ventricular end-diastolic dimension (LVEDD \&gt; $70 \mathrm{~mm}$ ) from 2008 to 2018. 26 patients received early surgical aortic valve replacement (SAVR), whereas 13 patients received conventional treatment at the discretion of the Cardiologists. Propensity score analysis was done evaluating overall and cardiovascular survival rates between two groups.

\section{RESULTS:}

The patients had a mean age of $48.3 \pm 12.5$ years; $75 \%$ were men. Mean LVEF and LVEDD were $58.3 \pm 7.7 \%$ and $75.3 \pm 5.2 \mathrm{~mm}$, respectively. Kaplan-Meier analysis revealed that early AVR was associated with better 10 -year cardiovascular $(\mathrm{P}=0.034)$ survivals in the overall population. In the 12 propensity score-matched pairs, early AVR was still associated with a significantly better 10 -year overall survival ( $\mathrm{P}=0.018$ ). Furthermore, patients who underwent early AVR had significantly improved 3-, 5- and 10-year cardiovascular survival rates of 97.8, 96 and $93.2 \%$, respectively, compared with $90.5,84.5$ and $79.0 \%$ for those in the GDMT group $(\mathrm{P}=0.006)$.

\section{CONCLUSIONS:}

Early SAVR is associated with an improved long-term outcome in patients with asymptomatic severe AI with LVEF $\geq 50 \%$ and LVEDD \&gt;70 mm. Our data is new evidence to strongly support current guidelines, which recommend a strategy of early operative intervention.

Aortic Root Enlargement using native untreated

Pericardium

Anurag Garg

Smruti Hindaria

ABSTRACT:

Aortic Root Enlargement is an invaluable operative technique with which every cardiac surgeon performing Aortic Valve Replacement should be familiar.

We present the technique using native untreated Pericardium for Aortic Root Enlargement in a series of patients. It allows for insertion of appropriate-sized mechanical or biological prosthesis in Aortic position to avoid patient/prosthesis mismatch. Native untreated pericardium is as safe as bovine pericardium with comparable results. It's a boon where finances are restricted or in emergency situations when alternatives are not available. The technique is safe and reproducible when performed meticulously.

De Vegas Suture Annuloplasty - a retrospection in to our Experience

B.V. Sai Chandran

Jayakrishnan S.

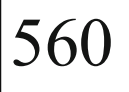

\section{ABSTRACT:}

TITLE- De Vega's Suture Annuloplasty - a retrospection in Experience AUTHORS: Dr Jayakrishnan S, Dr Saichandran B V, Dr Ramsankar P, Dr D P Rath

\section{BACKGROUND:}

Patients with long standing Rheumatic mitral and aortic valve disease have associated functional Tricuspid valve regurgitation mostly secondary to right ventricular dilation or pulmonary hypertension. Optimal surgical management of Tricuspid regurgitation remains a matter of debate. Surgeons advocate various methods including. Devega's suture annuloplasty, Ring annuloplasty, or tricuspid valve replacement. We at JIPMER use De vega's suture annuloplasty for functional tricuspid regurgitation.

OBJECTIVE:

We evaluated the efficacy of DeVega's Suture Annuloplasty in 95 Cases of Functional Tricuspid Regurgitation by echocardiography, clinical status, and long term results.

\section{MATERIALS AND METHODS:}

Retrospective study of all cases mitral valve surgery with (CASES) or with out (CONTROL) De Vegas annuloplasty done from 2012 January to December 2016 was done. Preoperative and 2 year postoperative 
echocardiographic measurements included Right ventricular and right atrial diameters, extend and direction of color flow of TR, its grade (severity), ejection fraction and Pulmonary artery pressure were done and compared pre and post operative data with control group.

\section{RESULTS:}

A Total of 95 cases were studied,44, controls and 51 cases, Right atrium and ventricular diameters, pulmonary artery pressure and ejection fraction were decreased post operatively in patients who underwent De Vegas annuloplasty(P\&1t;0.05). Our results indicate that De Vegas Annuloplasty is effective and should be considered in all cases of mitral valve surgeries which have associated functional moderate and severe TR CONCLUSION:

De Vegas suture Annuloplasty is effective in controlling progression of functional tricuspid disease and is recommended in all cases of functional TR in mitral valve surgeries

\author{
Successful Open Heart Surgery in HIV \\ patient - A reality in the public hospital of India \\ B.V. Sai Chandran \\ Prabhas Teja
}

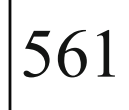

\begin{abstract}
:
India has the third largest HIV epidemic in the world and in 2017, 2.1 million people are living with HIV. With the emergence of ART there is now increased chance of survival these patients and the risk of developing age related conditions like cardiovascular disease. Our CTVS team at JIPMER, a quaternary care public hospital in Pondicherry has successfully performed an open heart surgery in a HIV positive individual in the month of October, 2018. This is a report of a 54 year old male who had been living with AIDS on antiretroviral drug therapy. He was admitted with complaints of DOE NYHA III and palpitations as a case of RHD with Severe MS requiring mitral valve replacement. Breaking the stigma and discrimination against the HIV positive individuals, we proceeded with the plan despite many limitations of a public hospital. Patient underwent specific evaluation to assess the status of immunodeficiency. Posted as the first case in the morning hours with thoroughly informed team, patient had an uneventful surgery. Extubated on POD3. Post-operatively he developed bed sore, persistent fever which was managed suspecting atypical pneumonia. ART was restarted and patient was discharged. We indeed would like to let the world know that OHS can be successfully performed in HIV positive patients with universal safety precautions, sound technique and an experienced team with all the complications and risks anticipated of the patient and to the medical team. HIV being prevalent globally and ART increasing the lifespan, we may expect more number of patients requiring cardiac/vascular surgery in the future.
\end{abstract}

\section{Long term survival (30 years) after Aortic valve replacement with Starr Edwards Aortic valve Priyanka Jindal; Kotturathu Mammen Cherian Chandrasekhar Verma}

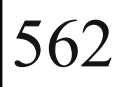

\section{ABSTRACT: \\ INTRODUCTION:}

Starr Edwards (SE) mechanical heart valve was one of the earliest heart valves that heralded a revolution in the surgical management of valvular heart disease. Long term results of this valve has made it a benchmark in the field of valvular surgery. Anecdotal reports are seen, of patients with these valves surviving for a long duration sometimes even without anti-coagulation. We report a patient who was re-operated 30 years after the initial implantation of SE aortic valve, the longest reported survival in India.

\section{CASE REPORT:}

Starr Edwards 9A aortic valve was implanted in 1988 for severe calcific aortic stenosis. Patient had optimal valve function despite inadequate medical followup and compliance to medication (T.Warfarin). He had a CVA in 1998 with complete recovery. He developed anterior wall MI in
2011 (?embolic). In 2018, 30 years after initial implantation, he was found to have prosthetic valve dysfunction with a high trans-valvular gradient needing replacement. Intra-operatively he was found to have pannus causing stenosis. Re-do AVR was done by sub-coronary implantation of a cryopreserved aortic homograft, in view of thrombo-embolic events and poor compliance with anti-coagulation.

On analysis of previous aortic SE valve it was found that the pannus was encroaching into the sewing ring causing severe stenosis, the silastic ball was slightly yellow in colour but showed no features of ball variance.

\section{CONCLUSION:}

Long term durability and good hemodynamic performance has been reported with more than 200000 SE caged ball valves implanted worldwide. Review of literature of the long term survival of SE valve patients has shown that these valves are a bench mark for mechanical heart valves.

\section{THE POSTERIOR ENLARGEMENT IN THE SMALL AORTIC ANNULUS : A SURGICAL SAFE PROCEDURE \\ Samir Kapoor \\ Rajiv Kumar Gupta}

\section{ABSTRACT:}

OBJECTIVE:

To avoid prosthesis patient mismatch surgical aortic root enlargement (ARE) during Aortic valve replacement (AVR) allows for larger prosthesis implantation and may be an important adjunt to surgical AVR.

\section{METHODS:}

During the period from January 2010 to January 2018, 90 patients underwent aortic valve replacement (AVR) with $19 \mathrm{~mm}$ or $18 \mathrm{~mm}$ for aortic stenosis. 12 patients (13\%) with 11/12 women, 18 to 72 years old (mean age 44.2 years) with aortic stenosis, underwent AVR applying posterior enlargement of the small aortic annulus with an effective aortic valve area $0.7 \pm 0.2 \mathrm{~cm} 2$. In addition, mitral valve replacement (MVR) was performed in 3 patients. All patients expect one had mechanical valves.

The defect after the enlargement was closed with either autologous gluteraldehyde treated pericardium or bovine pericardium and strengthened with Dacron patch. The follow-up period was more than 6 months. RESULT:

Patients undergoing AVR+ ARE were more likely to be female and in worse New York Heart Association status (P\&lt;0.001). Only one patient received bioprosthetic valve. Mean prosthesis size implanted was either $18 \mathrm{~mm}$ or $19 \mathrm{~mm}$ size in patients with ARE. There was no operative or hospital mortality. The length of CPB; Aortic cross clamping and duration of mechanical ventilation increased. The AVR + ARE was not associated with an increased risk of postoperative adverse events. There was one early death in the very first patient in whom MVR was the additional procedure. There was significant decrease in gradient and aortic valve orifice size was $1.2 \pm 0.5 \mathrm{~cm} 2$.

CONCLUSION:

Surgical ARE is a safe adjunct to AVR in the modern era. Although, the number of patients is small female patients seem to be the usual candidate for this procedure.

Rupture of left ventricle Post re-do mitral valve replacement - Repair with decellularised bovine pericardium

Srinivas Aaluri; K.M. Cherian

Anil Kumar

\section{ABSTRACT:}

INTRODUCTION:

Rupture of the left ventricle postMVR is a known and near fatal complication. We report a case of LV rupture post re-doMVR that presented with sudden bleeding of POD 1 and complete heart block. 


\section{CASE REPORT:}

Following a bio-prosthetic heart valve dysfunction, a 54 year - old lady underwent re-do MVR with a $27 \mathrm{~mm}$ bio-prosthetic heart valve. Intraoperatively the degenerated valve was encased in a fibrous sheath ( preserved fibrotic posterior mitral leaflet). Post-extubation on POD1, she had sudden bleeding through the drains. She was re-explored and was placed on emergent CPB. She developed complete heart block. She had pulsatile bleeding from the posterior $\mathrm{AV}$ groove to the right of the crux. Intra cardiac, there was a haematoma along the mitral annulus, abutting the right fibrous trigone, typeI rupture of the posterior wall of the left ventricle forming a track with an exit point in the right $\mathrm{AV}$ groove, to the right of the crux. The prosthetic mitral valve was removed and the posterior wall of the LV was repaired with a triangular patch of decellularised bovine pericardium sutured to the intact posterior wall, beyond the lacerated edges of the rupture in the LV, up to the annulus. The prosthetic valve was then re-sutured to the mitral annulus, incorporating the bovine pericardial patch posteriorly. She was weaned off CPB (with ventricular pacing). She regained $\mathrm{AF} /$ controlled ventricular rate late post-op. Post-operative recovery was otherwise uneventful with discharge on day10.

CONCLUSIONS:

Rheumatic heart disease, older age, hemodialysis and LVEDD\&lt; 50mm have been implicated in the rupture of LV post MVR, along with excessive debridement, traction and over-sizing of the valve. Preservation of PML is proposed to prevent this complication, however, PML can be fibrotic and should be carefully separated at the time of re-replacement of mitral valve.

Quadricuspid aortic valve and dilated ascending aorta Abdelhadi Ismail

Sajiram Sarvananthan

\section{ABSTRACT: \\ INTRODUCTION:}

Quadricuspid aortic valves (AV) are extremely rare and its clinical significance is unknown. Here we present a case with AR and dilated aortic root, where the aortic valve is quadricuspid.

\section{BACKGROUND:}

The anatomy of the AV is well described and the association of bicuspid aortic valve with root dilation is well documented. However, the AV with quadricusps is rare and represents $0.1 \%$ of overall incidence. It was first reported in 1862 and its association with root dilatation is not frequently documented.

CASE:

A 61 years gentleman presented with exertional shortness of breath and chest pain. He is an ex-smoker and had no significant co-morbidities. On examination, he was in SR with an end diastolic murmur and had no signs of HF. His ECG showed SR with RBBB, with some ST changes in leads V1-V3

Transthoracic echocardiography demonstrated a moderate-severe AR with a dilated root $(5.87 \mathrm{~cm})$ and ascending aorta.

CT angiogram showed mildly diseased coronaries and the morphology of AV was clearly shown to be quadricuspid with dilated aortic root $(4.5) \mathrm{cm}$ and mild ascending aortic dilatation $(4.3 \mathrm{~cm})$. Hence, he underwent aortic valve replacement with a $27 \mathrm{~mm}$ tissue valve prosthesis and a $32 \mathrm{~mm}$ dacron tube graft to replace both the root and ascending aorta. The surgery and subsequent recovery were uneventful.

\section{CLINICAL RELEVANCE:}

High quality imaging modality is a useful tool in planning an operative stagey. The decision to replace the valve was due insufficient data on repairing quadricuspid aortic valves.

Decellularised, sterile-stabilised bovine pericardium (DSBP) scaffolds as future alternative for glutaraldehyde-fixed tissue engineered pericardial patch material.
J.J. van den Heever

Francis E. Smit

\section{ABSTRACT: \\ OBJECTIVE:}

Fibrous encapsulation of tissue engineered pericardial patches causing thickening of the patch tissue remains a challenge. The long-term effects on material characteristics like flexibility and degeneration remains unclear and thus may limit its use in valve substitutes or repairs. Fibrous encapsulation, tensile strength and calcification potential were compared between the UFS's proprietary decellularised sterilised bovine pericardial (DSBP)-scaffolds, using no glutaraldehyde, and two commercial tissue engineered bovine pericardial patches (CardioCel, Admedus and St Jude Encap, Glycar) in a juvenile ovine model after 180 days implantation. Glycar uses glutaraldehyde fixation and detoxification processes and the tissue engineered CardioCel pericardial patch combines decellularisation, modified glutaraldehyde fixation and detoxification.

\section{METHODS:}

The DSBP-scaffolds, CardioCel and Glycar patches were implanted in three groups of six juvenile Dorper sheep $(n=18)$ for 180 days. In each sheep both a pulmonary and an aorta implant were performed. Baseline microscopy (including H\&amp;E, modified von Gieson (MvG), von Kossa (VK) and quantitative calcium, Youngs Modules (YM) and tensile strength (TS) testing were performed pre- and post-implantation.

\section{RESULTS:}

In both the pulmonary and aorta explants a macroscopic fibrous peel covered the Glycar and CardioCel patches, but was absent in the explanted DSBP-scaffolds. In all the CardioCel and Glycar patches H\&amp;E demonstrated fibrous encapsulation, which included minimal recellularisation and collagen formation. The DSBP-scaffolds demonstrated full thickness scaffold recellularisation with fibroblasts and collagen formation and endothelial cover. MvG demonstrated absence of elastin and von Kossa confirmed no calcification in all explants. No statistical difference could be demonstrated in the TS, YM or quantitative calcium between the explant groups.

\section{CONCLUSION:}

The non-glutaraldehyde DSBP scaffolds, demonstrated in-vivo recellularisation, collagen formation, reendothelialisation, comparative TS-retention, lack of calcification and absence of fibrous encapsulation and warrants further investigation for use in cardiovascular applications.

\section{Markers for predictor for death in pediatric double valve surgeries \\ Prasanna Simha Mohan Rao \\ P. Varun Gautam}

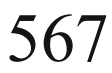

\section{ABSTRACT: INTRODUCTION:}

Aortic and Mitral valve surgeries are common in children and pose a challenge. Double valve procedures are associated with an increased risk of mortality and increased morbidity. Not many studies exist as of today that focus on outcomes after pediatric double valve procedures. hence, the need for the study.

\section{MATERIALS AND METHODS:}

Patients less than 18 years of age, who underwent mitral valve repair with concomittant autopericardial aortic valve reconstruction with or without tricuspid valve repair were included in the study. Special focus was laid on the pre-operative echo findings including the $\mathrm{Z}$ scores for the various parameters.

\section{RESULTS:}

12 patients less than 18 years of age who undewent a double valve procedure were included in the study. Of them, 4 patients had a pre-operative $\mathrm{Z}$ score for left ventricular dimensions ( LVIDd/s) more than +7 . All 4 of them had a difficult wean off Carido-pulmonary bypass and died in the 
immediate peri-operative period. The remaining 8 patients are on a regular followup.

\section{Conclusion:}

Pre-operative $\mathrm{Z}$ score value for left ventricular dimensions of +7 or greater can be considered as an independent marker for mortality in pediatric population undergoing double valve procedures. However, a larger sample study would be required to confirm the same.

\section{Transventricular Mitral valve repair} for Ischemic mitral regurgitation- effective results in scarred $L V$

Tejaswi N Marla

Srikanth bhumana

\section{ABSTRACT: \\ INTRODUCTION:}

Ischemic MR remains the most important and unresolved aspects in the management of ischemic heart disease. $20-25 \%$ of the patients after myocardial infarction develop significant MR, which affects the prognosis.

\section{AIM:}

Mitral valve repair through scarred inferior wall by addressing both the valve and scarred inferior wall same time

\section{MATERIALS AND METHODS:}

A 55 yr old male patient with old IWMI IN 2013 and primary PCI to RCA, presented with dyspnea on exertion (NYHA III) and palpitations. 2D ECHO showed severe eccentric MR due to chordal rupture, along with akinetic basal and inferior wall of LV, EF of 0.50 . Coronary angiogram done revealed patent RCA stent and normal LAD, circumflex.

After median sternotomy, LV showed large inferior wall scar. CPB initiated. LV inferior wall scar opened and mitral valve showed $\mathrm{P} 2$ chordal rupture with PML prolapse and annular dilatation. Neochordal reconstruction was done using CV-4 sutures. PML annuloplasty band was prepared by using GORETEX patch. Pledgeted sutures were passed through PML annulus from LV side avoiding injury to chordae. Annuloplasty band was placed on LA side and sutures tightened on LA side. LV inferior wall scar plicated with CV-2 sutures without pledgets.

\section{RESULTS:}

Patient maintained stable hemodynamics in the post op period. No rhythm disturbances noted during post op period. POD 7 2D echocardiogram showed mild MR. patient got discharged on day 8 on diuretics and beta blockers.

After one month, review echocardiogram showed trivial MR and EF of 0.55 . cardiac status was NYHA II.

3 year follow up, patient was asymptomatic, NYHA I-II, Echo showed trivial MR( central jet).

\section{CONCLUSION:}

Transventricular approach to repair mitral valve avoids separate atriotomy incision and gives good view of the subvalvular apparatus there by identifying damaged chordae and effective repair can be accomplished. Plication of the inferior wall scar helps in improving LV function.

\section{Successful repair of left ventricle rupture after} mitral valve replacement by intracardiac patch- a case report

Divya Arora; Apresh Sanyal; Shamsher Singh Lohchab Ashish Manmohan Agrawal

\section{ABSTRACT: \\ INTRODUCTION:}

Left ventricular rupture is rare but one of the most catastrophic complications after mitral valve replacement. The present report describes successful repair ofa type II LV rupture, which occurred after mitral valve replacement.
55 yrs male underwent MVR for Severe MS. There was bilateral commissural fusion with calcification. PML was fibrosed with severesub valvular fusion. Both leaflets excised with decalcification. Base of PML preserved. Chordae of Both papillary muscles fixed at commissural annulus. \#33 sizer St Jude passed easily although was hesitant to use \#31 prosthesis yet chosen it. After 1/2 hour of decannulation there was brisk bleeding posteriorly. LV rupture was suspected. Patient was taken on CPB and arrested. There was type II LV tear about $2 \mathrm{~cm}$ in size. Tear was closed externally with Teflon Felt. After weaning off from CPB again there was bleeding. Patient was again taken on CPB and heart was arrested and and LA was opened. A small tear Lateral to stitched part noted externally. Prosthesis was explanted. A large piece of pericardium was stitched to healthy myocardium surrounding the rupture internally up to posterior annulus.\#27 prosthesis was reimplanted. On weaning off from CPB there was no bleeding.

\section{RESULTS:}

Patient extubated after 12 hrs. Rest of recovery was uneventful and discharged on 7th PO day and is doing well after 9 months of follow up. CONCLUSION:

We successfully treated LV rupture by intracardiac patch repair. We believe this procedure is one of the most effective approachesfor treating LV rupture.

Change of Pulmonary Artery Pressure after Mitral Valve Replacement in patients with rheumatic mitral valve disease and pulmonary hypertension Mayank Acharya; Dharmendra Joshi; Niraj Bhattarai; Samir Azam Sunny Mohammed Aslam Hossain

\section{ABSTRACT:}

\section{BACKGROUND:}

The prevalence of Rheumatic heart disease is still a burden in developing nations. About $75 \%$ of the cases of rheumatic heart disease are from developing nations. One of the causes of significant morbidity in patients having rheumatic mitral valve disease is pulmonary hypertension.

\section{METHODS:}

In this study, the change of mean pulmonary artery pressure has been evaluated in 67 patients who had mean pulmonary artery pressure above $30 \mathrm{mmHg}$ who underwent mitral valve replacement at Banglabandhu Sheikh Mujib Medical University and Lubana Hospital, Dhaka, Bangladesh.

\section{RESULTS:}

The mean CPB and cross clamp time was $70.3 \pm 28.5$ and $40.8 \pm 17.3$ minutes. The preoperative mean pulmonary artery pressure was $63.6 \pm$ $20.1 \mathrm{mmHg}$. The post-operative mean pulmonary artery pressure at $1 \mathrm{st}$ POD was $33.6 \pm 11.6 \mathrm{mmHg}$ and after 3 months was $30.3 \pm 9.6 \mathrm{mmHg}$. Post-operative mortality was $5.9 \%(\mathrm{n}=4)$.

\section{CONCLUSION:}

Significant decrease in PAP is noticed after mitral valve replacement in patients with previously high PAP.

\section{Management of tricuspid valve regurgitation \\ Anurag Garg \\ Smruti Hindaria}

\section{ABSTRACT:}

Addressing tricuspid valve regurgitation during surgery on the heart for other pathologies is a complicated decision. TR is associated with left sided valvular diseases more commonly with mitral valve than with aortic valve. It is also associated with left to right shunts of long standing duration like atrial septal defect very commonly.

We are discussing the problem of secondary tricuspid dilatation with or without regurgitation. The question is whether it should be repaired or left untreated. 
There is no reliable method to judge how much of TR is reversible after corrective left heart surgery or ASD closure. Also, there are divided opinions regarding repairing mild to moderate $\mathrm{TR}$.

At our centre, we have been repairing tricuspid valve in all patients with severe TR and moderate TR with or without annular dilatation. In patients with mild TR also we repair the valve if there is annular dilatation. We are following up patients and results have been reassuring.

We advocate repairing all patients with tricuspid regurgitation except those with mild TR without annular dilatation.

\section{Iatrogenic intraoperative ostial obstruction of right coronary artery during aortic valve replacement (AVR) - a rare complication Divya Arora; Ashok Chahal; Apresh Sanyal; Shamsher Singh Lohchab \\ Ashish Manmohan Agrawal}

\section{ABSTRACT: \\ INTRODUCTION:}

Coronary obstruction intraoperative is a rare but lethal complication. This case report highlights challenges in making intra operative diagnosis of this rare complication and its management

\section{BACKGROUND:}

Incidence of ostial coronary artery obstruction intra operative in AVR only few case reports were available in literature. Whenever surgeon face a difficulty in weaning cardiopulmonary bypass (CPB) support main culprit thought goes to prolonged residual effect of cardioplegia solution or ischemic time leading to myocardial dysfunction.

CASE:

Symptomatic 48 years female, severe aortic stenosis, aortic valve replaced with $18 \mathrm{~mm}$ Medtronic Open Pivot 360 AP bileaflet mechanical prosthesis in supraannular position. Weaning attempt from CPB failed due to right ventricle (RV) distension, full CPB support reinstituted with active venting, 2nd attempt to wean off also failed, ionotropic support increased and RV contractility transiently improved, possibility of RCA ostial obstruction suspected in view of low lying coronary ostia and narrow root, after weaning off $\mathrm{CPB}, \mathrm{RCA}$ proximal arteriotomy done and there was little retrograde flow, no antegrade flow. Saphenous vein graft was anastomosed to proximal RCA on beating heart without coronary stabilizer and proximal anastomosis on ascending aorta with partial clamp. With restoration of RCA flow RV contractility improved and RV distension resolved. At 6 months of follow up her condition is stable and NYHA class II.

\section{CONCLUSION:}

We have learned that ostial obstruction of coronary artery in AVR an avoidable complication by following these steps: 1) preoperative assessment of low lying coronary ostia as advocated for Transcatheter AVR. 2) large prosthesis in small aortic root should be avoided and if needed aortic root enlargement should be performed.

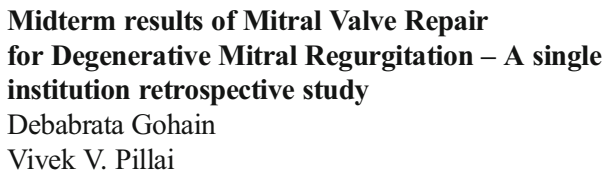

\section{ABSTRACT: \\ BACKGROUND:}

We retrospectively analyzed 81 patients who underwent mitral repair for degenerative MR and who had a follow-up period of 5 years.

\section{OBJECTIVES}

The objective of the study was to analyze event free survival, progression of MR, functional status, durability of repair based on the different techniques and freedom from reoperation.

\section{MATERIAL AND METHODS:}

Retrospective study of 81 patients who underwent mitral repair from 2011 to 2015. Functional class assessment, echocardiographic data, surgical procedure performed and statistical analysis using SPSS software and KaplanMeir curves.

\section{RESULTS:}

$75 \%(60 / 81)$ of patients were male with $90 \%(72 / 81)$ having a preoperative functional NYHA class 3. Preopeartive MR $(90 \%, 72 / 81)$ ) was Grade 4 with $90 \%$ (71/81)of patients in sinus rhythm.57 patients with isolated PML prolapse, 15 isolated AML prolapse and 9 patients bileaflet prolapse. Surgical techniques included triangular/quadrangular resections, Alfieri stitches, chordal transfers, PTFE neochoradal reconstructions and ring annuloplasty by flexible/rigid rings. Post operative TEE used to confirm adequacy of repair in all cases. Follow up of 81 patients at 5 years, 17 (21\%) had no MR,40 (49\%) had mild (1-2+) MR, 16 (19\%) had mild (2+) MR and $8(10 \%)$ had moderate (3+) MR. Improvement in functional class was seen in $96 \%$ of the patients. 4 were readmitted for various reasons and 1 had reoperation for MR progression.

\section{CONCLUSION:}

Mitral Valve Repair is a durable operation with excellent mid term results regardless of the type of leaflet pathology and annuloplasty ring implanted. Transesophageal echo confirmation of immediate postprocedural outcome targeted at trivial grade MR is essential for long term durability.

Femoro femoral and Ileofemoral crossover grafts in complex aortoiliac occlusive disease. Pares Bandyopadhyay

Binoy K. Sarkar

\section{ABSTRACT: INTRODUCTION:}

The extra-anatomic femoro-femoral bypass procedure is an effective surgical procedure for high-risk patients with an acceptable operative mortality and morbidity to improve the quality of life. Apart from severely debilitated and at high risk for direct aorto-iliac reconstruction, this procedure was also found to be effective in good-risk patients with severe claudication, ischemic restpain or trophic lesions. We have used femorofemoral bypass and crossover iliofemoral bypass utilising trans-obturator approach in this setting in poor risk patients with impending gangrene, rest pain, or early tissue compromise of a leg with gratifying results.

\section{METHODS:}

We have retrospectively studied nine patients with complex aorto-ileofemoral occlusive disease presented with critically ischaemic limb who were operated over last 7 years in our Institute of NRS Medical college and Hospital at Kolkata. All these patients had extra anatomic crossover bypass grafts.

\section{RESULTS:}

We had 7 elderly male and two female patients. All patients recovered well postoperatively. There was no significant morbidity on the donor limb side with significant improvement of symptoms and wound healing in all these cases. Three patients had wound infection with delayed wound healing. There was no mortality. Prosthetic bypass grafts were used in all cases.

\section{CONCLUSION:}

This study demonstrates that the extra-anatomic femoro-femoral bypass procedure is an effective alternative approach for high-risk patients with an acceptable operative morbidity to improve the quality of life. description here.

Median sternotomy scar outcome: Our experience Dharmendra Kumar Srivastava;

Subhash Singh Rajput; A.P. Jain

Navneet Kumar Srivastva 


\section{ABSTRACT: \\ INTRODUCTION:}

Median sternotomy scar is one of the most painful reminders of an open heart surgery. We have evaluated the different scar features after one year of surgery.

\section{METHOD:}

We have evaluated scar outcome in 60 patients with median sternotomy operated from may 2016 to april 2017 using the Patient and Observer Scar Assessment Scale (POSAS) and photographs. POSAS is a questionnaire for the patient as well as the clinician.

\section{RESULTS:}

Median age of the patient was 35.38 years. Out of the 60 patients, all of Asian origin $(100 \%), 29(48.33 \%)$ were male and $31(51.66 \%)$ female. Twelve patients (20\%) complained of pruritus over scar and two $(3.33 \%)$ with pain. Eighteen patients $(30 \%)$ presented with poor scar healing and six $(10 \%)$ were not satisfied with the cosmesis of the scar. The clinicians evaluated the color, increased contour and poor healing of the scars. Twelve patients $(20 \%)$ presented with hypertrophic scarring.

\section{CONCLUSION:}

This study shows that hypertrophic scarring can develop in 1 in 5 patients and 1 in 3 can present with poor healing. Living with scars can be a challenge in a society that values physical attractiveness so it is important for a clinician to examine and provide proper preventive measures to ensure good scar outcome.

\section{ACTUAL 10 YEAR FOLLOW UP OF PATIENTS AFTER HEART VALVE REPLACEMENT -OUTCOME DATA FROM A SINGLE CENTRE Chandrasekar Padmanaban; \\ Muralidharan Srinivasn \\ Muthukumar Sundaram}

\section{ABSTRACT: \\ BACKGROUND:}

This study looked at all patients who underwent isolated heart valve replacements in our centre in the year 2007and analyzed their outcome at the end of 10 years

\section{METHODS:}

Case records of 166 (n) consecutive patients who underwent isolated heart valve replacements in the year 2007 at various positions- Mitral (MVR - mechanical valve -X1, tissue valve $\mathrm{X} 2$ ), aortic (AVR mechanical Y1, tissue -Y2), Double valvemitral and aortic (DVR Z1-mechanical Z2- tissue valve) were analyzed. The primary end points were death from any cause and valve related complication including MAACE(Mortality, Cerebrovascular Accident, Prosthetic valve thrombosis, Structural valve dysfunction, Reoperation ), NYHA class and Echo (prosthetic valve function and LV function) were analyzed.

RESULTS:

Mean Age was 42.97 (Range $78-14$ years). Ten year survival for X1- $86.9 \%$ X2-75.1\%, Y1-90.6\% Y2- 57.1\% Z1-80\% Z2$66 \%$. Incidence of MACCE were calculated per 100 patient year for X1- 3.13 X2-2.7, Y1-10.1, Y2-2.53, Z1-1.1, Z2-0.5 per 100 patient years. Event free survival for X1-67.5\%, X2-55\% Y1$87.2 \%$ Y2 $25.3 \%$ Z1-83.3\%Z2-41\% per 100 patient years. Overall early mortality (\&lt; 30days)-1.8\%9(n=3 ) and late mortality was $6.62 \%(\mathrm{n}=11) .6 .02 \%(\mathrm{n}=10)$ last followup. Mean INR for X1- 2.1 y1- $1.8 \mathrm{Z} 1-2.68$. Valve related readmission were $32.6 \%$. Bio-prosthetic valve degeneration were $15.2(n=5)$. Mechanical valve dysfunction were $4.47 \% \quad(n=6)$

\section{CONCLUSION:}

Event free 10 year survival is best with Mechanical aortic Valve followed by DVR mechanical valves and worst with AVR tissue valves. Inspite of sub therapeutic INR values in our population the incidence of thromboembolic events are surprisingly low. We need a valve registry covering more years to rule out skewed results due to small numbers with Tissue AVR, Tissue DVR and Tissue MVR.

Intraperitoneal hemorrhage - A rare complication following cardiac surgery

A. Sivaraman; Manimaran;

Saravana Krishnu Raja

P.A. Abinayavallaban

\section{ABSTRACT:}

\section{INTRODUCTION:}

Cardiac surgery has many well known complications but gastrointestinal complications that too intraperitoneal hemorrhage is very rare. Mortality rate following intraperitoneal hemorrhage ranges from $14 \%-100 \%$ if not diagnosed early. So far only three cases have been reported with complication of intraperitoneal hemorrhage following cardiac surgery. We present this case report because of the rare complication that occurs after cardiac surgery.

\section{CASE REPORT:}

A $42 \mathrm{Yr}$ female who is a known case of RHD-MS/AS/AR presented with c/o breathlessness - NYHA class III and Palpitations admitted for double valve replacement in our ward. She underwent routine workup process and was posted for double valve replacement after anesthesia fitness. She underwent Double valve replacement with $25 \mathrm{~mm}$ St Judes in mitral and $19 \mathrm{~mm} \mathrm{St}$ Judes in aortic position. Immediate Post operative period patient had persistent hypotension and decreased urine output, inspite of high inotropic support. So emergency reexploration done to rule cardiac tamponade but there were no features of tamponade and chest was closed. After few hours patient developed abdominal distension and four quadrant aspiration showed bloody aspirate and so Surgical gastroenterology opinion sought and advised emergency laparotomy which showed $1.5 \mathrm{~L}$ hemoperitoneum with bleeding from splenic hilar region and was found to be ruptured splenic artery pseudoaneurysm with Splenic abscess was noted. Spleenectomy was done. Patient vitals stabilized following the procedure and weaned and extubation done on 3rd POD and discharged on 17 th POD.

\section{CONCLUSION:}

Cardiac surgical procedures involve multiple cardiac complications but one should also think of other system complications even though they are rare. We present this case because of the rarity.

The Myocardial Protective Effects of Adenosine additive Potassium Blood Cardioplegia D. Janardhan Reddy; K.V. Muralidharan; P.S.N. Raju; Ramakrishnan; Masthanamma Chengali; C. Srinivasan; Srikanth Bhumanna; Ashok Sampath Thejaswi N. Marla

\section{ABSTRACT:}

\section{INTRODUCTION:}

Hypothermic depolarizing hyperkalemic $(\mathrm{K}+20 \mathrm{mEq} / \mathrm{L})$ blood cardioplegia is the "gold standard" in cardiac surgery. $\mathrm{K}+$ has been associated with deleterious consequences, eg, intracellular calcium overload. An alternative approach to arresting the heart is to maintain the transmembrane electrical potential in a polarized state, which locks the ion channels in a "closed" state. Therefore, ionic imbalances and subsequent consequences are likely to be avoided. Adenosine triphosphate-sensitive potassium channel opening (PCO) agents (eg, nicorandil and aprikalim) have been used to achieve polarized or hyperpolarized arrest. However, PCO agents have been reported to increase postischemic arrhythmias and myocardial oxygen demand on reperfusion and to produce profound 
systemic hypotension. For these reasons, hyperpolarized arrest with PCO agents as the arresting agent has not been adopted as a clinical cardioplegia strategy. Adenosine activates adenosine triphosphatesensitive potassium channels by A1-receptor-mediated mechanisms.

\section{AIM:}

This study aims to elucidate the hypothesis that elective arrest in a polarized state with adenosine $(300 \mu \mathrm{mol} / \mathrm{L}$ via adenosine triphosphatesensitive potassium channel opening) as the arresting agents in blood cardioplegia provides cardioprotection comparable to standard hypothermic K+-blood cardioplegia.

\section{MATERIALS AND METHODS:}

100 patients who had undergone mitral valve replacement for stenotic and noncompetent mitral valves were analysed for post operative outcome in the form of myocardial ischaemia, arrhythmia, CPB span, postoperative stay and mortality.

\section{RESULTS:}

The patients with Adenosine augmented Potassium blood cardioplegia had better outcome in terms of myocardial protection with decreased depression of ventricular function, seen in around $3.5 \pm 1 \%$ arrhythmias in less than $5.2 \pm 2 \%$ of the study population. CONCLUSION:

Blood cardioplegia offers better myocardial protection and operating conditions than intermittent ischemia or continuous coronary perfusion. Adenosine as an alternative approach to arresting the heart to maintain the transmembrane electrical potential in a polarized state defers the deleterious effects of potassium blood cardioplegia and showered better outcome.

\section{Acute aortic prosthetic valve endocarditis - our surgical experience. \\ C.S. Hiremath}

Naveen Pandarinathan

\section{9}

\section{ABSTRACT: \\ INTRODUCTION:}

Prosthetic valve endocarditis is a rare complication of valve replacement surgery and is associated with high morbidity and mortality rates.

Here, we present a case of acute prosthetic aortic valve endocarditis who presented with NYHA IV symptoms 12 years after prosthetic aortic valve replacement who underwent early surgery and recovered successfully.

CASE:

A 39 years male patient who underwent prosthetic aortic valve replacement with \#23 TTK chitra valve in 2005 presented with NYHA IV symptoms. ECG showed sinus tachycardia. CXR showed cardiomegaly with features of pulmonary edema. ECHO showed severe AR with prosthetic valve dehiscence with mild MR. Lab parameters showed anaemia, elevated ESR, leucocytosis with deranged bleeding parameters. $\mathrm{CT}$ angio showed normal coronaries. Blood culture grew streptococcus species and was started on appropriate antibiotics. As he was in heart failure, with in 3 days of admission, patient underwent early surgery. Intraoperatively, there was prosthetic valve dehiscence and aortomitral discontinuity with LCC and RCC outpunches. Under femorofemoral bypass, patient underwent valve explantation, thorough annular tissue debridement, outpunches closed with gelfoam, recreation of aortomitral continuity and with subannular sutures, \#21 TTK chitra valve was implanted. Postoperatively, patient had complete heart block and PPI was inserted after 3 weeks. His blood cultures became negative after 3 weeks and was treated with IV antibiotics for 6 weeks and discharged with oral antibiotics for 4 weeks. Discharge echo showed normal prosthetic valve function with gradient 25/ $15 \mathrm{mmhg}$, mild MR, good LV function.

\section{CONCLUSION:}

Prosthetic valve endocarditis is a rare and serious complication of valve replacement surgery. Early surgery in patients with NYHA IV symptoms despite positive cultures can be done with successful results.

Clinical analysis of cardiovascular reoperations on a total-206-case report Tao Hong

Yu Shi

\section{OBJECTIVE:}

To summarize and explore risk factors on the mortality and recovering process of patients undergoing various repeated heart surgeries in 3 years recently.

\section{METHODS:}

Clinical data of 206 consecutive patients who underwent secondary heart valve surgery from May 2015 to August 2017 in Zhongshan Hospital of Fudan University was retrospectively analyzed. There were 106 male patients and 100 female patients aging from 14 to 83 years old. Following variables were collected: preoperative morbidity, heart function, initial surgical strategies, internal time between operations, indications, reasons of the secondary operation, ICU time, postoperative morbidity and mortality. Multivariate logistic regression was used to analyze the risk factors of death and recovering time of patients undergoing secondary surgery.

\section{RESULT:}

The main reasons of the first operation include the transplantation and repair of the valve, correction of congenital heart disease, and operations for the great vessels and the second are unavoidable because of the valve neoplasm, perivalvular leak, emerging tricuspid insufficiency and the great vessel lesion and etc. 17 patients died in perioperative period with the overall mortality $8.3 \%$ at the condition of 190 patients with series of complications The main reasons of the perioperative period death included low cardiac output syndrome, respiratory failure, and renal insufficiency. Multivariate logistic regression showed that patients have postoperative LCOS $(\mathrm{OR}=5.514$, $\mathrm{P}=0.043)$, postoperative respiratory failure $(\mathrm{OR}=0.009, \mathrm{P} \& 1 \mathrm{lt} ; 0.001)$, postoperative renal insufficiency $(\mathrm{OR}=18.135, \mathrm{P}=0.001)$ are independent risk factors of perioperative death of patients underwent reoperations. Besides of the 3 reasons before, what affects the ICU time are preoperative neurological disorders( $\mathrm{P} \& 1 \mathrm{t} ; 0.001)$, preoperative heart functions $(\mathrm{P}=0.09)$ and postoperative pleural effusion $(\mathrm{P}=0.02)$ at the regression of the linear analysis.

\section{CONCLUSION:}

Adequate operation time and effective assessment and preservation for myocardial, respiratory and renal functions play an important role in improving prognosis. Postoperative LCOS, respiratory failure, renal insufficiency are risk factors of perioperative death.

Solo Smart stentless bioprosthetic valve produces superior effective orifice area index in aortic valve replacement

Ryoi Okano; Yuko Gatate; Kenichi Kamiya;

Masaomi Fukuzumi

Tadamasa Miyauchi

\section{ABSTRACT:}

BACKGROUND:

Solo Smart (Solo), a new stentless biological valve, has been reported to offer larger effective orifice area (EOA) than stented bioprosthetic valve. However, there have been no reports for Asian patients.

\section{MATERIALS:}

We evaluated 167 consecutive patients who underwent aortic valve replacement between 2014 and 2018. Valve orifice areas were compared postoperatively between Group A (before April 2016; $\mathrm{n}=74$ ) and Group 
B (after April 2016; $n=96$ ). Group B was subdivided into the Solo group $(\mathrm{n}=37)$ and the stented bioprosthetic valve group $(\mathrm{n}=33)$.

\section{RESULTS:}

Body surface area (BSA) was significantly smaller in the Solo subgroup compared with the other groups. The mean size of the transplanted valves was larger in Group B than in Group A (A: $22.3 \pm 1.8$ vs. B: $23.5 \pm 2.0 \mathrm{~mm}$, $\mathrm{p} \& \mathrm{lt} ; 0.01)$. Within the group $\mathrm{B}$, the valve size was significantly smaller in the Solo group compared with the stented valve group ( $22.7 \pm 1.9$ vs. $24 \pm$ $2.0 \mathrm{~mm})$. However, EOAI was larger in Solo than stented valve (1.28 \pm 0.33 vs. $1.13 \pm 0.27, p$ \&lt; 0.05 ) and additionally, at each size, Solo was significantly superior $(21 \mathrm{~mm}$, EOAI $1.18 \pm 0.23$ vs. $1.01 \pm 0.22 ; 23 \mathrm{~mm}$, EOAI $1.31 \pm 0.35$ vs. $0.97 \pm 0.30$; and $25 \mathrm{~mm}$, EOAI $1.48 \pm 0.22$ vs. $1.17 \pm 0.25$ ). CONCLUSION:

Even though recent technological advancement enabled us to use bigger prosthetic valve, Solo Smart offers larger EOAIs for patients with smaller annuli.

Sternal pull up - A new ingenious and simple technique for the congenital heart surgeon C. Ananthanarayanan; Kartik Patel; Pankaj Garg; Tushar Gajjar; Amit Mishra; Chirag Doshi;

Vishal Agarwal; Ravi Patel; Rahul Kumar

\section{INTRODUCTION:}

Delayed sternal closure is a valuable tool in the armamentarium of congenital heart surgeons. It comes with its own disadvantages of prolonged ventilation, intensive care unit stay, sepsis and wound infection. We present our own technique of closing the sternum primarily or early using a sternal pull up technique to avoid or reduce the duration of open chest and its attendant complications.

\section{RESULTS:}

From 2016 to 2018 , we have used this technique in a total of 25 patients. This includes patients undergoing various surgical procedures such as Rastelli operation $(n=7)$, Conduit for pulmonary atresia with ventricular septal defect $(n=4)$, truncus arteriosus $(n=5)$, arterial switch operation $(n=9)$. All patients were successfully weaned off the sternal pull up. Two patients died of ventilator associated pneumonia and one due to sepsis. None of the patients had sternal instability or wound infection (superficial or deep sternal).

CONCLUSION:

Sternal pull up is an ingenious, easily reproducible and cheap technique with multiple benefits and can be used safely in many congenital heart surgeries.

Early and mid-term outcomes following off-pump coronary artery bypass grafting in patients with end stage renal disease

Sayedur Rahman Khan; Jahangir Kabir

Sonjoy Biswas

\section{ABSTRACT:}

Introduction and Objectives:

End stage renal disease (ESRD) is considered to be an independent risk factor for early and late survival following coronary artery bypass grafting. In this study, we assessed early and mid-term outcomes in ESRD patients after OPCAB with complete revascularization and identified the predictors for postoperative death and cardiac events.

MATERIALS AND METHODS:

A retrospective review of 27 consecutive dialysis-dependent patients scheduled for OPCAB was conducted between January, 2015 and November, 2018 at United Hospital Limited, Dhaka, Bangladesh. Clinical characteristics, mortality and post-operative complications were evaluated.

\section{RESULTS:}

Complete revascularization was successfully performed in all patients. The mean number of anastomoses was 4.1. Thirty-day mortality was $7.4 \%$. In-hospital mortality was $3.7 \%$. At a mean followup of 18 months, there were four late deaths including one cardiac death, and three non-cardiac events. Actuarial survival rate at one and three years was $88.8 \%$ and $77.0 \%$, respectively. Cardiac event free rate at one and three years was $85.9 \%$ and $72.6 \%$, respectively. Multivariate analysis revealed that preoperative left ventricular ejection fraction $(\mathrm{P}=0.003)$ and smoking history $(\mathrm{P}=0.026)$ were significant predictors for mid-term mortality, and co-existing peripheral vascular disease (PVD) was a significant predictor for cardiac events $(\mathrm{P}=0.033)$.

\section{CONCLUSIONS:}

Complete revascularization is safe with acceptable early and mid-term outcomes in off-pump techniques in ESRD patients.

Long Term Outcome of Off-Pump Coronary

Artery Bypass Grafting in Patients with In-Stent

Restenosis

Saydur R. Khan; Sonjoy Biswas;

S.M. Zakir Khaled; Jahangir Kabir

Syed Nahian

\section{ABSTRACT: \\ OBJECTIVE:}

One of the major complications of percutaneous coronary intervention is in-stent restenosis (ISR). Now-a-days, it has become more challenging problem for both the cardiologists and the cardiac surgeons. We reviewed the long-term outcome of patients with ISR who underwent off-pump coronary artery bypass grafting $(\mathrm{CABG})$.

\section{METHODS:}

Symptomatic patients $(\mathrm{n}=130)$ having Canadian Cardiovascular Society (CCS) score II-IV with ISR and multi-vessel disease were identified using cardiac catheterization laboratory data between January 2010 and November 2015. All patients underwent off -pump CABG for the management of ISR. The interval from the latest intervention ranged from 4 to 96 months. Clinical outcome events of interest included death, myocardial infarction (MI), target vessel revascularization (TVR) and combined end point of these major adverse cardiovascular events (MACE). Followup periods ranged from 6 to 60 months.

\section{RESULTS:}

There were no operative deaths. Age distribution ranges from 48 years to 65 years, among them 119 were male while 11 were female. In the long term follow up there were only 2 mortalities the cause of which was non cardiac and there were no reported incidence of MI. All patients in the follow-up were at a Canadian Cardiovascular Society (CCS) class 0 or 1. CONCLUSIONS:

Off-pump bypass surgery for patients with ISR and multi-vessel disease can be safely performed and provides a favorable long-term clinical outcome.

In-Stent Restenosis, Coronary Artery Bypass Grafting (CABG)

Lateral Translocation of Main Pulmonary artery to Left Pulmonary artery in a case of Tetralogy of Fallot with anamolous origin of Right Coronary artery from Left Coronary sinus: A case report

Saket Agarwal

Sayyed Ehtesham Hussain Naqvi

\section{ABSTRACT:}

\section{BACKGROUND:}

Tetralogy of Fallot with anamolous origin of coronary arteries is a rare subset of congenital artery disease $(0.7-1 \%)$. Reconstruction/ Translocation of the neo-ostium, Opening of the intramural component 
(if any), Coronary artery bypass grafting are some of the described surgical options. Translocation of Main pulmonary artery to Left pulmonary artery is an excellent surgical option if the two coronary ostia are close to each other but sometimes is technical difficult in case of hypoplastic pulmonary artery. We report one such case in which successful translocation of Main pulmonary artery (MPA) wa done.

CASE REPORT:

A 5 years old male with cyanosis and clubbing pesented to us diagnosed as a case of Tetralogy of Fallot with pulmonary stenosis and was planned for Intra cardiac repair. On evaluation CT angiography revieled subaortic VSD, infundibular and valvular pulmonary stenosis with anamolous origin of Right Coronary artery (RCA) from Left Coronary Sinus with intra mural course. Patient was operated and succesful intracardiac repair with translocation of MPA to left Pulmonary artery was done to relieve compression from the RCA.

\section{DISCLOSURE:}

The case report has not been presented but the complete case report and the review of literature is under consideration for publication.

Double orifice mitral valve with Supramitral ring with Mitral Stenosis with Ventricular septal defect with aortic overide with Sub-aortic membrane and severe pulmonary artery hypertension: A rare case scenario Vishnu Datt

Sayyed Ehtesham Hussain Naqvi

\section{ABSTRACT: \\ BACKGROUND:}

Double orifice mitral valve (DOMV) is an uncommon congenital cardiac anomaly and is characterized by a mitral valve with a single fibrous annulus with two orifices that open into the left ventricle, each having an independent chordal attachment to the papillary muscle. Frequently associated lesions are atrioventricular septal defect, Coarctation of aorta, Interrupted aortic arch, Patent Ductus Arteriosus, Primum atrial septal defect, Tetralogy of Fallot, Ebstein anomaly, left-sided obstructive lesions, moderate to severe MS and MR. Association of DOMV supramitral ring, Sub Aortic Membrane causing severe PAH is rare with potential for cardiac morbidity and mortality and hence hereby reported. CASE REPORT:

A 4 years, male child, weighing $13 \mathrm{~kg}$ presented to us with a history of recurrent fever, chest infection and failure to thrive. Transthoracic echocardiography revealed dilated left atrium and left ventricle, mitral valve showed 2 separate valve orifices separated by a fibrous bridge with a supra mitral ring causing, mitral stenosis. Mean gradient across right and left sided orifice were $7.7 \mathrm{mmHg}$ and $12 \mathrm{~mm} \mathrm{Hg}$ respectively. A Sub aortic membrane, large subaortic VSD with bidirectional shunt and aortic over ride were noted. There was severe PAH(RVSP- $86 \mathrm{mmHg}$ ). Patient was operated for removal of Supramitral ring, Sub aortic membrane and dacron patch closure of VSD with creation of PFO in view of severe PAH.

However postoperatively the patient developed an irreversible fatal pulmonary hypertensive crisis (PHC), immediately after transferring the patient to the cardiac intensive care unit (ICU) from the operating room (OR). The PHC was refractory to intravenous and inhaled milrinone and nitroglycerine and intravenous adrenaline, dobutamine, norepinephrine, and vasopressin, patent foramen oval (PFO) and $\mathrm{CPB}$ support. The management of DOMV and perioperative pulmonary hypertension is a rare case scenario and hence hereby presented.

DISCOSURE:

Complete case report not presented but is under consideration for publication.

Results of surgical repair of aortopulmonary window with interrupted aortic arch

Shiv Kumar Choudhary
Sachin Talwar

\section{ABSTRACT: \\ OBJECTIVE:}

Aortopulmonary Window (APW) with Interrupted aortic arch (IAA) is a rare congenital cardiac malformation. We discuss our experience with surgery for this condition.

\section{METHODS:}

Between 2005 to 2019, 6 patients underwent repair of APW with IAA at our institute. We studied anatomic characteristics, and early and late results of surgery for these patients.

RESULTS:

Of the 6 patients, 4 were male. Mean age was 4.5 months (10 days-15 months). Subtypes of APW were type $1(n=3)$, type $2(n=2)$, and type 3 $(n=1)$. IAA was type A $(n=5)$ and type $B(n=1)$. APW was repaired with sandwich technique using polytetrafluoroethylene patch in all patients and the IAA under Deep hypothermia and circulatory arrest (TCA) with end-to-end anastomosis in 5 and aortic homograft aortic reconstruction $(\mathrm{n}=1)$. Mean CPB, cross-clamp and TCA times were 146, 111 \&amp; 32 minutes respectively. There were 2 early deaths ( 1 each due sepsis \&amp; neurological injury). Intensive care unit (ICU) stay was mean of 4.5 (2-16 days) and was complicated by severe pulmonary hypertension (PAH) requiring nitric oxide in two and tracheostomy in patient. Mean followup was $64(20-120)$. months There were no late deaths.

\section{CONCLUSIONS:}

Diagnosis of APW with IAA should always be entertained in the presence of a large patent arterial duct with APW. This rare and high risk subset of patients requires complex reconstruction. Late results of surgical repair are acceptable after a period of with intense and prolonged ICU) stay.

Results of surgical repair of double aortic arch Shiv Kumar Choudhary; Sachin Talwar

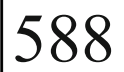

\section{ABSTRACT:}

\section{OBJECTIVE:}

Double aortic arch (DAA) is rare but is the commonest type of vascular ring amenable to surgical repair. In this paper, we discuss our experience with its surgical management.

\section{METHODS:}

We studied the anatomic characteristics and results of surgery in 20 patients undergoing repair of DAA at our institute between January 2005 July 2018.

\section{RESULTS:}

Of 20 patients, 11 were male. Mean age was 12 years ( 2 months-31 years). Eighteen patients had dominant right arch, 1 patient had dominant left arch and 1 patient had a codominant arch. Symptoms manifested as tracheoesophageal compression $(n=15)$, only respiratory compression $(n=4)$, only esophageal compression $(n=1)$. Associated lesions were Tetralogy of Fallot (TOF, $n=1$ ), TOF with absent left pulmonary artery (LPA, $n=2)$ Ruptured sinus of Valsalva $(n=1)$, Ventricular septal defect $(n=1)$, univentricular heart $(n=1)$, and patent foramen ovale $(n=2)$. Division of non-dominant arch was performed through left thoracotomy $(\mathrm{n}=18)$ (Video), right thoracotomy $(\mathrm{n}=1)$ and median sternotomy $(\mathrm{n}=1)$. Five patients including TOF with absent LPA underwent thoracotomy for DAA followed by sternotomy for intracardiac lesions in same sitting; in 1 patient with TOF, repair was staged after initial period of division and anastomosis of left arch to LPA followed by TOF repair after few months. There were no early or late deaths.

Follow-up was mean of 56 months $(2-140)$ and all patients are asymptomatic with no surgical residua.

\section{CONCLUSIONS:}

Repair of DAA is associated with satisfactory early and late results. The method of repair needs to be individualized when associated defects are present. 
Early surgical intervention for Infective Endocarditis: Analysis of factors influencing outcome

Praveen Varma; Kirun Gopal
Sujit Kumar Mohanty

\section{ABSTRACT:}

Immunologic reactions to protamine sulphate during cardiac surgery are very rare. The frequency and outcome of such adverse reaction is unclear. Clotting of distal anastomosis sites and proximal site (in aorta) is very rare. We report a case of unusual life threatening reaction to protamine that occurred in a Non-diabetic patient following the uneventful off pump coronary artery bypass grafting operation.

Outcomes after mechanical mitral valve replacement in patients aged 20 to 70 years

Kurian V.M.; Sowmya S.

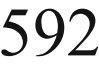

\section{ABSTRACT:}

\section{OBJECTIVE:}

To provide data on major morbidities following mechanical mitral valve replacement.

\section{METHODS:}

Retrospective analysis of 81 patients who underwent isolated mechanical MVR in our department, a single surgeon experience, from 2012 to 2017. Patient's preoperative comorbidities, rhythm, LV function, intraoperative bypass, cross clamp time, postoperative duration of ICU and hospital stay, rhythm changes, INR values, mitral valve gradients, MACCE events, formation of thrombus, pannus, IE, redo surgeries, SVD, NSVD, bleeding complications were monitored. Patient's 1st follow up was taken at 1 year following discharge and last follow up was last visit to hospital. Exclusion criteria were concomitant $\mathrm{CABG}$ or valve replacements or repair of any other valve, congenital cardiac history or procedures.

RESULTS:

Mean age was 46.46+/- 22.56. 63 patients had RHD, 17 mitral valve prolapse and 1 HOCM. 28 were in AF, 52 in sinus rhythm and 1 in PVC preoperatively. LV function was $60+/-14$. BSA was $1.62+/-0.36$, AXC time 47.7+/-32.2, CPB time 76.3+/-54.2. Mean ICU stay was $3.19+/-1.62$ and hospital stay $10.03+/-3.16$. Mean gradients at the time of discharge were $3.64+/-2.94$, at end of one year were $4.97+/-10.1$, last follow up 4.11+/-3.94. 1 had paravalvular leak, 3 patients had MACCE events, 1 SVD, 3 NSVD, 4 thrombus, 2 pannus, 3 central thromboembolic events, 1 IE and 4 bleeding events during follow up. 9 patients underwent redo MVR.

\section{CONCLUSION:}

Postoperative morbidities are acceptable and these findings inform decision making in patients, particularly those basing prosthesis selection, lifestyle choices rather than access to health care or contraindications to lifelong anticoagulation.

Immediate and Short-term Outcomes of Aortic Valve Replacement with Aortic Root Enlargement for Small Aortic Root

Tejas R. Shah

Utkarsh Sanjay Sanghavi

\section{ABSTRACT:}

\section{BACKGROUND:}

Aortic root enlargement (ARE) during aortic valve replacement (AVR) allows for larger prosthesis implantation and can be an important adjunct to surgical AVR. The technique although proven to prevent patient prosthesis mismatch (PPM) still isn't a routine standard of care due to bias among the surgeons regarding its benefits justifying the increased operative time and cardio-pulmonary bypass $(\mathrm{CPB})$ duration compared to isolated AVR.
Unusal life threatening protamine reaction in a patient after off pump CABG. A case report Sunil Kumar Satpathy 
To analyze the immediate and short-term outcomes of ARE using death and adverse events as end points.

\section{METHODS:}

61 patients with aortic valve disease were operated on between January 2015 and December 2017 in the Department of Cardio-Thoracic \&amp; Vascular Surgery, Sri Sathya Sai Institute of Higher Medical Sciences, Whitefield, Bangalore. 12(19.67\%) patients underwent ARE. Root enlargement was selectively performed in patients at risk for PPM [indexed effective orifice area (iEOA) less than $0.85 \mathrm{~cm} 2 / \mathrm{m} 2$ ]. Patients were put in $\mathrm{AVR}$ and AVR + ARE group respectively.

\section{RESULTS:}

The mean age was $38.2 \pm 14.16$ years in AVR group and $36.75 \pm 14.97$ years in AVR+ARE group. The mean size of the prosthetic valve implanted was 22.43 \pm 2.3 in AVR group and $24.33 \pm 1.8$ in AVR+ARE group ( $p$ \&lt;0.001). ARE required an average of 45 minutes of additional aortic clamp time (p\&lt;0.001).

\section{CONCLUSION:}

Aortic root enlargement can be performed readily with minimal added risk relative to standard AVR. It is a safe and effective procedure and should be considered particularly in patients with a small aortic annulus as it reduces post-operative gradients and the incidence of patientprosthesis mismatch
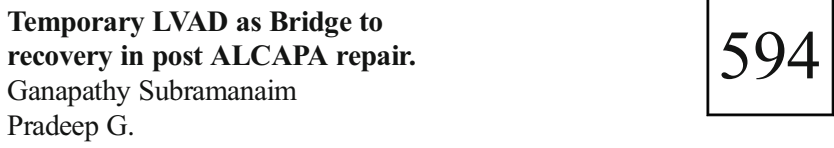

\section{ABSTRACT:}

Temporary LVAD as bridge to recovery in post ALCAPA repair. Dr.Pradeep, Dr.ganapathy Subramaniam, Dr.K.R.Balakrishnan, Dr.Suresh RAo

Fortis Malar Hospitals, Chennai.

\section{INTRODUCTION:}

ALCAPA presenting with left ventricular dysfunction is associated with high degree of mortality in the early post operative period. We are presenting a series of 3 consecutive patients supported with Temporary LVAD at least for first 48 hours as bridge to recovery.

\section{METHODOLOGY:}

3 patients ranging from newborn to 4 months underwent ALCAPA repair in the last 6 months. Considering the previous experience it was decided to support the left ventricle with Temporary LVAD. Tempororay LVAD was structured using inflow cannula from Left atrium and Outflow cannula into ascending aorta. Outflow cannula was inserted in 2 cases with a $5 \mathrm{~mm}$ gore-tex interposition graft considering the size of aorta and to facilitate cannula removal and re insertion. Both Heparinisd and non heparinised circuits were used and coagulation profile was adjusted accordingly. Maquet Rotaflow pump was used in all 3 cases. Left ventricle was supported for a minimum of 48 hours and gradually weaned off monitoring the function using Echo cardiogram.

\section{CONCLUSION:}

All 3 patients had an unventful recovery with no end organ or neurological issues and was discharged before 10th day. Gradual exposure of systemic vascular resistance to dilated and failed LV with mechanical circulatory support alleviate the dreadful complication like low cardiac output state in immediate post operative period.

\section{OUTCOMES AFTER MECHANICAL AORTIC VALVE REPLACEMENT \\ V.M. Kurian; Rajan}

S.; Anjith Prakash; Sowmya S.

Shilpa Shree

\section{ABSTRACT:}

OBJECTIVE:

To determine the postoperative morbidity following mechanical aortic valve replacement.

\section{METHODS:}

92 patients underwent mechanical aortic valve replacement between 2012 to 2016. Patient's preoperative comorbidities, LV function with echo analysis, intraoperative bypass and cross clamp time; postoperative duration of ICU and hospital stay, INR values, aortic valve gradients, MACCE events, formation of pannus, thrombus, IE, redo surgeries, SVD, NSVD, bleeding complications were monitored. Patient's first and last follow up details were taken. Redo AVR, combined surgeries, incomplete surveys were excluded from study.

\section{RESULTS:}

Mean age was $49.4+/-30.46 .37$ patients presented with angina, 26 with syncope and 60 with dyspnoea. 55 patients had RHD, 18 bicuspid valve, 9 degenerative diseases, 2 Infective endocarditis and 1 had takayasu arteritis as the etiology. Mean EF was 53.96+/-26.1. Mean preoperative gradients were $46.979+/-52.32$. Mean AR slope was 483.13 . Mean ICU stay was $3.03+/-2.4$ and hospital stay was $7.94+/-5.2$. Mean prosthetic valve gradient during 1 st follow up was 11.26+/-10.46. Mean gradient at last follow up was 13.14+/-20.64. 2 patients died due to stuck valve and 1 due to RTA. One developed infective endocarditis, 1 developed CCF, 3 major bleeding episodes, 1 intrapericardial haematoma, 5 developed CVA, 2 had stuck valves and thrombolysed, 1 underwent redo AVR with hemiarch replacement due to aortic root abscess.

\section{CONCLUSION:}

Provides knowledge of outcomes beyond what is known about the mortality benefit, to provide insight into the morbidity burden of modern-day AVR.

\section{A Comparative Study of Arsenic Deposition in Human Nails and Myocardial Tissue Fahmida Binta Wali; Nazmul Hosain}

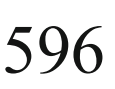

\section{ABSTRACT: \\ INTRODUCTION AND OBJECTIVE:}

Arsenicosis is a major problem in many areas of the World including parts of India and Bangladesh. Arsenic (As) is a toxic element present in environment particularly in water. If drinking water or food contains high level of Arsenic, this may be deposited in various tissues in abnormal levels particularly in hair, nail and skin causing damage and health hazards. The susceptibility of myocardium to Arsenic deposition and its adverse effects hasn't been studied well. Objective of this study is to figure out the relationship between the level of Arsenic deposition in human nails and myocardium.

\section{MATERIALS AND METHODS:}

Nail and cardiac tissue samples were collected from 50 adult patients fulfilling the criteria and undergoing open heart surgical procedures at Chittagong Medical College Hospital, Chittagong and National Heart Foundation Hospital Dhaka Bangladesh between July 2017 and June 2018. Nail samples (1-3mm breadth) were collected from all the fingers and toes of the patient on the day before surgery. A small piece of myocardium was harvested from the right atrial appendage within of venous cannulation purse string site. Arsenic measurement was performed by Hydride Generation Atomic Absorption Spectrometry (HG-AAS) in ICDDRB, a reputed international organization engaged in health research activities in Dhaka, Bangladesh.

RESULT:

Mean+SE of Arsenic concentration found in the nails was 436.30+ $35.17 \mathrm{ppb}$ whereas that in atrial myocardium was $4.07+0.40 \mathrm{ppb}$. Pearson correlation test Showed that there is a significant positive linear 
relationship between the level of Arsenic concentration in nails and that in atrial myocardium $(\mathrm{r}=0.501, \mathrm{p}=\& 1 \mathrm{lt} ; 0.005)$.

\section{CONCLUSION:}

A significant positive linear relationship exists between the level of Arsenic concentration in nails and that in atrial myocardium.

Total Anomalous Pulmonary Venous Connection (TAPVC) - Our experience from a single center Neelam B. Desai

Wahaj Ali

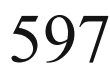

\section{ABSTRACT: \\ BACKGROUND:}

TAPVC is a cardiac malformation in which there is no direct connection between any pulmonary vein and the left atrium; rather, all the pulmonary veins connect to the right atrium or one of its tributaries.

\section{METHODS:}

A retrospective study of 27 patients of TAPVC was done who underwent surgical correction at our institute between August 2010 to January 2018. There were 16 male (59\%) and 11 female (41\%). Twelve patients (44\%) were less than 10 year of age. 17 patients (63\%) had a Supracardiac TAPVC, 7 patients $(26 \%)$ had Cardiac TAPVC and 3 patients $(11 \%)$ had Mixed TAPVC. All patients (n-27) underwent elective surgery. Median age at operation was 11 years (range, 3 to 46). All (100\%) underwent preoperative transthoracic echocardiography. Nine patients (33\%) underwent cardiac catheterization. Fifteen patients $(56 \%)$ had severe pulmonary artery hypertension. Twenty five patients $(93 \%)$ were correctly diagnosed before treatment. All patients were treated surgically. The defect was approached through the right atrium in all the patients (100\%).

\section{RESULTS:}

Of 2 deaths, 1 died due to severe biventricular dysfunction (about 1 month after the initial surgery), and the other due to postoperative multi organ failure (on POD-7). All survivors were surviving well at last follow up.

\section{CONCLUSIONS:}

The adverse events and deaths encountered in this study are during early postoperative period. Long term Survival after TAPVC repair in the adolescent and adult age group is excellent with significant improvement in quality of life.

\section{Surgical management of Cardiac}

Myxomas - a single center experience

Hiremath C.S

Dinesh Kumar R.

\section{ABSTRACT: \\ BACKGROUND:}

Primary cardiac myxoma is the commonest benign neoplasm of the heart. Surgical excision of the cardiac myxomas offers the best long term results.

\section{OBJECTIVE:}

We report our 10-year surgical experience in management of cardiac myxoma.

\section{METHODS:}

102 patients with cardiac tumors were operated between February 2008 - August 2018 in the Department of Cardio-Thoracic \&amp; Vascular Surgery, Sri Sathya Sai Institute of Higher Medical Sciences, Whitefield, Bangalore of which 98 patients had histologically proven myxoma.

\section{RESULTS:}

In our series mean age was 41.6 years, female preponderance noted (64.28 \%). 84 patients had LA myxoma, 1 had RV myxoma remaining patients had RA myxoma. 7 patients presented with stroke and 1 with gangrene over toes. 67 patients presented with pulmonary arterial hypertension and 20 with ventricular dysfunction. Anaemia and increased erythrocyte sedimentation rate was found in 77 and 76 patients respectively. 84 patients had myxomas arising from interatrial septum, 6 from left atrial wall, 2 from left atrial roof, 3 from right atrial wall, 1 each from pulmonary valve, mitral valve, eustachian valve. Concomitant coronary artery bypass procedure was done in 2 patients, mitral valve repair in 11 , tricuspid valve repair in 9, pulmonary valve reconstruction in 1 . There were no perioperative strokes, myocardial infarction, rhythm disturbances, deaths. The mean follow-up was 9.70 months $+/-3.73$ months. 89 patients were followed up $(90.81 \%)$ who had no recurrences, arrhythmias.

\section{CONCLUSION:}

Surgical excision of cardiac myxomas offers best results. In our institute, we prefer trans septal approach for myxomas arising from the inter atrial septum which helps not only to excise the myxoma completely without any residues but also to inspect all four chambers of the heart. Trans-septal approach does not require two separate atrial incisions thus decreasing the possibility of arrhythmias.

\section{The outcomes of surgical repair in Ebstein} anomaly at SSSIHMS, Whitefield.

Hiremath C.S

Dinesh Kumar R.

\section{ABSTRACT:}

\section{BACKGROUND:}

Ebstein anomaly is a congenital heart defect in which tricuspid valve leaflet is adherent to the underling myocardium due to failure of delamination. The incidence of Ebstein anomaly occurs in about one to five per 200,000 live births

\section{OBJECTIVE:}

To review the outcomes of surgery in patients with Ebstein anomaly at our institution.

\section{METHODS:}

10 patients with Ebstein anomaly were operated between March 2012 August 2018 in the Department of Cardio-Thoracic \&amp; Vascular Surgery, Sri Sathya Sai Institute of Higher Medical Sciences, Whitefield, Bangalore.

\section{RESULTS:}

Mean age in our series was 22.7 years with female preponderance $(70 \%)$. 5 patients had cyanosis, 6 patients were of carpentier type B classification, 3 type D, 1 type C. 8 patients presented with severe tricuspid regurgitation, 3 with ventricular dysfunction, 8 with associated ostium secundum atrial septal defect. Of 10 patients, 6 underwent biventricular repair in whose mean NYHA functional class improved from $2.66+/-0.31$ to $1.16+/-0.40$. Of 3 patients who underwent Uni-ventricular repair in whose mean NYHA functional class improved from $3.66+/-0.577$ to $2.33+/-0.57 .9$ patients were followed up with mean follow-up of 21 months $+/-8.48$ months.

\section{CONCLUSION:}

Though the surgical correction for Ebstein anomaly varies from patient to patients, depending on the nature of tricuspid valve, volume and functional status of right ventricle, surgical repair helps symptomatic relief and improves the patient's condition of life.

OPCAB Complete Arterial Revascularisation with Double Inflow for high risk coronary artery disease Y.A. Nazer

Pradeep Kumar Radhakrishnan

\section{ABSTRACT:}

\section{INTRODUCTION:}

With the application of coronary stents being widened, increasingly high-risk patients are being encountered in surgical practice, the need to offer $\mathrm{CABG}$ techniques that improve long-term 
survival at the same time reduces short-term complications is the need of the hour.

\section{Material and Methods:}

Multiple inflows and those in which vein graft was used or performed on pump this period (Oct 2016-Oct 2018) were excluded from the study. All conduits were fully skeletonized. LIMA was anastomosed to anterior zone vessels- LAD, Diagonals and High intermediate branches, with RIMA in tandem with radial artery extension being anastomosed to lateral zone and inferior zone vessels. The nondominant radial artery was used after Doppler flow evaluation of the arm. Elective use of milrinone and noradrenaline as inotropes which was tapered off in 24 hours. The institutionalized operative technique performed on the last 238 patients will be discussed.

\section{RESULTS:}

The Achilles heel of surgical revascularisation strategies has been the saphenous vein graft, which was successfully overcome with the operative design of total arterial complete revascularisation for vessels with critical occlusion. The major criticism of SYNTAX trial, the neurological injury could be overcome by eliminating aortic manipulation and cardiopulmonary bypass. To address these concerns and design the best possible revascularisation strategy we performed beating heart total arterial complete revascularisation with double inflow.

\section{CONCLUSIONS:}

Revascularization by this method is simple, reproducible, and amenable to minimally invasive methods and is thus trendy and future compatible, addressing issues of short-term complications and improved long-term survival.

\section{OUR EXPERIENCE OF TYPE A ACUTE AORTIC DISSECTION \& THEIR OUTCOMES IN 17 CASES Ankit Jain}

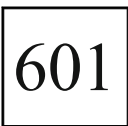

\section{ABSTRACT:}

AIM:

TYPE A AORTIC DISSECTION IS AN EMERGENCY. METICULUS PLANNING \& PROMPT SURGICAL INTERVENTION GIVE SATISFACTORY \& REPRODUCIBLE RESULTS.

\section{MATERIAL AND METHODS:}

17 CASES ADMMITTED FROM JAN 2016- OCT 2018 WERE INCLUDED OF TYPE A AORTIC DISSECTION

RESULTS:

Mean age was 49 (range 24 to 63 ) years.

Mean time to extubation was 16 (range13 to 29,) hours. Mean time Pleural drainage was $210(180-470) \mathrm{ml}$, and mean ICU stay was 6.27(range 4-9) days. Total hospital stay was $9.8(8-15)$ days. Operative procedure Bentall(13) + Bentall /CABG(3)+BENTALL/TOTAL ARCH REPLACEMENTG(1). There were no late deaths or thromboembolic complications. One patient required Rexploration. 161 patients underwent axillary cannulation only one patient underwent femoral cannulation. One of the patient had reintubation. One of the patient had rehospitalisation. There wasone early death.

CONCLUSION:

PROMPT SURGICAL INTERVENTION \&amp; SAFE SURGICAL TECHNIQUES GIVE SATISFACTORY RESULTS.

Why to avoid coronary stents in specific subsets - Is it time for a shift in reference frame? Sanal Kumar V.R.

Pradeep Kumar Radhakrishnan

ABSTRACT:

INTRODUCTION:
In-Silico studies sing CFD using viscous flow probes by us have shown that Venturi effect in partially blocked arteries creates cavitations and shock waves followed by pressure overshoot leading to hemorrhage and heart attack with plaque rupture inside the artery having a higher relaxation modulus.

\section{MATERIALS AND METHODS:}

We have proved that the likelihood of the aerodynamic flow-choking in the blockage region of any internal flow system including the human artery occurs when the differential pressure $(\Delta \mathrm{P})$ equals the product of the differential density $(\Delta \rho)$ and the square of the axial velocity (V axial) at the blockage region. Attaining this significant physical condition in any internal flow system is very dangerous owing to the fact that it invites shock waves if the downstream cross-sectional area of the duct is higher than the blockage region where the choking occurs. This limiting condition arises when the Venturi effect acting on the blood flow through micro CD nozzle throat effect causes a decrease of the blood pressure beyond the restriction

\section{RESULTS:}

In vitro studies done by us showed that the boundary-layer blockage occurs while attaining the aerodynamic flow-choking condition. This leads to instent narrowing and higher reinterventions rates

\section{CONCLUSION:}

Boundary layer choking was noted to higher in vessels with distal bifurcation patterns, smaller vessels, and longer blocks. Critical MI index and predictive priori can be derived from the above studies Clearly, coronary bypass surgery which avoids the diseased area would be a better longterm choice scientifically. The results would find applications in SSTO vehicles and Hybrid rockets.

Dual inflow total arterial composite grafts are safer than single inflow composite grafts: Short term SPECT Analysis

Sunil Basavaraj

Pradeep Kumar Radhakrishnan

\section{ABSTRACT:}

Title Dual inflow total arterial composite grafts are safer than single inflow composite grafts: Short term SPECT AnalysisR Pradeep Kumar MS, Mch (AIIMS), Post-doctoral fellow (SCTIMST), Senior Consultant AHRI Kerala India, Sunil Basavaraj Mch MNAMS, Square Hospitals Bangladesh

OBJECTIVES:

Composite arterial grafts can be done with single or dual inflow systems. Behavioural patterns of these grafts at stress and at rest needs analysis, as often a decision needs to be made whether these have to be done as a routine or by choice in select situations only.

\section{METHODS:}

50 patients who underwent revascularisation by composite arterial grafts underwent a retrospective evaluation. Conduit non availability or porcelain aorta were indications of this procedure. Intra operative flow measurements were used in all cases. Perioperative transthoracic Doppler ultrasound, exercise test at 12 months, sestamibi scitigraphy at rest and during induced hyperaemia and 64 slice CT were recorded.

RESULTS:

Perioperative and rest flows were adequate in 196 distal anastomoses fashioned as composite grafts.22 patients showed stress test that was positive. Dipyridamole stress induced SPECT imaged showed inability to meet requirements at maximal hyperaemia. Stress scores observed and coronary flow reserve noted would be discussed.

\section{CONCLUSIONS:}

Single inflow grafts should be discouraged as the first option in daily surgical practice. This is a choice in patients without other alternative options. Larger studies over more prolonged periods are needed to confirm the results of this study. 
A case series of HOCM - our experience

Giridhar Kamalapurkar

Shilpa Suresh
An unusual case of dysphagia- vascular

ring- A case report.

J. Nageswar Rao;

Praveen C.H..; Anup H.S.

\section{ABSTRACT: \\ INTRODUCTION AND OBJECTIVES:}

Hypertrophic cardiomyopathy (HOCM) is a primary myocardial disease characterized by left ventricular hypertrophy in the absence of other etiologies. Surgical septal myectomy is the gold standard for the treatment of patients with severe drug-refractory symptoms or LVOT obstruction. However, it involves the risk of incomplete resection of septal muscles or iatrogenic septal perforation depending. We present our experience of successful septal myectomy in HOCM.

\section{MATERIALS AND METHODS:}

Retrospective analysis of all cases of HOCM who underwent septal myectomy were included in the study. All the patients underwent preoperative tranthoracic echocardiography, intraoperative transesophageal echocardiography and postoperative transthoracic echocardiography. The total number of cases were 20 from January 2015 to october 2018. The postoperative mean LVOT gradient was 12 . One patient had a postoperative VSD, two patients needed pacemaker and the mortality was 1 . CONCLUSION:

Septal myectomy remains the treatment of choice in HOCM. In our experience, we could achieve good postoperative LVOT gradients with simple and safe surgery.

Role of Pre-operative Evaluation in Successful

Outcome of Vascular Access AV Fistula

Vinay Krishna; Anumeet Bagga

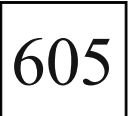

\section{ABSTRACT: \\ INTRODUCTION:}

Haemodialysis treatment for end stage renal disease(ESRD) requires reliable vascular access. Optimal access is provided via a functional arteriovenous (AV) fistula, which compared with other forms of vascular access, provides superior long term patency, requires few interventions, has low thrombosis and infection rates and cost. However, it has been estimated that around $20-60 \%$ of fistulas never mature sufficiently to enable haemodialysis treatment.

\section{MATERIALS AND METHODS:}

This study was conducted in 400 consecutive patients who came to CVTS department of our Institute for vascular access AV fistula between September 2017 to September 2018. Thorough preoperative arterial evaluation was done by -pulse examination, modified Allen's test, Duplex ultrasound for arterial size (diameter) and flow rates and CT angiography. Venous evaluation was done by Duplex ultrasound, contrast venography. Patients were followed up on 15th day, at 1 month and 6 months of AV fistula creation.

\section{RESULTS:}

Out of the 400 patients, preoperative evaluation by physical examination was done in all of them. In 368 patients only Duplex ultrasound was used as the imaging modality, whereas in 28 patients only contrast venography was performed, while both Duplex and contrast venography were used in 50 patients. In 4 patients, no imaging modality was used (due to time and availability issues). In 15 patients, CT Angiography was done. Out of the $400 \mathrm{AV}$ fistulas, 362 were created on the radial artery with cephalic vein (end to side). Good patency rates were obtained where the diameter of cephalic vein was $\& \mathrm{gt} ; 2 \mathrm{~mm}$ and that of radial artery \&gt; $2 \mathrm{~mm}$ and minimum flow of $20 \mathrm{ml} / \mathrm{min}$. In 344 patients, the AV fistulas matured successfully.

\section{CONCLUSION:}

It was found that Duplex scan was a superior imaging modality and helped in successful AV fistula creation. Contrast angiography is problematic in patients with renal damage.

\section{ABSTRACT: \\ ntroduction:}

Extrinsic compression of esophagus produced by vascular structures is a rare cause of dysphagia and numerous congenital anomalies of great arteries exist which are of clinical importance when they compress the trachea and esophagus.

CASE:

An 8 year old female child with history of device closure of ventricular septal defect was brought with complaints of Dysphagia, mainly to solid food since 6 months and no respiratory symptoms. Clinical examination and 2D ECHO was unremarkable. CT aortogram suggested right sided aortic arch with mirror image branching of aortic arch vessels and aberrant left subclavian artery. Cardiac Catheterization study was consistent with findings of CT aortogram and ruled out Coarctation of aorta and patent ductus arteriosus. Barium swallow suggested luminal compromise of esophagus at D4 level, consistent with dysphagia lusoria secondary to aortic diverticulum. A left lateral thoracotomy approach to the vascular ring was done and ligamentum arteriosum and vascular ring was dissected, looped and divided. Patient had an uneventful post operative recovery. Patient followed up post operatively at 3 month, was asymptomatic. Barium swallow was done post operatively suggested satisfactory excision of diverticulum and satisfactory dynamic flow at the upper thoracic esophagus with no significant post operative adhesion or diverticula.

\section{CONCLUSION:}

Vascular ring is a rare clinical entity with myriad of presentation ranging from stridor to dysphagia. This case report highlights early prompt diagnosis and management of vascular ring that can reduce the morbidity and mortality of the disease.

Bilateral Internal Mammary Arteries:

How to do it, The Finer Points

Vaibhav Mishra

\section{Abstract:}

Most of studies have conclusively proven the advantage of Bilateral Mammary arteries in CABG in terms of enhanced graft patency which then translates into improved survival, more so in Diabetic Patients.

Despite this the Use of B/L IMA's is only $6 \%$ in US [STS ] and $10 \%$ in Europe and similar percentages would be expected in India.

There are many challenges to use of B/ L IMAs, concerns of Sternal wound dehiscence, Increased operative times, and unfamiliarity with doing $\mathrm{Y}$ and Sequencing, Harvesting conduits length of conduits, coupled with inadequate training make it a challenging operation. Also one has to be mindful of Competitive flow and degree of stenosis.

Truth remains that most surgeons don't get to do or see this operation as residents and have to develop this skill on there own.

There are many points that need to be evaluated if this operation needs to be done successfully.

Age can be a relative contraindication only but if the patient is fit it can be done in any age, specially it saves the long incisions on legs and healing problems and early mobilisation is possible.

It can be done in Mod and Severe LV dysfunction as well, provided its not Ac MI.

In diabetics the concern of wound infection is there but most trails have shown as no difference in wound healing, and this can be reduced by meticulous skeletonization of both IMAs without damaging the vein 
With the help of operative videos i will demonstrate step by step how to perform this safely and how to reduce the learning curve of B/L IMAs.

Repair of the Rheumatic mitral valve: our experience Vishal Khante

\section{ABSTRACT: \\ BACKGROUND:}

Repair techniques for myxomatous valves have been well established, however repair of rheumatic mitral valves has always been seen as being difficult and attempted by few. Recent development of reproducible techniques has led to adoption of the procedure. We review our early experience in repairing the rheumatic mitral valve.

\section{METHODS:}

The rheumatic mitral repair program started in November 2017 at our unit. All mitral valve procedures including double valve disease have been included on an intention to treat basis. Myxomatous mitral repairs have been excluded from the analysis. The data was collected prospectively. All procedures were performed by a single surgeon with a standard approach (comissurotomy, peeling, sharp dissection of edges and annuloplasty) at different sites. On table TEE/TTE were performed to ensure valve competency, valve was replaced when more than mild regurgitation was present. Data is presented as mean $\pm \mathrm{SD}$.

\section{RESULTS:}

A total of 220 mitral procedures have been performed between November 2017 to date. 25 patients were non-rheumatic. Male to female ratio is $2: 3$. Average age of patients was $46.5( \pm 16.8) \mathrm{yrs}$; mean BSA was $1.57 \pm 0.22 \mathrm{~m} 2$; mean CPB time was $102.4 \pm 49.2$ min and mean $\mathrm{X}$ clamp time was $70.2 \pm 29.8 \mathrm{~min} .13$ patients needed valve replacement during the procedure when repair result was not acceptable (5.9\%). 3 patients required at a later period( 2 days-3months). One patient died in the immediate post op period.

\section{CONCLUSIONS:}

Rheumatic mitral repair is possible with a success rate of $7.2 \%$ and low mortality. Long-term data is still awaited. Standardisation of techniques makes the procedure reproducible.

\section{BRONCHO-ESOPHAGEAL FISTULA CAUSED BY FOREIGN BODY \\ Lanza Giovanni \\ Alberto Oliaro}

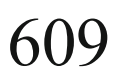

\section{ABSTRACT: \\ OBJECTIVES:}

The bronco-esophageal fistula can be caused by esophagitis, specially esophageal candidiasis, esophageal cancer, burns and caustic injury and inhaled foreign body. This is an infrequent condition and few cases caused by foreign body aspiration are described in the literature. This video shows a singular case of bronco-esophageal fistula caused by inhaled insulin needle.

\section{VIDEO DESCRIPTION:}

The patient, cocaine and heroin addicted, accidentally inhaled an insulin needle which settled in the main left bronchus. However he remained asymptomatic for almost one year. The needle then caused a fistula between the left bronchus and the esophagus; consequently the patient started to suffer from cough and dysphagia. A first therapeutic approach with an endoscopic clipping device was undertaken, but failed to close the fistula. The patient was then selected for surgical repair. Even if the preoperative radiological and endoscopic studies demonstrated a narrow fistula tract, the surgical findings showed a large communication between the bronchus and the esophagus. In this case endoscopic clipping would have never closed the esophageal defect because of its dimensions: only surgical repair can ensure a successful and permanent closure. The access to be preferable is a right postero-lateral thoracotomy: through this access the esophagus can be easily isolated and completely exposed. The use of staplers is recommended because can guarantee reliable esophageal and bronchial suture.

\section{CONCLUSIONS:}

The bronco-esophageal fistula should be treated by open surgical repair through a right postero-lateral thoracotomy; the use of staplers is preferable to ensure durable suture. The use of endoclips is not recommended.

\section{ROBOTIC LIGATION OF THORACIC DUCT FOR CHYLOTHORAX: A Video Presentation}

Rane N.; Thombare B.; Khan A.Z.

ABSTRACT:

\section{BACKGROUND:}

We present a video of 2 cases of Robotic Ligation of Thoracic Duct and Pleurectomy for Chylothorax

\section{METHODS:}

An 83-year-old male patient presented with recurrent bilateral chylothorax following a diagnosis of chronic lymphoid leukemia. Conservative treatment had been continued for 6 weeks and had failed. We performed a robotic ligation of thoracic duct with pleurectomy. The thoracic duct was identified and ligated by tying with a suture and clipping. The Thoracic duct was then disconnected. A Robotic pleurectomy was performed on the right side and a VATS pleurectomy was done on the left side

\section{RESULTS:}

The drains were removed on 4 th postoperative day. The patient was discharged home the following day.

\section{CONCLUSION:}

Robotic ligation of thoracic duct is technically feasible. The robotic technique allows you good dexterity to dissect and tie the duct. In addition a pleurectomy can also be performed easily.

Robotic resection of 2 nd rib tumor: video presentation Thombare B, Rane N, Khan AZ

\section{ABSTRACT:}

OBJECTIVE:

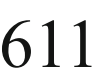

We present a video of robotic resection of 2 nd rib tumor. We show a new technique of cutting of 2 nd rib using wire saw by minimally invasive technique

\section{METHODS:}

An 18 year old male patient, during a routine naval pre selection medical screening, was found to have a tumor located on the posterior aspect of the 2 nd rib. Open resection would entail a thoracotomy and a definite medical test failure for naval selection. We did a robotic resection of the 2 nd rib tumor using innovative techniques to cut the two ends of the ribs by a wire saw. The specimen was delivered out through the port site with minimal chest wall injury.

\section{RESULTS:}

This patient was eventually selected for the Indian Navy, having passed his medical test with flying colors and is now a training naval officer.

\section{CONCLUSIONS:}

Robotic resection of chest wall tumor is possible with minimal damage to chest wall and quick return to work. Innovative thinking and surgical techniques can benefit patients and help them to return to normal life and to choose fulfilling careers.

\section{ENDOSCOPIC RESECTION} OF WHOLE RIB TUMOR

Rane N.; Thombare B.; Shaiwal Khandelwal; Rajnish Kumar; Anand Sharma; Tarun Piplani; Dhiraj Gautam; Ali Zamir Khan 


\section{ABSTRACT: \\ OBJECTIVE:}

We describe a minimally invasive technique of endoscopic resection of rib involved completely by the tumour (fibrous dysplasia)

\section{METHODS:}

30 years male patient presented with left 9 th rib tumor. The radiology was suggestive of fibrous dysplasia and needed resection of the whole rib. Endoscopic resection was performed by making three incisions of size about $1.5 \mathrm{~cm}$ over the rib and a subcutaneous plane was developed using a glove finger mounted over a metallic suction cannula and inflated using normal saline. A long sleek retractor was used to retract the skin and further dissection was continued using routine endoscopic instruments. Repair of the chest wall was done using prolene mesh following rib resection. The similar technique used to extract a bullet from the chest wall impacted at the undersurface of scapula.

\section{RESULTS:}

The patient was discharged on postoperative day 2 . The morbidity was minimal and the cosmesis was excellent.

\section{CONCLUSION:}

This technique of minimally invasive surgery can be used to resect chest wall lesions with minimal morbidity and excellent cosmetic outcomes using routine endoscopic instruments.

\section{Evolution of Heart Transplant: Three decades} of experience from Heart Transplantation in Toronto

Mitesh Badiwala

Thiruthani Kumaran M.M

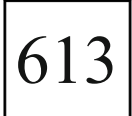

\section{ABSTRACT: \\ BACKGROUND:}

Heart transplantation remains the standard of care for eligible patients with advanced heart failure. This study reports on 30 years of experience in a single quaternary hospital with an analysis of donor and recipient profile and its outcomes.

\section{RESULTS:}

We performed a retrospective analysis of heart transplant donors and recipient profile between 1985 and 2016. During this period, 650 patients were transplanted; mean recipient age ( $49 \pm 11$ years) which did not differ significantly between decades but the donor age increased significantly over the decades $(31+/-10$ years to $35+/-15$ years).

Traumatic brain injury and cerebrovascular accident accounted for the main causes of donor deaths. In the recipient profile, non-ischemic Cardiomyopathies and Congenital etiologies increased over the decades from 27 to $58 \%$ and 1 to $10 \%$, respectively. Ischemic cardiomyopathies decreased from 63 to $22 \%$ over time. There was a significant increase in cold ischemic time across the decades (1st122 \pm 84 , 2nd126 \pm 84 , 3rd145 \pm 68 minutes; p\&lt;0.001) but didn’t affect the outcome.

Graft rejection per patient decreased during the most recent decade (first 1.0 \pm 2.4 , second $4.6 \pm 5$, third $3.5 \pm 3.1$ ). Patients listed as high priority in the last decade increased compared to the second decade (Chi-square, p\&lt;0.001). Additionally, the number of patients bridged with left-ventricular assist devices (LVAD) increased. Patient cause of death did not differ significantly across decades, with graft failure $(23.7 \%)$ and infection (14\%).

Patient survival progressively improved between decades (5-year survival: 1 st66\%, 2nd76\%, 3rd86\%, 10-year survival: 1 st $46 \%$, 2nd67\%, 3rd73\%; p\&1t;0.001).

\section{CONCLUSION:}

An improvement in recipient survival has been noted despite a higher risk donor profile being accepted for transplantation. Improvements in peri- and post-operative patient care may have led to improved outcomes.
Extending donor heart acceptability may help mitigate the worldwide issue of organ shortage and decrease waitlist mortality.

\section{Outcome of $\mathbf{4 0}$ consecutive cases of Modified Ross procedure using novel Dacron valved conduit Rajarshi Ghosh; Santosh Kumar Pandey; Sujoy Chatterjee; Hemant Kumar Nayak; Saurabhi Das; Satyajit Bose; Srirup Chatterjee Lakshmi Kumari Sankhyan \\ ABSTRACT:}

The Ross procedure is an established option for aortic valve disease in children. Due to limited availability of pulmonary homografts we devised a novel technique for right ventricular outflow tract (RVOT) reconstruction by preparing indigenous Dacron valved conduit. Forty consecutive cases of Modified Ross procedure done at our centre (2013-2018) were analysed. Thirty-seven patients $(95 \%)$ were followed up with median duration of 2.5 (0.08-5.5) years. Median age was 12 (5-39) years. Nineteen (47.5\%) patients had rheumatic aortic valve disease, while 19 $(47.5 \%)$ had congenital aortic valve disease. Aortic root replacement with pulmonary autograft was performed in all patients. Dacron conduit for RVOT reconstruction was used with on table sewn bileaflet valve using dacron patch $(n=22)$, PTFE membrane $(n=10)$, bioprosthetic valve $(n=4)$ and pericardium $(n=1)$. Additional surgical procedures included mitral valve repair $(\mathrm{n}=10)$, septal myectomy $(\mathrm{n}=2)$, ascending aorta replacement $(n=1)$, ruptured sinus of valvsalva (RSOV) repair $(n=1)$ and VSD closure $(n=1)$. There was one in-hospital mortality while one late death occurred at 3.5 years postoperatively. The neoaortic valve regurgitation on echocardiographic evaluation at follow up was - trivial $(n=28)$, mild $(n=7)$ and moderate $(n=2)$. Mild RVOT obstruction was present in 8 patients while 18 patients had mild pulmonary regurgitation. No patient required reintervention during follow up. Our early results of Modified Ross procedure are encouraging, however long term follow up is required.

\section{Challenges in surgical correction of adult Tetralogy} of Fallot: outcomes with targeted care

Nayem Raja; Tarun Raina Ramman; Sumir Girotra; Sushil Azad; Sitaraman Radhakrishnan;

Parvathi U. Iyer; Krishna S. Iyer

Kuntal Roy Chowdhuri

\section{ABSTRACT:}

\section{INTRODUCTION:}

Unrepaired adult Tetralogy of Fallot (TOF) is not uncommon in developing countries. Published literature reports discharge mortality of $5-15 \%$. Our discharge mortality from 1995- 2005 was $2 / 81(2.5 \%)$. Risk factors for death were - severe polycythemia, RV dysfunction and collaterals. Subsequently, following strategies were instituted : 1) Aggressive search and preoperative intervention for collaterals 2) staging BT shunt for persistent severe thrombocytopenia 3) Multiple preoperative phlebotomies 4) preoperative neuroimaging to detect infarcts and bleeds 5) Controlled RVOT resection 6) Preemptive management of RV dysfunction. Our aim is to assess whether these strategies resulted in improved outcomes.

\section{PATIENTS AND METHODS:}

From Jan 2006 to June 2018, 117 patients (Median age 21 years, range 16 - 50) with diagnosis of TOF $(n=108)$, DORV [TOF type] $(n=5)$, TOF with Absent Pulmonary valve $(n=4)$ were operated. Preoperative MAPCA coiling was required in 21 and 30 received multiple preoperative phlebotomies. 58 patients received transannular patch, 6 required pulmonary valve 
replacement, 2 tricuspid valve repair, 1 aortic and 1 mitral valve replacement. 4 underwent a staging BT shunt for persistent severe thrombocytopenia. Median preoperative hematocrit value was $55 \%$ (range 34-78)

\section{RESULTS:}

In hospital mortality was $0 / 117$ with no late deaths. Median duration of ventilation, ICU and hospital stay was 17 hours, 42 hours and 8 days respectively. 5/117 underwent reexploration for bleeding and one required multiple craniotomies for recurrent subdural hematoma. 2 underwent subsequent pulmonary valve replacement after 5 and 8 years. CONCLUSION:

Early outcomes after surgery for adult TOF can be greatly improved with highly individualized management despite multiple perioperative challenges.

\section{Novel Technique of Vascular Anastomosis: Posterior Continous and Anterior Interrupted Technique. \\ Hari Bipin Radhakrishnan; Simran Kundan}

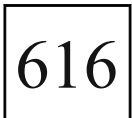

\begin{abstract}
:
AIM:

Vascular anastomosis in children very exacting, with very little margin for error. There is an ever present potential for stenosis and many ways have been devised to enlarge the anastomosis.
\end{abstract} METHODS:

In our practice, we have devised a novel way for all vascular anastomosis, which involves a continuous sutureline along the posterior wall, once the corners are turned, the continuous sutureline is interuppted and completed with simple interrupted sutures. We have applied this to all our ASO's, for the neoaortic and neo pulmonary artery, Glenn anatomosis, neo SVC-RA junction in Warden's procedure. We have applied this method for Warden's and Muller's procedure.

RESULTS:

Out of the 462 ASO's we have had 10 reoperations for supravalvar pulmonary stenosis out of which 8 had augmentation with anterior pericardium and only $2 \mathrm{pt}$ had tissue to tissue continuity. None of the Glenn had stenosis. Average gradient in the neo SVC-RA junctions in Warden's procedure has been $2.3 \mathrm{mmHg}$. Muller's subgroup, the average gradient has been $1 \mathrm{mmHg}$ and all have been laminar flow on long term follow up. This method of Anatomosis, maximizes the effective orifice area, it allows for even match of unequal diameter vessels. It is especially useful in the coronary transfer in the closed arterial technique in ASO, when the coronaries lie across the neo-Aortic sutureline. We have never revised or misjudged a coronary for its lie and orientation with this method. CONCLUSION:

The technique of posterior continous and anterior interrupted, maximizes the growth potential of the small structures, the anterior interrupted sutures breaks down the fibrotic process allowing for growth inbetween the adjoining sutures.

Timing of coronary artery bypass after acute myocardial infarction- does it matter?

Mrinalendu Das; Anton Prem

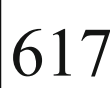

\section{ABSTRACT:}

\section{OBJECTIVE:}

The optimal timing of coronary artery bypass grafting (CABG) operations in patients with recent acute myocardial infarction (AMI) remains unclear. In this study, we aim to assess the influence of timing on postoperative outcomes in patients undergoing CABG following AMI.

\section{METHODS:}

In this retrospective analysis 12046 consecutive patients undergoing CABG were included. 2479 patients $(20.5 \%)$ had a history of AMI. Based on timing of CABG patients were divided into 3 groups: Group I (those operated within 7 days of AMI) group II ( \&gt; 7 days but $\leq 30$ days) and group III (those operated after 30 days. The 3 groups were compared in terms of baseline, intra-operative and post-operative morbidity and mortality. Multivariate analysis was carried out to assess the independent influence of timing of CABG on outcomes.

\section{RESULTS:}

The 3 groups were well matched in terms of body mass index ( $\mathrm{p}=0.91)$, hypertension $(p=0.11)$, peripheral vascular disease $(p=0.77)$, airway disease $(\mathrm{p}=0.079)$, diabetes $(\mathrm{p}=0.14)$, ejection fraction $(\mathrm{p}=0.07)$. hypothyroidism $(0.05)$, renal failure $(0.54)$ and neurological events $(0.55)$. Both elective and emergency conversions to cardiopulmonary bypass was significantly higher in group I ( $\mathrm{p} \& 1 \mathrm{t} ; 0.001)$. Transfusion requirements $(\mathrm{P}=0.004)$ and deep sternal wound infections $(\mathrm{P}=0.007)$ were significantly more common in group I while risk of renal failure and 30-day mortality were significantly more common in group II (P\&lt;0.001). Multivariate analysis showed that timing of operation was an independent risk factor for renal failure (Odds ratio $0.482,95 \% \mathrm{CI} 0.31$ to 0.73 ) and 30 day mortality (Odds ratio $0.59,95 \%$ CI $0.38-0.91$ ).

\section{CONCLUSION:}

Based on this study we conclude that timing of CABG after AMI does influences outcomes and carries the least risk when carried out after a delay of 4 weeks and should be preferred in patients who are stable.

Porcine versus bovine bioprosthetic valves in mitral position: does choice really matter???

\section{ABSTRACT: \\ BACKGROUND:}

Bioprosthetic valve options are increasingly used for surgical Mitral valve replacement (MVR). However, current decision making regarding the type of bioprosthetic valve choice is not data-driven given the incomplete information on comparative long-term outcomes after bovine (BoMVR) vs porcine (PoMVR). As such, this study sought to examine the effect of bioprosthetic valve choice on long-term survival and reoperation after MVR.

\section{METHODS:}

A retrospective analysis of all bioprosthetic MVRs, with or without Coronary Artery Bypass Grafting (CABG) procedures, at a single tertiary referral institution from jan 2005 to dec 2008 was conducted using a prospectively maintained database. Procedures were classified as BoMVR or PoMVR. The outcomes were defined based on STS guidelines for valve related mortality and morbidity. The patients were followed up for ten years. The age group was from $40-70$ years.

\section{RESULTS:}

We identified 166 BoMVR patients, and 138 PoMVR patients who were matched based on propensity random sampling matching. The final sample came down to 154 BoMVR and 120 PoMVR patients. kaplan meier survival and cox proportional hazards model were used for corresponding statistical analysis. Freedom from re operation (all cause), freedom from non structural valve deterioration, freedom from structural valve deterioration, freedom from heart failure, freedom from infective endocarditis were $96.4 \pm 0.08 \%$ (p\&lt;0.05 9.6-10.01), 97.1 $\pm 0.07 \%$ (p\&lt;0.04,9.69.9), 98.6 $\pm 0.04 \%$ (p\&lt;0.06,9.8-10.02), 100\% (p\&lt;0.03 9.8-10.08), 98.6 $\pm 0.06 \%$ (p\&lt;0.07,9.7-10.03) in PoMVR respectively and 94.6 $\pm 0.09 \%$ (p\&lt;0.45 9.5-9.9), 91.6 $\pm 0.08 \%$ (p\&lt;0.04,9.5-9.8), 94.6 $\pm 0.04 \%$ (p\&lt;0.06,9.7-9.9), 94 $\pm 0.08 \%$ (p\&lt;0.03,9.7-10.2) 92.8 $\pm 0.08 \%$ (p\&lt; $0.07,9.8-10.1$ ) in BoMVR groups respectively at the end of $10 \mathrm{yr}$ follow up.

\section{CONCLUSIONS:}

For patients undergoing MVR with a bioprosthetic valve, with or without CABG, the choice of PoMVR vs BoMVR favours more in favour of PoMVR as evidenced by the outcome results. Probably long term follow up with more patients might throw further light on the debatable topic. 
Transplantation of oversized hearts in pediatric age group.-outcomes and lessons learnt.

Pradeep, Ganapathy subramaniam,

K.R.Balakarishnan, Suresh Rao

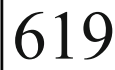

\section{ABSTRACT: \\ INTRODUCTION:}

In cardiac transplantation the generally accepted upper limit of donor recipient weight ratio is 1.2. Higher incidence of requirement of ECMO support and Mortality has been reported when the ratio is more than 2 . However strict adherence to this criteria limits the availability of organs for patients who needs transplantation at the earliest. we report our experience on Transplantation done on patients whose donors weight were more than 2 times of the recipients.

Our Experience:

$50 \%$ of pediatric donors have over sized hearts of \&gt; $2: 1$. Out of $5 / 40$ deaths $-12.5 \%$ early mortality.

2 late deaths -after initial big heart syndrome.

1 due to high PVR and RV dysfunction

2 - big heart syndrome

1 - primary graft dysfunction.

Donor heart size measurement - biatrial, SVC junction to apex, Posterior mitral to to anterior RV in PLAX view.

IN all DCM who are not redo's, one can cut down on pericardium up to phrenic and even incise the pericardium posterior to phrenic.

Leaving chest open -3 patients - closed the next day in all three with immediate good outcome and discharged. The anterior surface of the RV which bulges due to high PVR immediate post $\mathrm{CPB}$, and effect of ischemia usually settles down by next day.

Leaving PFO - RV adjusts better.

Traction suture - a suture which goes through the skin and the sternum which can be used to pull up the sternum to prevent compression of RV. CONCLUSION:

In the era of organ shortage creating space, RV managed uncompressed, effective management of Big Heart syndrome will benefit pediatric recipients from donors with weight even more than three to four times them.

\section{Weight gain potential- a neglected} entity during valve replacement

Karthik Raman; Anbarasu Mohanraj;

Jacob Jamesraj; V.M. Kurian; Rajan Sethuratnam;

Ejaz Ahmed Sheriff; Anjith Prakash; Jeswin Moses; Shilpashree

Vijayanand Palanisamy

\section{ABSTRACT: \\ INTRODUCTION:}

During valve replacement, appropriate valve size will be chosen based on the weight of the patient at time of surgery, neglecting the potential of the patient for gaining weight. We aimed at evaluating the weight gain potential and its effect on hemodynamics in post mitral valve replacement (MVR) patients. Material and methods In 118 post-MVR patients, Demographic and echocardiographic data at the time of discharge and follow-up (minimum of 6months) were obtained and analyzed. Primary aim of study is to analyze the hemodynamics of patients based on weight gain and weight loss. Secondary aim is to evaluate the same in Patientprosthesis mismatch (PPM) subgroup and to evaluate the study population for the potential to gain/loss weight.

\section{RESULTS:}

Among 118 patients, 87 patients(73.7\%) gained weight and 31 patients $(26.3 \%)$ had weight loss. In 87 weight gained patients, left atrial (LA) size $(\mathrm{p}=0.011)$ and pulmonary artery systolic (PASP) pressure $(p=0.028)$ at follow-up were significantly higher than the discharge values.
Among 53 PPM patients (incidence-44.9\%), 34 patients gained weight and their PASP was found to be elevated (discharge Vs follow-up) $(p=0.021)$ whereas weight lost group doesn't show any significant difference (discharge Vs follow-up)( $\mathrm{p}=0.972)$.

Frequency of weight gain was more among 20 to 30 years group patients $(\mathrm{p}=0.043)$. No sex predilection was noted( $\mathrm{p}=0.149) .28$ out of 30 patients $(93.3 \%)$ who weighed \&1t;50 kg preoperatively, gained weight during follow $u p(p=0.013)$.

\section{CONCLUSION:}

In post-MVR patients, weight gain has definitive influence over the hemodynamics. In PPM subgroup, weight gained patients had significantly increased PA systolic pressure at follow-up whereas weight lost group doesn't show significant difference. Young and $\& 1 t ; 50 \mathrm{~kg}$ weighed patients were more prone for weight gain and should be advised to maintain their weight post MVR for better hemodynamics.

One year outcomes of off- and on-pump coronary artery bypass grafting: a randomized multicentric study Kunal Sarkar

Lokeswara Rao Sajja

\section{ABSTRACT:}

\section{BACKGROUND:}

Early this year we reported 3 months graft patency and clinical outcomes of Prospective Randomized comparison of Off-pump and On-pump Multivessel cOronary artery bypass surgery To Evaluate outcomes and graft patency (PROMOTE patency trial). We now report major adverse cardiac and cerebrovascular events (MACCE) at one year between patients who underwent coronaryartery bypass grafting (CABG) performed using off-pump technique and on-pump technique.

\section{METHODS:}

Prospectively we enrolled 320 patients with multivessel coronary artery disease from March 2016 through March 2017 at 6 centers and were randomly assigned to undergo either off-pump CABG $(n=158$ patients) or on-pump CABG $(n=162$ patients). We analysed the outcomes and evaluated the graft patency at 3 months. 316 patients were followed up for one year outcomes (3 patients lost to follow up) and the outcomes were analysed in 313 patients.

\section{RESULTS:}

At one year, one mortality occurred in on-pump group $(0.63 \%)$ and none in off-pump group, $(\mathrm{p}=1.00)$. There was no difference between the groups in the outcomes of non-fatal myocardial infarction $(p=0.49)$, cerebro vascular accident $(p=1.00)$ repeat revascularization (percutaneous coronary intervention or redo $\mathrm{CABG}$ ) $(p=1.00)$ and chronic kidney disease requiring renal replacement therapy $(p=0.49)$. Kaplan Meier curves showed better outcomes in off-pump CABG compared to on-pump CABG in terms of MACCE.

\section{CONCLUSIONS:}

At 1 year there was no significant difference in MACCE between offpump and on-pump CABG.

Off-pump CABG $(\mathrm{n}=155)$

On-pump CABG $(\mathrm{n}=158)$

$P$ Value

Mortality

Myocardial infarction

Chronic kidney disease requiring renal replacement therapy

Cerebro vascular accident

Repeat revascularization

*Fisher exact test was used

One year outcomes of off-pump and on-pump CABG 
Autologous pericardial aortic valve reconstruction: Early experience

Prasanna Simha; Rakesh Naik;

Yousuf Rafi; Jeeva Vijayan

\section{ABSTRACT: \\ INTRODUCTION:}

Autopericardial aortic valve reconstruction is an attractive option compared to prosthetic valve replacement due to absence of anticoagulation, biocompatibility and excellent left ventricular regression.

\section{OBJECTIVE:}

We wanted to share the early results of autopericardial aortic valve reconstruction from our centre

\section{MATERIALS AND METHODS:}

Between August 2016 to June 2018, 20 patients underwent aortic valve reconstruction as per the techniques described by Ozaki et al. The surgery was done for aortic stenosis as well as regurgitation or a combination of both. A comprehensive echocardiographic evaluation was done pre-discharge and at 6 months. The post-operative echocardiographic parameters that were evaluated were aortic valve gradient, aortic valve area, peak and mean velocity, left ventricular diameters, left ventricular mass regression, length of coaptation and presence or absence of leaflet flutter and buckling. Other post operative parameters were also evaluated. The results were compared to that of prosthetic valve replacement. All the parameters were measured by a single expert. Statistical analysis was done using Fischer exact test or by Mann-Whitney U test.

RESULTS:

No patients were converted to prosthetic valve replacement. Auto pericardial aortic valve reconstruction has several favorable results including better left ventricular mass regression and aortic valve gradient. None of the patients required anticoagulation.

CONCLUSION:

Autopericardial aortic valve reconstruction is a feasible alternative to prosthetic valve replacement with several advantages.

Variable morphologic features and results of surgery for Hemitruncus Shiv Kumar Choudhary; Sachin Talwar

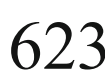

\section{ABSTRACT: \\ BACKGROUND:}

Hemitruncus, now appropriately renamed "Anomalous origin of one pulmonary artery branch from the aorta (AOPA)" is rare. We report our single-institution surgical experience with this condition.

\section{PATIENTS AND METHODS:}

Between January 1994-October 2018, 29 patients (age: Median 7 month, range 1 month-25 years) with AOPA underwent surgery. Twenty patients had anomalous origin of the right pulmonary artery (RPA) while 9 had anomalous origin of the left pulmonary artery (LPA) from the aorta. In patients with anomalous RPA, 14 patients had proximal type and 5 patients had distal type of AOPA. Seven patients had associated Tetralogy of Fallot (TOF), 1 patient had associated atrial septal defect and another 1 had a ventricular septal defect. In 23 patients, direct implantation into the main pulmonary artery was performed, while 6 patients required interposition of a graft (Dacron tube in 3, saphenous vein homograft in 1 and aortic homograft in 1). In 16, the procedure was without cardiopulmonary bypass $(\mathrm{CPB})$ and 13 required $\mathrm{CPB}$.

\section{RESULTS:}

There were 2 early deaths due to persistent hypoxemia in a 7-month old with TOF with anomalous LPA from aorta and another patient with AOPA and TOF had severe right ventricular dysfunction due to persistent pulmonary arterial hypertension. At a median follow-up of 36.5 months (range: 1-302 months), all 26 survivors were asymptomatic with no residual lesions. There were no late deaths or re-interventions.

\section{RESULTS:}

Repair of AOPA is associated with acceptable early and long-term results with good early \&amp; long-term survival without re-intervention.

New strategy for managing the first Blalock-Tausssig

Shunt in Infants With Patent Ductus Arteriosus

Dependent Circulation.

Naoki Masaki; Sadahiro Sai

ABSTRACT:

\section{OBJECTIVES:}

We evaluated the validity of our current strategies for perioperative management in the modified Blalock-Taussig shunt (mBTS) for patients with pulmonary atresia (PA) or critical pulmonary stenosis (PS) with patent ductus arteriosus (PDA) dependent circulation.

\section{METHODS:}

Our first step was dose reduction or discontinuation of lipo-prostaglandin E1 1 week preoperatively followed by nitrogen inhalation if necessary, targeting oxygen saturation ( $\mathrm{SpO} 2)$ of $70-80 \%$ to avoid cardiac failure by reducing preload. Second was administration of phosphodiesterase enzyme 3 inhibitor and nitroglycerin after anesthesia induction to stabilize $\mathrm{Qp} / \mathrm{Qs}$ by reducing afterload. Forty-two infants (PA 39 critical PS 8) who underwent the first mBTS between July 2008 and July 2018 were divided into two groups by strategy: conventional treatment (group C; n=20) and current strategies (group $\mathrm{S} ; \mathrm{n}=27$ ). Median age and body weight were 38 days (11-70) and $3.4 \mathrm{~kg}(2.4-4.0)$. Primary endpoints were postoperative excessive or low Qp events such as cardiopulmonary resuscitation, PDA ligation, shunt clipping, extracorporeal membrane oxygenation installation, and in-hospital mortality. Secondary outcomes were preoperative changes in brain natriuretic peptide (BNP) level, and postoperative peak lactate in each group.

\section{RESULTS:}

There were 2 deaths, 6 postoperative excessive Qp events and 2 low Qp events in group C, but no deaths, 1 excessive Qp and 3 low Qp events in group S. Preoperative SpO2 and BNP levels decreased within 1 week in group S: BNP, $468 \pm 451$ at 1 week preoperatively and $214 \pm 203 \mathrm{pg} / \mathrm{mL}$ intraoperatively (p\&lt;0.05). Intraoperative BNP was $344 \pm 617 \mathrm{pg} / \mathrm{mL}$ in group C. Peak lactate level differed significantly between the two groups: peak lactate $6.3 \pm 7.5 \mathrm{mmol} / \mathrm{L}$ in group $\mathrm{C}$; peak lactate $2.7 \pm 1.0 \mathrm{mmol} / \mathrm{L}$ (p\&lt;0.05) in group $\mathrm{S}$.

\section{CONCLUSIONS:}

These new strategies could reduce postoperative mortality and morbidity at the first mBTS in infants with PA or critical PS with PDA dependent circulation who are at risk of adverse events perioperatively.

Long-term Outcomes of Senning Procedure

Mariko Kobayashi; Toshihide Asou;

Sadamitsu Yanagi; Hideaki Ueda;

Seiyo Yasui; Yasunori Ohkawa

\section{ABSTRACT:}

\section{BACKGROUND:}

Long-term outcomes after the Senning procedure remain unclear. We reviewed the surgical outcomes of the Senning procedure and conducted a questionnaire survey with 36-item Short-Form (SF36) to survivors in order to clarify the current patients' quality of life (QOL).

\section{METHODS:}

Twenty consecutive patients underwent the Senning procedure performed by one surgeon between 1984 and 1988. Cardiac anomalies were transposition of great arteries (TGA (I)) in 15 patients, TGA (II) in 4, and criss- 
cross heart in 1 . The median follow-up period was 31.1 years ( 1 day 34.5 years).

\section{RESULTS:}

There were one operative death with right ventricular failure (RVF) and four late term deaths with RVF, pneumonia, and arrhythmia. The survival rate was $73.2 \pm 10.3 \%$ at 30 years. There were no reoperations for baffle stenosis. Freedom from pacemaker implantation was $65.3 \pm 12.6 \%$ at 30 years. Trace $\sim$ trivial tricuspid valve regurgitation (TR) were found in 7 , mild TR in 8 , and no moderate TR at $19.9 \pm 7.2$ years. Eight patients $(80 \%)$ replied to the questionnaire survey reporting that three women had a baby and three patients had taken one medication. In the results of the SF-36, the physiological, mental, and social QOL were not significantly different from age adjusted norms.

\section{CONCLUSION:}

In the 30-year follow-up, the outcomes of the Senning procedure were favorable in terms of morbidity, functional status and social integration with a similar score of norms in QOL and low incidence of reoperation, RVF, and TR, while the rate of PMI was relatively high. Careful observation for arrhythmia is mandatory in the long-term follow-up.

Rumors, Truth And Reality:

A Retrospective Study On Coronary Endarterectomy. Asit Baran Adhikary

Redoy Ranjan

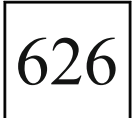

\section{ABSTRACT: \\ BACKGROUND:}

This retrospective study observed the consequences, survival and graft patency rate following coronary endarterectomy technique for diffuse coronary artery disease in a single surgeon's practice. METHODS:

Total 1138 patients were evaluated for 1403 endarterectomized coronary artery with coronary artery bypass graft surgery in the year of January 2005 to December 2017. Mean LV ejection fraction was $51 \%$, and about $66 \%$ of patients were at CCS Class (Canadian Cardiovascular Society) 3 or 4 . An angiographic evaluation was performed at 1 year following surgery.

\section{RESULTS:}

About two-third endarterectomy was performed on left coronary territory, most commonly (43.05\%) in LAD artery. The mean age of the study population was $61 \pm 2.5$ years, and OPCABG was performed in $88.05 \%$ study population. However, mean arteriotomy length was $9.5 \pm 5.5 \mathrm{~mm}$, and post-operative ICU mortality rate was only $1.6 \%$. Moreover, new onset of atrial fibrillation and perioperative myocardial infarction rate was only $14.7 \%$, and $3.2 \%$ respectively. Furthermore, freedom from all-cause mortality was $98.1 \%$ and $88.9 \%$ at 1 year and 5 years follow up respectively, and angina free survival rate was $87.5 \%$ at median follow-up of 5 years. CT angiography evaluation demonstrates graft patency rate was $94.8 \%$ at 1 year follow up.

\section{CONCLUSION:}

In diffuse coronary artery disease, concurrent coronary endarterectomy with $\mathrm{CABG}$ surgery triumph in favorable myocardial revascularization, and satisfactory graft patency rate.

\section{Is biventricular support necessary}

\section{for advanced heart failure?}

Kan Nawata; Osamu Kinoshita; Haruo Yamauchi; Shogo Shimada; Mitsutoshi Kimura; Masahiko Ando; Yoshifumi Itoda; Hyoe Komae; Yasuhiro Hoshino Minoru Ono
Abstract:

OBJECTIVE:

Biventricular assist device (BVAD) is reported to be used in 10$15 \%$ of VAD treatment of advanced heart failure. Our strategy is to stabilize INTERMACS profile 1 or unstable profile 2 patients by implanting paracorporeal VAD, central ECMO or IABP before proceeding to continuous-flow VAD (cf-VAD) implantation. We examined validity of this strategy by analyzing BVAD necessity and patient survival.

\section{PATIENTS AND METHODS:}

Consecutive 161 patients (39 females, 41 years of age) were implanted with cf-VAD between 2007 and 2018 for bridge to transplantation. DCM was seen in 110 , ICM in 13 , dHCM in 12 , post-myocarditis CM in 6 , ARVC in 3, RCM in 3 and others in 14.127 patients underwent primary cf-LVAD implantation. Pre-implant profiles of those patients were 2 in 62,3 in 59 and 4 in 6.34 patients were converted from other type of mechanical circulatory support (MCS). Actuarial survival was calculated by Kaplan-Meier analysis and comparisons were made using Log-rank method.

RESULTS:

No patient required permanent/temporary BVAD. 34 (83\%) out of 41 patients who were in severe pre-implant condition were successfully converted to cf-LVAD. Average support duration was 767 days. During follow-up 55 patients reached heart transplantation, 8 weaned from VAD due to recovery and 78 had on-going support. 1-, 2-, 3-year survival of 161 patients was $92.5 \%, 89.1 \%$ and $85.4 \%$. There were no statistical differences between subgroups, such as sex, pre-implant profiles, body size and primary/redo sternotomy.

\section{CONCLUSIONS:}

We successfully implanted cf-LVAD without even temporary RVAD support in consecutive 161 patients including those who were severely ill in pre-implant stage. Our strategy for stabilizing profile 1 and unstable profile 2 patients by other types of MCS seemed to be safe and effective.

Anomalous Origin of Right Coronary Artery from Pulmonary Artery: Rare yet Serious Jlanwen Liang; Dianyuan Li

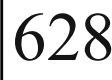

ABSTRACT:

OBJECTIVE:

Anomalous origin of the right coronary artery from the pulmonary artery (ARCAPA) is a rare yet severe congenital coronary artery anomaly. The study aims to summarize the main characteristics of ARCAPA for clinical vigilance and identification.

\section{METHODS:}

The study enrolled 16 ARCPA patients from Beijing International Hospital and The Eighth Affiliated Hospital of Sun Yat-sen University during February 1998 and April 2018. The demographics, clinical presentations, physical examination findings, surgical approach and follow-up of the subjects were analyzed.

\section{RESULTS:}

During the recent 20 years, 16 patients were identified ARCAPA and successfully performed surgery. 9 out of 16 ARCAPA (56.3\%) were male, ranging from 0.3 to 59 , three cases were diagnosed in infants $\leq 1$ year, 8 were diagnosed in children $\leq 18$ years of age, 2 were adults \&gt; 50 years of age. However, male tended to be identified earlier than female $[(10.9 \pm 15.9)$ vs. $(26.6 \pm 23.2), \mathrm{P}=0.07] .30 \%$ presented with recurrent pneumonia, $20 \%$ with symptoms associated with other cardiac defect, $10 \%$ with angina, $20 \%$ first with cardiac murmur and the rest were asymptomatic. Moreover, 6 were found complicated by other cardiac defect, including aortopulmonary window, atrial/ventricular septal defect, patent ductus arteriosus and mitral regurgitation. No pulmonary hypertension was found by right-heart 
catheterization and all subjects were successfully performed surgery for reimplantation of the anomalous RCA to an anterior aortic, meanwhile, associated cardiac defect was fixed. The left ventricular ejection fraction (LVEF) remain normal before and after surgery $[(56.9 \pm 12.1) \%$ vs. $(60.1 \pm 11.7) \%, \mathrm{P}=0.46]$, postoperative LVID declined significantly compared with preoperative [(41.9 \pm 9.4$) \mathrm{mm}$ vs. $(38.6 \pm 9.3), \mathrm{P}=0.036]$. The average ward day was $8.38 \pm 2.8$, the mean follow-up was $67.7 \pm 48.9$ months and no cardiac death happen.
CONCLUSION:

ARCAPA is a rare congenital coronary anomaly. Surgical reimplantation is the effective treatment and should be considered as soon as possible for this potentially fatal condition.

Publisher's note Springer Nature remains neutral with regard to jurisdictional claims in published maps and institutional affiliations. 